WHOI-92-16

1991

Copy 2

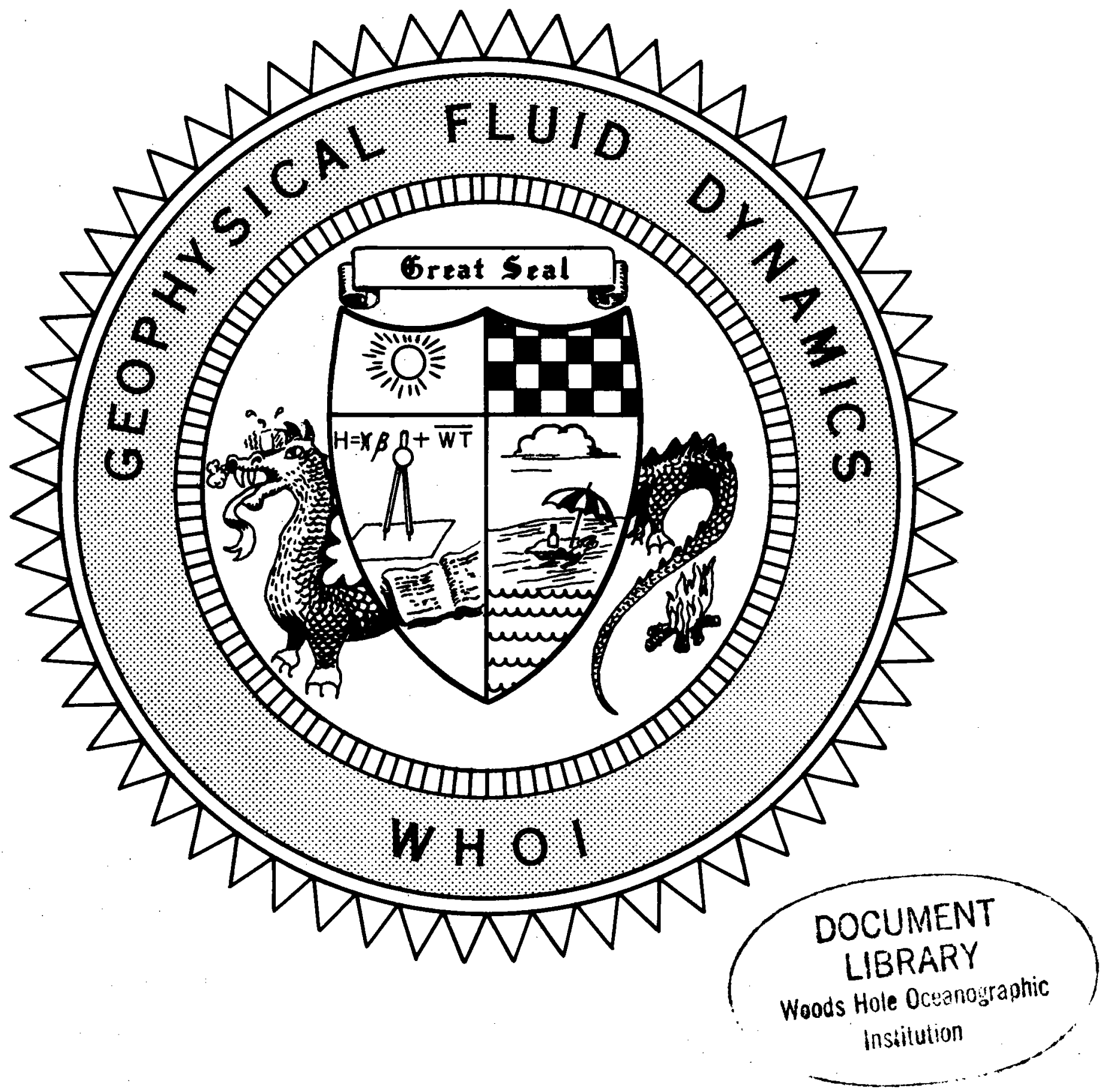

Course Lectures Abstracts of Participants Fellows Project Reports 


\title{
1991 Summer Study Program \\ in \\ Geophysical Fluid Dynamics \\ Patterns in Fluid Flow
}

\author{
Bill Young, Director \\ Stephan Fauve, Principal Lecturer \\ Barbara Ewing DeRemer, Administrator \\ Steve Meacham, Editor \\ Woods Hole Oceanographic Institution \\ Woods Hole, Massachusetts 02543
}

September 1991

\section{Technical Report}

Funding was provided by the National Science Foundation through Grant No. OCE 8901012.

Reproduction in whole or in part is permitted for any purpose of the United States Government. This report should be cited as:

Woods Hole Oceanog. Inst. Tech. Rept., WHOI-92-16.

Approved for publication; distribution unlimited.

Approved for Distribution:

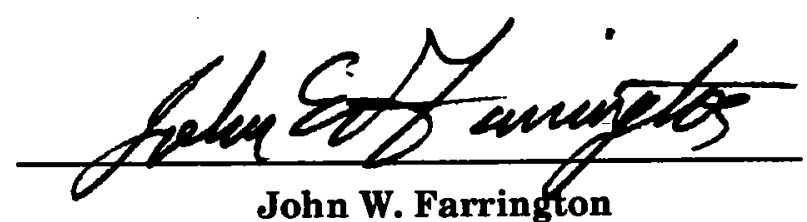

Associate Director for Education

Dean of Graduate Studies 


\section{Preface}

The GFD program in 1991 focused on pattern forming processes in physics and geophysics. The principal lecturer, Stephan Fauve, discussed a variety of systems, including our old favorite, Rayleigh-Bénard convection, but passing on to exotic examples such as vertically vibrated granular layers. Fauve's lectures emphasize a unified theoretical viewpoint based on symmetry arguments. Patterns produced by instabilities can be described by amplitude equations, whose form can be deduced by symmetry arguments, rather than the asymptotic expansions that have been the staple of past Summer GFD Programs. The amplitude equations are far simpler than the complete equations of motion, and symmetry arguments are easier than asymptotic expansions. Symmetry arguments also explain why diverse systems are often described by the same amplitude equation. Even for granular layers, where there is not a universally accepted continuum description, the appropriate amplitude equation can often be found using symmetry arguments and then compared with experiment.

Our second speaker, Daniel Rothman, surveyed the state of the art in lattice gas computations. His lectures illustrate the great utility of these methods in simulating the flow of complex multiphase fluids, particularly at low Reynolds numbers. The lattice gas simulations reveal a complicated phenomenology much of which awaits analytic exploration.

The fellowship lectures cover broad ground and reflect the interests of the staff members associated with the program. They range from the formation of sand dunes, through the theory of lattice gases, and on to two dimensional-turbulence and convection on planetary scales. Readers desiring to quote from these reports should seek the permission of the authors (a partial list of electronic mail addresses is included on page $\mathrm{v}$ ). As in previous years, these reports are extensively reworked for publication or appear as chapters in doctoral theses. The task of assembling the volume in 1991 was at first facilitated by our newly acquired computers, only to be complicated by hurricane Bob which severed electric power to Walsh Cottage in the final hectic days of the Summer. 
Our program was graced with the presence of Henry Stommel who spent many hours on the Walsh cottage porch discussing thermohaline regulators, scientific biography and the theory of billiards. His close association with Walsh Cottage, as a principal lecturer, enthusiastic participant and a founder of the program, began in 1958. He designed our enduring logo, with its great seal and convective dragon. His good sense is illustrated by the one position he never assumed: that of director. With his recent death this winter past, Henry bequeaths us the memory of a convivial spirit in service of a free-ranging intellectual curiosity which marks the GFD program at its finest.

We are indebted to the Ocean Sciences Division of the National Science Foundation and to the Office of Naval Research for their financial support of the program. The hospitality of the Woods Hole Oceanographic Institution is appreciated. The Woods Hole Education Office, particularly Barbara Ewing DeRemer and Jake Peirson, were responsible for the smooth operation of our administration.

\section{W.R. Young}

February, 1992 
I PARTICIPANTS $\ldots \ldots \ldots \ldots \ldots \ldots \ldots \ldots \ldots \ldots \ldots \ldots \ldots \ldots \ldots \ldots \ldots, \quad \mathbf{v}$

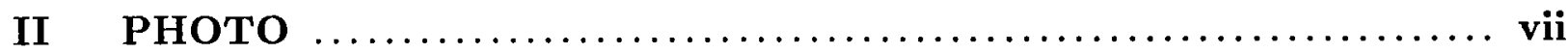

III PRINCIPAL LECTURES - Stephan Fauve

1. Pattern Forming Instabilities $\ldots \ldots \ldots \ldots \ldots \ldots \ldots \ldots \ldots \ldots \ldots \ldots \ldots, 2$

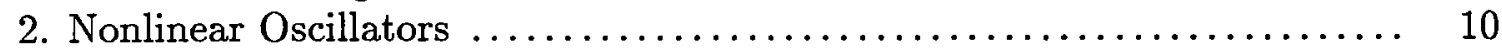

3. Nonlinear Waves in Dispersive Media ........................ 26

4. Cellular Instabilities: A canonical example, Rayleigh-Bénard convection . 39

5. Amplitude Equations in Dissipative Systems ................... 54

6. Secondary Instabilities of Cellular Flows: Eckhaus and zig-zag instabilities 68

7. Drift Instabilities of Cellular Patterns . . . . . . . . . . . . . . . . . . . . 86

8. Nonlinear Localized Structures $\ldots \ldots \ldots \ldots \ldots \ldots \ldots \ldots \ldots \ldots \ldots \ldots . . . \ldots \ldots$

IV SPECIAL LECTURES - Dan Rothman

Simple Models of Complex Fluids $\ldots \ldots \ldots \ldots \ldots \ldots \ldots \ldots \ldots \ldots \ldots \ldots . \ldots \ldots$

V PAPERS

When Rayleigh-Bénard meets zebra-leopard

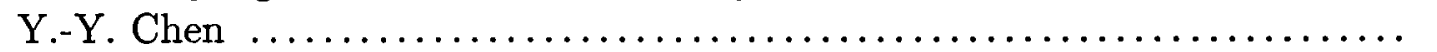

The Phase-Diffusion Mean Drift Equations for Natural Convection Patterns

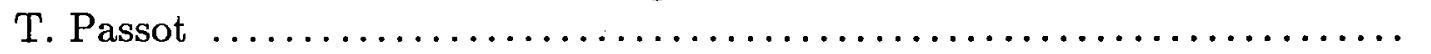

Slow methods for simple people

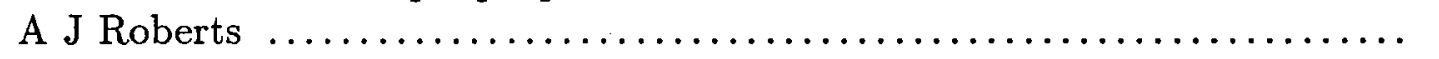

Rational mathematical modelling: shear dispersion and planform selection

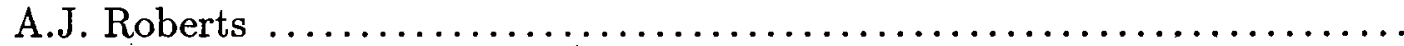

Fundamentally discrete patterns in geomorphology

B. Werner

\section{FELLOWS REPORTS}

Surface tension in immiscible lattice gases

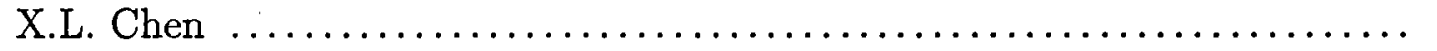

A continuum model for Aeolian sand ripples

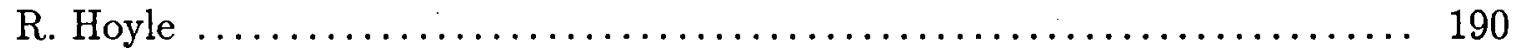

Convection patterns at zero Prandtl number

K. Kumar ........................................... 203

Ray theory of water waves on a current

P.A. Milewski 
A simple model of pressure effects on mush growth

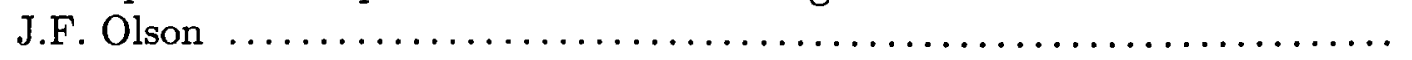

Boussinesq convection in the equatorial region of a rotating spherical shell

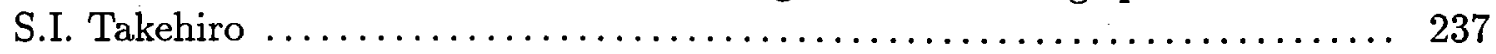

Forced vortex merger

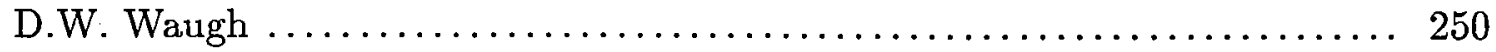

Primitive equation models of Gulf Stream meanders

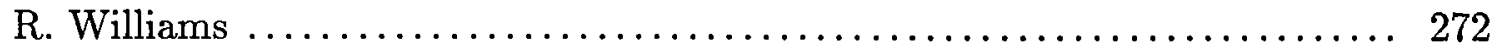

Shear dispersion and thermal reconnection

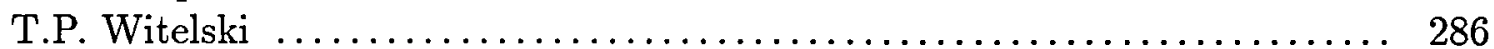

\section{ABSTRACTS OF SEMINARS}

Almost symmetric solitary eddies in two-layer models

G.G. Sutyrin and W.K. Dewar ............................ 306

Applications of contour dynamics to eddies

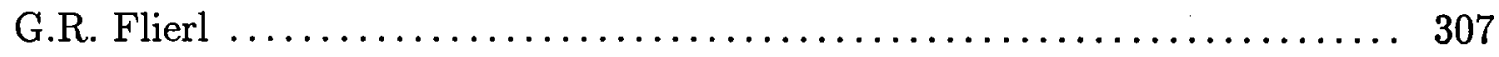

Surface tension

J. Keller .............................................. 310

Molecular dynamics of interfaces and spreading drops

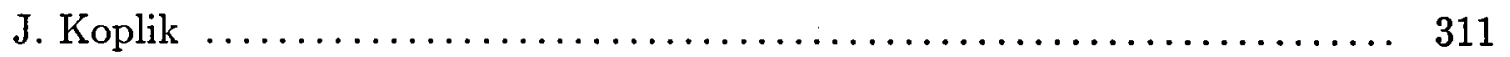

Influence of periodic and stochastic modulation on transcritical and flip

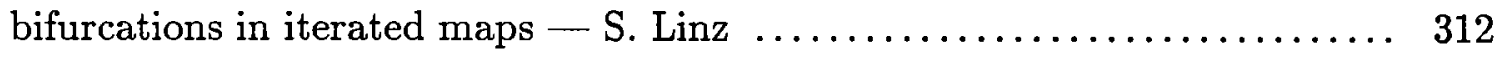

Three-dimensional, quasigeostrophic vortices

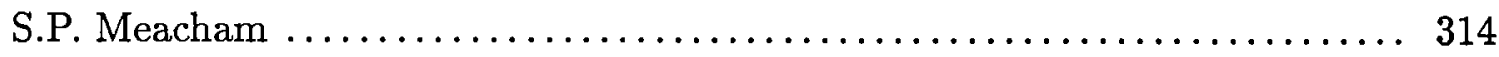

The merger of three-dimensional, quasigeostrophic vortices

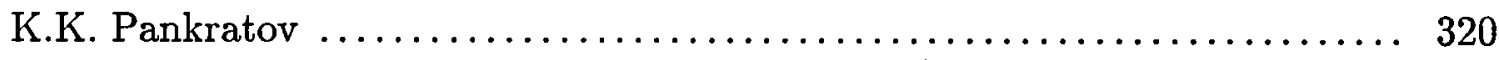

Development of axisymmetric aingular solutions to the incompressible 3-D Euler

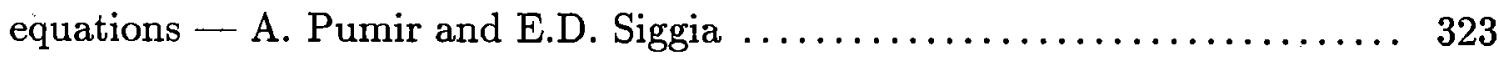

Zero Prandtl number convection

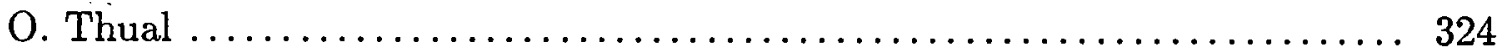

Instability, finite-amplitude and unsteady salt fingers

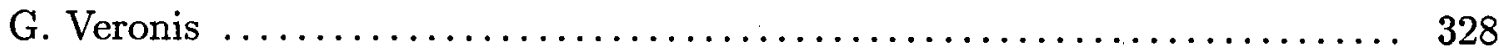

The efficiency of vortex merger

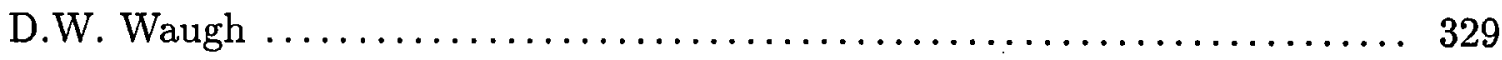

Two-dimensional decaying structured turbulence

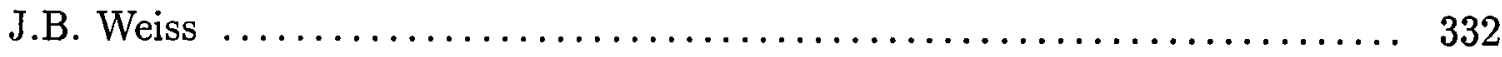

Hydraulics of stratified and planetary flows

A.W. Woods $\ldots \ldots \ldots \ldots \ldots \ldots \ldots \ldots \ldots \ldots \ldots \ldots \ldots \ldots \ldots \ldots \ldots \ldots \ldots, 334$ 


\section{Participants}

\begin{tabular}{|c|c|c|c|}
\hline The Fellows & Country & Institution & e-mail address \\
\hline Xiao-Liang Chen & P.R.C. & Cornell University & xchen@ionvax.tn.cornell.edu \\
\hline Rebecca Bryony Hoyle & U.K. & University of Cambridge & $r b h 11 @ p h x . c a m . a c . u k$ \\
\hline Krishna Kumar & India & Ecole Norm. Superieure de Lyon & \\
\hline Paul Antoine Milewski & France & M.I.T. & brasil@math.mit.edu \\
\hline Shin-Ichi Takehiro & Japan & University of Tokyo & takepiro@gpnws.geoph.s.u-tokyo.ac.jp \\
\hline Darryn W. Waugh & N.Z. & University of Cambridge & dw@atm.amtp.cam.ac.uk \\
\hline Thomas Peter Witelski & U.S. & Cooper Union & witelski@cco.caltech.edu \\
\hline Richard James Williams & U.K. & U. Cal/Scripps & rwilliams@ssurf.ucsd.edu \\
\hline John Forest Olson & Canada & M.I.T. & john@segovia.mit.edu \\
\hline \multicolumn{4}{|l|}{ The Staff and Visitors } \\
\hline James L. Anderson & US & Stevens Inst. Tech & \\
\hline Antoine Badan-Dangon & Mexico & CICESE & \\
\hline Axel Brandenberg & Germany & Nordita & \\
\hline Paola Cessi & Italy & U. Cal/Scripps & cessi@dalek.ucsd.edu \\
\hline Y. Y. Chen & Taiwan & Cal Tech & yyc@styx.caltech.edu \\
\hline William K. Dewar & U.S. & Florida State University & bill@ocean.ocean.fsu.edu \\
\hline Stephan Fauve & France & Ecole Norm. Superieure de Lyon & \\
\hline Glenn Flierl & U.S. & M.I.T. & glenn@pimms.mit.edu \\
\hline Glenn R. Ierley & U.S. & Michigan Tech University & grierley@mtus5.cts.mtu.edu \\
\hline Richard A. Jarvis & U.K. & Yale University & jarvis\%climat@venus.ycc.yale.edu \\
\hline Richard Jennings & U.K. & University of Cambridge & \\
\hline Keith Julien & U.K. & University of Colorado at Boulder & \\
\hline Vladimir Kamenkovich & USSR & M.I.T. & v.kamenkovich@omnet.bitnet \\
\hline Joseph Keller & U.S. & Stanford University & \\
\hline
\end{tabular}




$$
\text { vi }
$$

\begin{tabular}{|c|c|c|c|}
\hline Joel Koplik & U.S. & The Levich Institute/CCNY & \\
\hline Stefan Linz & Germany & Northwestern University & linz@casbah.acns.nwu.edu \\
\hline Willem Malkus & U.S. & M.I.T. & wvrm@math.mit.edu \\
\hline Josep Maria Massaguer & Spain & University Politecnica & \\
\hline Sean McNamara & US & U Cal/Scripps & \\
\hline Stephen Meacham & U.K. & M.I.T. & meach@tri.ocean.fsu.edu \\
\hline Thierry Passot & France & University of Arizona & \\
\hline Michael R.E. Proctor & U.K. & University of Cambridge & \\
\hline Alain Pumir & France & Cornell University & \\
\hline A.J. Roberts & Aust & University of Adelaide & aroberts@spam.ua.oz.au \\
\hline Claes G. Rooth & Sweden & University of Miami & \\
\hline Daniel H. Rothman & U.S. & M.I.T. & dan@segovia.mit.edu \\
\hline Rick Salmon & U.S. & U Cal/Scripps & \\
\hline Edward A. Spiegel & U.S. & Columbia University & eas@cuphyd.phys.columbia.edu \\
\hline Melvin Stern & U.S. & Florida State University & \\
\hline Olivier Thual & France & N.C.A.R. & thual@ncar.ucar.edu \\
\hline George Veronis & U.S. & Yale University & \\
\hline J. B. Weiss & U.S. & N.C.A.R. & jweiss@ncar.ucar.edu \\
\hline Brad Werner & U.S. & U Cal/Scripps & werner@coast.ucsd.edu \\
\hline Andrew Woods & U.K. & University of Cambridge & $a w w 1 @ p h x . c a m . a c . u k$ \\
\hline William Young (Dir) & Aust & U Cal/Scripps & bill@dalek.ucsd.edu \\
\hline Stephane Zaleski & France & Universite de Paris & \\
\hline Gianluigi Zanetti & Italy & Princeton University & \\
\hline
\end{tabular}




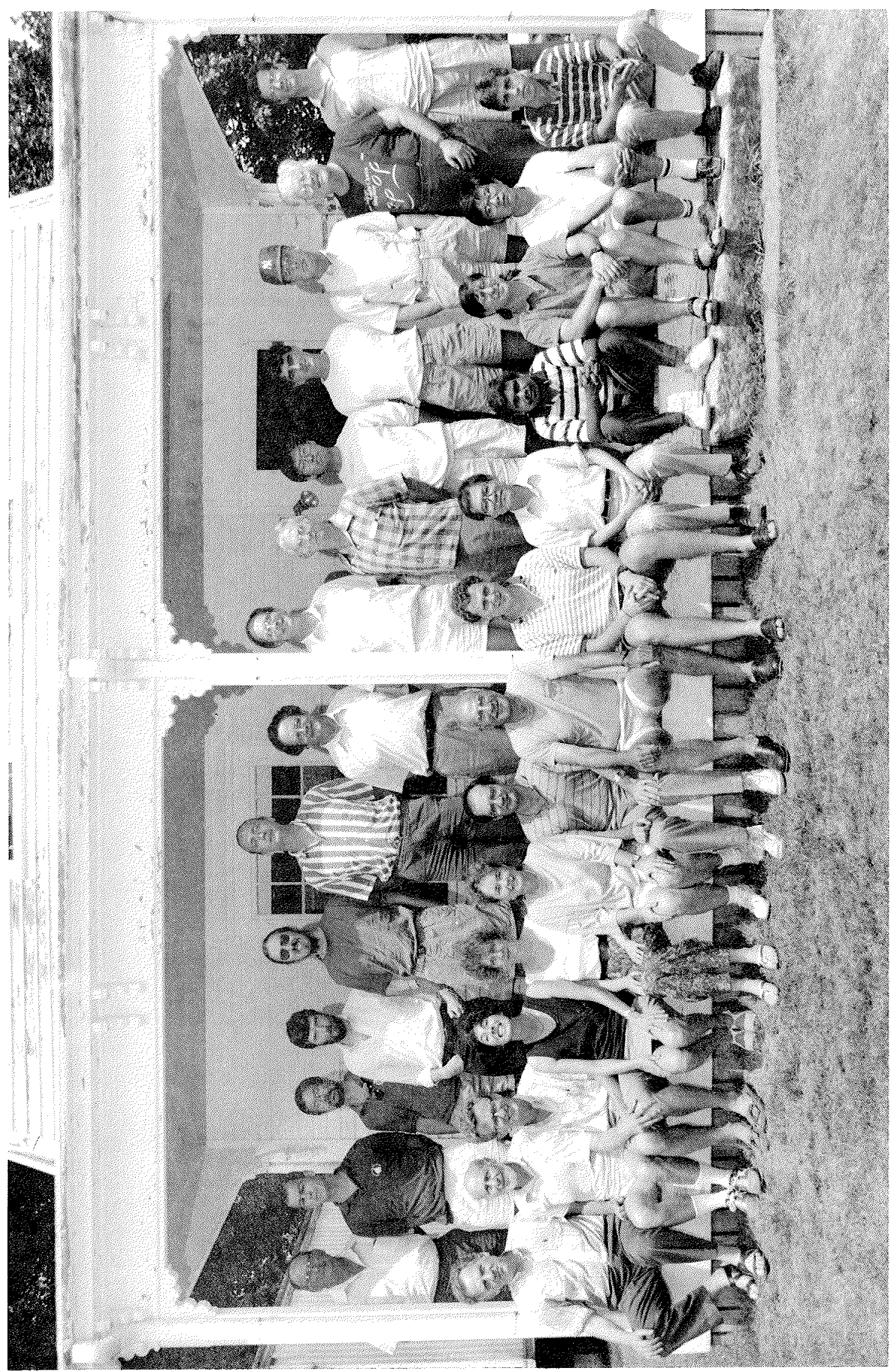

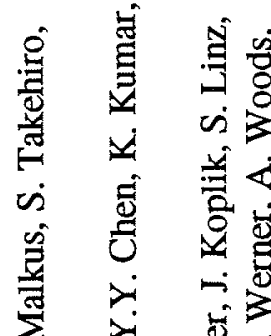

$>\quad$ 导

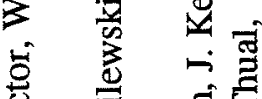

ह

(x)

¿ 总 की

咅 $>$

ல 0 递

㐘 ญ

焉证

$\therefore$ क त

总产离

- 0 棺

๑ 击

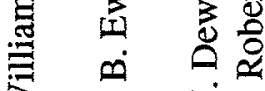

3 के

$\approx$ त्र

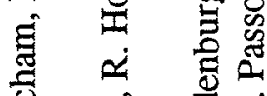

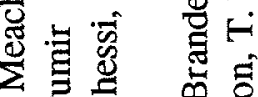

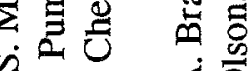

काष

节

纪 ن它是

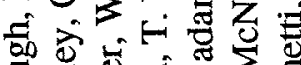
密武的

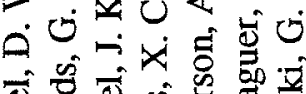

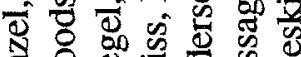

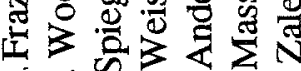

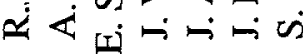

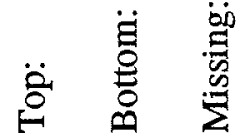





\section{Principal Lectures}




\section{Pattern Forming Instabilities}

Instabilities in nonlinear systems driven far from equilibrium often consist of a transition from a motionless state to one varying periodically in space or time. Various examples, widely studied in the past years, are, Rayleigh-Benard convection, Couette-Taylor flow, waves in shear flows, instabilities of liquid crystals, oscillatory chemical reactions, ... The appearance of periodic structures in these systems driven externally by a forcing homogeneous in space or constant in time, corresponds to a bifurcation, characterized by one or several modes that become unstable as a control parameter is varied. Linear stability analysis of the basic state gives the critical value of the control parameter for the primary instability onset, the nature of the most unstable modes and their growth rate above criticality. Many examples have been studied for a long time, and can be found for instance in the books of Chandrasekhar (1961) or Drazin and Reid (1981). However, linear stability analysis does not describe the saturation mechanism of the primary instability, and thus a nonlinear analysis should be performed to determine the selected pattern, its dynamics and in particular the secondary instabilities that occur as the control parameter is increased above criticality. Before considering these problems, we present some examples of the characteristic phenomena that occur above a pattern-forming instability onset.

\subsection{Example: The Faraday instability}

As a first example, consider a cylindrical vessel containing a liquid and its vapor (or any other gas), vertically vibrated at frequency $\omega_{e}$ (Figure 1.1).

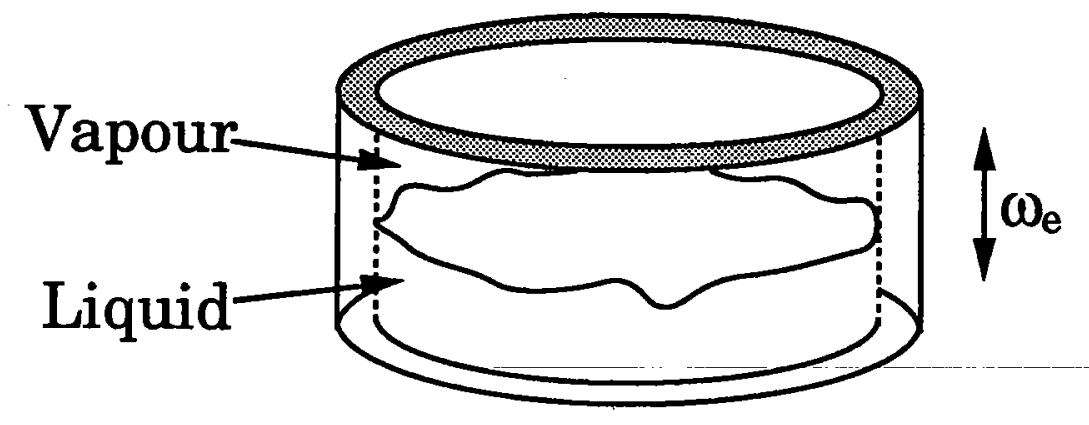

Figure 1.1. Sketch of the apparatus for the Faraday instability. 

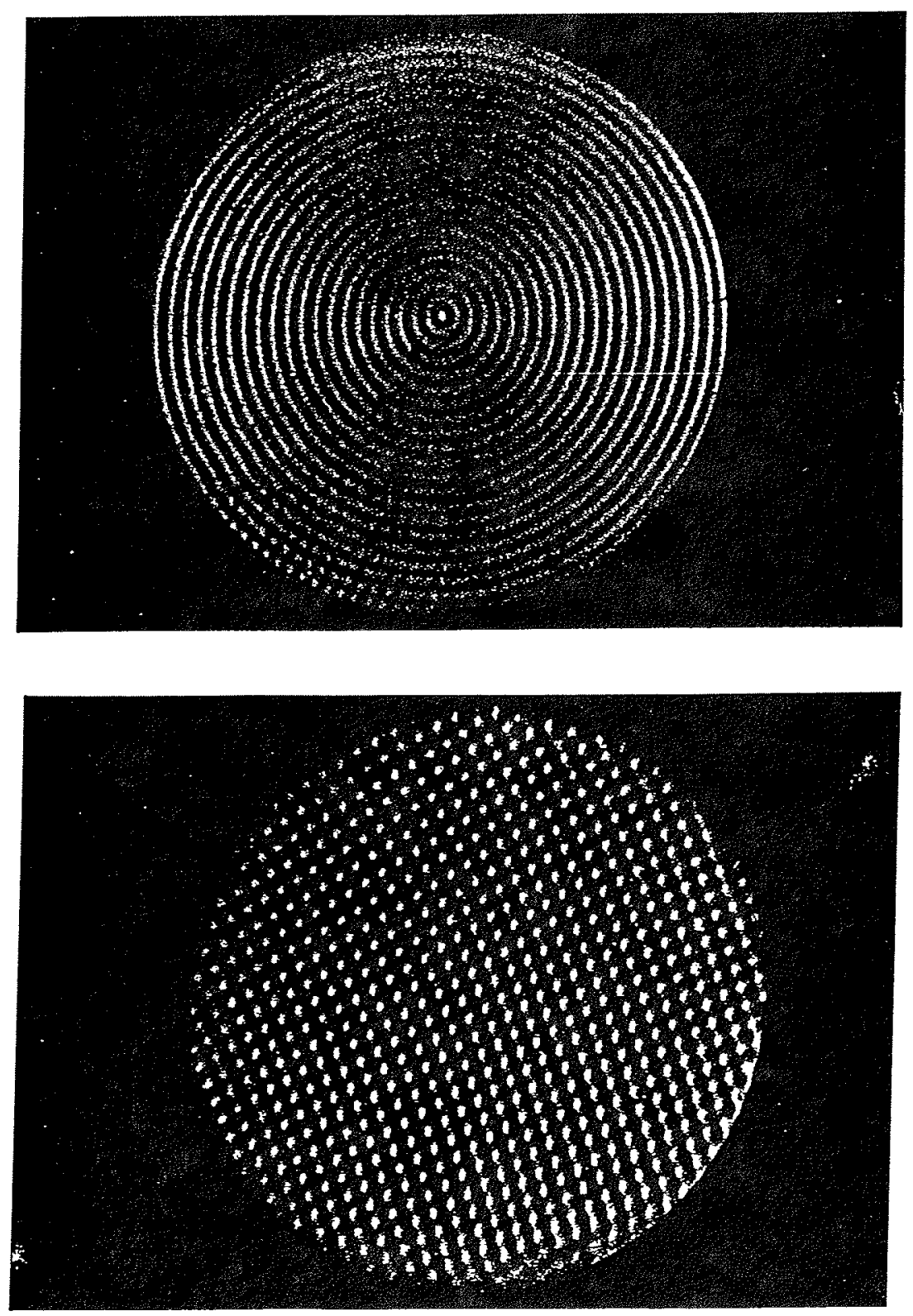

Figure 1.2. (a) Concentric circular standing waves at the interface between liquid $\mathrm{CO}_{2}$ and its vapor submitted to vertical vibrations. This pattern is only observed as a transient regime at instability onset; it is unstable to modulations perpendicular to the wave crests (already apparent) and the final stable stationary standing wave pattern is the square array (b).

It was discovered by Faraday (1831) that when the vibration amplitude exceeds a critical value, the flat liquid-gas interface becomes unstable to standing waves. Figures 1.2 a and 1.2 b display the surface waves excited by vertical shaking, seen from above. The axisymmetric standing wave of Figure $1.2 \mathrm{a}$ is observed as a transient at instability onset; the shape of this mode is due to the circular geometry of the vessel. This is the most unstable linear eigenmode of the flat interface. However, this axisymmetric pattern is not nonlinearly stable; in a large 
enough container, nonlinear interactions select the square pattern of Figure $1.2 \mathrm{~b}$, which is the stable stationary state just above the instability onset. Thus, from this first example, one observes that the patterns ultimately selected by the instability do not generally correspond to the most unstable linear mode. Here, nonlinear effects are strong enough to overcome the boundary effects that trigger the axisymmetric pattern.

We can think of the square pattern as the result of two sets of counter-propagating waves, perpendicular to each other and of equal amplitudes. When we have a liquid-vapor system, we can approach the critical point at which the liquid-vapor interface disappears. Near this critical point, the nonlinear interaction between the intersecting waves changes so that they cannot both remain stable, and one set vanishes. We then get a one-dimensional standing wave pattern (Figure 1.3). Thus nonlinear effects not only saturate the growth of the linearly unstable modes but they also act as a selection mechanism for the pattern.

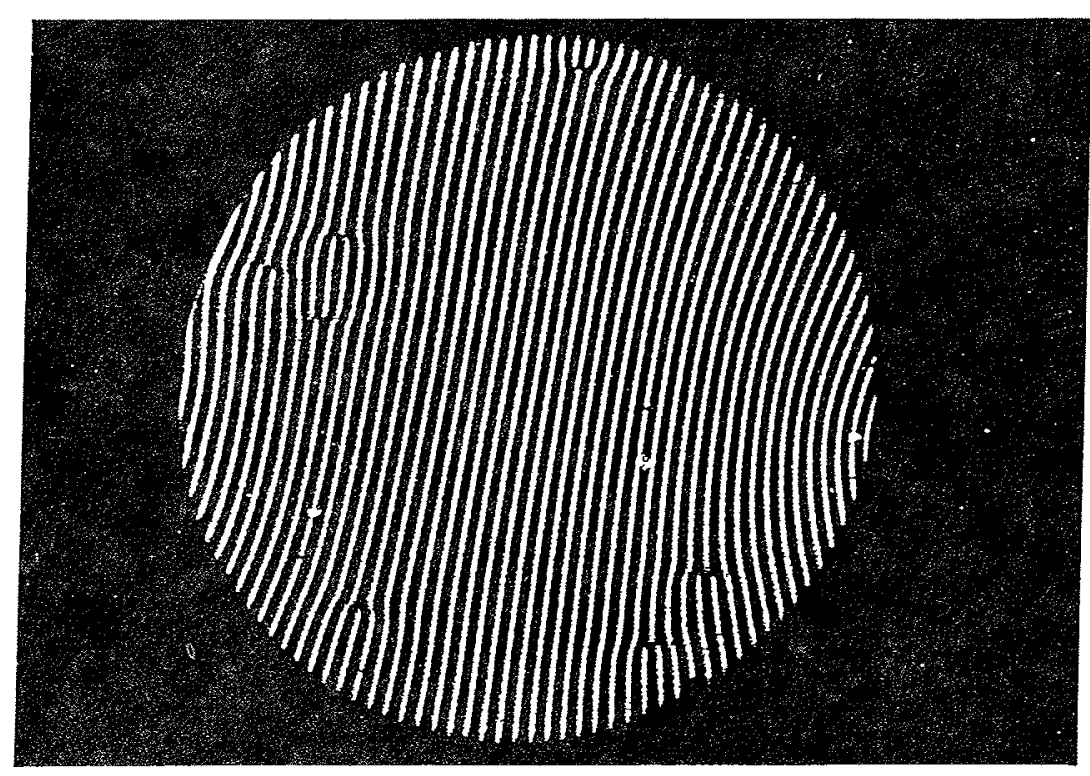

Figure 1.3. Near the critical liquid-vapor point of $\mathrm{CO}_{2}$, only one of the perpendicular sets of waves can remain. Note the defects in the wave pattern.

In Figure 1.3 we see that some of the wave-crests do not reach all the way across the vessel. The endpoint of a crest is called a defect. If one integrates the phase-gradient along a closed curve around one of these defects, the integral comes to $\pm 2 \pi$ instead of 0 , because one passes more waves on one side of the defect than on the other. At the defect itself, the amplitude of the wave vanishes, so that at this point the phase is undefined. Defects are widely observed when a periodic pattern undergoes a secondary instability. In the above example, they nucleate or annihilate by pair or at the lateral boundary and during their lifetime move in the underlying periodic pattern. Their dynamics plays an important rôle in the transition to spatiotemporal disorder. Their shape traces back to the symmetries of the pattern. For instance, defects of the square standing wave pattern consist of lines instead of points; they separate two regions of the wave-field that oscillate out of phase (Figure 1.4). 


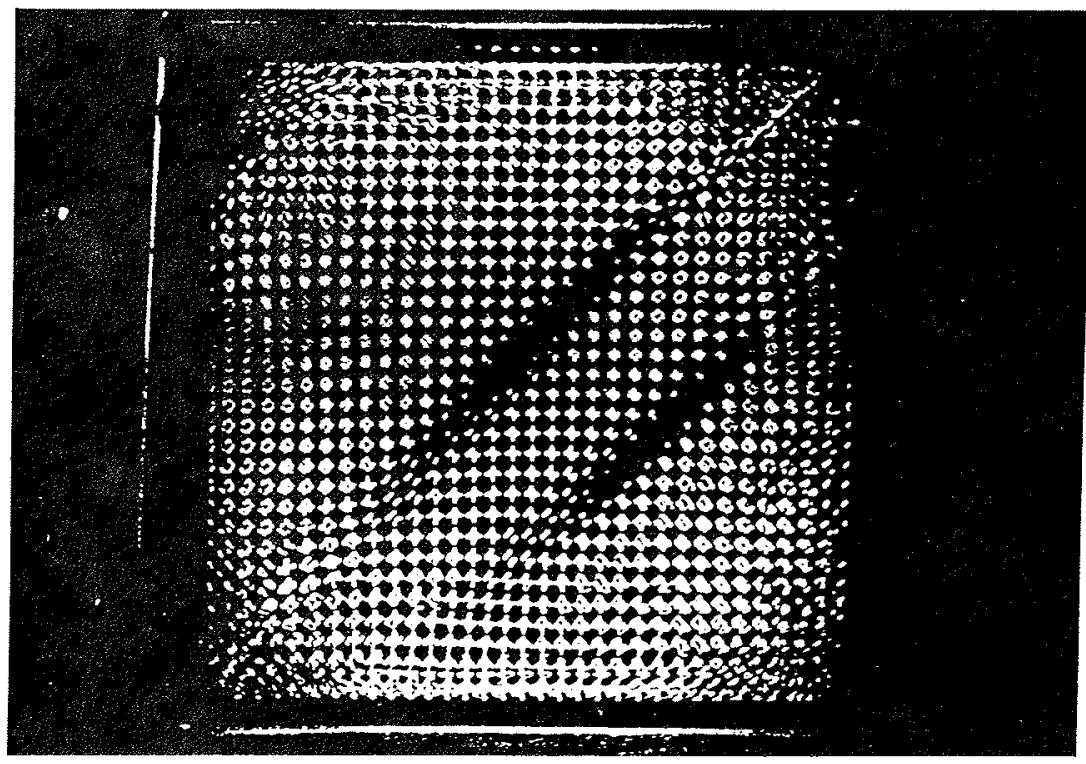

Figure 1.4. Line-defects in surface waves on vertically-shaken mercury. The regions on opposite sides of the lines are out of phase with one another.

Secondary instabilities of periodic patterns do not always generate defects. An important class of secondary instabilities consists of long-wavelength modulations of the primary pattern. The surface wave pattern in an elongated rectangular geometry or in an annular container, i.e. when a one-dimensional wave is forced by the boundary conditions, exhibits a secondary instability consisting of a long wavelength spatiotemporal modulation of the primary pattern wavelength (Figure 1.5).

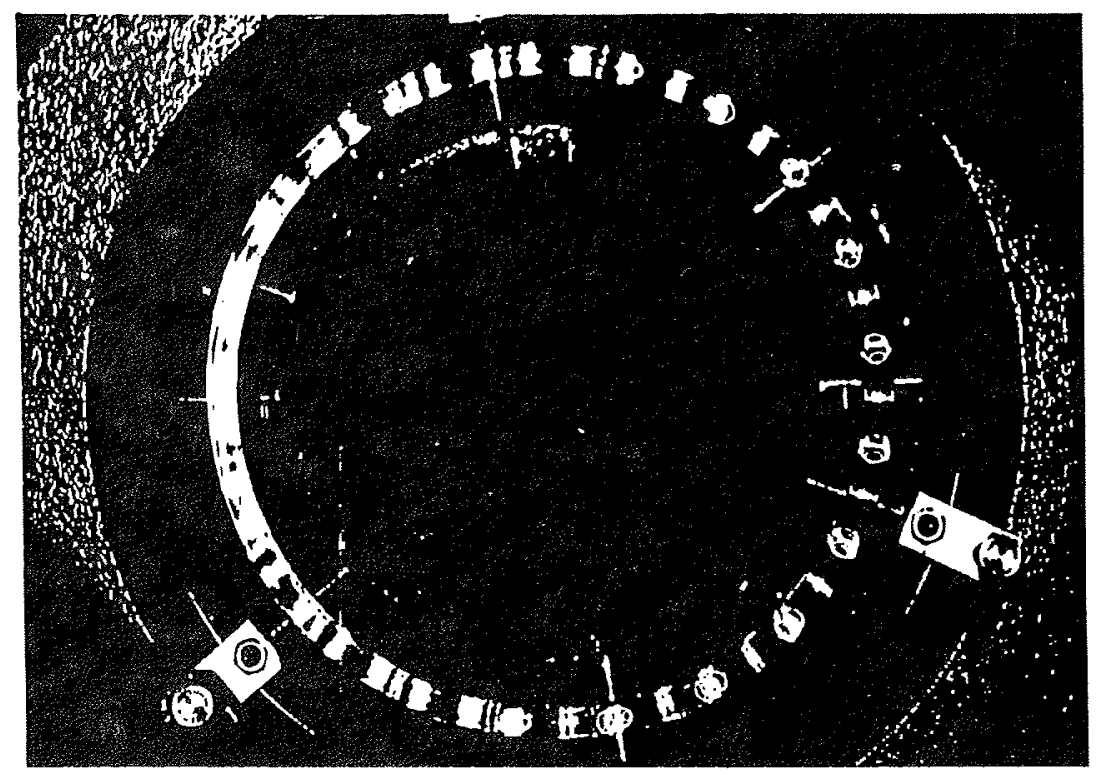

Figure 1.5. Snapshot of the long wavelength modulation of the basic standing wave in an annular geometry. The basic standing wave consists of 21 wavelengths and the wavelength of the modulation is equal to the perimeter of the annulus. 


\subsection{Analogy with phase-transitions: amplitude equations}

The different phenomena described above are not particular to parametrically generated surface waves; long-wavelength instabilities or defect-dynamics are widely observed above the onset of most pattern-forming instabilities (Wesfreid and Zaleski, 1984 ; Wesfreid et al., 1988). An obvious unifying description consists of looking for evolution equations for the amplitude and the phase, i.e. for the complex amplitude of the periodic pattern generated by the primary instability. Indeed, at the primary instability onset, the critical modes have, by definition, a vanishing growth rate; we will show that adiabatic elimination of all the other (faster) modes leads to nonlinear partial differential equations that govern the amplitude of the critical modes, and so describes the slow modulations in space or time of the periodic structure envelope: these are the amplitude equations. We will see that the form of the amplitude equations can be derived simply from symmetry considerations, and that the underlying detailed equations are needed only to evaluate parameters in the amplitude equations. Another possible way is to use experiments to determine the parameters in an amplitude equation, because we can deduce the form of the equation without knowing all the details of the microscopic dynamics. This is similar to the description of a fluid flow using Navier-Stokes equation the form of which traces back to conservation laws; the coefficients, viscosity for instance, depend on the microscopic dynamics and might be computed using the Boltzmann equation; it is however simpler and more reliable to use the experimentally measured coefficients.

We will mainly be studying amplitude equations, rather than the microscopic governing equations of the underlying systems. We take this approach because similar patterns are observed in a wide range of systems, and their behaviour is a result of the broken symmetries at the primary instability onset, rather than that of the microscopic dynamics. Systems with different microscopic description frequently exhibit, on a macroscopic level, similar patterns which are governed by the same amplitude equation. The situation is analogous to the one we encounter in phase-transitions in condensed-matter physics where the behaviour of the order parameter is governed by symmetries and does not depend on the "chemical details" of the system. The close analogy between instabilities in nonlinear systems driven far from equilibrium and phase transitions is now well documented experimentally as well as theoretically. This idea was fathered by Landau (1941), and developed by several people in the context of hydrodynamics, electric circuits, nonlinear optics and chemical instabilities. In this context, the complex amplitude of the periodic pattern plays the role of an order

parameter and characterizes the broken symmetries at instability onset. Amplitude equations are analogous to the Ginzburg-Landau description of phase transitions. 


\subsection{Long wavelength neutral modes: phase dynamics}

The second area that we will study is the disorganization of the primary pattern through secondary instabilities. When the primary instability saturates nonlinearly and gives rise to a finite amplitude periodic pattern, only its phase remains neutral in the long-wavelength limit. Indeed, a spatially uniform modification of the phase corresponds to a shift of the periodic pattern, and thus is neutral because of translational invariance in space. Likewise, other broken symmetries, translational invariance in time at the onset of an oscillatory instability, Galilean invariance at the onset of a pattern-forming instability, etc ..., may generate long-wavelength neutral modes, i.e. modes that are neither dissipated nor amplifiied at zero wavenumber. These modes are analogous to Goldstone modes in particle physics or condensed-matter physics, and often lead to secondary instabilities of the primary pattern. Because their growth rate vanishes in the long wavelength limit, we eliminate adiabatically the other fast modes in order to obtain evolution equations that describe pattern-dynamics through slowly varying phases. Thus, contrary to the situation at instability onset the pattern amplitude is no longer a neutral mode above criticality; for a perfectly periodic pattern, it saturates at a finite value. However, phase instabilities, that usually occur at zero wavenumber do not always saturate in the long wavelength limit; they often cascade to short scales, leading to defect nucleation in the primary pattern. Although non-neutral, the pattern amplitude locally vanishes, thus breaking the long-wavelength approximation. A consistent description of this type of pattern-dynamics is still an open problem.

\subsection{Localized nonlinear structures}

Shock-waves or solitons are well known examples of nonlinear localized structures. Defects of periodic patterns are another class of localized structures. Although incompletely, we will define the main characteristics of these objects, try to classify them and to understand their dynamics.

Another type of localized structure is well known in fluid dynamics and illustrated by plate 109 in Van Dyke (1982), reproduced here as Figure 1.6. It is a turbulent spot, i.e. a region of turbulent flow advected in a laminar flow. This type of structure is widely observed in pipe flows or boundary layers. The turbulent region can expand when moving, but there are also situations where the spot keeps a nearly constant size.

Similarly, pattern forming instabilities can display localized structures consisting of a region in the bifurcated state surrounded by the basic state. This occurs when there is a parameter range in which the system has two stable states of different form; then one might observe both of them in separate regions. Thermal convection of a binary fluid mixture in an annulus, studied by Kolodner, Bensimon and Surko (1988), displays such localized patterns (Figure 1.7).

An earlier example is the localized standing wave observed in Faraday instability (Wu, Keolian and Rudnick, 1984). In the limit of small dissipation, we will show that these structures trace back to the solitary waves of conservative systems. 


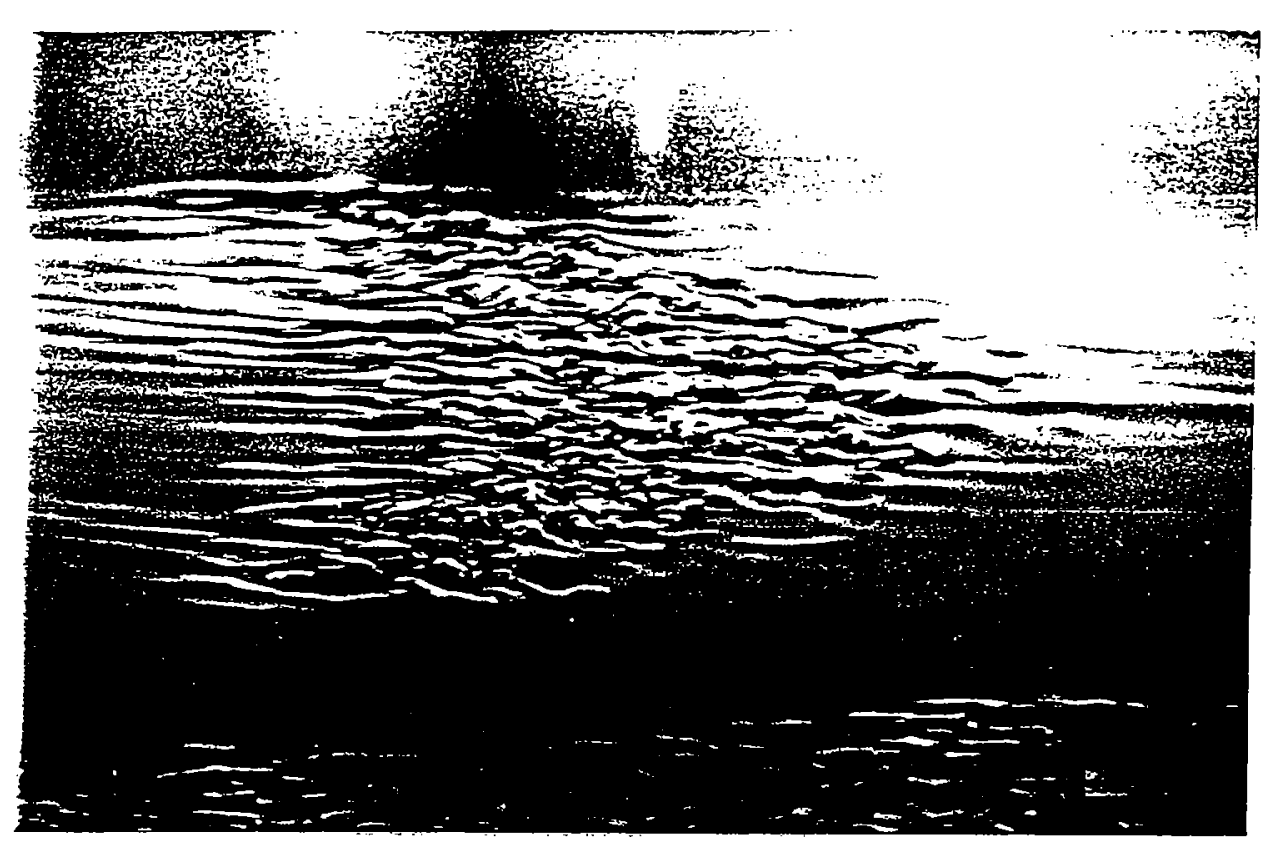

Figure 1.6. Emmons spot in the boundary layer over a flat plate.

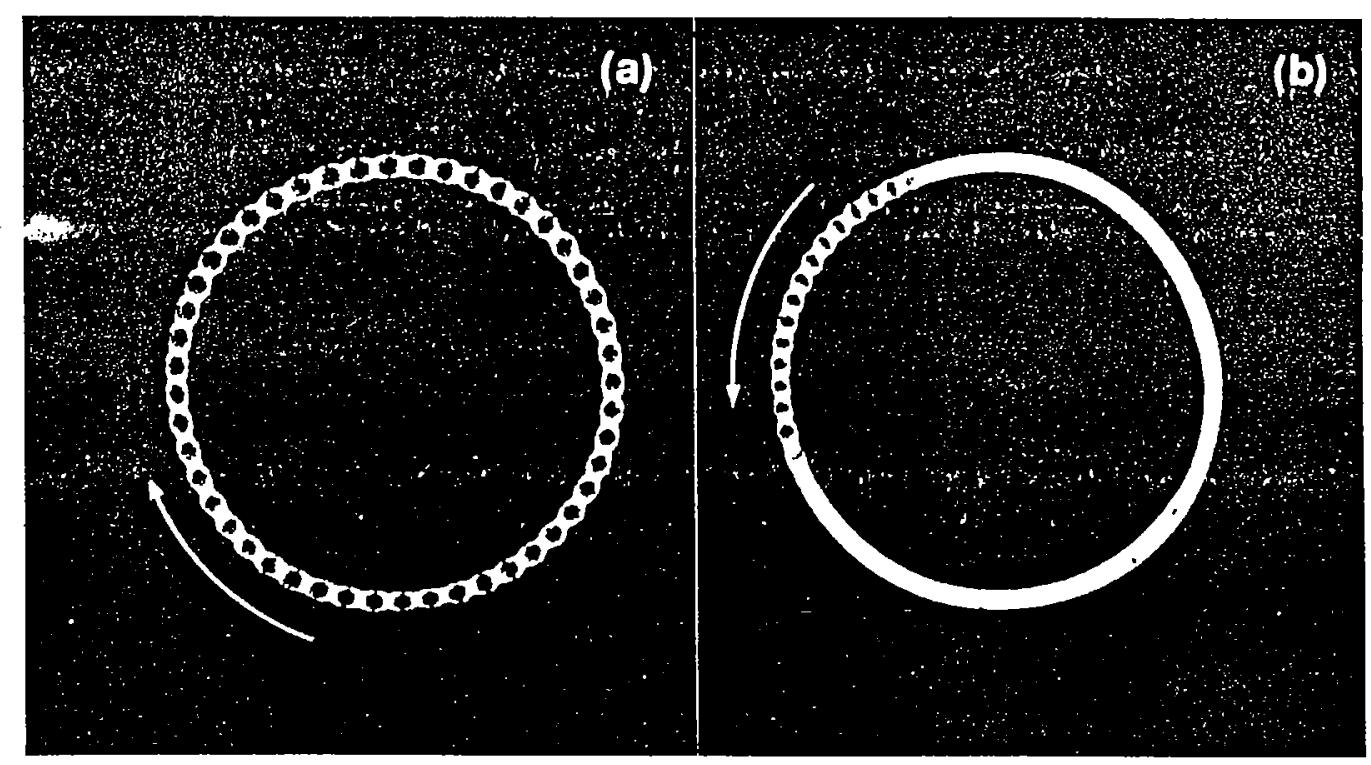

Figure 1.7. (a) Periodic pattern of travelling convection rolls (b) The travelling rolls fill only part of the annulus; both the conducting and convecting states are stable and coexist in different regions of the annulus. 


\section{References}

Chandrasekhar, S. (1961) Hydrodynamic and Hydromagnetic Stability, Clarendon Press, Oxford.

Drazin, P. G. and Reid, W. H. (1981) Hydrodynamic Stability, Cambridge University Press. Faraday, M. (1831) Phil. Trans. R. Soc. London, 52, 319.

Kolodner, P., Bensimon, D. and Surko, C.M. (1988). Travelling-wave convection in an annulus, Phys. Rev. Lett. 60, 17.

Landau, L., (1967) On the theory of phase transitions, On the problem of turbulence, Collected papers of L. Landau, Gordon and Breach .

Van Dyke, M. (1982). An Album of Fluid Motion

Wesfreid, J. E. and Zaleski, S., (1984) Cellular Structures in Instabilities, Lectures Notes in Physics, Springer Verlag

Wesfreid, J. E., Brand, H. R., Manneville, P., Albinet, G. and Boccara, N., (1988) Propagation in Systems far from Equilibrium, Springer Verlag.

Wu, J., Keolian, R. and Rudnick, I. (1984). Phys. Rev. Lett. 52, 1421. 


\section{Nonlinear Oscillators}

In this lecture we study dissipative nonlinear oscillators as the simplest examples of temporal patterns, i.e. periodic limit cycles, quasiperiodic regimes and frequency locking phenomena. Two different amplification mechanisms are considered to balance dissipation and sustain an oscillatory regime:

- "negative dissipation", for which the system is autonomous,

- parametric forcing, where an external time dependent perturbation is applied.

Our objective is to find an amplitude equation for the amplitude and phase of the oscillation. We show that its form depends on the amplification mechanism, because the broken symmetries at the oscillatory instability onset are different, but that universal behavior of the oscillation amplitude and the frequency exists in both types of oscillator. We next consider a negative dissipation oscillator with an external time dependent forcing, and study frequency-locking phenomena.

\subsection{Van der Pol Oscillator}

We begin with an autonomous system with negative dissipation. The canonical example is the Van der Pol oscillator, introduced in electronics a long time ago (Van der Pol, 1934). The governing equation is

$$
\ddot{u}-2 \lambda \dot{u}+u^{2} \dot{u}+\omega_{0}^{2} u=0 .
$$

Here, the dissipation is negative if $\lambda>0$ and the nonlinear term causes the system to saturate when $u^{2} \sim \lambda$.

\subsubsection{Global or linear stability}

Some insight into the behavior of this equation can be obtained by studying the total energy of the system, which is here a simple example of Liapunov functional. This is a useful first step before attempting a more detailed analysis. The sum of potential and kinetic energies is

$$
E=\omega_{0}^{2} \frac{u^{2}}{2}+\frac{\dot{u}^{2}}{2}
$$

and the time derivative of the energy is

$$
\dot{E}=\omega_{0}^{2} u \dot{u}+\dot{u} \ddot{u}=\left(2 \lambda-u^{2}\right) \dot{u}^{2} .
$$

Thus if $\lambda<0$ (linear damping), $\dot{E}<0$. Moreover, there is a lower bound, $E=0$, which occurs if and only if $u=\dot{u}=0$, and consequently the motionless state is globally stable. 
We are interested in the case $\lambda>0$. A linear stability analysis about $\mathrm{u}=0$ is done by talking $u \propto \exp (\eta t)$, and if $\operatorname{Re}(\eta)>0$ the system is unstable. Substituting into (1) and linearizing gives the characteristic polynomial

$$
\eta^{2}-2 \lambda \eta+\omega_{0}^{2}=0
$$

or $\eta=\lambda \pm \sqrt{\lambda^{2}-\omega_{0}^{2}}$. If we let $\lambda=\mu \epsilon$ where $\epsilon$ is small, $\eta \approx \mu \epsilon \pm i \omega_{0}^{2}$ and the system becomes unstable as $\lambda$ changes sign; this is a Hopf bifurcation and the system's behavior changes from a damped oscillation to an amplified oscillation as $\lambda$ increases. Near the bifurcation point, the growth rate is small and the time scale of the growth is $T \sim 1 / \epsilon$.

\subsubsection{Nonlinear effects}

The linear analysis predicts exponential growth, but this is eventually checked by the nonlinear term, which leads to saturation when $u^{2} \approx \epsilon$. We can see this by studying the energy balance of the system. We suppose that there is a harmonic limit cycle when $\lambda$ is small and so we are close to the bifurcation, and we also presume that the amplitude of the oscillation varies on a slow time scale, thus

$$
u \approx a(t) \cos \omega_{0} t \text { and } \dot{u} \approx-\omega_{0} a(t) \sin \omega_{0} t
$$

The energy balance over one cycle requires

$$
\frac{1}{T} \int_{0}^{T} \dot{E} d t=0 \quad \text { or } \quad 2 \lambda \overline{\dot{u}^{2}}-\overline{u^{2} \dot{u}^{2}}=0,
$$

where the overbar denotes averaging over one cycle.

Using the above expressions for $u$, we find $a^{2}=8 \lambda=8 \mu \epsilon$, and so the scaling of the oscillation amplitude above criticality is $u \sim \sqrt{\epsilon}$.

A second rôle of the nonlinearity is the production of higher harmonics and the subsequent shift of the fundamental frequency. We show next that these effects are connected. If the governing equation is multiplied by $u$ and averaged over one cycle, the terms involving $\dot{u}$ all average to zero and we are left with the virial equation

$$
\overline{\dot{u}^{2}}=\omega_{0}^{2} \overline{u^{2}}
$$

Note that both the nonlinear and dissipative terms have averaged to zero. However, the effect of nonlinearity still enters, since we assume that $u$ contains some higher harmonic components:

$$
u=\sum_{n=1}^{\infty} a_{n} \cos \left(n \omega t+\phi_{n}\right) .
$$

When this is substituted into the virial equation, we obtain

$$
\sum n^{2} \omega^{2} a_{n}^{2}=\omega_{0}^{2} \sum a_{n}^{2}
$$


and this can be written as

$$
\frac{\left(\omega^{2}-\omega_{0}^{2}\right)}{\omega_{0}^{2}}=-\frac{\sum\left(n^{2}-1\right) a_{n}^{2}}{\sum n^{2} a_{n}^{2}} .
$$

Assuming that the amplitude of successive harmonics decreases fast enough with increasing $n$, the frequency shift, $\Delta \omega \equiv \omega-\omega_{0}$, is to leading order,

$$
\frac{\Delta \omega}{\omega_{0}} \approx-\frac{3}{2} \frac{a_{2}^{2}}{a_{1}^{2}}-4 \frac{a_{3}^{2}}{a_{1}^{2}}+\cdots
$$

Thus the shift of the fundamental frequency is related to the existence of higher harmonics. For the Van der Pol equation, there is no quadratic nonlinearity and $a_{2}=0$; thus there is no frequency correction to leading order. Note however that the relationship between the fundamental frequency shift and the amplitude of the higher harmonics, is in general not so simple as the one above. For instance, replace $\omega_{0}^{2} u$ by $\omega_{0}^{2} \sin u$ in the Van der Pol equation and try the same exercise.

\subsubsection{Amplitude equations : the multiple-scale method}

In cases where the amplitude varies much more slowly than the underlying oscillation, we can use the disparity in temporal scale to obtain a simplified description of amplitude variations independently of the faster time scale. This is sketched schematically in Figure 2.1. The oscillation has time scale $t$. We introduce a second time variable, $T=\epsilon t$, to describe the slow variation in amplitude; indeed, in the vicinity of instability onset, $\lambda=\mu \epsilon$, the instability growth rate scales like $\epsilon$.

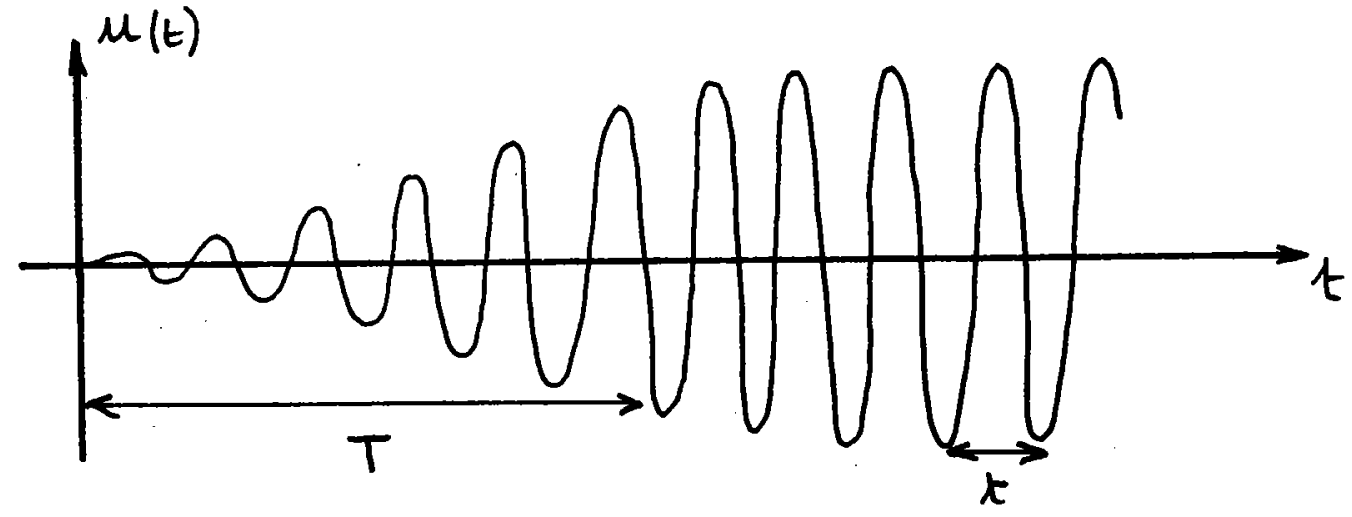

Figure 2.1. Amplitude varying much more slowly than the oscillation gives multiple time scales.

We have already seen that the saturation amplitude for $u$ scales like $\sqrt{\epsilon}$, so we take

$$
u(t) \rightarrow \sqrt{\epsilon} \tilde{u}(t, T) .
$$

Using

$$
\frac{\partial}{\partial t} \rightarrow \frac{\partial}{\partial t}+\epsilon \frac{\partial}{\partial T}
$$


we rewrite the Van der Pol equation:

$$
\epsilon^{1 / 2} \frac{\partial^{2} \tilde{u}}{\partial t^{2}}+2 \epsilon^{3 / 2} \frac{\partial^{2} \tilde{u}}{\partial t \partial T}+\epsilon^{5 / 2} \frac{\partial^{2} \tilde{u}}{\partial T^{2}}+\left(\epsilon \tilde{u}^{2}-2 \mu \epsilon\right)\left(\epsilon^{1 / 2} \frac{\partial \tilde{u}}{\partial t}+\epsilon^{3 / 2} \frac{\partial \tilde{u}}{\partial T}\right)+\epsilon^{1 / 2} \omega_{0}^{2} \tilde{u}=0
$$

We can factor $\sqrt{\epsilon}$ out of every term in this equation; then to order $\epsilon^{0}$, we have a simple harmonic oscillator:

$$
\left(\frac{\partial^{2}}{\partial t^{2}}+\omega_{0}^{2}\right) \tilde{u}=0
$$

It will prove convenient to define $\mathcal{L}=\frac{\partial^{2}}{\partial t^{2}}+\omega_{0}^{2}$. We can now introduce a perturbation expansion

$$
\tilde{u}=\tilde{u}_{0}+\epsilon^{1 / 2} \tilde{u}_{1}+\epsilon \tilde{u}_{2}+\ldots
$$

and collect terms in increasing powers of $\epsilon$ :

$$
\begin{gathered}
\epsilon^{0}: \mathcal{L} \tilde{u}_{0}=0 \\
\epsilon^{1 / 2}: \mathcal{L} \tilde{u}_{1}=0 \\
\epsilon^{1}: \mathcal{L} \tilde{u}_{2}=-2 \frac{\partial^{2} \tilde{u}_{0}}{\partial t \partial T}-\tilde{u}_{0}^{2} \frac{\partial \tilde{u}_{0}}{\partial t}+2 \mu \frac{\partial \tilde{u}_{0}}{\partial t}
\end{gathered}
$$

and so forth. The $\epsilon^{0}$ equation is easily solved:

$$
\tilde{u}_{0}=A(T) e^{i \omega_{0} t}+\bar{A}(T) e^{-i \omega_{0} t}
$$

where $A(T)$ is the slowly-varying amplitude of the oscillation. We could write such a solution to the $\epsilon^{1 / 2}$ equation as well, but since $\tilde{u}_{1}$ does not appear in the equation for $\tilde{u}_{2}$, we don't need it until we go to higher order. Substituting the leading order solution into the $\epsilon^{1}$ equation yields

$$
\mathcal{L} \tilde{u}_{2}=2 i \omega_{0}\left(\mu A(T)-\frac{d A}{d T}\right) e^{i \omega_{0} t}-i \omega_{0}\left(|A|^{2} A e^{i \omega_{0} t}+A^{3} e^{3 i \omega_{0} t}\right)+c c
$$

"cc" in this (and subsequent) equations refers to the complex conjugate of the preceding expression.

In order to avoid secular growth of $\tilde{u}_{2}$, it is necessary to set the resonant terms-the terms in $e^{i \omega_{0} t}$ - to zero. Doing so yields

$$
\left[2\left(\mu A(T)-\frac{d A}{d T}\right)-|A|^{2} A\right] e^{i t}-c c=0
$$

Since $e^{i t}$ and $e^{-i t}$ are linearly independent, we can set the displayed expression and its complex conjugate to zero independently. Hence,

$$
\frac{d A}{d T}=\mu A-\frac{1}{2}|A|^{2} A
$$


Taking $A=R e^{i \theta}$, factoring out $e^{i \theta}$ and separating real and imaginary parts, gives

$$
\begin{gathered}
\frac{d R}{d T}=\mu R-\frac{1}{2} R^{3} \\
\frac{d \theta}{d T}=0 .
\end{gathered}
$$

The amplitude $R$ approaches $\sqrt{2 \mu}$ when $\mu>0$ and the phase $\theta$ does not vary with the slow time scale at all. This second feature is not generic for a Hopf bifurcation and traces back to the absence of frequency shift to leading order. As a simple exercise, you can again consider the Van der Pol equation with $\omega_{0}^{2} \sin u$ instead of $\omega_{0}^{2} u$, and show that a term proportional to $R^{2}$ occurs the $\theta$ equation.

Generically, the amplitude equation for a Hopf bifurcation is of the form

$$
\frac{d A}{d T}=\mu A-\beta|A|^{2} A
$$

with $\beta=\beta_{r}+i \beta_{i}$ some complex number. Then, the equations for $R$ and $\theta$ become

$$
\frac{d R}{d T}=\left(\mu-\beta_{r} R^{2}\right) R, \quad \frac{d \theta}{d T}=-\beta_{i} R^{2}
$$

If $\beta_{r}>0$, the leading order nonlinearity saturates the instability and the bifurcation is said "direct" or "supercritical". Above instability onset, the oscillation amplitude is proportional to $\sqrt{\mu}$. Otherwise, the bifurcation is "inverse" or "subcritical". In the supercritical situation the phase increases linearly in time, indicating the fundamental frequency shift proportional to $\mu$. Oscillatory instabilities observed experimentally often display this characteristic behavior for the oscillation amplitude and frequency slightly above criticality. The corresponding measurements are a useful check that the system undergoes a Hopf bifurcation. Note however, that the frequency $\omega_{0}$ itself usually depends on the experimental control parameter (proportional to $\mu$ ); this dependence is generally linear to leading order and adds to the one due to $\beta_{i}$. This makes $\beta_{i}$ difficult to measure precisely.

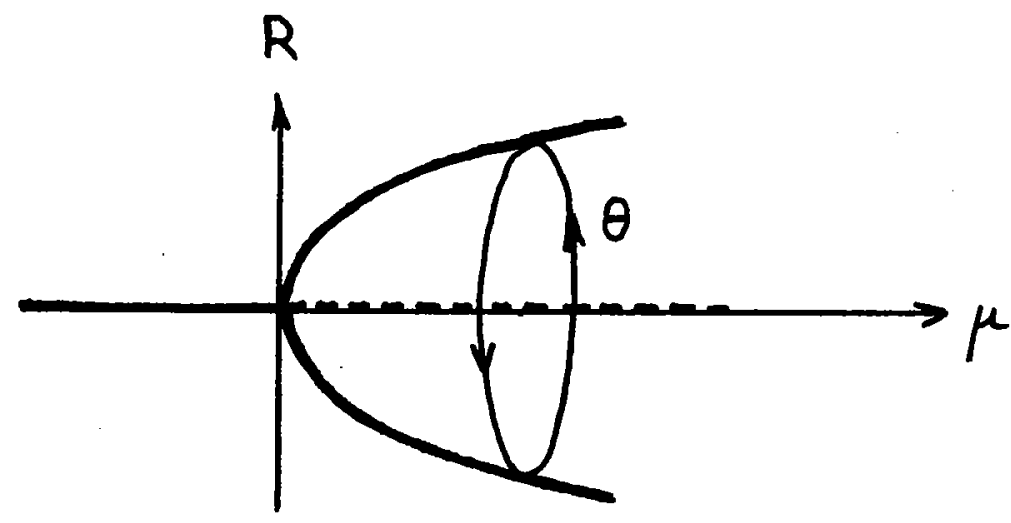

Figure 2.2. Diagram of the supercritical Hopf bifurcation. 


\subsubsection{Symmetry arguments}

We show next that the form of the evolution equation for the oscillation complex amplitude $A$ is determined by symmetry constraints. It is clear that the original Van der Pol equation

$$
\ddot{u}-2 \lambda \dot{u}+u^{2} \dot{u}+\omega_{0}^{2} u=0
$$

describes an autonomous system, i.e. it is invariant under translation in time and is unaffected by a change of variables $t \rightarrow t+\theta$. This does not mean that any solution should be translationally invariant in time; any oscillatory solution, and in particular the expansion we used for $u$

$$
u=\sqrt{\epsilon}\left[A(T) e^{i \omega_{0} t}+\bar{A}(T) e^{-i \omega_{0} t}\right]+\epsilon u_{1}+\cdots,
$$

obviously breaks translational invariance in time. However, the ensemble of possible solutions should have this invariance. In other words, any image of an oscillatory solution under the transformation corresponding to the broken symmetry must be a solution of the autonomous system. Thus, if $u(t)$ is an oscillatory solution, so is $u(t+\theta)$. This obvious requirement is enough to determine the form of the amplitude equation, if one assumes, and this is a crucial assumption, that $d A / d T$ can be expanded in powers of $A$ and $\bar{A}$ in the vicinity of instability onset.

Making the transformation $t \rightarrow t+\theta / \omega_{0}$, one get

$$
u\left(t+\theta / \omega_{0}\right)=\sqrt{\epsilon}\left[A(T) e^{i \omega_{0} t} e^{i \theta}+\bar{A}(T) e^{-i \omega_{0} t} e^{-i \theta}\right]+\epsilon u_{1}+\cdots
$$

The dynamics of the transformed $u$ is to be the same as the original $u$, therefore the dynamics of $A$ should be invariant under the transformation,

$$
A(T) \rightarrow A(T) e^{i \theta}
$$

This transformation selects the combinations of $A$ and $\bar{A}$ that can appear in the amplitude equation: only those which also transform with a factor of $e^{i \theta}$ will do. Consider all the possibilities up to cubic terms:

$$
\begin{gathered}
A \rightarrow A e^{i \theta} \quad \bar{A} \rightarrow \bar{A} e^{-i \theta} \\
A^{2} \rightarrow A^{2} e^{2 i \theta}, \quad A \bar{A} \rightarrow A \bar{A}, \quad \bar{A}^{2} \rightarrow \bar{A}^{2} e^{-2 i \theta}, \\
A^{3} \rightarrow A^{3} e^{3 i \theta}, \quad A^{2} \bar{A} \rightarrow A^{2} \bar{A} e^{i \theta}, \quad A \bar{A}^{2} \rightarrow A \bar{A}^{2} e^{-i \theta}, \quad \bar{A}^{3} \rightarrow \bar{A}^{3} e^{-3 i \theta},
\end{gathered}
$$

Only the boxed terms scale appropriately, so only they can appear in the amplitude equation. Hence, we could have deduced the form of the amplitude equation simply from the symmetry of the original equation and the solution to the zeroth-order equation that fixes the brokensymmetry. 
Note that we consider $t$ and $T$ as independent variables and did not change $A(T) \rightarrow A(T+$ $\left.\epsilon \theta / \omega_{0}\right)$. This is only approximately correct, and if we sought higher order perturbations, we would have to make the more complete substitution.

\subsubsection{Discussion about symmetry arguments}

A. Roberts pointed out that symmetry is not sufficient to reduce the possibilities to the few mentioned above. Rather, there is an implicit assumption that there are no memory effects. He presented an example term

$$
A(T) \int_{-\infty}^{T} A\left(T^{\prime}\right) \bar{A}\left(T^{\prime}\right) K\left(T-T^{\prime}\right) d T^{\prime}
$$

which would satisfy the symmetry requirement but include memory effects.

The locality assumption is obviously made when one looks for $d A / d T$ as an expansion in powers of $A$ and $\bar{A}$. It would be interesting to work out a simple example with memory effects.

R. Salmon argued about the relevance of the symmetry requirement. He said that a differential equation has many symmetries, and that any solution can break some of these symmetries. He felt that the reason we consider time translation symmetry and not some other symmetry is physical rather than mathematical.

The symmetries that have to be considered are the ones that are broken by the linearly unstable solution. The symmetry requirement for the ensemble of possible bifurcated solutions constrains the form of the amplitude equation. A symmetry of the original problem that is not broken by the linearly unstable mode gives no constraint on the amplitude equation. Amplitude equations can also have symmetries that are not forced by the original equation. As we will see when studying frequency-locking phenomena, the form of the linearly unstable mode is of crucial importance; an amplitude equation is meaningless without the expression of the original field as a function of the amplitude.

\subsection{Parametric Oscillators}

We will now discuss the bifurcation structure of parametric oscillators. Parametric amplification occurs widely in physical situations. Examples include Langmuir waves in plasmas, spin waves in ferromagnets, surface waves on a ferrofluid in a time dependent magnetic field, or on a liquid dielectric in an alternating electric field. As shown in the first section, parametric amplification of surface waves on a horizontal layer of fluid vertically vibrated, is a simple experimental model of pattern dynamics. Let us mention also that parametric amplifiers were widely used in electronics. We study here the simplest example which is a pendulum whose support is vibrated. The pendulum angle $u(t)$ from the vertical axis, is governed by the damped Mathieu equation

$$
\ddot{u}+2 \lambda \dot{u}+\omega_{0}^{2}\left(1+f \sin \omega_{e} t\right) \sin u=0
$$


where $\lambda$ is the damping, $\omega_{0}$ is the natural frequency, $f$ is the forcing amplitude and $\omega_{e}$ is the external forcing frequency. The resonance points of the Mathieu equation are well known to occur whenever $n \omega_{e}-\omega_{0}=\omega_{0}$ and are shown in Figure 2.3.

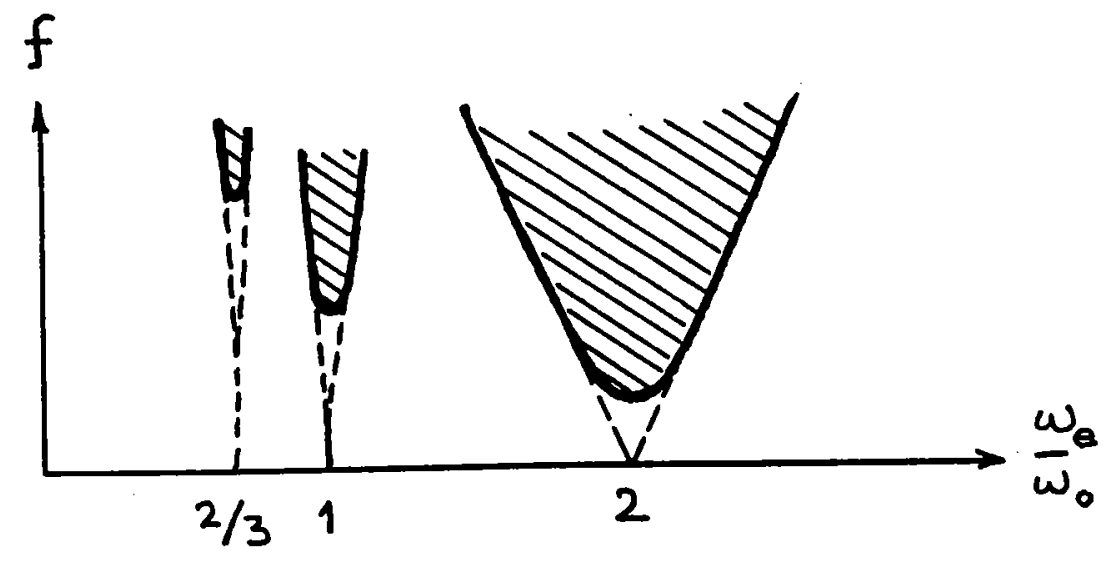

Figure 2.3. Resonance tongues of the Mathieu equation (unstable regions are hatched).

We now examine the strongest resonance, $\omega_{e}=2 \omega_{0}$, in detail. We obtain the amplitude equation by the method of multiple scales. The three parameters in the equation are damping, forcing and the detuning $\delta=\frac{\omega_{0}^{2}}{\omega_{e}^{2}}-\frac{1}{4}$. To bring in all the effects at the same order we use the scaling $\delta=\epsilon \Delta, \lambda=\epsilon \Lambda, f=\epsilon F$ and let

$$
u(t)=\sqrt{\epsilon}\left[u_{0}(t, T)+\sqrt{\epsilon} u_{1}(t, T)+\ldots\right]
$$

where the slow time is $T=\epsilon t$.

After substituting these in the equation and collecting terms we obtain at the zeroth order

$$
\mathcal{L} u_{0} \equiv\left(\frac{d^{2}}{d t^{2}}+\omega_{0}^{2}\right) u_{0}=0
$$

which has the solution

$$
u_{0}=A(T) e^{i \omega_{0} t}+\bar{A}(T) e^{-i \omega_{0} t}
$$

The first order equation is the same and its solution can be incorporated into $u_{0}$. The second order $\left(\epsilon^{1}\right)$ problem is

$$
\mathcal{L} u_{2}=-2 \frac{\partial^{2} u_{0}}{\partial t \partial T}-2 \Lambda \frac{\partial u_{0}}{\partial t}-\omega_{0}^{2} F u_{0} \sin \omega_{e} t+\frac{1}{6} \omega_{0}^{2} u_{0}^{3}
$$

Using the fact that $\omega_{e}=2 \omega_{0}-4 \epsilon \Delta \omega_{0}+O\left(\epsilon^{2}\right)$ the solvability condition to eliminate resonant terms is

$$
\frac{d A}{d T}=-\Lambda A+\frac{\omega_{0} F}{4} \bar{A} e^{-4 i \Delta \omega_{0} T}-\frac{\omega_{0}}{4} i A^{2} \bar{A}
$$


By moving to a frame of reference rotating with $\omega_{e} / 2$ (instead of $\omega_{0}$ ) with the transformation $A=B e^{-2 i \Delta \omega_{0} T}$ we obtain the autonomous amplitude equation

$$
\frac{d B}{d T}=(-\Lambda+i \nu) B+\mu \bar{B}+i \beta|B|^{2} B
$$

where $\nu=2 \Delta \omega_{0}, \mu=\omega_{0} F / 4$ and $\beta=-\omega_{0} / 4$.

The form of this equation could have been guessed by using symmetry arguments, the relevant symmetry of the Mathieu equation being $t \rightarrow t+\frac{2 \pi}{\omega_{e}}$, which restricts terms in the amplitude equation to be invariant under $B \rightarrow-B$. This symmetry is a much weaker restriction than the one for the Van der Pol equation $\left(A \rightarrow A e^{i \theta}\right)$. The term proportional to $i B$ in the amplitude equation corresponds to a rotation of $B$ at constant velocity in the complex plane and thus to a detuning. In other words, $\nu \neq 0$ indicates that $\omega_{e} / 2$ and $\omega_{0}$ ) are slightly different, thus that the forcing frequency is not exactly at parametric resonance. Moreover, collecting all the terms with pure imaginary coefficients, $i\left(\nu+\beta|B|^{2}\right) B$, shows that $\beta|B|^{2}$ is a nonlinear detuning. It is associated with the amplitude dependence of the oscillator frequency, and this nonlinear effect is the one that saturates the instability, by shifting the oscillator away from parametric resonance. This is to be contrasted to the Van der Pol oscillator where the instability is saturated by nonlinear damping. The term $\mu \bar{A}$, that breaks rotational invariance in the complex plane of the amplitude equation, is precisely the one that results from the parametric forcing.

Let us now study the linear stability of the solution $u=0$. Writing $B=X+i Y$ and inserting a mode proportional to $e^{\eta t}$ we obtain the following quadratic for the eigenvalues:

$$
\eta^{2}+2 \Lambda \eta+\left(\Lambda^{2}-\mu^{2}+\nu^{2}\right)=0
$$

Since the damping, $\Lambda$, is positive, we see that there is no Hopf bifurcation contrary to the Van der Pol case. There is a stationary bifurcation at a threshold forcing amplitude $\mu_{c}=\sqrt{\Lambda^{2}+\nu^{2}}$. This is shown in Figure 2.4 which reproduces the $2: 1$ resonance curve of Figure 2.1.

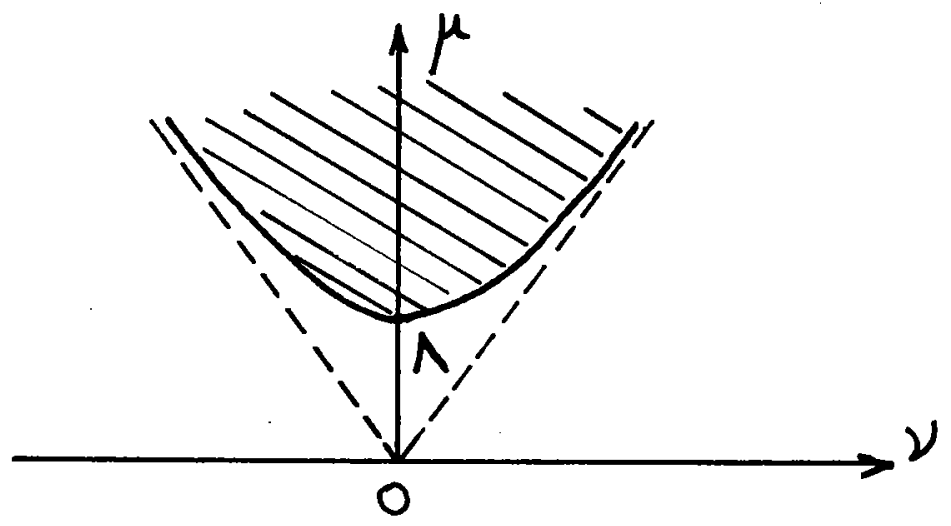

Figure 2.4. Linear stability in parameter space of $u=0$ with $2: 1$ forcing 
The nonlinear stability is only slightly more complicated. We write $B=R e^{i \theta}$ in (2.5) and obtain the equations

$$
\begin{aligned}
& \frac{d R}{d \tau}=(-\Lambda+\mu \cos 2 \theta) R, \\
& \frac{d \theta}{d \tau}=\nu-\mu \sin 2 \theta+\beta R^{2} .
\end{aligned}
$$

To find the stationary solutions we set the right hand side of the equations to zero. Defining the finite amplitude stationary solution to be $R_{0}$ we obtain

$$
\beta R_{0}^{2}=-\nu \pm \sqrt{\mu^{2}-\Lambda^{2}}
$$

Without loss of generality we take $\beta>0$ (otherwise we consider the complex conjugate equation). For real solutions we need $\mu>\Lambda$. Then if $\nu>0$ only the positive sign is valid and there is one solution for the amplitude, i.e. two solutions with different phases labeled here by $2 \times 1$ ). If $\nu<0$, then for $\mu<\sqrt{\nu^{2}+\Lambda^{2}}$ we can have 4 solutions ( 2 solutions with 2 phases). Figure 2.5 shows these different regions.

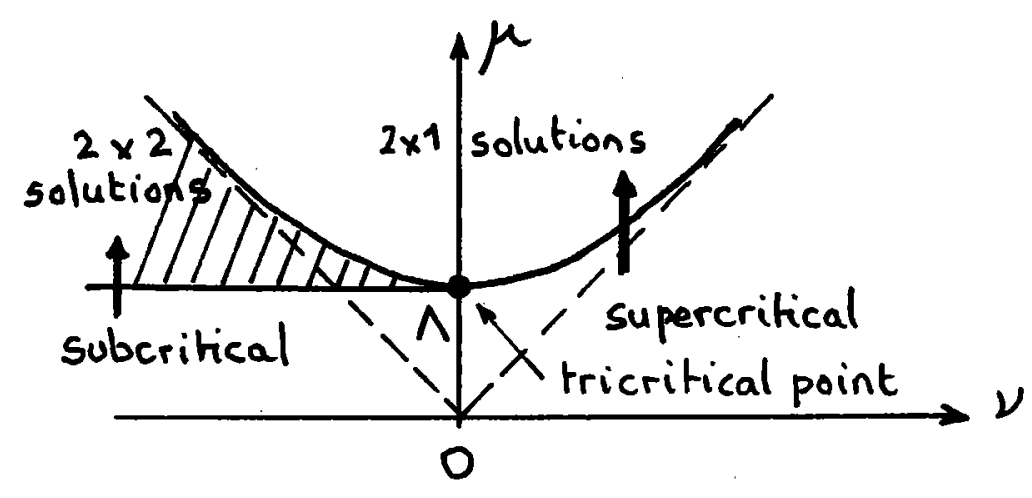

Figure 2.5. Nonlinear solutions in parameter space with $2: 1$ forcing.

The behavior is made clearer in the bifurcation diagrams. For $\nu>0$ (Figure 2.6 a) we have a supercritical bifurcation at $\mu_{c}=\sqrt{\nu^{2}+\Lambda^{2}}$. For $\nu<0$ (Figure $2.6 \mathrm{~b}$ ) we have a subcritical bifurcation which is why we have $2 \times 2$ non-zero solutions. The point $\nu=0, \mu=\Lambda$ is a tricritical point (in the language of phase transitions). As usual in subcritical bifurcations one of the solutions is unstable (shown by the dashed branch in the diagram). The stability of the branches can be derived by perturbing the finite amplitude solutions. If we write $R=R_{0}+r$ and $\theta=\theta_{0}+\phi$, then the eigenvalue $\sigma$ of the perturbation satisfies

$$
\sigma^{2}+2 \Lambda \sigma+4 \beta R_{0}^{2}\left(\nu+\beta R_{0}^{2}\right)=0 .
$$

Thus, the bifurcated solutions are stable if $\nu+\beta R_{0}^{2}>0$.

Scaling behaviors above criticality display an interesting feature: for the tricritical point at $\nu=0$, if one writes $\mu=\mu_{c}+\epsilon$, the amplitude scales as $R_{0} \sim \epsilon^{\frac{1}{4}}$; for the supercritical case $R_{0} \sim \epsilon^{\frac{1}{2}}$ as for the Hopf bifurcation. For $\nu \approx 0$, one expects a cross-over between the two behaviors. The bifurcation diagram of the parametric oscillator is richer than the one of the Hopf bifurcation. This is because parametric forcing involves two control-parameters, the forcing amplitude and its frequency, instead of one for the Hopf bifurcation. 

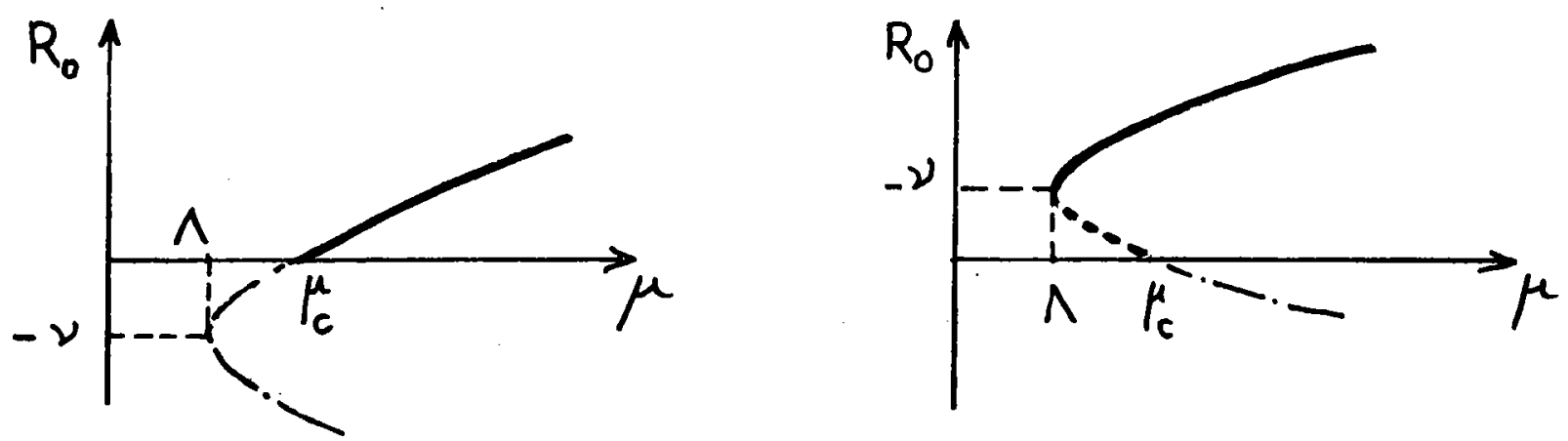

Figure 2.6. Bifurcation diagram for the parametric oscillator (a) $\nu>0$, (b) $\nu<0$.

\subsection{Frequency locking}

Two independent oscillators have generically incommensurate frequencies. In simple words, this means that if one sets-up two clocks, even as similar as possible, they oscillate at slightly different frequencies and thus finally indicate a different time if one waits long enough. It has been known since Huyghens that a coupling, even very small, can lock the phases of the oscillators i.e. force them to oscillate at the same frequency, or more generally with commensurate frequencies. In the phase-locking process, the system thus bifurcates from a quasiperiodic to a periodic regime. A similar situation exists in crystallography for spatial patterns, known as the commensurate-incommensurate transition.

Let us first show experimental results in Rayleigh-Benard convection. Convective rolls in a horizontal layer of mercury heated from below become unstable to an oscillatory motion as the temperature difference across the layer is increased above a critical value. The mercury temperature thus oscillates at a "natural" frequency $\omega_{0} / 2 \pi$ (Figure 2.7 a). We apply an external periodic forcing by rotating the mercury layer about its vertical axis, with a sinusoidal angular velocity of frequency $\omega_{e} / 2 \pi$. Figure 2.7 displays the different flow regimes when the external frequency is about twice the natural one. One can observe locked (b) or quasiperiodic regimes $(\mathrm{c}, \mathrm{e})$. These regimes are located in the experimental parameter space displayed in Figure 2.8. At small forcing amplitude the locked regime is observed within a tongue (the "Arnold tongue") that begins at twice the natural frequency for vanishing external forcing amplitude. When the detuning is increased there is a transition from the locked to the quasiperiodic regime. For large forcing amplitude the natural oscillation is completely inhibited and the temperature oscillates at the forcing frequency ("forced" regime).

The transitions between these regimes can be modeled by considering the simpler system of a forced Van der Pol oscillator that is externally forced. The governing equation for this system is as considered in section 2, except for an additional forcing term $\sqrt{\epsilon} f \cos \omega_{e} t$. Assuming that the dissipation is small $(\lambda=\mu \epsilon)$ and rescaling $u$ by $\sqrt{\epsilon}$ we have

$$
\ddot{u}+\omega_{0}^{2} u=\epsilon\left(2 \mu-u^{2}\right) \dot{u}+f \cos \omega_{e} t .
$$




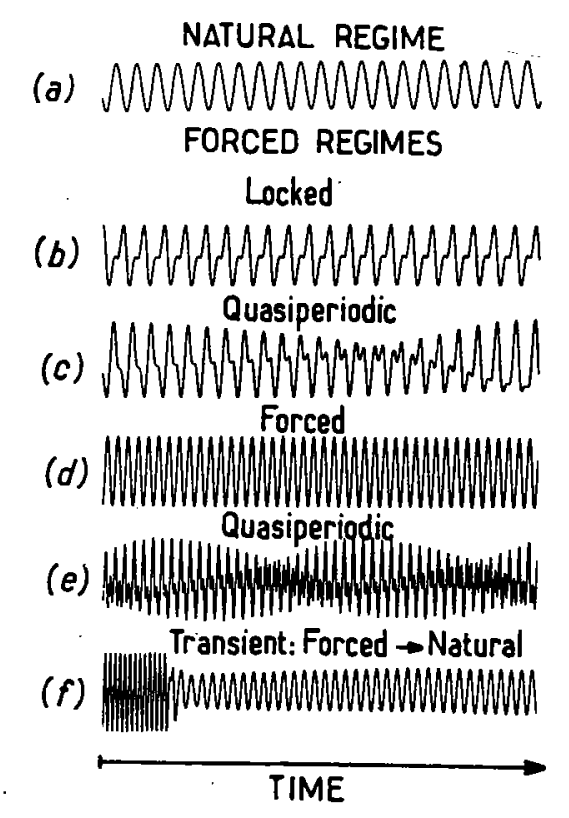

Figure 2.7. Temperature-time records of a layer of mercury heated from below, for different forcing amplitude and frequency, see Chiffaudel and Fauve (1987) for details.

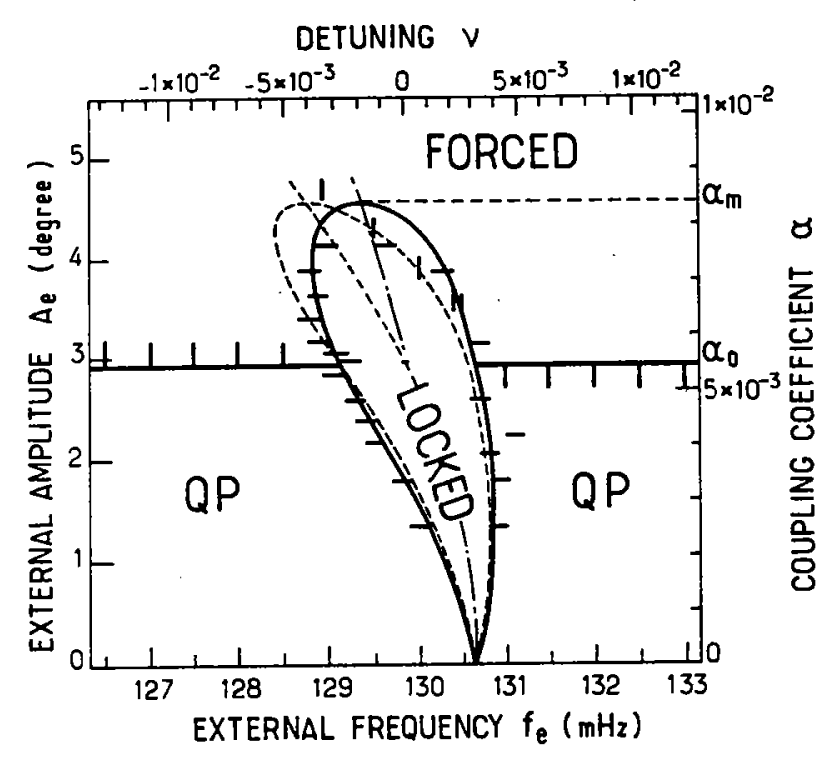

Figure 2.8. The locked, quasiperiodic and forced regimes as external forcing amplitude and frequency are varied. The bars correspond to experimentally measured transitions, the solid and dashed curves are calculated transition curves using an amplitude equation (the two theoretical curves correspond to different coefficients, see Chiffaudel and Fauve (1987) for details.) 
The method of multiple time scales is used to determine the amplitude equation for this system in precisely the same manner as for the previous systems.

We first consider non-resonant forcing (i.e. $\omega_{e}$ and $\omega_{0}$ incommensurable). The leading order term in $u$ is

$$
u_{0}=A(T) \mathrm{e}^{i \omega_{0} t}+\text { c.c. }+\frac{f}{\omega_{0}^{2}-\omega_{e}^{2}} \cos \omega_{e} t
$$

where the last term is due to the external forcing. At the next order we have

$$
\mathcal{L} u_{1}=\left(2 \mu-u_{0}^{2}\right) \frac{\partial u_{0}}{\partial t}-2 \frac{\partial^{2} u_{0}}{\partial t \partial T} .
$$

The solvability condition then gives

$$
\frac{\mathrm{d} A}{\mathrm{~d} T}=\xi A-\frac{1}{2}|A|^{2} A,
$$

where

$$
\xi=\mu-\frac{1}{4}\left(\frac{f}{\omega_{0}^{2}-\omega_{e}^{2}}\right)^{2} .
$$

This amplitude equation is same as in the unforced case except for the form of the coefficients (when $f=0, \xi=\mu$ ). The additional forcing term shifts the onset of instability to larger $\mu$ by reducing $\xi$, see Figure 2.9. There are other well known examples of stabilization by applying a periodic forcing; for instance, the unstable up position of a pendulum can be stabilized by vibrating the point of support.

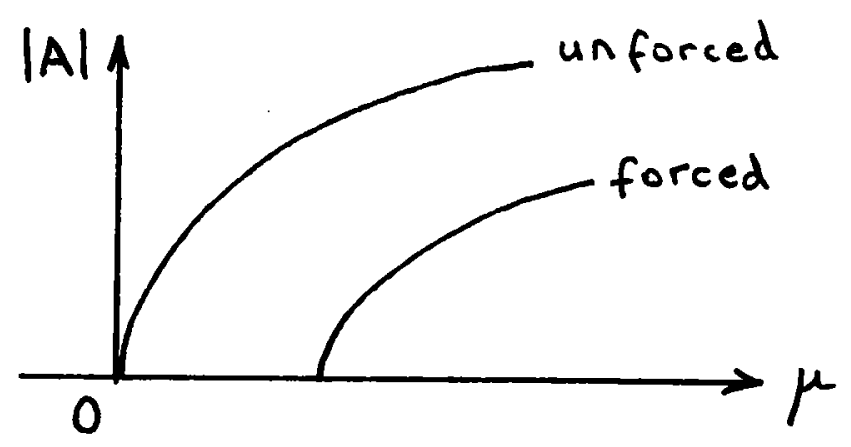

Figure 2.9. Variation of amplitude $|A|$ with $\mu$ for unforced (a) and forced (b) Van der Pol oscillators. 
Note the symmetry, $t \rightarrow t+\frac{2 \pi n}{\omega_{e}}\left(n\right.$ is an integer), imposes the invariance $A \rightarrow A e^{i 2 \pi n \frac{\omega_{0}}{\omega_{e}}}$, on the terms in the amplitude equation. For $\omega_{0}$ and $\omega_{e}$ incommensurate, this is a constraint as strong as the rotation in the complex plane, $A \rightarrow A e^{i \theta}$. Thus, the form of the amplitude equation is the same as in the unforced case, and phase-locking terms that break the $A \rightarrow$ $A e^{i \theta}$ invariance, cannot be found at any order in the amplitude equation. This is due to the form of the leading order solution, $u_{0}$.

Indeed, let us consider small amplitude forcing with $\omega_{e}=\omega_{0}+\epsilon \sigma$ and $f=\epsilon F$. As the forcing is of order $\epsilon$ there is no term due to the forcing in $u_{0}$, and the leading order solution is

$$
u_{0}=A(T) \mathrm{e}^{i \omega_{0} t}+\text { c.c. }
$$

However, the additional term $F \cos \left(\omega_{0} t+\sigma t\right)$ at the next order changes the solvability condition so that

$$
\frac{\mathrm{d} A}{\mathrm{~d} T}=\mu A-\frac{1}{2}|A|^{2} A-\frac{i F}{4 \omega_{0}} \mathrm{e}^{i \sigma T} .
$$

Writing $A=B \mathrm{e}^{i \sigma T}$, gives the amplitude equation

$$
\frac{\mathrm{d} B}{\mathrm{~d} T}=(\mu-i \sigma) B-\frac{1}{2}|B|^{2} B-\frac{i F}{4 \omega_{0}} .
$$

This transformation amounts to writing

$$
u_{0}=B(T) \mathrm{e}^{i \omega_{e} t}+\text { c.c. }
$$

and thus to looking for an amplitude equation in the "reference frame" of the external oscillator. For this new choice of $u_{0}$, the symmetry $t \rightarrow t+\frac{2 \pi n}{\omega_{e}}$, only requires $B \rightarrow B$ ! There is no constraint on the amplitude equation, and indeed the rotation symmetry in the complex plane is broken to leading order by the forcing through the constant term $i F / 4 \omega_{0}$.

This simple example shows how important is the choice of the leading order solution, $u_{0}(t)$. The second choice is called "resonant forcing" although with a non-zero detuning $\sigma$ one can describe a quasiperiodic regime. However, to leading order the response is assumed to be at the forcing frequency. It is the correct choice if one wants to describe frequency-locking phenomena.

Let us now generalize to the case : $\omega_{e}=n \omega_{0} / p$ ( $n, p$ are integers), where the system is invariant under discrete translation in time $t \rightarrow t+2 \pi / \omega_{e}$. The equation for the amplitude $B$ of

$$
u_{0}=B(T) \mathrm{e}^{i \frac{p}{n} \omega_{e} t}+\text { c.c. }
$$

must be invariant to the rotation $B \rightarrow B \mathrm{e}^{i 2 \pi p / n}$. Therefore an additional term $\bar{B}^{n-1}$ related to the forcing is allowed, and the amplitude equation is of the form

$$
\frac{\mathrm{d} B}{\mathrm{~d} T}=(\mu+i \nu) B-\beta|B|^{2} B-\alpha \bar{B}^{n-1} .
$$


The coefficient $\nu$ represents the detuning, while $\alpha$ is related to the forcing. When $n=1,2,3$ and 4 the forcing term is of at least the same order as the $\beta$ term, and these are known as strong resonances.

The three different regimes correspond to:

$1 B=0$, the forced regime,

$2 B=$ const, the locked regime,

$3 B$ time dependent, the quasiperiodic regime.

We consider the specific case $n / p=2$. For simplicity we choose $\beta$ to be real and equal to unity. The linear stability of the the forced regime $A=0$ is exactly the same as that of the parametric oscillator considered earlier. It is stable when $\mu<0$ and $\alpha^{2}<\mu^{2}+\nu^{2}$. The boundary at $\alpha^{2}=\mu^{2}+\nu^{2}$ corresponds to a stationary bifurcation (in the reference frame of the external oscillator), while the boundary at $\mu=0$ is a Hopf bifurcation provided that $\nu^{2}>\alpha^{2}$ ), and corresponds to the boundary between the forced and quasiperiodic regimes. Substituting $B=R \mathrm{e}^{i \theta}$ into the amplitude equation we have that the locked regime $(\mathrm{d} \theta / \mathrm{d} t=0)$ occurs when $\sin 2 \theta=\nu / \alpha$, hence when $|\nu|<|\alpha|$. When this inequality is not satisfied, there exists no constant non-zero solution for $B$ and the system bifurcates to the quasiperiodic regime through a saddle-node bifurcation.

Figure 2.10 shows the three time-forced regimes on the $(\alpha, \nu)$ plane.
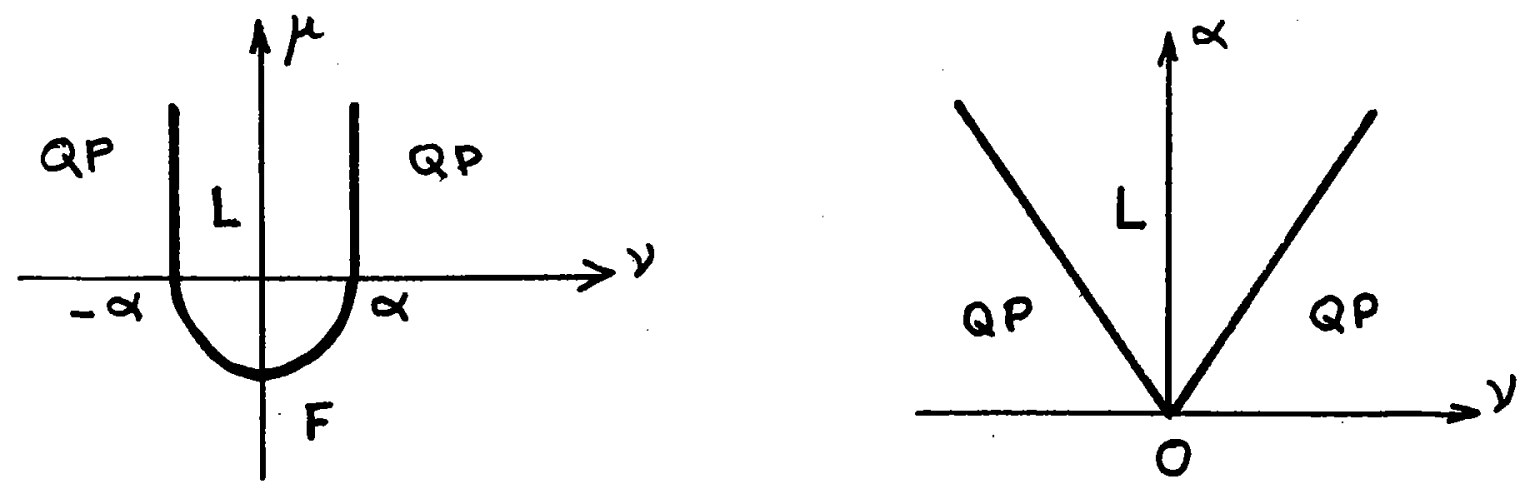

Figure 2.10. The three regimes for a forced Van der Pol oscillator. There is a stationary bifurcation between the forced $(F)$ and locked $(L)$ regimes, a Hopf bifurcation between the forced and quasiperiodic (QP) regimes, and a saddle-node bifurcation between the quasiperiodic and locked regimes. There are codimensiontwo points at $( \pm \mu, 0)$. 


\section{References}

Arnold V. I. (1983) Geometrical Methods in the Theory of Ordinary Differential Equations, Springer Verlag.

Bender, C. M. and Orszag, S. A., (1978) Advanced Mathematical Methods for Scientists and Engineers, McGraw-Hill.

Chiffaudel, A. and Fauve, S., (1987) Strong Resonance in Forced Oscillatory Convection, Phys. Rev.A 35, 4004-4007.

Guckenheimer, J. and Holmes, P., (1984) Nonlinear Oscillations, Dynamical Systems and Bifurcations of Vector Fields, Springer Verlag.

Nayfeh A. H., (1973) Perturbation Methods., Wiley-Interscience.

Van der Pol, B., (1934) Proc. I. R. E. 22, 1051-1086. 


\section{Nonlinear Waves in Dispersive Media}

In this section we consider the propagation of a quasi-monochromatic wave and study the dynamics of dispersion and nonlinearity. To wit, the objective is to find an evolution equation for the slowly varying amplitude and phase of the wave. Using this amplitude equation we can then study the long wavelength stability of periodic waves, and look for solitary wavetrains.

We begin with a simple example, namely the array of pendula shown in figure (3.1). Each pendulum oscillates in the plane perpendicular to the axis of the array and is coupled to its neighbors by torsion springs.

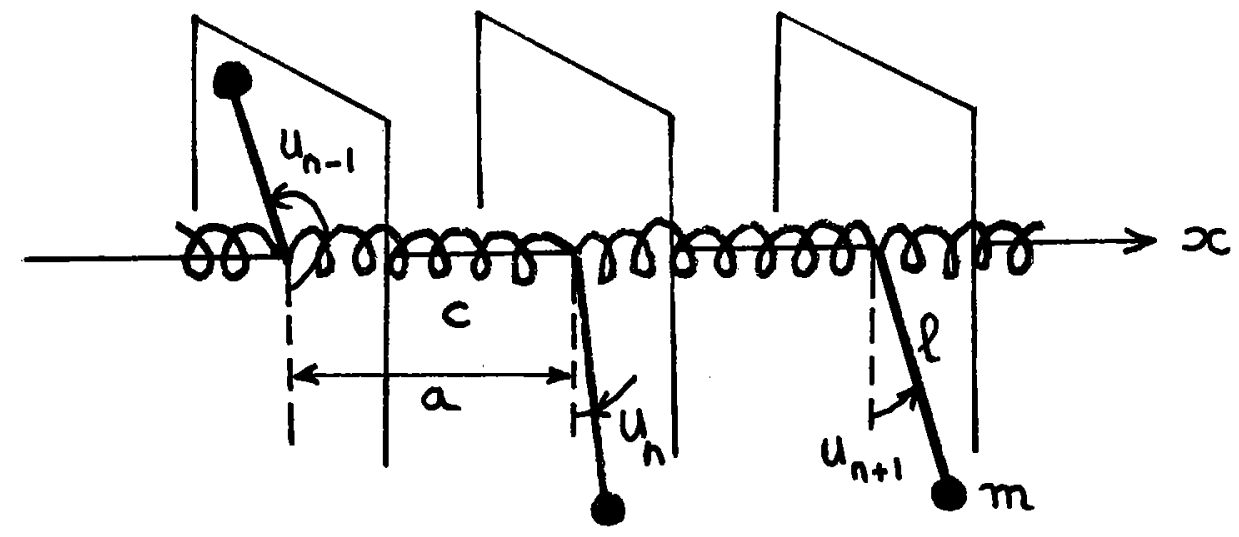

Figure 3.1. The array of coupled pendula

The equation governing the angle from the vertical, $u_{n}(t)$, of the $n^{t h}$ pendulum is,

$$
m l^{2} \frac{d^{2} u_{n}}{d t^{2}}=-m g l \sin u_{n}+c\left(u_{n-1}-2 u_{n}+u_{n+1}\right),
$$

where $m$ is the mass of a pendulum, $l$ is its length and $c$ is the spring torsion constant. We want to investigate phenomena on a lengthscale $\lambda>>a$, where $a$ is the distance between two pendula. In this case we can take the continuous limit of the above equation, rescaling time and space, to obtain:

$$
\frac{\partial^{2} u}{\partial t^{2}}=-\sin u+\frac{\partial^{2} u}{\partial x^{2}}
$$

This is the Sine-Gordon equation, which is also found in nonlinear optics, where it models the propagation of pulses in resonant media, in condensed-matter physics where it describes charge-density waves in periodic pinning potentials or propagation along Josephson transmission lines, and in field theory where it was used to describe elementary particles. However, it is also a long wavelength approximation of our array of pendula and it will be helpful to keep this example in mind to understand the results of this section. 


\subsection{Evolution of a wave packet}

Consider a wave packet which is peaked around $k=k_{0}$, and can be represented as

$$
u(x, t)=\int_{-\infty}^{\infty} F(k) e^{i(\omega(k) t-k x)} d k
$$

Linearizing (3.1) about $u=0$ and substituting for $u$ using (3.2) gives the dispersion relation,

$$
\omega^{2}=1+k^{2}
$$

The group velocity, $U(k)$, is given by

$$
U=\frac{d \omega}{d k}=\frac{k}{\omega}
$$

Notice that

$$
\frac{d^{2} \omega}{d k^{2}}=\frac{\omega-U k}{\omega^{2}}=\frac{1-U^{2}}{\omega} \neq 0
$$

so that the medium is dispersive.

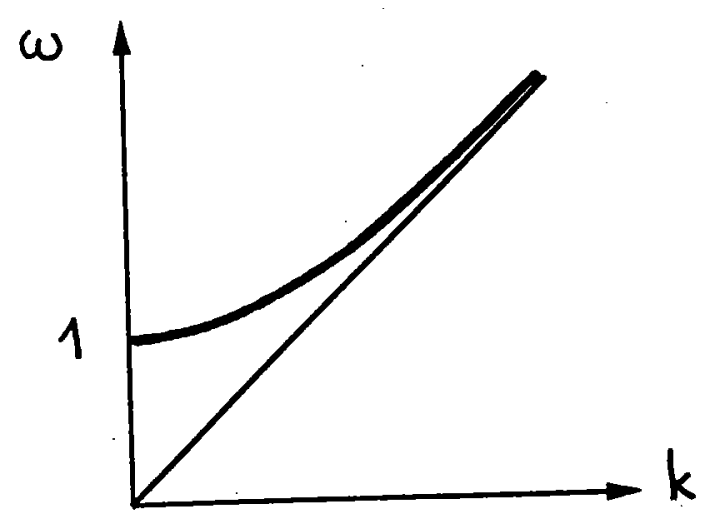

Figure 3.2. The dispersion relation for the wave packet described by equation (3.2).

The largest contribution to the integral in equation (3.2) will come from $k$ in the neighborhood of $k_{0}$ and we make the approximation

$$
u(x, t) \approx e^{i\left(\omega\left(k_{0}\right)-k_{0} x\right)} \int_{-\infty}^{\infty} F\left(k_{0}+K\right) e^{i(\Omega(K)-K x)} d K,
$$

where

$$
\begin{gathered}
\Omega(K)=U_{0} K+\frac{\omega_{0}^{\prime \prime} K^{2}}{2}+\cdots \\
U_{0}=U\left(k_{0}\right), \\
\omega_{0}^{\prime \prime}=\frac{d^{2} \omega}{d k^{2}}\left(k_{0}\right) .
\end{gathered}
$$


This gives us

$$
u(x, t) \approx A(x, t) e^{i\left(\omega_{0} t-k_{0} x\right)}+c . c .
$$

where the envelope is

$$
A(x, t)=\int_{-\infty}^{\infty} \hat{A}(K, t) e^{-i K x} d K
$$

and

$$
\hat{A}(K, t)=F\left(k_{0}+K\right) e^{i \Omega(K) t} .
$$

Now using (3.3) gives

$$
i \Omega \hat{A}=i U_{0} K \hat{A}+\frac{i \omega_{0}^{\prime \prime} K^{2} \hat{A}}{2}+\cdots
$$

Taking the inverse Fourier transform we see that this corresponds to

$$
\frac{\partial A}{\partial t}=-U_{0} \frac{\partial A}{\partial x}-\frac{i \omega_{0}^{\prime \prime}}{2} \frac{\partial^{2} A}{\partial x^{2}}
$$

Note that $A$ is slowly varying in space compared to $2 \pi / k_{0}$ since the wave packet is peaked around $k_{0}$. Thus it is clear from (3.5) that $A$ is also slowly varying in time compared to $2 \pi / \omega_{0}$ (where $\omega_{0}=\omega\left(k_{0}\right)$ ). Equation (3.5) describes the amplitude of a slowly varying wave. The first term on the right hand side represents the propagation of amplitude perturbations at the group velocity, and can be removed if we transform to a reference frame moving at $U_{0}$. We then get a Schrödinger equation

$$
\frac{\partial A}{\partial t}=i \alpha \frac{\partial^{2} A}{\partial x^{2}}
$$

where $\alpha=-\omega_{0}^{\prime \prime} / 2$ represents dispersion. It is easily seen that $\xi=x^{2} / 4 i \alpha t$ is a similarity variable and that $\int|A|^{2} d x$ is conserved. Thus, a self-similar solution is,

$$
A \propto(4 i \alpha t)^{-1 / 2} f(\xi)
$$

In particular, with an initial condition of the form

$$
A(x, 0) \propto \exp \left(-x^{2} / x_{0}^{2}\right)
$$

we have

$$
A(x, t) \propto\left(x_{0}^{2}+4 i \alpha t\right)^{-1 / 2} \exp \left[-x^{2} /\left(x_{0}^{2}+4 i \alpha t\right)\right],
$$

and we get a well-known result: dispersion causes the amplitude of the wave-packet to decrease like $t^{-1 / 2}$. This is valid for large $t$ and $x_{0}$, since $A$ is assumed to vary slowly in time and space. Notice that here the approximation is included at the stage of formulating the amplitude equation.

The next step is to include nonlinear terms in the evolution equation of the amplitude $A$. Assuming that these terms are monomials in $A$ and $\bar{A}$, we proceed using symmetry 
arguments in a similar way as we did for nonlinear oscillators. The Sine-Gordon equation (3.1) is invariant to translations in both time and space,

$$
t \rightarrow t+\theta, \quad x \rightarrow x+\phi
$$

and from (3.4) we see that this corresponds to

$$
A \rightarrow A e^{i \psi}
$$

where $\psi$ can vary through all reals. Considering all possible nonlinear terms, we find that the lowest order term with the right transformation property is $|A|^{2} A$. So we can write

$$
\frac{\partial A}{\partial t}=-U_{0} \frac{\partial A}{\partial x}-i \frac{\omega_{0}^{\prime \prime}}{2} \frac{\partial^{2} A}{\partial x^{2}}+\beta|A|^{2} A
$$

There are two further symmetries: time reversal and space reflection. In the general case these can be applied separately, but we have taken the particular form of $u$ given by (3.4) that consists only of waves propagating to the right, and this constrains us to applying both transformations together (see below for the general case).

Applying the symmetries together implies the invariance of the amplitude equation under the transformation

$$
t \rightarrow-t, \quad x \rightarrow-x \quad A \rightarrow \bar{A}
$$

and applying this to (3.6) gives

$$
-\frac{\partial \bar{A}}{\partial t}=U_{0} \frac{\partial \bar{A}}{\partial x}+i \alpha \frac{\partial^{2} \bar{A}}{\partial x^{2}}+\beta|A|^{2} \bar{A}
$$

However the complex conjugate of (3.6) is

$$
\frac{\partial \bar{A}}{\partial t}=-U_{0} \frac{\partial \bar{A}}{\partial x}-i \alpha \frac{\partial^{2} \bar{A}}{\partial x^{2}}+\bar{\beta}|A|^{2} \bar{A}
$$

Hence $\bar{\beta}=-\beta$, and $\beta$ is pure imaginary, so we can replace $\beta$ with $-i \beta$ in (3.6). If we also transform to a frame moving with the group velocity, $U_{0}$, we obtain

$$
\frac{\partial A}{\partial t}=i \alpha \frac{\partial^{2} A}{\partial x^{2}}-i \beta|A|^{2} A
$$

This is the nonlinear Schrödinger equation. It shows that the dynamics of the wave-packet consists of a balance between dispersion, $i \alpha A_{x x}$, and nonlinearity, $-i \beta|A|^{2} A$, that traces back in this problem to the amplitude dependence of the frequency of each oscillator. These points are illustrated by the particular solution

$$
\begin{gathered}
A=Q e^{i(\Omega t-q x)} \\
\Omega=-\alpha q^{2}-\beta Q^{2}
\end{gathered}
$$


which corresponds to shifting $\omega_{0} \rightarrow \omega_{0}+\Omega, k_{0} \rightarrow k_{0}+q$. Thus, nonlinearity and dispersion act in antagonistic ways if $\alpha \beta<0$.

We now derive the nonlinear Schrödinger equation from the Sine-Gordon equation using a multiple-scale expansion. Considering an initial condition which is a slowly modulated wave in space, we take as a small parameter the typical modulation wavenumber compared to the wavenumber of the carrier wave, thus

$$
\frac{\partial}{\partial x} \rightarrow \frac{\partial}{\partial x}+\epsilon \frac{\partial}{\partial X}
$$

As discussed above, we expect two characteristic timescales, one corresponding to the propagation of the wave envelope at the group velocity, and the other to the dispersion of the wave-packet, thus

$$
\frac{\partial}{\partial t} \rightarrow \frac{\partial}{\partial t}+\epsilon \frac{\partial}{\partial T_{1}}+\epsilon^{2} \frac{\partial}{\partial T_{2}}
$$

We need now to scale the oscillation amplitude $u\left(x, t, X, T_{1}, T_{2}\right)$ to be able to handle the nonlinear term of (3.1) perturbatively. There does not exist a correct scaling versus a wrong one. If the amplitude is scaled too small, we get to leading order an amplitude equation with only linear terms, which is correct; if it is scaled too large, we get nonlinear terms at a lower order than dispersion, which is also true if the amplitude is large. One generally considers that the most interesting situation consists of having both effects, nonlinearity and dispersion, at the same order in the amplitude equation; this fixes the scale for the amplitude, and

$$
u(x, t)=\epsilon\left[A\left(X, T_{1}, T_{2}\right) e^{i\left(\omega_{0} t-k_{0} x\right)}+c . c .\right]+\epsilon u_{1}\left(x, t, X, T_{1}, T_{2}\right)+\epsilon^{2} u_{2}\left(x, t, X, T_{1}, T_{2}\right)+\cdots
$$

At $O\left(\epsilon^{2}\right)$, the solvability condition is

$$
\frac{\partial A}{\partial T_{1}}=-U_{0} \frac{\partial A}{\partial X}
$$

which leads us to take $A \equiv A\left(X-U_{0} T_{1}, T_{2}\right)$. Then at $O\left(\epsilon^{3}\right)$, the solvability condition gives

$$
\frac{\partial A}{\partial T_{2}}=i \alpha \frac{\partial^{2} A}{\partial X^{2}}-i \beta|A|^{2} A
$$

with $\alpha=-\omega_{0}^{\prime \prime} / 2$ and $\beta=1 / 4 \omega_{0}$. Note that $\alpha \beta<0$ so that dispersion and nonlinearity are antagonistic. We have recovered the nonlinear Schrödinger equation (3.7). We could have taken two slow lengthscales and one slow timescale, and this would have resulted in a different form of the nonlinear Schrödinger equation which is widely used in nonlinear optics.

To deal with the general case we must consider both left and right-propagating waves, and begin the expansion with

$$
u=A e^{i\left(\omega_{0} t-k_{0} x\right)}+B e^{i\left(\omega_{0} t+k_{0} x\right)}+c . c .
$$


This leads to the two coupled amplitude equations

$$
\begin{gathered}
\frac{\partial A}{\partial t}=-U_{0} \frac{\partial A}{\partial x}-i \frac{\omega_{0}^{\prime \prime}}{2} \frac{\partial^{2} A}{\partial x^{2}}-i \beta|A|^{2} A-i \gamma|B|^{2} A, \\
\frac{\partial B}{\partial t}=U_{0} \frac{\partial B}{\partial x}-i \frac{\omega_{0}^{\prime \prime}}{2} \frac{\partial^{2} B}{\partial x^{2}}-\left.i \gamma|A-i \beta| B\right|^{2} B .
\end{gathered}
$$

Translational invariances in time and space constrain the form of the leading order nonlinear terms. Space reflection symmetry implies the invariance under the transformation

$$
x \rightarrow-x, \quad A \rightarrow B, \quad B \rightarrow A,
$$

and shows that the coefficients of the similar nonlinear terms should be the same in both equation. Time reversal symmetry implies

$$
t \rightarrow-t, \quad A \rightarrow \bar{B}, \quad B \rightarrow \bar{A}
$$

so that the coefficients of the nonlinear terms are pure imaginary. Note that one can also check that the coefficient of the propagative term is real whereas the one of the dispersive term is pure imaginary.

A slight problem arises if one tries to get these coupled equations with a multiple-scale expansion. Indeed, one cannot remove both propagative terms by transforming to a frame moving at the group velocity. It is straightforward to change the scaling of the amplitude in order to bring nonlinear terms at the same order as propagative ones, but dispersive terms are smaller, and one should not in principle keep them. This is obviously a bad choice since one does not expect dispersion to become negligible because of the presence of counterpropagating waves. The problem here is that one small adjustable parameter is not enough to balance all the relevant terms allowed by symmetries. One way out is to look for another small parameter, here obviously the group velocity, however this restricts our study to the case of small carrier wave frequency. Another way is to keep the dispersive terms; but should we scale amplitude as for the nonlinear Schrödinger equation, or in order to bring leading order nonlinear terms with propagative terms, and then keep higher order nonlinearities at the order of dispersive terms? The two different scalings might be relevant, one when the counter-propagating wave-packets are far apart, the other when they collide. There is perhaps no rigorous way to describe that situation with amplitude equations.

The nonlinear Schrödinger equation is the generic evolution equation that governs the complex amplitude of a nonlinear wave in dispersive media. It has been widely used to describe surface waves and light propagation in optical fibers. It should be modified in the vicinity of a caustic where the group velocity is stationary and correspondingly dispersive effects are small. It also occurs that the wave amplitude is coupled to a mean field. This is a general situation when there exists a neutral mode at zero wave number and we will discuss that later; as a simple example derive the nonlinear Schrödinger equation for the envelope of a quasi-monochromatic wave governed by the Korteweg-de Vries equation. Note also that when a conservative system undergoes a dispersive instability, such as the Kelvin-Helmholtz instability, the amplitude of the unstable waves is not governed by the nonlinear Schrödinger equation (see section 4 ). 


\subsection{The side-band or Benjamin-Feir instability}

We now use the nonlinear Schrödinger equation to study the stability of a quasi-monochromatic wave. The original motivation was to understand the instability of Stokes waves. When a wave train of surface gravity waves is generated with a paddle oscillating at constant frequency, one observes that if the fluid layer is deep enough compared to the wavelength, the quasi-monochromatic wave is unstable and breaks into a series of pulses.

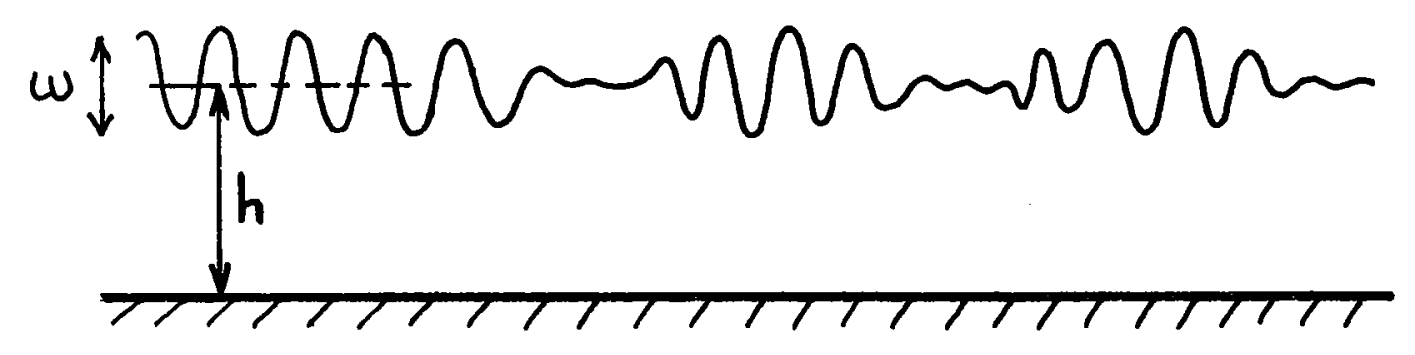

Figure 3.3. A deep layer of water, forced by an oscillating paddle, exhibits the side-band instability. Benjamin-Feir (1967), Lake et al. (1977), Melville (1982).

Return to the nonlinear Schrödinger equation (3.7),

$$
\frac{\partial A}{\partial t}=-i \alpha \frac{\partial^{2} A}{\partial x^{2}}-i \beta|A|^{2} A,
$$

and consider the particular solution

$$
A_{0}=Q e^{i \Omega t}, \quad \Omega=-\beta Q^{2},
$$

that represents a quasi-monochromatic wave of amplitude $Q$, wavenumber $k_{0}$ and frequency $\omega_{0}+\Omega$. If we perturb $A_{0}$ slightly, so that

$$
A=[Q+r(x, t)] e^{i[\Omega t+\theta(x, t)]}
$$

we obtain

$$
\frac{\partial r}{\partial t}+i \Omega(Q+r)+i(Q+r) \frac{\partial \theta}{\partial t}=-i \beta(Q+r)^{3}+i \alpha\left[\frac{\partial^{2} r}{\partial x^{2}}+2 i \frac{\partial r}{\partial x} \frac{\partial \theta}{\partial x}+i(Q+r) \frac{\partial^{2} \theta}{\partial x^{2}}-(Q+r)\left(\frac{\partial \theta}{\partial x}\right)^{2}\right] .
$$

Linearizing, and separating real and imaginary parts, gives

$$
\begin{gathered}
\frac{\partial r}{\partial t}=-\alpha Q \frac{\partial^{2} \theta}{\partial x^{2}} \\
\frac{\partial \theta}{\partial t}=-2 \beta Q r+\frac{\alpha}{Q} \frac{\partial^{2} r}{\partial x^{2}}
\end{gathered}
$$


Taking $\left(\begin{array}{l}r \\ \theta\end{array}\right) \propto e^{\eta t-i K x}$, one finds for the dispersion relation

$$
\left|\begin{array}{cc}
-\eta & \alpha Q K^{2} \\
-2 \beta Q-\alpha Q^{-1} K^{2} & -\eta
\end{array}\right|=0
$$

or

$$
\eta^{2}=-\left[2 \alpha \beta Q^{2} K^{2}+\alpha^{2} K^{4}\right],
$$

which is always negative for $\alpha \beta>0$, but has a positive region for $\alpha \beta<0$ as shown in figure (3.4). Thus if $\alpha \beta>0$ then $\eta$ is pure imaginary, and the quasi-monochromatic wave (3.4) is a stable solution.

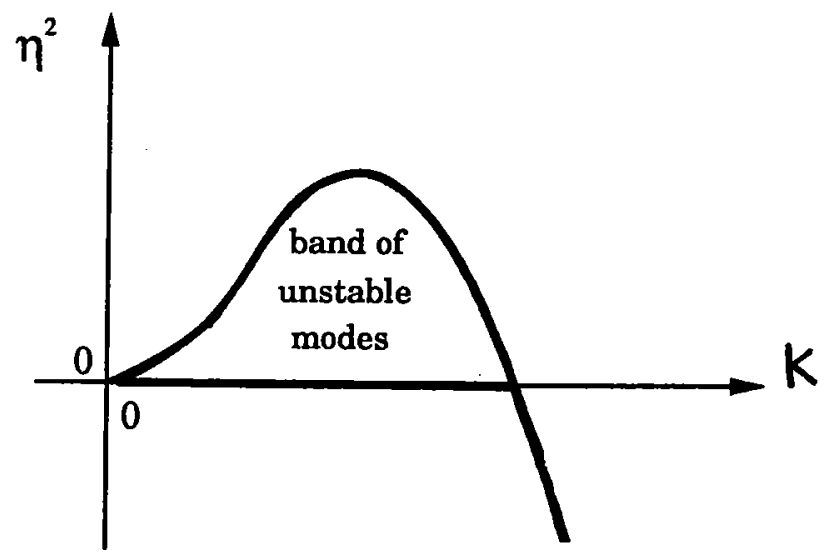

Figure 3.4. The dispersion relation for the perturbation to $A_{0}$

On the other hand if $\alpha \beta<0$ then in the long wavelength region, $\eta$ has both a negative and a positive root. When $\eta$ is positive, there is an instability, the Benjamin-Feir or side-band instability. It has the name "side-band" because if one takes a band of frequencies centered on $\omega_{0}$ as shown in figure (3.5), the interaction of one side-mode with the second harmonic is resonant with the other side-mode, causing it to be amplified, i.e. $2 \omega_{0}-\left(\omega_{0}-\Omega\right)=\omega_{0}+\Omega$. As an exercise, write the perturbation to $A_{0}$ as the sum of two side-band modes and see how their coupling generates the instability.

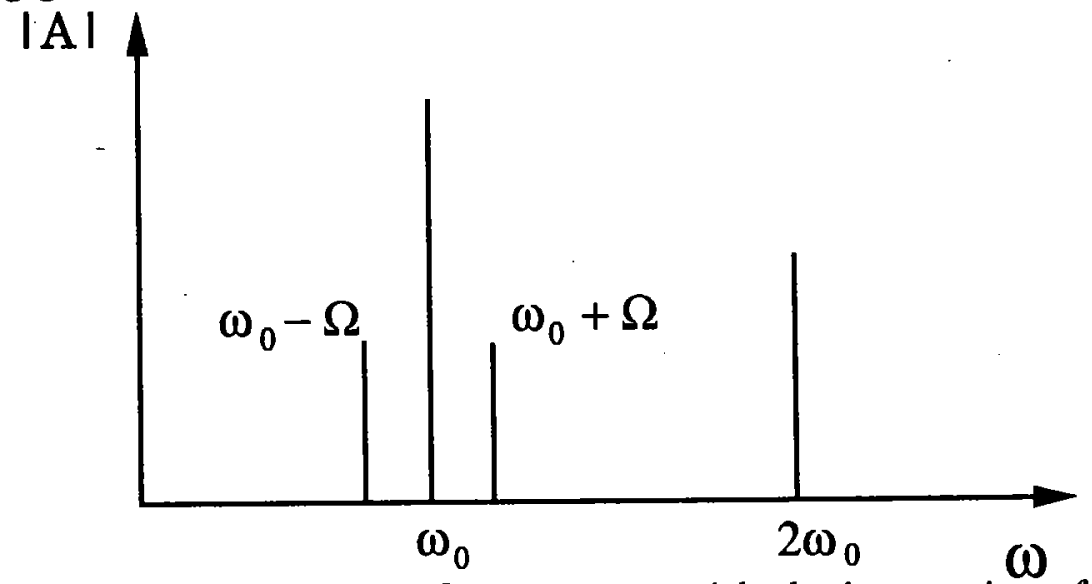

Figure 3.5. The mode at $\omega=\omega_{0}-\Omega$ is resonant with the interaction of the modes at $\omega_{0}+\Omega$ and $2 \omega_{0}$ 
Another way to understand this instability is to combine (3.8) and (3.9) and find

$$
\frac{\partial^{2} \theta}{\partial t^{2}}=2 \alpha \beta Q^{2} \frac{\partial^{2} \theta}{\partial x^{2}}-\alpha^{2} \frac{\partial^{4} \theta}{\partial x^{4}}
$$

So if $\alpha \beta<0$, the phase of the wave obeys an unstable propagation equation (the propagation velocity is imaginary).

Time reversal and space-reflection symmetries imply,

$$
t \rightarrow-t, x \rightarrow-x, \theta \rightarrow-\theta
$$

and thus determine the lowest order nonlinear term in the equation for the phase. The result is

$$
\frac{\partial^{2} \theta}{\partial t^{2}}=2 \alpha \beta Q^{2} \frac{\partial^{2} \theta}{\partial x^{2}}-\alpha^{2} \frac{\partial^{4} \theta}{\partial x^{4}}+g \frac{\partial \theta}{\partial x} \frac{\partial^{2} \theta}{\partial x^{2}}
$$

This is the Boussinesq equation. It has well-known solitary wave solutions consisting of a localized region with a non zero phase-gradient, thus a localized region with a different local wavenumber for the wave train. We consider in the next section these localized structures as solitary waves solutions of the nonlinear Schrödinger equation.

(Try this exercise: set $\theta=A_{1}(x, t) e^{i\left(\omega_{1} t-k_{1} x\right)}+c . c$. , in the Boussinesq equation, we would find that $A_{1}$ satisfies the nonlinear Schrödinger equation with coefficients depending on those of the nonlinear Schrödinger equation which was the ancestor of the Boussinesq equation. Derive the mapping between the old and new coefficients. Is there a fixed point? If yes, what does this would mean? Find other similar examples using symmetry arguments to guess the form of the successive equations.)

\subsection{Solitary waves}

Nonlinear wave equations sometimes have solitary wave solutions with locally concentrated amplitudes. These waves propagate without changing their shape owing to the balance between nonlinearity and dispersion. In this section, we discuss solitary wave solutions of the nonlinear Schrödinger equation.

\subsubsection{Solitary wave solutions in the Benjamin-Feir unstable regime}

First, we solve the nonlinear Schrödinger equation to get solitary wave solutions in the Benjamin-Feir unstable case, i.e. $\alpha \beta<0$. For simplicity, we select the parameters to be $\alpha=1$ and $\beta=-2$ with appropriate scalings of space and amplitude. Then the nonlinear Schrödinger equation is

$$
\frac{\partial A}{\partial t}=i \frac{\partial^{2} A}{\partial x^{2}}+2 i|A|^{2} A,
$$

We assume the form of the solution is $A_{s}(x, t)=R(x) \exp (i \Omega t)$. Substituting this into (3.10), we get

$$
R_{x x}=-\frac{\partial V}{\partial R}
$$


where the potential $V(R)$ is

$$
V(R) \equiv-\frac{1}{2} \Omega R^{2}+\frac{1}{2} R^{4}
$$

Figure 3.6 shows the profile of the potential $V(R)$. Equation (3.11) is the equation of motion of a particle in the potential $V(R)$ with $x$ considered as time and $R$ as the position of the particle. Multiplying by $R_{x}$ and integrating, we have

$$
\frac{1}{2} R_{x}^{2}+V(R)=E
$$

where $E$ is a constant. In the case of $E<0$, the solution of (3.12) corresponds to the periodic motion between $R_{1}<R<R_{2}$ in figure 3.6. Thus, we get periodic solutions with respect to $x$ as shown in figure 3.7. These solutions are called cnoidal waves. In the case of $E=0$, the solution corresponds to the motion of the particle which starts with $R=0$ at $t \rightarrow-\infty$, reaches $R=R_{0}$ and returns to $R=0$ as $t \rightarrow \infty$. Therefore, we get a solitary wave solution whose profile tends to zero as $x \longrightarrow \pm \infty$. There is a simple analytic form for this special case:

$$
A_{s}=\sqrt{\Omega} \operatorname{sech}(\sqrt{\Omega} x) \exp (i \Omega t)
$$

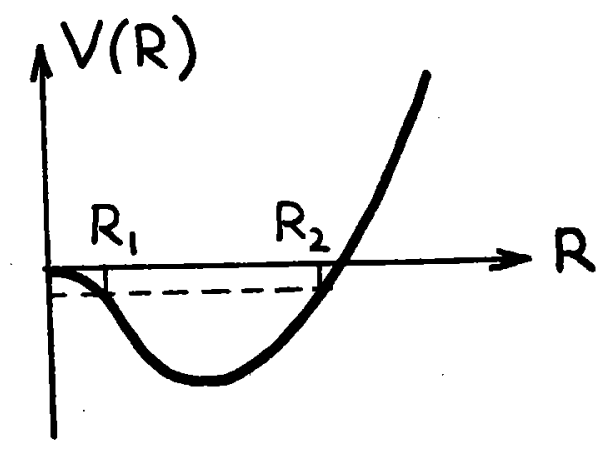

Figure 3.6. The potential $V(R)$

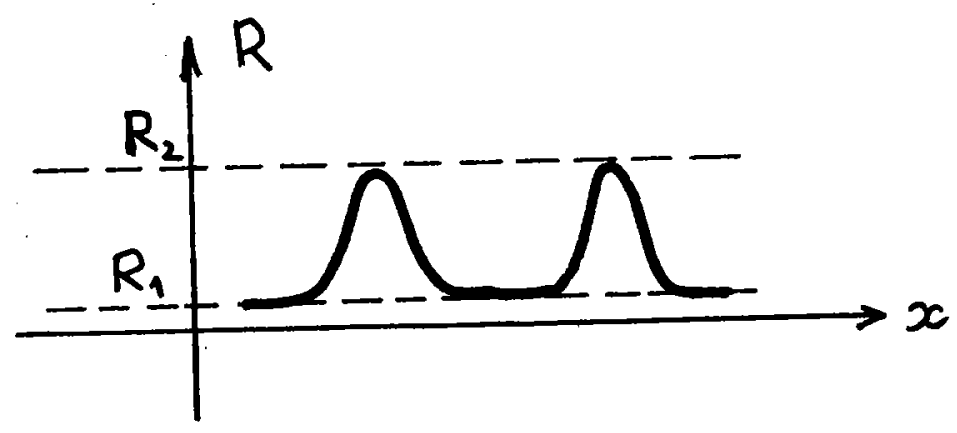

Figure 3.7. (a) Cnoidal wave $(E<0)$ 


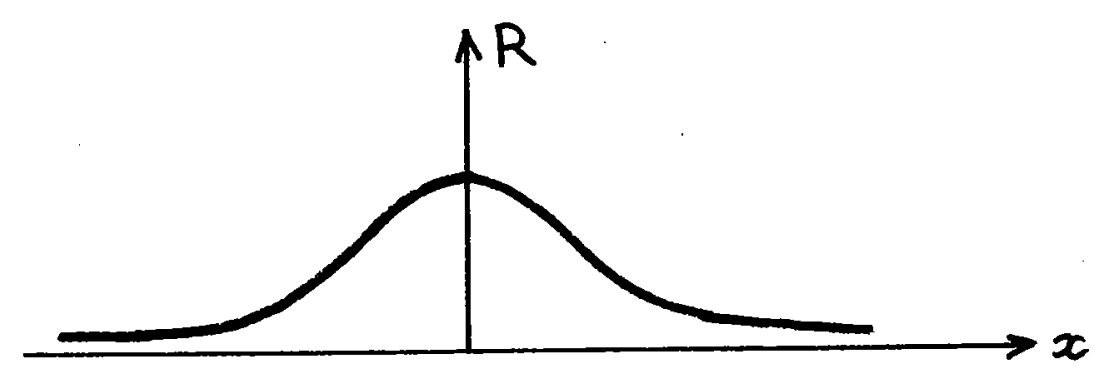

Figure 3.8. (b) Solitary wave $(E=0)$

\subsubsection{Symmetries and other solitary wave solutions}

By symmetry argument, we can derive other solitary waves from one simple solitary wave. The nonlinear Schrödinger equation has the following symmetry properties,

$$
\left\{\begin{array}{l}
x \longrightarrow \lambda x \\
t \longrightarrow \lambda^{2} t \\
A \longrightarrow \lambda^{-1} A
\end{array}\right.
$$

Applying this symmetry to the simple solitary wave solution $A_{s 1}=\operatorname{sech} x \exp (i t)$, we have

$$
A_{s 2}=\sqrt{\Omega} \operatorname{sech}(\sqrt{\Omega} x) \exp (i \Omega t)
$$

Further, using another symmetry

$$
\left\{\begin{array}{l}
x \longrightarrow x+v t \\
A \longrightarrow A \exp \left(-i \frac{v}{2} x+i \frac{v^{2}}{4}\right) t
\end{array}\right.
$$

we get a further solitary wave solution,

$$
A_{s 3}=\sqrt{\Omega} \operatorname{sech}[\sqrt{\Omega}(x+v t)] \exp i\left[\frac{1}{2} v x+\left(\Omega-\frac{v^{2}}{4}\right) t\right] .
$$

One can also consider translational invariance in space, thus replacing $x$ by $x-x_{0}$, and rotational invariance in the complex plane that leads to an arbitrary phase factor in $A$. The important point to notice is that continuous families of solutions are associated with the invariance properties of the evolution equation. We will use this later to study the dynamics of localized structures.

\subsubsection{Solitary wave solutions in the Benjamin-Feir stable regime}

Next we consider solitary wave solutions in the Benjamin-Feir stable case, i.e. $\alpha \beta>0$. For simplicity, we select the parameters to be $\alpha=1$ and $\beta=2$.

$$
\frac{\partial A}{\partial t}=i \frac{\partial^{2} A}{\partial x^{2}}-2 i|A|^{2} A
$$


We assume the form of the solution is $A_{s}=R(x) \exp (i \Omega t+\theta(x))$. Substituing it into (3.13), we get

$$
\begin{gathered}
\Omega R=-2 R^{3}+R_{x x}-R \theta_{x}^{2}, \\
0=-2 R_{x} \theta_{x}-\theta_{x x} R=-\left(R^{2} \theta_{x}\right)_{x}
\end{gathered}
$$

We can eliminate $\theta$ by integrating (3.15) and substituting into (3.16),

$$
\begin{aligned}
R_{x x} & =-\frac{\partial V}{\partial R}, \\
V(R) & =\frac{1}{2}\left(-\Omega R^{2}-R^{4}+\frac{h^{2}}{R^{2}}\right),
\end{aligned}
$$

where $h \equiv R^{2} \theta_{x}$ is constant.

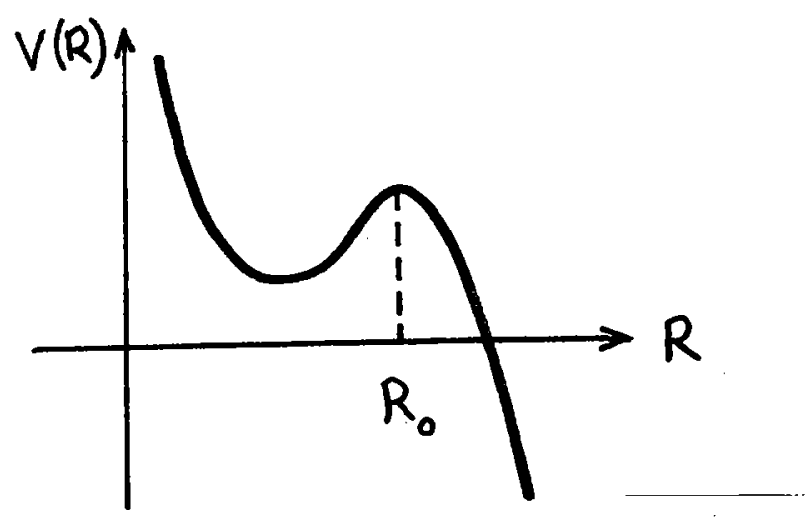

Figure 3.9. The potential $V(R)$

In the same manner as for the Benjamin-Feir unstable case, we can find the solution by selecting the maximum value of the potential (figure 3.9),

$$
R^{2}=R_{0}^{2}-\frac{a^{2}}{\cosh ^{2} a x}
$$

where $R=R_{0}$ gives the maximum of $V(R)$, and the parameters are

$$
\Omega=-3 R_{0}^{2}+a^{2} \quad h^{2}=R_{0}^{4}\left(R_{0}^{2}-a^{2}\right) .
$$

This solution is called an "envelope hole soliton", or a "dark soliton" in optics since it consists of a region with a smaller oscillation amplitude. Note that the local wavenumber is changes according to the relation $R^{2} \theta_{x}=$ constant. 


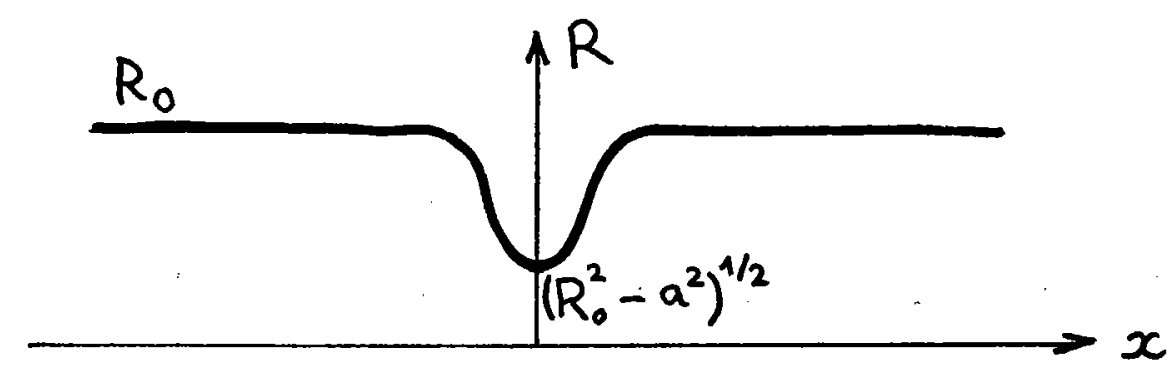

Figure 3.10. An envelope hole soliton or dark soliton

In particular, when we choose $h=0, a=R_{0}$, we have

$$
R^{2}=R_{0}^{2} \tanh ^{2} R_{0} x .
$$

In the pendulum model, this represents a non-oscillating location that separates two regions that oscillate out of phase.

\section{References}

Benjamin, T. B. and Feir, J. E. (1967) The disintegration of wave trains on deep water, J. Fluid Mech. 27, 417-430.

Lake, B.M., Yuen, M.C., Rungaldier, M., and Ferguson, W.E. (1977) Nonlinear deep-water waves: theory and experiment. Part 2. Evolution of a continuous wave train, J. Fluid Mech. 83, 49-74.

Melville, W.K. (1982) The instability and breaking down of deep-water waves, J. Fluid Mech. 115, 165-185.

Newell, A. C. (1985) Solitons in Mathematics and Physics, SIAM

Stuart, J. T. and Di Prima, R. C. (1978) The Eckhaus and Benjamin-Feir resonance mechanisms, Proc. Roy. Soc. London A 362, 27-41.

Whitham, G.B.(1974) Linear and Nonlinear Waves Wiley 


\section{Cellular Instabilities: A canonical example, Rayleigh- Bénard convection}

Various fluid flows display instabilities that generate cellular structures. Examples are the Couette-Taylor flow, Rayleigh-Bénard convection, the Faraday instability and many shear flow experiments. The fluid can sometimes assumed to be inviscid but we will first consider situations where dissipation cannot be neglected. These "dissipative instabilities" are described by simpler amplitude equations, in particular when they generate stationary patterns, such as convective rolls for instance. A canonical example of stationary cellular instability is Rayleigh-Bénard convection that we will study in this section.

\subsection{Rayleigh-Bénard convection}

\subsubsection{Convection in the Rayleigh-Bénard geometry}

Thermal convection widely occurs in geophysical and astrophysical flows: in the earth's mantle, it is responsible for the motion of tectonic plates; in the earth's core, it generates the earth magnetic field by dynamo effect; in the sun or other stars, it is the advection mechanism for the energy generated in the core. Thermal convection has been also studied extensively in laboratory experiments, both for engineering purposes and also as one of the simplest example of hydrodynamic instability that displays pattern formation and transition to turbulence." There exist many reviews about thermal convection, for instance, Spiegel (1971, 1972), Palm (1975), Normand et al. (1977), Busse (1978, 1981); the reader may also look at the book by Gershuni and Zhukovitskii (1976).

Convection in the Rayleigh-Bénard geometry is achieved by uniformly heating from below a horizontal layer of fluid (Figure 4.1). For small temperature gradients, the fluid remains in a stable heat-conducting state, with a linear temperature profile and no fluid motion. However, if the fluid has a negative thermal expansion coefficient, $-\alpha$, the thermal gradient generates a density stratification with cold heavy fluid above warm light fluid. For sufficiently large temperature differences, the resulting buoyancy force overcomes dissipative effects due to viscosity and heat diffusivity, causing less dense warmer fluid to rise and cooler fluid to sink. With appropriate boundary conditions, periodic parallel convection rolls result from the circulation of the fluid. 


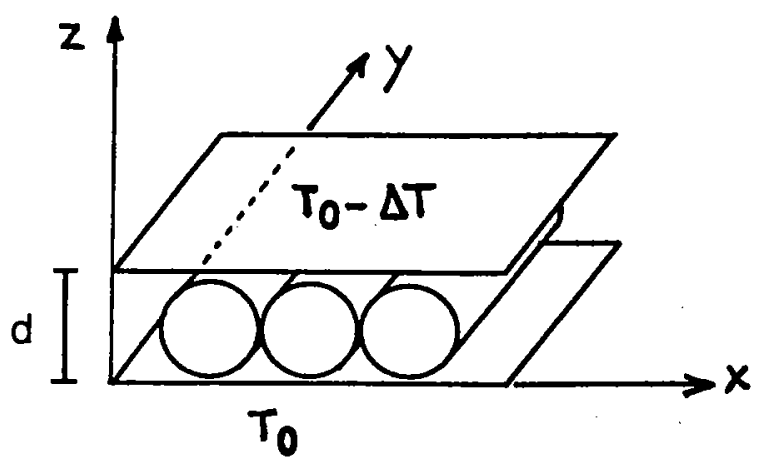

Figure 4.1. Sketch of a Rayleigh-Bénard experiment

At the onset of convection, any field $u(x, y, z, t)$ representing the state of the system, i.e. one of the velocity components, the temperature fluctuation from the heat-conduction profile or the pressure fluctuation from hydrostatic equilibrium, takes the form

$$
u(x, y, z, t)=\left[A(X, Y, T) \exp \left(i k_{c} x\right)+\text { c.c. }\right] f(z)+\cdots
$$

This represents periodic convection rolls perpendicular to the $\mathrm{x}$-axis with a slowly varying complex amplitude $A(X, Y, T)$. The modulus of $A$ accounts for the convection amplitude whereas the phase of $A$ is related to the local wavenumber difference from its critical value, $k_{c}$. The vertical structure of the convection mode is described by $f(z)$ and depends on the boundary conditions at the lower and upper plates (see below).

Our objective is to find the amplitude equation, i.e. the evolution equation for $A$, and to use it to describe pattern dynamics near the onset of convection.

\subsubsection{The Boussinesq approximation}

The Boussinesq approximation is reasonably accurate in usual experimental situations. In this approximation, the fluid behaves as though it were incompressible, the density varying only as a consequence of changes in temperature; the density variation about its mean value is taken into account only in the buoyancy force term; the mechanical dissipation rate is neglected in the heat equation, and the fluid parameters, viscosity, heat diffusivity and heat capacity are assumed to be constant. These a priori physical assumptions can be replaced by a rigorous asymptotic expansion of the conservation equations of mass, momentum and energy (see the review by Malkus, 1964); the resulting Boussinesq equations are,

$$
\begin{gathered}
\nabla \cdot \mathbf{v}=0 \\
\rho_{0}\left[\frac{\partial \mathbf{v}}{\partial t}+(\mathbf{v} \cdot \nabla) \mathbf{v}\right]=-\nabla p+\rho_{0} \nu \nabla^{2} \mathbf{v}-\rho(T) g \hat{\mathbf{z}} \\
\frac{\partial T}{\partial t}+\mathbf{v} \cdot \nabla T=\kappa \nabla^{2} T
\end{gathered}
$$


where $\nu$ is the fluid kinematic viscosity and $\kappa$ is the heat diffusivity; $\rho_{0}$ is the fluid density at a reference temperature and $T$ is the temperature difference from that reference. Thus,

$$
\rho(T) \approx \rho_{0}(1-\alpha T)
$$

Defining $\theta$ as the temperature fluctuation from the heat-conducting profile,

$$
T=T_{0}-\frac{\Delta T}{d} z+\theta
$$

where $\Delta T$ is the temperature difference across the layer of height $d$, and using $d, d^{2} / \kappa$ and $\Delta T$ as scales for length, time and temperature, one get

$$
\begin{gathered}
\nabla \cdot \mathbf{v}=0 \\
\frac{\partial \mathbf{v}}{\partial t}+(\mathbf{v} \cdot \nabla) \mathbf{v}=-\nabla \pi+P \nabla^{2} \mathbf{v}+R P \theta \hat{\mathbf{z}} \\
\frac{\partial \theta}{\partial t}+\mathbf{v} \cdot \nabla \theta=\mathbf{v} \cdot \hat{\mathbf{z}}+\nabla^{2} \theta
\end{gathered}
$$

where $P=\nu / \kappa$ is the Prandtl number, and $R=g \alpha \Delta T d^{3} / \nu \kappa$ is the Rayleigh number. These two dimensionless numbers, together with the boundary conditions, characterize the convection problem in the Boussinesq approximation. Let us mention that the small or large Prandtl number or large Rayleigh number limits of equations $(4.6,4.7 ., 4.8)$ are usually considered without any caution in the literature, although these limits might invalidate the Boussinesq approximation.

The Prandtl number is the ratio of the timescales of the two diffusive processes involved in convection, heat diffusion and momentum diffusion. Depending on the microscopic mechanisms of transport, the Prandtl number varies over many orders of magnitude in different convective flows of interest.

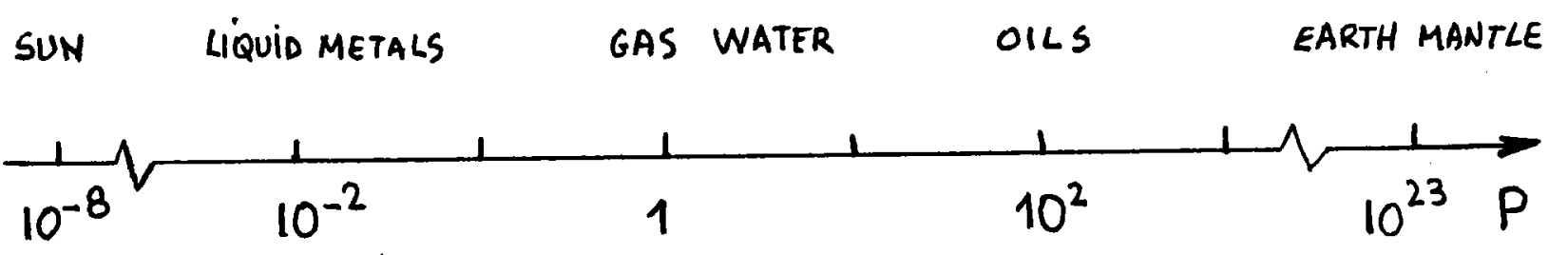

Figure 4.2. Typical values of the Prandtl number 
The Rayleigh number is proportional to the temperature difference across the fluid layer, and relates the strength of the driving mechanism to dissipative processes. It is the control parameter in a convection experiment.

\subsubsection{Boundary Conditions}

We need now to specify the boundary conditions. We consider a fluid layer of infinite horizontal extent or periodic lateral boundary conditions. At the upper and lower boundaries, the temperature and the heat flux are assumed to be continuous. There exist two simple limit situations:

- boundaries with high heat conductivity

$$
\left.\theta\right|_{B}=0
$$

- insulating boundaries

$$
\left.\frac{\partial \theta}{\partial z}\right|_{B}=0
$$

Depending on the nature of the boundaries, the boundary condition for velocity can be either "no-slip" or "stress-free". If the boundary is a rigid plate, the "no-slip" boundary condition is applicable for viscous fluids, i.e.

$$
\left.\mathbf{v}\right|_{B}=0 .
$$

We separate the velocity into horizontal and vertical components, $\mathbf{v}=\mathbf{v}_{h}+w \hat{\mathbf{z}}$, where $\mathbf{v}_{h}$ is the horizontal velocity, and $w$ is the $z$ component. Since $\mathbf{v}_{h}$ must vanish identically at $z=z_{B}$, it's horizontal derivatives also vanish at $z=z_{B}$. Using $\nabla \cdot \mathbf{v}=0$, we then have

$$
\left.w\right|_{B}=0,\left.\quad \frac{\partial w}{\partial z}\right|_{B}=0 .
$$

If the boundary is an interface with another fluid or a free surface open to the air, boundary conditions have to account for the continuity of both the normal velocity $w$ and of the tangential stress in the plane of the interface. Assuming that surface tension effects are not involved and that the interface remains flat, then we have the following "stress-free" boundary conditions:

$$
\left.w\right|_{B}=0,\left.\quad \frac{\partial \mathbf{v}_{h}}{\partial z}\right|_{B}=0 .
$$

Again using the incompressibility condition $\nabla \cdot \mathbf{v}=0$, we get

$$
\left.w\right|_{B}=0,\left.\quad \frac{\partial^{2} w}{\partial z^{2}}\right|_{B}=0 .
$$

One example of a "stress-free" experimental boundary condition consists of a convection layer of oil sandwiched between layers of mercury and gaseous helium (Goldstein and Graham, 1969). The "stress-free" boundary conditions can be applied since the viscosity of oil is much larger than the one of mercury or helium, and the temperature fluctuation should be zero 
at the oil-mercury interface, while the fluctuation heat flux should be zero at the oil-helium interface.

Finally, let us mention the crucial effect of temperature boundary conditions on the convective regime observed at onset. As buoyancy is the driving mechanism, the length scale of the convection pattern is primarily fixed by the characteristic scale for the temperature disturbances. In the case of boundaries with a high heat-conductivity compared to the one of the fluid, the temperature disturbances should vanish on the boundaries and the relevant length scale is the height of the layer $d$.

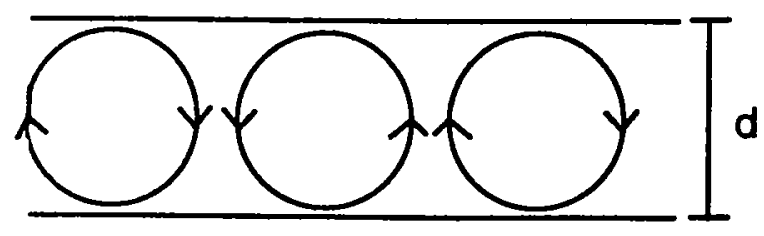

Figure 4.3. Convective regime at onset with boundaries of high heat conductivity

For insulating boundaries, the isotherms can penetrate into the boundaries and the temperature can vary on a very large length scale compared to $d$. The pattern wavelength goes to infinity in the insulating limit, i.e. there is only one roll in the fluid container (figure 4.4).

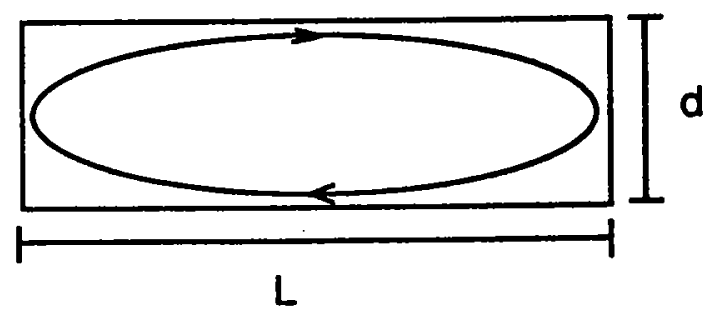

Figure 4.4. Convective regime at onset with insulating boundaries 


\subsection{Linear Stability Analysis}

The linear stability of the motionless heat-conducting state can be studied analytically using the Boussinesq equations $(4.6,4.7,4.8)$ with stress-free (4.12) and perfectly conducting (4.9) boundary conditions.

We first eliminate the pressure field by applying the operators curl and curl curl to the momentum equation (4.7) and we get the evolution equations for the vertical vorticity, $\zeta$, and the vertical velocity, $w$, by projecting on the vertical axis:

$$
\begin{gathered}
\frac{\partial \zeta}{\partial t}+\hat{\mathbf{z}} \cdot \nabla \times[(\mathbf{v} \cdot \nabla) \mathbf{v}]=P \nabla^{2} \zeta, \\
\frac{\partial}{\partial t} \nabla^{2} w-\hat{\mathbf{z}} \cdot \nabla \times \nabla \times[(\mathbf{v} \cdot \nabla) \mathbf{v}]=P \nabla^{4} w+R P \nabla_{h}^{2} \theta,
\end{gathered}
$$

where $\nabla_{h}^{2}$ stands for the Laplacian operator in the horizontal plane. Note that, at the linear stage, the vertical vorticity decouples and obeys a diffusion equation. Thus, the vertical vorticity modes can be ignored in the linear stability analysis; however, they should be kept in the study of finite amplitude convection since they are nonlinearly coupled to the linear convection modes. Neglecting the nonlinear terms in equations (4.14) and (4.8) yields the coupled linearized system for $w$ and $\theta$.

$$
\begin{array}{r}
\frac{\partial}{\partial t} \nabla^{2} w=P \nabla^{4} w+R P \nabla_{h}^{2} \theta, \\
\frac{\partial \theta}{\partial t}=w+\nabla^{2} \theta .
\end{array}
$$

From the requirement of spatial periodicity in the horizontal plane, we consider a normal mode of the disturbances $(w, \theta)$ of the form

$$
\begin{array}{r}
w(x, y, z, t)=W(z) \exp [i \mathbf{k} \cdot \mathbf{r}+\sigma t] \\
\theta(x, y, z, t)=\Theta(z) \exp [i \mathbf{k} \cdot \mathbf{r}+\sigma t]
\end{array}
$$

where $\mathbf{r}$ is the position vector in the horizontal plane, and $\mathbf{k}$ is the pattern (horizontal) wavevector. Boundary conditions $(4.9,4.12)$ together with equations $(4.15)$ require that $W$ and all its even derivatives vanish for $z=0$ and $z=1$. It follows that

$$
W(z)=W_{0} \sin n \pi z \quad \text { with } \quad n=0,1, \ldots
$$

Using (4.15), (4.16) and (4.17) we obtain the dispersion relation for the growth rate $\sigma$ of the normal mode $k$

$$
\sigma^{2}+q_{n}^{2}(1+P) \sigma+\left(P q_{n}^{4}-\frac{R P k^{2}}{q_{n}^{2}}\right)=0
$$

where $q_{n}^{2}=k^{2}+n^{2} \pi^{2}$. 
A stationary instability occurs when the constant term in $\sigma$ of the dispersion relation vanishes and becomes negative. Thus, as the Rayleigh number is increased, a mode with $n=1$ bifurcates first for $R=R_{c}(k)$ with

$$
R_{c}(k)=\frac{\left(\pi^{2}+k^{2}\right)^{3}}{k^{2}}
$$

This defines the marginal stability curve on which a mode with $n=1$ and horizontal wavenumber $k$ has a zero growth rate (Figure 4.5 ).

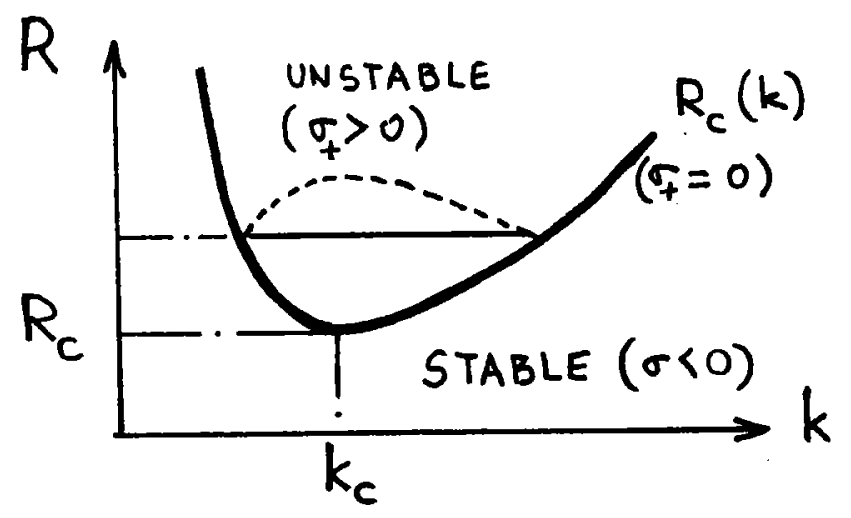

Figure 4.5. The marginal stability curve $R_{c}(k)$; the dashed curve represents the growth rate of the unstable modes for $R>R_{c}$.

The critical Rayleigh number $R_{c}$ and the critical wavenumber $k_{c}$ at convection onset correspond to the minimum of the marginal stability curve (4.19),

$$
R_{c}=\frac{27 \pi^{4}}{4}, \quad k_{c}=\frac{\pi}{\sqrt{2}}
$$

These critical values depend on the boundary conditions; in particular, as said above, $k_{c}$ vanishes in the limit of thermally insulating upper and lower boundaries.

Slightly above criticality we expand the positive solution $\sigma_{+}$of the dispersion relation (4.18) and get the growth rate of the unstable modes

$$
\sigma_{+}(R, k) \approx\left(\pi^{2}+k_{c}^{2}\right) \frac{P}{1+P} \frac{R-R_{c}(k)}{R_{c}(k)}
$$

which is proportional to the distance from criticality. Using

$$
R_{c}(k)=R_{c}+\frac{\left(k-k_{c}\right)^{2}}{2}\left(\frac{\partial^{2} R_{c}}{\partial k^{2}}\right)_{c}+\cdots
$$

we get to leading order in $R-R_{c}$ and $k-k_{c}$,

$$
\sigma_{+}(k, R)=\left(\pi^{2}+k_{c}^{2}\right) \frac{P}{1+P} \frac{\left(R-R_{c}\right)}{R_{c}}-\alpha\left(k-k_{c}\right)^{2}+\cdots,
$$


with

$$
\alpha=\left(\pi^{2}+k_{c}^{2}\right) \frac{P}{1+P} \frac{1}{2 R_{c}}\left(\frac{\partial^{2} R_{c}}{\partial k^{2}}\right)_{c} .
$$

Note that the expansion of $\sigma_{+}(k, R)$ does have a term proportional to $\left(R-R_{c}\right)\left(k-k_{c}\right)$, but this is of higher order than both the terms in (4.20). Specifically, because the marginal stability curve is locally a parabola close to its minimum, we have $\left(k-k_{c}\right) \sim\left(R-R_{c}\right)^{1 / 2}$. Thus, for $R$ larger than $R_{c}$, there exists a band of unstable modes with growth rates determined by equation (4.20) (Figure 4.5).

Linear analysis gives the critical Rayleigh number $R_{c}$ for instability onset and determines the modulus $k_{c}$ of the critical wavevector $\mathbf{k}$ of the unstable modes. The direction of $\mathbf{k}$ is arbitrary; this orientational degeneracy is obviously related to the isotropy in the horizontal plane. There is also a translational degeneracy which is related to the translational invariance of the layer of infinite horizontal extent. These degeneracies do not result from the linear approximation but from the symmetries of the Rayleigh-Bénard geometry; thus, they will persist in the nonlinear analysis. On the other hand, there is a pattern degeneracy that results from the linear approximation; indeed, any superposition of normal modes

$$
w(\mathbf{r}, z)=\sum_{p} c_{p} \exp \left(i \mathbf{k}_{p} \cdot \mathbf{r}\right)
$$

with $\left|\mathbf{k}_{p}\right|=k_{c}$ and where the $c_{p}$ 's are constant coefficients, is a solution of the linear problem with a zero growth rate at criticality. In order to represent a real field $w$, we must impose the conditions, $c_{-p}=\bar{c}_{p}$ and $\mathbf{k}_{-p}=-\mathbf{k}_{p}$, but the number of non zero $c_{p}$ 's, i.e. the shape of the pattern, and their modulus, i.e. the amplitude of the convection velocity, remain undeterminate. Three basic examples of the cellular pattern described by (4.22), that involve respectively one, two and three wavevectors, are sketched in Figure 4.6.
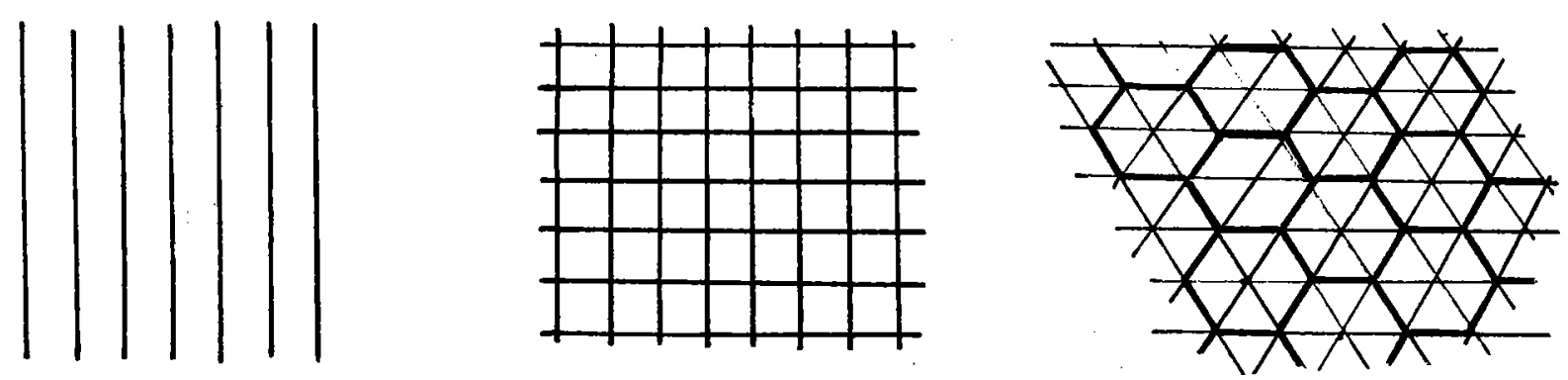

Figure 4.6. Rolls, squares and hexagons 
Nonlinear interactions between the modes with different wave vectors generally select one pattern at instability onset and determine the amplitude above criticality. However, it happens sometimes that no stationary pattern exists even immediately above a stationary instability onset; the nonlinear regime is then time-periodic or chaotic.

Another problem results from the existence of a continuous band of unstable modes above criticality as described by equation (4.20). Linear analysis only determines the one with the highest growth rate, but the wavenumber selected by nonlinear interactions may correspond to a different one. The interaction of two (or several) modes within the unstable band gives rise to a spatial modulation of the periodic pattern on a large length scale compared to the pattern wavelength. The inverse of this length scale is of order $\left(k-k_{c}\right)$, thus within a multiple-scale expansion procedure, it corresponds to a "slow scale" $X$ such that

$$
X=\left(R-R_{c}\right)^{1 / 2} x
$$

Let us recall, that close to the instability onset, the slow time scale $T$ that corresponds to the vanishing growth rate of the unstable mode $(4.20)$ is

$$
T=\left(R-R_{c}\right) t
$$

\subsection{Nonlinear saturation of the critical modes}

\subsubsection{Nonlinear saturation of a roll pattern: the Landau equation}

We first show how nonlinear terms saturate the amplitude of the convection velocity of a roll pattern above $R_{c}$. We use a stream function, $\psi(x, z, t)$, and write the velocity field

$$
\mathbf{v}=\left(-\partial_{z} \psi, 0, \partial_{x} \psi\right)
$$

Equations $(4.6,4.7,4.8)$ become

$$
\begin{aligned}
\frac{\partial}{\partial t} \nabla^{2} \psi+J\left(\psi, \nabla^{2} \psi\right) & =P \nabla^{4} \psi+R P \frac{\partial \theta}{\partial x} \\
\frac{\partial \theta}{\partial t}+J(\psi, \theta) & =\frac{\partial \psi}{\partial x}+\nabla^{2} \theta
\end{aligned}
$$

where $J$ is the Jacobian,

$$
J(f, g) \equiv \frac{\partial f}{\partial x} \frac{\partial g}{\partial z}-\frac{\partial f}{\partial z} \frac{\partial g}{\partial x} .
$$

Assuming stress-free perfectly conducting boundary conditions at $z=0,1$, the marginal mode that describes rolls perpendicular to the $x$-axis for $R=R_{c}$ reads

$$
\begin{array}{r}
\psi(x, z)=\left[A \exp \left(i k_{c} x\right)+c . c .\right] \sin \pi z \\
\theta(x, z)=\frac{k_{c}}{k_{c}^{2}+\pi^{2}}\left[i A \exp \left(i k_{c} x\right)-c . c .\right] \sin \pi z .
\end{array}
$$


The problem is to determine how the convection amplitude, $|A|$, saturates above criticality because of nonlinear interactions. This has been shown by Gorkov (1957) and Malkus and Veronis (1958) with a Poincaré-Lindstedt expansion. We will use a multiple-scale expansion, which is only slightly different, in order to keep the time-dependence of $A$. We don't consider in this section a possible modulation of $A$ on a slow length scale (see section 4.3.3).

We expand $\psi$ and $\theta$

$$
\psi=\sum_{n=1} \epsilon^{n} \psi_{n}, \quad \theta=\sum_{n=1} \epsilon^{n} \theta_{n}
$$

where $\epsilon$ is a small parameter related to the distance to criticality by

$$
R=R_{c}+\sum_{n=1} \epsilon^{n} R_{n}
$$

The Boussinesq equations have a symmetry: $z \rightarrow-z, \psi \rightarrow-\psi$, and $\theta \rightarrow-\theta$. Thus to leading order, $R_{1}=0$, and

$$
R-R_{c} \approx \epsilon^{2} R_{2}
$$

Using the result of the linear theory for the growth rate of the unstable mode, we obtain for the convection mode time scale

$$
\partial_{t}=\epsilon^{2} \partial_{T}
$$

To leading order in $\epsilon$, equations $(4.25,4.26)$ give the linear problem, and the solutions for $\psi_{1}$ and $\theta_{1}$ are given by the linear modes (4.27). To the next order we get,

$$
\begin{array}{r}
\psi_{2}=0 \\
\theta_{2}=-\frac{k_{c}^{2}}{2 \pi\left(k_{c}^{2}+\pi^{2}\right)} \sin 2 \pi z
\end{array}
$$

that describes how nonlinear temperature advection deforms the vertical temperature profile. The solvability condition at the next order gives the evolution equation for $A$,

$$
\frac{d A}{d T}=\mu A-\beta|A|^{2} A
$$

with

$$
\begin{array}{r}
\mu=\left(\pi^{2}+k_{c}^{2}\right) \frac{P}{1+P} \frac{\left(R-R_{c}\right)}{\epsilon^{2} R_{c}}, \\
\beta=\frac{k_{c}^{2}}{2} \frac{P}{1+P} .
\end{array}
$$

Note that, since $R-R_{c}$ is of order $\epsilon^{2}$, all the terms of the amplitude equation (4.28) are of order one. However, we can easily write this equation using original unscaled variables for time, amplitude and distance to criticality, $R-R_{c}$; we can check on the unscaled form that all terms are of order $\left(R-R_{c}\right)^{3 / 2}$.

As previously shown for nonlinear oscillators, the form of the amplitude equation is here also determined by symmetry constraints. Translational invariance in the horizontal plane 
implies that, if $\left(\psi_{0}(x, z), \theta_{0}(x, z)\right)$ represents a roll solution, $\left(\psi_{0}\left(x+x_{0}, z\right), \theta_{0}\left(x+x_{0}, z\right)\right)$ is another solution; this amounts to shift the rolls in the horizontal plane, or to a change in the origin of the $x$-axis. From equation (4.27) this transformation corresponds to a rotation in the complex plane for $A$, and so the amplitude equation should be invariant under the transformation

$$
A \rightarrow A \mathrm{e}^{i \phi}, \text { for any real } \phi .
$$

As shown for nonlinear oscillators, the only allowed nonlinear term up to third order in amplitude is thus $|A|^{2} A$, and the amplitude equation reads

$$
\frac{d A}{d T}=\mu A-\beta|A|^{2} A,
$$

where $\beta$ is a priori a complex number.

However, there is here an additional symmetry, space-reflection: $x \rightarrow-x$. From equation (4.27) this transformation corresponds to

$$
A \rightarrow \bar{A}
$$

for the complex amplitude. Taking the complex conjugate of the amplitude equation, applying the reflection transformation and comparing to the original amplitude equation, gives $\beta=\bar{\beta}$, thus $\beta$ is real. The form of the amplitude equation (4.28) is determined by symmetry constraints. The perturbative calculation starting from the Boussinesq equations is only useful to get the sign of $\beta$ and shows that the bifurcation is supercritical. This can be also shown using variational methods (Sorokin, 1953), and for a large variety of boundary conditions, the motionless state is globally stable below $R_{c}$ in the Boussinesq approximation. Above $R_{c}$, the convection velocity amplitude increases continuously from zero and scales as $\left(R-R_{c}\right)^{1 / 2}$. It is the order parameter of the transition, the corresponding broken symmetry being translational invariance in space. Note finally that equation (4.28) can be written in a variational form

$$
\frac{d A}{d T}=-\frac{\partial V}{\partial \bar{A}},
$$

where

$$
V(A, \bar{A})=-\mu A \bar{A}+\frac{1}{2} \beta A^{2} \bar{A}^{2},
$$

is the "Landau free-energy" in the vicinity of the transition.

\subsubsection{Pattern selection}

We now consider the problem of pattern selection via nonlinear interactions. As said above, in the slightly supercritical range, any superposition (4.22) of marginal modes has the same growth rate. Let us consider two examples, squares and hexagons.

For squares, we have

$$
\psi(x, y, t)=\epsilon\left(\left[A_{1} \exp \left(i k_{c} x\right)+c . c .\right]+\left[A_{2} \exp \left(i k_{c} y\right)+c . c .\right]\right) \sin \pi z+\cdots,
$$


where $A_{1}(T)$ and $A_{2}(T)$ are the complex amplitudes of the two sets of perpendicular rolls. Using symmetry considerations, the amplitude equations read

$$
\begin{aligned}
& \frac{d A_{1}}{d T}=\mu A_{1}-\left[\beta\left|A_{1}\right|^{2}+\gamma\left|A_{2}\right|^{2}\right] A_{1}, \\
& \frac{d A_{2}}{d T}=\mu A_{2}-\left[\gamma\left|A_{1}\right|^{2}+\beta\left|A_{2}\right|^{2}\right] A_{2} .
\end{aligned}
$$

It is an easy exercise to show that for $\mu>0$, stationary squares $\left(\left|A_{1}\right|=\left|A_{2}\right|\right)$ are stable when $|\gamma|<\beta$, i.e when the cross-coupling nonlinear term is small enough so that the two sets of rolls weakly interact; when their interaction is too strong, more precisely when, $\gamma>\beta$, one of the two sets of rolls nonlinearly damps out the other, and rolls are the stable nonlinear state. This is the situation for Boussinesq convection with stress-free perfectly heat-conducting boundary conditions. On the contrary, with insulating boundaries, squares are observed.

For hexagons, we have

$$
\psi(x, y, t)=\epsilon \sum_{p=1}^{3}\left[A_{p} \exp \left(i \mathbf{k}_{p} \cdot \mathbf{r}\right)+c . c .\right] \sin \pi z+\cdots,
$$

with $\left|\mathbf{k}_{p}\right|=k_{c}$ and $\mathbf{k}_{1}+\mathbf{k}_{2}+\mathbf{k}_{3}=0$, and where the $A_{p}(T)$ 's are the complex amplitudes of the three sets of rolls. Using symmetry considerations, the amplitude equations read

$$
\frac{d A_{l}}{d T}=\mu A_{l}-\left[\beta\left|A_{l}\right|^{2}+\delta\left(\left|A_{m}\right|^{2}+\left|A_{n}\right|^{2}\right)\right] A_{l}, \quad \text { cyclic permutations of }(l, m, n) .
$$

Note that a term proportional to $\bar{A}_{m} \bar{A}_{n}$ in the evolution equation for $A_{l}$ respects the translational and reflection $(x \rightarrow-x)$ symmetries, but is forbidden here because of the additional Boussinesq symmetry $(z \rightarrow-z, \theta \rightarrow-\theta, \psi \rightarrow-\psi)$. A possible exercise at this stage is to determine the stability domains of rolls, squares and hexagons as a function of the real coupling constants $\beta, \gamma, \delta$ and $\mu$. Show also that the square-hexagons transition is "first order" and relate that to a symmetry argument.

The general problem of pattern selection is much more difficult to solve and has been studied by Schlüter et al. (1965) for the case of rigid perfectly conducting boundary conditions. They have found that rolls are the only stable stationary pattern just above the onset of convection. Using a similar analysis, Riahi (1983) has found stationary squares in the case of thermally insulating rigid boundaries.

\subsubsection{Slowly varying amplitude of a roll pattern: the Ginzburg-Landau equation}

We now consider the problem of the existence of a band of unstable modes above $R_{c}$. For simplicity we assume stress-free perfectly heat conducting boundaries, so that the pattern consists of parallel rolls. To take into account the modes around $k_{c}$, we consider a wave packet

$$
\psi(x, z)=\left[A(X, Y, T) \exp \left(i k_{c} x\right)+c . c .\right] \sin \pi z+\cdots,
$$


where $A(X, Y, T)$ represents the slowly varying envelope of the roll pattern. We first consider modulations only along the $x$-axis, thus $A=A(X, T)$. We have

$$
A(X, T)=\int \hat{A}(K, \Sigma) \exp (\Sigma T+i K X) \delta(\Sigma-\Sigma(\mu, K)) d K d \Sigma
$$

where

$$
\Sigma(\mu, K)=\mu-\alpha K^{2}+\cdots,
$$

is the dispersion relation (4.20) in terms of scaled (order one in $\epsilon$ ) variables, i.e. $K=$ $\left(k-k_{c}\right) / \epsilon$. The Fourier-Laplace transform of the dispersion relation, (4.36), gives the linear part of the evolution equation for the amplitude $A(X, T)$,

$$
\frac{\partial A}{\partial T}=\mu A+\alpha \frac{\partial^{2} A}{\partial X^{2}}
$$

Taking into account the leading order nonlinear term, we get

$$
\frac{\partial A}{\partial T}=\mu A+\alpha \frac{\partial^{2} A}{\partial X^{2}}-\beta|A|^{2} A
$$

We now consider modulations also along the rolls axis, thus $A=A(X, Y, T)$. We have

$$
\mathbf{k}=\left(k_{c}+\delta k_{x}\right) \hat{\mathbf{x}}+\left(\delta k_{y}\right) \hat{\mathbf{y}}
$$

and the generalization of the dispersion relation (4.20) that respects rotational invariance in the horizontal plane, is

$$
\sigma(k, R)=\left(\pi^{2}+k_{c}^{2}\right) \frac{P}{1+P} \frac{\left(R-R_{c}\right)}{R_{c}}-\xi_{0}^{2}\left(\mathbf{k}^{2}-k_{c}^{2}\right)^{2}+\cdots
$$

with $4 k_{c}^{2} \xi_{0}^{2}=\alpha$. We have

$$
\left(\mathbf{k}^{2}-k_{c}^{2}\right)^{2}=\left(2 k_{c} \delta k_{x}+\left(\delta k_{x}\right)^{2}+\left(\delta k_{y}\right)^{2}\right)
$$

Thus, the relevant scalings are

$$
\delta k_{x}=\epsilon K_{x}, \quad \delta k_{y}=\epsilon^{1 / 2} K_{y}
$$

Slower $y$-modulations do not affect the amplitude equation to leading order, whereas modes corresponding to modulations on shorter scales are too strongly damped to be marginal. In terms of scaled variables, the dispersion relation reads

$$
\Sigma=\mu-\alpha\left[K_{x}+\frac{1}{2 k_{c}} K_{y}^{2}\right]^{2}+\cdots
$$


Taking its Fourier-Laplace transform and adding the leading order nonlinear term, gives

$$
\frac{\partial A}{\partial T}=\mu A+\alpha\left(\frac{\partial}{\partial X}-\frac{i}{2 k_{c}} \frac{\partial^{2}}{\partial Y^{2}}\right)^{2} A-\beta|A|^{2} A
$$

In terms of unscaled variables, all the terms of (4.38) are of order $\epsilon^{3}$. This equation has been obtained by Newell and Whitehead (1969) and Segel (1969), using a multiple-scale expansion both in space and time. Note that partial derivatives in $X$ and $Y$ are not involved symmetrically because the roll pattern breaks the rotational invariance in the horizontal plane.

We can write the amplitude equation (4.38) in variational form,

$$
\frac{\partial A}{\partial T}=-\frac{\delta \mathcal{L}}{\delta \overline{\mathcal{A}}}
$$

where

$$
\mathcal{L}[A]=\int\left[-\mu|A|^{2}+\frac{\beta}{2}|A|^{4}+\alpha\left|\left(\frac{\partial}{\partial X}-\frac{i}{2 k_{c}} \frac{\partial^{2}}{\partial Y^{2}}\right) A\right|^{2}\right] d X d Y,
$$

is analogous to a "Ginzburg-Landau" free-energy.

In the following lectures we will study pattern dynamics governed by this "Ginzburg-Landau" equation, also named in the context of convection, the Newell-Whitehead-Segel equation. In the case of stress-free boundary conditions, this equation is incorrect because it does not take into account the nonlinear interaction with vertical vorticity modes. We will discuss this effect later. With different coefficients than the ones derived above, the Ginzburg-Landau equation is correct to leading order for the description of slowly modulated roll-patterns in convection with rigid thermally conducting boundaries. More generally, it describes slowly modulated one-dimensional patterns that occur via a stationary bifurcation in a dissipative system, invariant under translations, rotations and space reflections in the horizontal plane, when no other marginal mode than the roll-mode at wavenumber $k_{c}$ is involved. 


\section{References}

Busse, F. H. (1978) Nonlinear Properties of Convection, Rep. Prog. Phys. 41, 1929-1967.

Busse, F. H. (1981) Transition to Turbulence in Rayleigh-Bénard Convection, Hydrodynamic Instabilities and the Transition to Turbulence, Swinney, H. L. and Gollub, J. P. Editors, Topics in Applied Physics 45, 97-137, Springer Verlag.

Gershuni, G. Z., Zhukovitskii, E. M., (1976) Convection Stability of Incompressible Fluids, Ketter Publications.

Goldstein, R. J., Graham, D. J. (1969) Stability of a Horizontal Fluid Layer with Zero Shear Boundaries, Phys. Fluids 12, 1133-1137.

Gorkov, L. P., (1957) Stationary Convection in a Plane Liquid Layer Near the Critical Heat Transfer Point, Soviet. Phys. JETP 6, 311-315.

Malkus, W. V. R., (1964) Boussinesq Equations and Convection Energetics, W. H. O. I. Tech. Rep., 11-37.

Malkus, W. V. R., Veronis, G. (1958) Finite amplitude cellular convection, J. Fluid Mech. 4, 225-260.

Newell, A.C., Whitehead, J.A. (1969) Finite bandwidth, finite amplitude convection, J. Fluid Mech. 38,279-303.

Normand, C., Pomeau, Y. Velarde, M. G. (1977) Convective Instability: a Physicist Approach, Rev. Mod. Phys. 49, 581-624.

Palm, E. (1975) Nonlinear Thermal Convection, Ann. Rev. Fluid Mech. 7, 39-61.

Riahi, N. (1983) Nonlinear Convection in a Porous Layer with Finite conducting Boundaries, J. Fluid Mech. 129, 153-171.

Schlüter, A., Lortz, D., Busse, F. (1965) On the Stability of Steady Finite Amplitude Convection, J. Fluid Mech. 23, 129-144.

Segel, L. A., (1969) J. Fluid Mech. 38, 203-224.

Sorokin, V. S. (1953) Variational Method in the Theory of Convection, Prikl. Mat. Mekh. $\mathbf{1 7}, 39$.

Spiegel, E. A. (1971) Convection in Stars, Ann. Rev. Astron. Astrophys. 9, 323-352.

Spiegel, E. A. (1972) Convection in Stars, Ann. Rev. Astron. Astrophys. 10, 261-304. 


\section{Amplitude Equations in Dissipative Systems}

We consider a dissipative system governed by a nonlinear partial differential equation

$$
\frac{\partial U}{\partial t}=L_{\mu}(\nabla) \cdot U+N(\nabla, U),
$$

where $U(\mathbf{r}, t)$ represents a set of scalar or pseudoscalar fields. The system is driven externally by a control parameter $\mu$ constant in space and time (except in section 5.3 where we consider parametric instabilities). Its basic state is thus homogeneous in space and constant in time, and corresponds to $U=0$, say. $L_{\mu}(\nabla)$ is a linear operator which involves spatial derivatives, and $N(\nabla, U)$ represents nonlinear terms. We assume that equation (5.1) is invariant under continuous translations in space and time (except in section 5.3 where it is invariant under continuous translations in space and discrete translations in time). We also assume in some cases, space-reflection symmetry or invariance under galilean transformations.

For a critical value of $\mu$, the basic state, $U=0$, loses its stability. The linear stability analysis consists of solving the eigenvalue problem

$$
L_{\mu}(\nabla) \cdot U=\eta U .
$$

The basic state is stable when all the eigenvalues $\eta$ have a negative real part. The instability onset, or the bifurcation of $U=0$, is characterized by one or several eigenvalues with a zero real part. The corresponding eigenfunctions, $U_{i, \mathbf{k}}(\mathbf{r}, t)$, are the critical modes, and characterize the temporal or spatial pattern that sets in at the instability onset, and breaks spontaneously some of the invariances listed above. In the vicinity of the instability onset, the amplitudes $A_{i}$ of the critical modes vary on a time scale much slower than that of the other modes, and thus contain all the information about the asymptotic time dependence of $U$. More precisely, the non-critical (damped) modes don't vanish only because they are forced by the critical (slightly unstable) modes through nonlinear interactions; thus, they follow adiabatically critical modes; adiabatic elimination of fast modes leads to amplitude equations. We observed in the previous lectures that symmetry constraints determine their form. We give now a catalogue of the most frequent situations. 


\subsection{Stationary instability}

In the previous lecture we studied the example of Rayleigh- Bénard convection. A stationary instability corresponds to a marginal mode with a real growth rate. We shall discuss the case when the critical wave number, $k_{c}$, is nonzero; situations where the critical wave number is vanishingly small will be considered in section 5.5 .

\subsubsection{One-dimensional pattern}

Let us first consider the situation where the nonlinear terms select a one-dimensional "rollpattern". In the vicinity of the instability onset, $r \approx r_{c}$, the growth rate is a real function of $k$ with a maximum around $k_{c}$ (Figure 5.1)

$$
\sigma=r-r_{c}+\frac{1}{2}\left(\frac{\partial^{2} \sigma}{\partial k^{2}}\right)_{c}\left(k-k_{c}\right)^{2}+\cdots
$$

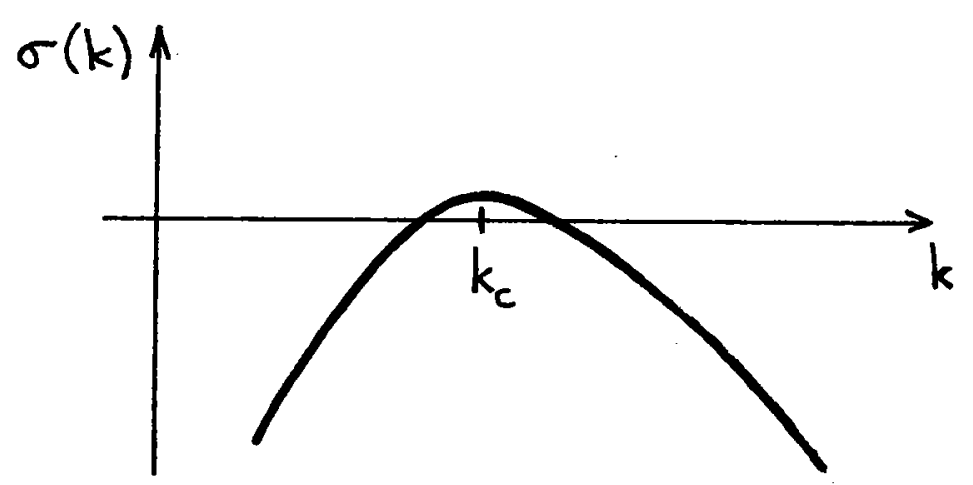

Figure 5.1. Growth rate versus wave number for a stationary instability at $k=k_{c}$

We take the distance to criticality, $r-r_{c}$, of order $\epsilon^{2}(\epsilon \ll 1)$, accordingly the instability growth rate is of order $\epsilon^{2}$; this is the time scale for the slow critical modes. Correspondingly, there is a large spatial scale generated by modes interaction in the unstable wavenumber band; as $\sigma(k)$ is locally a parabola, $k-k_{c} \leq O(\epsilon)$, and the large spatial scale corresponds to $k-k_{c}=\epsilon K$. Thus, the scaled dispersion relation is

$$
\Sigma(K)=\mu-\alpha K^{2}+\cdots,
$$

where $\Sigma=\sigma / \epsilon^{2}, \mu=\left(r-r_{c}\right) / \epsilon^{2}, \alpha=-\frac{1}{2}\left(\frac{\partial \sigma}{\partial k}\right)_{c}$, and $K$, are all of order one.

We first consider modulations of the pattern only along the $x$-axis, and write

$$
\mathbf{U}(x, t)=\epsilon\left[A(X, T) \exp \left(\mathrm{ik}_{\mathrm{c}} \mathrm{x}\right)+c . c .\right] \tilde{\mathbf{U}}_{k_{c}}+\cdots,
$$

where $\tilde{\mathbf{U}}_{k_{c}} \exp \left(i k_{c} x\right)$ is the critical mode. As shown in previous lectures, the linear evolution equation for $A$ is the Fourier-Laplace transform of (5.4), and the leading order nonlinear term compatible with translational invariance in space is $|A|^{2} A$. Moreover, its coefficient $\beta$ 
is real if the system is invariant under space-reflection symmetry, $x \rightarrow-x$. Thus, to leading order, $A$ obeys the Ginzburg-Landau equation

$$
\frac{\partial A}{\partial T}=\mu A+\alpha \frac{\partial^{2} A}{\partial X^{2}}-\beta|A|^{2} A .
$$

The nonlinear term saturates the instability growth if $\beta>0$, and the bifurcation is supercritical. In terms of the original scaled variables, the critical mode amplitude scales like $\epsilon$ i.e. like $\left(r-r_{c}\right)^{1 / 2}$. The slow spatial scale can be understood as a coherence length and diverges at criticality like $\left(r-r_{c}\right)^{-1 / 2}$.

If $\beta<0$, higher order nonlinear terms should be taken into account, and a simple model for a subcritical bifurcation is

$$
\frac{\partial A}{\partial T}=\mu A+\alpha \frac{\partial^{2} A}{\partial X^{2}}-\beta|A|^{2} A-\gamma|A|^{4} A,
$$

with $\gamma>0$. However, in terms of scaled variables, $\beta$ should be small in order to get the two nonlinear terms at the same order; thus (5.7) is asymptotically correct only in the vicinity of a tricritical point.

Like the Ginzburg-Landau equation, (5.7) can be put in variational form

$$
\frac{\partial A}{\partial T}=-\frac{\delta \mathcal{L}}{\delta \overline{\mathcal{A}}}
$$

where

$$
\begin{gathered}
\mathcal{L}[A]=\int\left[\alpha\left|\frac{\partial A}{\partial X}\right|^{2}-V(A, \bar{A})\right] d X, \\
V(A, \bar{A})=\mu|A|^{2}-\frac{\beta}{2}|A|^{4}-\frac{\gamma}{3}|A|^{6} .
\end{gathered}
$$

$\mathcal{L}[A]$ is a Lyapunov functional; indeed, multiplying (5.7) by $\partial \bar{A} / \partial T$, and adding to the complex conjugate expression, yields

$$
\frac{d}{d t} \mathcal{L}[A]=-2 \int\left|\frac{\partial A}{\partial T}\right|^{2} d X<0 .
$$

Thus $\mathcal{L}[A]$ is a decreasing function, bounded from below by the constant $A$ 's that are the maxima of $V(A, \bar{A})$. For a supercritical bifurcation $(\beta>0$, fifth order term neglected), the uniform state $A=0$ is globally stable for $\mu<0$, whereas perfectly periodic patterns corresponding to $A=\sqrt{\mu / \beta} \exp (i \phi)$, with constant $\phi$, are globally stable for $\mu>0$. The degeneracy in $\phi$ is obviously related to translational invariance in space, but a more subtle effect that involves the phase might happen when the system is not invariant under reflection symmetry, $x \rightarrow-x$. 
When the system is not invariant under reflection symmetry, $x \rightarrow-x$, the coefficient $\beta$ is in general a complex number. Writing $A(X, T)=R(X, T) \exp i \phi(X, T)$, equation (5.6) yields

$$
\begin{array}{r}
\frac{\partial R}{\partial T}=\left[\mu-\alpha\left(\frac{\partial \phi}{\partial X}\right)^{2}\right] R+\alpha \frac{\partial^{2} R}{\partial X^{2}}-\beta_{r} R^{3} \\
R \frac{\partial \phi}{\partial T}=2 \frac{\partial R}{\partial X} \frac{\partial \phi}{\partial X}+R \frac{\partial^{2} \phi}{\partial X^{2}}-\beta_{i} R^{3}
\end{array}
$$

Consequently, a homogeneous roll-pattern of amplitude $R_{0}$ has its phase that linearly increases in time

$$
\phi_{0}=-\beta_{i} R_{0}^{2} T
$$

Thus, from (5.5), one observes that this "stationary" instability gives rise to a travelling pattern

$$
\mathbf{U}_{0}(x, t)=\epsilon\left[R_{0} \exp i\left(k_{c} x-\epsilon^{2} \beta_{i} R_{0}^{2} t\right)+c c\right] \tilde{\mathbf{U}}_{k_{c}}+\cdots
$$

due to the externally broken reflection symmetry. We will study a similar effect when the reflection symmetry is spontaneously broken; a secondary instability then generates a drifting pattern from a stationary one (see lecture 7 ).

Note that the absence of a term proportional to $\partial A / \partial X$ in (5.6) is not related to reflection symmetry. Indeed, a term of the form $i \partial A / \partial X$ is compatible with the $x \rightarrow-x$ symmetry, and is present as soon as one expands $\mathbf{U}$ in (5.5) at $k \neq k_{c}$. This corresponds to the change of variable

$$
A=B \exp (i q X)
$$

that gives

$$
\frac{\partial B}{\partial T}=\left(\mu-\alpha q^{2}\right) B+2 i q \alpha \frac{\partial B}{\partial X}+\alpha \frac{\partial^{2} B}{\partial X^{2}}-\beta|B|^{2} B,
$$

thus showing that the growth rate of the mode $k=k_{c}+\epsilon q$ is $\mu-\alpha q^{2}$, i.e. in scaled terms, $r-r_{c}-\alpha\left(k-k_{c}\right)^{2}$, in agreement with the dispersion relation. The absence of a term in $i \partial A / \partial X$ in (5.6) is thus only related to the fact that the first unstable mode $k_{c}$ is the one with the maximum growth rate $\sigma(k)$.

A simple model, that mimics the formation of a one-dimensional pattern is the SwiftHohenberg equation for a field $u(x, y, t)$

$$
\frac{\partial u}{\partial t}=\left[r-r_{c}-\left(k_{c}^{2}+\Delta\right)^{2}\right] u-u^{3} .
$$

As an exercise consider a one-dimensional field in space, $u(x, t)$, and using a multiple-scale expansion technique, derive the Ginzburg-Landau equation in the vicinity of the instability onset, $r-r_{c} \approx \mu \epsilon^{2}$. Try the same exercise with the model

$$
\frac{\partial u}{\partial t}=\left[r-r_{c}-\left(k_{c}{ }^{2}+\Delta\right)^{2}\right] u-a u^{2}-u \frac{\partial u}{\partial x},
$$


which is not invariant under the $x \rightarrow-x$ reflection symmetry, and show that $\beta$ is complex. Find the nature of the bifurcation as a function of $a$, and derive (5.7) in the vicinity of the tricritical point.

Finally, let us consider a situation where the spatial phase is quenched. This occurs in the convection problem for instance, if one takes stress-free boundary conditions also at the lateral boundaries, $x=0$ and $x=2 n \pi / k_{c}$, where $n$ is an integer. Then, the phase of the pattern is fixed,

$$
\mathbf{U}(x, t)=\epsilon R(X, T) \sin \left(k_{c} x\right) \tilde{\mathbf{U}}_{k_{c}}+\cdots,
$$

and its amplitude is governed by a real Ginzburg-Landau equation

$$
\frac{\partial R}{\partial T}=\mu R+\alpha \frac{\partial^{2} R}{\partial X^{2}}-\beta R^{3} .
$$

The broken symmetry at the instability onset is not translational invariance, which is here externally broken because of the lateral boundary conditions, but the $R \rightarrow-R$ symmetry of Boussinesq convection.

\subsubsection{Two-dimensional modulations of a one-dimensional cellular pattern}

We now consider the dynamics of two-dimensional modulations of a one-dimensional rollpattern, parallel to the $x$-axis. In isotropic systems, the growth-rate depends on $\mathbf{k}^{2}$ and is maximum for $\mathbf{k}^{2}=k_{c}{ }^{2}$; thus, for $|\mathbf{k}| \approx k_{c}$

$$
\sigma(\mathbf{k})=r-r_{c}-\xi_{0}{ }^{2}\left(\mathbf{k}^{2}-k_{c}{ }^{2}\right)^{2}+\cdots,
$$

and as shown in Lecture 4, the amplitude equation reads

$$
\frac{\partial A}{\partial T}=\mu A+\alpha\left(\frac{\partial}{\partial X}-\frac{i}{2 k_{c}} \frac{\partial^{2}}{\partial Y^{2}}\right)^{2} A-\beta|A|^{2} A
$$

or in variational form

$$
\frac{\partial A}{\partial T}=-\frac{\delta \mathcal{L}}{\delta \overline{\mathcal{A}}}
$$

with

$$
\mathcal{L}[A]=\int\left[-\mu|A|^{2}+\frac{\beta}{2}|A|^{4}+\alpha\left|\left(\frac{\partial}{\partial X}-\frac{i}{2 k_{c}} \frac{\partial^{2}}{\partial Y^{2}}\right) A\right|^{2}\right] d X d Y
$$

For anisotropic systems, the growth-rate is maximum for $|\mathbf{k}|=k_{c}$ with $\mathbf{k}$ along a preferred axis, $x$ say. Thus

$$
\sigma(\mathbf{k})=r-r_{c}-\alpha\left(k_{x}-k_{c}\right)^{2}+\alpha^{\prime}{k_{y}}^{2}+\cdots,
$$

and

$$
\Sigma(\mathbf{K})=\mu-\alpha K_{X}^{2}+\alpha^{\prime} K_{Y}^{2}+\cdots
$$


so that the Ginzburg-Landau equation takes the form

$$
\frac{\partial A}{\partial T}=\mu A+\alpha \frac{\partial^{2} A}{\partial X^{2}}+\alpha^{\prime} \frac{\partial^{2} A}{\partial Y^{2}}-\beta|A|^{2} A
$$

\subsubsection{Two-dimensional patterns}

We have already considered two-dimensional patterns in the previous lecture. Let us take the example of hexagons or more precisely of patterns with three basic wave-vectors, $\mathbf{k}_{1}, \mathbf{k}_{2}, \mathbf{k}_{3}$, such that $\mathbf{k}_{p}=k_{c}$ and $\mathbf{k}_{1}+\mathbf{k}_{2}+\mathbf{k}_{3}=0$,

$$
\mathbf{U}(x, y, t)=\epsilon \sum_{p=1}^{3}\left[A_{p} \exp \left(i \mathbf{k}_{p} \cdot \mathbf{r}\right)+c . c .\right] \tilde{\mathbf{U}}_{k Q c}+\cdots
$$

but without the $U \rightarrow-U$ invariance. Using symmetry considerations, the amplitude equations read

$$
\frac{d A_{l}}{d T}=\mu A_{l}+\rho \bar{A}_{m} \bar{A}_{n}-\left[\beta\left|A_{l}\right|^{2}+\delta\left(\left|A_{m}\right|^{2}+\left|A_{n}\right|^{2}\right)\right] A_{l} .
$$

Note that this equation is asymptotically valid only if the $U \rightarrow-U$ symmetry is slightly broken, so that the quadratic and cubic nonlinearities are obtained at the same order. The quadratic nonlinearities correspond to the resonant triad interaction $\mathbf{k}_{1}+\mathbf{k}_{2}+\mathbf{k}_{3}=0$. Although one can change the phase $\phi_{p}$ of each wave by shifting the origin in the horizontal plane along $\mathbf{k}_{p}$, the above relation implies

$$
\phi_{1}+\phi_{2}+\phi_{3}=\Phi=\text { constant }
$$

$\Phi$ determines the shape of the pattern (hexagons, triangles, ...). Using translational and reflection symmetry, one can restrict $\Phi$ to the interval $[0, \pi / 2]$. Note that the leading order amplitude equations (5.25) select $\Phi=0$.

\subsection{Oscillatory instability}

We now consider situations where the instability growth-rate has an imaginary part $\omega(k)$; thus, the unstable mode has an oscillatory behavior with a pulsation $\omega\left(k_{c}\right)$ at onset. We begin with the case $k_{c}=0$.

\subsubsection{Oscillatory instability at zero wavenumber}

When the real part of the growth-rate is maximum at zero wavenumber, we have

$$
\eta(k)=\sigma(k)+i \omega(k)
$$

with

$$
\begin{array}{r}
\sigma(k)=r-r_{c}+\frac{1}{2}\left(\frac{\partial^{2} \sigma}{\partial k^{2}}\right)_{0} k^{2}+\cdots \\
\omega(k)=\omega_{0}+\left(\frac{\partial \omega}{\partial k}\right)_{0} k+\frac{1}{2}\left(\frac{\partial^{2} \omega}{\partial k^{2}}\right)_{0} k^{2}+\cdots
\end{array}
$$


In the vicinity of the instability onset, $r-r_{c}=\mu \epsilon^{2}$,

$$
\mathrm{U}(x, t)=\epsilon\left[A(X, T) \exp \left(\mathrm{i} \omega_{0} \mathrm{t}\right)+c . c .\right] \tilde{\mathbf{U}}_{0}+\cdots,
$$

where $\tilde{\mathbf{U}}_{0} \exp \left(i \omega_{0} t\right)$ is the critical mode. As previously observed, the Fourier-Laplace transform of the dispersion relation gives the linear part of the amplitude equation, and translational invariance in time determines the form of the leading order nonlinear term, $|A|^{2} A$. We get

$$
\frac{\partial A}{\partial T}=\mu A-c \frac{\partial A}{\partial X}+\alpha \frac{\partial^{2} A}{\partial X^{2}}-\beta|A|^{2} A, .
$$

where $c=(\partial \omega / \partial k)_{0}$ is the group velocity, $\alpha_{r}=-\frac{1}{2}\left(\partial^{2} \sigma / \partial k^{2}\right)_{0}$ is related to the diffusion of space-dependent perturbations $\left(\alpha_{r}>0\right), \alpha_{i}=-\frac{1}{2}\left(\partial^{2} \omega / \partial k^{2}\right)_{0}$ corresponds to the dispersion, $\beta_{r}$ is the nonlinear dissipation, and $\beta_{i}$ is related to the nonlinear amplitude-dependence of the frequency. Note that, like for the nonlinear Schrödinger equation, one needs two slow time scales $T_{1}$ and $T_{2}$ when deriving (5.30) with an asymptotic expansion. Transforming to the reference frame moving at the group velocity yields

$$
\frac{\partial A}{\partial T}=\mu A+\alpha \Delta A-\beta|A|^{2} A .
$$

Equation (5.31) is a Ginzburg-Landau equation with complex coefficients $\alpha$ and $\beta$. This is a crucial difference from (5.6) for stationary cellular instabilities, that involves real coefficients. Indeed, no variational formulation is known for (5.31). Thus, unlike (5.6) $A$ does not evolve in order to minimize a functional $\mathcal{L}[A]$, but displays in some parameter range, periodic or even chaotic behaviors in space and time. Equation (5.31) has two simple limits, a "variational" one for $\alpha$ and $\beta$ real

$$
\frac{\partial A}{\partial T}=-\frac{\delta \mathcal{L}}{\delta \overline{\mathcal{A}}}, \quad \frac{d}{d t} \mathcal{L}[A]=-2 \int\left|\frac{\partial A}{\partial T}\right|^{2} d X<0,
$$

and a conservative limit (the nonlinear Schrödinger equation), for $\alpha$ and $\beta$ pure imaginary,

$$
\frac{\partial A}{\partial T}=-\frac{\delta \mathcal{L}}{\delta \overline{\mathcal{A}}}, \quad \frac{d}{d t} \mathcal{L}[A]=0 .
$$

One method is to investigate (5.31) perturbatively, starting from one of these limit situations (lecture 9).

\subsubsection{Oscillatory instability at finite wavenumber}

Hydrodynamic instabilities often lead to time dependent cellular patterns. The CouetteTaylor flow between concentric cylinders (DiPrima and Swinney, 1981), thermal convection in the presence of a salinity gradient or in binary fluid mixtures (Turner, 1973), thermal convection in a layer of fluid rotating about a vertical axis (Chandrasekhar, 1961), display a Hopf bifurcation at a finite wavenumber $k_{c} \neq 0$. The growth-rate of the marginal modes is

$$
\eta(k)=\sigma(k)+i \omega(k)
$$


with

$$
\begin{array}{r}
\sigma(k)=r-r_{c}+\frac{1}{2}\left(\frac{\partial^{2} \sigma}{\partial k^{2}}\right)_{c}\left(k-k_{c}\right)^{2}+\cdots \\
\omega(k)=\omega_{0}+\left(\frac{\partial \omega}{\partial k}\right)_{c}\left(k-k_{c}\right)+\frac{1}{2}\left(\frac{\partial^{2} \omega}{\partial k^{2}}\right)_{c}\left(k-k_{c}\right)^{2}+\cdots
\end{array}
$$

In the vicinity of the instability onset, $r-r_{c}=\mu \epsilon^{2}$,

$$
\begin{array}{r}
\mathbf{U}(x, t)=\epsilon\left[A(X, T) \exp \left(i \omega_{0} t-k_{c} x\right)+c . c .\right] \tilde{\mathbf{U}}_{-k_{c}} \\
\quad+\epsilon\left[B(X, T) \exp \left(i \omega_{0} t+k_{c} x\right)+c . c .\right] \tilde{\mathbf{U}}_{k_{c}}+\cdots,
\end{array}
$$

where $A$ and $B$ are the complex amplitudes of the waves propagating to the right and to the left. These obey the following amplitude equations

$$
\begin{aligned}
& \frac{\partial A}{\partial T}=\mu A-c \frac{\partial A}{\partial X}+\alpha \frac{\partial^{2} A}{\partial X^{2}}-\left(\beta|A|^{2}+\gamma|B|^{2}\right) A, \\
& \frac{\partial B}{\partial T}=\mu B+c \frac{\partial B}{\partial X}+\alpha \frac{\partial^{2} B}{\partial X^{2}}+\left(\gamma|A|^{2}+\beta|B|^{2}\right) B .
\end{aligned}
$$

Equations (5.35) are invariant under the transformations

$$
\begin{aligned}
A \rightarrow A \exp (-i \phi), & B \rightarrow B \exp (i \phi), \\
A \rightarrow A \exp (i \theta), & B \rightarrow B \exp (i \theta),
\end{aligned}
$$

that reflect translational invariance in space and time, and under the transformation

$$
X \rightarrow-X, \quad A \rightarrow B, \quad B \rightarrow A,
$$

that traces back to space-reflection symmetry, $x \rightarrow-x$. Note that terms of the form $i \partial A / \partial X$, $i \partial B / \partial X$, satisfy symmetry requirements but are not involved because $\sigma$ is maximum for $k=k_{c}$. Let us also remark that the group velocity, $c$, should be small in order to get all the terms of (5.35) at the same order of an asymptotic expansion (see the discussion about counter-propagating waves in lecture 3 ).

Restricting the discussion to spatially homogeneous solutions, it is easy to check that (5.35) describes either:

- propagating waves $(|A| \neq 0,|B|=0$, or $|A|=0,|B| \neq 0)$, which are stable if $\gamma_{r}>\beta_{r}>0$,

- standing waves $\left(|A|=|B| \neq 0\right.$, which are stable if $\beta_{r}>\gamma_{r}$.

When $\beta_{r}<0$ or $\gamma_{r}<-\left|\beta_{r}\right|$, the bifurcation is subcritical. 


\subsection{Parametric instability}

Parametric instabilities in spatially extended systems also generate waves. Let us consider for example the one-dimensional array of coupled pendula, already studied in lecture 3 , with an additional damping and a parametric forcing. In the long-wavelength limit, the governing equation is

$$
\frac{\partial^{2} u}{\partial t^{2}}+2 \lambda \frac{\partial u}{\partial t}+\left(1+f \sin \omega_{e} t\right) \sin u=\frac{\partial^{2} u}{\partial x^{2}} .
$$

In the limit of small dissipation $(\lambda \ll 1)$, the dispersion relation of the unforced array of pendula is

$$
\omega(k)^{2}=1+k^{2} .
$$

The strongest parametric response being at $\omega_{e} / 2$, a wavenumber $k_{c}$ is selected by the dispersion relation, such that

$$
\omega_{e} \equiv 2 \omega \approx 2 \omega\left(k_{c}\right)
$$

In the vicinity of the instability onset,

$$
u(x, t)=\epsilon\left[A(X, T) \exp i\left(\omega_{0} t-k_{c} x\right)+B(X, T) \exp i\left(\omega_{0} t+k_{c} x\right)+c . c .\right]+\cdots .
$$

The symmetry requirements are:

- continuous translational invariance in space, that implies the invariance under the transformation

$$
A \rightarrow A \exp (-i \phi), \quad B \rightarrow B \exp (i \phi)
$$

- discrete translational invariance in time, $t \rightarrow t+2 \pi / \omega_{e}$, that implies

$$
A \rightarrow-A, \quad B \rightarrow-B
$$

- space-reflection symmetry, $x \rightarrow-x$, that implies

$$
X \rightarrow-X, \quad A \rightarrow B, \quad B \rightarrow A .
$$

All the terms of (5.35) respect these requirements, but additional terms are allowed due to the less restrictive requirement of translational invariance in time (discrete instead of continuous). To leading order, two additional terms, proportional to the forcing, are allowed, $F \bar{A}$ and $F \bar{B}$. We get

$$
\begin{aligned}
& \frac{\partial A}{\partial T}=(-\Lambda+i \nu) A+F \bar{B}-c \frac{\partial A}{\partial X}+\alpha \frac{\partial^{2} A}{\partial X^{2}}-\left(\beta|A|^{2}+\gamma|B|^{2}\right) A \\
& \frac{\partial B}{\partial T}=(-\Lambda+i \nu) B+F \bar{A}+c \frac{\partial B}{\partial X}+\alpha \frac{\partial^{2} B}{\partial X^{2}}-\left(\gamma|A|^{2}+\beta|B|^{2}\right) B,
\end{aligned}
$$

where $\Lambda$ is proportional to the dissipation and $\nu$ is proportional to the detuning from parametric resonance. Equations (5.39) show that the right-going wave is forced by the left-going wave, and vice-versa, so that only standing waves are parametrically generated in the vicinity of the instability onset. In other words, because the phase of the parametric response is quenched by the external forcing in time, the only possibility at onset is a standing wave. 
5.4 Neutral modes at zero wavenumber. Systems with Galilean invariance

Symmetry properties or conservation laws often imply the existence of neutral modes at zero wavenumber. Consider the following model of one-dimensional stationary cellular instability

$$
\frac{\partial u}{\partial t}=\frac{\partial^{2} u}{\partial x^{2}}+r \frac{\partial^{4} u}{\partial x^{4}}+\frac{\partial^{6} u}{\partial x^{6}}-u \frac{\partial u}{\partial x} .
$$

The growth-rate $\sigma(k)$ for a perturbation of wavenumber $k$ around the $u=0$ solution reads

$$
\sigma(k)=-k^{2}\left(1-r k^{2}+k^{4}\right)
$$

and is displayed on Figure 5.2. For $r \approx r_{c}=2$, the null state undergoes a stationary instability to a cellular structure of wavenumber $k_{c}$. The local behavior of the growth-rate around $k_{c}$ is thus similar to the one of the Swift-Hohenberg model (5.13).

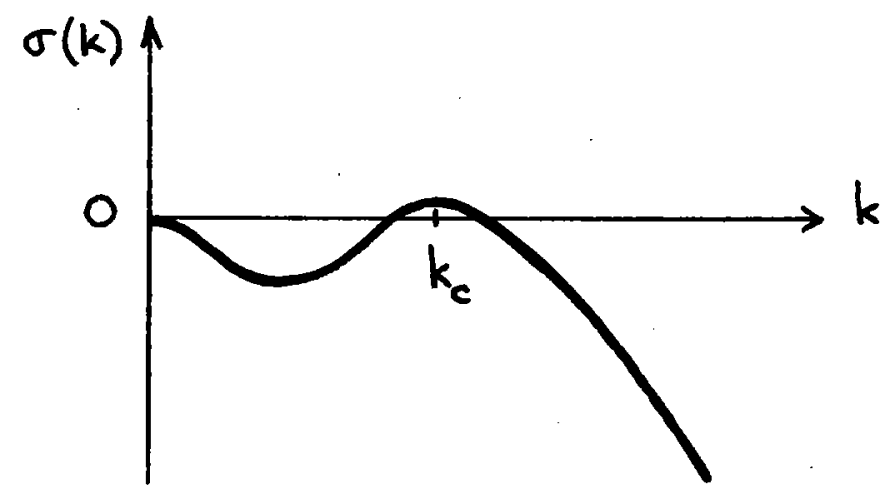

Figure 5.2. Growth-rate versus wave number for the model (5.40)

However, an important difference is that (5.40) can be written in a conservative form

$$
\frac{\partial u}{\partial t}=\frac{\partial}{\partial x}\left(\frac{\partial u}{\partial x}+r \frac{\partial^{3} u}{\partial x^{3}}+\frac{\partial^{5} u}{\partial x^{5}}-\frac{1}{2} u^{2}\right)
$$

If $u$ is considered as a velocity field along the $x$-axis, this traces back to the Galilean invariance of (5.40), i.e. the invariance under the transformation

$$
x \rightarrow x-v t, \quad u \rightarrow u+v .
$$

This implies the existence of marginal modes at zero wavenumber, as shown by the dispersion relation of figure (5.2). In the vicinity of the instability onset, the amplitude of these slow modes should be taken into account, since it is generally coupled to the amplitude of the critical modes at $k_{c}$. Thus, we write

$$
u(x, t)=\epsilon[A(X, T) \exp (i x)+\text { c.c. }+B(X, T)]+\cdots,
$$


and get coupled evolution equations for $A$ and $B$,

$$
\begin{gathered}
\frac{\partial A}{\partial T}=\mu A+4 \frac{\partial^{2} A}{\partial X^{2}}-\frac{1}{36}|A|^{2} A-i A B \\
\frac{\partial B}{\partial T}=\frac{\partial^{2} B}{\partial X^{2}}-\frac{\partial}{\partial X}|A|^{2} .
\end{gathered}
$$

Note that all the terms in (5.42) and (5.43) cannot be obtained at the same order of an asymptotic expansion, and one should again use two different time scales. If $u$ is considered as a velocity field, $B$ is a large-scale flow that advects the cellular pattern, thus shifting its phase through the term $i A B$ in (5.41); in turn, the amplitude inhomogeneities of the cellular pattern, generates the large-scale flow $B$ in (5.42). Note that $u$ is a "compressible velocity field", but a similar effect occurs with Boussinesq convection rolls when two-dimensional perturbations are taken into account (see lecture 7). The important point to remember is that the amplitude of neutral modes couples to the one of the critical modes, and modifies the dynamics described by the Ginzburg-Landau equation.

\subsection{Conserved order parameter}

Another class of stationary cellular instabilities where additional symmetries modify the form of the evolution equation for the amplitude of the critical modes, is when the amplitude obeys a conservation law. This situation is similar to a phase transition with a conserved order parameter, spinodal decomposition for instance (Langer, 1975). Let us consider again the example of Rayleigh-Bénard convection, but this time with upper and lower perfectly insulating boundaries. The Boussinesq equations are

$$
\begin{gathered}
\nabla \cdot \mathbf{v}=0 \\
\frac{\partial \mathbf{v}}{\partial t}+(\mathbf{v} \cdot \nabla) \mathbf{v}=-\nabla \pi+P \nabla^{2} \mathbf{v}+R P \theta \hat{\mathbf{z}} \\
\frac{\partial \theta}{\partial t}+\mathbf{v} \cdot \nabla \theta=\mathbf{v} \cdot \hat{\mathbf{z}}+\nabla^{2} \theta
\end{gathered}
$$

with

$$
\begin{gathered}
\frac{\partial \theta}{\partial z}=0 \\
\mathbf{v}=0
\end{gathered}
$$

at the boundaries, $z=0$ and $z=1$. We consider rigid boundaries in order to avoid the coupling with a neutral mean flow at instability onset. We first consider a solution of equations (5.44 - 5.48),

$$
\mathbf{U}_{0}=\left[\mathbf{v}_{0}(\mathbf{r}, t), \theta_{0}(\mathbf{r}, t), \pi_{0}(\mathbf{r}, t)\right],
$$

and note that

$$
\mathbf{U}_{\Theta}=\left[\mathbf{v}_{0}, \theta_{0}+\Theta(t), \pi_{0}+R P \Theta(t) z\right]
$$


is also a solution if

$$
\frac{\partial \Theta}{\partial t}=0
$$

In other words, $D \mathbf{U}=(0,1, R P z)$ is an eigenmode with a zero eigenvalue. Thus, there is a neutral mode at zero wavenumber, that traces back to the existence of the continuous family of solutions above. To investigate the stability of the static state, $\mathrm{U}=0$, we consider

$$
\mathbf{U}=\Theta(X, Y, T) D \mathbf{U}+\cdots,
$$

where $\Theta(X, Y, T)$ is slowly varying in space and time. When $R$ is small, the space-dependent buoyancy in equation (5.45) is small and generates a small velocity field; thus, the coupling with velocity in equation (5.46) is weak, and $\Theta$ is damped because of thermal diffusion. The growth-rate is negative and the static state is stable in the long wavelength limit (see figure 5.3). However, when $R$ increases above a critical value $R_{c}$, the effective diffusivity in the equation for $\Theta$ changes sign due to the coupling with the velocity field that enhances the temperature perturbation $\Theta$; we get a stationary instability at zero wavenumber (see figure 5.3). The evolution equation for $\Theta(X, Y, T)$ can be obtained with a multiple-scale expansion (Chapman, 1978). We want to point out here that it should have the form of a conservation equation. Indeed, using the boundary conditions, the vertical average of (5.46) reads

$$
\frac{\partial}{\partial t} \int_{0}^{1} \theta d z+\nabla_{h} \cdot\left[\int_{0}^{1}\left[(\theta-z) \mathbf{v}_{h}-\nabla_{h} \theta\right]\right]=0 .
$$

In the vicinity of the instability onset, $R-R_{c}=\epsilon^{2} R_{c} r$, the evolution equation for $\Theta$ is at leading order

$$
\frac{\partial \Theta}{\partial T}=-r \nabla_{h}^{2} \Theta-\kappa \nabla_{h}^{4} \Theta+\gamma \nabla_{h} \cdot\left[\left(\nabla_{h} \Theta\right)^{2} \nabla_{h} \Theta\right] .
$$

The absence of terms that involve explicitly $\Theta$ is related to the freedom of a constant shift in temperature, that we have with the Boussinesq equations with insulating boundary conditions. The even number of space-derivatives is due to $x$ and $y$-reflection symmetries in the horizontal plane. The absence of quadratic nonlinearities traces back to the Boussinesq symmetry. We will find many similar examples of stationary instability at zero wavenumber when studying phase instabilities of cellular patterns (lecture 6). 


\subsection{Conservative systems and dispersive instabilities}

Another additional symmetry that affects the form of the amplitude equation is the time reversal symmetry of conservative systems. A well-known example is that of two inviscid fluid layers of different densities, possibly moving at different velocities, in which KelvinHelmholtz or Rayleigh-Taylor instabilities can occur.

We restrict our attention to the Kelvin-Helmholtz instability. A layer of density $\rho^{\prime}$ moves at velocity $U^{\prime}$ above a layer of density $\rho,\left(\rho>\rho^{\prime}\right)$, moving at velocity $U$. The stability analysis leads to the following dispersion relation (Chandrasekhar, 1961)

$$
\frac{\eta}{k}=-i \frac{\rho U+\rho^{\prime} U^{\prime}}{\rho+\rho^{\prime}} \pm i\left[\frac{g}{k} \frac{\rho-\rho^{\prime}}{\rho+\rho^{\prime}}-\frac{\rho \rho^{\prime}}{\left(\rho+\rho^{\prime}\right)^{2}}\left(U-U^{\prime}\right)^{2}+\frac{T}{\rho+\rho^{\prime}} k\right]^{\frac{3}{2}}
$$

where $\mathrm{k}$ is the wavenumber and $\mathrm{T}$ is the surface tension. Instability occurs when the term in the square brackets is negative, a result of the shear being large enough. The first term in the square brackets shows that the gravitational restoring force stabilizes low wavenumbers, whereas the last term shows that surface tension stabilizes high wavenumbers. The net effect is that with both a density step and surface tension, there is a critical velocity difference $\left(\Delta U^{2}\right)_{c}$ for the onset of instability, at which a critical wavenumber $k_{c}$ becomes unstable first; at slightly higher values of $\Delta U^{2}$ there is a narrow band of unstable wavenumbers.

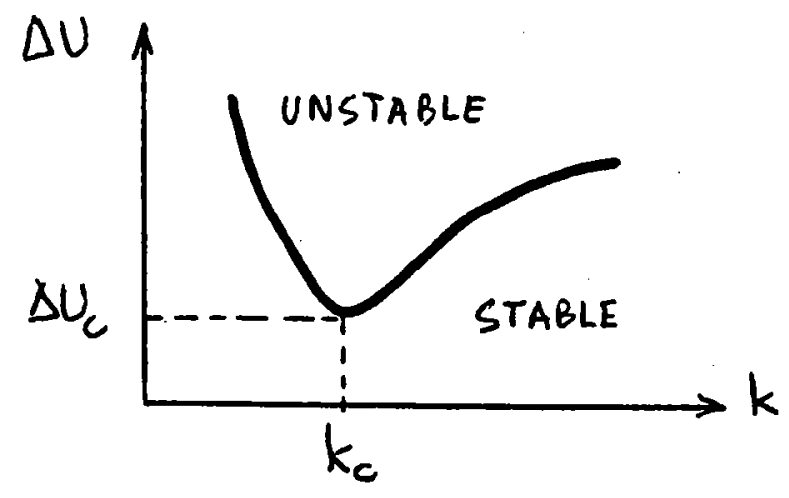

Figure 5.3. Marginal stability curve for the Kelvin-Helmholtz instability

This looks very similar to Rayleigh-Bénard convection, although the growth-rate for the Kelvin-Helmholtz has an imaginary part that corresponds to the frequency of the unstable waves, but the main difference is related to the conservative nature of the present problem. At the onset of the Kelvin-Helmholtz instability, four pure imaginary eigenvalues collide by pair and give rise to four complex eigenvalues, $( \pm \eta, \pm \bar{\eta})$, whereas for an instability in a dissipative system, a real eigenvalue, or pairs of complex-conjugate eigenvalues cross the imaginary axis. The former situation is a dispersive instability, and is related to time reversal symmetry; indeed, this implies that, if $\eta$ is an eigenvalue, then $-\eta$ is another eigenvalue. As the original problem involves real quantities, $\pm \bar{\eta}$ are also eigenvalues, and a conservative 
system involves either, pure imaginary eigenvalues (stable range), or complex eigenvalues $( \pm \eta, \pm \bar{\eta})$ (unstable range).

In the unstable range, the amplitude equation is also modified by the additional time reversal symmetry constraint. Let us consider for instance the following conservative model of SwiftHohenberg type.

$$
\frac{\partial^{2} u}{\partial t^{2}}=\left[\mu-\left(1+\frac{\partial^{2}}{\partial x^{2}}\right)^{2}\right] u+u \frac{\partial u}{\partial x}
$$

When $0<\mu \ll 1$, the system is unstable about $k_{c}=1$ and the linear growth-rate around $k_{c}$ is

$$
\eta^{2} \approx \mu-\alpha\left(k-k_{c}\right)^{2}
$$

Using the scalings

$$
\begin{array}{r}
\frac{\partial}{\partial x}=\frac{\partial}{\partial x}+\sqrt{\mu} \frac{\partial}{\partial X} \\
\frac{\partial}{\partial t}=\sqrt{\mu} \frac{\partial}{\partial T},
\end{array}
$$

one can show that the amplitude equation is

$$
\frac{\partial^{2} A}{\partial T^{2}}=\mu A+\alpha \frac{\partial^{2} U}{\partial X^{2}}-\beta|A|^{2} A .
$$

This is a nonlinear Klein-Gordon equation.

\section{References}

Chandrasekhar, S. (1961) Hydrodynamic and Hydromagnetic Stability, Clarendon Press, Oxford.

Chapman, C. J. (1978) Nonlinear Rayleigh-Bénard Convection Between Poorly Conducting Boundaries, Woods Hole Oceanographic Institution Technical Report.

Coullet, P., Fauve, S. (1985) Collective Modes of Periodic Structures, Combustion and Nonlinear Phenomena, Les Editions de Physique.

DiPrima, R. C., Swinney, H. L., (1981) Instabilities and Transition in Flow between Concentric Rotating Cylinders, Hydrodynamic Instabilities and the Transition to Turbulence, Swinney, H. L. and Gollub, J. P. Editors, Topics in Applied Physics 45, 97-137, Springer Verlag.

Fauve, S. (1985) Large Scale Instabilities of Cellular Flows, Woods Hole Oceanographic Institution Technical Report.

Langer, J. S. (1975) Spinodal Decomposition, Fluctuations, Instabilities, and Phase Transitions, T. Riste Editor, NATO Advanced Study Institutes Series, Plenum Press.

Turner, J. S. (1973) Buoyancy Effects in Fluids, Cambridge University Press. 


\section{Secondary Instabilities of Cellular Flows: Eckhaus and zig-zag instabilities}

\subsection{Broken symmetries and neutral modes}

We have so far considered the onset of cellular structures as a control parameter is changed. We now consider the instability of such structures which occurs as the parameter is changed further. We call this a secondary instability of the system. In many cases, a secondary instability arises from a neutral mode associated with a symmetry of the governing equations broken by the primary instability.

For example, consider convection in container of infinite extent in the horizontal plane, or with periodic boundary conditions. The onset of convection breaks translational symmetry in the direction perpendicular to the rolls, the $x$-axis, say. However, one can imagine pushing the rolls along the $x$-axis without any expenditure of energy, since this only amounts to a shift of the $x$-axis origin. This translation is a neutral mode. In other words, one roll solution breaks translational invariance, but the ensemble of all the possible roll solutions should be invariant according to the Curie principle. Thus, as we noticed earlier, the phase of the periodic structure above the instability onset is arbitrary. Changing the phase amounts to a move along the orbit of all the possible roll solutions. This "motion" does not require any energy and is a neutral mode of the periodic structure, i.e. it has a zero growth rate.

Let us illustrate this concept with Swift-Hohenberg-type models. We first consider the form

$$
\frac{\partial u}{\partial t}=\left[\mu-\left(1+\frac{\partial^{2}}{\partial x^{2}}\right)^{2}\right] u-u^{3} .
$$

Suppose there exists a periodic solution $u_{0}(x)$ to the full nonlinear equation (6.1), that is,

$$
L \cdot u_{0}=u_{0}^{3},
$$

where $L=\left[\mu-\left(1+\frac{\partial^{2}}{\partial x^{2}}\right)^{2}\right]$. Taking a derivative of the equation above yields

$$
L \cdot\left(\frac{d u_{0}}{d x}\right)=3 u_{0}^{2} \frac{d u_{0}}{d x} .
$$

Now, in order to investigate the linear stability of $u_{0}$ we write

$$
u=u_{0}(x)+\epsilon v(x, t)
$$

and we get from equation (6.1)

$$
\frac{\partial v}{\partial t}=L \cdot v-3 u_{0}^{2} v+O\left(\epsilon^{2}\right)
$$


Comparison of equation (6.2) with equation (6.3) shows that

$$
v=\frac{d u_{0}}{d x}
$$

is an eigenmode with a zero eigenvalue. A perturbation, $v$, proportional to this eigenmode corresponds to a translation $u_{0}(x+\epsilon)$. This can be shown differently by looking for a solution in the form

$$
u(x, t)=u_{0}[x+\phi(t)]
$$

where $u_{0}$ is our steady state solution, and $\phi(t)$ is a time dependent phase. Substituting this into equation (6.1) yields

$$
u_{0}^{\prime} \frac{d \phi}{d t}=L \cdot u_{0}-u_{0}^{3}
$$

where $u_{0}^{\prime}$ is the derivative of $u_{0}$ with respect to its argument. We get

$$
\frac{d \phi}{d t}=0
$$

which confirms that the translational perturbation is a neutral mode of equation (6.1).

Translational invariance is of course not the only possible broken symmetry at the onset of a pattern-forming instability. Consider for instance the model (5.40) of lecture 5

$$
\frac{\partial u}{\partial t}=\frac{\partial^{2} u}{\partial x^{2}}+r \frac{\partial^{4} u}{\partial x^{4}}+\frac{\partial^{6} u}{\partial x^{6}}-u \frac{\partial u}{\partial x}
$$

which is Galilean invariant, i.e. invariant under the transformation

$$
x \rightarrow x-c t, \quad u \rightarrow u+c
$$

We consider a periodic solution $u_{0}(x)$ of equation (6.5) and look for a perturbation in the form

$$
u=u_{0}[x+\phi(t)]-\psi(t)
$$

We get from (6.4)

$$
\frac{d \phi}{d t} u_{0}^{\prime}-\frac{d \psi}{d t}-\psi u_{0}^{\prime}=0
$$

thus,

$$
\begin{aligned}
\frac{d \phi}{d t} & =\psi \\
\frac{d \psi}{d t} & =0 .
\end{aligned}
$$

Two neutral modes are now involved, and they trace back to the broken translational and Galilean invariances. The form of the coupling between $\phi$ and $\psi$ can be understood as follows: if one pushes the pattern $u_{0}(x)$ at a constant velocity $\psi$ along the $x$-axis, one observes a spatial phase $\phi$ that increases linearly in time. 


\subsection{Phase dynamics}

We could have imposed perturbations of the form (6.3) but with the phase $\phi$ slowly varying both in time and space, $\phi(X, T)$, where $X=\epsilon x$ and $T=\epsilon^{\xi} t$ are slow variables (for a diffusive behavior for instance, $\xi=2$ ). More generally, a long wavelength perturbation of the perfectly periodic pattern $u_{0}(x)$ can be written in the form

$$
u(x, t)=u_{0}[x+\phi(X, T)]+u_{\perp}(X, T),
$$

where

$$
u_{0}[x+\phi(X, T)]-u_{0} \approx \phi(X, T) \frac{d u_{0}}{d x}
$$

represents perturbations along the orbit of the symmetry group corresponding to translational invariance, i.e. phase perturbations, and $u_{\perp}$ consists of perturbations transverse to the group orbit, i.e. amplitude perturbations. A constant phase perturbation is neutral, and correspondingly weakly damped in the long wavelength limit. By contrast, amplitude perturbations decay on a faster time scale when the perfectly periodic pattern is stable. Thus, adiabatic elimination of the amplitude modes leads to an evolution equation for the phase. This "phase equation" can be derived assuming that an expansion in powers of the gradients of the phase

$$
\frac{\partial \phi}{\partial T}=\mathcal{F}\left[\frac{\partial \phi}{\partial X}, \frac{\partial^{2} \phi}{\partial X^{2}}, \ldots\right] .
$$

The linear part of this equation is the Fourier-Laplace transform of the dispersion relation for the growth rate of the phase modes. The growth rate is zero at zero wavenumber due to translational invariance. If it is negative in the long wavenumber limit, the phase perturbation is damped. However, due to the coupling with amplitude modes, the growth rate may be positive in the long wavelength limit, thus describing a phase instability of the perfectly periodic pattern.

In the case of model (6.5), long wavelength perturbations should be considered in the form

$$
u(x, t)=u_{0}[x+\phi(X, T)]-\psi(X, T)+u_{\perp}(X, T) .
$$

This leads to phase equations of the form

$$
\begin{array}{r}
\frac{\partial \phi}{\partial T}=\psi+\mathcal{F}\left[\frac{\partial \phi}{\partial X}, \frac{\partial^{2} \phi}{\partial X^{2}}, \ldots\right], \\
\frac{\partial \psi}{\partial T}=\mathcal{G}\left[\frac{\partial \phi}{\partial X}, \frac{\partial^{2} \phi}{\partial X^{2}}, \ldots\right] .
\end{array}
$$

Thus phase dynamics is second order in time, and propagative modes may result from the coupling between the phases associated with broken translational and Galilean invariances.

Before we pursue phase dynamics further, it is worth pointing out that it is only useful for secondary instabilities with wavenumbers much smaller than those of the primary instability. 
In other words, we can only investigate variations of the underlying pattern which are much larger in scale than the basic pattern wavelength. Furthermore, we had earlier said that amplitude and phase ought to vary together, but here we have considered phase variations in isolation. This turns out to be reasonable when the secondary instability is well separated from the onset of the primary instability. In this case, the amplitude follows adiabatically the phase gradients, so no additional equation for the amplitude is needed. However, in the vicinity of the primary instability, coefficients in the phase equation diverge, and we must use the full amplitude equation instead.

Other symmetries besides translation and Galilean invariance can be found in the basic equations, but all symmetries do not, in general, lead to slowly varying local dynamics, and so are not amenable to the method of phase dynamics. For example, rotational and dilatational invariances, regardless how small the rate of transformation, both result in arbitrarily large effects at sites sufficiently distant from the center, and do not lead, in general, to additional phase modes.

\subsection{Eckhaus Instability}

\subsubsection{Compression mode of a one-dimensional pattern}

We considered stationary cellular instability earlier, and derived the amplitude equation, that reads in the supercritical case with appropriate amplitude and length scales,

$$
\frac{\partial A}{\partial T}=\mu A+\frac{\partial^{2} A}{\partial X^{2}}-|A|^{2} A
$$

Equation (6.12) has stationary solutions

$$
A_{q}=Q \exp (i q X) \text { where } Q^{2}=\mu-q^{2},
$$

that represent all the possible roll solutions which correspond to the unstable band of modes above the instability onset.

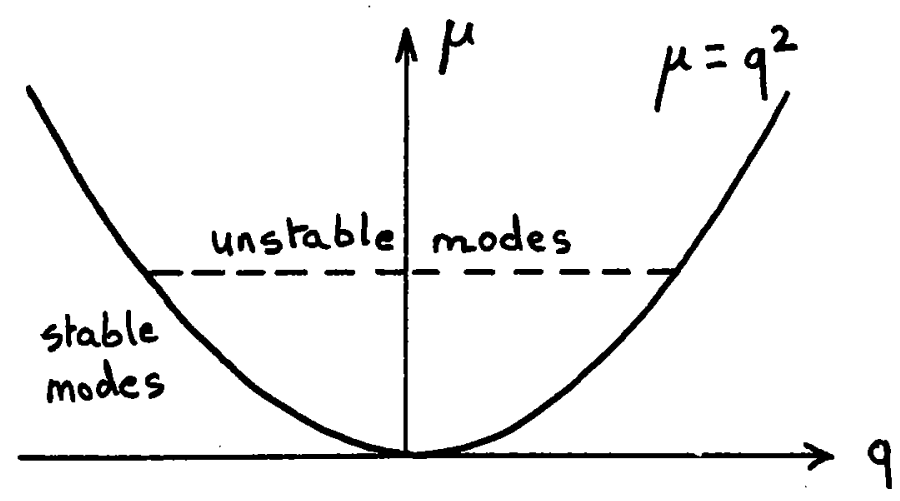

Figure 6.1. Cellular Instability: Cellular patterns exist only for parameters within the parabola. 
We investigate the stability of these stationary patterns by perturbing their amplitude and phase, thus writing

$$
A=[Q+r(X, T)] \exp i[q X+\phi(X, T)]
$$

in equation (6.12). Expanding and separating real and imaginary parts yields

$$
\begin{array}{r}
\frac{\partial r}{\partial T}=\mu[Q+r]+\frac{\partial^{2} r}{\partial X^{2}}-[Q+r]\left[q+\frac{\partial \phi}{\partial X}\right]^{2}-[Q+r]^{3} \\
{[Q+r] \frac{\partial \phi}{\partial T}=2 \frac{\partial r}{\partial X}\left[q+\frac{\partial \phi}{\partial X}\right]+[Q+r] \frac{\partial^{2} \phi}{\partial X^{2}}}
\end{array}
$$

If we linearize in $r$ and $\phi$, and use $Q^{2}=\mu-q^{2}$, we obtain

$$
\begin{array}{r}
\frac{\partial r}{\partial T} \approx-2 Q^{2} r+\frac{\partial^{2} r}{\partial X^{2}}-2 Q q \frac{\partial \phi}{\partial T}, \\
\frac{\partial \phi}{\partial T} \approx 2 \frac{q}{Q} \frac{\partial r}{\partial X}+\frac{\partial^{2} \phi}{\partial X^{2}} .
\end{array}
$$

We consider modes proportional to $\exp (\eta t+i K X)$ and get from equation (6.15) the dispersion relation

$$
\left|\begin{array}{cc}
\eta+2 Q^{2}+K^{2} & 2 i Q q K \\
-2 \frac{q}{Q} i K & \eta+K^{2}
\end{array}\right|=0
$$

which has solutions

$$
\eta(K)=-\left(Q^{2}+K^{2}\right) \pm \sqrt{Q^{4}+4 K^{2} q^{2}} .
$$

Thus, for small $K$, we have two different branches:

- the "amplitude modes", $\eta_{-}(K)=-2 Q^{2}+O\left(K^{2}\right)$, that are damped,

- the "phase modes", $\eta_{+}(K)=-K^{2}\left(1-2 q^{2} / Q^{2}\right)+O\left(K^{4}\right)$, that are marginal.

The phase modes

$$
\eta_{+}(K)=-\left(\frac{\mu-3 q^{2}}{\mu-q^{2}}\right) K^{2}-2 \frac{q^{4}}{\left(\mu-q^{2}\right)^{3}} K^{4}+O\left(K^{6}\right)
$$

lead to an instability when

$$
D_{\|}=\left(\frac{\mu-3 q^{2}}{\mu-q^{2}}\right)<0,
$$

so when $q^{2}<\mu<3 q^{2}$; this is the Eckhaus instability. We can thus nest this secondary instability curve in the earlier stability diagram. 


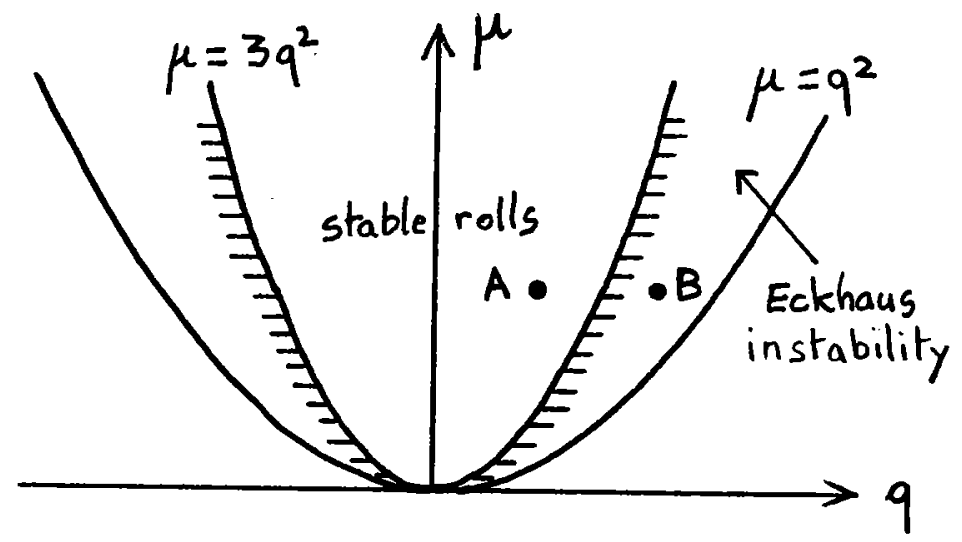

Figure 6.2. Eckhaus instability diagram. The pattern generating primary instability occurs inside the outer parabola, but the resulting pattern is stable only within the inner parabola.

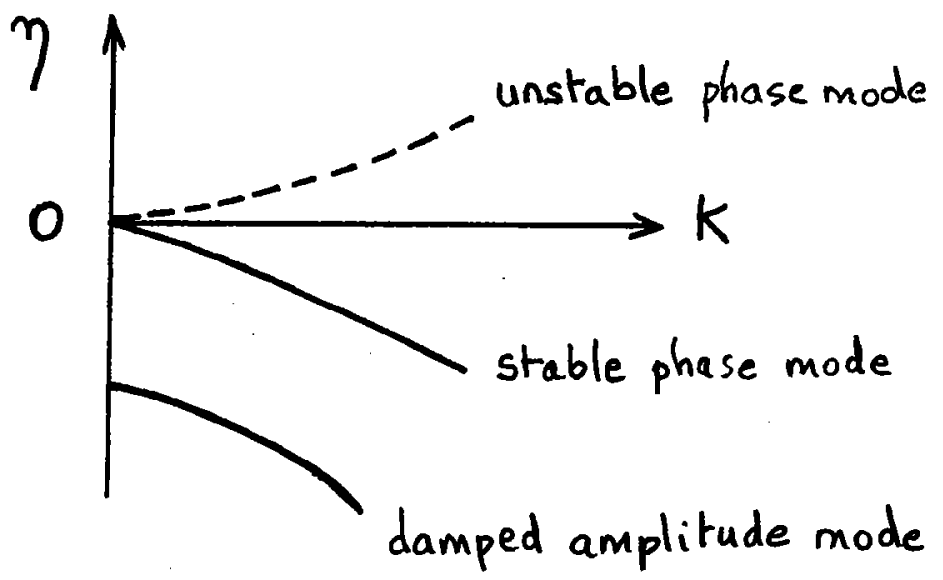

Figure 6.3. Amplitude and phase perturbations have different timescales in the long wavelength limit.

\subsubsection{Nonlinear phase equation for the Eckhaus instability}

We now derive the phase equation of the Eckhaus instability in order to describe pattern $\mathrm{dy}$ namics through the evolution of its slowly varying phase. As said above, this is possible in the long wavelength limit because we have two types of perturbations with different timescales (see Figure 6.3); adiabatic elimination of the amplitude modes leads to an evolution equation for the phase.

For a better understanding, let us consider again the linear equations (6.15). For spatially homogeneous perturbations, as already observed, the amplitude perturbation $r$ decays exponentially whereas the phase perturbation $\phi$ is neutral. For perturbations slowly varying in space, $r$ is nonzero only because it is forced by phase gradients; thus, the amplitude follows adiabatically the phase gradients, and the dominant balance in the evolution equation for $r$ in $(6.15)$ is

$$
2 Q^{2} r \approx-2 Q q \frac{\partial \phi}{\partial T}
$$


Substituting this into the phase equation of (6.15) we obtain

$$
\frac{\partial \phi}{\partial T} \approx\left(1-\frac{2 q^{2}}{Q^{2}}\right) \frac{\partial^{2} \phi}{\partial X^{2}} \equiv D_{\|} \frac{\partial^{2} \phi}{\partial X^{2}} .
$$

We see that the Eckhaus instability shows up as a negative diffusivity in the phase equation. When the primary pattern wavenumber is far enough from that of the critical one, the smallscale flow generated by the primary instability acts as a negative diffusivity for large scale perturbations.

The higher order linear terms of the phase equation can be obtained from higher order balances in (6.15), or in a simpler way from the Fourier-Laplace transform of the small $K$ expansion of the dispersion relation (6.17),

$$
\frac{\partial \phi}{\partial T}=D_{\|} \frac{\partial^{2} \phi}{\partial X^{2}}-\kappa \frac{\partial^{4} \phi}{\partial X^{4}}
$$

where $\kappa=2 q^{4} / Q^{6}$. In the vicinity of the Eckhaus instability onset, $D_{\|} \approx 0$, then $\kappa \approx \frac{3}{4 \mu}$.

We next derive the leading order nonlinear term of the phase equation. Let us first see from symmetry arguments what form the nonlinear term might have. Translational invariance, $x \rightarrow x+x_{0}$, implies that the phase equation should be invariant under $\phi \rightarrow \phi+\phi_{0}$. This precludes any terms explicitly depending on $\phi$ such as $\phi^{2}$ or $\phi \phi_{X}$. Reflection invariance $(x \rightarrow-x)$ implies $\phi \rightarrow-\phi$, eliminating terms such as $\phi_{X} \phi_{X}$. The lowest order term possible is therefore $\phi_{X} \phi_{X X}$. The weakly nonlinear phase equation would then be (if the coefficient of this nonlinear term does not vanish)

$$
\frac{\partial \phi}{\partial T}=D_{\|} \frac{\partial^{2} \phi}{\partial X^{2}}-\kappa \frac{\partial^{4} \phi}{\partial X^{4}}+g \frac{\partial \phi}{\partial X} \frac{\partial^{2} \phi}{\partial X^{2}} .
$$

Factoring the diffusive term, $\left(D_{\|}+g \phi_{X}\right) \phi_{X X}$, we see that the nonlinearity acts as an effective space dependent diffusivity; however, we will see that it cannot compensate the negative diffusivity in the unstable regime, and it does not saturate the Eckhaus instability which is thus a subcritical one whatever the sign of $g$. To find the coefficient $g$ one can proceed formally with a multiple scale expansion from the nonlinear equations for $r$ and $\phi$. There is however a much simpler procedure: we first notice that $\phi=p X$ is a particular solution of (6.18), that simply represents a homogeneous roll solution of wavenumber $k_{c}+\epsilon(q+p)$. The linear stability of this homogeneous roll solution is governed by the dispersion relation

$$
\eta=-D_{\|}(q+p) K^{2}+O\left(K^{4}\right)
$$

We can also compute $\eta$ by linearization of (6.18) near $\phi=p X$; we get

$$
\eta=-\left[D_{\|}(q)+g p\right] K^{2}+O\left(K^{4}\right)
$$


Identifying the two expressions to leading order in $p$, we obtain

$$
g=\frac{\partial D_{\|}}{\partial q} .
$$

We see that at the Eckhaus instability onset, $D_{\|}=0$, all the coefficients of the higher order terms of the phase equation (6.18), diverge as $\mu \rightarrow 0$. As already mentioned, this divergence arises because near the primary cellular instability the amplitude mode also becomes neutral, and thus cannot be eliminated. The full amplitude equation (6.12) should be used to capture the correct behavior. However, one expects that for long wavelength perturbations of rolls, the form of the phase equation (6.18) remains valid along the Eckhaus instability curve, even for rather large values of $r-r_{c}$ out of the range of validity of the amplitude equation.

Let us now analyze the behavior of the phase equation (6.18) in the vicinity of the Eckhaus instability onset. If we look at the linear dispersion relation for a mode $\exp (\eta T+i K X)$ we find (see Figure 6.4) that when $D_{\|}<0$ there is a band of unstable modes for $0<K<K_{*}$.

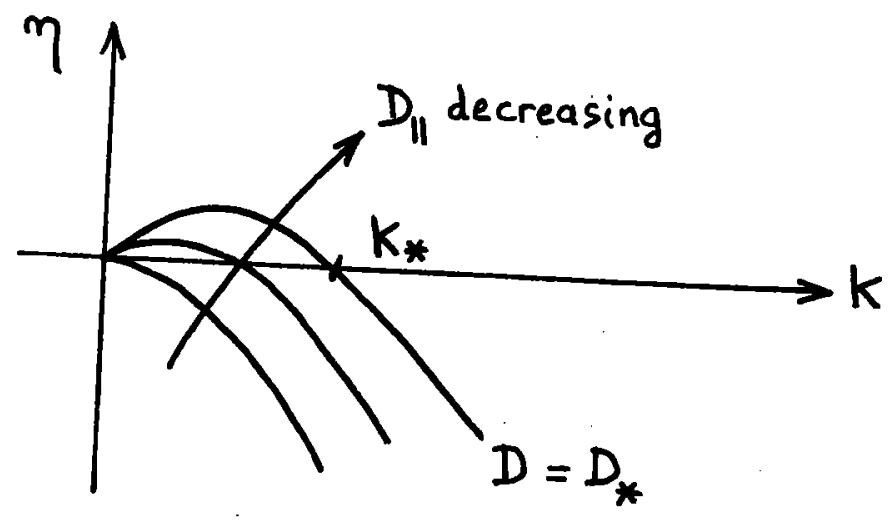

Figure 6.4. Dispersion relation for the phase equation.

We will focus on the neutral mode at $K=K_{*}$ since it is the first to go unstable in an experiment with periodic boundary conditions (the first unstable mode is such that $K_{*}=$ $2 \pi / L$, where $L$ is the size of the periodic domain). The finite geometry thus delays the instability until $D_{\|}=-\left|D_{*}\right|$ when the mode $K_{*}$ becomes neutral. The pattern is then unstable to the compression mode of wavenumber $K_{*}$; let us now study the finite amplitude behavior of this mode.

We obtain this amplitude equation as usual, by inserting a mode

$$
\phi=a(T) \exp \left(i K_{*} X\right)+c . c .
$$

The resulting equation for $a(T)$ is

$$
\frac{d a}{d T}=-\left(D_{\|}-D_{*}\right) K_{*}^{2} a+2 \frac{\mu}{9} g^{2} K_{*}^{2}|a|^{2} a .
$$

We see that there is no stabilization from the nonlinear term (since its coefficient is positive). This is shown on the bifurcation diagram (Figure 6.5). 


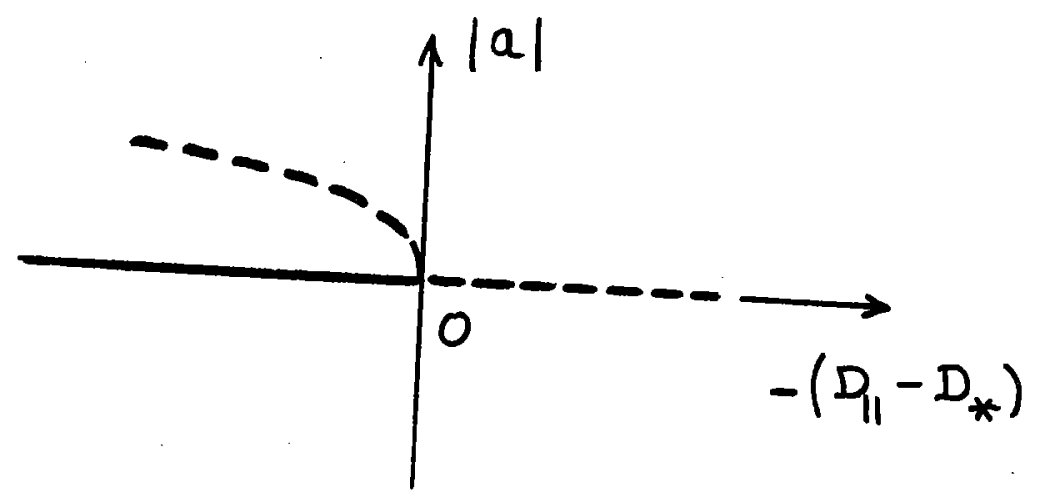

Figure 6.5. Bifurcation diagram of Eckhaus instability

Thus linearly stable rolls $\left(D_{\|}>D_{*}\right)$ can be nonlinearly unstable to finite amplitude perturbations, such as a localized compression or dilatation of the pattern. We will evaluate the critical size of the perturbation that generates the finite amplitude Eckhaus instability; this is analogous to a nucleation energy in first order phase transitions.

We look first for stationary solutions of the phase equation (6. 18). Setting $\partial \phi / \partial T=0$, we obtain by integrating (6.18)

$$
\lambda=D_{\|} \psi-\kappa \frac{\partial^{2} \psi}{\partial X^{2}}+\frac{g}{2} \psi^{2}
$$

Here $\psi$ is the phase gradient,

$$
\psi=\frac{\partial \phi}{\partial X}
$$

i.e. the variation of the local wavenumber, and $\lambda$ is a constant of integration related to the wavenumber at infinity. We can recast the problem as the motion of a particle of mass $\kappa$ in a potential $U(\psi)$ such that

$$
\begin{array}{r}
\kappa \frac{\partial^{2} \psi}{\partial X^{2}}=-\frac{d U}{d \psi} \\
U(\psi)=\lambda \psi-\frac{D_{\|}}{2} \psi^{2}-\frac{g}{6} \psi^{3} .
\end{array}
$$

The potential $U$ can be simplified by eliminating the quadratic term with the transformation $\tilde{\psi}=\psi+D_{\|} / g$. We obtain

$$
U(\tilde{\psi})-U_{0}=\left(\lambda+\frac{D_{\|}^{2}}{2 g}\right) \tilde{\psi}-\frac{g}{6} \tilde{\psi}^{3}
$$

where $U_{0}$ is a constant. This potential is indicated in Figure 6.6. The solutions are graphically obvious, but it should be recalled that these correspond to the $X$ dependence of steady solutions. Nothing has been said as yet about their stability. We have two stationary solutions homogeneous in space (points A and B in Figure 6.6), and oscillatory solutions around $\mathrm{B}$ that represent patterns with a periodically modulated wavenumber. One solution of particular interest is the limiting solitary wave solution, where one imagines the particle travelling from the local maximum to the left and taking an infinite time to return. This 
motion is shown in Figure 6.7 and we see that it corresponds to a localized compression of the rolls.

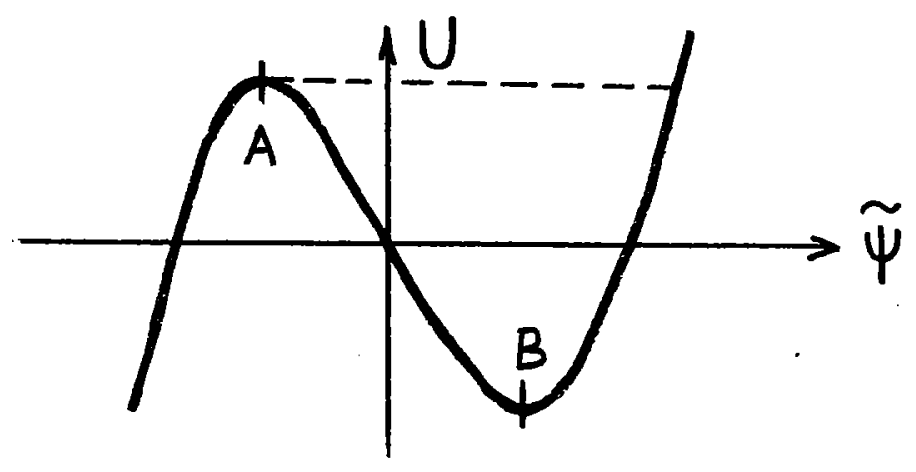

Figure 6.6. Potential well of stationary solutions of the phase equation.

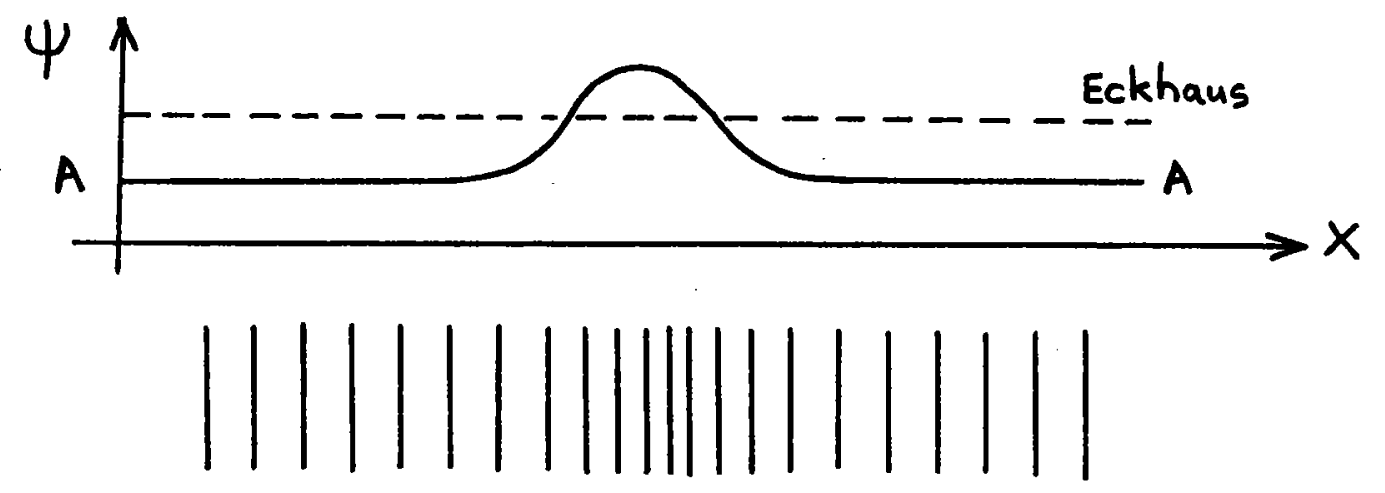

Figure 6.7. Solitary compression wave in the roll pattern.

To study the stability of the above stationary solutions, we find a Lyapunov functional. To wit, multiply equation (6.18) by $\partial \phi / \partial T$ and integrate over a wavelength in $X$. Integrating by parts we obtain

$$
\int_{0}^{L}\left(\frac{\partial \phi}{\partial T}\right)^{2} d X=\int_{0}^{L}\left[-D_{\|} \frac{\partial \phi}{\partial X} \frac{\partial^{2} \phi}{\partial X \partial T}-\kappa \frac{\partial^{3} \phi}{\partial X^{2} \partial T} \frac{\partial^{2} \phi}{\partial X^{2}}-\frac{g}{2}\left(\frac{\partial \phi}{\partial X}\right)^{2} \frac{\partial^{2} \phi}{\partial X \partial T}\right] d X .
$$

Thus,

$$
\frac{d}{d T} \mathcal{F} \equiv \frac{d}{d T} \int_{0}^{L}\left[\frac{D_{\|}}{2}\left(\frac{\partial \phi}{\partial X}\right)^{2}+\frac{\kappa}{2}\left(\frac{\partial^{2} \phi}{\partial X^{2}}\right)^{2}+\frac{g}{6}\left(\frac{\partial \phi}{\partial X}\right)^{3}\right] d X=-\int_{0}^{L}\left(\frac{\partial \phi}{\partial T}\right)^{2} d X \leq 0
$$

$\mathcal{F}[\phi(X, T)]$ is decreasing during the phase dynamics but is not a proper Lyapunov functional because it is not bounded from below. Moreover, we should take into account an additional 
constraint imposed by the conservation form of the phase equation (6.18), or of the equation for the phase gradient $\psi$

$$
\frac{\partial \psi}{\partial T}=\frac{\partial^{2}}{\partial X^{2}}\left[D_{\|} \psi-\kappa \frac{\partial^{2} \psi}{\partial X^{2}}+\frac{g}{2} \psi^{2}\right] .
$$

This imposes the constraint

$$
\frac{d}{d t} \int \psi d X=0
$$

on the evolution, i.e. the conservation of the mean wavenumber. Therefore, the form of the phase equation shows that a pattern cannot evolve by continuously changing its wavelength.

When $\mathcal{F}[\phi(X, T)]$ has a local minimum, we can study the dynamics by minimizing this functional where the above constraint enters with its Lagrange multiplier $\lambda$,

$$
\mathcal{F}_{\lambda}=\int_{0}^{L}\left[\frac{D_{\|}}{2} \psi^{2}+\frac{\kappa}{2}\left(\frac{\partial \psi}{\partial X}\right)^{2}+\frac{g}{6} \psi^{3}-\lambda \psi\right] d X .
$$

Thus,

$$
\mathcal{F}_{\lambda}=\int_{0}^{L}\left[\frac{\kappa}{2}\left(\frac{\partial \psi}{\partial X}\right)^{2}-U(\psi)\right] d X,
$$

and, to minimize $\mathcal{F}_{\lambda}$, we should maximize $U$. This means that the local maximum of $U$ in Figure 6.6 is metastable whereas the local minimum is unstable. Indeed, points A and B in Figure 6.6 correspond to the same points on the Eckhaus stability diagram (Figure 6.2). Note that the oscillatory solutions about $B$ are unstable. The solitary wave solution represents the "critical nucleus", i.e. the critical localized perturbation to the linearly stable pattern represented by A, that generates the finite amplitude Eckhaus instability. The corresponding value of $\mathcal{F}_{\lambda}$ minus its evaluation for the homogeneous stable pattern, represents the "energy barrier" of a pattern changing process from the homogeneous pattern A; this barrier vanishes at the linear Eckhaus instability onset. These points will be further covered next by studying the amplitude equation (6.12) directly.

\subsubsection{Localized solutions of the one-dimensional Ginzburg-Landau equation}

We now investigate in more detail the amplitude equation (6.12). We first rescale the time scale so that (6.12) can be written as

$$
A_{T}= \pm A+\frac{\partial^{2} A}{\partial X^{2}}-|A|^{2} A,
$$

where the plus or minus sign corresponds to the sign of $\mu$. As already mentioned in lecture $5,(6.24)$ is a variational problem, i.e.

$$
\begin{array}{r}
\frac{d}{d T} \mathcal{L}[A]=-2 \int_{0}^{L}\left|\frac{\partial A}{\partial T}\right|^{2} d X \leq 0, \\
\mathcal{L}[A]=\int_{0}^{L}\left[\left|\frac{\partial A}{\partial X}\right|^{2} \mp|A|^{2}+\frac{1}{2}|A|^{4}\right] d X .
\end{array}
$$


The Lyapunov functional $\mathcal{L}$ is a decreasing function and has a minimum value for the homogeneous solution $\left(A_{X}=0\right)$ that maximizes $U(A)= \pm A^{2}-\frac{1}{2} A^{4}$. For $\mu<0$, the stationary solutions of (6.24) are

$$
A=Q \exp (i q X), \text { with } Q^{2}=1-q^{2},
$$

and the Lyapunov functional (6.25) may be rewritten as

$$
\mathcal{L}=\int_{0}^{L} l(q) d X
$$

where

$$
l(q)=-\frac{1}{2}\left(q^{2}-1\right)^{2} .
$$

We check that the periodic pattern with the critical wavenumber $k_{c}$, i.e. $q=0$, corresponds to the absolute minimum of $l(q)$. The inflection points of $l(q)$ correspond to $l^{\prime \prime}(q)=-2\left(3 q^{2}-\right.$ 1) $=0$, which is the Eckhaus instability limit $q_{E}^{2}=1 / 3$. In solid state physics $l(q)$ is the free energy density and $l^{\prime \prime}(q)<0$ corresponds to negative compressibility. Thus, the Eckhaus instability corresponds to a negative compressibility of the periodic pattern (Figure 6.8).

Figure 6.8. Plots of $\mu(q)$ and $l(q)$

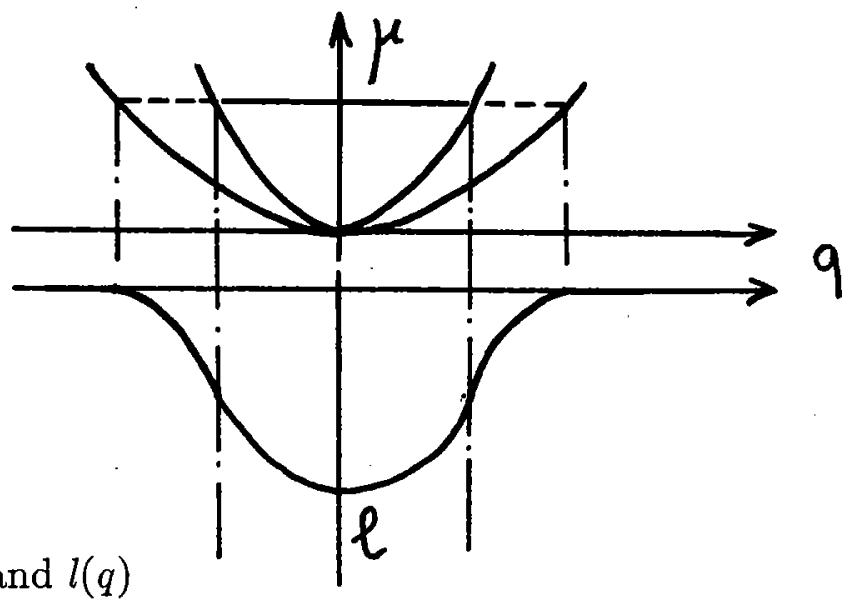

We now investigate the wavenumber changing process. Substituting $A=R e^{i \phi}$ into the amplitude equation (6.24) and equating real and imaginary parts we get

$$
\begin{array}{r}
\frac{\partial R}{\partial T}=R-R^{3}+\frac{\partial^{2} R}{\partial X^{2}}-R\left(\frac{\partial \phi}{\partial X}\right)^{2}, \\
R \frac{\partial \phi}{\partial T}=2 \frac{\partial R}{\partial X} \frac{\partial \phi}{\partial X}+R \frac{\partial^{2} \phi}{\partial X^{2}} .
\end{array}
$$

For stationary solutions the second equation gives

$$
R^{2} \frac{\partial \phi}{\partial X}=h,
$$

where $h$ is a constant. This conservation of "angular momentum" arises from the rotational invariance of the amplitude equation. The constraint (6.28) is the reason why the pattern 
wavenumber changing process cannot occur by homogeneously modifying the wavenumber at constant amplitude.

Substituting (6.28) into (6.27), we get for stationary solutions

$$
\frac{\partial^{2} R}{\partial X^{2}}=-R+R^{3}+h^{2} / R^{3},
$$

which is of the form

$$
\begin{array}{r}
\frac{\partial^{2} R}{\partial X}=-\frac{\partial V}{\partial R}, \\
V(R)=\frac{1}{2} R^{2}-\frac{1}{4} R^{4}+\frac{h^{2}}{2 R^{2}},
\end{array}
$$

thus corresponding again to the motion of a particle in the potential $V(R)$ (Figure 6.9 a). Bounded solutions exist for $h^{2}<4 / 27$; the two extrema $R_{ \pm}$of $V$ correspond to homogeneous stationary solutions, such that

$$
h^{2}=R_{ \pm}^{4}\left(1-R_{ \pm}^{2}\right) .
$$

Their stability can be determined using the Lyapunov functional (6.25); $R_{+}$is the stable solution corresponding to the point $\mathrm{A}$ in Figures 6.2 and 6.6 , and $R_{-}$is the unstable one corresponding to $\mathrm{B}$.
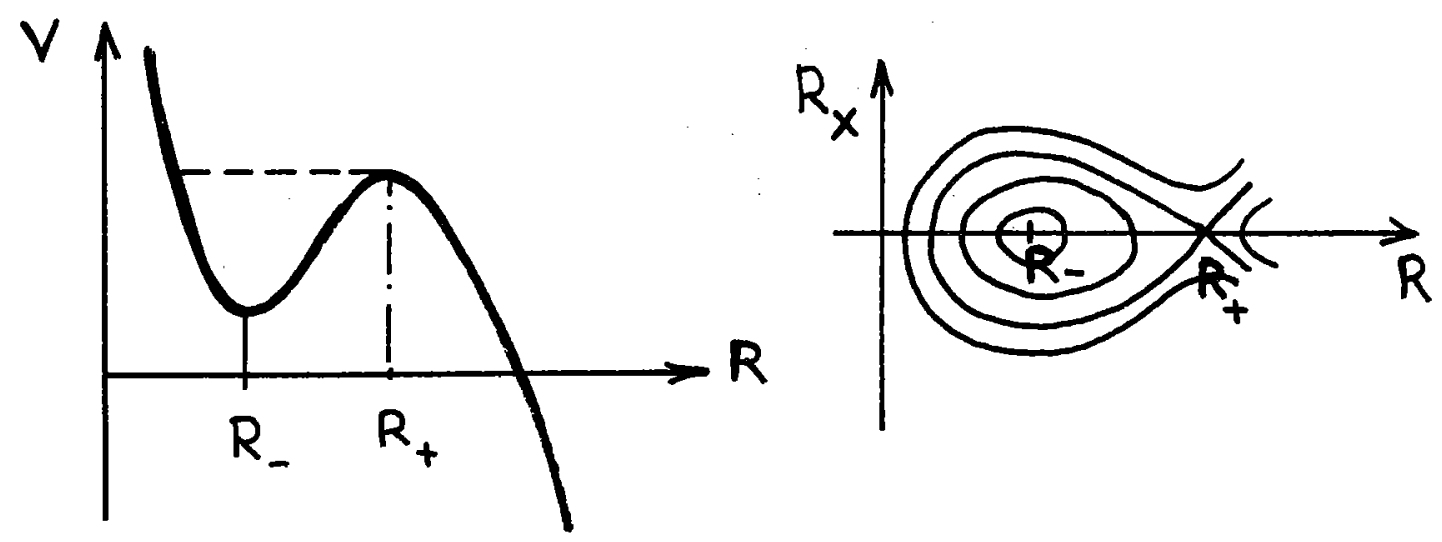

Figure 6.9. Potential well $V(R)$ for stationary solutions of the amplitude equation (6.24) and corresponding phase space

Figure $6.9 \mathrm{~b}$ shows the phase space of the stationary solutions of amplitude equation (6.24). The homoclinic orbit passing through $R_{+}$corresponds to the solitary wave solution that describes a localized compression (or dilatation) of the pattern, already found within the phase equation approach. The constraint (6.28) shows that the amplitude $R$ decreases in these regions. When the localized compression (or dilatation) of the pattern is too large, the amplitude vanishes and this allows the annihilation (or creation) of pair of rolls; this wavenumber changing mechanism involves length scales comparable to the wavelength of the primary pattern, and is out of the range of validity of the amplitude equation (6.12) or of the phase equation (6.18). However, we were able to obtain the qualitative behavior of the Eckhaus instability from these long-wavelength approximations. 


\subsection{The zig-zag instability}

\subsubsection{Torsion mode of a one-dimensional pattern}

Like the Eckhaus instability, the zig-zag instability, shown in Figure 6.10 is associated with the broken translational invariance which a stationary roll solution exhibits. In this case however the perturbation to the rolls is a transverse one, and can be written in the long wavelength limit,

$$
u(x, y, t)=u_{0}[x+\phi(Y, T)]+u_{\perp}(X, Y, T) .
$$

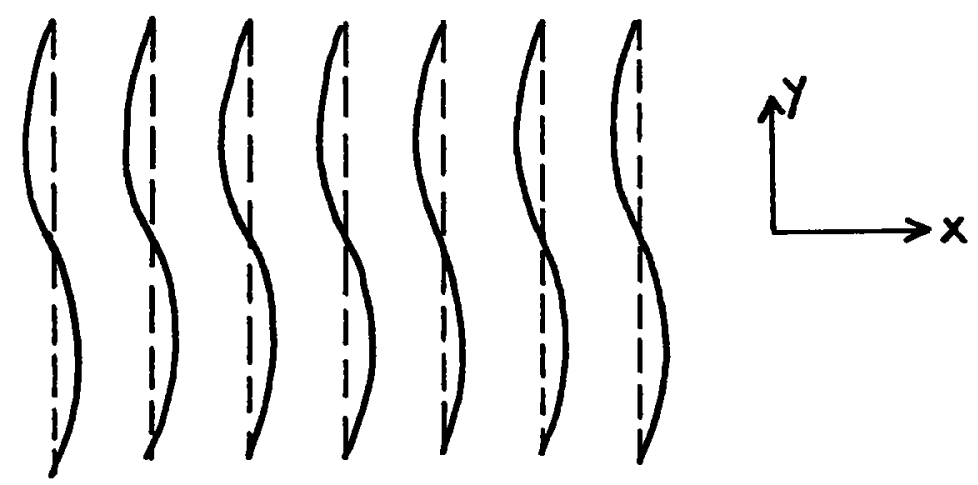

Figure 6.10. The form of the zig-zag instability: unperturbed rolls (dashed lines) and zig-zag mode (solid lines).

To investigate the dynamics of this torsion mode in the framework of the amplitude equation, we should use

$$
\frac{\partial A}{\partial T}=\mu A+\left(\frac{\partial}{\partial X}-\frac{i}{2 k_{c}} \frac{\partial^{2}}{\partial Y^{2}}\right)^{2} A-|A|^{2} A,
$$

obtained in lecture 5 .

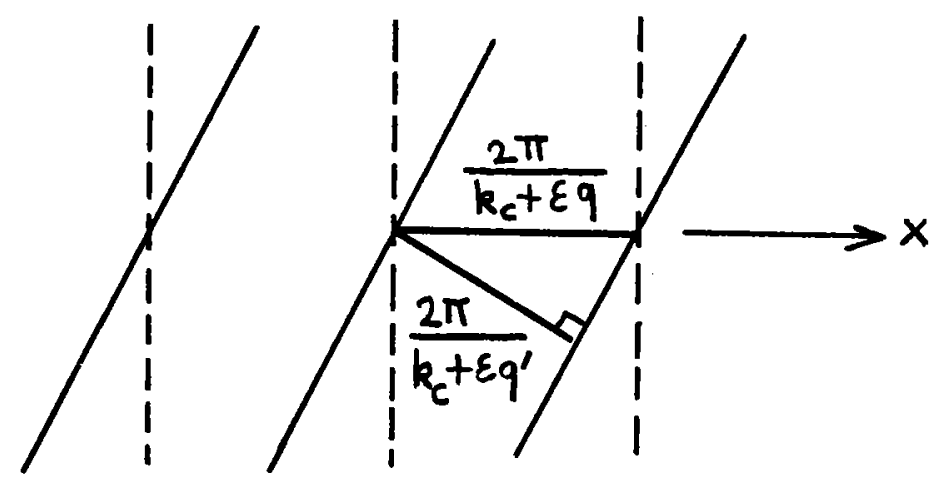

Figure 6.11. The effect of rotation of the roll planform 
Figure 6.11 shows that rotation of the rolls leads to a decrease in the wavelength of the pattern, and hence an increase in the wavenumber. The zig-zag mode locally corresponds to a rotation of the pattern, so we can deduce that it will result in a local increase of the wavenumber. Referring to Figure 6.8, we see that for $q<0$ an increase in $q$ corresponds to a decease in $l(q)$, the Lyapunov functional, so in this region the pattern is unstable to zig-zag perturbations. However, for $q>0, l(q)$ increases as $q$ increases, demonstrating that the pattern is stable to local rotations. The zig-zag instability thus occurs for $k<k_{c}$ in the framework of (6.31). Indeed, if we look for a perturbation varying slowly in $y$,

$$
A=[Q+r(Y, T)] \exp i[q X+\phi(Y, T)]
$$

we get to leading order

$$
\frac{\partial \phi}{\partial T} \approx D_{\perp} \frac{\partial^{2} \phi}{\partial Y^{2}}, \text { where } D_{\perp}=\frac{q}{k_{c}}
$$

\subsubsection{Nonlinear phase equation for the zig-zag instability}

Using symmetry considerations, translational invariance, that implies the invariance under $\phi \rightarrow \phi+\phi_{0}, x$-reflection symmetry, that implies the invariance under the transformation $X \rightarrow-X, \phi \rightarrow-\phi$, and $y$-reflection symmetry, that implies the invariance under the transformation $Y \rightarrow-Y$, we obtain to leading nonlinear order, the phase equation

$$
\frac{\partial \phi}{\partial T}=D_{\perp} \frac{\partial^{2} \phi}{\partial Y^{2}}-\kappa \frac{\partial^{4} \phi}{\partial Y^{4}}+g\left(\frac{\partial \phi}{\partial Y}\right)^{2} \frac{\partial^{2} \phi}{\partial Y^{2}}
$$

Note the scaling $\phi \sim O(1)$, contrary to the Eckhaus instability where $\phi$ should be small. Rearranging (6.32) results in

$$
\frac{\partial \phi}{\partial T}=\left[D_{\perp}+g\left(\frac{\partial \phi}{\partial Y}\right)^{2}\right] \frac{\partial^{2} \phi}{\partial Y^{2}}-\kappa \frac{\partial^{4} \phi}{\partial Y^{4}} .
$$

Now, $D_{\perp}<0$ for instability; if the instability is to be saturated, the effective diffusivity must be positive, and hence $g$ must be positive. Let us compute $g$, using a similar method as for the Eckhaus instability: first, note that $\phi=p Y$ is a particular solution of (6.32) that corresponds to a tilted roll pattern. Writing, $\phi=p Y+\tilde{\phi}$, and linearizing in $\tilde{\phi}$, results in

$$
\frac{\partial \tilde{\phi}}{\partial T}=\left(D_{\perp}+g p^{2}\right) \frac{\partial^{2} \tilde{\phi}}{\partial Y^{2}}-\kappa \frac{\partial^{4} \tilde{\phi}}{\partial Y^{4}}
$$

However, the tilted roll solution is simply another roll pattern with a new wavenumber, $q^{\prime}$, where $q^{\prime}=q+p^{2} / 2 k_{c}$. So we can also write

$$
\frac{\partial \tilde{\phi}}{\partial T}=D_{\perp}\left(q^{\prime}\right) \frac{\partial^{2} \tilde{\phi}}{\partial Y^{\prime 2}}-\kappa \frac{\partial^{4} \tilde{\phi}}{\partial Y^{\prime 4}}
$$


and matching the two previous equations gives

$$
g=\frac{1}{2 k_{c}} \frac{\partial D_{\perp}}{\partial q}=\frac{1}{2 k_{c}^{2}} .
$$

Hence $g>0$, and the zig-zag instability is supercritical in the framework of the amplitude equation (6.31).

We now use the phase equation (6.32) to study the pattern generated by the zig-zag instability in the supercritical regime. If we let $\phi \propto \exp (\eta T+i K Y)$ and linearize about $\phi=0$, we obtain

$$
\eta=-D_{\perp} K^{2}-\kappa K^{4}
$$

This dispersion relation is similar to the one of the Eckhaus instability (see Figure 6.4) it might be thought that the mode with the maximum growth rate, $k_{m a x}$, corresponds to the characteristic length scale of the zig-zag instability in the supercritical regime, but a nonlinear analysis will show that this is not so.

The stationary solutions of (6.32) satisfy

$$
\lambda=D_{\perp} \psi-\kappa \frac{\partial^{2} \psi}{\partial Y^{2}}+\frac{g}{3} \psi^{3}
$$

where

$$
\psi=\frac{\partial \phi}{\partial Y}
$$

is the phase gradient, and $\lambda$ is a constant of integration related to the wavenumber at infinity. This can be written

$$
\begin{array}{r}
\kappa \frac{\partial^{2} \psi}{\partial Y^{2}}=-\frac{d U}{d \psi}, \\
U(\psi)=\lambda \psi-\frac{D_{\perp} J}{2} \psi^{2}-\frac{g}{12} \psi^{4},
\end{array}
$$

showing again that the determination of the stationary solutions amounts to find the trajectories of a particle of mass $\kappa$ in the potential well $U(\psi)$. We look for a Lyapunov functional in order to determine the stability of these solutions. Multiplying (6.32) by $\partial \phi / \partial T$ and integrating by parts gives

$$
\begin{array}{r}
\frac{d}{d T} \mathcal{F}=-\int_{0}^{L}\left(\frac{\partial \phi}{\partial T}\right)^{2} d Y \leq 0, \\
\mathcal{F} \equiv \int_{0}^{L}\left[\frac{\kappa}{2}\left(\frac{\partial \psi}{\partial Y}\right)^{2}+\frac{D_{\perp}}{2} \psi^{2}+\frac{g}{12} \psi^{4}\right] d Y .
\end{array}
$$

Thus, $\mathcal{F}[\psi(Y, T)]$ decreases; however, the governing equation for $\psi$ has a conservative form

$$
\frac{\partial \psi}{\partial T}=\frac{\partial^{2}}{\partial Y^{2}}\left[D_{\perp} \psi+\frac{g}{3} \psi^{3}-\kappa \frac{\partial^{2} \psi}{\partial Y^{2}}\right]
$$


which implies the constraint

$$
\frac{d}{d T} \int_{0}^{L} \psi d Y=0,
$$

on the phase dynamics. Thus, $\lambda$ appears like a Lagrange multiplier, and we have to minimize

$$
\mathcal{F}_{\lambda}=\int_{0}^{L}\left[\frac{\kappa}{2}\left(\frac{\partial \psi}{\partial Y}\right)^{2}-U(\psi)\right] d Y .
$$

If we start from rolls perpendicular to the $x$-axis, i.e. $\psi=0$, in the unstable regime, $D_{\perp}<0$, we can take $\lambda=0$ and the potential $U(\psi)$ is symmetric (Figure 6.12).

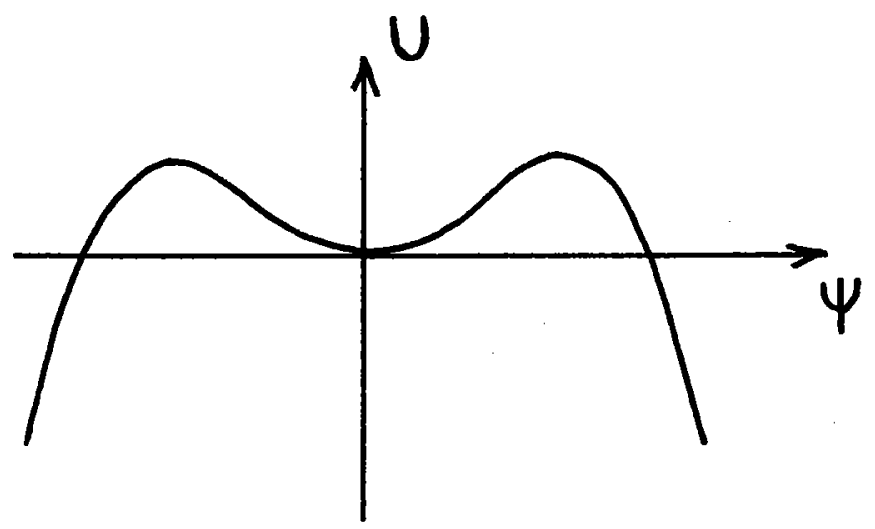

Figure 6.12. The potential $U(\psi)$ for $D_{\perp}<0$ and $\lambda=0$

The fastest growing mode is the one with wavenumber $k_{m a x}$ and generates a wavy pattern such as the one displayed in Figure 6.10. This pattern corresponds to an oscillatory solution about $\psi=0$ in the potential well of Figure 6.12; thus it is unstable. The stable pattern which satisfies the constraint (6.36) corresponds to the limiting solitary wave solution that connects the two opposite values $\psi= \pm \sqrt{3\left|D_{\perp}\right| J / g}$, i.e. two sets of rolls with different orientations, symmetric with respect to the $y$-axis (see Figure 6.13). Note that this solution cannot be observed if we assume periodic boundary conditions in $Y$; the dynamics lead to a two kink pattern in that case.

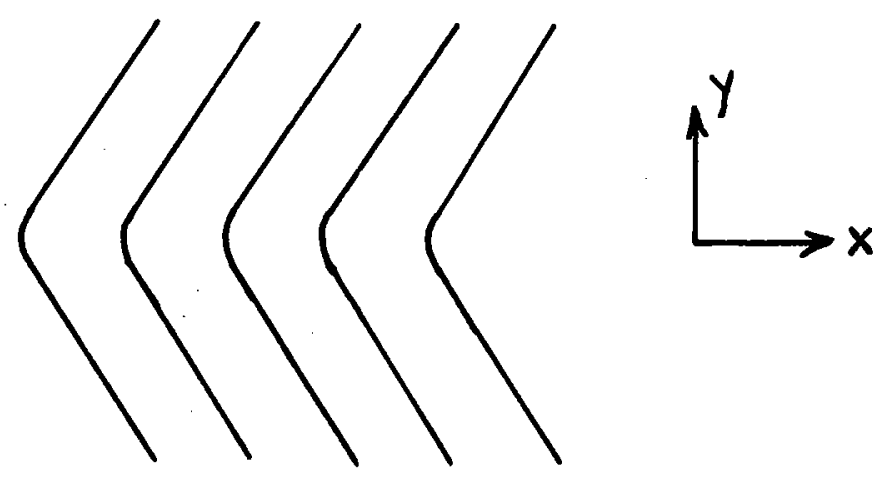

Figure 6.13. Two sets of rolls with different orientations 
Thus, in the nonlinear regime, the zig-zag instability generates the largest possible domains with parallel rolls, compatible with the mean wavenumber conservation and the constraints related to boundary conditions. Although the Eckhaus and the zig-zag instabilities have similar dispersion relations, their nonlinear dynamics are different: the linearly unstable perturbations cascade to short scales in the Eckhaus instability, whereas larger and larger domains are created during the nonlinear evolution of the zig-zag instability.

\section{References}

Eckhaus, V. (1965) Studies in Nonlinear Stability Theory, Springer Verlag.

Fauve, S. (1985) Large Scale Instabilities of Cellular Flows, Woods Hole Oceanographic Institution Technical Report.

Kramer, L., Zimmermann, W. (1985) On the Eckhaus Instability for Spatially Periodic Patterns, Physica D 16, 221.

Kuramoto, Y. (1984) Phase Dynamics of Weakly Unstable Periodic Structures, Prog. Theor. Phys. 71, 1182-1196

Pomeau, Y., Manneville, P. (1979) Stability and Fluctuations of a Spatially Periodic Convective Flow, J. Phys. Lettres 40, 609-612

Stuart, J. T., DiPrima, R. C., (1978) The Eckhaus and Benjamin-Feir Resonance Mechanisms, Proc. Roy. Soc. Lond. A 362, 27-41 


\section{Drift Instabilities of Cellular Patterns}

\subsection{Introduction}

Another type of secondary instability that occurs in many systems is the "drift instability". After a first bifurcation to a stationary cellular structure, $\mathbf{u}_{0}(x)$, further increase in the bifurcation parameter generates a secondary bifurcation to a traveling pattern of the form $\mathbf{u}_{D}(x \pm c t)$. The motion of the pattern in one of the preferential directions, $\pm x$, breaks the space reflection symmetry. As we observed in lecture 5 (model (5.14)), a stationary bifurcation may generate a traveling pattern at onset when the system is not invariant under reflection symmetry $x \rightarrow-x$. Here, we consider systems which are invariant under reflection symmetry and which give rise to a symmetric primary pattern; the reflection symmetry is spontaneously broken at finite amplitude when the static pattern undergoes the secondary drift bifurcation.

Drift instabilities of cellular patterns have been widely observed in various experimental situations. Couette flow between two horizontal coaxial cylinders with a partially filled gap (Mutabazi et al., 1988), displays transitions from stationary to traveling rolls ; as clearly noticed, the traveling rolls are tilted and the direction of the propagation is determined by this asymmetry. The traveling-roll state is either homogeneous in space, or there exist domains of inclined rolls with opposite tilt, and thus opposite propagation direction. Similar results have been found recently in a film draining experiment (Rabaud et al., 1990). Drift instabilities have been also observed in directional crystal growth experiments. Above the onset of the Mullins-Sekerka instability of liquid crystals, "solitary modes" propagating along the interface have been observed (Simon et al., 1988). These "solitary modes" consist of domains of stretched asymmetric cells that connect two regions with symmetric cells. Similarly, domains of tilted lamellae moving tranversally along the growth front have been observed during directional solidification of eutectics (Faivre et al., 1989), and the relationship of the tilt direction to the one of propagation has been also emphasized. Finally, a drift instability was observed recently for a standing surface wave excited parametrically in a horizontal layer of fluid contained in a thin annulus, submitted to vertical vibrations (Douady et al., 1989). It was observed that, as the driving amplitude is increased, the standing wave pattern either begins to move at a constant speed in one direction, or undergoes an oscillatory instability that corresponds to a compression mode of the periodic structure (i.e. a wavenumber modulation in space and time).

On the theoretical side, it is interesting to note that a secondary bifurcation that transforms a stationary structure into a traveling one has been predicted by Malomed and Tribelsky (1984) before the experimental results quoted above. They used a Galerkin approximation for model equations of the Kuramoto-Sivashinsky type, and pointed out that the drift instability 
arises in that case from the coupling between the spatial phase of the basic structure with the second harmonic generation. Recently this bifurcation was understood in a more general way from symmetry considerations (Coullet et al., 1989). Finally, a drift instability has been observed by numerical integration of the Kuramoto-Sivashinsky equation (Thual and Bellevaux, 1988).

We first propose a model that describes the drift instability of a stationary cellular pattern. We then show that a drift instability of a standing wave, such as the one observed in the Faraday experiment, can be understood as a secondary bifurcation described by the evolution equations for the amplitudes of the right and left propagating waves (equations 5.39). In all cases, we will show that the basic mechanism consists of the coupling between the spatial phase, $\phi$, of the primary pattern, with the order parameter, $V$, associated with the broken space-reflection symmetry. We will give the general governing equations for the drift bifurcation. (The notation of each section is independent.)

\subsection{A drift instability of stationary patterns}

In their Galerkin approximation of a model equation, Malomed and Tribelsky found that the drift instability occurs when the linear damping of the second harmonic of the basic pattern is not strong enough. We thus consider a situation where two modes $k$ and $2 k$ interact resonantly,

$$
\mathbf{u}(x, t)=[A(X, T) \exp (i k x)+\text { c.c. }] \mathbf{u}_{k}+[B(X, T) \exp (2 i k x)+\text { c.c. }] \mathbf{u}_{2 k}+\cdots
$$

From symmetry arguments (translational invariance in space), the evolution equations for $A$ and $B$ read, to third order,

$$
\begin{aligned}
& \frac{\partial A}{\partial T}=\mu A-\bar{A} B-\alpha|A|^{2} A-\beta|B|^{2} A, \\
& \frac{\partial B}{\partial T}=\nu B+\varepsilon A^{2}-\gamma|A|^{2} B-\delta|B|^{2} B .
\end{aligned}
$$

The quadratic coupling terms describe the resonant interaction between the modes $k$ and $2 k$. The coefficient of $\bar{A} B$ can be taken equal to -1 , making the transformation $\mathbf{u} \rightarrow-\mathbf{u}$, if necessary. The coefficient of $A^{2}$ is $\varepsilon$ and by scaling the amplitudes we can ensure that $\varepsilon$ equals either 1 or -1 . Positive values of $\alpha, \beta, \gamma$ and $\delta$ ensure global stability. The bifurcation diagram of equations (7.2) has been studied by several authors in the context of resonant wave interaction (see for instance, Proctor and Jones (1988) and the references therein). We thus refer to these papers for the mathematical aspects and discuss equations (7.2) in the restricted context of the "drift bifurcation".

Writing

$$
A=R \exp (i \phi), \quad B=S \exp (i \theta), \quad \Sigma=2 \phi-\theta,
$$

we get from (7.2)

$$
\frac{\partial R}{\partial T}=\left(\mu-\alpha R^{2}-\beta S^{2}\right) R-R S \cos \Sigma
$$




$$
\begin{gathered}
\frac{\partial S}{\partial T}=\left(\nu-\gamma R^{2}-\delta S^{2}\right) S+\varepsilon R^{2} \cos \Sigma \\
\frac{\partial \Sigma}{\partial T}=\left(2 S-\varepsilon \frac{R^{2}}{S}\right) \sin \Sigma \\
\frac{\partial \phi}{\partial T}=S \sin \Sigma
\end{gathered}
$$

In the context of our study we must take $\nu<0$ (the second harmonic is linearly damped) and increase the bifurcation parameter $\mu$. When $\mu$ becomes positive, the null state bifurcates to an orbit of stable stationary patterns related to each other by space translation:

$$
R=R_{0} \neq 0, \quad S=S_{0} \neq 0, \quad \Sigma=\Sigma_{0}=0, \quad \text { and } \quad \phi \text { arbitrary }
$$

A cellular pattern drifting with a constant velocity, corresponds to :

$$
\frac{\partial R}{\partial T}=0, \quad \frac{\partial S}{\partial T}=0, \quad \frac{\partial \Sigma}{\partial T}=0, \quad \frac{\partial \phi}{\partial T}=\text { constant } \neq 0 .
$$

This implies $2 S-\varepsilon R^{2} / S=0$, and thus $\varepsilon=1$. So the coefficients of the quadratic terms must have opposite signs in order to observe the drift instability. Note that this means that the second harmonic does not enhance the stationary instability near onset; indeed, for $\mu \approx 0$ and $\nu<0, B$ follows adiabatically $A\left(B \propto A^{2}\right)$, and the quadratic terms of equation (7.2) contributes to saturating the primary instability. The stationary pattern is destabilized when $2 S_{0}+R_{0}^{2} / S_{0}$ vanishes as $\mu$ is increased. This happens if the condition $1+\nu(2 \gamma+\delta)>0$ is satisfied, which corresponds to the condition that the second harmonic is not strongly damped $(|\nu|$ not too large). The system of equations $(7.3 \mathrm{a}, \mathrm{b}, \mathrm{c})$ then undergoes a supercritical pitchfork bifurcation. The two bifurcated stationary states are such that $R_{D}^{2}=2 S_{D}^{2}, \Sigma= \pm \Sigma_{D} \neq 0$. Above the instability onset, $\phi$ increases linearly in time according to equation (7. $3 \mathrm{~d}$ ). As noted earlier, this state represents traveling waves . The bifurcation from the stationary pattern to the traveling one has the following characteristics. The order parameter, $\Sigma=2 \phi-\theta$, undergoes a pitchfork bifurcation that breaks the basic pattern reflection symmetry. The coupling with the basic pattern spatial phase, $\phi$, induces the drift motion according to equation $(7.3 \mathrm{~d})$, and the direction of propagation is determined by the sign of $\Sigma$. Thus the mechanism described by Malomed and Tribelsky for their model equations appears to be a general one. We expect that this $k-2 k$ interaction mechanism is relevant for most of the experiments quoted above. 


\subsection{The drift instability of a parametrically excited standing wave}

Let us now consider the drift instability of a standing surface wave, generated by parametric excitation in a horizontal layer of fluid contained in a thin annulus, submitted to vertical vibrations (Douady et al., 1989). It was observed that, as the driving amplitude is increased, the standing wave pattern, either begins to move at a constant speed in one direction, or undergoes an oscillatory instability that corresponds to a wavenumber modulation in space and time. We first consider the drift bifurcation and then discuss the oscillatory instability.

Close to the onset of instability, we write the surface deformation in the form

$$
\xi(x, t)=A(X, T) \exp i(\omega t-k x)+B(X, T) \exp i(\omega t+k x)+\text { c.c. }+\cdots,
$$

where $A$ and $B$ are the slowly varying amplitudes of the right and left waves at frequency $\omega=\omega_{e} / 2$, where $\omega_{e}$ is the external driving frequency. The equations for $A$ and $B$ are at leading order (see lecture 5),

$$
\begin{aligned}
& \frac{\partial A}{\partial T}+c \frac{\partial A}{\partial X}=(-\lambda+i \nu) A+\mu \bar{B}+\alpha \frac{\partial^{2} A}{\partial X^{2}}+\left(\beta|A|^{2}+\gamma|B|^{2}\right) A \\
& \frac{\partial B}{\partial T}-c \frac{\partial B}{\partial X}=(-\lambda+i \nu) B+\mu \bar{A}+\alpha \frac{\partial^{2} B}{\partial X^{2}}+\left(\beta|B|^{2}+\gamma|A|^{2}\right) B
\end{aligned}
$$

where $\lambda$ is the dissipation ( $\lambda>0), \nu$ corresponds to the detuning between the surface wave frequency $\omega_{0}$ and $\omega_{e} / 2, \mu$ is proportional to the external forcing amplitude. The imaginary parts of $\beta$ and $\gamma$ describe the nonlinear frequency variation of the wave as a function of the amplitude, whereas the real parts correspond to nonlinear dissipation. $\alpha_{i}$ corresponds to dispersion.

When $\mu \geq 0$, a standing wave regime is observed. To analyze its stability we write,

$$
A=\exp (S+R) \exp i(\Theta+\Phi) \quad \text { and } \quad B=\exp (S-R) \exp i(\Theta-\Phi),
$$

and get from equations (7.5) for spatially homogeneous waves :

$$
\begin{gathered}
\frac{\partial S}{\partial T}=-\lambda+\cosh 2 R\left[\mu \cos 2 \Theta+\left(\beta_{r}+\gamma_{r}\right) \exp 2 S\right], \\
\frac{\partial \Theta}{\partial T}=\nu+\cosh 2 R\left[-\mu \sin 2 \Theta+\left(\beta_{i}+\gamma_{i}\right) \exp 2 S\right], \\
\frac{\partial R}{\partial T}=\sinh 2 R\left[-\mu \cos 2 \Theta+\left(\beta_{r}-\gamma_{r}\right) \exp 2 S\right], \\
\frac{\partial \Phi}{\partial T}=\sinh 2 R\left[\mu \sin 2 \Theta+\left(\beta_{i}-\gamma_{i}\right) \exp 2 S\right] .
\end{gathered}
$$

$\Theta$ and $\Phi$ are respectively the temporal and spatial phases of the pattern. Note that the equation for $\Phi$ decouples, because of the translation invariance of the system in space. The 
standing-wave solutions correspond to $\left(S_{0}, \Theta_{0}, R=0\right)$. Their stability with respect to spatially homogeneous perturbations is simple to investigate from equations (7.6). We assume $\beta_{r}+\gamma_{r}<0$ and that the detuning small enough $\left(|\nu|<\lambda\left|\beta_{r} / \beta_{i}\right|\right)$. Then, perturbations in $S$ and $\Theta$ are damped. Perturbations in $R$ and $\Phi$ obey the equations,

$$
\begin{gathered}
\frac{\partial \Phi}{\partial T}=2\left[\nu+2 \beta_{i} \exp 2 S_{0}\right] R+\cdots \\
\frac{\partial R}{\partial T}=2\left[-\lambda+2 \beta_{r} \exp 2 S_{0}\right] R+\cdots
\end{gathered}
$$

When the standing wave pattern amplitude $\exp \left(S_{0}\right)$ is small, $R$ is damped and the standing wave pattern is stable. As the driving amplitude is increased, $S_{0}$ increases and $R$ becomes unstable for $\exp \left(2 S_{0}\right)=\lambda / 2 \beta_{r}$, provided that $\beta_{r}>0$. A nonzero value of $R$ breaks the $x \rightarrow-x$ symmetry (see equation (7.4) and the expressions of $A$ and $B$ versus $S, R, \Theta, \Phi$ ). Thus $R$ is the order parameter of the "drift bifurcation" for this standing wave problem. The coupling with the spatial phase $\Phi$ generates the drift ( $7.7 \mathrm{a})$. The structure of the "drift bifurcation" is thus similar to that described previously for a stationary pattern. However, higher order terms in equations (7.7) show that the "drift bifurcation" is subcritical in this case. One can easily check this by noting that the drifting solution of equations (7.6), $\frac{\partial S}{\partial T}=\frac{\partial R}{\partial T}=\frac{\partial \Theta}{\partial T}=0, \frac{\partial \Phi}{\partial T} \neq 0$, exists for $\exp \left(2 S_{0}\right)<\lambda / 2 \beta_{r}$, i.e. only before the onset of the drift bifurcation. But additional terms of the form $|A|^{4} A,|B|^{4} B$, can stabilize the drifting solution, and even make the drift bifurcation supercritical.

\subsection{The drift bifurcation}

We observed in the above examples that the drift bifurcation consists of a secondary instability of the basic pattern that spontaneously breaks its reflection symmetry $x \rightarrow-x$. The eigenvalue $\lambda$ of the corresponding eigenmode $\mathbf{u}_{\pi}(x)$ vanishes at the bifurcation. However, we have a persistent zero eigenvalue associated with translational symmetry; the drift instability results from the coupling between the reflection symmetry-breaking amplitude mode, and the phase mode associated with translational invariance. In the vicinity of this instability onset, we write

$$
\mathbf{u}(x, t)=\mathbf{u}_{0}[x+\phi(X, T)]+V(X, T) \mathbf{u}_{\pi}(x)+\cdots,
$$

and look for coupled equations for $\phi$ and $V$. The form of these equations is given by symmetry arguments, translational invariance in space $\left(\phi \rightarrow \phi+\phi_{0}\right)$, and space reflection symmetry $(x \rightarrow-x, \phi \rightarrow-\phi, V \rightarrow-V)$. We get to leading orders in the gradient expansion

$$
\begin{gathered}
\frac{\partial \phi}{\partial T}=V \\
\frac{\partial V}{\partial T}=\lambda V-V^{3}+a \frac{\partial^{2} \phi}{\partial X^{2}}+b \frac{\partial^{2} V}{\partial X^{2}}+f V \frac{\partial \phi}{\partial X}+g V \frac{\partial V}{\partial X}+h \frac{\partial \phi}{\partial X} \frac{\partial^{2} \phi}{\partial X^{2}}+\cdots
\end{gathered}
$$

Higher-order terms in equation ( $7.9 \mathrm{a}$ ) can always be removed via a nonlinear transformation (Fauve et al. 1987). (The coefficient in equation $(7.9$ a) has been scaled in $V$ ). If the 
coefficients, $a$ and $b$, are positive, the $V=0$ solution first bifurcates when $\lambda$ vanishes and becomes positive. The homogeneous drifting pattern, $V_{0}= \pm \sqrt{\lambda}, \phi_{0}=V_{0} T$, bifurcates supercritically; however its stability to inhomogeneous disturbances of the form $\exp (\eta T+$ $i K X)$, is governed by the dispersion relation,

$$
\eta^{2}+\left(2 \lambda-i K g V_{0}+b K^{2}\right) \eta-i K f V_{0}+a K^{2}=0\left(K^{2}\right) .
$$

This that shows that the term $f V \partial \phi / \partial X$ destabilizes the homogeneous pattern independently of the sign of $f$. This may appear somewhat surprising, because at the drift instability onset, one bifurcates from the linearly stable static pattern to the linearly unstable static pattern without the appearance of stable drifting patterns; this behavior traces back to the existence of subcritical localized traveling solutions of equation (7.9), that describe localized drifting regions with tilted cells, widely observed in most experiments. Thus, even when the the drift bifurcation is supercritical if one only considers homogeneous patterns (as in section 7.2 for instance), the coupling term $f V \partial \phi / \partial X$ makes it generically subcritical when no restriction is imposed.

Another experimental observation can be understood in the framework of equations (7.9): it is the stationary pattern wavenumber selection often observed as the control parameter is increased. In most of the above quoted experiments, it is observed that a pattern wavenumber modification occurs by nucleation of a transient drifting domain that generates a phase gradient, say $K$, and leads to a new periodic pattern of wavenumber $k+\epsilon K$. Indeed, $V=0$, $\phi=K x$, is a particular solution of equations (7.9), for which the damping rate of perturbations in $V$ is $\lambda+f K$. Consequently the drift of this new pattern is inhibited if $f K<0$, for $|K|>\lambda / f$. The new periodic pattern thus remains stationary because of wavenumber modification. Within the framework of the model of section 7.2, this stabilization mechanism is associated to the increase of the second harmonic damping rate when the pattern wavenumber is increased.

\subsection{Oscillatory phase modulation of periodic patterns}

As said above, an oscillatory phase modulation of periodic patterns is observed as a secondary instability of parametrically generated surface waves (Douady et al., 1989). After this instability onset, the position of the wavecrests is modulated in space and time by a standing wave. This oscillatory instability is observed close to the "drift bifurcation" in the experimental parameter space. The numerical integration of equations (7.5) has shown that the oscillatory instability corresponds to a standing wave modulation of the basic pattern spatial and temporal phases, in agreement with the experimental observations.

We show that the coupling that generates the "drift bifurcation" is also a possible mechanism to describe phase modulation of periodic patterns, if the order parameter $V$ is destabilized at a finite wavenumber. We consider equations (7.9), that govern the space dependent perturbations of the basic periodic pattern, with $\lambda \approx-\lambda_{0}, \lambda_{0}>0$. Thus, the standing pattern 
is stable with respect to homogeneous perturbations. The growth rate of a perturbation of the form $\exp (\eta T+i K X)$, is governed by the dispersion relation,

$$
\eta^{2}+\eta\left(-\lambda+b K^{2}\right)+a K^{2}=o\left(K^{2}\right) .
$$

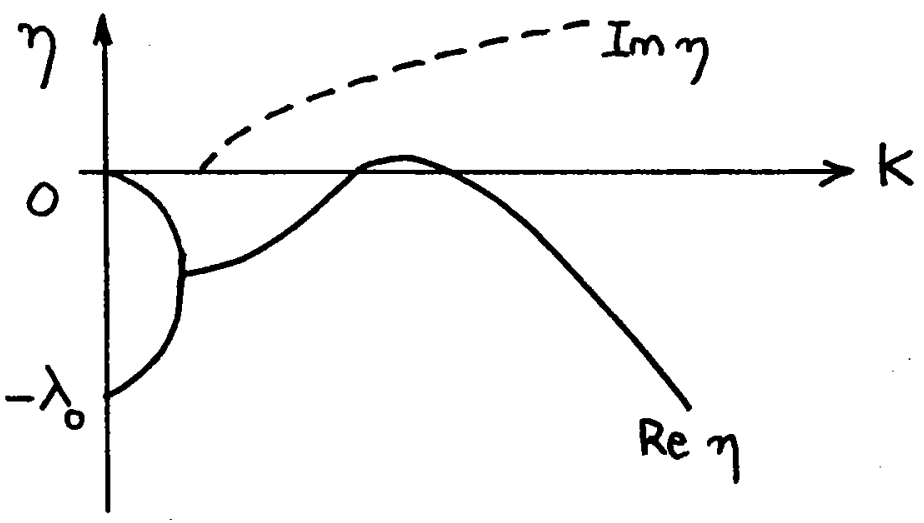

Figure 7.1. Perturbation growth rate as a function of the wavenumber of the oscillatory instability $(a>0, b<0)$.

Stability at short wavelength requires higher order terms (fourth order gradients). For $a>0$ and $b<0$ an oscillatory instabilty occurs first. The corresponding growth rate is displayed on figure 7.1 ; it shows that the oscillatory instability results from the interaction of the neutral mode, because of the translation invariance in space, with the slightly damped, reflection symmetry-breaking mode associated with the "drift bifurcation" before its onset value. An instability leading to a stationary modulation of the basic pattern wavelength can occur for $a<0$ and $b>0$.

We have thus shown that a variety of recent experimental observations of periodic pattern secondary instabilities can be understood in a simple framework: the coupling of the neutral mode associated with translational invariance in space, with a reflection symmetry-breaking bifurcation. Note that a similar singularity, with two zero eigenvalues at a secondary instability onset, occurs for the oscillatory instability of convection rolls and leads to traveling waves that propagate along their axis, although the underlying physical reasons are different (Fauve et al., 1987). Let us finally mention that the secondary instabilities described here fit in a general classification, proposed recently on the basis of symmetry arguments (Coullet and Iooss, 1989), but the present approach illustrates the simple physical mechanisms that generate these secondary instabilities. 


\section{References}

Coullet, P., Goldstein, R. E., Gunaratne, G. H. (1989) Phys. Rev. Letters 63, 1954-1957. Coullet, P., Iooss, G. (1989) Phys. Rev. Letters 64, 866.

Douady, S., Fauve, S., Thual, O., (1989) Europhysics Letters 10, 309-315.

Fauve, S., Bolton, E. W., Brachet, M., (1987) Physica 29 D, 202-214.

Faivre, G., de Cheveigné, S., Guthmann, C., Kurowski, P. (1989) Europhysics Letters 9, 779-784.

Malomed, B. A., Tribelsky, M. I., (1984) Physica 14 D, 67-87.

Mutabazi, I., Hegseth, J. J., Andereck, C. D., Wesfreid J. E., (1988) Phys. Rev. A38, 4752-4760.

Proctor, M. R. E., Jones, C., (1988) J. Fluid Mech. 188, 301-335.

Rabaud, M., Michalland, S., Couder, Y. (1990) Phys. Rev. Letters 64, 184.

Simon, A. J., Bechhoefer J., Libchaber, A. (1988) Phys. Rev. Letters 61, 2574-2577.

Thual, O., Bellevaux, C., (1988) Fifth Beer-Sheva Seminar on MHD Flows and Turbulence, AIAA Progress in Astronautics and Aeronautics 112, 332-354. 


\section{Nonlinear Localized Structures}

\subsection{Different types of nonlinear localized structures}

Nonlinear interactions usually transfer energy to higher harmonics, thus making steeper a wavefront. Nonlinear effects can be balanced by dissipation; this is usually the case for shock waves. Let us consider a simple example, the Burgers equation

$$
\frac{\partial \rho}{\partial t}+\rho \frac{\partial \rho}{\partial x}=\nu \frac{\partial^{2} \rho}{\partial x^{2}}
$$

We look for a traveling solution of the form $\rho=\rho(x-c t)$, that connects two constant values of $\rho, \rho_{1}$ and $\rho_{2}$ for $x \rightarrow \pm \infty$. Equation (8.1) becomes

$$
-c \rho^{\prime}+\rho \rho^{\prime}=\nu \rho^{\prime \prime} \text {. }
$$

Integrating once yields

$$
-c \rho+\frac{1}{2} \rho^{2}-\nu \rho^{\prime}=\text { constant }
$$

and consequently

$$
c=\frac{\rho_{1}+\rho_{2}}{2} .
$$

Thus, although the shape of $\rho(x-c t)$ depends on the dissipation $\nu$, this does not affect the shock velocity that depends only on the jump in $\rho$.

In conservative systems nonlinear effects may be balanced by dispersion: this is the basic mechanism that gives rise to solitary waves. We have already considered soliton solutions of the nonlinear Schrödinger equation, and note that they can travel at any velocity because of Galilean invariance. This is not of course the case for all solitary waves; consider for instance the Sine-Gordon equation

$$
\frac{\partial^{2} u}{\partial t^{2}}=\frac{\partial^{2} u}{\partial x^{2}}-\sin u
$$

which has kink type solitons of the form

$$
u(x, t)= \pm 4 \tan ^{-1}\left[\exp \left(\frac{x-x_{0}-c t}{\sqrt{1-c^{2}}}\right)\right] .
$$

Unlike the solutions of the nonlinear Schrödinger equation, there is a speed limit, 1, for the solutions (8.3). However, there is a more important characteristic, associated with the discrete symmetry

$$
u \rightarrow u+2 n \pi,
$$

of the Sine-Gordon equation; the soliton solution (8.3) can be considered as a localized structure, connecting in space two states $u=0$ and $u= \pm 2 \pi$, related by this discrete symmetry transformation (Figure 9.1). This is called a topological soliton. 


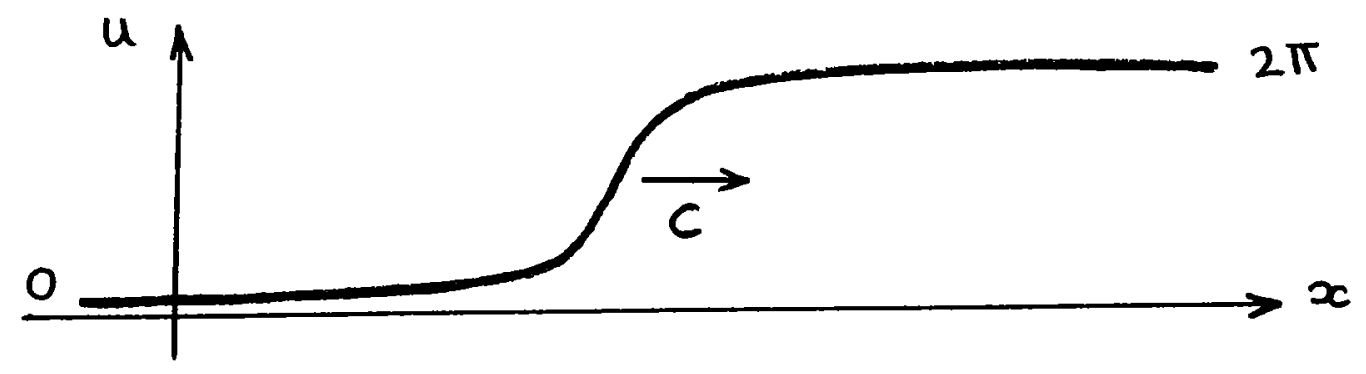

Figure 8.1. A kink soliton of the Sine-Gordon equation

Similar types of localized structures, from the point of view of symmetry, exist in phase transitions or in patterns generated by instabilities; they are called topological defects. As we already mentioned, the amplitudes of unstable modes are analogous to order parameters in phase transition theory; indeed, a nonzero amplitude is associated with the broken symmetry at the instability onset. Thus, in the supercritical regime, it is natural to use the term "defect," to denote a region in space where the amplitude vanishes. A defect is said topologically stable when a slight perturbation of the amplitude in space or time does not affect its characteristic shape (for instance only slightly translates it in space). We will illustrate this concept with some very simple examples (for a review, see Mermin 1979).

Let us consider the Ginzburg-Landau equation (5.16) for a real one-dimensional amplitude $R(X, T)$

$$
\frac{\partial R}{\partial T}=\mu R+\frac{\partial^{2} R}{\partial X^{2}}-R^{3} .
$$

The broken symmetry at the instability onset $(\mu=0)$ is the $R \rightarrow-R$ symmetry. Correspondingly, there exist two homogeneous solutions, $R_{0}= \pm \sqrt{\mu}$, that are related by this symmetry transformation. The space dependent solution,

$$
R(X)=\tanh \left(\sqrt{\frac{\mu}{2}} X\right)
$$

connects these two homogeneous solutions (Figure 8.2); it is a topological defect.

The tanh is also a solution for the complex Ginzburg-Landau equation,

$$
\frac{\partial A}{\partial T}=\mu A+\Delta A-|A|^{2} A \text {. }
$$

However, it is not a topological defect since one can easily remove it with phase perturbations. On the contrary, for a two-dimensional field, $A(X, Y, T)$, there are topologically stable point defects. Indeed, the complex amplitude $A(X, Y, T)$ vanishes if its real and imaginary parts vanish; at a given instant, $\operatorname{Re} A(X, Y, T)=0$ and $\operatorname{Im} A(X, Y, T)=0$ define one-dimensional curves in the $(X, Y)$ plane, that generically intersect at a point (Figure 8.3). If one slightly perturbs $A(X, Y, T)$, these curves move slightly and so does the defect, but the qualitative character is unchanged. Similarly, for a three-dimensional field, $A(X, Y, Z, T)$, topological defects of the complex Ginzburg-Landau equation are lines. 


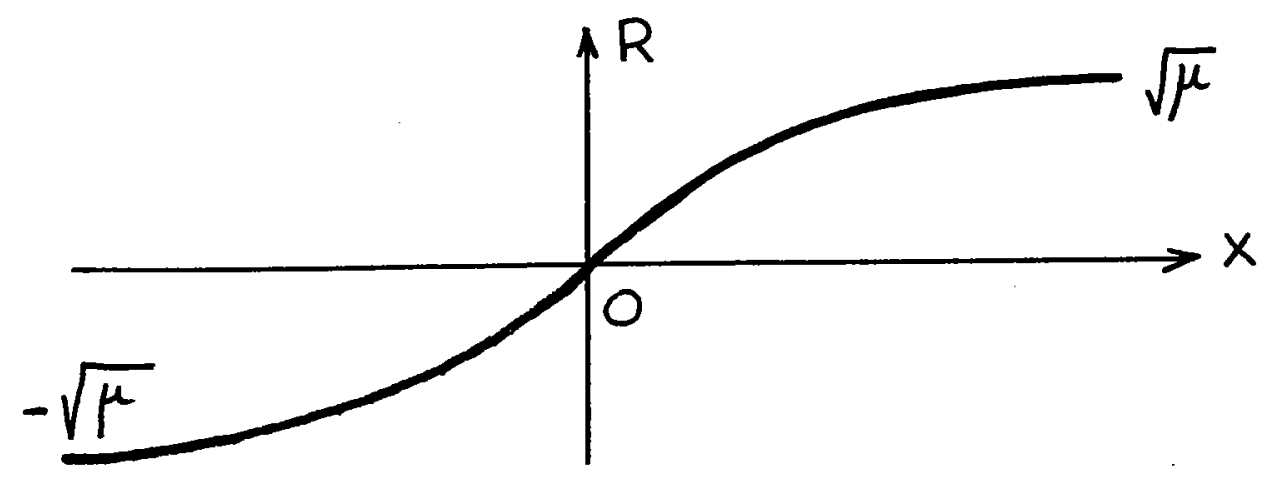

Figure 8.2. A topological defect for the real one-dimensional Ginzburg-Landau equation

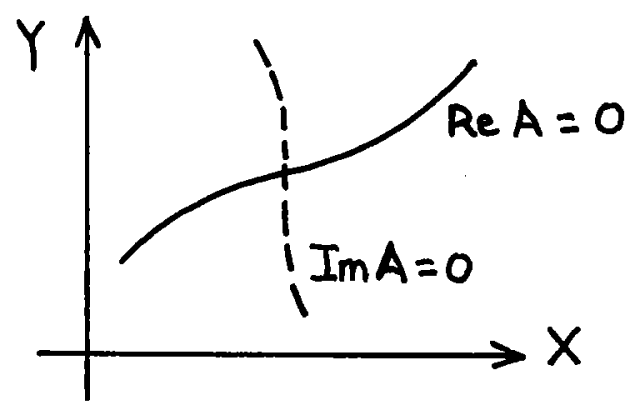

Figure 8.3. The location of a topological defect for a two-dimensional GinzburgLandau equation

If equation (8.5) is modified to

$$
\frac{\partial A}{\partial T}=\mu A+\Delta A+|A|^{2} A-|A|^{4} A,
$$

the bifurcation becomes subcritical. The interesting new feature is bistability; indeed, it is clear from the bifurcation diagram displayed in Figure 8.4 that two stable states coexist for $\mu$ negative. Obviously, these two solutions are not related by a symmetry transformation, but we can also consider an interface that separates in space these two stable states, or a droplet that consists of a region in one state surrounded by the other one. In a system with a Lyapunov functional, i.e. a free energy, the interface moves such that the lowest energy state increases in size. Contrary to the situation with shock waves, the interface velocity does not depend only on the energy difference between the two homogeneous solutions, but also on the shape of the interface. With nonvariational systems, interesting new phenomena occur (see section 8.3). 

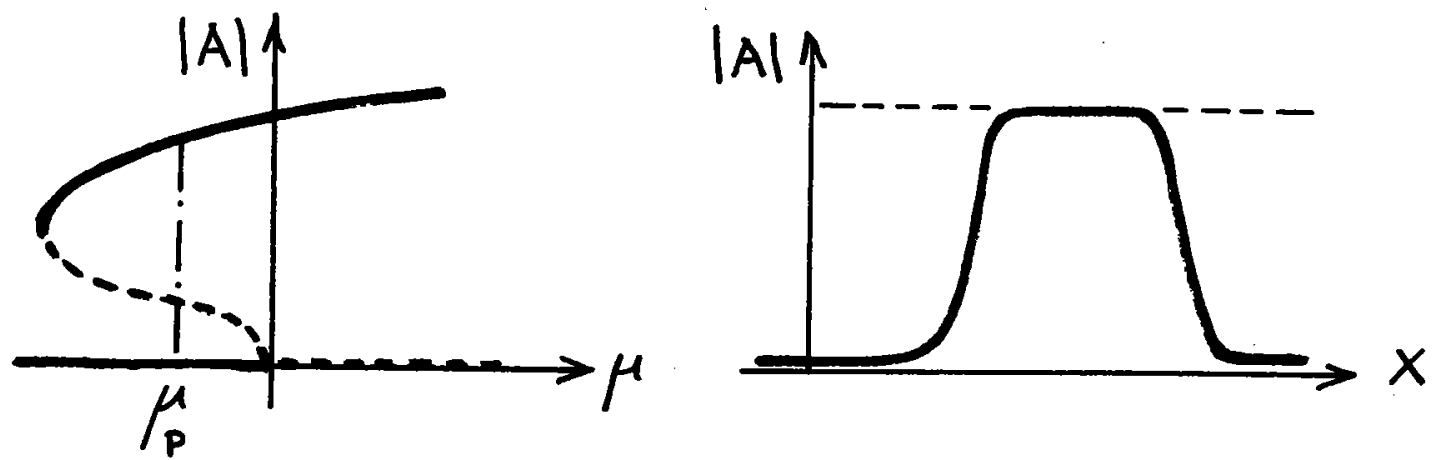

Figure 8.4. Bistability and localized structure in the vicinity of a subcritical bifurcation

\subsection{Kink dynamics}

We consider equation (8.4) in the supercritical regime $(\mu>0)$; taking appropriate time, space and amplitude scales, we get

$$
\frac{\partial R}{\partial T}=2 R\left(1-R^{2}\right)+\frac{\partial^{2} R}{\partial X^{2}}
$$

This equation admits a kink type solution or defect,

$$
R=\tanh X
$$

Consider a solution consisting of several kinks (Figure 8.4). Such a solution is unstable because the neutral translation mode, $\partial R / \partial X$, has a node; the only stable solution is the one with a single kink. What is the time evolution of such unstable structures? An interesting approach is to consider many topological defects, far one from the other, as a "gas" of such kinks and write an evolution equation for their density.

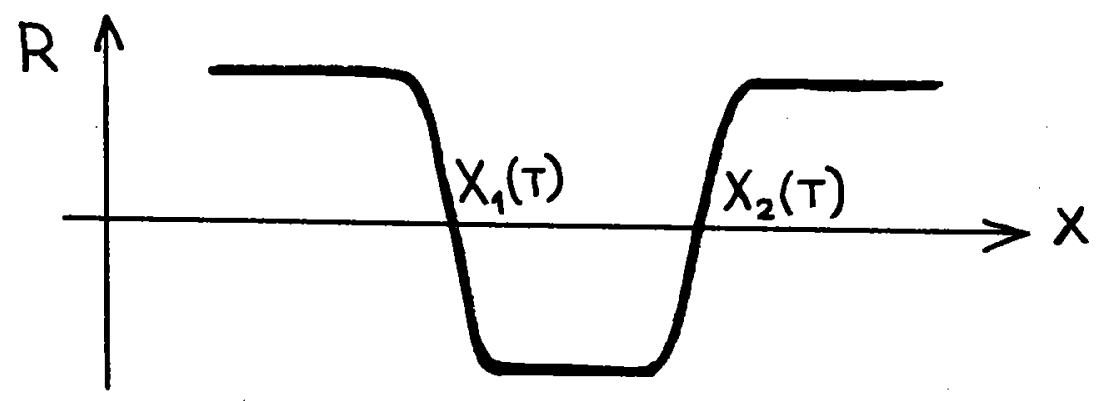

Figure 8.5. The two kink solution (8.8) 
We first derive an equation describing the interaction of two kinks that are far apart. Let us seek a solution in the form

$$
R(X, T)=R_{1}\left[X_{1}(T)\right] R_{2}\left[X_{2}(T)\right]
$$

where

$$
R_{j}\left[X_{i}(T)\right]=\tanh \left[X-X_{i}(T)\right]
$$

and $j$ is 1 or 2 . Substituting (8.8) in (8.7) we obtain the equation

$$
-\dot{X}_{1} R_{1}^{\prime} R_{2}-\dot{X}_{2} R_{1} R_{2}^{\prime}=2 R_{1} R_{2}\left(1-R_{1}^{2} R_{2}^{2}\right)+R_{1}^{\prime \prime} R_{2}+2 R_{1}^{\prime} R_{2}^{\prime}+R_{1} R_{2}^{\prime \prime}
$$

where a dot denotes time derivative and primes denote differentiation with respect to $X_{1}$ or $X_{2}$. By direct differentiation one can verify that

$$
\begin{array}{r}
R_{i}^{\prime}=1-R_{i}^{2} \\
R_{i}^{\prime \prime}=-2 R_{i}\left(1-R_{i}^{2}\right) .
\end{array}
$$

At $X=X_{i}(T)$ we have $R_{i}=0, R_{i}^{\prime}= \pm 1$ and $R_{j} \approx \pm 1$. Substituting $X=X_{i}$ in (8.10) we derive the equations of motion for the kinks

$$
\begin{array}{r}
\dot{X}_{1} \approx 2\left[1-R_{2}^{2}\left(X_{1}\right)\right] \approx 2 \exp \left(-2\left|X_{1}-X_{2}\right|\right), \\
\dot{X}_{2} \approx-2\left[\left(1-R_{1}^{2}\left(X_{2}\right)\right] \approx-2 \exp \left(-2\left|X_{1}-X_{2}\right|\right),\right.
\end{array}
$$

which show that each kink feels the effect of the exponentially decreasing tail of the other at large separations. We can generalize this to consider the interaction of many kinks in the nearest neighbor approximation:

$$
\dot{X}_{i} \approx 2 \exp \left(-2\left|X_{i+1}-X_{i}\right|\right)-2 \exp \left(-2\left|X_{i}-X_{i-1}\right|\right) .
$$

Define

$$
\rho_{i}=\exp \left(-2\left|X_{i+1}-X_{i}\right|\right)
$$

Then $-1 / \log \rho_{i}$ is the "kink density". Taking the logarithm of (8.12) and differentiating with respect to time, we have

$$
\dot{\rho}_{i}=-2\left(\dot{X}_{i+1}-\dot{X}_{i}\right) \rho_{i}
$$

and on using (8.11) and (8.12),

$$
\dot{\rho}_{i}=-4\left(\rho_{i+1}-2 \rho_{i}+\rho_{i-1}\right) \rho_{i} .
$$

Finally, passing to the continuum limit, we have the equation for a "rarefied kink gas"

$$
\frac{\partial \rho}{\partial T}=-8 \rho \frac{\partial^{2} \rho}{\partial X^{2}}
$$


We see that this is a nonlinear diffusion equation where the diffusion coefficient $D=-8 \rho$ is negative. It therefore describes a condensation process with all the kinks tending to clump together due to mutual attraction. Let us consider a train of kinks, equispaced with a distance $a$ apart. Then, $X_{i+1}^{(0)}-X_{i}^{(0)}=a$, where the superscript zero denotes the initial state. Setting, $X_{i}=X_{i}^{(0)}+u_{i}$, and looking for normal modes, $u_{n} \propto \exp (\eta T-i K n a)$, we have

$$
\eta=16 e^{-a} \sin ^{2}(K a / 2),
$$

which shows that the mode with the largest growth rate is at $K a=\pi$, i.e. an optical mode at twice the wavelength of the kink array.

The important point to note here is that we can describe a system at different approximation levels corresponding to equations (8.7), (8.11) and (8.16). Depending on the problem, one can consider localized structures as "particles" and use (8.11), or use a continuous description.

\subsection{Localized structures in the vicinity of a subcritical bifurcation}

Consider the following simple model equation describing a subcritical bifurcation:

$$
\frac{\partial A}{\partial T}=\mu A+\alpha \frac{\partial^{2} A}{\partial X^{2}}+\beta|A|^{2} A+\gamma|A|^{4} A .
$$

If the coefficients of this equation are real, the steady state is given by the solution which minimizes the Lyapunov functional

$$
\begin{array}{r}
\mathcal{L}=\int_{0}^{L} \alpha_{r}\left|A_{x}\right|^{2}-V(|A|) d X \\
V(|A|)=\mu|A|^{2}+\frac{\beta_{r}}{2}|A|^{4}+\frac{\gamma_{r}}{3}|A|^{6} .
\end{array}
$$

We are considering a subcritical bifurcation so that $\beta_{r}>0$ and $\gamma_{r}<0$. The three possible situations are shown in Figure 8.6. The two states have the same energy and thus can coexist only if $\mu=\mu_{P} ; \mu_{P}$ corresponds to the Maxwell plateau of first order phase transitions.

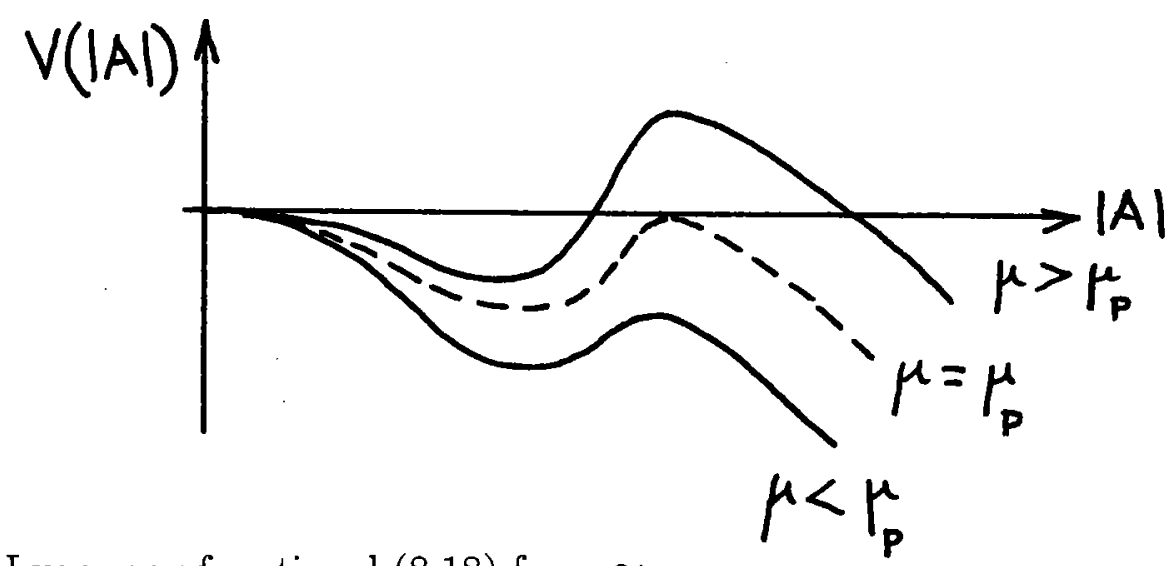

Figure 8.6. The Lyapunov functional (8.18) for $\mu \approx \mu_{P}$ 
Now consider the situation with $\alpha$ and $\beta$ complex so that there is no longer a Lyapunov functional for the problem. In this situation stable localized structures are possible (Thual and Fauve, 1989). The stabilization can be explained by the following rough argument. Substituting

$$
A=R(X, T) \exp [i \theta(X, T)]
$$

in equation (8.17) and equating real and imaginary parts we have

$$
\begin{array}{r}
\frac{\partial R}{\partial T}=\alpha_{r} \frac{\partial^{2} R}{\partial X^{2}}+\left[\mu-\alpha_{r}\left(\frac{\partial \theta}{\partial X}\right)^{2}\right] R+\beta_{r} R^{3}+\gamma_{r} R^{5}-\alpha_{i}\left(2 \frac{\partial R}{\partial X} \frac{\partial \theta}{\partial X}+R \frac{\partial^{2} \theta}{\partial X^{2}}\right) \\
R \frac{\partial \theta}{\partial T}=\alpha_{r}\left(2 \frac{\partial R}{\partial X} \frac{\partial \theta}{\partial X}+R \frac{\partial^{2} \theta}{\partial X^{2}}\right)+\alpha_{i} \frac{\partial^{2} R}{\partial X^{2}}-\alpha_{i} R\left(\frac{\partial \theta}{\partial X}\right)^{2}+\beta_{i} R^{3}+\gamma_{i} R^{5}
\end{array}
$$

If the imaginary part of the coefficients are zero, (8.19) is solved by $\partial \theta / \partial X=0$, and we have a variational problem for $R$. In the presence of a nonzero imaginary part, the amplitude and phase equations couple so that there is a nonzero $\partial \theta / \partial X$. This changes the effective value of $\mu$ in the coefficient of $R$ in (8.19); we define

$$
\mu_{\mathrm{eff}}=\mu-\left(\frac{\partial \theta}{\partial X}\right)^{2}
$$

The effect of the phase gradient is to decrease $\mu_{\text {eff }}$ in the outer region, thus stabilizing the zero solution, whereas the bifurcated solution is stabilized in the core of the localized structure.

These localized structures can be obtained perturbatively in the variational and conservative limits; in the conservative limit, let us write (8.17) as

$$
\begin{array}{r}
\frac{\partial A}{\partial T}=i \frac{\partial^{2} A}{\partial X^{2}}+2 i|A|^{2} A+\epsilon P(A) \\
P(A)=\mu A+\alpha_{r} \frac{\partial^{2} A}{\partial X^{2}}+\beta_{r}|A|^{2} A+\gamma_{r}|A|^{4} A .
\end{array}
$$

For $\epsilon=0$ this is simply the nonlinear Schrödinger equation and admits the one parameter family of solutions

$$
A_{s}=\Delta \operatorname{sech}(\Delta X) \exp \left(-i \Delta^{2} T\right) .
$$

The existence of such a one parameter family is due to the scale invariance of the nonlinear Schrödinger equation.

If $\epsilon$ is given a small but nonzero value, we look for slowly varying solitons of the form

$$
A(X, T)=\Delta(T) \operatorname{sech}[\Delta(T) X] \exp [-i \Theta(T)] .
$$

The temporal evolution of a soliton under the action of a perturbation $P(A)$ is a well-known problem of soliton theory and can be solved with the inverse scattering method (see for 
instance Lamb, 1980). The temporal evolution of $\Delta(T)$ can be found in a simpler way here: multiplying equation (8.20) by $\bar{A}$ and integrating on space leads to the evolution equation

$$
\frac{d}{d T} \int|A|^{2} d X=\epsilon \int\left[\mu|A|^{2}+\beta_{r}|A|^{4}+\gamma_{r}|A|^{6}-\alpha_{r}\left|A_{x}\right|^{2}\right] d X .
$$

Substituting (8.21) in (8.22), we get to leading order, an evolution equation for $\Delta$,

$$
\frac{1}{2} \frac{d \Delta}{d T}=\mu \Delta+\frac{4}{3}\left(2 \beta_{r}-\alpha_{r}\right) \Delta^{3}+\frac{128}{15} \gamma_{r} \Delta^{5}
$$

For $\alpha_{r}<2 \beta_{r}$, equation (8.23) has two nonzero solutions $\Delta_{ \pm}$for $\mu_{s}<\mu<0$, with $\mu_{s}=$ $5\left(-\alpha_{r}+2 \beta_{r}\right)^{2} / 96 \gamma_{r}$. Only the larger is stable, and gives the size of the selected pulse.

The above mechanism is a rather general one: the dissipative terms of equation (8.17) stabilize one of the soliton solutions among the continuous family (8.21) and select its size by breaking the scale invariance associated with the corresponding conservative problem.

\section{References}

Fauve, S., Thual, O. (1990) Phys. Rev. Lett. 64, 282-284

Hakim, V., Jakobsen, P. Pomeau, Y. (1990) Europhysics Lett.11, 19-24

Kawasaki, K., Ohta, T. (1982) Physica 116 A, 573

Lamb, G. L. (1980) Elements of Soliton Theory, Wiley

Mermin, N. D. (1979) The Topological Theory of Defects in Ordered Media, Rev. Mod. Phys. 51, 591-648

Thual, O., Fauve, S. (1988) J. Physique 49 , 1829-1833 


\section{Special Lectures}




\section{Simple models of complex fluids}

Daniel H. Rothman

Department of Earth, Atmospheric, and Planetary Sciences, Massachusetts Institute of Technology, Cambridge, Massachusetts 02139

\subsection{Abstract}

I show how discrete lattice gases may be constructed for the simulation of the hydrodynamics of a variety of complex fluids. After reviewing a lattice-gas model of two immiscible fluids, I show how variations of this basic model can simulate a number of considerably different physical systems. Simulations of phase separation, multiphase flow through porous media, emulsions, fluidized particles, and ferrofluids are described.

\subsection{Introduction}

Complex fluids differ from simple fluids, such as water, in that they contain structures of mesoscopic, or polyatomic, size (Witten, 1990). Examples include suspensions and emulsions, or, more generally, colloids, which are fluids containing either dispersed solid particles or liquid drops. Interfacial flows may also be considered examples of complex fluids, since the interfaces influence flow at an intermediate scale. Though widely varying, complex fluids are similar in that the mesoscopic structure within them gives rise to a variety of interesting, and often non-Newtonian, mechanical responses, in addition to examples of self organization. Complex fluids manifest themselves in a variety of ways ranging, for example, from dishwashing soap, glue, and paint, to atmospheric clouds, foam at the sea surface, mud, and magmatic mixtures in the earth's mantle.

In these lectures, I show how discrete lattice gases (Frisch et al., 1986; Frisch et al., 1987) may be constructed for the simulation of the dynamics of certain two-dimensional complex fluids. Although the breadth of hydrodynamic complexity is limited to multispecies fluid mixtures in which interfacial phenomena play a significant role, a wide variety of "complexfluid phenomenology" is nonetheless exhibited by these models.

Our archetypal example is a discrete model of two immiscible fluids, which is referred to as the immiscible lattice-gas (ILG) (Rothman and Keller, 1988). If prepared initially as a mixture (e.g., oil and water), the ILG spontaneously separates into two phases. Once the interfaces form, the patterns created by the interfacial flows can be quite interesting, as, for example, in the case of a gravitationally unstable interface separating a light fluid from a heavy fluid. Another example of interest concerns the shape of the front formed by the invasion of water into a porous medium filled with oil. 
After introducing the ILG, I show how simple modifications of it provide for two new models with qualitatively different physical behavior. One of the new models simulates an emulsion. The other is generically representative of a two-species fluid with competing interactions, in which the interactions are inspired by those of a ferrofluid under an applied magnetic field.

The model of emulsions is a suspension of liquid bubbles, each immiscible with each other, and also immiscible with the suspending fluid. Despite this simplicity, many interesting examples of nonequilibrium pattern formation are possible. For example, when the emulsion is sheared, large-scale structures are created by the collective interaction of the emulsified bubbles. In another example, when an upward buoyancy force is applied to the suspending fluid and an equal but downward buoyancy force is applied to the suspended bubbles, the bubbles act as fluidized particles and one may observe instabilities similar to those observed in fluidized beds.

Ferrofluids, the inspiration for the third model, are suspensions of small magnetic particles (Rosensweig, 1985). Ferrofluids are of technological interest because they flow hydrodynamically under application of a magnetic field. They are also of basic scientific interest because of the complex patterns formed by various ferrohydrodynamic instabilities (Rosensweig, 1985). These patterns are the result of competing interactions: one is a short range attractive interaction, and the other is a long-range repulsive interaction. The model I describe here is a discrete two-component fluid with competing surface-tension-like interactions. The model exhibits several morphological phase transitions that depend on relative interaction strength and concentration; the specific patterns observed range from hexagonally ordered bubbles to randomly oriented stripes.

\subsection{Immiscible lattice gases}

\subsubsection{The model}

Although it has been extensively described elsewhere (Rothman and Keller, 1988; Rothman and Zaleski, 1989; Rothman, 1990a; Rothman, 1990b; Gunstensen and Rothman, 1991a,b), to make these lectures self-contained I begin here with a review of the ILG and a short summary of the principal scientific results that have been obtained thus far in applications.

The ILG is a simple two-species extension of the original lattice gas introduced by Frisch, Hasslacher, and Pomeau (FHP) (Frisch et al., 1986). In the FHP model, identical particles of unit mass move with unit speed from site to site on a triangular lattice. When particles meet at a site, they obey simple collision rules that conserve mass and momentum. The microscopic behavior of this lattice gas has been shown to be very close to the incompressible Navier-Stokes equations (Frisch et al., 1987; Wolfram, 1986; Kadanoff et al., 1989; Zanetti, 1989, Doolen et al., 1990, Doolen, 1991).

The two species of the ILG are termed "red" and "blue." Each site on the two-dimensional triangular lattice may contain red particles, blue particles, or both, but at most one particle (red or blue) may move in each of the six directions $\mathbf{c}_{1}, \ldots, \mathbf{c}_{\mathbf{6}}$. Each site may also have a seventh stationary, or rest, particle moving with velocity $c_{0}$ and subject to the same exclusion 
rule. The configuration at a site $\mathbf{X}$ is thus described by the Boolean variables $r=\left\{r_{i}\right\}$ and $b=\left\{b_{i}\right\}$, which indicate the presence (1) or absence (0) of a red or blue particle, respectively, moving with velocity $\mathrm{c}_{i}$. Note that the exclusion rule prevents $r_{i}$ and $b_{i}$ from both being equal to one.

Surface tension in the ILG arises from the collision rules that describe the interaction of two colors at a single site with the particles situated at the nearest-neighbor sites. Specifically, at a site $\mathbf{x}$ undergoing' a collision, a color flux $\mathbf{q}$ is defined to be the difference between the red momentum and the blue momentum:

$$
\mathrm{q}[r(\mathbf{x}), b(\mathbf{x})] \equiv \sum_{i=1}^{6} \mathbf{c}_{i}\left[r_{i}(\mathbf{x})-b_{i}(\mathbf{x})\right]
$$

We also define the local color gradient

$$
\mathbf{f}(\mathbf{x}) \equiv \sum_{i} \mathbf{c}_{i} \sum_{j}\left[r_{j}\left(\mathbf{x}+\mathbf{c}_{i}\right)-b_{j}\left(\mathbf{x}+\mathbf{c}_{i}\right)\right]
$$

The ILG collision rule is anti-diffusive: it maximizes the flux of color in the direction of the local color gradient. The result of a collision, $r \rightarrow r^{\prime}, b \rightarrow b^{\prime}$, is the configuration that maximizes

$$
\mathbf{q}\left(r^{\prime}, b^{\prime}\right) \cdot \mathbf{f}
$$

such that the number of red particles and the number of blue particles is conserved,

$$
\sum_{i} r_{i}^{\prime}=\sum_{i} r_{i}, \quad \sum_{i} b_{i}^{\prime}=\sum_{i} b_{i}
$$

and so is the total momentum:

$$
\sum_{i} \mathbf{c}_{i}\left(r_{i}^{\prime}+b_{i}^{\prime}\right)=\sum_{i} \mathbf{c}_{i}\left(r_{i}+b_{i}\right)
$$

Figure 1 demonstrates the nonequilibrium behavior of these dynamics. A $256 \times 256$ lattice is initialized with density $\rho=7 d=4.9$ particles per site, with $50 \%$ of the particles red, and $50 \%$ blue. As time progresses, the domains of red and blue grow larger, eventually resulting in a steady state in which one thick blue stripe is parallel to an equally thick red stripe. (It is actually one stripe because the doubly periodic boundary conditions create a 2-D torus.) 

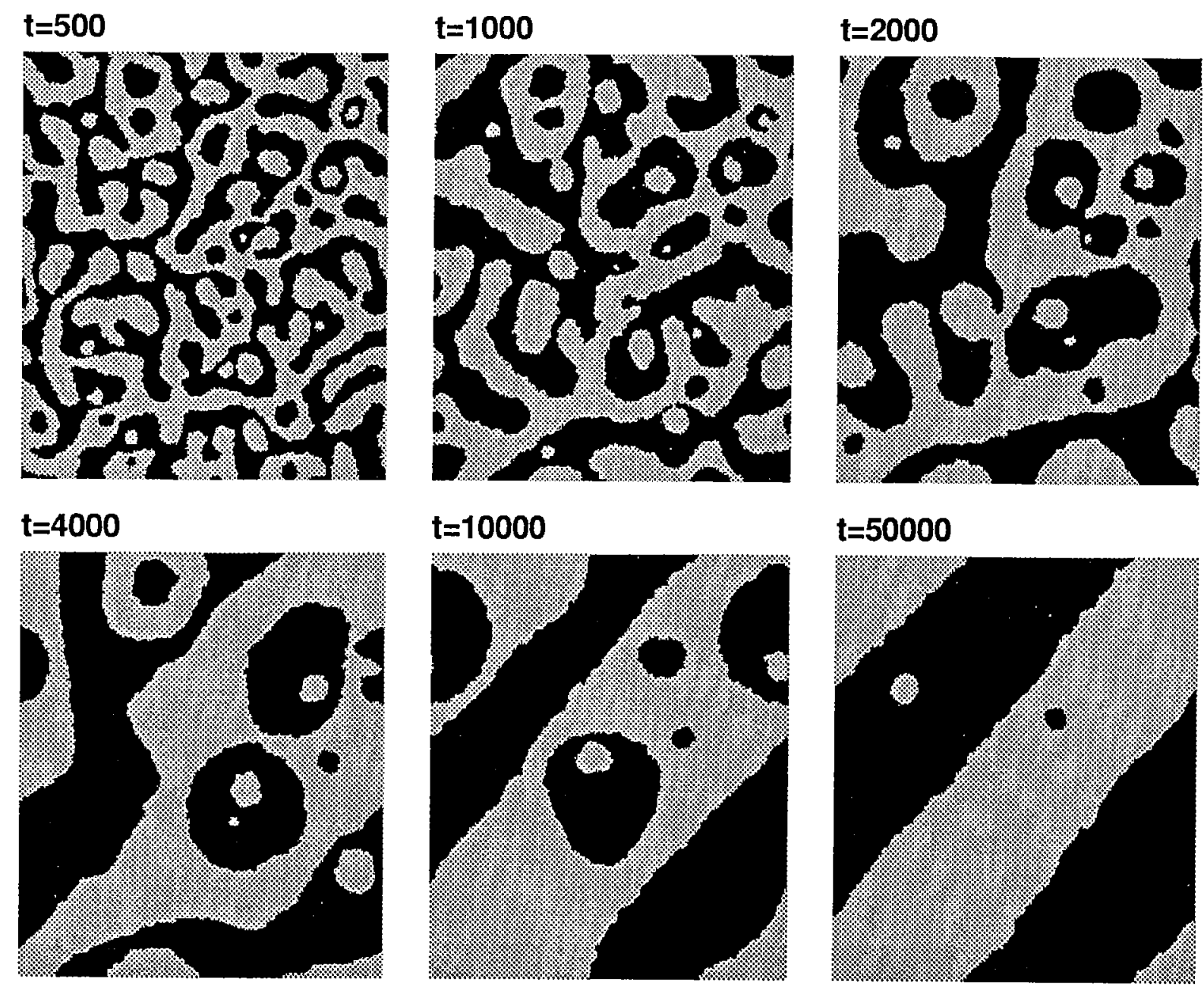

Figure 9.1. Nonequilibrium behavior of the immiscible lattice gas, where here blue corresponds to black, and red to grey. The configuration at time step 0 is a homogeneous mixture. Boundaries are periodic in both directions.

Detailed empirical studies of the ILG have shown that the collision dynamics create surface tension at interfaces (Rothman and Keller, 1988; Rothman, 1990a; Gunstensen and Rothman, 1991a). Theoretically, one may predict the magnitude of the surface tension by noting that the collision rule creates an anisotropic stress near interfaces. Such a calculation has already been carried out successfully for a Boltzmann-equation-based model of surface tension (Gunstensen et al., 1991). A similar calculation for the ILG is currently being pursued by X. L. Chen (see this report).

The ILG has thus far proven useful for a number of scientific applications. Two of these applications are briefly reviewed below.

\subsubsection{ILG studies of phase transitions and hydrodynamics}

Figure 1 qualitatively shows a phase-separation transition: a mixture, initially prepared at some "temperature" $T>T_{c}$, is subjected to a deep quench at time $t=0$. The resulting 
patterns are expected to be self-similar with respect to time (Gunton et al., 1983). In other words, the size distribution of bubbles at early times is related to the distribution at late times by a simple scaling. Empirical analysis (Rothman, 1990b) reveals just such a scaling relation. Additional studies (Rothman and Zaleski, 1989) have given further support, both theoretical and empirical, to the idea that the ILG may be used to simulate the dynamics of phase transitions. Thus, because the model already simulates the Navier-Stokes equations in homogeneous regions, it may be used to study how the phase separation process interacts with hydrodynamical effects. Thus far, studies of phase separation in a simple shear (Rothman, 1990b) have revealed that hydrodynamic interactions give rise to ordered patterns similar to those found in smectic liquid crystals, in addition to detailing a complex, time-dependent rheology (Rothman, 1991). [See also the laboratory experiments of Chan et al. (1988).] A related study by Stockman et al. (1990) has used the ILG to simulate the segregation of two fluids of different viscosity during channel flow.

\subsubsection{ILG studies of multiphase flow through porous media}

The basic idea of this second area of application is illustrated in Figure 2, which shows a simulation of the invasion of a $2-D$ porous medium (formed by the gray squares) by a nonwetting fluid (black), when the medium is initially filled with a wetting fluid (white). Here the viscosities are matched and the capillary number (viscosity $\times$ characteristic velocity / surface tension) is about $10^{-2}$. The dominance of capillary forces creates the ramified front, a phenomenon known as capillary fingering (Lenormand et al., 1983).
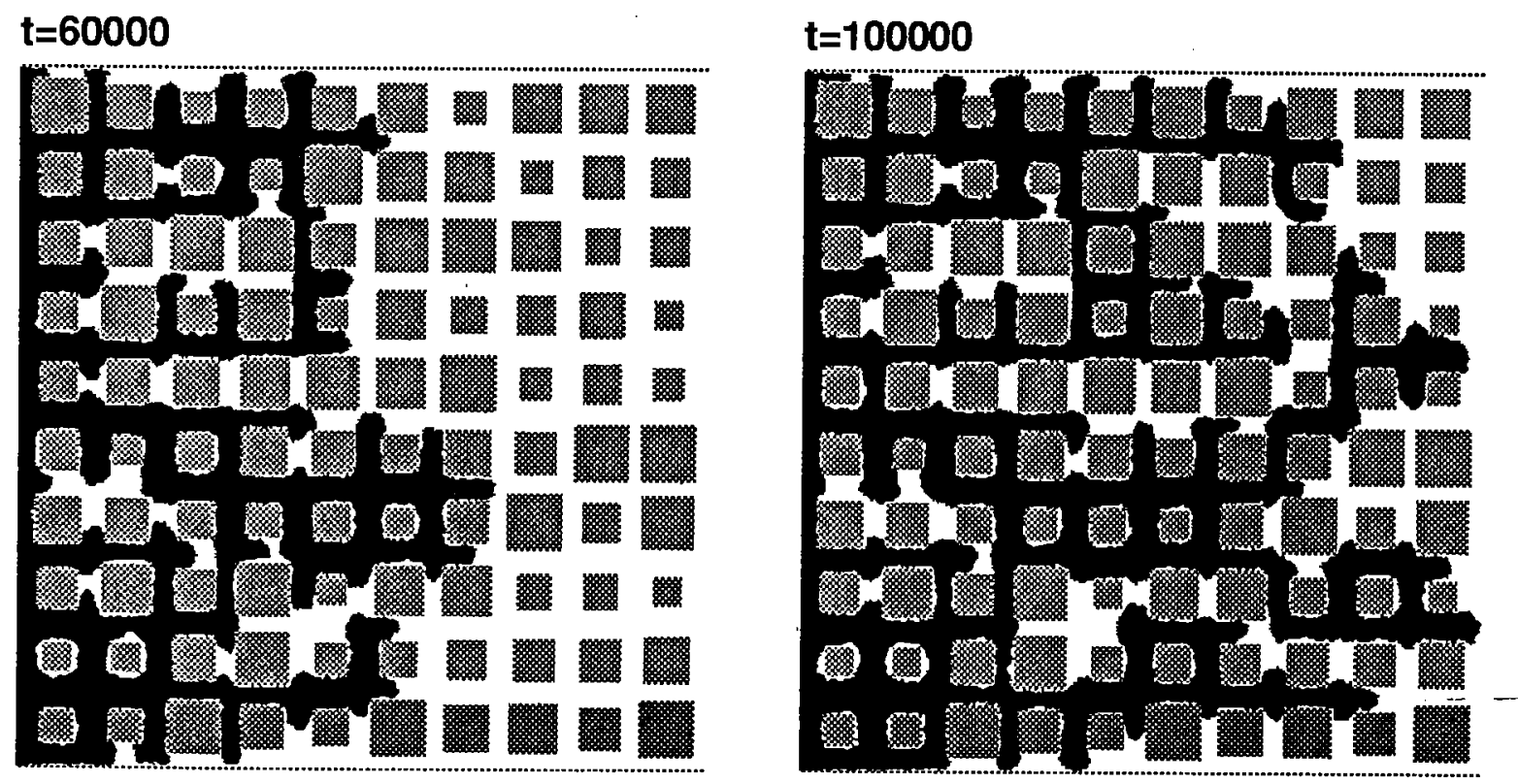

Figure 9.2. Immiscible displacement of a wetting fluid (white) by a nonwetting fluid (black) in a 2-D microscopic model of a porous medium. Time $t$ is in time steps. The nonwetting fluid is injected with constant pressure on the left. Note the trapping of wetting fluid. The simulation was performed by Andrew Gunstensen of MIT. 
The objective of ILG simulations of porous flow is to investigate numerically the macroscopic, or constitutive, equations that describe the volume-averaged flow. From considerations of irreversible thermodynamics, one is led to postulate a coupled linear response (e.g., de Gennes, 1983),

$$
J_{i}=\sum_{j} L_{i j} X_{j}
$$

where $J_{i}$ is the volume-average flux of the $i$ th fluid, $X_{j}$ is the force applied to the $j$ th fluid, and $L_{i j}$ is a matrix of phenomenological, or "relative permeability" coefficients. Empirical investigation of these flows in two dimensions (Rothman, 1990a) shows that capillary forces create nonlinear effects that are not predicted by equation (9.6). However, at sufficiently high forcing, the flow does reach a linear regime, and Onsager's reciprocal relation $L_{i j}=L_{j i}$ is observed. Work in progress by Andrew Gunstensen in three dimensions [using a lattice-Boltzmann method (Gunstensen et al., 1991) confirms these conclusions, but goes considerably further in elucidating the principal physical mechanisms that govern the aggregate flow.

\subsubsection{Other lattice-gas models of phase separation}

In concluding this review of the ILG, it is useful to contrast it with other lattice-gas models of phase separation that have recently appeared in the literature. Rem and Somers (1989) and Somers and Rem (1991) have developed a model qualitatively similar to the ILG, but without the need for non-local interactions. Their purely local collision rule results from coloring "holes" in addition to particles. Chen et al. (1991) have further pursued this idea. A model qualitatively different from the ILG was introduced by Appert and Zaleski (1990) and further described by Appert et al. (1991). Their model consists of only one species of particle, but with two thermodynamic phases: liquid and gas. The liquid-gas transition results from a nonlocal collision rule that exchanges momentum between pairs of sites but conserves the total momentum. This momentum exchange creates an addition term in the model's equation of state such that a dense phase (liquid) and a dilute phase (gas) can coexist.

\subsection{Emulsions, suspensions, and fluidized particles}

\subsubsection{Preliminaries}

Our starting point for a model of emulsions is the three-phase ILG described by Gunstensen and Rothman (1991b). In addition to red and blue particles, we now also have a third species, "green." Let $\phi_{i}^{(k)}=1$ if the $k$ th species is moving with velocity $\mathbf{c}_{i}$ at a given site, and 0 otherwise. The same exclusion rule used before still holds: no more than one particle (of any color) may move with velocity $\mathbf{c}_{i}$ at any site at any time.

The $k$ th color flux is given by

$$
\mathbf{q}_{k}(\mathbf{x}) \equiv \sum_{i=1}^{6} \mathbf{c}_{i} \phi_{i}^{(k)}, \quad k=1,2,3
$$


and the $k$ th color gradient is

$$
\mathbf{f}_{k}(\mathbf{x}) \equiv \sum_{i} \mathbf{c}_{i} \sum_{j} \phi_{j}^{(k)}\left(\mathbf{x}+\mathbf{c}_{i}\right), \quad k=1,2,3 .
$$

The result of a collision, $\phi_{i}^{(k)} \rightarrow \phi_{i}^{(k)}$, maximizes

$$
\sum_{k=1}^{k=3} \alpha_{k} \mathbf{f}_{k} \cdot \mathbf{q}_{k}\left(\phi_{i}^{\prime(k)}\right)
$$

subject, as before, to the conservation of species

$$
\sum_{i} \phi_{i}^{(k)}=\sum_{i} \phi_{i}^{(k)}, \quad k=1,2,3
$$

and conservation of total momentum:

$$
\sum_{i} \mathrm{c}_{i} \sum_{k} \phi_{i}^{(k)}=\sum_{i} \mathbf{c}_{i} \sum_{k} \phi_{i}^{(k)} .
$$

The $\alpha_{k}$ are parameters that allow specification of the three surface tensions $\sigma_{12}, \sigma_{13}$, and $\sigma_{23}$. For example, if $\alpha_{1}=\alpha_{2}=\alpha_{3}$, then $\sigma_{12}=\sigma_{13}=\sigma_{23}$, and three-phase contact points are stable. However, if the $\alpha_{k}$ 's are not all equal, three-phase contact points might not be stable. Of particular interest here is the case $\alpha_{1}=\alpha_{2}=1$, and $\alpha_{3}=-0.5$, which for a sufficiently large concentration of species 3 , results in an equilibrium state in which a bubble of fluid 1 and a bubble of fluid 2 reside in a "sea" of fluid 3 (Gunstensen and Rothman, 1991b).

\subsubsection{The emulsion model}

Emulsions are suspensions of liquid bubbles. An emulsion is said to be stable if the bubbles remain dispersed and undergo neither flocculation nor phase separation. In real emulsions, stability usually depends on electrostatic repulsion (due, for example, to the presence of a surfactant) or some form of polymer stabilization (Russel et al., 1989). Nothing so elaborate is attempted here. Instead, I create a bath of bubbles, each immiscible with each other, and also immiscible with the emulsifying fluid. The model is simply an $N$-species extension of equations (9.7) - (9.11), where $1 \leq k \leq N, \alpha_{1}=-0.5, \alpha_{k}=1$ for $2 \leq k \leq N$, and $N$ is arbitrarily large.

Such a model, however, is clearly unwieldy if equations (9.7) - (9.11) are directly implemented with $N$ greater than about 4 . How may this problem be surmounted? First, note that when there is at most one rest particle, no more than 7 species may collide at any one site at any time. Furthermore, only 3 species are significant: one corresponds to the emulsifying fluid, and the other two correspond to two bubbles at their point of approach. Thus the $N$-phase model may be mapped site-by-site to a 3 -phase collision rule.

The procedure is as follows. Each particle moving with velocity $\mathbf{c}_{i}$ is indexed by an nonnegative integer $n_{i} \leq N$ indicating its species code. At each site, the particle codes $\left\{n_{i}\right\}$ 
are transformed to Boolean variables $\phi_{i}^{(k)}$, where $k=1$ corresponds to the emulsifying species, and $k=2$ and $k=3$ correspond to the first and second most numerous emulsified species, respectively. (Cases of equal populations are dealt with deterministically.) All other emulsified species are considered neutral, or "colorless," and are assigned $k=4$. The outcome of a collision then follows from equations (9.7) - (9.9) precisely as before, and from the conservation equations (9.10) and (9.11) in which the case $k=4$ is also included. After the collision, the Boolean variables $\phi_{i}^{\prime(k)}$ are remapped to integer codes $n_{i}^{\prime}$, based on the previously stored correspondence between the four possible values of $k$ and the $N$ possible values of $n_{i}$.

My implementation of the 2-D emulsion model on a Sun Sparcstation 2 computer was based on a 21-bit lookup table. For each of 7 velocities per site, the transformed particle code was stored as an integer between 0 and 4 , inclusive, thus requiring 3 bits of memory each. Various tables were then constructed that depend in some way on the 21-bit configuration code. Clearly there is waste in this scheme, and a more memory-efficient algorithm could be designed if required by specialized computer architectures. The code, written in $\mathrm{C}$, runs at about 25,000 site updates per second, about one-fourth the speed of the two-phase ILG.

\subsubsection{Some preliminary simulations of emulsions}

No quantitative work has yet been done with the emulsion model, but a few illustrations of simulations serve to indicate the possibilities for future scientific computations.

Figure 3 shows two snapshots of a simulated emulsion, in which the emulsified phase fills $50 \%$ of the volume. The early time is shown shortly after the emulsion was initialized as a square grid of bubbles. At the later time, statistical fluctuations inherent to the lattice gas have created a pattern of bubbles that is independent of the initial configuration. Clearly one phenomenon accessible to study with the model is the collective Brownian motion in a densely concentrated emulsion.

Figure 4 shows the model's response to a simple shear. The shear is in the form of a "V"shaped velocity profile, with periodic boundary conditions, such that

$$
u_{y}=C(x-W / 4) \quad 0 \leq x<W / 2
$$

and

$$
u_{y}=-C(x-3 W / 4) \quad W / 2 \leq x<W,
$$

where $u_{0}$ is the peak velocity at the end points, $W$ is the total distance in the horizontal (shear) direction, and the shear rate $C=4 u_{0} / W$. Here $u_{0}=0.15, W=512 \sqrt{3} / 2$, and the area fraction of the emulsified phase is $50 \%$. The shear rate and the bubble size are sufficiently large for viscous forces to dominate Brownian diffusion. At the early time, the emulsified bubbles clearly show the influence of their initial configuration as a square network of bubbles and also of the "V"-shaped velocity profile. Note particularly how the bubbles have become elliptically elongated, with their major axes at about 45 degrees from the direction of the 

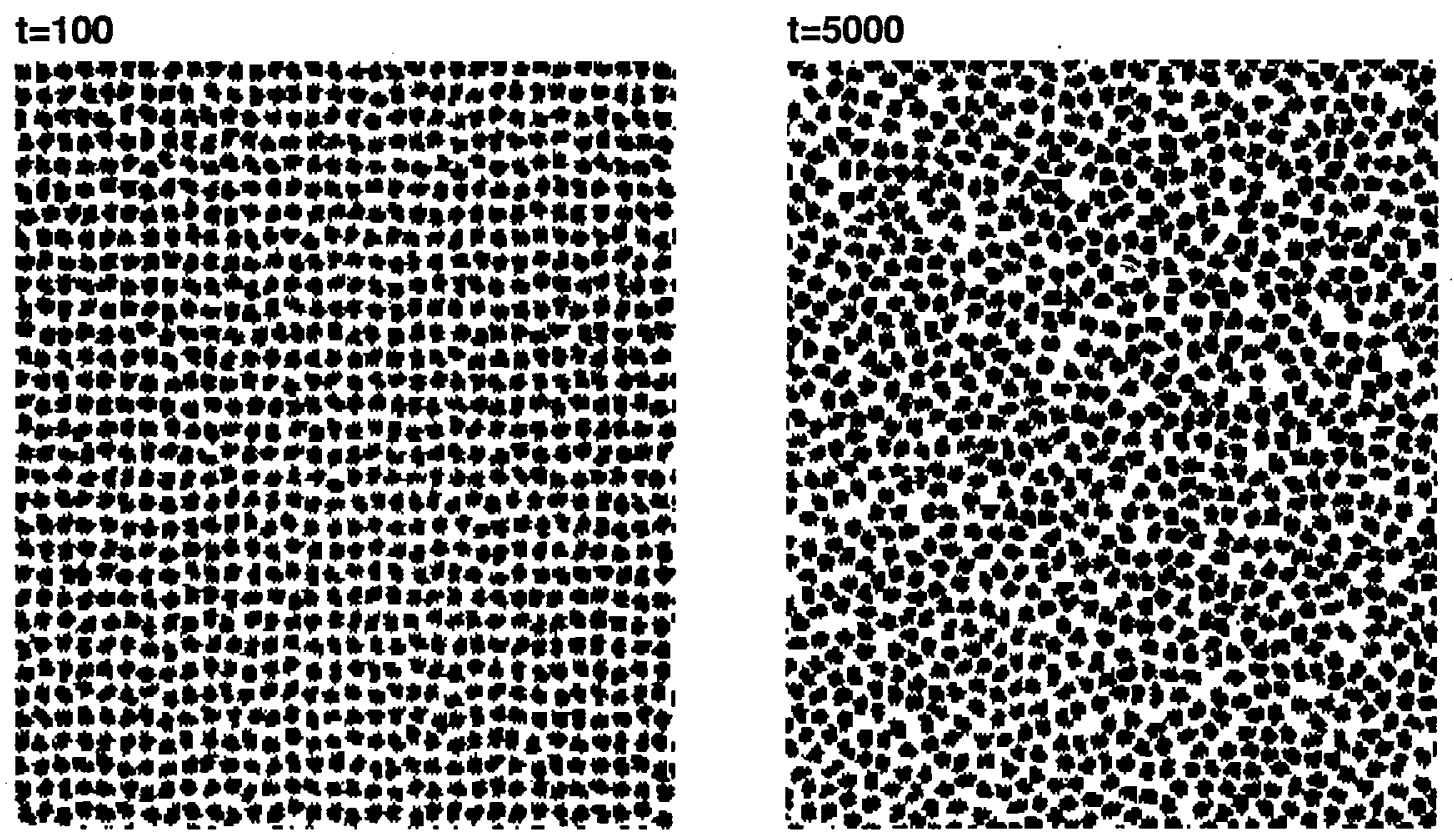

Figure 9.3. A simulated emulsion. There are approximately 1000 bubbles, each with a diameter of about 6 lattice units. The early time is shown after initialization with a square grid of bubbles. Fluctuations result in the random configuration shown at the later time.

flow and the shear. This orientational ordering is a consequence of stretching along the principal axis of strain. The pattern formed by the bubbles at the later time, however, is surprising. Here there is clear evidence of substantial long-range correlations that have formed independently of the initial configuration. These correlations appear related to the smecticlike correlations found in sheared phase separation (Chan et al., 1988; Rothman, 1990). Similar ordered structures are also known in granular flows (Hopkins and Louge, 1991) and even in molecular dynamics (Evans et al., 1984; Loose and Hess, 1989). Here, it is likely that hydrodynamic interactions between bubbles are creating the ordered structures, but more work is necessary to substantiate this hypothesis.

\subsubsection{Fluidized particles}

At first glance, emulsions seem to have little in common with fluidized particles, but I argue here that the emulsion model may indeed be used to study certain aspects of the dynamics of fluidized beds.

What are fluidized beds? Imagine a container of macroscopic particles (e.g., grains of sand). At the bottom of the container is a porous grating, with a mesh spacing sufficiently fine to support the particles yet sufficiently wide to allow a substantial rate of air flow through it. Imagine that an updraft of air can be forced through the grating. If the flow rate is small enough, the grains of sand remain more or less static, acting as an unconsolidated porous medium through which the air flows. If the flow rate is large enough, however, the drag force induced by the flow becomes equal to the net weight of the particles themselves. The bed of 
$\mathbf{t}=\mathbf{2 0 0}$

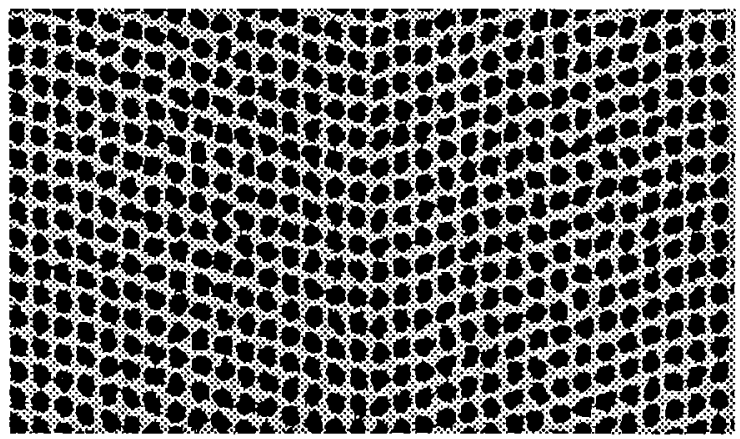

$t=12000$

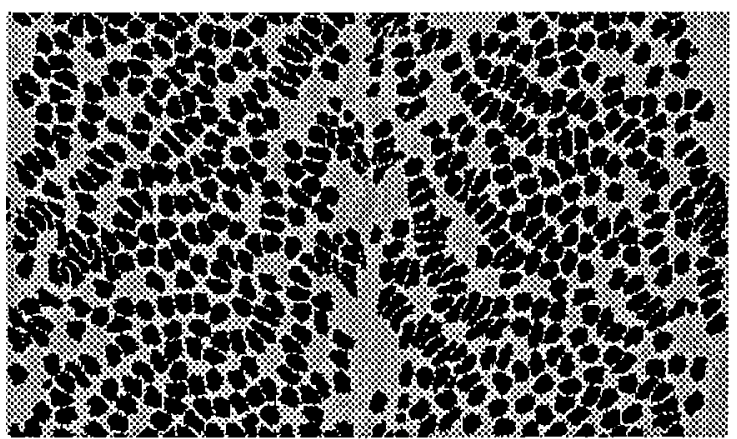

Figure 9.4. Simulation of a 2-D emulsion in a "V"-shaped shear flow. The early time is shown shortly after initialization as a square grid of bubbles. Note the substantial long-range order, independent of the initial conditions, that has developed at the later time. At the later time, the bubbles avoid the middle and sides of the simulation because of the discontinuity in the slope of the velocity profile.

particles then expands, and is said to be fluidized. At a scale much larger than the particle size, a uniformly fluidized bed of particles acts as a fluid itself, albeit with a complicated constitutive equation (e.g., Davidson and Harrison, 1963; Batchelor, 1988).

Fluidized beds are typically studied in the context of chemical-engineering applications, such as the design of efficient chemical reactors. However, fluidized beds also occur widely in nature. One of the best known examples is quicksand, which occurs when unconsolidated soil is fluidized by upwelling groundwater. A less obvious but very intriguing manifestation of fluidization is thought to occur in certain hot giant stars (Prendergast and Spiegel, 1973) and even possibly on the moon (Murray, et al., 1969).

Of particular interest here are the instabilities that occur in uniformly fluidized beds (Davidson and Harrison, 1963; Batchelor, 1988). Instabilities typically manifest themselves as the formation of "bubbles" of fluidizing fluid, which, due to their anomalous buoyancy, rise quickly through the bed. Decades of work have attempted to better understand the mechanisms giving rise to these instabilities. A recent theory due to Batchelor (1988) places much of this work in perspective in addition to deriving a new theory for the instability of a one-dimensional concentration wave. A basic understanding of fluidization instabilities is sufficiently challenging, however, that no comprehensive theory has yet been developed that describes the formation of voidage bubbles. Numerical simulations, therefore, can potentially offer important insight into this problem.

To study fluidized particles with the emulsion model, first note that our emulsion is a suspension of liquid bubbles, each with density equal to that of the suspending fluid, while fluidized particles are usually not only solid but also typically of much greater density than the fluidizing fluid. In this context, liquid bubbles differ from solid spheres in that liquid bubbles can deform, they do not rotate like a solid body, and flows can occur within them. 
These differences all diminish, however, as the viscosity within the bubbles and the surface tension increases. Moreover, liquid bubbles are similar to solid spheres in that their boundary conditions are the same: for both, there is no slip relative to the motion of their interfaces.

However, the restriction of the emulsion model to matched densities is potentially more problematic. Buoyancy forces may still be included, but only by accelerating the bubbles downward and the fluidizing fluid upward. This amounts to a Boussinesq approximation, in which density differences are included only insofar as they give rise to a gravitational force. As noted in early lattice-gas work by Burges and Zaleski (1987), this is equivalent to a fictitious world in which the inertial masses of all microscopic lattice-gas particles are equal, but the gravitational masses of lattice-gas particles of different species may differ.

So, in physical terms, we are left with a suspension of density-matched liquid bubbles in which the liquid bubbles are accelerated downward and the suspending fluid is accelerated upward. What happens? Figure 5 shows such a system at different stages of its evolution. There are no walls, and boundary conditions are periodic in both directions. The area fraction of fluidizing fluid is $60 \%$, the lattice has $256 \times 256$ sites, and there are approximately 1000 fluidized particles (i.e., emulsified bubbles). The frame of reference is such that the total upward buoyancy force applied to the fluidizing fluid equals that of the total downward buoyancy force applied to the fluidized particles; thus the total vertical (and horizontal) momentum at any time is approximately zero. The Reynolds number based on particle size is about 1 . At early stages of the process the particles are uniformly fluidized; i.e., coarse grained averages of the concentration of fluidized particles show only slight variations. At later stages, however, "voidage cracks" appear in the system, and later grow into "voidage bubbles". The voidage cracks are remarkably similar to those occurring in the 2-D fluidization experiments of Fortes et al. (1987). The voidage bubbles coalesce when they collide, and thus appear to grow with time. Indeed the entire process appears as a mechanically-induced phase separation. In this process, the local concentration of the fluidized particles can increase, apparently by the drafting of fluidized particles into the wake of particles that are leading a downward plunge (Fortes et al, 1987). As time increases this effect amplifies. Whether there is a preferred maximum scale of the voidage bubbles remains to be determined.

Precisely how closely this fluidization model corresponds to real fluidization also remains to be determined. Aside from the discrepancies already pointed out, it is also important to note that this lattice-gas model, like most others, is not Galilean-invariant. However, this is only a relevant issue at Reynolds numbers greater than about 1 (see, e.g., Gunstensen and Rothman, 1991a); thus the early stages of fluidization instabilities may still be reasonably well-captured by the model. 


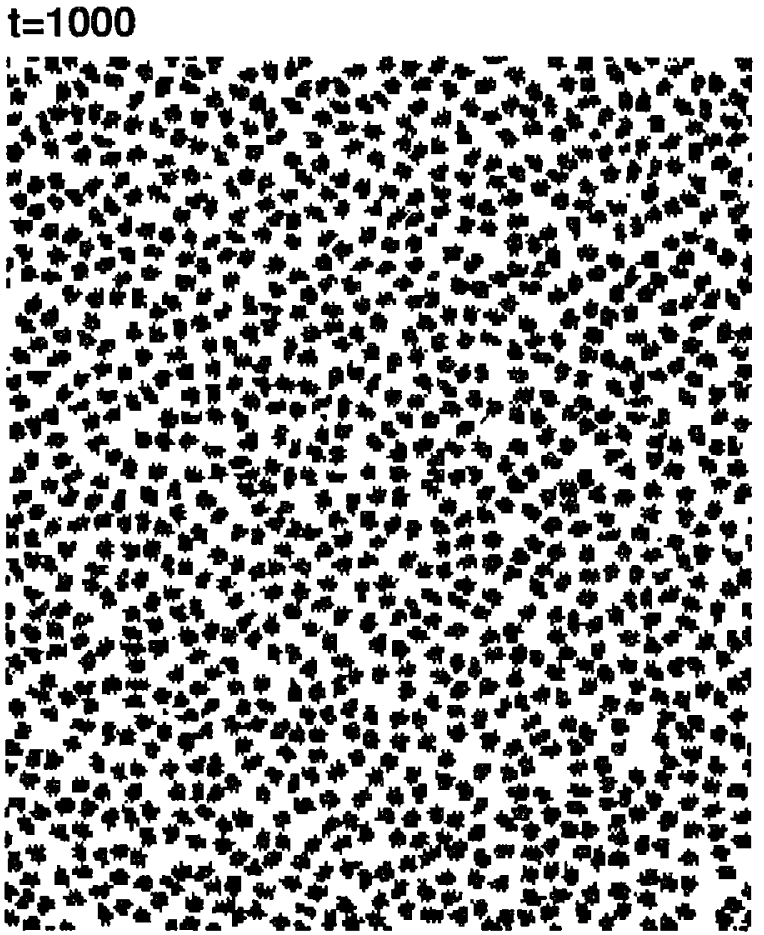

\section{$t=27000$}

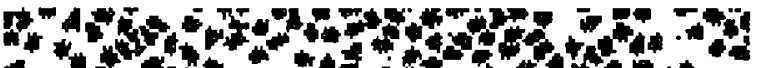
$+44^{2}+t^{2}+2+4$

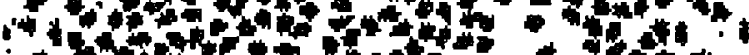

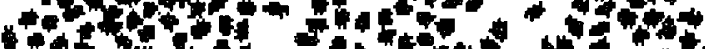

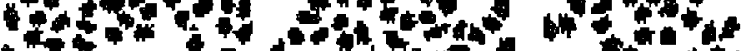

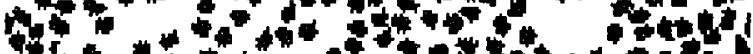

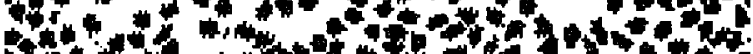

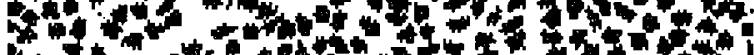

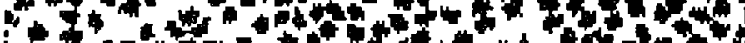

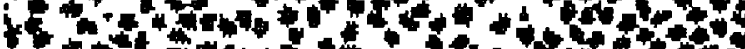

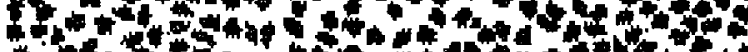

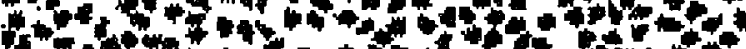

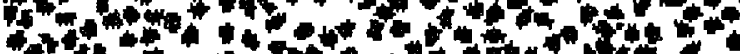

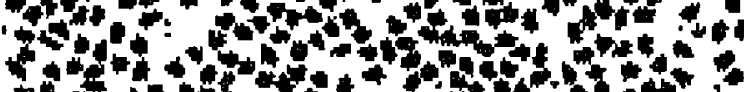

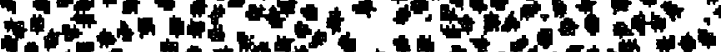

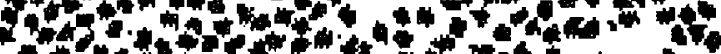

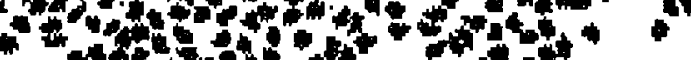

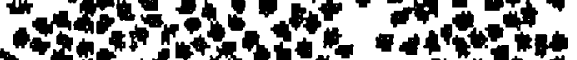

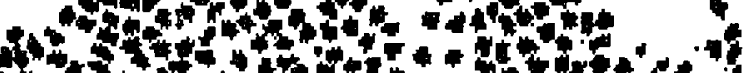

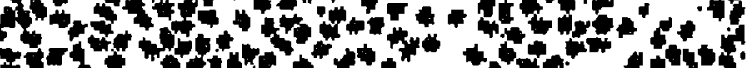

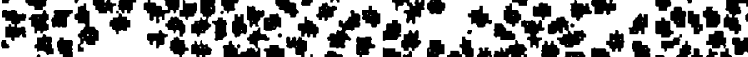

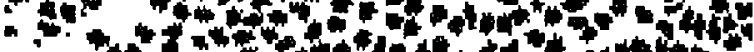

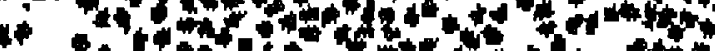

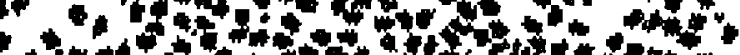

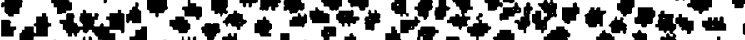

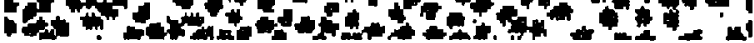

$t=14000$

Fn

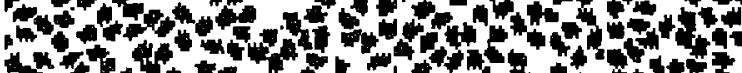

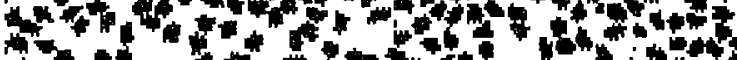

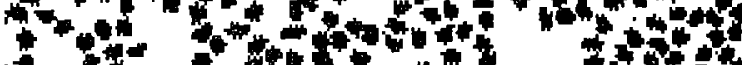

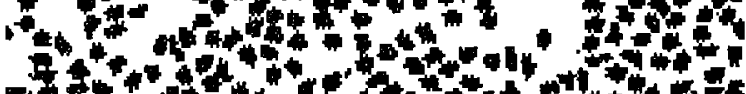

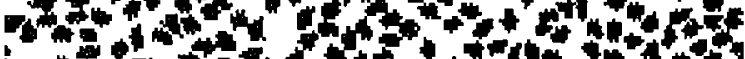

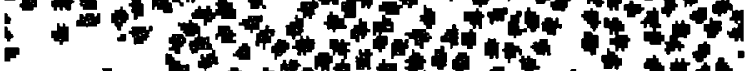

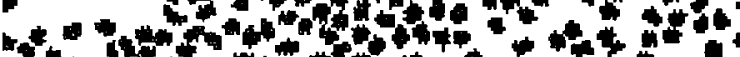

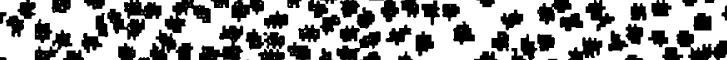

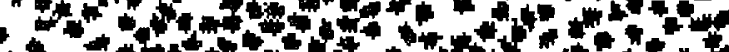

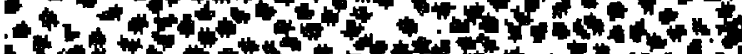

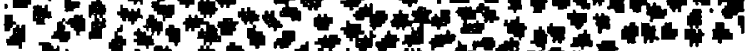

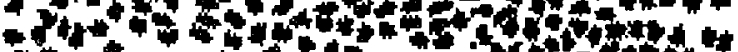

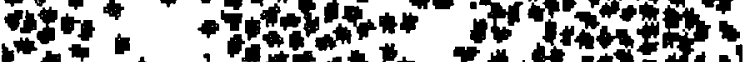

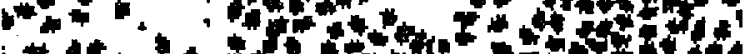

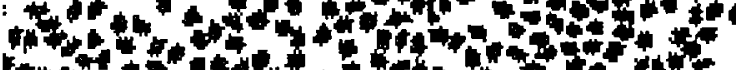

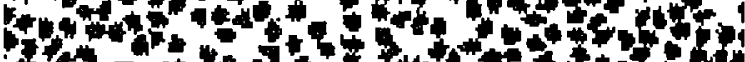

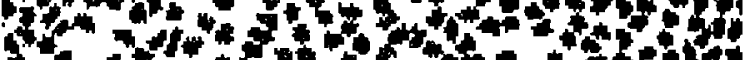

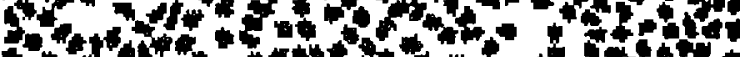

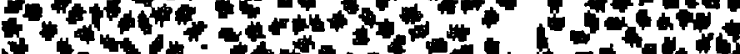

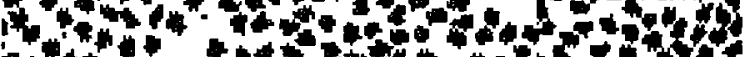

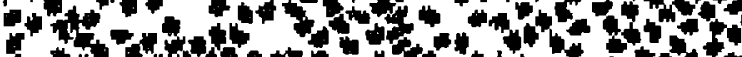

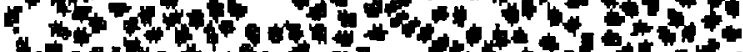

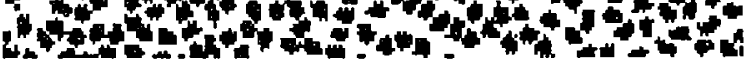

\section{$\mathrm{t}=\mathbf{4 5 0 0 0}$}

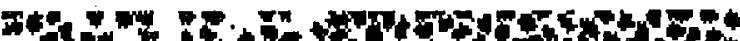

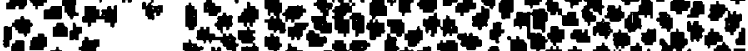

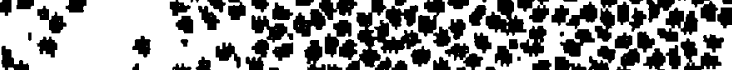

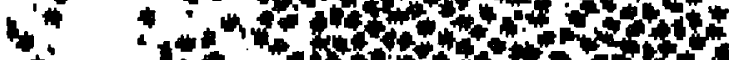

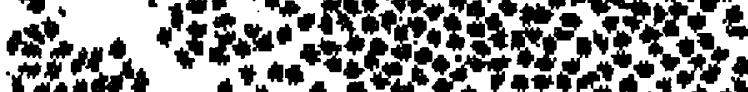

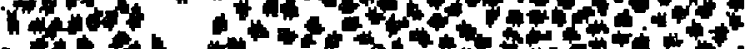
I

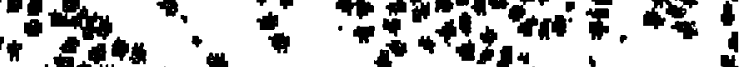

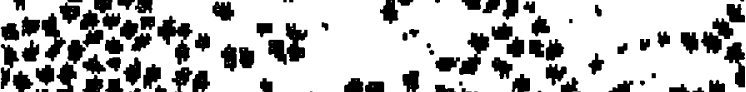

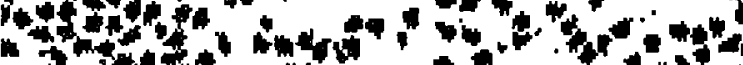

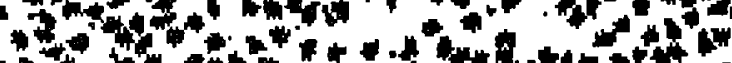

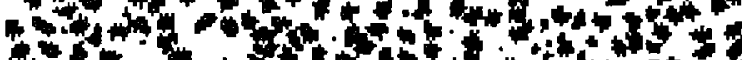

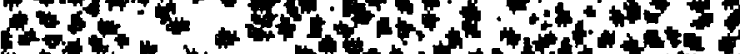

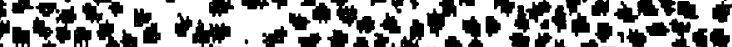

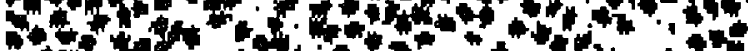

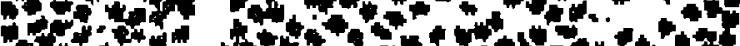

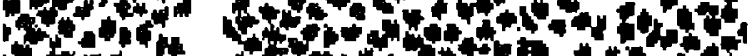

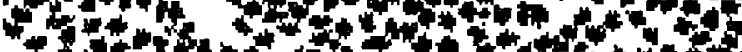

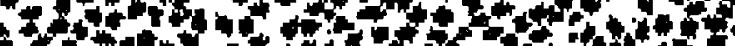

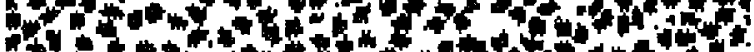

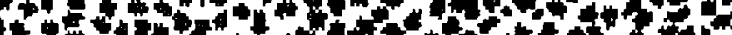

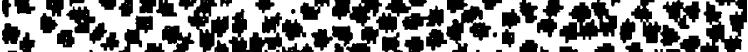

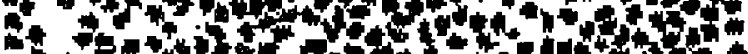

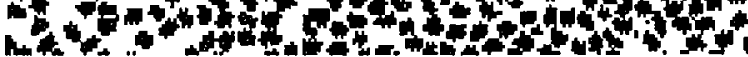

Figure 9.5. A simulation of fluidized particles, using the emulsion model. Boundary conditions are periodic in both directions. The fluidizing fluid is white and the fluidized particles are black. Note the development of voidage bubbles as the simulation evolves. 


\subsection{Competing interactions in a binary fluid}

\subsubsection{Ferrofluids and other striped systems}

The final model I describe is also an extension of the ILG, but its phenomenology is distinct not only from the ILG but also the emulsion model described in the previous section. Here, I define two ILG-like interactions that oppose each other. One of the interactions is the surfacetension rule already defined above. The other interaction, to be defined below, is repulsive rather than attractive, and depends on distant neighbors rather than nearest neighbors.

The motivation for such a model comes from observations of a quasi-two-dimensional ferrofluid subjected to a magnetic field applied normal to the plane of the fluid. Figure 6 shows the labyrinth pattern that results from such an experiment. Note that the orientations of the stripes exhibit short-range order but not long-range order.

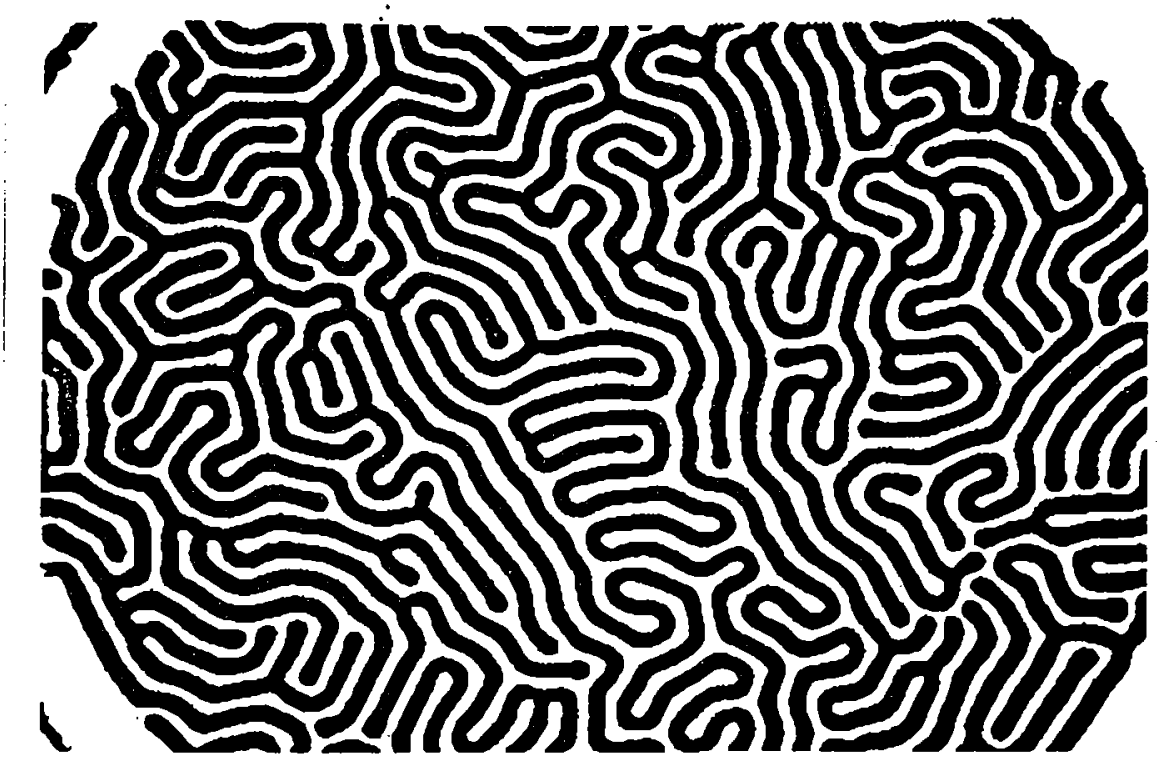

Figure 9.6. Random stripes formed by a drop of ferrofluid (black) between two horizontal glass plates about a millimeter apart, after a magnetic field has been applied in the orthogonal direction. The thickness of the stripes is about equal to the separation of the plates. From Witten (1990); experiment performed by R.E. Rosensweig.

A ferrofluid is a colloidal suspension of ferromagnetic particles, typically about $100 \AA$ in size (Rosensweig, 1985). The ferrofluid acts as a typical fluid, except when a magnetic field is applied. The ferrofluid flows in response to an applied magnetic field, which makes it qualitatively different from any known natural fluid.

There have been a number of technological applications of ferrofluids (Rosensweig, 1985), but our interest here is not specifically ferrofluids themselves but the patterns, such as the one in Figure 5, that they can exhibit. Here the ferrofluid-the black fluid-is between two glass plates, separated by a distance about equal to the width of a stripe. The ferrofluid is 
initially placed between the plates as a circular drop surrounded by air. When a magnetic field is applied in the direction normal to the plates, magnetic repulsion attempts to break up the drop. However, surface tension at the ferrofluid-air interface tries to retain the drop's original circular shape. The striped pattern of Figure 6 is thus the result of this competition between the short-range attractive forces of surface tension and the long-range repulsive forces due to magnetic interactions within the ferrofluid.

Such striped patterns are not unique to ferrofluids. Indeed, there is a wide range of systems with similar morphological phases but different microphysics. For example, striped patterns have been observed in thin uniaxial magnetic films (Garel and Doniach, 1982) and also Langmuir monolayers of polar molecules such as surfactants, fatty acids, or lipids (Andelman et al., 1987). In both of these instances there is also competition between short-range attraction and long-range repulsion. In the former case the long-range repulsion is still magnetic in origin, while for the latter it arises from electrostatic interactions due to the presence of polar molecules. Random stripes such as those in Figure 6 are also observed in certain block copolymers (Bates, 1991; Witten, 1991). Here again, surface energy plays the attractive role, while "polymer stretch" energy acts to oppose it. Finally, one can cite Rayleigh-Benard convection for the case in which the vertical dimension of a 3-D layer of fluid is much smaller than the other two dimensions. Here the fluid is heated from below, and competition between thermal diffusion and buoyancy effects due to thermal expansion can create a 2-D striped pattern of thermal "rolls" having much in common with Figure 6 [see, for example, the experiments illustrated in Heutmaker et al. (1985) or the simulations of the Swift-Hohenberg equation in the book by Manneville (1990)].

\subsubsection{A lattice-gas collision rule with competing interactions}

Here I show how random stripes (in fact, much more) can be obtained from a simple collision rule in a lattice-gas mixture: Although the construction of the model is inspired by interactions in ferrofluids, the rules introduced here are intended to be a generic example of competing short-range and long-range interactions, with no specificity intended. Thus a principal goal of the work is to offer some insight into the unifying structure that underlies the diverse systems outlined above.

The outcome of a collision depends on two competing interactions, one of which acts at short range and is attractive, while the other opposes it and acts at long range. The attractive interaction is the ILG collision rule described by equations (1.1)-(1.5). This may be considered to be the physical mechanism that, in the case of the ferrofluid, tries to keep circular drops circular, or, in general, attempts to minimize the total length of all interfaces in the 2-D system.

To model the long-range interactions in a ferrofluid accurately, one would need to model the repulsive dipole interaction over the entire system, with interaction strength dropping off like 
$r^{-3}$. Such a scheme would be unwieldy at best. Instead I choose to define a coarse-grained color gradient

$$
\langle\mathbf{f}(\mathbf{x})\rangle_{l} \equiv \sum_{i} \mathbf{c}_{i} \sum_{j}\left[r_{j}\left(\mathbf{x}+l \mathbf{c}_{i}\right)-b_{j}\left(\mathbf{x}+l \mathbf{c}_{i}\right)\right],
$$

where the parameter $l$ defines the scale of coarse-graining. The result of a collision, $r \rightarrow r^{\prime}$, $b \rightarrow b^{\prime}$, is now the configuration that maximizes

$$
\mathbf{q}\left(r^{\prime}, b^{\prime}\right) \cdot\left(\mathbf{f} /|\mathbf{f}|-\alpha\langle\mathbf{f}\rangle_{l} /\left|\langle\mathbf{f}\rangle_{l}\right|\right)
$$

where $\alpha \geq 0$ is a parameter, $\mathbf{q}$ is given by equation (1.1) and mass, momentum, and color are conserved according to equations (1.4) and (1.5).

The consequences of such a rule may be perceived by first considering the extreme case in which $\alpha=\infty$, i.e., only the long range interaction is active. Because the dynamics are essentially the same as in the ILG, but with reversed sign and a coarse-grained gradient, one still expects surface tension at interfaces. However, the interfaces form in response to color information at a distance $l$ from the interface, not immediately adjacent to it. If the interaction were positive, the effect would simply be thick interfaces. Because the interaction is negative, however, the interfaces separate alternating regions of blue and red, about $l$ lattice units apart. In the absence of the attractive interaction, these are parallel stripes. When $\alpha$ is small, however, the usual surface-tension interaction attempts to minimize surface lengths, causing some stripes to curl, thus breaking long-range order and creating random stripes.

\subsubsection{Some preliminary simulations}

Figure 7 shows results from the stripe model, with $\alpha=0.25$ and $l=5$, on a lattice of size $128 \times 128$. Here the concentration of blue is $50 \%$ and the density of particles is $d=0.9$. The initial condition was fully mixed; the striped phase was attained after only about 100 time steps. The collision rule is the one described above, but the computation of $\langle\mathbf{f}\rangle_{l}$ involves the sum of 12 points around a circle rather than just 6, which appears necessary to make the orientations of the stripes isotropic.

One interesting aspect of the stripes of Figure 7 are their fluctuations: one sees that considerable change in the pattern has resulted between the two time steps shown. The change is gradual: over short time scales, fluctuations occur over just a few stripes, whereas the accumulation of the short-period fluctuations results in entirely new patterns over long time scales. Preliminary measurements show that the pattern fluctuations (determined by measurements of color fraction versus time at various points) have a $1 / f$ spectrum over one decade in frequency. 

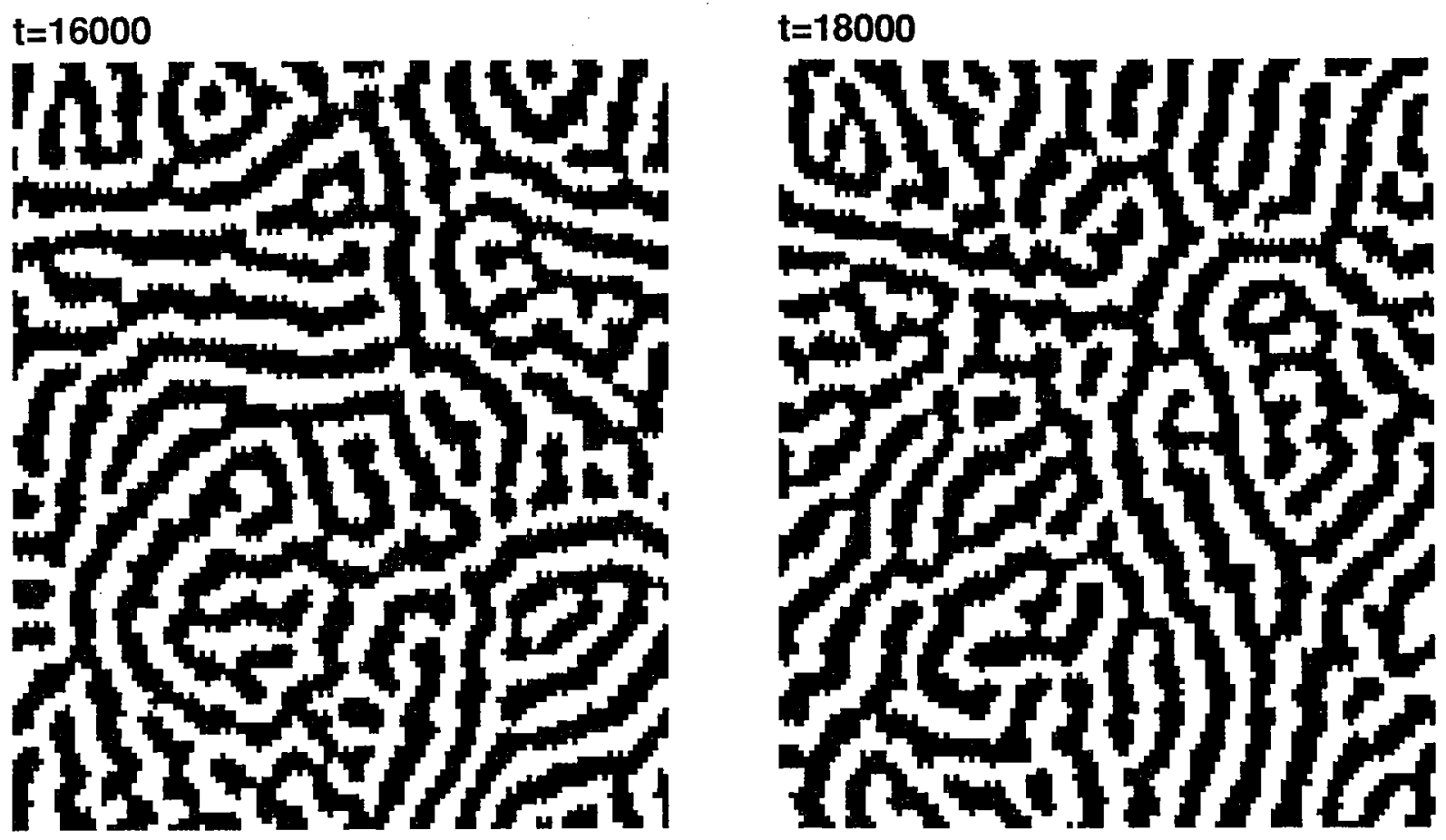

Figure 9.7. Competing-interaction model on a $128 \times 128$ lattice, with $\alpha=0.25$ and $l=5$. The initial state was fully mixed, with equal concentrations of both fluids. Note how fluctuations change the pattern over time.

A second interesting aspect of the model is its behavior with respect to $\alpha$, the relative strength of the long range interaction. Figure 8 shows some results with $\alpha=1.0$, twice as high as that in Figure 7. Now the stripes are parallel, rather than randomly oriented, due to the relative insignificance of the short-range attraction. Thus there is a change in morphology from random to parallel stripes as $\alpha$ increases. In this case, fluctuations result in the frequent appearance (and disappearance) of dislocations.

Lastly, it is interesting to point out the dependence of the pattern on the relative concentration of the phases. The previous examples were for concentrations of 50\%; in Figure 9, however, we see results for a blue concentration of $33 \%$. Rather than stripes, we now obtain a hexagonally symmetric distribution of bubbles, each spaced about $l$ lattice units from each other. The orientation of this hexagonal network appears to be independent of the underlying lattice. Close inspection of the figure also reveals dislocations in the hexagonal structure. This pattern is relatively free of fluctuations. 

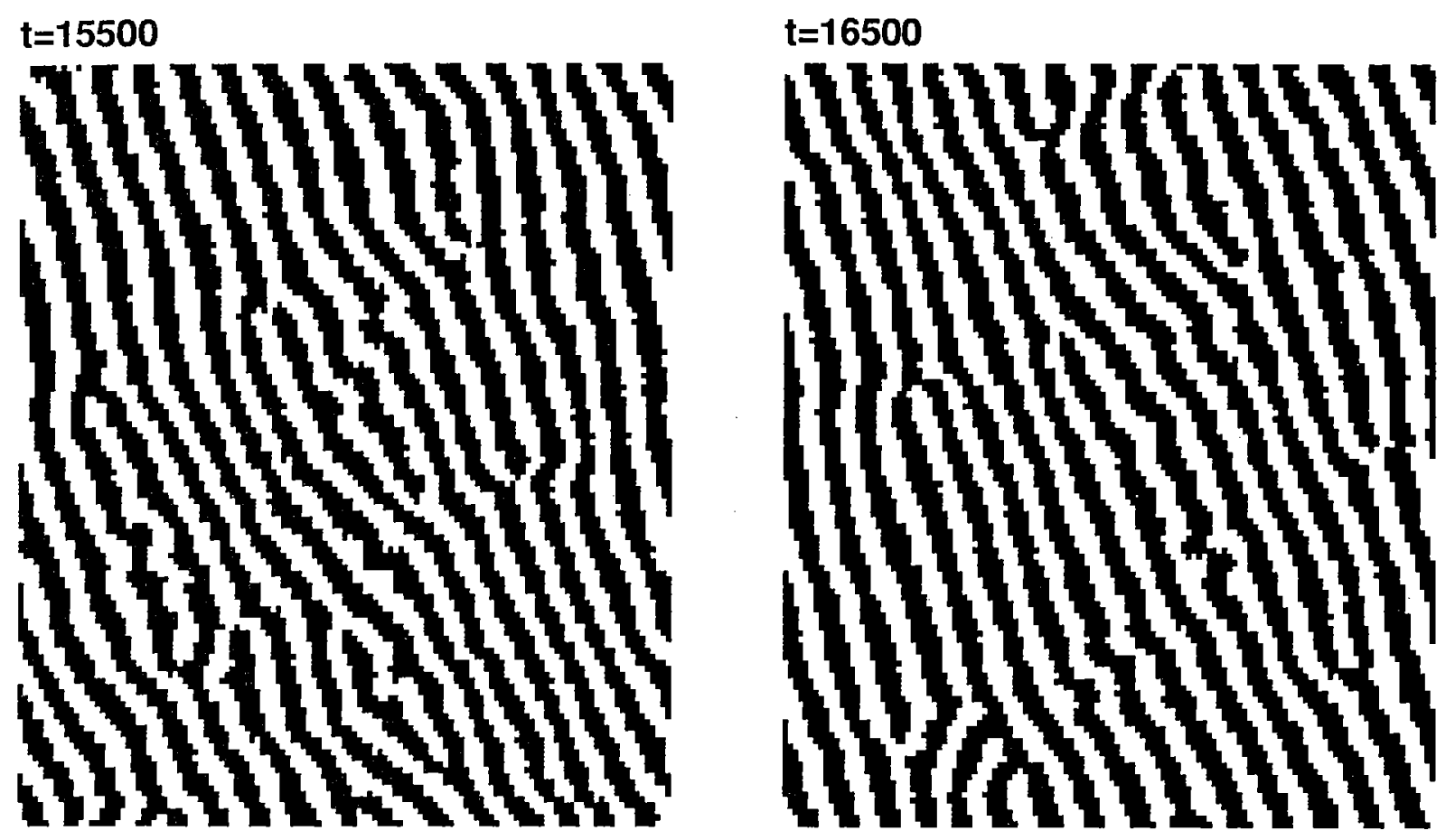

Figure 9.8. Competing-interaction model on a $128 \times 128$ lattice, with $\alpha=1.0$ and $l=5$. The initial state was fully mixed, with equal concentrations of both fluids. Compare to Figure 7, where the strength of the long range interaction was weaker. Note how fluctuations result in a number of transient dislocations.

\subsection{Conclusions}

These lectures have shown how one may specify simple collision rules in multi-species lattice gases to produce models of a wide variety of complex fluids. The archetypal model is the immiscible lattice gas, a model in which two species interact with nearest neighbors to produce surface tension at interfaces. Phase separation and complex flows such as two-phase flow in porous media have been fruitfully studied with the model.

Simple extensions of the ILG have been shown to allow simulations of emulsions, fluidized particles, and the striped patterns formed by ferrofluids. For these problems, work has only just begun, and much remains to be done to ascertain how closely these lattice-gas models mimic real complex fluids.

\subsection{Acknowledgments}

The work on stripes was initiated during a visit to The University of Chicago, where discussions with L. Kadanoff, S. Langer, J. Marko, T. Witten, and others were both inspirational and helpful. Discussions with E. Spiegel motivated the work on fluidization. I also thank W. Young, S. Zaleski, G. Zanetti, and other participants in the 1991 Woods Hole Geophysical Fluid Dynamics Program for interesting discussions. This work was funded in part by the MIT Porous Flow Project and NSF Grant 9017062-EAR. 


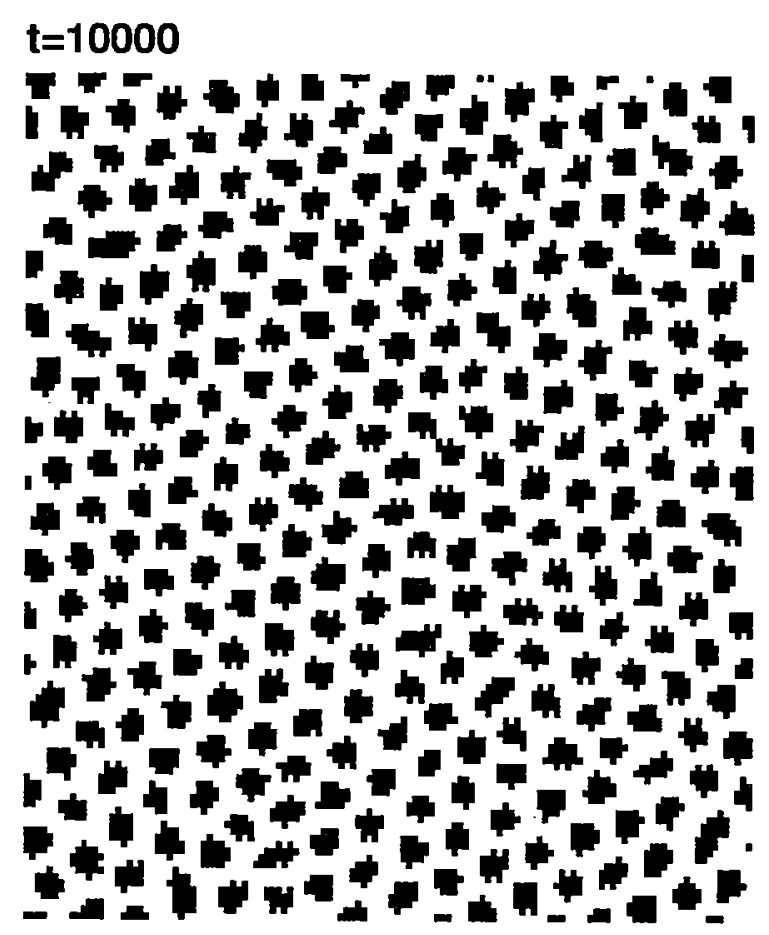

Figure 9.9. Competing-interaction model on a $128 \times 128$ lattice, with $\alpha=1.0$ and $l=5$. The initial state was fully mixed. The concentration of black is $33 \%$. The change in the pattern relative to that of Figure 8 is due entirely to the change in concentration.

\section{References}

Andelman, D., Brochard, F., and Joanny, J.-F., Phase transitions in Langmuir monolayers of polar molecules, J. Chem. Phys. 86, 3673-3681, 1987.

Appert, C. and Zaleski, S., A lattice gas with a liquid-gas transition, Phys. Rev. Lett. 64, $1-4,1990$.

Appert, C., Rothman, D.H., and Zaleski, S., A liquid-gas model on a lattice, Physica D 47, 85-96, 1991.

Batchelor, G.K., A new theory of the instability of a uniform fluidized bed, J. Fluid Mech. 193, 75-110, 1988.

Bates, F.S., Polymer-polymer phase behavior, Science 251, 898-905, 1991.

Burges, C. and Zaleski, S., Buoyant mixtures of cellular-automaton fluids, Complex Systems, $1,31-50,1987$.

Chan, C.K., Perrot, F., and Beysens, D., Effect of hydrodynamics on growth: spinodal decomposition under uniform shear flow, Phys. Rev. Lett. 61, 412-415, 1988.

Chen, S., G. D. Doolen, K. Eggert, D. Grunau, and E. Y. Loh, Local lattice-gas model for immiscible fluids, Phys. Rev. A 43, 7053-7056, 1991.

Davidson, J.F. and Harrison, D., Fluidized particles Cambridge University Press, New York, 1963. 
Doolen, G., Frisch, U., Hasslacher, B., Orszag, S, and Wolfram, S., eds., Lattice-gas methods for partial differential equations, Addison-Wesley, New York, 1990.

Doolen, G., ed., Lattice-gas methods for PDE's, North Holland, Amsterdam, 1991.

Evans, D.J., Hanley, H.J.M., and Hess, S., Physics Today 37(1), 26, 1984.

Fortes, A.F., Joseph, D.D., and Lundgren, T.S., J. Fluid Mech. 177, 467-483, 1987.

Frisch, U., Hasslacher, B., and Pomeau, Y., Lattice-gas automata for the Navier-Stokes equation, Phys. Rev. Lett., 56, 1505-1508, 1986.

Frisch, U., d'Humières, D., Hasslacher, B., Lallemand, P., and Pomeau, Y., and Rivet, J-P., Lattice-gas hydrodynamics in two and three dimensions, Complex Systems, 1, 648-707, 1987.

Garel, T. and Doniach, S., Phase transitions with spontaneous modulation - the dipolar Ising ferromagnet, Phys. Rev. B 26, 325-329, 1982.

de Gennes, P.G., Theory of slow biphasic flows in porous media, Physico-Chem. Hydrodyn., 4, 175-185, 1983.

Gunstensen, A. and Rothman, D.H., A Galilean-invariant immiscible lattice-gas, Physica $D$ 47, 53-63, 1991a.

Gunstensen, A. and Rothman, D.H., A lattice-gas model for three immiscible fluids, Physica $D$ 47, 47-52, $1991 \mathrm{~b}$.

Gunstensen, A., Rothman, D.H., Zaleski, S., and Zanetti, G., Lattice Boltzmann model of immiscible fluids, Phys. Rev. A 43, 4320-4327, 1991.

Gunton, J.D, San Miguel, M. and Sahni, P.S., in Phase Transitions and Critical Phenomena, edited by C. Domb and J.L. Lebowitz (Academic, New York, 1983).

Heutmaker, M.S., Fraenkel, P.N., and Gollub, J.P., Convection patterns: time evolution of the wave-vector field, Phys. Rev. Lett. 54, 1369-1371, 1985.

Hopkins, M.A. and Louge, M.Y., Inelastic microstructure in rapid granular flows of smooth disks, Phys. Fluids A 3, 47-57, 1991.

Kadanoff, L., McNamara, G., and Zanetti, G., From automata to fluid flow: comparisons of simulation and theory, Phys. Rev. A 40, 4527, 1989.

Lenormand, R., Zarcone, C., and Sarr, A., Mechanisms of the displacement of one fluid by another in a network of capillary ducts, J. Fluid Mech., 135, 337-353, 1983.

Loose, W. and Hess, S., Rheology of dense model fluids via nonequilibrium molecular dynamics: shear thinning and ordering transition, Rheologica Acta 28, 91-101, 1989.

Manneville, P., Dissipative structures and weak turbulence Academic Press, Boston, 1990.

Murray, J.D., Spiegel, E.A., and Theys, J., Fluidization on the Moon(?), Comm. Ap. Sp. Sci. 1, 165-171, 1969.

Prendergast, K.H. and Spiegel, E.A., Photon bubbles, Comm. Ap. Sp. Sci. 5, 43-49, 1973.

Rem, P. and Somers, J., Cellular automata on a transputer network, in Discrete kinetic theory, lattice-gas dynamics, and foundations of hydrodynamics, R. Monaco, ed., 268-275, World Scientific, 1989.

Rosensweig, R.E., Ferrohydrodynamics, Cambridge University Press, New York, 1985.

Rothman, D.H., Macroscopic laws for immiscible two-phase flow in porous media: results from numerical experiments, J. Geophys. Res. 95, 8663-8674, 1990a. 
Rothman, D.H., Deformation, growth, and order in sheared spinodal decomposition, Phys. Rev. Lett. 65, 3305-3308, 1990 b.

Rothman, D.H., Complex rheology in a model of a phase separating fluid, Europhys. Lett. 14, 337-342, 1991.

Rothman, D.H. and Keller, J.M., Immiscible cellular-automaton fluids, J. Stat. Phys., 52, 1119-1127, 1988.

Rothman, D.H. and Zaleski, S., Spinodal decomposition in a lattice-gas automaton, $J$. Physique (France), 50, 2161-2174, 1989.

Russel, W.B., Saville, D.A., and Schowalter, W.R., Colloidal Dispersions, Cambridge University Press, New York, 1989.

Somers, J. and Rem, P., Analysis of surface tension in two-phase lattice gases, Physica $D$ 47, 39-46, 1991.

Stockman, H.W., Stockman, C.T., and Carrigan, C.R., Modelling viscous segregation in immiscible fluids using lattice-gas automata, Nature 348, 523-525, 1990.

Witten, T.A., Structured fluids, Physics Today, 21-28, July 1990.

Wolfram, S., Cellular automaton fluids 1: Basic theory, J. Stat. Phys., 45, 471-526, 1986.

Zanetti, G., The hydrodynamics of lattice-gas automata, Phys. Rev. A, 40,1539-1548, 1989. 


\section{Papers}




\title{
10. When Rayleigh-Bénard Meets Zebra-leopard
}

\author{
Yih-Yuh Chen \\ Condensed Matter Physics 114-36, California Institute of Technology \\ Present address: Institute of Physics, Academia Sinica, Nankang, Taipei, \\ Taiwan, R.O.C.
}

A physicist might know something about the roll pattern exhibited in Rayleigh-Bénard convection. He might also have noticed the beautiful coat markings displayed by the many animals in the zoo when his three-year-old niece took him there last time. And when a physicist is so obsessed with the universality of the Nature, as many of them indeed are, you can bet that sooner or later he is bound to claim that a convection system is just like an animal. An animal? Hmmm... (Fig. 1)

Of course, it takes more motivation than this superficial observation to make a funny claim like that. Actually, the similarity between the pattern formation in a Rayleigh-Bénard cell and the animal coat markings wouldn't be that surprising if you happened to have played with the nodal lines of the eigenfunctions of the Laplacian operator $-\nabla^{2}$ before. (Once, out of boredom, I was drawing the nodal lines for fun during office hours when my boss, Prof. Mike Cross, came in from behind and saw the pictures. My unwarranted embarassment immediately evaporated the moment I heard him say "Working hard on Rayleigh-Bénard convection, huh?") Yes, I mean to say that the two apparently different systems do share a common feature that is conveyed by the Laplacian. To show you how this works, let's look at the convection problem first. Suppose you fill a shallow tank with water and seal it up, then impose a temperature gradient across the top and the bottom plates in such a way that the bottom is warmer than the top. When the temperature gradient is not too big you will not see any fluid motion inside this cell. You know the system is in a stable pure (thermal) conducting state, and the temperature decreases linearly from the bottom to the top. But when you increase the temperature gradient beyond a certain limit, all of a sudden the fluid will become unstable - because now the buoyancy experienced by a fluid element which happens to be hotter (and hence lighter) than its environment due to uncontrollable fluctuations is strong enough to overcome the resisting viscosity - and convective rolls will start to form spontaneously. Shine a beam of light from the bottom and look down from above. You will see bright bands intervened by dark ones. The dark bands are there because the light rays passing through the hotter rising fluid are refracted and defocused. The bright bands correspond to the regions where fluid is sinking to the bottom. This is what the box 
in Fig. 1 is showing. (The circles and the arrows indicate the fluid motion you would see when you look at it from the side.)

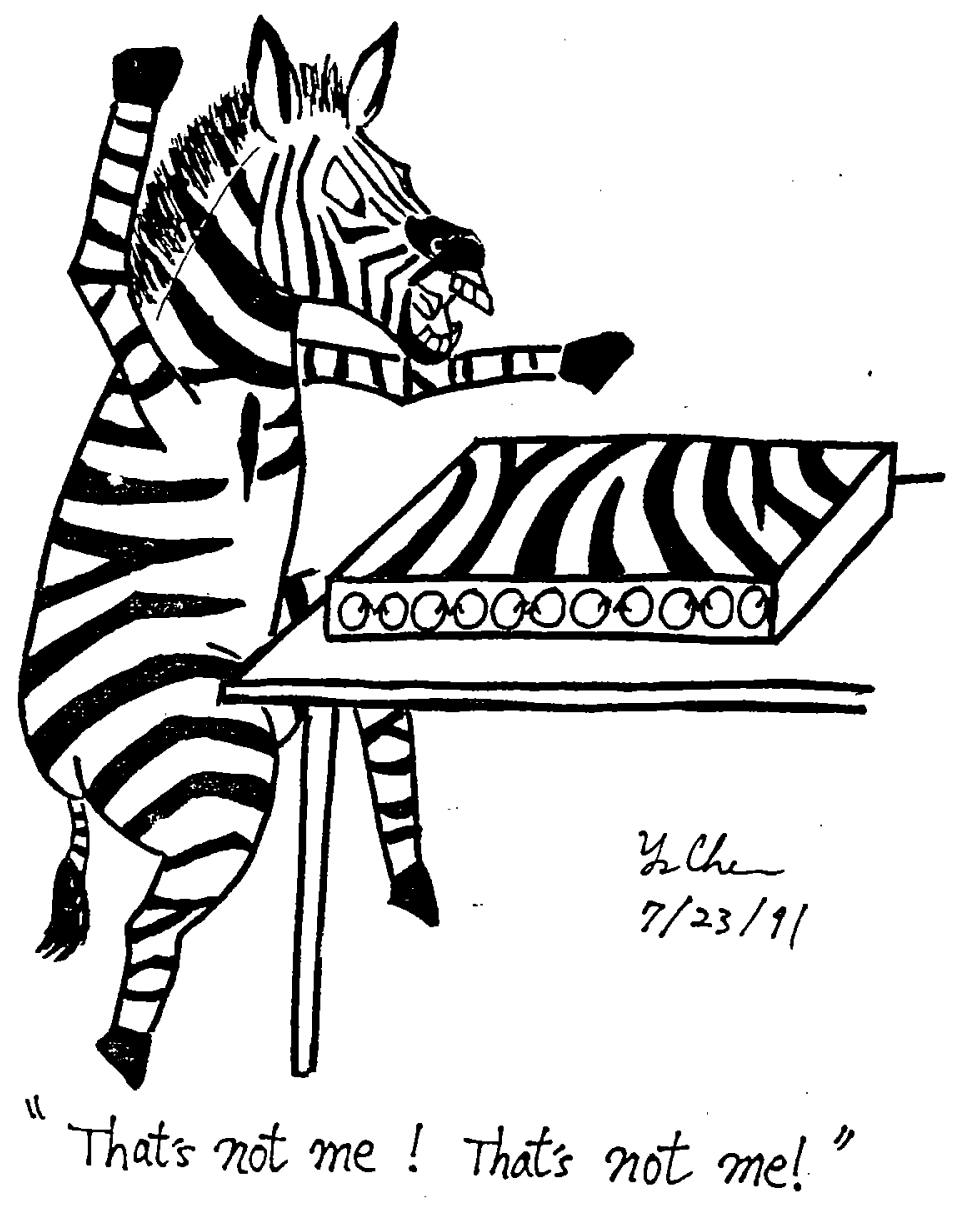

Figure 10.1. Entirely different systems can exhibit similar spatial patterns. But does this mean they are related?

The linearized equations which govern this system can be conveniently written in the following nondimensional form:

$$
\begin{aligned}
& \frac{\partial \mathbf{u}}{\partial t}=\sigma \nabla^{2} \mathbf{u}+\sigma \theta \hat{e}_{z}-\nabla p \\
& \frac{\partial \theta}{\partial t}=\nabla^{2} \theta+R u_{z} \quad\left(u_{z} \equiv \mathbf{u} \cdot \hat{e}_{z}\right) \\
& \nabla \cdot \mathbf{u}=0 \quad \text { (incompressibility condition) }
\end{aligned}
$$

Here $\mathbf{u}$ is the fluid velocity, $p$ the fluid pressure, and $\theta$ is the deviation of temperature from the pure conducting state. Eqn. (1) is the Navier-Stokes equation, or Newton's law $\mathbf{F}=m$ a if you like, with the parameter $\sigma$ representing the coefficient of fluid viscosity and the buoyancy approximated by the term $\sigma \theta \hat{e}_{z}$. The buoyancy is proportional to $\theta$ because it is 
proportional to minus density fluctuation, which itself is proportional to minus the deviation of temperature. (The $\sigma$ in front of $\theta$ is there because of the particular scaling we have chosen for the temperature.) Eqn. (2) is the heat transport equation. $R$ is called Rayleigh number, which is just the scaled temperature gradient across the fluid layer. Suppose you sit still inside the fluid and there is an upward flow with velocity $u_{z}$. Then after a short time interval $\delta t$ the fluid element that was at a distance $u_{z} \delta t$ beneath you will have reached where you are. That fluid element is hotter than the fluid it displaces by an amount $R u_{z} \delta t$, because the former is closer to the bottom than the latter. This means the temperature at the point you are sitting at must increase by the same amount. This is the reason why $R u_{z}$ is there in Eqn. (2). Eqn. (3) is the mathematical statement of conservation of mass when, to the lowest order, the fluid densitity is assumed constant in the bulk.

Although it is generally true that linear equations are easier to solve than nonlinear equations, that does not necessarily mean that we will know how to solve linear problems in the first place. The convection problem we are dealing with is just one example. It is at this point that Lord Rayleigh came in and saved us. This is basically what he said: "Look, if you don't know how to solve this set of horrible PDE's, why don't you just replace all the $\nabla^{2}$ by some number $-\mu$, and reduce the whole problem to solving algebraic equations only?" If you think about it, this is quite a reduction in labor, because it is equivalent to solving the PDE's without actually solving them! Of course, replacing the Laplacian by a scalar simply means that you are assuming that all the variables in this problem are linear functions of $\varphi, \frac{\partial \varphi}{\partial x}, \frac{\partial \varphi}{\partial y}, \ldots$, etc., where $\varphi$ is an eigenfunction of the Laplacian $-\nabla^{2}$ with the corresponding eigenvalue $\mu$. This is fine except for one difficulty, namely, the boundary conditions suitable for the original problem in general can not be satisfied when you do this substitution! Just how we can retain Rayleigh's idea while making the realistic boundary conditions satisfied will constitute a major part of this lecture later. For now let's just pretend that everything is all right, so that loosely speaking you know the solution for the convection problem, e.g. the temperature $\theta$, is proportional to $\varphi$. Now increase $R$ so that the system becomes unstable. The most unstable mode, which is necessarily an eigenfunction $\varphi$ of the Laplacian, will grow exponentially. An immediate consequence of this analysis is that the system will pick up this configuration, and the dark bands (positive $\theta$ ) are separated from the bright bands (negative $\theta$ ) by the nodal lines of $\theta$. In other words, the pattern you see in a convection cell corresponds nicely to the nodal lines of the Laplacian eigenfunction $\varphi$ ! (Question: You probably are aware that the nodal lines of a Laplacian eigenfunction usually are orthogonal to each other. Does this have anything to do with the experimental observation that a convective roll tends to end perpendicular to the sidewall of a convection cell?)

Fine. But how about the zebra? The idea goes back to Turing. According to him, it is the reacting and diffusing chemicals inside a developing embryo that will cause an instability which then triggers the laying down of coat patterns we see on some animals. For example, it might be that the function of (at least) one of the chemicals is to activate a certain gene to cause the sedimentation of color pigments when its concentration exceeds certain threshold. The exact details of how this all works out doesn't concern us here. The important idea 
is that if we can map out the concentration distribution of that particular chemical and determine where the concentration exceeds that threshold, then we probably can tell where we expect to see a spot (as in leopard) or a stripe (as in zebra) to appear on the animal's skin. So here goes Turing's simple model. Suppose all the chemicals originally are in a uniform equilibrium state. Is this state stable against small perturbations? If not, then the chemical concentrations will grow exponentially (in the linear regime), and eventually that special chemical will exceed the threshold and cause the generation of spatial pattern. In the language of mathematics, then, we will have to solve

$$
\frac{\partial \psi}{\partial t}=\hat{D} \nabla^{2} \psi+\hat{A} \psi
$$

where $\psi$ is an $m \times 1$ column vector representing the deviations of the concentration of $m$ chemicals from equilibrium, $\hat{D}$ is a diagonal matrix with diffusion constants of the chemicals as its matrix elements, and $\hat{A}$ is an $m \times m$ matrix that characterizes the linearized reactions among these chemicals. To solve this equation, Turing said: "Replace $\nabla^{2}$ by $-\mu$, and that's it!" So you see, this is just variation on the same theme: Laplace is the name, replace is the game! Fortunate for us that this time we don't really have to worry about the boundary conditions, because the most natural boundary condition in this case is the no-flux condition $\left(\frac{\partial \psi}{\partial n}=0 ; \mathbf{n} \equiv\right.$ outward normal), which is automatically satisfied if, in the substitution process, we require the Laplacian eigenfunction $\varphi$ to satisfy the Neumann boundary condition $\frac{\partial \varphi}{\partial n}=0$. Specifically, we can assume that it's possible to find an $m \times 1$ constant column vector $\bar{u}$ so that

$$
\psi=\varphi \bar{u} .
$$

(This means all spatial variation is carried by $\varphi$ alone.) Substitute this into Eqn. (4), then the requirement that a nontrivial solution to the resulting matrix equation exists will give us the following dispersion relation for the growth rate $\lambda$

$$
\operatorname{det}(-\mu \hat{D}+\hat{A}-\lambda)=0 \text {. }
$$

If the two matrices $\hat{A}$ and $\hat{D}$ are suitably chosen, then $\lambda(\mu)$ will always have a negative real part unless $\mu$ equals some number $\mu_{c}$, in which case the system becomes marginally stable, i.e., $\operatorname{Re} \lambda\left(\mu_{c}\right)=0$. Plot the real part of $\lambda$ as a function of $\mu$, and you will get a picture that looks like Fig. 2.

But you also know that the Laplacian eigenvalue $\mu$ scales with system size $L$ like $L^{-2}$. For instance, in a one-dimensional problem $\mu$ will be something like $(j \pi / L)^{2}$, where $j$ is an integer. Therefore, you get a curve of $\operatorname{Re}(\lambda)$ versus $L$ that goes up and touches the $L$-axis at $L_{1}=\pi /\left(\mu_{c}\right)^{1 / 2}$, and then comes down again for larger $L$, if you choose $j$ to be unity. Similarly, the $j=2$ curve will touch the $L$-axis at $L_{2}=2 \pi /\left(\mu_{c}\right)^{1 / 2}, \ldots$ etc.. Now superpose all the curves $j=1,2,3, \ldots$, and you obtain Fig. 3 . 


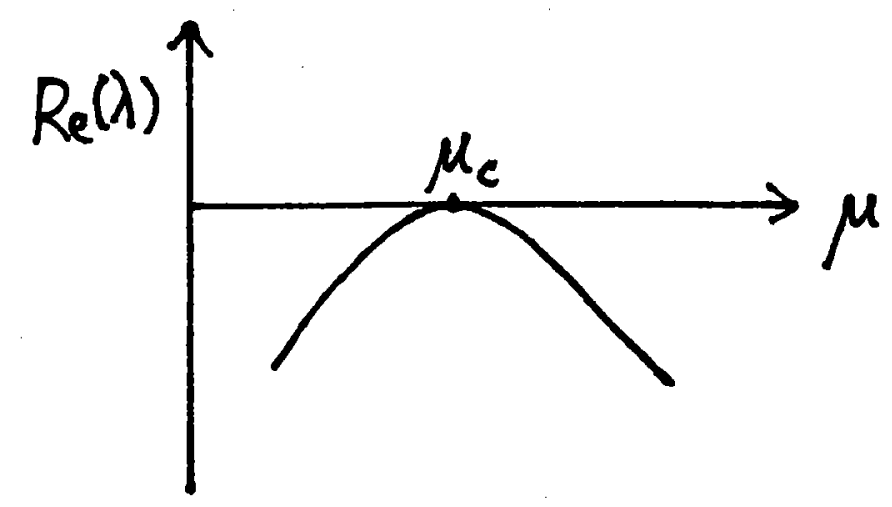

Figure 10.2.

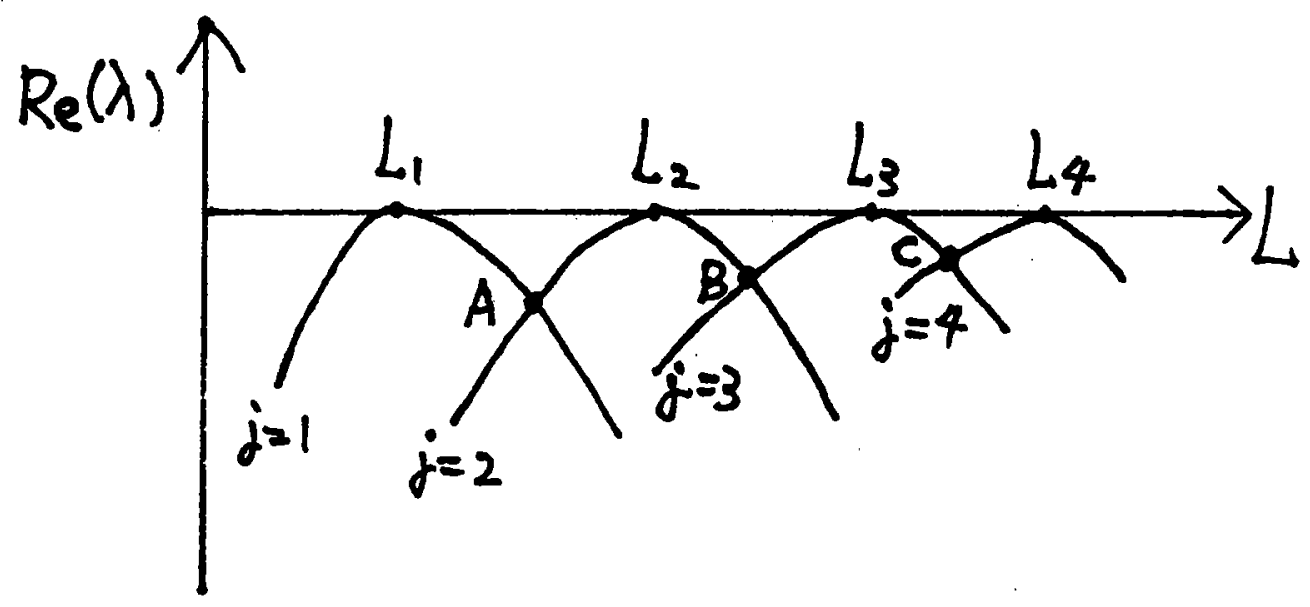

Figure 10.3.

The implication of this figure is quite interesting: if everything is right, then a developing embryo which gradually grows in size $L$ will exhibit the pattern associated with the $j=1$ mode when $L=L_{1}$, because the "cell" becomes unstable at this size, then due to the regain of stability the pattern disappears upon further growth until $L$ reaches $L_{2}$, in which case it will pick up the pattern associated with the $j=2$ mode,...,etc.. In short, the pattern acquired by the embryo through this mechanism depends on when the actual pattern is laid down! Hence, the different patterns you observe in animals can be explained by one and the same mechanism. And, again, you see that the pattern actually corresponds nicely to the nodal lines of a Laplacian eigenfunction! This is the basic reason why a physicist might tell you that "Rayleigh-Bénard $\approx$ zebra-leopard," an equality that most likely will be ridiculed by all people less naive than that physicist! As attractive as this biological pattern formation model might sound, it would be irresponsible of me if I didn't tell you that there still is no good experimental evidence indicating that this is the right mechanism. Because of the speculative nature of this model, and because this is a summer school on patterns in fluid dynamics rather than biological systems, I will leave this subject here and refer you to 
the book by Murray and the ample references cited therein. The reason I chose to introduce this topic here is to show you that very different objects might have the same origin in some context, and sometimes we do benefit from studying a seemingly unrelated problem. As a matter of fact, in the following we shall borrow the idea learned from this simple biological model and apply it to the Rayleigh-Bénard convection, which is more relevant to the theme of this summer school.

You can easily check that Rayleigh's ansatz for the convection problem is the same as assuming that the solution can be written as

$$
\begin{aligned}
\psi \equiv\left(\begin{array}{c}
u_{x} \\
u_{y} \\
u_{z} \\
\theta
\end{array}\right) & =\left(\begin{array}{c}
\bar{u} \frac{\partial \varphi}{\partial x} \\
\bar{u} \frac{\partial \varphi}{\partial y} \\
\bar{u}_{z} \frac{\partial \varphi}{\partial z} \\
\bar{\theta} \frac{\partial \varphi}{\partial z}
\end{array}\right), \\
p & =\bar{p} \varphi
\end{aligned}
$$

where $\bar{u}, \bar{u}_{z}, \bar{\theta}$ and $\bar{p}$ are some constants, and $\varphi$ is the same Laplacian eigenfunction we encountered in Turing's model. This solution is ok for a cell which satisfies the following boundary conditions: 1) the two horizontal plates are kept at a fixed temperature $(\theta=0)$, 2 ) the sidewall is perfectly insulating $\left.\left(\frac{\partial \theta}{\partial n}=0\right), 3\right)$ the normal velocity at the sidewall is zero, and 4) all the boundaries are "rotation-free," i.e.,

$$
\hat{e}_{\|} \cdot\left(\frac{\partial \mathbf{u}}{\partial n}+(\mathbf{u} \cdot \nabla) \hat{n}\right)=0
$$

where $\hat{e}_{\|}$is any vector parallel to the boundary. (This boundary condition guarantees that the vertical vorticity at the sidewall is always zero, and is different from the familiar stressfree condition, because the second term, which involves the curvature of the boundary, has the wrong sign.*) It is the last one that is causing the trouble - this is not a physically realizable boundary condition. A real fluid should satisfy the no-slip condition $(\mathbf{u}=0)$ instead. Besides, in a real experiment one might also want to learn how the sidewall thermal property affects the system, in which case condition 2) will not suffice. Before we tackle this difficulty, I should remind you that, just like in Turing's model, this ansatz does allow us to write down the dispersion relation in a closed form like Eqn. (6) quite easily, and if the Rayleigh number $R$ is tuned just right, i.e., if $R$ equals the critical Rayleigh number for an infinite cell $R_{C \infty}$, then the growth rates $\lambda$ as functions of cell size $L$ will look very muck like what is shown in Fig. 3. The importance of this simple picture cannot be overemphasized, because later we will come back to this figure and derive some general property from it.

Now we get to the fun part of the whole business: how do you make out something from the unrealistic solution we got before? The idea won't be foreign to you - by perturbation, of course! At first sight this is hardly encouraging at all since we all know that perturbation

* I am indebted to Bill Young for bringing this point to my attention. 
calculation sometimes can get so nasty that only poor students who have to earn their degree would do it. Call ourselves lucky! We don't have to work out that kind of perturbation to extract the information we want! Indeed, we won't even expand any messy Taylor series when we do the perturbation. The secret lies in the fact that this system has a very nice variational characterization whose restricted version was discovered by the early pioneers Pelew \& Southwell and Sorokin, and had been used extensively by others to compute the critical Rayleigh number accurately. Here, we will go one step further and 1) make use of the variational principle to actually facilitate the analysis of the effects of the sidewall on the stability, and 2) generalize the variational principle so that it applies to a convection cell with a real sidewall.

To begin with, we will rescale $\theta$ by $\theta \rightarrow \sqrt{\frac{R}{\theta}} \theta$ so that Eqns. (1) and (2) become

$$
\begin{aligned}
\mathcal{L} \psi & =\frac{\partial \psi}{\partial t}=\lambda \psi \\
\mathcal{L} \psi & \equiv\left\{\left(\begin{array}{cccc}
\sigma & 0 & 0 & 0 \\
0 & \sigma & 0 & 0 \\
0 & 0 & \sigma & 0 \\
0 & 0 & 0 & 1
\end{array}\right) \nabla^{2}+\left(\begin{array}{cccc}
0 & 0 & 0 & 0 \\
0 & 0 & 0 & 0 \\
0 & 0 & 0 & \sqrt{\sigma R} \\
0 & 0 & \sqrt{\sigma R} & 0
\end{array}\right)\right\} \psi-\left(\begin{array}{c}
\frac{\partial p}{\partial x} \\
\frac{\partial p}{\partial y} \\
\frac{\partial p}{\partial z} \\
0
\end{array}\right) \\
& \equiv\left\{\hat{D} \nabla^{2}+\hat{A}\right\} \psi-\left(\begin{array}{c}
\nabla p \\
0
\end{array}\right),
\end{aligned}
$$

where $\hat{D}$ and $\hat{A}$ are $4 \times 4$ matrices defined in an obvious way, $\lambda$ is the eigenvalue of this problem, and

$$
\psi \equiv\left(\begin{array}{l}
\mathbf{u} \\
\theta
\end{array}\right)
$$

If we define inner product between $\psi$ and $\psi^{\prime}$ by

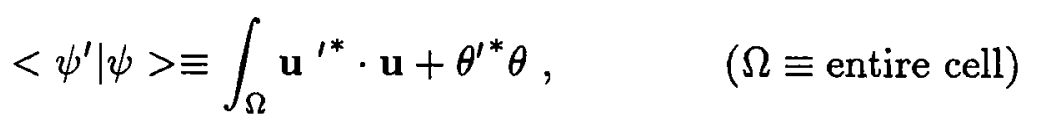

where ${ }^{*}$ indicates complex conjugation, then it is easy to show that $\mathcal{L}$ is a self-adjoint operator whose eigenvalues are real-valued, provided the boundary conditions for $\mathbf{u}$ and $\theta$ are homogeneous in the following sense

$$
\begin{gathered}
u_{n} \equiv \mathbf{u} \cdot \hat{n}=0 \\
\hat{e}_{\|} \cdot\left(\frac{\partial \mathbf{u}}{\partial n}+(\mathbf{u} \cdot \nabla) \hat{n}+\alpha \mathbf{u}\right) \equiv \hat{e}_{\|} \cdot\left(\frac{\partial \mathbf{u}}{\partial n}+\hat{\alpha} \cdot \mathbf{u}\right)=0 \\
\frac{\partial \theta}{\partial n}+\beta \theta=0 .
\end{gathered}
$$

In the above, $\alpha$ and $\beta$ are scalars that can vary on the boundary $\partial \Omega$, though throughout this lecture we shall assume that, for the sake of illustration, they are constants on each of the two horizontal plates and the sidewalls. I would like to remind you that you shouldn't 
be bothered by the artificial boundary condition expressed in Eqn. (13), because it is just a trick we shall use in the analysis to extract information on the real problem in which the velocity is required to vanish $(\alpha \rightarrow \infty ; \mathbf{u} \rightarrow 0)$ on the sidewall. (However, Eqn. (13) still has an interesting physical interpretation when $\alpha=0$. It means that the vertical vorticity near the sidewall actually vanishes. Because of this property, we will call this special boundary condition the "rotation-free" condition from now on.) But the same is not true for Eqn. (14). It turns out that $\beta$ is an effective thermal conductivity of the sidewall. This is especially true when the sidewall is very thin. But we won't say another thing about this thermal boundary condition until the very end of this lecture. You should also remember that in a real experiment people usually control the temperatures on the two horizontal plates so that they are always fixed at a prescribed value. This means the boundary condition for the temperature deviation $\theta$ on both plates is $\theta=0$. I will call a convection cell which satisfies the kind of boundary conditions specified by Eqns. (12)-(14) a "surrogate system."

Lest you become lost later in the lecture, let me tell you in advance what is awaiting you next. We will try to understand how the growth rate $\lambda$ or the critical Rayleigh number $R_{C}$ is affected by the tuning parameters $\alpha$ and $\beta$. Once we get a hold of its behavior we can simply let $\alpha \rightarrow \infty$ so that the no-slip condition is recovered. What this amounts to is that indeed we can "solve" the real problem with this approach. We can also let $\beta \rightarrow \infty$ if we are interested in the case with a perfectly conducting sidewall. In order to make the demonstration more transparent, however, we will only work with the perturbation on $\beta$ from now on, though it should be borne in mind that whenever something works for $\beta$ then quite often it will work for $\alpha$ as well. Now we are ready for the first variational principle.

\section{Variational Principle I}

Define a variational functional

$$
I[\psi] \equiv \frac{-\left(\int_{\Omega} \sigma|\nabla \mathbf{u}|^{2}+|\nabla \theta|^{2}\right)+2 \sqrt{\sigma R} \int_{\Omega} u_{z} \theta-\left(\int_{\partial \Omega} \sigma \mathbf{u} \cdot \widehat{\alpha} \cdot \mathbf{u}+\beta|\theta|^{2}\right)}{\int_{\Omega}|\mathbf{u}|^{2}+|\theta|^{2}},
$$

where $\psi$ must satisfy $\nabla \cdot \mathbf{u}=0$ in the convection cell $\Omega$ and $u_{n}=0$ on the boundary $\partial \Omega$. Then $I[\psi]$ takes a stationary value if and only if 1 ) on the boundary $\partial \Omega \mathbf{u}$ and $\theta$ satisfy both Eqns. (13) and (14), and 2) that

$$
\mathcal{L} \psi=\lambda \psi
$$

for some constant $\lambda$. In other words, $\psi$ is an eigenfunction of $\mathcal{L}$ with Eqns. (13) and (14) as the boundary conditions, and the eigenvalue $\lambda$ is given precisely by $I[\psi]$.

In fact, $I[\psi]$ has an infinite number of eigenvalues $\lambda_{1} \geq \lambda_{2} \geq \lambda_{3}, \ldots$, and $\lambda_{1}$ is characterized by

$$
\lambda_{1}=\max _{\psi} I[\psi] .
$$

This is very useful because it tells us how to characterize the critical Rayleigh number $R_{C}$ (三 the lowest $R$ that makes this system unstable) as well. This can be seen as follows. Suppose 
you adjust your $R$ in the experiment so that $R=R_{C}$, then by definition the most unstable mode $\lambda_{1}$ must vanish (so that you get a marginally stable state). Then by Eqns. (15) and (17) you immediately get

\section{Variational Principle II}

$$
\frac{1}{\sqrt{R_{C}}}=\max _{\psi} \frac{2 \int_{\Omega} u_{z} \theta}{\int_{\Omega}|\nabla \mathbf{u}|^{2}+|\nabla \theta|^{2}+\int_{\partial \Omega} \mathbf{u} \cdot \widehat{\alpha} \cdot \mathbf{u}+\beta|\theta|^{2}} \equiv \max _{\psi} J[\psi],
$$

where we have absorbed $\sqrt{\sigma}$ into $\mathbf{u}$.

You can do a lot of wonderful things with the variational principles. For example, you can easily prove that increasing the sidewall conductivity from $\beta$ to a higher value $\beta^{\prime}$ always increases the stability, a result that seems intuitively trivial because higher sidewall conductivity means it is able to dissipate heat faster, thus stablizing the system. The actual proof goes like this. Let $\psi_{\beta}$ be the eigenfunction corresponding to the first eigenvalue when the thermal boundary is characterized by $\beta$. We will also attach a subscript $\beta$ to $I[\psi]$ to remind ourselves of the explicit dependence of $I[\psi]$ on $\beta$. Then

$$
\begin{aligned}
\lambda_{1}\left(\beta^{\prime}\right) & \equiv I_{\beta^{\prime}}\left[\psi_{\beta^{\prime}}\right]=I_{\beta}\left[\psi_{\beta^{\prime}}\right]-\left(I_{\beta}\left[\psi_{\beta^{\prime}}\right]-I_{\beta^{\prime}}\left[\psi_{\beta^{\prime}}\right]\right) \\
& =I_{\beta}\left[\psi_{\beta^{\prime}}\right]-\left(\beta^{\prime}-\beta\right) \frac{\int_{\partial \Omega}|\theta|^{2}}{\int_{\Omega}|\mathbf{u}|^{2}+|\theta|^{2}} \\
& \leq \max _{\psi} I_{\beta}[\psi]-\left(\beta^{\prime}-\beta\right) \frac{\int_{\partial \Omega}|\theta|^{2}}{\int_{\Omega}|\mathbf{u}|^{2}+|\theta|^{2}} \\
& \equiv \lambda_{1}(\beta)-\left(\beta^{\prime}-\beta\right) \frac{\int_{\partial \Omega}|\theta|^{2}}{\int_{\Omega}|\mathbf{u}|^{2}+|\theta|^{2}}
\end{aligned}
$$

Hence $\lambda_{1}\left(\beta^{\prime}\right) \leq \lambda_{1}(\beta)$. See? The proof is almost trivial if you use this approach. In fact, you can easily convince yourself that in the case when $\lambda_{1}$ is not degenerate you can even conclude that

$$
\frac{\partial \lambda_{1}}{\partial \beta}=-\frac{\int_{\partial \Omega}|\theta|^{2}}{\int_{\Omega}|\mathbf{u}|^{2}+|\theta|^{2}} \leq 0 .
$$

With a little bit more work, it is possible to prove that the results we derived above actually hold for all $\lambda_{j}(j=1,2,3, \ldots)$. And it goes without saying that you get a similar answer when you tune $\alpha$.

Ok, so I hope I have convinced you that the eigenvalues must increase when you lower the value of $\beta$. But if you think about it, something funny is happening here! Why? Well, let's suppose that you decrease $\beta$ from $+\infty$ to $-\infty$. You know that if you just follow a particular eigenvalue, then it will become bigger and bigger as you proceed, because I just showed you that it must be so. Yet physically you know the two cases $\pm \infty$ must correspond to the same problem in which the boundary condition is $\theta=0$ (Cf: Eqn. (14)). This means that after the whole process is over, you have actually come back to the system you started with. So 
why is it that our eigenvalues don't come back at all? The answer is: an eigenvalue will displace another one that was previously above it, while the top one(s) will go to infinity at the end of this cyclic process. This phenomenon is shown in Fig. 4, and is related to the so-called "Berry's phase" in quantum mechanics. (See the book by Shapere and Wilczek if you are interested in this subject.) You can easily see why at least one eigenvalue must "run away" to infinity when $\beta \rightarrow-\infty$. All you have to do is take any trial function whose $\theta$ is not identically zero on $\partial \Omega$ and substitute it into Eqn. (15). Clearly, $I[\psi]$ must be very large when $\beta$ is very negative. So from $\lambda_{1} \geq I[\psi]$ we conclude that at least one eigenvalue $\left(\lambda_{1}\right)$ blows up if $\beta \rightarrow-\infty$.

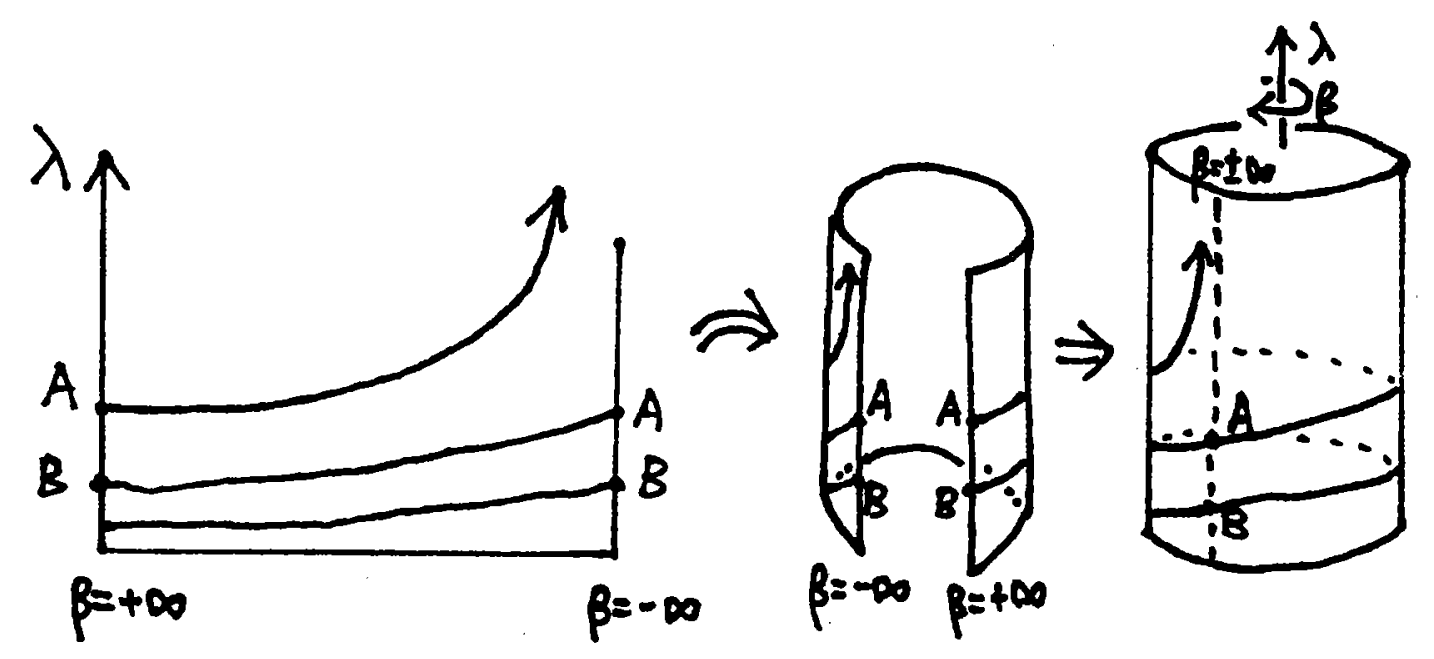

Figure 10.4. The identification of $\beta= \pm \infty$ in the figure on the left is made easy by rolling it up to form the cylinder on the right.

It gets even more interesting when degeneracy happens while only one eigenvalue runs away. Let's suppose that the two most unstable states $\lambda_{1}$ and $\lambda_{2}$ become degenerate at some $\beta_{0}$, and denote this degenerate eigenvalue by $\lambda_{0}$. This time I want to convince you that one of the $\lambda_{0}$ 's will simply get stuck at this value and is reluctant to change at all if you tune $\beta$. The reasoning goes like this. In Fig. 5 we show the two states as functions of $\beta$. Notice that the second unstable state at $\beta=+\infty$ becomes the most unstable state when $\beta$ is decreased beyond the degenerate point $\beta_{0}$, and it simply disappears at infinity when $\beta \rightarrow \infty$. So curve 1 actually represents the most unstable state when $\beta=-\infty$. But we also know we should identify the most unstable states at $\beta= \pm \infty$. This leaves us with only one possibility: curve 1 is level all over the range of $\beta$, because I showed you before that the curve is a nonincreasing function of $\beta$. In other words, this eigenvalue does not change with $\beta$. 


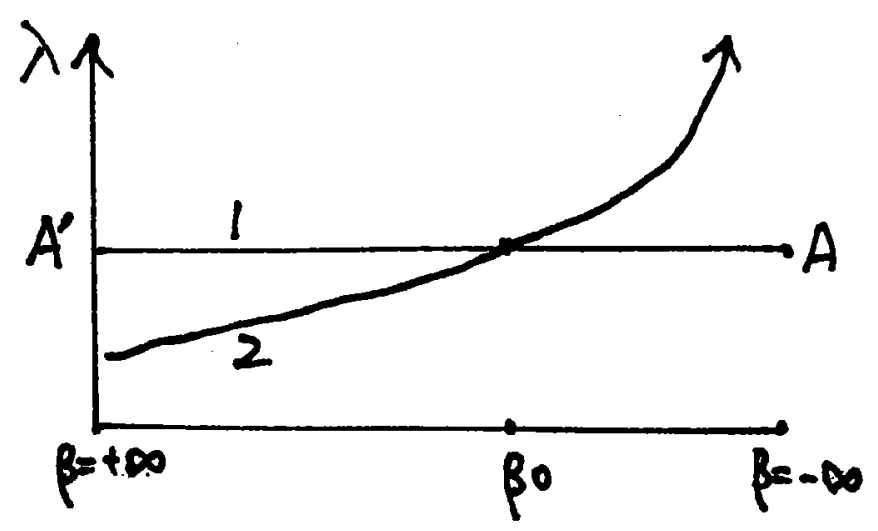

Figure 10.5. The monoticity of $\lambda(\beta)$ implies curve 1 is actually flat because $A^{\prime}$ and $A$ must be identified.

At this point you might think that this whole thing is only of academic interest and is not related to our understanding of the finite-size effect on the convective instability. Sorry, it is. Let's consider a cylindrical cell and control the Rayleigh number $R$ so that $R=R_{C \infty} \equiv$ critical Rayleigh number for an infinite cell. Because of the rotational symmetry, the solutions of this problem can be classified according to their azimuthal quantum number $m$, i.e., the angular dependence of the solutions in each class is of form $e^{i m \phi}$, where $m$ is an integer. If you fix $m$, then the eigenvalues for this class of solutions look just like what's shown in Fig. 3 , and the intersections $A, B, C, \ldots$ can be analytically calculated if one so desires. Now apply the previous argument to these intersections. The conclusion? Again, we get those hard-line eigenvalues who simply refuse to change at all! Because we are talking physics here, not the fashionable politics, this turns out to be a happy situation for us. Indeed, with the existence of these fixed points, we are able to show that the growth rate $\lambda$ is inversely proportional to the system size $L: \lambda \propto-L^{-2}$, because theses fixed points have the same scaling property. And the reason why the fixed points obey this scaling law is very simple, too. To see this, we observe that, in Fig. 3 , near $\lambda=0$ the $j$-th curve can be approximated by a parabola:

$$
\lambda \approx \frac{1}{2} \frac{\partial^{2} \lambda}{\partial \mu_{c}^{2}}\left(\frac{k_{j}^{2}}{L^{2}}-\mu_{c}\right)^{2},
$$

where $k_{j}^{2} / L^{2}$ is nothing but the $j$-th eigenvalue of the two-dimensional Laplacian $-\nabla_{h}^{2}$ on the horizontal cross-section of the cell subject to the Neumann boundary condition, so that the intersection size $L_{i n t}$ of the $j$-th and the $j+1$-th curves can be solved from

$$
\left(\frac{k_{j}^{2}}{L_{i n t}^{2}}-\mu_{c}\right)^{2}=\left(\frac{k_{j+1}^{2}}{L_{i n t}^{2}}-\mu_{c}\right)^{2} \text {. }
$$

After trivial algebra we get

$$
\lambda \approx \frac{1}{2} \frac{\partial^{2} \lambda}{\partial \mu_{c}^{2}} \mu_{c}^{2}\left(\frac{k_{j+1}^{2}-k_{j}^{2}}{k_{j+1}^{2}+k_{j}^{2}}\right)^{2} \approx \frac{1}{2} \frac{\partial^{2} \lambda}{\partial \mu_{c}^{2}} \frac{\left(k_{j+1}-k_{j}\right)^{2}}{L_{i n t}^{2}},
$$


which clearly shows that it is inversely proportional to $L_{i n t}^{2}$ since $k_{j+1}-k_{j} \approx \pi$ for large enough $j .{ }^{*}$ But if these fixed points scale like $L^{-2}$, then there is no reason why the resulting $\lambda(L)$ curve doesn't scale as such when you turn on the perturbation of $\beta$, because, after all, it always contains these fixed points. (Fig. 6) So you get an interesting quantitative prediction without really doing much calculation at all! Déjà vu, right? Certainly, a more ambitious claim will be that $\lambda(L)$ actually scales like $L^{-2}$ for almost all nonnegative $\alpha$ and $\beta$ ! But to show this would require a little bit more algebra, which is not our main concern here since you already got the idea. Because the principal lectures of this summer school are on the amplitude equation, I think you may be happy to learn that this scaling law can be derived via the amplitude equation, too! For a derivation based on this approach, see the papers by Ahletrs et al. and by Cross et al..
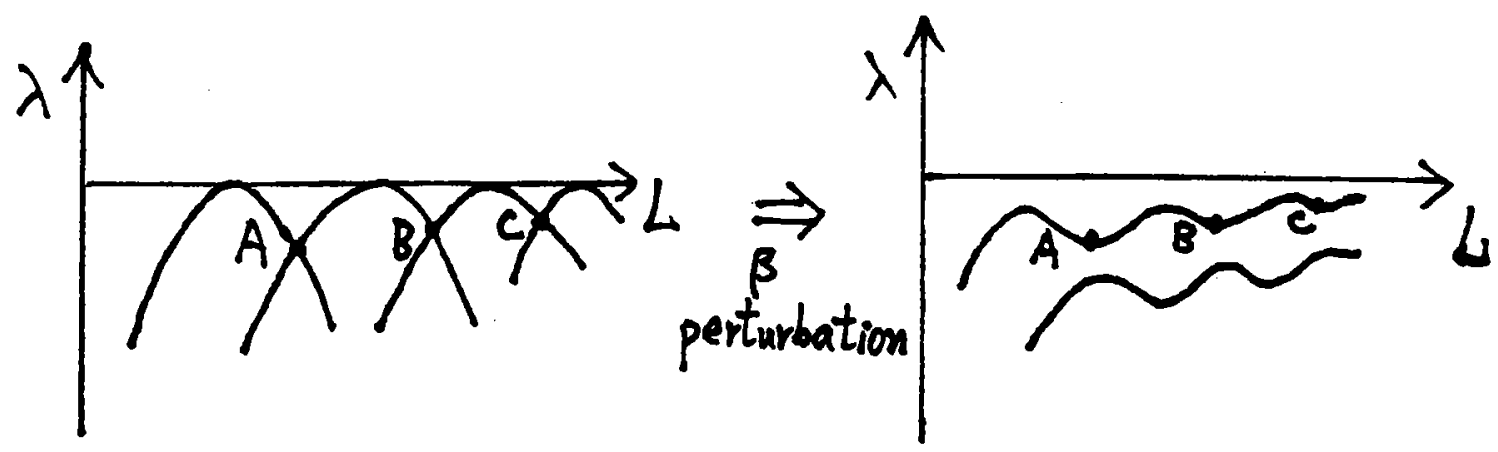

Figure 10.6. The points $A, B, C, \ldots$ are not perturbed by $\beta$.

What about convection cells of other shapes? Can we still derive a similar scaling law without sweating, just like before? The answer is yes. For our purpose, I will use the variational principle to prove it again, though you should be aware that it is possible to get a sharper result if you actually carry out a perturbation calculation. (You can get more if you are willing to sweat a little bit, that is.) Let $\Omega$ be a cell which contains $\Omega^{\prime}$. Suppose their sidewalls are both rigid and conducting $(\mathbf{u}=\theta=0)$. Assume $\psi^{\prime}$ is the ground state solution for $\Omega^{\prime}$, then we can define a trial function $\psi$ for $\Omega$ by taking $\psi \equiv \psi^{\prime}$ in their common domain $\Omega^{\prime}$, and $\psi \equiv 0$ otherwise. Then by Variational Principle I we have

$$
\lambda_{1}^{\prime} \equiv I\left[\psi^{\prime}\right]=I[\psi] \leq \lambda_{1},
$$

i.e., the larger cell $\Omega$ is not more stable than the smaller cell $\Omega^{\prime}$. Now if you are interested in the scaling law for a large cell $\Omega$ whose geometry is not easy to analyze, all you have

* For a fixed $m$, the Laplacian $\nabla_{h}^{2}$ is approximately equal to $d^{2} / d r^{2}$ at a large radius $r \approx L$. So long as $r$ doesn't change much in the region we are considering, we can conclude that its $j$-th eigenfunction is proportional to $\cos \left(\frac{k_{j} r}{L}+c\right)$ for some constant $c$. Imposing the Neumann boundary condition at $r=L$ immediately gives $k_{j}=j \pi+c^{\prime}$ for some constant $c^{\prime}$. This proves $k_{j+1}-k_{j} \approx \pi$. 
to do is sandwich it by two large concentric cylindrical cells $\Omega_{1}$ and $\Omega_{2}$ in such a way that $\Omega_{1} \subset \Omega \subset \Omega_{2}$. Then our prediction of the $L^{-2}$ behavior for $\Omega_{1}$ and $\Omega_{2}$ and the theorem expressed by Eqn. (23) dictate that $\Omega$ itself must satisfy the same scaling property. Once more, to show that this is true for other boundary conditions will require a little bit standard perturbation theory, which we choose not to go into.

Having spent so much time on the surrogate cell, it is only fair if we say something about the real system, such as how the thermal condition Eqn. (14) is justified. More generally, how do we include the real sidewall in our analysis? The answer actually is very simple. All we need to do is append the temperature variable $\theta_{w}$ inside the wall to the abstract vector $\psi$ to form a "bigger" vector $\Psi$ :

$$
\Psi=\left(\begin{array}{c}
\psi \\
\theta_{w}
\end{array}\right)
$$

This done, you can easily show that, for instance, the critical Rayleigh number $R_{C}$ is associated with the following variational principle.

\section{Variational Principle II'}

$$
\frac{1}{\sqrt{R_{C}}}=\max _{\Psi} \frac{2 \int_{\Omega} u_{z} \theta}{\int_{\Omega}|\nabla \mathbf{u}|^{2}+|\nabla \theta|^{2}+\int_{\partial \Omega} \mathbf{u} \cdot \widehat{\alpha} \cdot \mathbf{u}+\int_{\Omega_{w}} r \kappa_{w}\left|\nabla \theta_{w}\right|^{2}} \equiv J^{\prime}[\Psi],
$$

where $\Omega_{w}$ signifies the (arbitrarily shaped) wall region, and $r$ and $\kappa_{w}$ denote the ratio of the sidewall specific heat to that of the fluid and the sidewall thermal diffusivity, respectively.

If you compare Eqn. (18) with Eqn. (25), then it is clear that somehow $r \kappa_{w}$ term in the latter is analogous to the $\beta$ boundary term in the former. Because of this similarity, you could have guessed that $\beta$ must really have captured the physics characteristic of the sidewall thermal property. And, not surprisingly, you can copy the proof we gave before for $\beta$ and show that increasing the sidewall conductivity indeed raises the stability of the system, i.e., $R_{C}$ is an increasing function of $\kappa_{w}$. To add more to your enjoyment, you can prove with equal ease that increasing the thickness of the sidewall also makes the system more stable.

So, the only question left is: just exactly how does $\beta$ represent $\kappa_{w}$ ? To answer this question in a satisfactory way will take us beyond the happy and unsophisticated ending I had in mind for this already long dragging story. So I'll simply give you a straight answer: when the sidewall is arbitrarily shaped, it is possible to find two constants $c_{1}$ and $c_{2}$ and define $\beta_{j} \equiv c_{j} r \kappa_{w}(j=1,2)$ such that $R_{C}^{\text {surrogate }}\left(\beta_{1}\right) \leq R_{C}^{\text {real system }}\left(\kappa_{w}\right) \leq R_{C}^{\text {surrogate }}\left(\beta_{2}\right)$. This inequality simply says that, roughly speaking, the stability of the real system is always in between that of two surrogate systems. Therefore it is quite all right to study the stability of a surrogate system alone when you discover that solving the real system is very horrifying, if not impossible. As you can see, this inequality is quite in line with the basic philosophy I 
am conveying throughout the whole lecture - sometimes you really can avoid dealing with the real thing by cheating!

Acknowledgment. Besides letting me get away easy with the office doodling, Prof. Cross also allowed me the luxury of complete freedom in exploring all the wild things as well as stealing his insights in physics. This work is but one of those things that would be impossible without his guidance.

For elementary discussions on the variational principles in the convection problem, see Chandrasekhar and Joseph. My two papers listed below contain generalizations and more technical details that are only briefly touched upon in this lecture. They should be attempted after dinner only if indigestion is not a health concern.

\section{References}

Ahlers, G., Cross, M.C., Hohenberg, P.C. and Safran, S. 1981 J. Fluid Mech. 110, 297.

Chandrasekhar, S. 1961 Hydrodynamic and Hydromagnetic Stability, pp.27ff, Oxford Univ. Press.

Chen, Yih-Yuh 1991a, submitted to J. Fluid Mech.

Chen, Yih-Yuh 1991b, submitted to Phys. Rev. A.

Cross, M.C., Daniels, P.G., Hohenberg, P.C. and Siggia, E.D. 1983 J. Fluid Mech. 127, 155.

Joseph, D.D. 1976 Stability of Fluid Motions, Vols. I \& II, Springer-Verlag.

Murray, J.D. 1989 Mathematical Biology, Chapters 14 and 15, Springer-Verlag.

Pellew, A. and Southwell, R.V. 1940 Proc. Roy. Soc. (London) A, 176, 312.

Lord Rayleigh 1916 Phil. Mag. (6) 32, 529.

Shapere, A. and Wilczek, F. (ed.) 1989 Geometric Phases in Physics, World Scientific.

Sorokin, V.S. 1953 Prikl. Mat. Mekh. 17, 39.

Turing, A.M. 1952 Phil. Trans. Roy. Soc. London B237, 37. 


\section{The Phase-Diffusion Mean Drift Equations for Nat- ural Convection Patterns}

T. Passot

Department of Mathematics, University of Arizona, Tucson AZ, 85721

\subsection{Introduction}

Many interesting behaviors of pattern-forming systems described by non-linear partial differential equations can be captured by standard perturbative techniques when the stress parameter is small and/or there exists a spectral gap between the microscopic and the macroscopic scales. We are mainly interested here in the latter case, and more particularly in the case of Rayleigh-Bénard convection where only rolls are formed.

A physical system close to a bifurcation can be described by a low-dimensional dynamics if the number of modes whose eigenvalue cross the imaginary axis at the bifurcation threshold (the order parameters) is small. Although these order parameters, which determine the macroscopic order of the system, still interact with the damped modes, the "slaving principle", (rigorously demonstrated for ODE's), states indeed that these decaying modes can be eliminated and explicitly expressed by the order parameters. In the class of systems leading to pattern formation, this may be compromised due to the spatial extension of the system (an infinite number of modes are squeezed in the immediate vicinity of the critical point). When one direction can be privileged (either because the system is not rotationaly invariant as it is the case for liquid crystals, or because one chooses to look at a particular direction only), it is possible to derive amplitude equations (such as the Ginzburg-Landau or the Newell-Whitehead-Segel equations (Newell and Whitehead, 1969 and Segel, 1969), which describe the behavior of the order parameters on a slow time scale. These equations contain slow spatial derivatives to account for the mode coupling in the bandwidth around each normal mode and thus are called envelope equations. They can be reduced to a generic form depending only on the type of bifurcation considered and the symmetries of the problem. Another approach can be adopted to treat the fully two-dimensional case (Bestehorn and Haken, 1990), where equations for the order parameters are derived which contain the fully spatio-temporal dynamics on a fast varying scale (such as in the Swift-Hohenberg equation (Swift and Hohenberg, 1977)).

When the stress parameter is well above its critical value leading to the onset of the spatial pattern under investigation, it is only possible to derive a large-scale description of the dynamics in terms of phase variations. The undisturbed pattern is indeed translationally invariant, and thus the phase mode is still a marginal mode. The amplitude of the nonlinear 
waves building the pattern is however always slaved to phase gradients, except possibly near defects. An exact derivation of the phase equation for rolls with no privileged direction has been achieved recently in the case of Rayleigh-Bénard convection by Cross and Newell (1984) and Newell, Passot and Souli (1990a, 1990b). The technique was already known in other fields: for modulated nondissipative nonlinear train waves as developed by Whitham (1974) or for modulated traveling waves in reaction-diffusion equations as exposed by Howard and Kopell (1977) (see also Kuramoto (1984)). In all these examples, use is made of the inverse aspect ratio $\epsilon$ as an expansion parameter, and not of the stress parameter as previously. This relies on the observation that almost everywhere in the convection pattern, a local wavevector $\mathbf{k}$ can be defined which changes slowly throughout the container. Therefore, ignoring the dependence in the vertical direction, the field variables can be represented by locally periodic functions $f\left(\theta ; R_{(i)}\right)$ where $f$ is $2 \pi$ periodic in $\theta$ and $R_{(i)}$ represents the collection of stress parameters of the system. The field variable $f$ varies over distances of the order of the roll size whereas the amplitude $A$ (the norm of $f$ e.g.) or the wavevector (the gradient of $\theta$ ) vary over distances of the size of the box. This fact allows for the definition of a large-scale phase $\Theta(X, Y, T)=\epsilon \theta$ where $X=\epsilon x, Y=\epsilon y$ and $T=\epsilon^{2} t$ are the scales of the long wavelength disturbances. Note also that in contrast to the case of the Newell-Whitehead-Segel equations, direction $Y$ is here scaled in the same way as direction $X$ since we want to preserve rotational invariance. To leading order, and when no mean flow effects are present, the phase obeys a universal quasi-linear diffusion equation

$$
\tau(k) \Theta_{T}+\nabla \cdot \mathbf{k} B(k)=0
$$

where $\tau(k)>0$ and $B(k)$ are calculable functions of the wavenumber $k=|\mathbf{k}|$. To this order of approximation, the amplitude $A$ of the rolls is slaved to the wavenumber $k$ through the relation

$$
A^{2}=\mu^{2}(k)
$$

Equation (1) generalizes the fixed orientation equation of Pomeau and Manneville (1979). At finite Prandtl numbers a large scale horizontal mean flow, generated by the curvature of the rolls, advects the phase contours. Its presence is due to the existence of another marginal mode, namely a large-scale varying pressure field. The next section is devoted to a derivation of these coupled phase-mean drift equations and Section 3 is concerned with some predictions made on the behavior of convective patterns using the previous equations. In particular it is shown that all previous theories are contained in the present formalism and that moreover some new instabilities can be analyzed, such as the one of circular target patterns.

The difficulty with equation (11.1) is that it is ill-posed for some values of the wavenumber, outside the nonlinear stability region. Section 4 is devoted to the definition of a regularizing scheme for that equation, illustrated on a microscopic pattern forming model, the SwiftHohenberg equation and Section 5 is the conclusion. 


\subsection{The phase diffusion-mean drift equations}

The starting point of the calculation is the determination of stable fully nonlinear straight parallel roll solutions of the following Oberbeck-Boussinesq equations:

$$
\begin{gathered}
\sigma\left(\partial_{t} \mathbf{u}+\mathbf{u} \cdot \nabla \mathbf{u}\right)=-\nabla p+T \hat{\mathbf{z}}+\nabla^{2} \mathbf{u} \\
\partial_{t} T+\mathbf{u} \nabla T=R w+\nabla^{2} T \\
\nabla \cdot \mathbf{u}=0
\end{gathered}
$$

where the parameter $R$ represents the Rayleigh number and $\sigma$ is the inverse Prandtl number. The temperature is $T$, the pressure $p$ and the velocity $\mathbf{u}$ has components $(u, v, w)$. Rigidrigid boundary conditions are considered which state that $\mathbf{u}=T=0$ at the top and bottom boundaries: $z= \pm \frac{1}{2}$. The existence and stability of a one parameter family of $2 \pi$ periodic solution $\mathbf{v}=\mathbf{f}(\theta, z ; k)$ (where $\mathbf{v}=(u, v, w, T, p)$ and $\theta=k x$ ) is assured by the previous work of Busse and Colleagues (see e.g. Busse $(1967,1978)$ and references therein); $f$ is calculated, using a Galerkin technique as in Busse (1967).

Now we must look for modulated solutions and solve the linear equations obtained after inserting in (11.3) - (11.5) the following expansions in powers of the inverse aspect ratio $\epsilon$ :

$$
\begin{aligned}
& \partial_{z} \rightarrow \partial_{z} \\
& \partial_{\mathbf{x}} \rightarrow \mathbf{k} \partial_{\theta}+\epsilon \nabla \mathbf{X} \\
& \partial_{t} \rightarrow \epsilon \Theta_{T} \partial_{\theta}+\epsilon^{2} \partial_{T} \\
& \mathbf{v}=\mathbf{v}_{\mathbf{0}}+\epsilon \mathbf{v}_{1}+\epsilon^{2} \mathbf{v}_{\mathbf{2}}+\cdots
\end{aligned}
$$

Cross and along the roll velocities are also introduced: $\tilde{u}=\hat{\mathbf{k}} \cdot \mathbf{u}$ and $\tilde{v}=(\hat{\mathbf{k}} \times \mathbf{u}) \cdot \hat{\mathbf{z}}$ where $\hat{\mathbf{k}}=\frac{\mathbf{k}}{k}$. We end up at order $\epsilon$ and $\epsilon^{2}$, with linear systems of the form $M \mathbf{v}_{\mathbf{j}}=\mathbf{g}_{\mathbf{j}} \quad(j=1,2)$, where $M$ is the operator obtained by linearizing (11.3) - (11.5) about $\mathbf{v}=\mathbf{f}$. This operator is singular since due to translational invariance, $\frac{\partial f}{\partial \theta}$ is element of its kernel. We remark that there is another marginal mode corresponding to the fact that the pressure is at order 0 defined up to a large-scale varying constant $p_{s}$. When solving for $\mathbf{v}_{\mathbf{1}}$ (resp. $\mathbf{v}_{\mathbf{2}}$ ), solvability conditions have thus to be applied to $\mathrm{g}_{1}$ (resp. $\mathrm{g}_{2}$ ), leading respectively to the phase and mean drift equations. The compatibility conditions state that the right-hand sides of the linear systems must be orthogonal to the kernel of the adjoint operator $M^{\dagger}$. Since $M$ is not self-adjoint, we must solve for the homogeneous adjoint boundary value problem separately. One of its solution, corresponding to the pressure mode is trivial; it reads $\mathbf{v}^{\dagger}=(0,0,0,0,1)$. The corresponding solvability condition is satisfied for $j=1$ but becomes nontrivial for $j=2$ : it states that the "large-scale" divergence of the field $\left\langle\mathbf{u}_{\mathbf{1}}\right\rangle(<>$ denotes averaging over $\theta$ and $z$ ) must be zero, but we know that $\left\langle\mathbf{u}_{1}\right\rangle$ will contain non divergence-free terms due to the contributions of the slow horizontal Reynolds stresses: $\sigma \partial_{X} u_{0}^{2}+\sigma \partial_{Y}\left(u_{0} v_{0}\right)$ and $\sigma \partial_{X}\left(u_{0} v_{0}\right)+\sigma \partial_{Y} v_{0}^{2}$. It appears clearly now why me must include the slowly varying pressure $p_{s}$ at order 0 , whose gradients contribute at order $\epsilon$ in the resolution of the velocity field: 
the pressure $p_{s}$ is precisely determined by requiring that $\mathbf{u}_{1}$ be solenoidal and this equation is just another form of the mean drift equation that we usually prefer to write in terms of a stream function $\psi$ after eliminating $p_{s}(\langle\mathbf{u}\rangle=(\hat{\mathbf{k}} \times \nabla \psi) \cdot \hat{\mathbf{z}})$. We also see that at infinite Prandtl number no Reynolds stress is present to drive the mean flow.

The system is closed but the difficulty is that we must solve for the fluctuating parts of $\mathbf{v}_{\mathbf{1}}$; no approximation for the vertical structure of the mean flow will suffice in order to get a reasonable agreement with experiment, especially at low Prandtl numbers. We have to use an extremely robust inversion method to solve these singular equations and we use a technique based on a singular value decomposition of the matrix obtained after projecting the operator $M$ on the appropriate basis. At this order of approximation, the equations read:

$$
\begin{gathered}
\Omega(A, k, R, \sigma)=0, \\
\Theta_{T}+\rho(k) \mathbf{V} \cdot \nabla \Theta+\frac{1}{\tau(k)} \nabla \cdot \mathbf{k} B(k)=0, \\
\hat{\mathbf{z}} \cdot \nabla \times \hat{\mathbf{k}} \alpha(k)(\hat{\mathbf{k}} \times \nabla \psi) \cdot \hat{\mathbf{z}}-\nabla \cdot \hat{\mathbf{k}} \beta(k)(\hat{\mathbf{k}} \cdot \nabla \psi) \\
=\hat{\mathbf{z}} \nabla \times\left(\sigma \mathbf{k} \nabla \cdot \mathbf{k} A^{2}-\frac{\hat{\mathbf{k}}}{\tau_{\alpha}(k)} \nabla \cdot \mathbf{k} B_{\alpha}(k)\right)-\nabla \cdot \hat{\mathbf{k}}\left(\nabla \times \mathbf{k} B_{\beta}(k)\right) \cdot \hat{\mathbf{z}}
\end{gathered}
$$

where $\mathbf{k}=\nabla \Theta, \mathbf{V}=\nabla \times \psi \hat{\mathbf{z}}$, the quantities $\rho(k), B(k), \tau(k), \alpha(k), \beta(k), B_{\alpha}(k), \tau_{\alpha}(k)$, $B_{\beta}(k)$ are all functions of $k$ which are explicitly calculated. The first equation, otained by fixing the periodicity of the basic roll solution, gives the amplitude $A$ as a function of the wavenumber $k$, given the Rayeigh and Prandtl numbers. The vertical structure of the mean drift velocity is different for the "along the roll" or the "across the roll" components and this is why the left-hand side of equation (11.8) is not simply a Laplacian. The first term of the right-hand side comes from the horizontal Reynolds stress whereas the second and third terms essentially come from the vertical Reynolds stress. The appropriate boundary conditions are that $\psi$ is a streamline and that the roll axis is perpendicular to the boundary: $\hat{\mathbf{k}} \cdot \hat{\mathbf{n}}=0$. For the special case of circular target patterns however, the correct boundary condition is rather: $\hat{\mathbf{k}} \times \hat{\mathbf{n}}=0$. These equations are translationaly and rotationaly invariant and also Galilean invariant, even though the original system is not. In the next section we will show that the preceding equations can predict the nonlinear stability region of straight parallel rolls (usually called the Busse balloon) and that moreover they can also predict the stability of circular target patterns. 


\subsection{Some results}

Linearizing equations $(11.6)-(11.8)$ about straight parallel rolls $\left(\Theta_{0}=k_{0} X, \psi_{0}=0\right)$, it is easy to find the loci (in the $(\mathrm{R}, \mathrm{k}$ ) plane, for a given $\sigma$ ) of the Eckhaus (for a purely $X$ dependent perturbation), zig-zag (for a purely $Y$ dependent perturbation) or skewedvaricose border, limits of the nonlinear stability domain. The skewed-varicose instability occurs when the maximum of the growth rate corresponds to a perturbation containing both $X$ and $Y$ dependences. It is the modification of the Eckhaus instability in presence of mean drift and generally leads to formation of pairs of dislocations.

However, in natural patterns, the influence of sidewall boundaries is such that the rolls tend to form circular patches. It is then natural to investigate the stability of exactly circular target patterns. Moreover there is a possibility to check our calculations and conjectures with laboratory experiments performed by Steinberg, Ahlers and Cannell (1985). These authors reproduce this model, using a lateral forcing on the sidewalls to initiate the circular pattern. For an exactly circular pattern (11.7) becomes:

$$
\Theta_{T}+\frac{1}{\tau r} \frac{\partial}{\partial r}(r k B)=0
$$

where $r$ is the radius in polar coordinate. It is easy to see that equation (11.9) leads to stationary solutions with $k=k_{B}$ such that $B\left(k_{B}\right)=0$, the focus acting as a source of new rolls if $k<k_{B}$ and as a sink if $k>k_{B}$. Therefore the curvature is acting as a wavenumber selection mechanism, first observed by Pomeau and Manneville (1981), even in natural patterns where fractions of foci singularities are present (especially in the corners of the containers). Using a functional which is shown to decrease almost every time (except perhaps when dislocations play a significant role) it can be argued (Newell, 1982) that on the time scale $\epsilon^{-2}$, the horizontal diffusion time, patches form which satisfy the boundary condition $\mathbf{k} \cdot \mathbf{n}=0$ along portions of the boundary, and in which $k \rightarrow k_{B}$ almost everywhere.

When non-axisymmetric perturbations are superimposed on the circular target $\Theta_{0}=k_{B} r$, a non-zero mean-drift field will be generated which tends to increase the deformation and opposes to the action of the diffusive terms. Above a certain value of the Rayleigh number (for a given Prandtl number), the circular patch will be linearly unstable. Nonlinear saturation is experimentally observed to occur which leads to an off-centered target. This "focus instability" is important in initiating time dependence (even in natural patterns), as observed when the selected wavenumber lies well inside the Busse balloon. At the point where $k_{B}$ crosses the skewed-varicose boundary we rather suggest that dislocations would be nucleated all over the container and that the spatial coherence will be lost. The values of the critical Rayleigh number at which the focus instability appears, as calculated by our theory, is consistent with experiments (Steinberg, Ahlers and Cannell, 1985), but corrections to account for the finiteness of the box are certainly necessary. Moreover it appears that in the center of the patch the amplitude of the rolls is no longer slaved (Newell, passot and Souli, 1990b) and this is important to calculate a precise value of the instability threshold. The study of this latter point requires the determination of coupled PDE's governing the 
behavior of both the phase and amplitude variables. This point is now addressed in the following Section.

\subsection{Regularization of the phase equation}

For the sake of general discussions, let us introduce a typical pattern forming equation of the form:

$$
\partial_{t} w+F\left(\frac{\partial}{\partial x}, R, w\right)=0
$$

which admits the null solution and a periodic basic solution of the form $w_{0}\left(\theta+\theta_{0} ; R, k\right)$. (The amplitude $A$ instead of $k$ can also be used to parametrize its family of solutions.)

The phase diffusion equation (11.1) can also be obtained for this equation; it was previously derived as a solvability condition of the equation which governs the correction $w^{\prime}$ to the almost periodic field $w_{0}$. This solvability condition is made necessary by the existence of a periodic null solution $v_{1}=\frac{d w_{0}}{d \theta_{0}}=\frac{\partial w_{0}}{\partial \theta}$ of the operator obtained by linearizing the original microscopic equations about the field $w_{0}$. The null solution $v_{1}$ exists because of the translational invariance. There is also a second null solution, $v_{2}=\frac{d w_{0}}{d A}=\frac{\partial w_{0}}{\partial A}+\frac{\theta}{2 k^{2}} \frac{\partial w_{0}}{\partial \theta} \frac{\partial k^{2}}{\partial A}$, but, because of the presence of the second term, $v_{2}$ is not periodic and thus in general does not lead to an additional solvability condition. It is however periodic at the boundaries of the marginal stability curve given by $A^{2}=\mu^{2}(R, k)=0$ so that for values of $k$ at which $\mu^{2}$ is zero, there is indeed a second solvability condition which turns out to involve a correction to the amplitude equation (11.2). We shall see shortly why this correction is necessary.

The difficulty with (11.1) is that it is ill-posed in the sense that

$$
\nabla \cdot \mathbf{k} B=\left(B+\frac{\theta_{x}^{2}}{k} \frac{d B}{d k}\right) \theta_{x x}+2 \frac{\theta_{x} \theta_{y}}{k} \frac{d B}{d k} \theta_{x y}+\left(B+\frac{\theta_{y}^{2}}{k} \frac{d B}{d k}\right) \theta_{y y}
$$

$\left(\mathbf{k}=\nabla \theta=\left(\theta_{x}, \theta_{y}\right)\right)$, is only negative definite and therefore (11.1) is only stable when both $B(k)$ and $\frac{d}{d k} k B$ are negative. This occurs for a band of wavenumbers $k_{Z}(R)<k<k_{E}(R)$ $\left(B\left(k_{Z}\right)=0,\left.\frac{d}{d k} k B\right|_{k=k_{E}}=0\right)$ which is the nonlinear stability domain or "Busse balloon". As soon as the wavenumber $k$ wanders outside the Busse balloon, there is a rapid growth of the short scales and unphysical instabilities. We must therefore regularize the phase diffusion equation.

The nature of the instabilities when $k<k_{Z}$ or $k>k_{E}$ are different (Fauve, 1987): At the zigzag border the instability is a supercritical-type bifurcation and it saturates when the rolls develop a zig-zag pattern. The regularization of (11.1) can thus simply be achieved by going to the next orders of the expansion, which consists in adding to the phase diffusion equation terms proportional to $\epsilon^{2} \nabla^{2} \nabla \cdot \mathbf{k}$. There are other nonlinear terms but this one is the most important, capable of achieving a balance with $\nabla \mathbf{k} B$ after introducing the scaling $\tilde{Y}=\epsilon^{\frac{1}{2}} y$ in the "along the roll direction". On the other hand, at the Eckhaus border, the instability leads to a readjustment of the roll wavelength (or for the skewed-varicose instability when mean flow effects are present, it leads to formation of dislocations), during which process 
the amplitude approaches zero locally and the wavenumber wanders way outside the right border of the marginal curve. It appears clearly now that even though the preceding type of regularization is sufficient at the beginning of the instability process, it is not at all sufficient to describe the whole story.

It will be necessary to supplement the phase diffusion equation by a Newell-Whiteheadtype equation when $k$ approaches $k_{r}$, the right border of the marginal stability curve. This is possible precisely because at $k_{r}$ the second solvability condition leads to a correction to the amplitude equation. But, in order to match properly the "generalized Ginzburg-Landau equation" derived at $k_{r}$ and the phase diffusion equation with the bilaplacian term, valid close to $k_{E}$, one must find coupled PDE's for the phase and an amplitude mode valid in the range $k_{E}<k<k_{r}$. This system does not need to contain exact higher order terms because the process which leads $k$ to approach and exceed $k_{r}$ is very rapid. The solution appears to be attracted to a robust singular solution of the reverse nonlinear heat equation in which the high $k$ modes are slaved to the low $k$ ones. This is analogous to the regularization of strictly hyperbolic equations: none of the large scale dynamics (shock strength and speed) are affected by the choice of the artificial viscosity.

How do we modify the amplitude equation which to this point is algebraic, which means the amplitude is slaved? In the case of the complex Swift-Hohenberg equation,

$$
\partial_{t} w+(1+\Delta)^{2} w-R w+w^{2} w^{*}=0
$$

the solution is obvious because we have the exact functional form of $w_{0}=A \exp (i \theta)$ which when substituted in (11.11) gives exactly:

$$
\begin{gathered}
A \Theta_{T}+\mathcal{L}_{1}^{1} A+\epsilon^{2} \mathcal{L}_{3} A=0 \\
R-A^{2}-\left(1-k^{2}\right)^{2}=\frac{\epsilon^{2}}{A}\left(A_{T}+\mathcal{L}_{2}^{1} A+\epsilon^{2} \mathcal{L}_{4} A\right) .
\end{gathered}
$$

where

$$
\begin{aligned}
& \mathcal{L}_{0}=\left(1+k^{2} \partial_{\theta}^{2}\right)^{2} \\
& \mathcal{L}_{1}=\left(1+k^{2} \partial_{\theta}^{2}\right) D_{1}+D_{1}\left(1+k^{2} \partial_{\theta}^{2}\right) \\
& \mathcal{L}_{2}=\left(1+k^{2} \partial_{\theta}^{2}\right) D_{2}+D_{2}\left(1+k^{2} \partial_{\theta}^{2}\right)+D_{1}^{2} \partial_{\theta}^{2} \\
& \mathcal{L}_{3}=D_{1} D_{2}+D_{2} D_{1} \\
& \mathcal{L}_{4}=D_{2}^{2}
\end{aligned}
$$

and

$$
\begin{aligned}
& D_{1}=2 \mathbf{k} \cdot \nabla+\nabla \cdot \mathbf{k} \\
& D_{2}=\nabla^{2} .
\end{aligned}
$$


$\mathcal{L}_{j}^{p}$ means that we replace $\frac{\partial^{2}}{\partial \theta^{2}}$ in $\mathcal{L}_{j}$ by $-p^{2}$.

In the simple case of second order equations, coupled equations for the phase and amplitude can also be obtained (Luke, 1966; Kogelman and Keller, 1973, Newell, 1985) starting from an explicit (nonperiodic) solution of the linear equation obtained at each order of the expansion, and imposing solvability as well as periodicity conditions.

In general however we will not have the luxury of such exact solutions and an alternative approach is to project the equation onto the left eigenmodes $\xi_{0}^{\dagger}, \xi_{1 S}^{\dagger}$ of the linear operator obtained after linearizing the original microscopic equations about $w_{0}$. ( $\xi_{0}$ corresponds to the phase mode of eigenvalue 0 and $\xi_{1}$ is the mode with the smallest nonzero eigenvalue $\lambda_{1}$, which, close to $k_{r}$, corresponds to the amplitude mode). The first equation obtained is the usual phase diffusion equation containing the dominant correction terms and the second one is a correction to the eikonal equation. In the low amplitude limit, one obtains:

$$
\begin{gathered}
\Theta_{T}+\frac{1}{\tilde{\tau}(k) A^{2}} \nabla \cdot\left(\mathbf{k} A^{2} \tilde{B}(k)\right)+O\left(\epsilon^{2}\right)=0 \\
\epsilon^{2} A_{T}+g(k) A\left(A^{2}-\mu^{2}(k)\right)+O\left(\epsilon^{2}\right)=0
\end{gathered}
$$

For the real Swift-Hohenberg equation at small amplitudes, where we can approximate $w$ by a one Galerkin mode expansion for the basic roll solution (i.e. $w=\sqrt{2} A \cos (\theta)$ ), we have $\mu^{2}(k)=\frac{2}{3}\left(R-\left(1-k^{2}\right)^{2}\right), \tilde{\tau}(k)=1, \tilde{B}(k)=2\left(1-k^{2}\right)$ and $g(k)=\frac{\mathbf{3}}{2}$.

One can also write the Newell-Whitehead-Segel equation derived for wavenumber $k_{r}$ and values of the Rayleigh $R$ well away from $R_{c}$. Considering eq. (11.10), we will denote by $\lambda(R, k)$ the eigenvalue of the operator $L_{0}=\left.\frac{\delta F}{\delta w}\right|_{w=0}$ for a mode whose wavenumber is slightly inside the marginal stability curve $\left(\lambda(R, k)\right.$ and $-\lambda_{1 S}(k) / 2$ are equivalent in the limit $k$ goes to $\left.k_{r}\right)$. It is easy to see that the complex amplitude equation for the marginal mode takes the form:

$$
\begin{gathered}
\partial_{\tau} \mathcal{A}+\left.\frac{\partial \lambda}{\partial R}\right|_{0}\left(R-R_{0}\right) \mathcal{A}+\left.\frac{\partial \lambda}{\partial\left(i k_{j}\right)}\right|_{0} \nabla_{j} \mathcal{A}+\left.\frac{\epsilon}{2} \frac{\partial^{2} \lambda}{\partial\left(i k_{j}\right) \partial\left(i k_{l}\right)}\right|_{0} \nabla_{j} \nabla_{l} \mathcal{A} \\
+g_{0}|\mathcal{A}|^{2} \mathcal{A}+O\left(\epsilon|\mathcal{A}|^{4} A\right)+O\left(\epsilon^{2}\right)=0
\end{gathered}
$$

where $\partial_{\tau}$ stands for $\partial_{T_{1}}+\epsilon \partial_{T_{2}}, T_{j}=\epsilon^{j} t$. The index 0 means that the expression has to be evaluated at $k_{r}$. One has of course $\lambda\left(R, k_{r}\right)=0$ and $g_{0}$ identifies here with the Landau constant which is also equal to $g\left(k_{r}\right)$, the limit as $k$ goes to $k_{r}$ of $-\frac{\lambda(R, k)}{\mu^{2}(k)}$. The term containing first order derivatives in $\mathcal{A}$ is at the origin of an amplitude instability of the $\mathcal{A}=$ constant solution, leading to a decrease of the wavenumber towards values closer to the band center. It is thus natural to look for an equation governing the evolution of $W=\mathcal{A} e^{i k_{r} x}$; it will read:

$$
\partial_{t} W+\Lambda_{0}\left(-\nabla^{2}\right) W+|W|^{2} \Gamma_{0} W=0
$$

where $\Lambda_{0}\left(-\nabla^{2}\right) W$ is the inverse Fourier transform of $\lambda\left(k^{2}\right) \hat{W}$ and $\Gamma_{0}=g_{0}$. 
Equations (11.14), (11.15) and (11.17) are equivalent close to $k_{r}$ in the sense that at smallest order, the asymptotic equations derived from (11.17) using the Cross-Newell formalism will identify with $(11.14),(11.15)$. It will be convenient now to extend the validity of (11.17) by writing an equation of a similar form but valid for any value of $k$ and such that its phase diffusion equation is exactly (11.1). Morover we can also demand that the first eigenvalue $\lambda_{1}$ be the same both for the microscopic equation and for the model.

We propose an equation of the form:

$$
\partial_{t} U+\Lambda\left(-\nabla^{2}\right) U+\left(U+U^{* 2}\right) \Gamma\left(-\nabla^{2}\right) U=0
$$

where $U=A \cos \theta$ is our real order parameter, and $U^{*}$ is calculated from $U$ by demanding that $U^{2}+U^{* 2}$ be the large scale squared amplitude of the rolls. Eq. (11.18) then admits monochromatic solutions. The functions $\Lambda$ and $\Gamma$ are determined by demanding that the phase diffusion equation derived from (11.18) be exactly the one we derive from the microscopic equations. Moreover, in order to match also the first nonzero eigenvalue $\lambda_{1}$ (or $g(k)$ ), we introduce a function $\beta(k)$ which relates the wavenumber $k$ of the pattern to the wavenumber $q$ measured form the solution $U$, by the relation: $q^{2}=\int^{k^{2}} \beta^{2}(x) d x$.

Preliminary numerical simulations of both the real Swift-Hohenberg equation considered as our microscopic equation and the model equation (11.18), show a very good agreement up to values of the stress parameter of $R=10$. Moreover it appears crucial to solve for a real order parameter $U$ and not for $W=A \exp (i \theta)$. Our equation is capable of capturing more involved processes, such as resonnances between $k$ and $k / 3$ modes leading for example to the breaking of a dislocation into two desinclinations. This work will be presented in a forthcoming paper (Passot and Newell, 1991).

\subsection{Conclusion}

As a first concluding remark we would like to mention that the equation (11.18) we derived in Section 4, or its extension taking into account the mean drift term (in progress), is certainly quite general and bridges the gap which existed between qualitative and quantitative models. The first ones, such as the Swift-hohenberg equation, or its generalizations (Manneville, 1983; Greenside, Cross and Coughran, 1988) give a good overall picture of convective patterns but do not allow for exact comparisons with experiments, and cannot extend to large Rayleigh numbers. The second ones were up to now restricted to the case of simple patterns, close to onset (e.g. the Newell-Whitehead-Segel equation), or to the case of "defect-free" patterns (the phase diffusion equation as presented in Newell, Passot and Souli 1990b).

The equation we present here must include a small scale behavior of the order parameters $A$ and $\Theta$ since it can reproduce the formation and dynamics of defects. But it does not need to capture all the small-scale features of the underlying pattern, such as boundary layers. In this sense it is much easier to handle than the microscopic model! 
Acknowledgments: We thank the organizers of the Woods Hole GFD Summer School for their hospitality. Research support from the Arizona Center for Mathematical Sciences, sponsored by AFOSR Contract FQ8671-900589 (AFOSR-90-0021) with the University Research Initiative Program at the University of Arizona, is gratefully acknowledged.

\section{References}

M. Bestehorn and H. Haken (1990) Traveling waves and pulses in a two-dimensional largeaspect-ratio system, Phys. Rev. A 42, 7195.

F.H. Busse (1967) On the stability of two-dimensional convection in a layer heated from below, J. Math. Phys. 46, 149.

F.H. Busse (1978) Nonlinear properties of convection, Rep. Prog. Phys. 41, 1929.

M.C. Cross and A.C. Newell (1984) Convection Patterns in Large Aspect Ratio Systems, Physica 10D, 299.

P. Coullet, L. Gil and J. Lega (1989) Defect mediated turbulence, Phys. Rev. Lett. 62, 1619.

S. Fauve (1987), Large Scale Instabilities of Cellular Flows, in "Instabilities and Nonequilibrium Structures", 63-88, Eds. E. Tirapegui and D. Villaroel, D. Reidel Publishing Company. H.S. Greenside, M.C. Cross and W.M. Coughran, Jr. (1988) Mean Flows and the Onset of Chaos in Large-Cell Convection, Phys. Rev. Lett. 60, 2269.

M.S. Heutmaker and J.P. Gollub (1987) Wave--vector field of convective flow patterns, Phys. Rev. A 35, 242.

L.N. Howard and N. Kopell (1977) Slowly Varying Waves and Shocks Structures in ReactionDiffusion Equations,S. Appl. Math. 56, 95.

S. Kogelman and J.B. Keller (1973) Asymptotic Theory of Nonlinear Wave Propagation SIAM J. Appl. Math. 24, 352.

Y. Kuramoto (1984), Chemical Oscillations, Waves and Turbulence, Springer Series in Synergetics 19, H. Haken Ed., Springer-Verlag.

J.C. Luke (1966) A Perturbation Method for Nonlinear Dispersive Wave Problems, Proc. Roy. Soc. London Ser. A 292, 403.

P. Manneville (1983) A two-dimensional model for three-dimensional convective patterns in wide containers, J. Physique 44, 759.

A.C. Newell (1982) Two Dimensional Convection Patterns in Large Aspect Ratio Systems, Lecture Notes in Num. Appl. Anal. 5, 205.

A.C. Newell (1985) Solitons in Mathematics and Physics, CBMS-NSF vol. 48, SIAM.

A.C. Newell, T. Passot and M. Souli (1990a) Convection at Finite Rayleigh Numbers in Large-Aspect-Ratio Containers, Phys. Rev. Lett 64, 2378.

A.C. Newell, T. Passot and M. Souli (1990b) The phase diffusion and mean drift equations for convection at finite Rayleigh numbers in large containers, J. Fluid. Mech. 220, 187-252. 
A.C. Newell, J.A. Whitehead (1969) Finite bandwidth, finite amplitude convection, J. Fluid Mech. 38, 279.

T. Passot and A.C. Newell (1991) The regularization of the phase diffusion equation for natural convective patterns, submitted to J. Physique.

Y. Pomeau and P. Manneville (1979) Stability and fluctuations of a spatially periodic convective flow, Journal de Physique- Lettres 23, L609.

Y. Pomeau and P. Manneville (1981) Wavelength selection in axisymmetric cellular structures, J. Physique Lett. 42, 1067.

L.A. Segel, (1969) J. Fluid Mech. 38, 203.

V. Steinberg, G. Ahlers and D.S. Cannell (1985), Pattern formation and wavenumber selection by Rayleigh-Bénard convection in a cylindrical container, Physica Scripta, T13, 135.

J. Swift and P.C. Hohenberg (1977) Hydrodynamic fluctuations at the convective instability, Phys. Rev. A 15, 319.

G.B. Whitham (1974) Linear and nonlinear waves, Wiley-Interscience, New-York. 


\title{
12. Slow methods for simple people
}

\author{
A J Roberts \\ University of Adelaide
}

Centre manifold reduction and its generalisations form a systematic approach to the derivation of approximate evolution equations of dynamics. The procedure presented here is simply a variation of that proposed by Coullet \& Spiegel (1983) and explained more simply by Roberts (1985). Two well known examples which will be examined are: the derivation of a Landau equation for the slowly-varying oscillations of the Van der Pol equation; and the derivation of the nonlinear Scrödinger equation for nonlinear dispersive waves in the Klein-Gordon equation. The method of multiple-scales has been used widely in this sort of context. However, it suffers from many significant defects such as: an inability to find higher-order corrections; the initial difficulty in deducing appropriate scalings; and its failure to handle interacting wavetrains except in contrived regions of parameter space. Methods based on centre manifold reduction are much more flexible, and can form the basis of a complete approach to modelling as will be described in the lecture on rational mathematical modelling.

\subsection{Van der Pol oscillations}

We will analyse the nonlinear equation

$$
\ddot{u}-2 \lambda \dot{u}+u^{2} \dot{u}+u=0
$$

based on $\lambda \approx 0$ where the the onset of regular oscillations takes place. Linearly, if $\lambda=0$ and $u$ is small we find the oscillatory solution $u=a e^{i t}+\bar{a} e^{-i t}$ for some complex coefficient $a$. It is the weak nonlinearity and the effects of non-zero $\lambda$ which modulate this pure oscillation and which can be captured by slowly varying $a$ in time.

For the application of a centre manifold type of analysis it is more convenient to rewrite the equations as the set of first-order differential equations

$$
\dot{u}=v, \quad \dot{v}=-u+2 \lambda v-u^{2} v
$$

which can be written as a vector equation for $\mathbf{u}(t)=(u(t), v(t))^{T}$. The centre manifold ansatz is to seek solutions of the form

$$
\mathbf{u}=\mathbf{w}(a, \theta)
$$


where $\theta$ is the phase of the rapid oscillations, here $\theta=t$ but more generally it is $\omega_{0} t$, and where $a$ is the slowly-varying complex amplitude which evolves according to

$$
\dot{a}=g(a) \text {. }
$$

By the chain rule, time derivatives transform as follows $\dot{\mathbf{u}}=\mathbf{w}_{\theta} \dot{\theta}+\mathbf{w}_{a} \dot{a}=\mathbf{w}_{\theta}+\mathbf{w}_{a} g(a)$ where subscripts denote differentiation. Thus the Van der Pol equation becomes

$$
\mathcal{L} \mathbf{w}=-\mathbf{w}_{a} g+\mathcal{N}, \quad \mathcal{L}=\left[\begin{array}{cc}
\partial_{\theta} & -1 \\
1 & \partial_{\theta}
\end{array}\right], \quad \mathcal{N}=\left[\begin{array}{c}
0 \\
2 \lambda v-u^{2} v
\end{array}\right]
$$

Here all the linear terms are on the left, while all the weak and nonlinear effects are represented on the right-hand side. The term $\mathbf{w}_{a} g$ represents the slow time variations of the amplitude of the oscillation and this is where the difference lies between the algebra of centre manifold reduction and that of the method of multiple scales (where this term would be represented as $\left.\epsilon^{2} e^{i t} \partial a / \partial T\right)$.

In general this last equation can only be solved asymptotically. The small parameters of the asymptotics are the oscillation amplitude $a$ and the bifurcation parameter $\lambda$. In principle we can choose the two parameters to be of arbitrary magnitude relative to each other; however, in practise, this is both cumbersome and of little net benefit. Instead, we choose $a$ be a quantity of "order 1" (i.e. of a size $\epsilon^{1}$ where $\epsilon$ is a measure of smallness), $\lambda$ be of "order 2" (i.e. of a size $\epsilon^{2}$ ) and then try an infinite series

$$
\mathbf{w} \sim \sum_{n=1}^{\infty} \mathbf{w}^{(n)}(a, \theta), \quad g \sim \sum_{n=2}^{\infty} g^{(n)}(a)
$$

where quantities with superscript $(n)$ contain terms of order $n$ only. Substituting into the equation and grouping all terms of the the same order we obtain the infinite hierarchy

$$
\mathcal{L} \mathrm{w}^{(1)}=0, \quad \mathcal{L} \mathbf{w}^{(n)}=-\sum_{m=1}^{n-1} \mathbf{w}_{a}^{(n-m)} g^{(m+1)}+\mathcal{N}^{(n)}, \quad n=2,3, \ldots
$$

where the nonlinear terms are $\mathcal{N}^{(2)}=0$ and

$$
\mathcal{N}^{(3)}=\left[\begin{array}{c}
0 \\
2 \lambda v^{(1)}-u^{(1)^{2}} v^{(1)}
\end{array}\right], \quad \mathcal{N}^{(4)}=\left[\begin{array}{c}
0 \\
v^{(2)}-u^{(1)^{2}} v^{(2)}-2 u^{(1)} u^{(2)} v^{(1)}
\end{array}\right], \cdots
$$

We solve these equations in succession $n=1,2, \ldots$ by choosing $g^{(n)}$ so that the right-hand side of the $n^{\text {th }}$ order equation is in the range of $\mathcal{L}$ (so that $\mathbf{w}^{(n)}$ is $2 \pi$-periodic in $\theta$ ), and then finding $\mathbf{w}^{(n)}$. This process may be automated and, using REDUCE to calculate and check, we may find that all even order quantities vanish, and that to fifth order

$$
u \sim a e^{i t}+\frac{i}{8} a^{3} e^{i 3 t}-\frac{1}{64} a^{3}\left(4 \lambda+|a|^{2}\right) e^{i 3 t}+\frac{5}{192} a^{5} e^{i 5 t}+\text { c.c. }
$$


where the slow evolution is determined by

$$
\dot{a} \sim\left(\lambda a-\frac{1}{2} a|a|^{2}\right)-i a\left(\frac{1}{2} \lambda^{2}-\lambda|a|^{2}+\frac{7}{16}|a|^{4}\right)-a|a|^{2}\left(\lambda^{2}-\frac{35}{32} \lambda|a|^{2}+\frac{37}{128}|a|^{4}\right)
$$

If the earlier ordering relationship between $a$ and $\lambda$ is varied then we get precisely the same terms in the asymptotic sums, it is just that they arrive at a different order in the expansion. This invariance of the terms in the equations under changes in the assumed ordering relationships is an important feature of the approach.

Of interest is whether the series, when extended to high-order, will converge or not. For the case when $\lambda=0$ precisely, the calculations simplify and the evolution equation can be found to $39^{\text {th }}$ order using REDUCE (taking an hour on a SPARCstation). Forming a DombSykes plot based on the resulting amplitude expansion indicates that it has zero radiusof-convergence. Thus these approximations to the Van der Pol dynamics are asymptotic only-as seems typical for nonlinear problems. Methods such as optimal truncation, just using the leading order or two for example, or Padè approximation (see Bender \& Orszag, 1978, Chapt.8) are then useful ways of summing the series.

\subsection{Nonlinear dispersive waves in the Klein-Gordon equation}

The Klein-Gordon equation with a cubic nonlinearity

$$
\frac{\partial^{2} u}{\partial t^{2}}=\frac{\partial^{2} u}{\partial x^{2}}-u+u^{3}
$$

is a simple equation which combines wave dispersion with nonlinearity. Linearly, there exist wave solutions $u=a e^{i(k x-\omega t)}+$ c.c. satisfying the dispersion relation $\omega^{2}=1+k^{2}$ for any complex amplitude $a$. The weak effects of the nonlinearity and any variations in wavenumber cause slow amplitude and phase variations which may be captured by slowly varying $a$ in both time $t$ and space $x$.

To use centre manifold reduction we rewrite it as the coupled first-order equations

$$
\frac{\partial u}{\partial t}=v, \quad \frac{\partial v}{\partial t}=\frac{\partial^{2} u}{\partial x^{2}}-u+u^{3}
$$

for the unknown $\mathbf{u}(x, t)=(u(x, t), v(x, t))^{T}$. The ansatz is to seek solutions of the form

$$
\mathbf{u}=\mathbf{w}(a, \theta)
$$

where $\theta=k x-\omega t$ is the phase of the waves so that $\mathbf{w}$ is $2 \pi$-periodic in $\theta$, and where $a(x, t)$ evolves slowly in both $x$ and $t$ according to

$$
a_{t}=g(a)
$$


where $g$ contains derivatives with respect to $x$ making this a partial differential equation for $a$. The time derivatives transform as before. The spatial differentiation, being of higherorder, is a little more involved and leads to $\partial^{2} u / \partial x^{2}=k^{2} u_{\theta \theta}+2 k u_{\theta a} a_{x}+u_{a a} a_{x}{ }^{2}+u_{a} a_{x x}$. Thus the nonlinear Klein-Gordon equation becomes

$$
\mathcal{L} \mathbf{w}=-\mathbf{w}_{a} g+\mathcal{N}, \quad \mathcal{L}=\left[\begin{array}{cc}
-\omega \partial_{\theta} & -1 \\
1-k^{2} \partial_{\theta}^{2} & -\omega \partial_{\theta}
\end{array}\right], \quad \mathcal{N}=\left[\begin{array}{c}
0 \\
2 k u_{\theta a} a_{x}+u_{a a} a_{x}^{2}+u_{a} a_{x x}+u^{3}
\end{array}\right]
$$

Again the dominant linear dynamics are on the left-hand side, while all the weak effects leading to slow modulation are on the right-hand side.

To solve this equation asymptotically we let both $a$ and spatial differentiation, $\partial_{x}$, be of "order 1". This is equivalent to the usual multiple-scale expansion of $X=\epsilon x$ and $a=\epsilon A$. Note that there is no need to decide upon a time-scale as the appropriate time-scale occurs naturally in the solution of the evolution equation. Then we try an infinite series

$$
\mathbf{w} \sim \sum_{n=1}^{\infty} \mathbf{w}^{(n)}(a, \theta), \quad g \sim \sum_{n=2}^{\infty} g^{(n)}(a)
$$

where again quantities with superscript $(n)$ contain terms of order $n$ only. Substituting into the equation and grouping all terms of the the same order we obtain the infinite hierarchy

$$
\mathcal{L} \mathbf{w}^{(1)}=0, \quad \mathcal{L} \mathbf{w}^{(n)}=-\sum_{m=1}^{n-1} \mathbf{w}_{a}^{(n-m)} g^{(m+1)}+\mathcal{N}^{(n)}, \quad n=2,3, \ldots
$$

where the nonlinear terms are, for example,

$$
\mathcal{N}^{(2)}=\left[\begin{array}{c}
0 \\
2 k u_{\theta a}^{(1)} a_{x}
\end{array}\right], \quad \mathcal{N}^{(3)}=\left[\begin{array}{c}
0 \\
u_{a a}^{(1)} a_{x}^{2}+u_{a}^{(1)} a_{x x}+u^{(1)^{3}}
\end{array}\right], \cdots
$$

As before we solve these equations in succession $n=1,2, \ldots$ by choosing $g^{(n)}$ so that $\mathbf{w}^{(n)}$ may be found to be $2 \pi$-periodic in $\theta$. Using REDUCE to calculate and check, we may find that

$$
u \sim a e^{i \theta}-\frac{1}{8} a^{3} e^{i 3 \theta}+c . c .
$$

where the complex amplitude evolves according to

$$
a_{t} \sim-\frac{k}{\omega} a_{x}+\left(\frac{i}{2 \omega^{3}} a_{x x}+\frac{3 i}{2} a|a|^{2}\right)-\left(\frac{k}{2 \omega^{5}} a_{x x x}+\frac{3 k}{2 \omega^{3}}\left(|a|^{2} a\right)_{x}\right) .
$$

These results are correct to fourth-order in $a$ and $\partial_{x}$, and if needed, they may be extended to high-order using computer algebra. They are obtained without the artifice of changing to a reference frame moving with the group velocity $k / \omega$.

The convergence of the above series is hard to establish; however, there are two accessible special cases. If there are no large-scale spatial variations in the amplitude of the nonlinear 
wave, i.e. $a_{x}=0$, then $g(a)$ is pure imaginary and is the convergent Taylor series for the amplitude dependence of the dispersion relation, at least for some range of $|a|$. If the waves are of small enough amplitude to be essentially linear then $g(a)$ becomes the convergent Taylor series for the linear dispersion relation $\omega(k)$ about the fixed wavenumber $k$.

The above analysis is for a single wavetrain. To study two interacting wavetrains we would substitute $\mathbf{u}(x, t)=\mathbf{w}\left(a_{1}, a_{2}, \theta_{1}, \theta_{2}\right)$ into the equations. Then the solvability condition that would give the equations for the evolution of the amplitudes $a_{i}$ are that $\mathbf{w}$ is $2 \pi$-periodic in both $\theta_{1}$ and $\theta_{2}$. This easily generalises to an arbitrary number of wavetrains or wave/meanfield interactions.

The analysis may be adapted to deal with spatial and temporal inhomegeneities in the coefficients of the equations, provided they are slow compared with the basic wave oscillation. It may also be extended to deal with the slow variations in phase and amplitude of strictly nonlinear waves if an exact family of solutions are known for uniformly propagating waves are known (see Kogelman \& Keller (1973) for a multiple scale treatment).

\subsection{Summary}

Much of the algebraic details in the above "centre manifold reduction" are identical to those in the method of multiple scales; however, it is the setting in which they are carried out which is different. This analysis of oscillations and waves is not strictly justified according to centre manifold theory (Carr, 1981) as there is no dissipation to drive the system to the centre manifold (technically, the above analysis constructs what are called sub-centre manifolds, see Sijbrand (1985)), none-the-less the results form models which we know are useful and also extends them significantly. These and further matters on the centre manifold reduction applied to nonlinear dispersive waves will be reported in more detail in a forthcoming paper (Roberts, 1991). It is interesting to note that the introduction of the phase variable $\theta$ makes the consequent derivation akin to that of the averaged Lagrangian approach developed by Whitham (1974) which is a very powerful method in conservative wave dynamics. 


\section{References}

C.M. Bender \& S.A. Orszag Advanced mathematical methods for engineers and scientists, McGraw-Hill (1978).

J. Carr "Applications of centre manifold theory" Applied Math Sci 35 (1981).

P.H. Coullet \& E.A. Spiegel "Amplitude equations for systems with competing instabilities" SIAM J Appl Math 43 (1983) pp776-821.

S. Kogelman \& J.B. Keller "Asymptotic theory of nonlinear wave propagation" SIAM J Appl Math 24 (1973) pp352-361.

A.J. Roberts "Simple examples of the derivation of amplitude equations for systems of equations possessing bifurcations" J Austral Math Soc Ser B 27 (1985) pp48-65.

A.J. Roberts "A sub-centre manifold description of the evolution and interaction of nonlinear dispersive waves" preprint (1991).

J. Sijbrand "Properties of center manifolds" Trans Amer Math Soc 289 (1985) pp431-469.

G.B. Whitham Linear and nonlinear waves (1974). 


\title{
13. Rational mathematical modelling-shear dispersion and planform selection
}

\author{
A J Roberts \\ University of Adelaide
}

The universe is an evolving dynamical system. To deal with its vast complexities we have to approximate. There are two types of approximation: one type would assert that a softball is roughly spherical; the other type is to treat its dynamics as if it were a simple point mass. It is this last type of approximation which I will address.

In order to understand any of the enormously complicated dynamics of the universe we only investigate small pieces. Even then we typically only investigate a limited range of the dynamics of the small pieces which we wish to study; for example, the incompressible approximation obliterates sound waves. In doing this we throw out of the system many of the degrees of freedom of its dynamics, to end up with a dynamical system of much lower dimensionality than the original. Centre manifold theory provides the inspiration for a systematic rationale for doing this. The aim is "not to throw out the baby with the bath water."

\subsection{A centre manifold approach to complete modelling}

This work using centre manifolds was started ten years ago by Coullet \& Spiegel (1983) at the GFD summer school of 1981. The material I described in the lecture slow methods for simple people is an application of those ideas, mixed with a little of multiple scales, to the approximation of nonlinear oscillations and dispersive waves. In the last $6-7$ years the geometric picture underlying centre manifold theory has lead to techniques which not only derive general low-dimensional models, but which also supply appropriate initial conditions, forcing and boundary conditions. In short, centre manifold theory provides the means to obtain complete models of dynamic systems.

\subsubsection{Centre manifold theory and applications}

The theory of centre manifolds addresses the long-term evolution in the vicinity of a reference equilibrium state. It is based on the linear dynamics. As an example (Roberts, 1985) consider the $2 \mathrm{D}$ dynamical system

$$
\dot{x}=-x y, \quad \dot{y}=-y+x^{2}-2 y^{2} .
$$

Linearly, $\dot{x} \approx 0$ and $\dot{y} \approx-y$, so exponentially quickly, like $e^{-t}$, the system evolves to the line $y=0$ and then remains there. Centre manifold theory asserts that this linear picture 
is largely correct; however, the nonlinearities bend the line to which the trajectories are attracted, to form the curved centre manifold which here is exactly $y=x^{2}$, and effect a slow evolution over long times on the manifold, here according to $\dot{x}=-x^{3}$. As can be seen in computer demonstrations and in the figure, the system settles exponentially quickly onto the $1 D$ dynamics on the manifold.

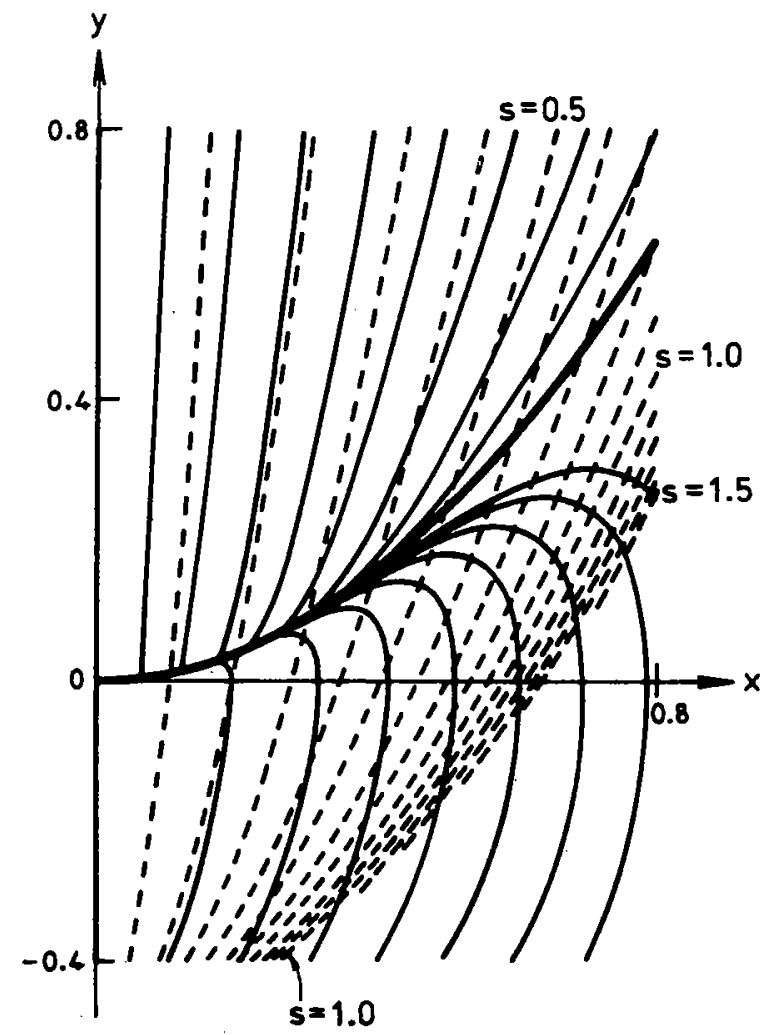

Figure 13.1. Trajectories of the system (13.1) are shown in solid; the dashed lines are the isochronic manifolds which are discussed later.

In physical dynamical systems we hope that a similar simplification may occur. Namely, that most of the dynamical modes, or degrees-of-freedom, are rapidly damped, as is. $y$ above, while relatively few modes are marginal and, like $x$ above, form the basis for a low-dimensional description of the long-term dynamics. For example, a softball may be modelled as a point mass with 6 degrees-of-freedom based on the visco-elastic decay of the internal modes of vibration (Muncaster, 1983). The theory for centre manifolds (Carr, 1981) asserts three important properties for the general system

$$
\dot{\mathbf{x}}=A \mathbf{x}+\mathbf{f}(\mathbf{x}, \mathbf{y}), \quad \dot{\mathbf{y}}=B \mathbf{y}+\mathbf{g}(\mathbf{x}, \mathbf{y})
$$

where $\Re\left\{\lambda_{A}\right\}=0, \Re\left\{\lambda_{B}\right\} \leq-\gamma<0$, and $\mathbf{f}$ and $\mathbf{g}$ are strictly nonlinear:

- existence-there exists a centre manifold $\mathbf{y}=\mathbf{h}(\mathbf{x})$ near the origin on which the evolution is governed by the lower-dimensional system

$$
\dot{\mathbf{x}}=A \mathbf{x}+\mathbf{f}(\mathbf{x}, \mathbf{h}(\mathbf{x}))
$$


- relevance-if $\mathbf{x}(0)$ and $\mathbf{y}(0)$ are sufficiently close to the origin then there exists a solution on the manifold, $\mathbf{X}(t)$ say, which is approached exponentially quickly: $\mathbf{x}(t)=\mathbf{X}(t)+O\left(e^{-\gamma t}\right)$ $\mathbf{y}(t)=\mathbf{h}(\mathbf{X}(t))+O\left(e^{-\gamma t}\right)$

- approximation -if an approximation to the shape of the manifold, $\phi(x)$ satifies $B \phi=$ $-g(\mathbf{x}, \phi)+\phi^{\prime}(\mathbf{x})[A \mathbf{x}+\mathbf{f}(\mathbf{x}, \phi)]+O\left(|\mathbf{x}|^{q}\right)$ for some $q$ then $\mathbf{h}(\mathbf{x})=\phi(\mathbf{x})+O\left(|\mathbf{x}|^{q}\right)$.

The requirement that $A$ has eigenvalues with zero real-part may seem unduly restrictive; however, by introducing a new variable which parameterises how far the eigenvalues are from having zero real-part, as in a bifurcation, the theory may be applied directly.*

These results have been used in a variety of contexts. Just some of which I am aware are: thermo-haline convection by Coullet \& Spiegel (1983); Couette-Taylor problem by Chossat \& Iooss (1985) and Laure \& Demay (1988); wave fronts by Eckmann \& Wayne (1990); tearing modes in magneto-hydrodynamics Grauer (1989); dispersion in rivers by Mercer \& Roberts (1990) and Young \& Jones (1991); rod equations in elasticity by Mielke (1988) and Roberts (1990); long time transients in Markov chains by Pollett \& Roberts (1990); Couette-Poiseuille flow by Renardy (1989); Kuramoto-Sivashinsky dynamics by Armbruster, Guckenheimer \& Holmes (1989) and Roberts (1990); synchronous machine dynamics by Kokotovic \& Sauer (1989); sideband stability theory by Cheng \& Chang (1990); and capturing chaos by Meron \& Procaccia (1986), Procaccia (1988) and Oats \& Roberts (1991). Most of these are straightforward applications of the theory. However, I have also developed extensions of the theory which make it applicable to an enormously large number of approximations.

\subsubsection{Slowly-varying space and time approximations}

One generalisation of centre manifold theory is to the derivation amplitude equations for systems with a continuum of marginal modes, rather than the finite number of discrete modes required by the simple theory. For example, the Taylor theory of shear dispersion in pipes and rivers, that

$$
\frac{\partial C}{\partial t}=-U \frac{\partial C}{\partial x}+D \frac{\partial^{2} C}{\partial x^{2}}
$$

where $C(x, t)$ is the cross-stream average of the detailed concentration $c(x, y, t)$, may be derived in Fourier space (Mercer \& Roberts, 1990) using the rigorous theory (where we also show convergence); however, it is more appealing to do it directly from the original physically based equations (Roberts,1988). The details of such a derivation are described in the lecture slow methods for simple people where the nonlinear Scrödinger equation is derived for the

* Centre manifold reduction may be generalised to dynamics where the retained long-term modes $\mathbf{x}$ also include exponential decaying or growing modes. For example, the system $\dot{x}=\lambda x-x y$ and $\dot{y}=-y+x^{2}-2 y^{2}$ has an invariant manifold $y=x^{2} /(1+\lambda)$ which plays the role of the centre manifold for small but finite $\lambda$. This approach is discussed in Roberts (1989) and leads to more flexibility at a cost of increased complexity in application. It is related to the concept of inertial manifolds, see Temam (1990). 
evolution of slowly-varying dispersive waves which are governed by the cubic-nonlinear KleinGordon equation.

The essence of the method is to imagine that the "amplitude" of the solution is slowly-varying in $x$, that is, $\partial / \partial x$ is analogous to a small ordering parameter. Such an application of the procedure would also be able to derive: the Kuramoto-Sivashinsky equation; the KortewegdeVries equation and Boussinesq equations for long waves; the Ginzburg-Landau equation for evolution and modulation of rolls.

\subsubsection{Initial conditions}

A question which is often overlooked when forming low-dimensional models is: what initial conditions should be used with the model? In general, a given initial condition for the full system will be off the low-dimensional centre manifold. Then the task is to project the given initial condition onto the centre manifold to provide an initial condition for the centre manifold model. The usual heuristic is simply to neglect the $\mathbf{y}$ component of the initial condition, this is equivalent to projecting parallel to the $\mathbf{y}$ axes. However, this does not take any account of the nonlinear dynamics away from the origin. For example, in shear dispersion this heuristic would lead to $C_{0}(x)$ being a delta-function for a point release in the river or pipe, but such a delta-function cannot predict some of the significant long-term effects of shear dispersion. A method of projecting which takes into account some of the dynamics of the system has to be used.

For example, the simple equation (13.1) may be integrated numerically to obtain the trajectories shown in the figure. Also plotted are the isochronic manifolds consisting of all the initial conditions which evolve exponentially quickly to the same long-term behaviour. Thus, any given initial condition for the full system should be projected along the appropriate isochronic manifold onto the centre manifold to give, implicitly, the correct initial condition for the low-dimensional model on the centre manifold.

In practise it is sufficiently accurate to project along the tangents of the isochronic manifold, evaluated where they cross the centre manifold. The algebraic details of finding such a projection is similar to that needed to find the centre manifold in the first place and may be found in the article by Roberts (1989). In the shear dispersion problem this technique for finding the appropriate initial condition gives

$$
C_{0}(x)=\overline{c_{0}}+\frac{\partial}{\partial x} \overline{v_{1}(y) c_{0}}+\frac{\partial^{2}}{\partial x^{2}} \overline{v_{2}(y) c_{0}}+\cdots
$$

where $c_{0}(x, y)$ is the initial given concentration, and $v_{1}(y)$ and $v_{2}(y)$ are derived cross-stream structure functions. The terms on the right-hand side are precisely what the Taylor model, and its higher order generalisations, require in order to accurately match the long-term behaviour of the displacement and the variance of the concentration distribution. 


\subsubsection{Forced dynamics}

Centre manifold theory traditionally applies only to autonomous differential equations. However, many practical problems involve a forcing of some sort. For simple systems such as (13.1) computer simulation again shows that the concept of the centre manifold as containing the long-term dynamics of the system still holds. But the location of the manifold is altered, and so is the low-dimensional dynamics on the manifold.

A simple argument to obtain the forced evolution of a low-dimensional model, described by $\dot{\mathbf{s}}=\mathbf{g}(\mathbf{s})+\mathbf{F}^{M}(\mathbf{s}, t)$, from the full forced dynamics, say $\dot{\mathbf{u}}=\mathbf{f}(\mathbf{u})+\mathbf{F}(t)$, is as follows. Imagine that the full forcing is decomposed into a series of delta-function impulses, $\mathbf{F}(t)=$ $\sum_{\ell} \mathbf{F}_{\ell} \delta\left(t-t_{\ell}\right)$. Between each impulse the system evolves autonomously and, according to centre manifold theory, approaches the centre manifold exponentially quickly and then evolves slowly. The effect of each impulse is to "kick" the system a small distance off the manifold so that each force-free interval is started a little distance away from the manifold. However, the previous subsection showed how to project an initial condition off the manifold back onto the manifold; thus each impulse of the full system corresponds to a derivable impulse of the low-dimensional centre manifold model. These in turn correspond to a forcing $\mathbf{F}^{M}(\mathbf{s}, t)$ for the model.

For example, if a forcing $\left(F_{x}(t), F_{y}(t)\right)$ is applied to the system (13.1) then the appropriate forcing of the one-dimensional centre manifold dynamics is $\dot{s}=-s^{3}+\left(1+2 s^{2}\right) F_{x}-s F_{y}$ where $x \approx s$ and $y \approx s^{2}$. If we consider the case of the weak vertical forcing, $F_{x}=0$ and $F_{y}=-\epsilon^{2}$ (which would normally be ignored in most approaches as it is entirely in the stable $y$ direction), then we find that the origin is destabilised in favour of two fixed points at $x= \pm \epsilon$. Astoundingly a small vertical force has made a relatively large change to the long-term dynamics. None-the-less this prediction is correct as may be readily seen in the full equations.

The above argument derives an appropriate change to the evolution equation in the presence of forcing. However, it does not determine where the attracting "centre manifold" has been moved. Arguments based on the natural consequences of the forcing have been developed and are reported in the article by Cox \& Roberts (1991).

\subsubsection{Boundary conditions}

A large number of mathematical models of dynamics have been based on the assumption that the critical determining amplitudes vary slowly in space, as discussed in an earlier subsection. These models are typically derived under the assumption that the domain of interest is arbitrarily large; however, typical solutions and physical problems of interest possess finite domains. What are the correct boundary conditions to be used at the edge of the domain for these classes of models?

Based on the treatment of boundary conditions for shear dispersion by Smith (1988), a general rational approach may be developed using centre manifold theory (Roberts, 1991). The essence of the approach is to understand a little of the dynamics of the boundary 
layers which occur at the boundaries of the domain due to any mismatch between the actual boundary conditions and the slowly-varying solutions of the centre manifold. To use the concepts described above we must interchange the role of space and time by imagining that the spatial variable $x$ is "time-like" and that the true time $t$ has the perturbation status of a slowly-varying parameter. Consequently boundary layers become exponential transients decaying towards the slow variations in the interior. Thus some boundary conditions for the model may be obtained by appropriately projecting the conditions at the end onto the centre manifold of the model - a technique that for temporal evolution is described above. Further boundary conditions for the model may be obtained by recognising that any rapid exponential transients present in the model equations do not actually model physical modes of the original dynamics and so additional conditions should be used to eliminate them. These two principles are generally sufficient to supply a complete set of boundary conditions for such slowly-varying low-dimensional models.

\subsection{Planform evolution as an embedded centre manifold}

In convection, the governing equation may be something like

$$
\frac{\partial \mathbf{u}}{\partial t}=R \mathbf{u}+\mathbf{u} \cdot \nabla \mathbf{u}+\nabla^{2} \mathbf{u}
$$

where the $R \mathbf{u}$ term represents unstable influences, $\mathbf{u} \cdot \nabla \mathbf{u}$ represents a nonlinearity, and $\nabla^{2} \mathbf{u}$ represents the dissipative terms. For small $R$ there is no motion, i.e. $\mathbf{u}=0$. For $R>R_{c}$, motion sets in and is typically in the form of convective rolls. But the equations are isotropic in the horizontal directions and so the rolls could have any orientation: which is chosen? Further, how do patches of rolls interact when the different patches have different alignment? What is the long-term pattern of the convection: rolls, squares, hexagons or chaos? In these questions, convection is a prototype of the self-organisation behaviour in dynamical systems with many competing modes.

The challenge is now to try to use centre manifold reduction to derive good models of the evolution of the convective planform. In doing so we find that a direct application fails, and in order to proceed an extension of the centre manifold approach has to be developed.

\subsubsection{Linear picture}

If we seek solutions of the form $\mathbf{u}=\mathbf{f}(z) \exp (\lambda t+i \mathbf{k} \cdot \mathbf{x})$ in the linearised equations we obtain the eigen-problem

$$
\lambda \mathbf{f}=R \mathbf{f}+\left(\partial_{z}^{2}-k^{2}\right) \mathbf{f} .
$$

For a given $R$, say just $>R_{c}$, the spectrum of eigen-values as a function of wavenumber $k$ are shown in the following figure. 


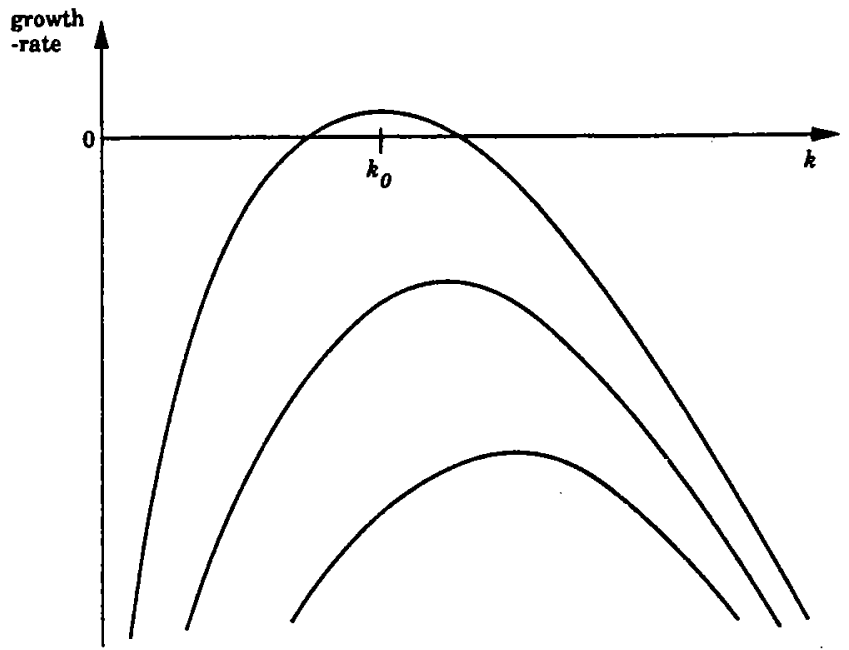

Figure 13.2. Spectrum of a typical convection problem near the onset of motion as a function of horizontal wavenumber $k$. The dashed line is the spectrum of a model discussed later.

For $R$ at the critical value $R_{c}$ the linearly marginal modes are those with some horizontal wavenumber, $\mathbf{f}(z) \exp (i \mathbf{k} \cdot \mathbf{x})$ such that $|\mathbf{k}|=k_{c}=1$ say. Often these marginal modes are called master modes as all other modes have a negative growth rate, are thus exponentially damped, and therefore only present due to relatively weak nonlinear forcing.

\subsubsection{The centre manifold ansatz implies zero-divisors}

The centre manifold ansatz is to pose that

$$
\mathbf{u}=\text { "master" }+\mathbf{h}(" \text { master") }
$$

where $\mathbf{h}$ is purely composed of the damped modes. Since the marginal modes all have a wavenumber with magnitude 1 , they can be parameterised by the angle $\phi$ of the wavenumber vector, for $0 \leq \phi<2 \pi$, and hence

$$
\mathbf{u}=\int_{0}^{2 \pi} A(\phi, t) \exp i(x \cos \phi+y \sin \phi) d \phi+\mathbf{h}[A(\phi, t)]
$$

where the independent amplitudes $A(\phi, t)$ evolve according to some functional equation

$$
\frac{\partial A}{\partial t}=G[A(\phi, t)]
$$

A consequence of the nonlinearity in the problem, say cubic, is that in order to find the nonlinear shape of the centre manifold we would have to solve an equation of the form

$$
\begin{gathered}
\mathcal{L} \mathbf{h}=\int G \exp i(x \cos \phi+y \sin \phi) d \phi+\iiint A\left(\phi_{1}, t\right) A\left(\phi_{2}, t\right) A\left(\phi_{3}, t\right) \times \\
\times \exp i\left[x\left(\cos \phi_{1}+\cos \phi_{2}+\cos \phi_{3}\right)+y\left(\sin \phi_{1}+\sin \phi_{2}+\sin \phi_{3}\right)\right] d \phi_{1} d \phi_{2} d \phi_{3}
\end{gathered}
$$


where the operator $\mathcal{L}$ is necessarily singular as it has the marginal modes as nontrivial homogeneous solutions. Following the standard centre manifold procedure, the right-hand side can be put into the range of $\mathcal{L}$ by choosing $G$ appropriately (and this determines the evolution of the amplitudes $A)$. But, there exist wavenumbers in the right-hand side, $\left(\cos \phi_{1}+\right.$ $\left.\cos \phi_{2}+\cos \phi_{3}, \sin \phi_{1}+\sin \phi_{2}+\sin \phi_{3}\right)$ arbitrarily close to the critical circle $|\mathbf{k}|=1$. Thus in inverting $\mathcal{L}$ to find the centre manifold, we would in essence be dividing by numbers arbitrarily close to zero. Hence the expansion has unbounded coefficients and is of no use.

Such divisions by (near) zero are a long-standing problem. They also occur in the nonlinear interactions among a continuous spectrum of waves.

\subsubsection{Embedding the centre manifold}

The problem in the above attempt to use centre manifold techniques is that there is no clear distinction between the long-lasting "master" modes, and the exponentially decaying "slave" modes. This can be seen in the earlier figure where the upper branch of eigen-values moves continuously from exponentially damped modes through the important marginal modes, and down again.

However, if we can construct a model whose linear spectrum is the dashed line shown in the earlier figure. That is, for wavenumbers near the critical, the model's spectrum must asymptotically match that of the exact system to some order, while away from marginal it is strictly negative. Thus, many of the branches in the original spectrum are definitely "slaves" and are ignored except for their modifying influence; while the principle branch is accurately modelled in the vicinity of the marginal modes. The modes of the principle branch which are away from critical are linearly inaccurate "dead wood" modes; however, our aim is to ensure these are accurate in the long-term evolution. In essence, the important "centre manifold" is embedded in a larger dynamical system which is none-the-less considerably simpler than the original full system.

A simple ordinary differential equation example is

$$
\dot{x}=-y(x+z), \quad \dot{y}=-\mu y+x^{2}-z^{2}, \quad \dot{z}=-z+x y .
$$

where $\mu$ is a parameter $0<\mu<1$. This system has a centre manifold given by $y \approx x^{2} / \mu$ and $z \approx x^{3} / \mu$. However, if $\mu$ is small so that it is hard to distinguish whether it is a long-lived master mode or an exponentially damped slave mode, then the centre manifold is highly curved as is shown by the small divisors. But we can embed the centre manifold in the much smoother embedding manifold $z \approx x y$, thus forming the two-dimensional model

$$
\dot{x}=-x y(1+y), \quad \dot{y}=-\mu y+x^{2}-x^{2} y^{2} \text {. }
$$

To do this embedding in general we resort to adiabatic iteration. Suppose the system may be written formally as

$$
\dot{\mathbf{x}}=A \mathbf{x}+\mathbf{f}(\mathbf{x}, \mathbf{z}), \quad \dot{\mathbf{z}}=C \mathbf{z}+\mathbf{h}(\mathbf{x}, \mathbf{z})
$$


where $\Re\left\{\lambda_{A}\right\} \leq 0$ and $\Re\left\{\lambda_{C}\right\} \leq-\gamma<0$. That is, the $\mathbf{x}$ modes contain some exponentially decaying "dead wood." Approximations to an embedding manifold $\mathbf{z}=\mathbf{Z}(\mathbf{x})$ may be found by the iteration scheme

$$
\mathbf{Z}^{(0)}(\mathbf{x})=\mathbf{0}, \quad C \mathbf{Z}^{(k+1)}=-\mathbf{h}\left(\mathbf{x}, \mathbf{Z}^{(k)}\right)+\frac{\partial \mathbf{Z}^{(k)}}{\partial \mathbf{x}}\left[A \mathbf{x}+\mathbf{f}\left(\mathbf{x}, \mathbf{Z}^{(k)}\right)\right] .
$$

As established in Roberts (1991) the approximate evolution equation

$$
\dot{\mathbf{x}}=A \mathbf{x}+\mathbf{f}\left(\mathbf{x}, \mathbf{Z}^{(k)}(\mathbf{x})\right)
$$

models accurately the long-term behaviour of the original system.

This supports the often used adiabatic approximation and gives it an appealing geometric picture.

\subsubsection{A toy convection problem}

To see how these ideas may apply to convection, consider the simple problem

$$
\begin{aligned}
& \frac{\partial a}{\partial t}=R a-\left(1+\nabla^{2}\right)^{2} a-a b \\
& \frac{\partial b}{\partial t}=R b-\left(1-\nabla^{2}\right)^{2} b+a^{2}
\end{aligned}
$$

which at the critical value of $R=0$ has a spectrum $\lambda_{a}=-\left(1-k^{2}\right)^{2}$ which becomes zero at the wavenumber $k=1$ and is negative everywhere else; and $\lambda_{b}=-\left(1+k^{2}\right)^{2}$ which is everywhere negative and bounded away from zero $(-\gamma=-1)$.

Simply pose that $b=\mathcal{B}(a)$ is the embedding manifold and iterate

$$
\mathcal{B}^{(0)}=0, \quad\left[\left(1-\nabla^{2}\right)^{2}-R\right] \mathcal{B}^{(k+1)}=a^{2}-\frac{\partial \mathcal{B}^{(k)}}{\partial a}\left[\left(R-\left(1+\nabla^{2}\right)^{2}\right) a-a \mathcal{B}^{(k)}\right]
$$

The first iterate is sufficient for many purposes and gives $\mathcal{B}^{(1)}=\mathcal{G} \star a^{2}$ where $\star$ denotes convolution and $\mathcal{G}$ is a radially symmetric Green's function. For example, when $R=0$, $\mathcal{G}(\mathbf{x})=K_{0}(|\mathbf{x}|) \star K_{0}(|\mathbf{x}|) /\left(4 \pi^{2}\right)$. Thus the accurate model is

$$
\frac{\partial a}{\partial t}=\left[R-\left(1+\nabla^{2}\right)^{2}\right] a-a \mathcal{G} \star a^{2}
$$

which has the unusual feature of being a nonlocal evolution equation.

The frequently used Swift-Hohenberg (1977) approximation replaces $\mathcal{G}$ by the delta-function spike with the same volume underneath (see Brand (1988), Cross \& Newell (1984), Cross \& Tesauro (1986), Greenside \& Coughran (1984), and Pomeau \& Manneville (1979)). Thus, here the Swift-Hohenberg approximation would be

$$
\frac{\partial a}{\partial t}=\left[R-\left(1+\nabla^{2}\right)^{2}\right] a-a^{3} /(1-R)
$$


In numerical solutions of the exact system and the embedded centre manifold model there was found to be almost no discernable difference in the evolution, see the figure below. In contrast the evolution of the Swift-Hohenberg model is markedly different to that of the exact system. Although they both seem to ultimately evolve towards a two-dimensional roll structure, they do it in significantly different ways. Firstly, the Swift-Hohenberg model of convection is of a significantly lower amplitude than the exact system. Secondly, the SwiftHohenberg model evolves to convective rolls much more quickly than the exact system which has a strong tendency to linger in a square pattern for a long time. Indeed the last defect in the Swift-Hohenberg model has just disappeared at the last time shown of $t=310$, while in both the exact system and the embedded centre manifold approximation the last defect does not disappear until after time $t=1000$.
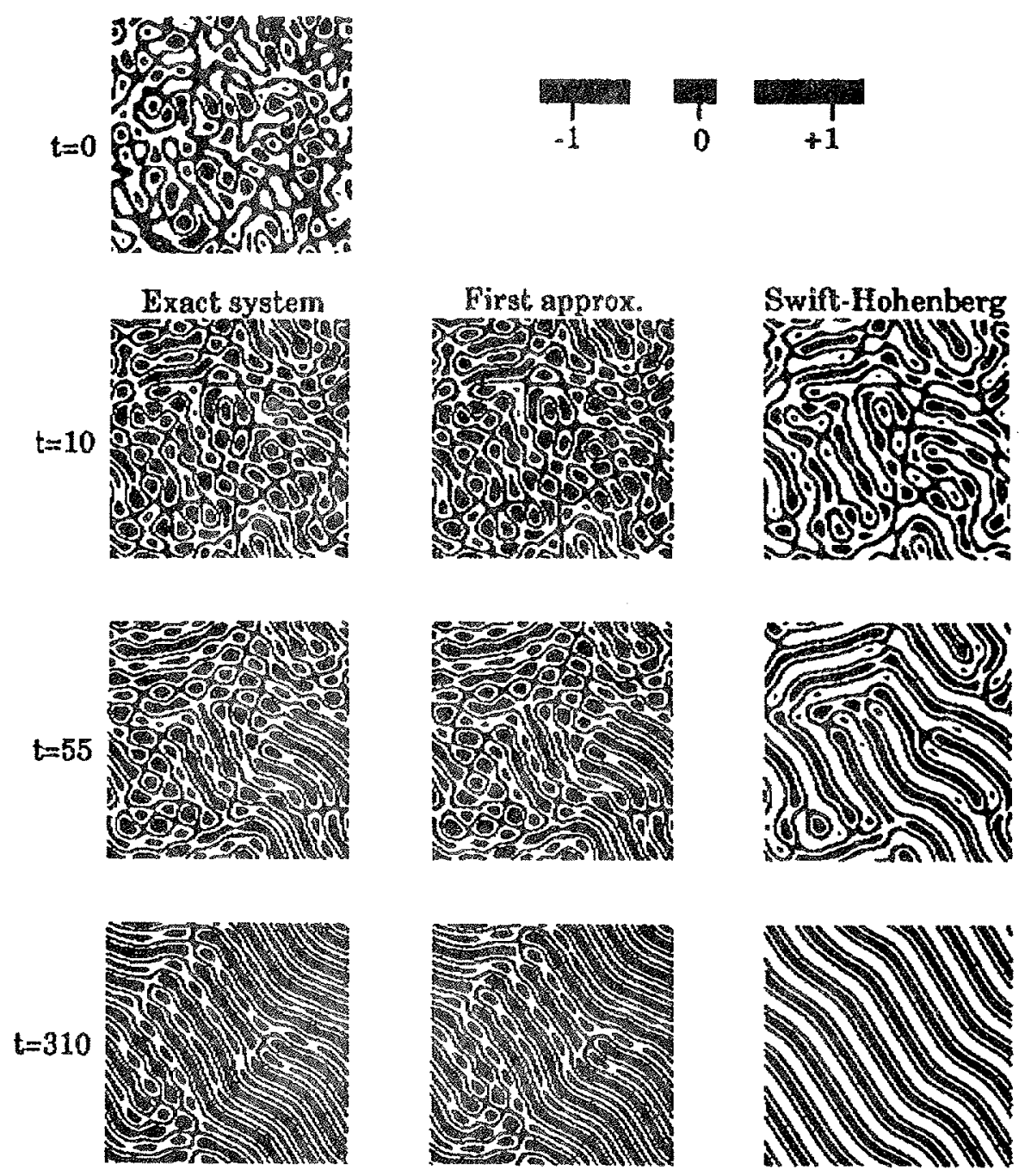

Figure 13.3. Comparison of the exact evolution of the planform and the two types of approximation: the nonlocal embedded centre manifold; and the localising SwiftHohenberg. All simulations were started at $t=0$ with the planform shown at the top-left. 


\subsection{Conclusion}

Centre manifold theory provides a systematic approach to the derivation of low-dimensional models. Not only is it supported by an appealing geometric picture, it is also superior to other methods in that it provides suitable initial conditions, boundary conditions and forcing.

In planform problems a direct application of centre manifold theory fails, but the process of embedding the centre manifold works very well. In using adiabatic iteration, this embedding of centre manifolds gives clear geometric support to the often used adiabatic approximation method. Lastly, the frequently used Swift-Hohenberg approximation may not be a good model of planform evolution.

\section{References}

D. Armbruster, J. Guckenheimer \& P. Holmes "Kuramoto Sivashinsky dynamics on the center-unstable manifold" SIAM J Appl Math 49 (1989) pp676-691.

H.R. Brand "Phase dynamics - a review and a perspective" pp206-224 in Propagation in systems far from equilibrium ed. by Wespeid et al, Springer-Verlag (1988).

P. Chossat \& G. Iooss "Primary and secondary bifurcations in the Couette-Taylor problem" Jap J Appl Math 2 (1985) 37-68.

P.H. Coullet \& E.A. Spiegel "Amplitude equations for systems with competing instabilities" SIAM J Appl Math 43 (1983) pp776-821.

M.C. Cross \& A.C. Newell "Convection patterns in large aspect ratio systems" Physica $D$ 10 (1984) pp299-328.

M.C. Cross \& G. Tesauro "Wavenumber selection and persistent dynamics in models of convection" Physica D 23 (1986) pp12-18.

J.P. Eckmann \& C.E. Wayne "Propagating fronts and the center manifold theorem" preprint (1990).

M. Cheng \& H.C. Chang "A generalised sideband stability theory via centre manifold projection" Phys Fluids A 2 (1990) pp1364-1379.

S.M. Cox \& A.J. Roberts "Centre manifolds of forced dynamical systems" J Austral Maths Soc Ser B 32 (1991) pp401-436.

R. Grauer "Nonlinear interactions of tearing modes in the vicinity of a bifurcation point of codimension two" Physica D 35 (1989) pp107-126.

H.S. Greenside \& W.M. Coughran, Jr. "Nonlinear pattern formation near the onset of Rayleigh-Bénard convection" Physical Review A 30 (1984) pp398-428.

P.V. Kokotovic \& P.W. Sauer "Integral manifolds as a tool for reduced-order modelling of nonlinear systems: a synchronous machine case study" IEEE Trans circuits \& Systems 36 (1989) pp403-410. 
P. Laure \& Y. Demay "Symbolic computation and equation on the centre manifold: application to the Couette-Taylor problem" Computers and fluids 16 (1988) pp229-238.

G.N. Mercer \& A.J. Roberts "The application of centre manifold theory to the dispersion of contaminant in channels with varying flow properties" SIAM J. Appl. Math. 50 (1990) pp1547-1565.

E. Meron \& I. Procaccia "Theory of chaos in surface waves: the reduction from hydrodynamics to few-dimensional dynamics" Phys Rev Lett 56 (1986) pp1323-1326.

A. Mielke "On Saint-Venant's problem and Saint Venant's principle in nonlinear elasticity" Trends in Appl Of Maths to Mech (1988) pp252-260.

R.G. Muncaster "Invariant manifolds in mechanics I: The general construction of coarse theories from fine theories" Arch. Rat. Mech. 84 (1983) pp353-373.

P.K. Pollett \& A.J. Roberts "A description of the long-term behaviour of absorbing continuous time Markov chains using a centre manifold" Adv in Appl Prob 22 (1990) pp111-128.

Y. Pomeau \& P. Manneville "Stability and fluctuations of a spatially periodic convective flow" J. Phys. Lett. 40 (1979) pp609-612.

I. Procaccia "Universal properties of dynamically complex systems: the organisation of chaos" Nature 333 (1988) pp618-623.

Y. Renardy "Weakly nonlinear behaviour of periodic disturbances in two-layer CouettePoiseuille flow" Phys Fluids A 1 (1989) 1666-1676.

A.J. Roberts "Simple examples of the derivation of amplitude equations for systems of equations possessing bifurcations" J. Austral. Math. Soc. Ser. B 27(1985) pp48-65.

A.J. Roberts "The application of centre manifold theory to the evolution of systems which vary slowly in space" J. Austral. Math. Soc. Ser. B 29(1988) pp480-500.

A.J. Roberts "Appropriate initial conditions for asymptotic descriptions of the long term evolution of dynamical systems" J. Austral. Math. Soc. Ser. B 31(1989) pp48-75.

A.J. Roberts "The utility of an invariant manifold description of the evolution of dynamical systems" SIAM J. of Math. Anal. 20 (1989) pp1447-1458.

A.J. Roberts "The invariant manifold of beam theory. Part 1: the simple circular rod" preprint (1990).

A.J. Roberts "Boundary conditions for approximate differential equations" $J$ Austral Math Soc $B$ (1991) to appear.

A.J. Roberts "Planform evolution in convection-an embedded centre manifold" $J$ Austral Math Soc B (1991) to appear.

R. Smith "Entry and exit conditions for flow reactors" IMA J Appl Math 41 (1988) pp1-40. J. Swift \& P.C. Hohenberg "Hydrodynamic fluctuations at the convective instability" Physical Review A 15 (1977) pp319-328.

R. Temam "Inertial manifolds" Math Intelligencer 12 (1990) pp68-74.

W.R. Young \& S.W. Jones "Shear dispersion" Phys Fluids A (1991). 


\section{Fundamentally Discrete Patterns in Geomorphol- ogy}

B.T. Werner

Center for Coastal Studies 0209 Scripps Institution of Oceanography La Jolla, California 92093-0209

\subsection{Introduction}

The Earth's surface largely is composed of material rearranged by processes associated with tectonic, fluvial, eolian and gravitational forces. These processes frequently are characterized by fluctuations and lack of regularity. In contrast, the resulting landscape often is observed to be in the form of regular patterns with a definite length scale or spacing. The focus of this paper will be a model in which strong feedbacks produce patterns of a definite size in the presence of fluctuations. In this model, the scale of fluctuations and the macroscopic scale remain coupled. Hence, a spatial continuum approximation for the evolution of the landscape pattern spacing fails to capture this key lack of separation between macroscopic and microscopic scales. The application of the model to landform development is illustrated with the example of wind ripples.

\subsection{Pattern Formation Model}

The following model describes a system in which a definite length scale, proportional only to the minimum discrete size of the system, emerges in a self-organized manner. The process by which this occurs is termed stochastic merging. The model and its results are discussed further in Werner et al. (1991).

Consider a ring containing discrete, uniform slots which are filled with links of extended objects termed here "worms," as illustrated in Figure 1. Each worm is characterized by the number of links comprising the worm, $w_{i}$. Time is measured in discrete steps of unit length. At each time step, the head of the ith worm moves forward (clockwise) one link with probability $\beta / w_{i}, 0<\beta<1$.

The evolution of this system can be described in terms of two opposing trends. The tendency is for the system to move toward worms of uniform size. For example (Figure 1), if a worm of length 2 is directly behind a worm of length 10 , the probability that the shorter worm's head moves forward while the larger worm's head remains stationary is $(\beta / 2)(1-\beta / 10)$. Conversely, the probability that the larger worm grows by one link in that time step is $(\beta / 10)(1-\beta / 2)$, roughly a factor of 5 less if $\beta<1$. The probabilities favor the larger worm decreasing in size and the smaller worm increasing in size until the sizes become equal. 


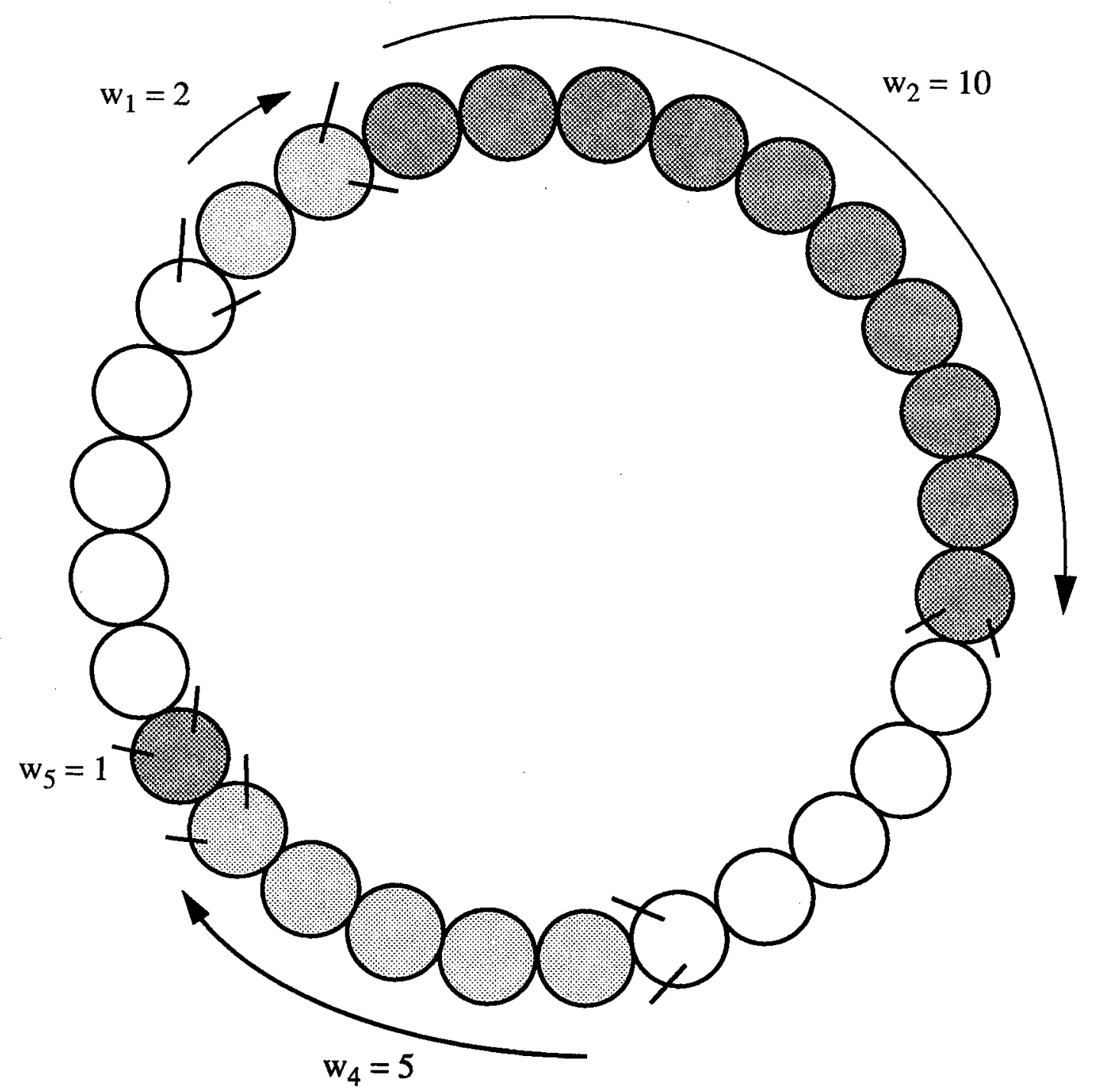

Figure 14.1. The worm model for stochastic merging. The head of a worm moves one link clockwise with probability $\beta / w$, with $w$ its length measured in number of links. The tendency is for worms to become more nearly equal in size. If the head of the worm of length 5 moves forward while the head of the worm of length 1 does not (with probability $(\beta / 5)(1-\beta)$ ), the two worms merge.

Fluctuations around this mean behavior can lead to reduction in the overall number of worms through merger between worms. For example, for a worm of length 1 followed by a worm of length 5 (Figure 1), there is a probability $(\beta / 5)(1-\beta / 1)$ that the shorter worm's length is reduced to zero on the next time step, representing an irreversible merger between the two worms. In this case, the number of worms on the ring is reduced by 1 from $N$ to $N-1$, and the mean size of a worm increases by the factor $N / N-1$.

The evolution of the system can be described in terms of two key variables: the mean size of a worm $\langle w\rangle=L / N$ and the standard deviation of the worm size $\sigma_{w}$. The former quantity documents the frequency of mergers, whereas the latter quantity measures the degree to 
which the system has achieved uniform worm size. Adopting the approximation that the behavior of a worm can be described in terms of its interactions with worms of the mean worm size only (roughly a mean field approximation), coupled with the assumption that $\beta<<1$, gives, via one-dimensional Markov Chain calculations:

$$
<w>=1 \cdot \ln (\beta t+k) \sigma_{w} / w=w^{-1 / 2},
$$

with $t$ the number of time steps and $k$ a time-integration constant. The 1 in front of the logarithm emphasizes that the mean worm size is measured in numbers of links. The mean worm size appears to stabilize because of the logarithm, depending proportionally only on the link size. In addition, the mean worm size becomes more uniform with time.

It is important to note that the evolution of the system is determined by mergers and that in the continuum limit, $w>>1$, the mergers cease: $w->$ constant in time and $\left(\sigma_{w} / w\right)->0$. A merger is an event which probes the smallest relevant scale of the system, i.e., a decision is made when $w=1$ whether to merge or not to merge. Hence, a continuum approximation does not incorporate the essential interesting feature of the worm system, namely the mergers. Scale separation between the smallest fluctuation size, the worm link size, and the mean size of the worms after a long period of time cannot be achieved. This contrasts with problems treatable by continuum mechanics, such as a fluid, where the macroscopic behavior is not sensitive to the microscopic details, except through bulk (averaged) parameters such as viscosity. Scaling the microscopic elements while keeping the bulk parameters constant has no effect on the fluid behavior. In contrast, doubling the size of the links precisely doubles the macroscopic scale (for constant $\beta$ ) in the stochastic merging worm model.

\subsection{Wind Ripples}

The motivation for developing the stochastic merging model described above came from studies of the mechanism by which wind ripples form. Grains on a sand surface are transported indirectly by the wind. The wind accelerates grains on long, low-angle hopping trajectories over the surface in saltation. Upon impact with the surface, these grains impart energy and momentum to the grains on the surface, causing a certain number to be ejected on short trajectories over which they have little interaction with the wind. This surface grain motion driven by the impacts of saltating grains is termed reptation. Theoretical and experimental arguments (e.g., Werner, 1990) strongly support the hypothesis that surface grains may be considered to be driven on short trajectories by randomly placed impacts of saltating grains.

Observations of the development of sand ripples from a flat bed exhibit the following approximate chronology (Sharp, 1963; Werner, 1987). First, the surface becomes mottled, with topography on the scale of a few grain diameters lacking significant visible organization. The topography develops into distinct grain piles which propagate in the direction of the wind and have a strong tendency to link with neighboring sand piles situated lateral to the wind direction. Two features of this propagation are important. First, the coherent propagation of a sand pile is made possible by the development of a shadow zone (Sharp, 1963) into 
which reptating grains moving over the ripple crest are deposited, and which is protected from direct impacts of saltating grains. Second, the mean propagation speed of a ripple is proportional to its cross-sectional size. Hence, a smaller ripple will begin to overtake and interact with a larger ripple located downwind.

The nature of this ripple-ripple interaction, when viewed in cross-section, is paramount in determining the evolution of the ripple field. When the overall size of the ripples is small, two interacting ripples often merge. As the ripples grow in size, the interaction tends to be in the form of an exchange of grains, where, as the small, trailing ripple climbs the upwind face of the large, leading ripple, it incorporates some fraction of the large ripple's grains, thereby increasing its size while decreasing the size of the large ripple. The originally large ripple, now the smaller of the two, then moves away from the trailing ripple. Frequently, this interaction will result in two ripples more nearly equal in size. These interactions have been observed both in the field (Werner, 1987) and in computer simulations (Werner, 1991). Tests performed in computer simulations of wind ripple development have shown that the mergers are driven by fluctuations in the rate of surface grain transport in reptation, related to the discrete, stochastic nature of the transport. The size of the ripples increases until it is large compared to the scale of the fluctuations of the sand surface elevation ( grain diameters). A plan view of a ripple formation simulation is shown in Figure 2.

\subsection{The Stochastic Merging Model Applied to Wind Ripples}

In order to relate the stochastic merging model described in the previous section to wind ripples, a wind ripple is idealized in cross-section as a triangular object of constant shape, meaning that the inclination angles of the upwind and downwind slopes of the ripple remain fixed throughout the evolution of the ripple field. A ripple propagates by shaving a thin slice from the upwind side and placing the same volume of sand on the downwind side in such a way as to preserve the upwind and downwind inclination angles, Figure 3a. The rate at which material is transported in this manner is determined by the reptation flux on the upwind side due to impacts of saltating grains.

In this model, merger between two ripples occurs when the depth of a trough between two ripple crests decreases to zero. Simulations and arguments based on the details of the model show that as the thickness of the slice transported on a ripple goes to zero, the probability of a merger over a fixed time period vanishes. Therefore, in the continuum approximation, there are no mergers.

The approximation that a worm is surrounded only by worms of the mean size is replaced in this idealized wind ripple model by the requirement that a ripple trough of depth $h$ is surrounded by ripple troughs of fixed depth equal to the mean ripple trough depth (or equivalently, the mean ripple height) $\langle h\rangle$, Figure $3 \mathrm{~b}$. Combining this assumption with the geometrical properties of triangular ripples leads to the following expression for the change in the trough depth $\Delta h$ in a time $\Delta t$ :

$$
\Delta h=2(\tan \alpha) F_{0} d^{3} s_{r} \Delta t\left(h^{-1}-<h>^{-1}\right),
$$




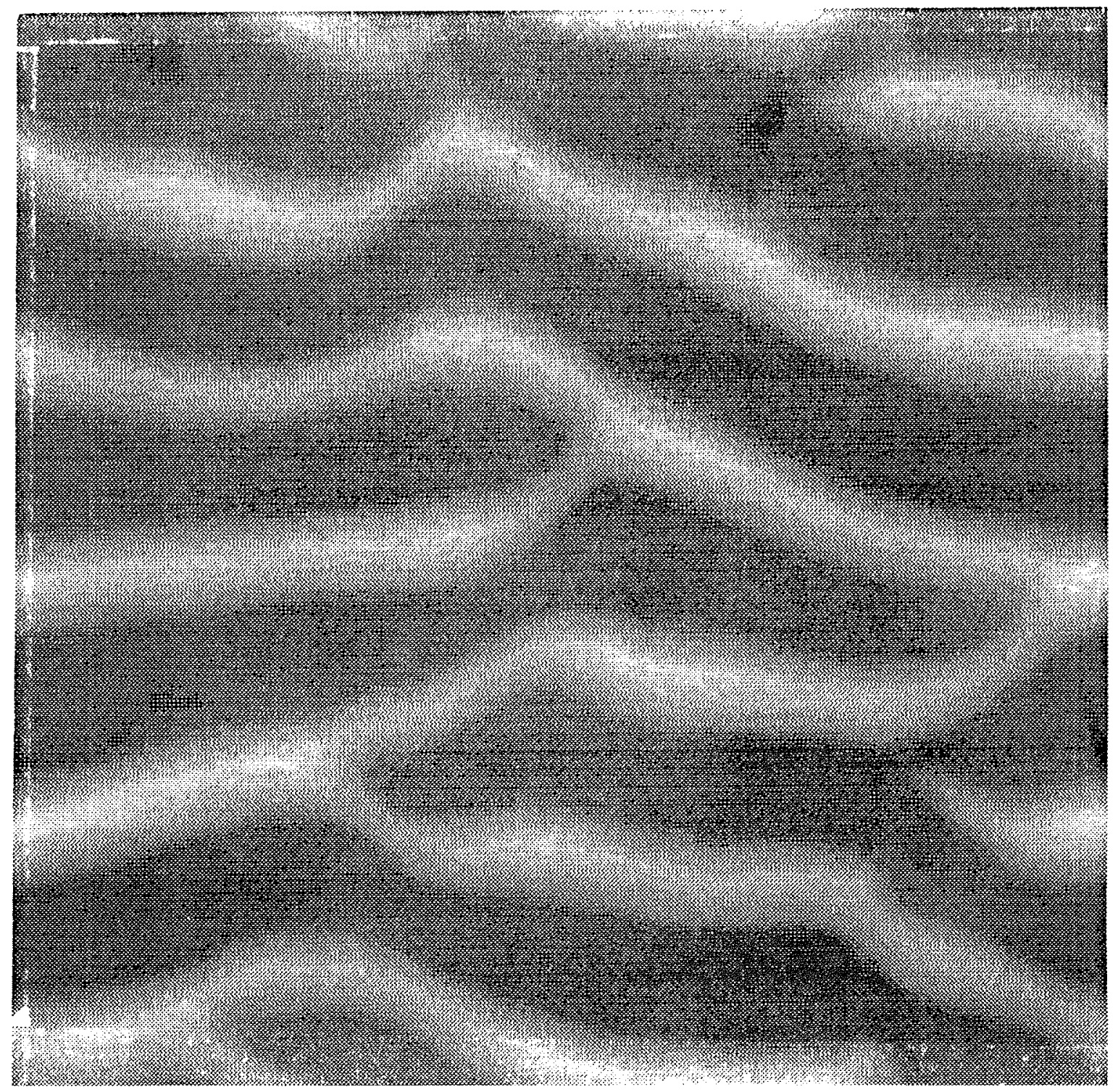

Figure 14.2. A plan view of three-dimensional ripples for a $1000 x 1000$ grain simulation after 20 saltating grain-bed impacts per surface grain. High topography is lighter. The saltating grains approach from bottom to top.

where $\alpha$ is the impact angle of saltating grains, $F_{0}$ is the saltating grain number flux, $d$ is the grain diameter and $s_{r}$ is the total distance surface grains are transported in reptation in the wind direction due to a single impact. The episodic, discrete nature of sand grain transport in reptation means that there is an effective minimum $\Delta h$, denoted by $D$. An approximate minimum value for $D$ is the grain diameter $d$, but various factors may increase $D$. The value appropriate for $D$ will be discussed in the next section. Assuming a particular value for $D$ is roughly equivalent to assuming that $h$ is a multiple of $D$. This leads to a mathematical equivalence between the time evolution of the depth of a ripple trough $h$ and the length of 


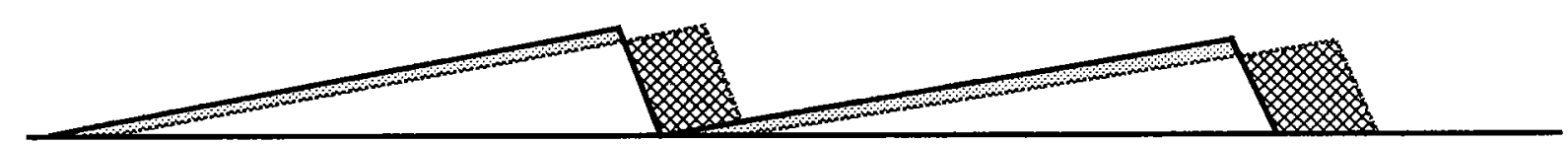

(a)

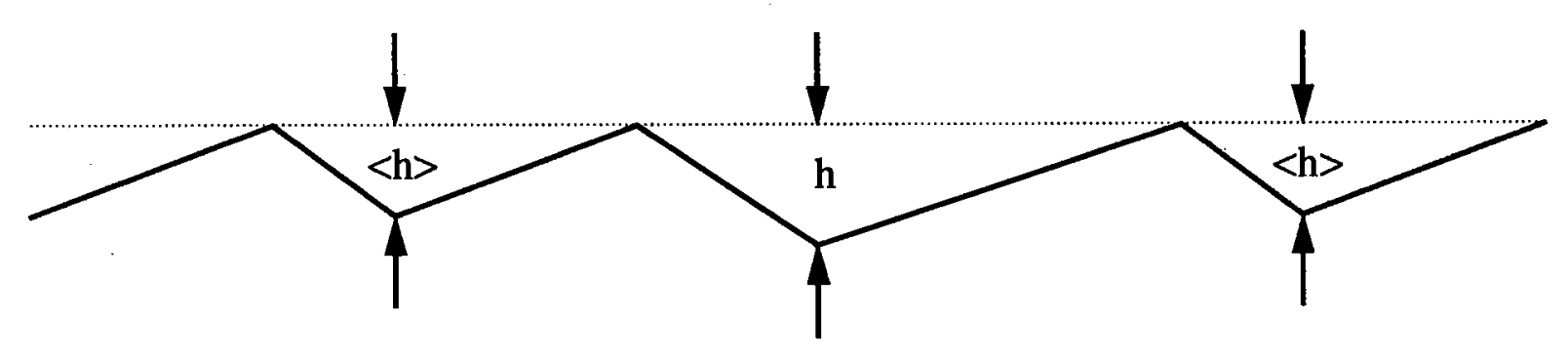

(b)

Figure 14.3. (a) Idealized cross-sectional ripple model. Ripples are approximated as triangles. A slab of sand is removed from the upwind face of the ripple (shaded) and deposited on the downwind face (cross- hatched). (b) Mean ripple approximation for the idealized cross-sectional ripple model. A single ripple trough of depth $h$ is surrounded by ripples forced to remain at the mean ripple trough depth (equivalently, the mean ripple height) $\langle h\rangle$. This model maps onto the stochastic merging worm model.

a worm $w$. Therefore, $\langle h\rangle$ increases logarithmically with time and the standard deviation of $h$ decreases relative to $\langle h\rangle$ :

$$
<h>=D \ln \left[2(\tan \alpha) F_{0} s_{r} d t+k\right], \sigma_{h} / h=<h>^{-1 / 2},
$$

with $t$ the time and $k$ a time-integration constant. Therefore, this model implies that the mean ripple height is directly proportional to the scale $D$, but that all other parameters of the problem are relatively unimportant for determining the height and therefore the spacing (for constant shape) of wind ripples.

\subsection{Discussion}

The stochastic merging model provides a means for obtaining the pattern of wind ripples characterized by a definite length scale without imposing that pattern or length scale directly on the system. One prediction is that the height (and therefore, roughly, the ripple spacing) should increase logarithmically with time. Figure 4 graphs the ripple spacing versus time for (1) a wind tunnel experiment at two wind speeds, (2) a field experiment and (3) a three-dimensional computer simulation experiment. The "time" in the computer simulation has been converted to minutes via some assumptions about saltation flux (Sorensen, 1985; 
Werner, 1991). The results of the wind tunnel and field experiments are to be considered preliminary in that the experimental setup and procedure were not described for the wind tunnel experiments and in that the wind was somewhat variable during the field experiment. Nonetheless, all three are roughly compatible with a logarithmic dependence of spacing on time. If it is assumed for the wind tunnel and field experiments that the ratio of spacing to height is 10 (a typical number), then the values of $D$ derived from the four graphs measured in grain diameters, $d$, (and rounded to the nearest grain diameter) are (1) wind tunnel experiment, $u *=30 \mathrm{~cm} / \mathrm{s}, D=1 d$, (2) wind tunnel experiment, $u *=41 \mathrm{~cm} / \mathrm{s}, D=4 d,(3)$ field experiment, $D=6 d$, and (4) computer simulation, $D=4 d$.

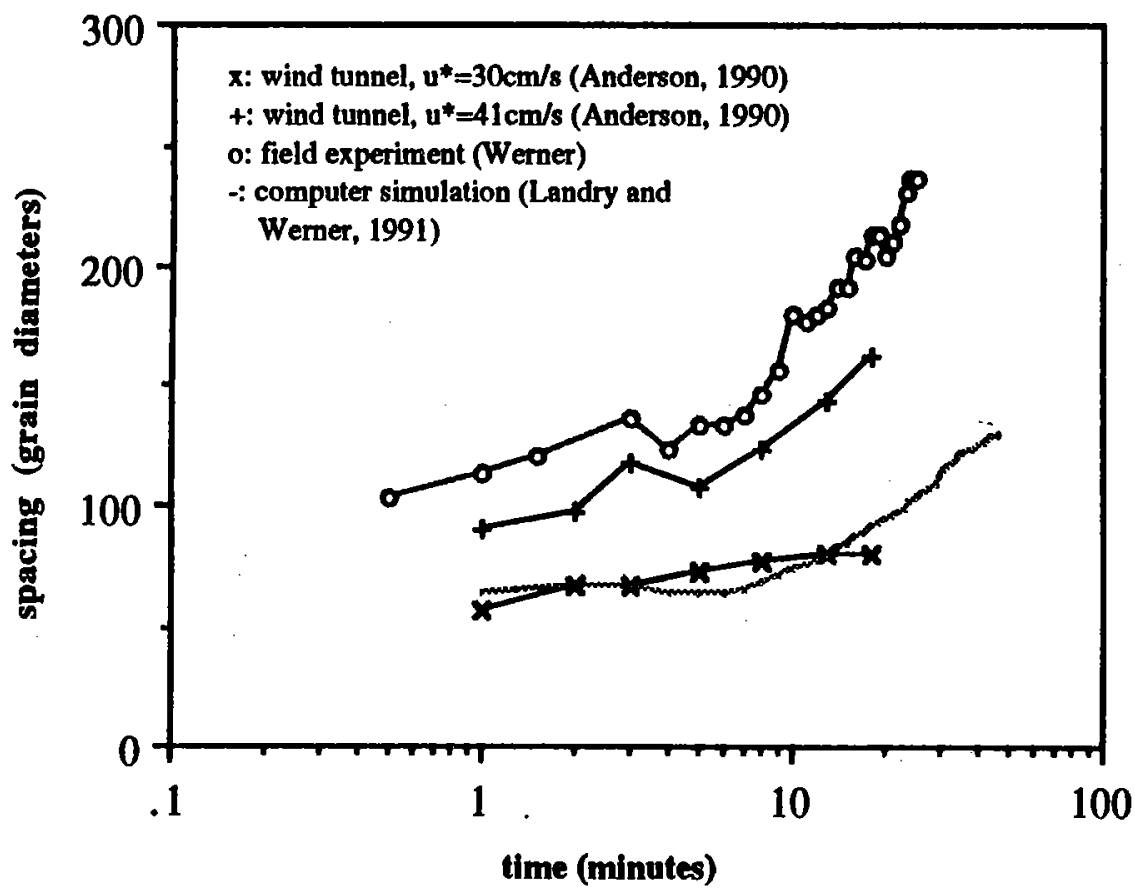

Figure 14.4. Mean ripple spacing versus time for four experiments: (a) Two wind tunnel experiments with $u *=30 \mathrm{~cm} / \mathrm{s}$ and $u *=41 \mathrm{~cm} / \mathrm{s}$ (Anderson, 1990); mean grain size $=0.03 \mathrm{~cm}$. (b) A field experiment (procedure described in Werner, 1987) at moderate, but varying wind speed (from $15-30 \mathrm{mph}$, mostly in the range $15-20 \mathrm{mph}$ ); mean grain size $=0.039 \mathrm{~cm}$. (c) A computer simulation experiment (Landry and Werner, 1991) with each grain-bed impact ejecting one grain 10 grain diameters "downwind." Number of grain-bed impacts in the simulation was converted to minutes of saltation using the data of Sorensen (1985) at $u *=40 \mathrm{~cm} / \mathrm{s}$.

The minimum physical value for $D$ is somewhat less than a grain diameter, because the ripple height can change only with the addition or removal of one grain to the ripple crest or to the ripple trough. However, the idealization to a fixed ripple shape used above limits the fluctuations in surface elevation to the ripple crest only. In Nature, the effect of fluctuations due to stochastic impacts acts in some integrated fashion to cause fluctuations in the positions of the ripple crest and trough around what the crest and trough positions would be in the 
absence of discrete, stochastic transport. Hence, values of $D$ greater than the grain diameter are expected. Moreover, ripple formation at high wind speeds should be characterized by a value of $D$ which is greater than at low wind speeds, because the range of saltating grain impact speeds increases with increasing wind speed, resulting in greater fluctuations in the ejection of grains into reptation and therefore in local elevation of the surface. The simulations also are expected to have a somewhat higher value of $D$ than the minimum observed in Nature (at low wind speeds) because the simulations confine grains on the surface to lie on a grid, which enforces local changes in topography to be order a grain diameter, in contrast to Nature, which can smooth granular surfaces to a greater degree. The observed values of $D$ are in agreement with these general comments and, although the experimental results are preliminary, the ability of stochastic merging to match the trends in the data, combined with the reliance in the simulations upon the basic, known physics of wind-blown sand transport support the stochastic merging model of wind ripple formation presented here.

Models of the development of other landform patterns have shown logarithmic dependence of landform size on time and a sensitivity of size scaling to fluctuations in transport. The patterns modeled include sorted stripes, alternating, downslope trending bands of fine and coarse grains which develop through grain transport by growth and toppling of needle ice (Werner and Hallet, 1991) and beach cusps, periodic arcuate scarping of the beach face in the swash zone under conditions of normally incident waves (Werner and Fink, 1991). The apparent ease with which systems develop patterns via stochastic merging observed in a wide variety of simulations by the author suggest that stochastic merging is a universal property of a certain class of physical systems. The interpretation given to the stochastic merging model here then implies that continuum models are not applicable to describing the pattern formation properties of these systems.

\subsection{Acknowledgements}

The author is grateful to his collaborators Daniel T. Gillespie, Peter K. Haff (on the worm model), Walter Landry, Jr. (on the wind ripple model) and to the attendees of the GFD program for their hospitality, comments and questions. Supported in part by the Mellon Foundation, the Office of Naval Research (N00014-91-J-1292) and the Vetlesen Foundation. 


\section{References}

R.S. Anderson (1990) Eolian ripples as examples of self- organization in geomorphological systems, Earth Science Reviews, 29, 77-96.

R.P. Sharp (1963) Wind ripples, J. Geology, 71, 617- 636.

M. Sorensen (1985) Estimation of some eolian saltation transport parameters from transport rate profiles, Proc. intl. workshop on the physics of blown sand, 1, Department of Theoretical Statistics, University of Aarhus, 141-190.

B.T. Werner (1987) A physical model of wind-blown sand transport, Ph.D. thesis, California Institute of Technology.

B.T. Werner (1990) A steady state model of wind-blown sand transport, J. Geology, 98, $1-17$.

B.T. Werner (1991) Computer simulation of sand surface self- organization in wind-blown sand transport, Sedimentology, in press.

B.T. Werner and T.M. Fink (1991) A model for beach cusps as self-organized patterns (Fall AGU abstract) EOS, submitted.

B.T. Werner and B. Hallet (1991) A stochastic model for sorted stripe formation, in preparation.

B.T. Werner, P.K. Haff and D.T. Gillespie (1991) A stochastic merging model of wind ripple formation, in preparation. 
Fellows Reports. 


\title{
15. Surface tension in immiscible lattice gases
}

\author{
Xiao-Liang Chen
}

Laboratory for Plasma Studies, Cornell University

\subsection{Abstract}

A surface tension formula is given for the immiscible lattice gases (Rothman and Keller, 1988, hereafter ILG). This formula explicitly shows that it is the nature of the two phase collision rules of the ILG which creates the surface tension. Preliminary results of this formula gives a reasonable estimate for the surface tension at high densities.

\subsection{Introduction}

Since macroscopic fluid equations are merely a reflection of microscopic conservation laws and symmetries, the macroscopic equations are the same for different types of fluids with different transport coefficients. Thus it is conceivable that an artificial micromodel with the constraints of basic conservation laws (momentum, mass) can give rise to the Navier-Stokes equations. This thought has led to recent successful applications of lattice gas automata simulations. The reason for using this alternative numerical simulation method is its simplicity and efficiency for certain problems. Due to the discreteness of this method, the state can be represented by Boolean variables, which are very efficiently stored and operated on by computers. However, this simulation only approaches the real world asymptotically, and there exists small scale noise. Thus it can only provide reliable information for large-scale averaged quantities. Also, it is difficult to extend the models, particularly the multiphase models from two to three dimensions. To remedy these problems of the LGA model, the Lattice Boltzmann model has been introduced (McNamara and Zanetti, 1988), in which the Boolean representation is replaced by ensemble averaged quantities. Of course you can always make the model more complex, but it is a trade-off with efficiency. Below we will first briefly review the single phase Frisch, Hasslacher and Pomeau(FHP) lattice gas model, the Rothman and Keller immiscible lattice gas (ILG) model, and the lattice Boltzmann model.

\subsubsection{FHP model}

There are three versions of FHP models. In these FHP models, the residing lattice is triangular with unit lattice constant as shown below. 


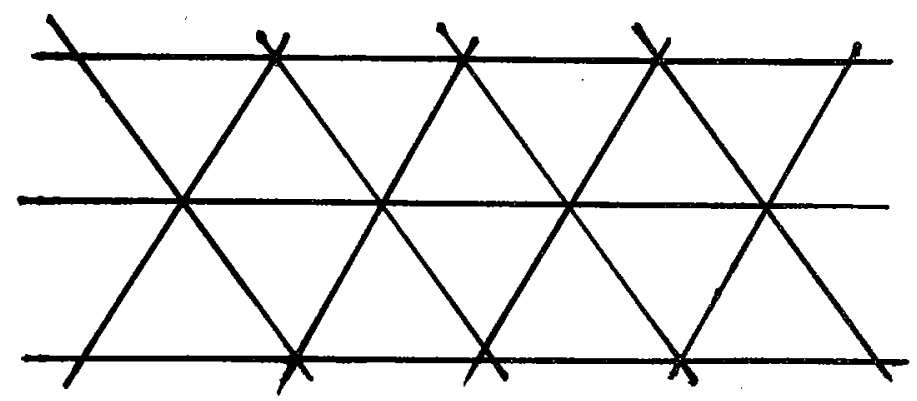

Figure 15.1. FHP Hexagon lattice structure
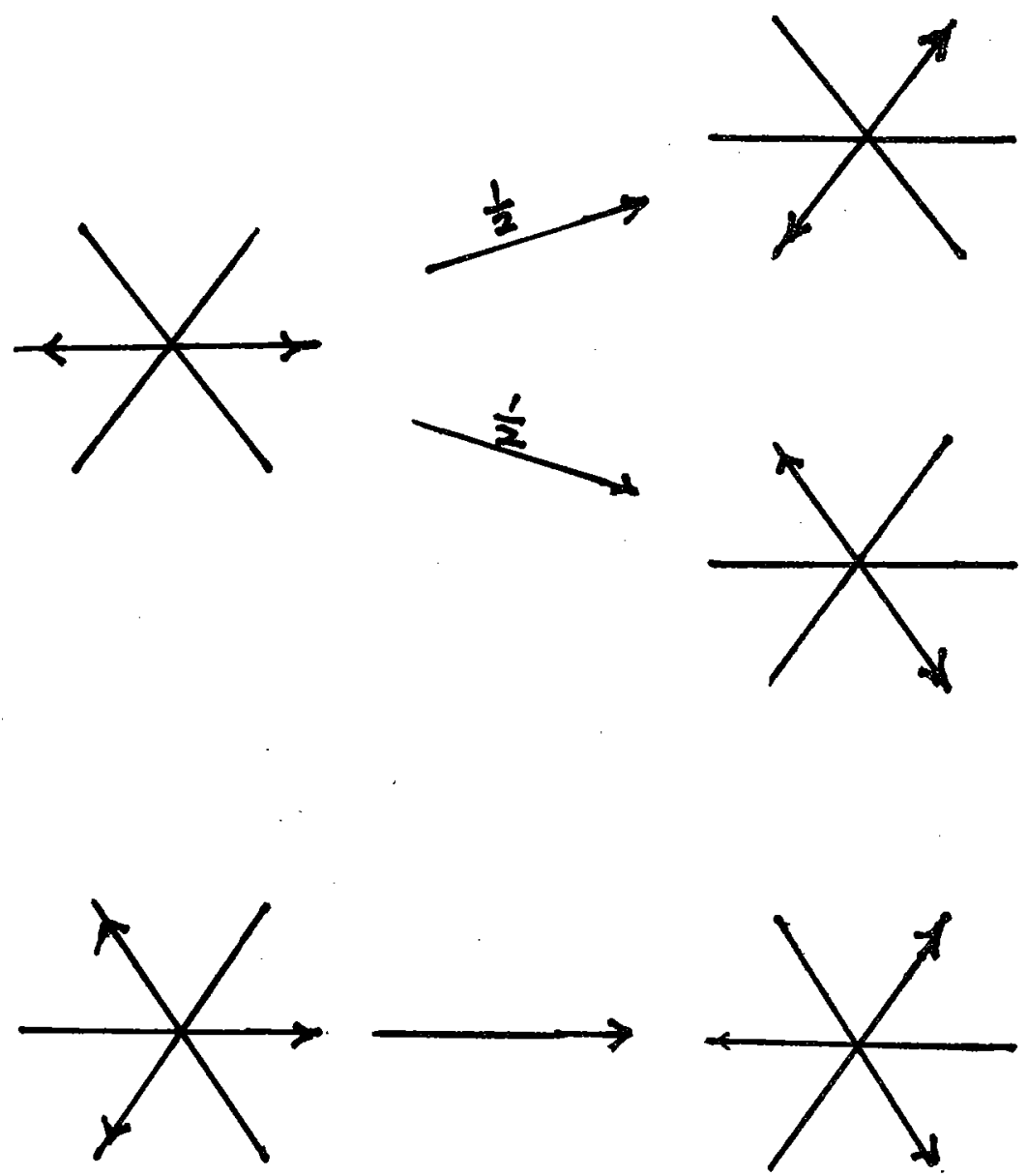

Figure 15.2. FHP-I collision rules

Each site is connected with its six neighbors by unit vectors $c_{i}(i=1, \cdots 6)$. Particles of unit mass and speed are moving along the lattice links and are located at the nodes at integer times. No more than one particle is to be found at a given time and node, moving in a given direction (exclusion principle). Thus a site can be described by a six bit state (or seven bit state if rest particles are included). For instance, $(1,0,0,0,0,0,1)$ means that at certain site there is a particle with unit velocity pointing at direction $i=1$, where $i$ is the index for seven states at a site, and there is another rest particle. Updating of the lattice involves 
propagation and collisions. Particles propagate to the nearest neighbour at the next time step according to their velocity directions, and then collide with other particles arriving at the same site. The collisions conserve momentum and particles. If collision rules involve only binary head-on collisions and triple collisions, it is called the FHP-I model (see Fig.1.2).

FHP-I is not invariant under duality (particle-hole exchange), but can be made so by inclusion of the duals of the head-on collisions (see Fig. 1.3).
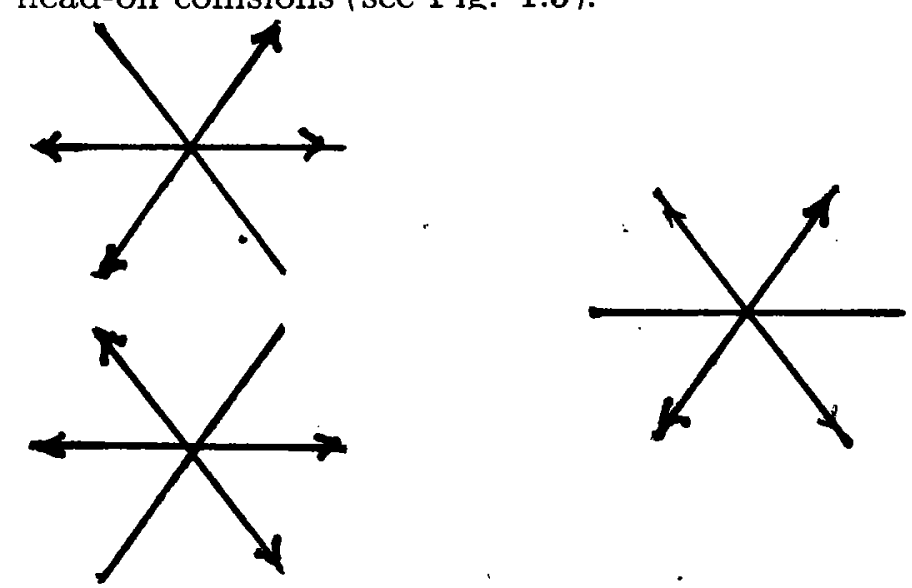

Figure 15.3. FHP-I dual head-on collision rules

The FHP-II model is a seven bit variant of FHP-I including a zero velocity "rest particles" (see Fig. 1.4, circle represents a rest particle).
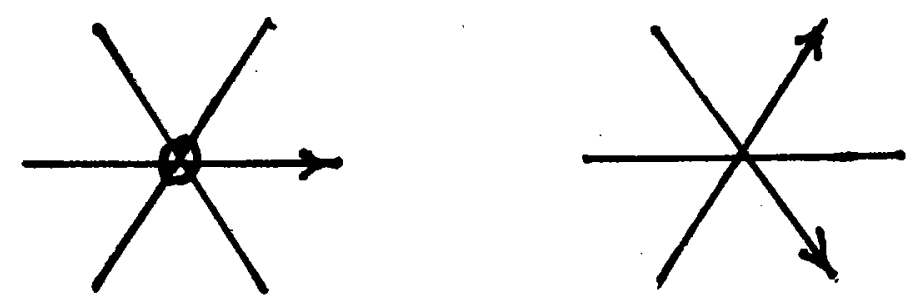

Figure 15.4. FHP-II collision rule with a rest particle

Finally, model FHP-III is a collision-saturated version of FHP-II, i.e. by inclusion of head-on collisions with a spectator (see Fig. 1.5). 

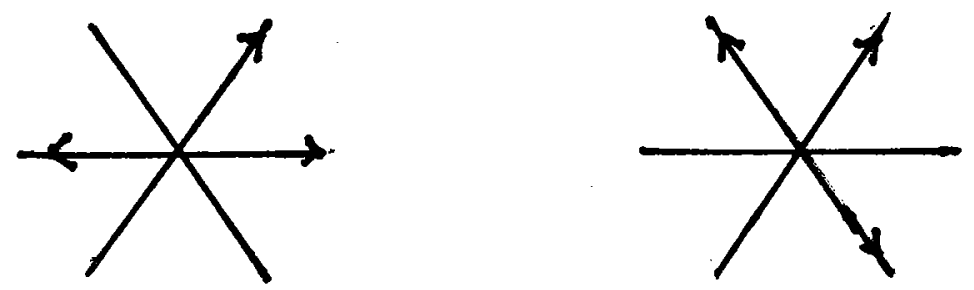

Figure 15.5. FHP-III collision with a "spectator"

The dynamics of the FHP models are invariant under all discrete transformations that conserve the triangula lattice: discrete translations, rotation by $\pi / 3$, and mirror symmetries with respect to a lattice line.

\subsubsection{ILG model}

Rothman and Keller extended the FHP-III model to immiscible two-phase flow with interfacial tension between fluid phases. Two phases are represented by two kinds of particles, referred to by color, "red" and "blue". The collisions not only conserve mass and momentum, but also number of reds and blues. Red and blue particles may simultaneously occupy the same site, but not with the same velocity. The configuration at a site is thus completely described by the two seven-bit variables

$$
r=\left[r_{i}, i=0 \cdots 6, r_{i}=0 \text { or } 1\right]
$$

and

$$
b=\left[b_{i}, i=0 \cdots 6, b_{i}=0 \text { or } 1\right] .
$$

Note that $r_{i}$ and $b_{i}$ cannot be both equal to zero. Due to the immiscible properties, like particles are attractive to each other while red and blue particles repulse each other. Since intermolecular forces in real fluid are very short ranged, only the influence of the nearest neighbours of a site $\mathrm{X}$ is accounted for in the ILG model. Specifically, a color flux and color field are defined and collision rules are designed such that the "work" performed by the flux against the field is minimized. The local color flux $\mathrm{q}[\mathrm{r}(\mathrm{X}), \mathrm{b}(\mathrm{X})]$ is defined to be the difference between the net red momentum and net blue momentum at a site $\mathrm{X}$,

$$
q[r(X), b(X)]=\sum_{i} \mathrm{c}_{\mathrm{i}}\left[r_{i}(X)-b_{i}(X)\right],
$$

and the local color field is defined to be the direction weighted sum of the difference between the number of reds and the number of blues at neiboring sites

$$
f(X)=\sum_{i} \mathbf{c}_{\mathbf{i}} \sum_{j}\left[r_{j}\left(X+\mathbf{c}_{\mathbf{i}}\right)-b_{j}\left(X+\mathbf{c}_{\mathbf{i}}\right)\right] .
$$


The work $\mathrm{W}$ performed by the flux against the field is then

$$
W=-f \cdot q .
$$

Obviously two phase collision rules results in particles of each color preferentially moving toward concentrations of like color, and away from concentrations of unlike color. If the solution is nonunique, the outcome of a collision is chosen with equal a priori weight from the set of solutions. A simple example is shown in Fig.1.6
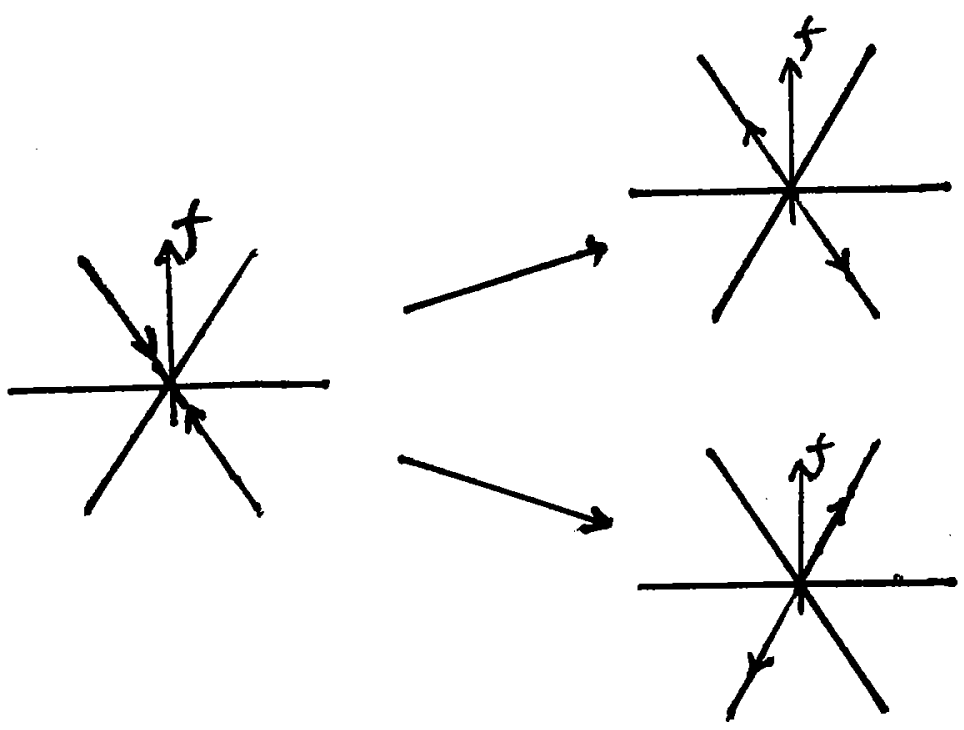

Figure 15.6. (a) A simple example to show the two phase collision rules.
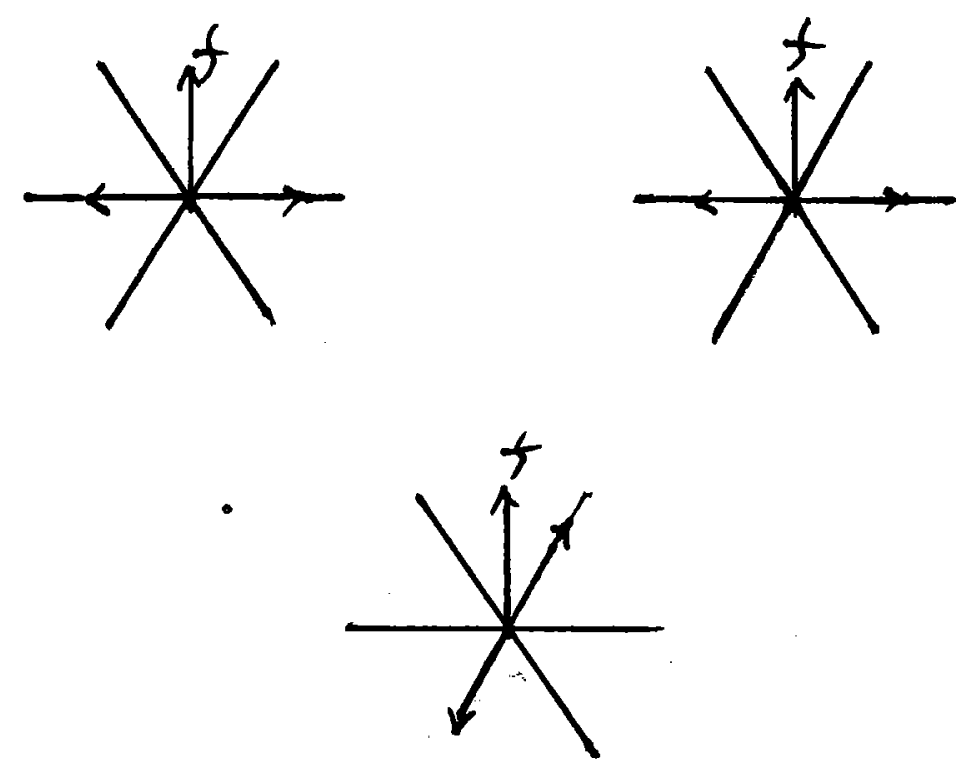

Figure 15.6. (b) Configurations in this group are forbidden due to the minimization of W defined in Eq.1.3 


\subsubsection{Lattice Boltzmann model}

The one phase Lattice Boltzmann model was first introduced by McNamara and Zanetti (1988). In the Boltzmann approximation, one assumes molecular chaos and that particles entering a collision process have no prior correlations. The Boolean variables $n_{i}(0$ or 1$)$ are replaced by the ensemble averaged mean population $N_{i}\left(0 \leq N_{i} \leq 1\right)$. The lattice Boltzmann model is very useful in deriving the transport coefficient for the lattice gas model. There are two versions of lattice Boltzmann equations. In the first version, the Boolean FHP collisions are expressed as an arithmetic nonlinear collision operator. Due to the high nonlinearity and floating point representation, this is not an efficient way to do the simulation. In the second version, the nonlinear operator is linearized about a local equilibrium. Both versions of lattice Boltzmann equation are free of statistical noise, but only the second version is easily implemented in three dimensions.

Gunstensen et al(1991) introduced an immiscible fluids lattice Boltzmann model, in which a two-step two-phase collision rule is employed. The first step of the two phase collision is to add a perturbation to the particle distribution near the interface which creates the surface tension dynamics. In the second step the mass is recolored to achieve zero diffusivity of one color into the other. A corresponding analytical surface tension formula is derived and agrees well with simulations. However this model is not a direct approximation of the ILG model, it is an independent model itself. The surface tension formula for Gunstensen's model predicts very different values from the original ILG measurement at high density, which falls off instead of linearly increasing with density. Thus a surface tension formula for the original ILG is needed. That is the task of the next section.

\subsection{A formula for ILG surface tension}

The mechanical definition of surface tension (Hirschfelder et al,1954) is

$$
\sigma=\int_{-\infty}^{\infty}\left(P_{N}-P_{T}\right) d z
$$

where $P_{N}$ and $P_{T}$ are the normal and tangential pressure, respectively, in the neigbourhood of an interface. The pressure tensor for the two dimensional lattice gas is given by(Frisch et al, 1987)

$$
P_{\alpha \beta}=\sum_{i} N_{i} c_{i \alpha} c_{i \beta}
$$

Hence

$$
P_{N}-P_{T}=\sum_{i} N_{i}\left(c_{i N}^{2}-c_{i T}^{2}\right) \equiv \sum_{i} N_{i} U_{i}
$$

where $U_{i} \equiv\left(c_{i N}^{2}-c_{i T}^{2}\right)$. If the particle distribution is isotropic, $P_{N}-P_{T}=0$, thus the surface tension is also a manifestation of an anisotropic distribution of particles near the interface.

In Gunstensen's model, the perturbation which creates the anisotropic particle distribution, and hence surface tension, is proportional to the density near the interface. Thus it is 
expected that surface tension will increase with density. In Rothman and Keller's original ILG model, the surface tension is due to the two phase collision operator which creates anisotropic particle distributions. As shown in Fig.1.6, red particles tend to align along the color field, while blue particles tend to align in the opposite direction of color field, provided the conservation laws are not violated. Thus there is higher probability for particles in the normal direction than in the tangential direction. When density is very high, it is difficult for the two phase collision operator to create anisotropic distributions. For instance, suppose $N_{i}=1(i=0, \cdots 6)$, i.e. there are seven particles at each site. No matter what collision rules are applied, output state $N_{i}^{\prime}=1$, due to the exclusion principle and conservation of particles. Thus no anisotropic distribution is created and the surface tension is zero in this extreme case. Also at very low density, say $d<0.14$, the collisions are predominately one particle collision due to the sparse distribution. Since two phase collision rules does not affect one particle collision due to constraint of momentum conservation. Surface tension is also expected to be zero at very low densities.

With the above basic understanding, below we will derive a surface tension formula for ILG.

\subsubsection{Basic equations}

For clarity, we start with a very simple example (see Fig.1.7) to show how the collision rules of one phase lattice gas model are expressed in terms of Boolean algebra.
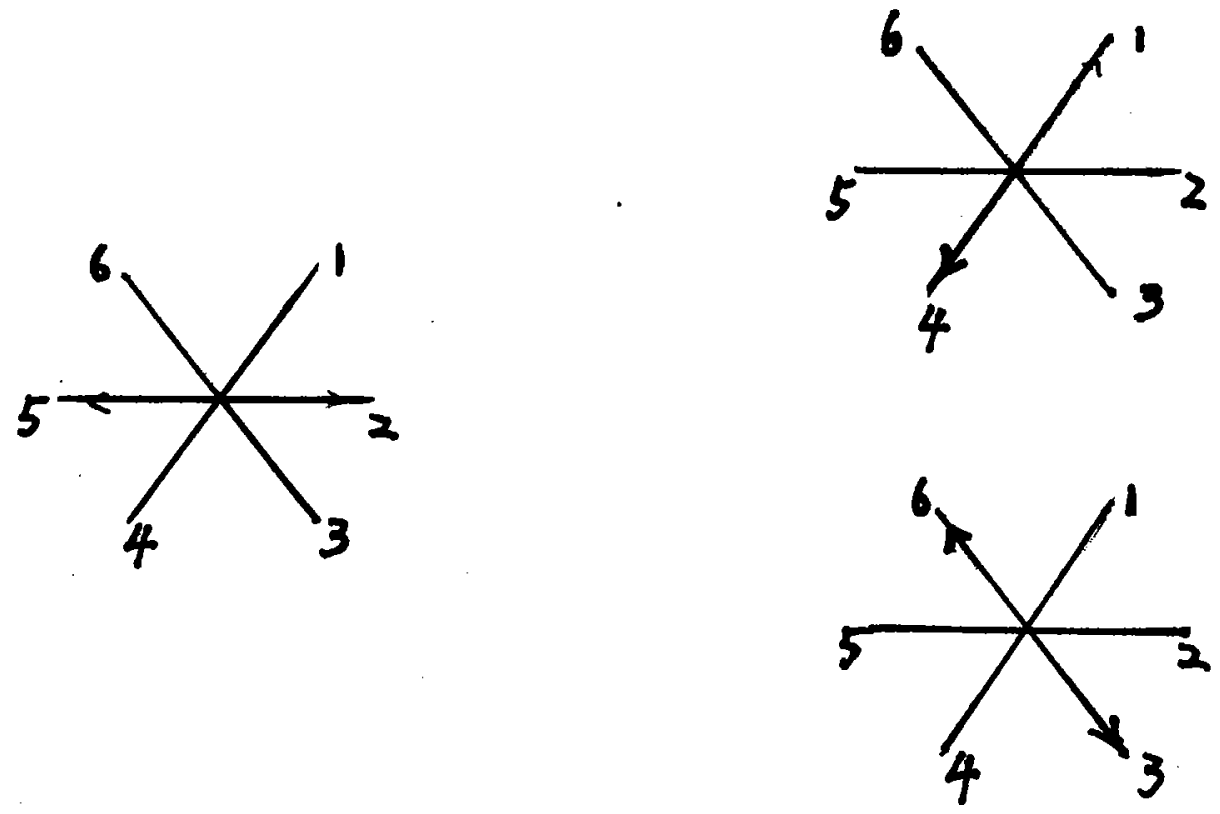

Figure 15.7. A simple one phase collision example

The microdynamic equation for the above collision and propagation process is

$$
\begin{gathered}
n_{1}\left(X+\mathbf{c}_{1}, t+1\right)=n_{1}^{\prime}(X, t)=\xi n_{2} n_{5}\left(1-n_{1}\right)\left(1-n_{3}\right)\left(1-n_{4}\right)\left(1-n_{6}\right)\left(1-n_{0}\right), \\
n_{3}\left(X+\mathbf{c}_{3}, t+1\right)=n_{3}^{\prime}(X, t)=(1-\xi) n_{2} n_{5}\left(1-n_{1}\right)\left(1-n_{3}\right)\left(1-n_{4}\right)\left(1-n_{6}\right)\left(1-n_{0}\right),
\end{gathered}
$$


where $\xi$ is the probability for one of the two possible output state. It is usually implemented by a Boolean variables taken either values 1 or 0 , which are randomly chosen such that probability of one half is assigned for each output state. There are another two similar sets of equations for $n_{4}$ and $n_{6}$ which are ommited here. In general, assume $\xi_{s s^{\prime}}$ as the probability between the transition of input state $s$ and output state $s^{\prime}$. If it is deterministic collision $\xi_{s s^{\prime}}=1$, otherwise equal probability is assigned for each possible output state, and

$$
\sum_{s^{\prime}} \xi_{s s^{\prime}}=1
$$

Then the general microdynamical equation can be expressed as

$$
n_{i}\left(X+c_{i}, t+1\right)=\sum_{s s^{\prime}} s_{i}^{\prime} \xi_{s s^{\prime}} \Pi_{j} n_{j}^{s_{j}}\left(1-n_{j}\right)^{1-s_{j}}
$$

The factor $s_{i}^{\prime}$ ensures the presence of a particle in the direction $i$ after the collision. Using Eq.(2.1) and the identity

$$
\sum_{s} s_{i} \Pi_{j} n_{j}^{s_{j}}\left(1-n_{j}\right)^{1-s_{j}}=n_{i}
$$

the microdynamic equations are rewritten as

$$
n_{i}\left(X+c_{i}, t+1\right)=n_{i}+\Delta_{i}(n)
$$

where $\Delta_{i}(n)$ is the change in direction $\mathrm{i}$ after collisions, and

$$
\Delta_{i}(n)=\sum_{s s^{\prime}}\left(s_{i}^{\prime}-s_{i}\right) \xi_{s s^{\prime}} \Pi_{j} n_{j}^{s_{j}}\left(1-n_{j}\right)^{1-s_{j}}
$$

The above microdynamical equations have the following conservation relations: particle conservation

$$
\sum_{i} n_{i}\left(X+\mathbf{c}_{\mathbf{i}}, t+1\right)=\sum_{i} n_{i}(X, t)
$$

momentum conservation

$$
\sum_{i} \mathbf{c}_{\mathbf{i}} n_{i}\left(X+\mathbf{c}_{\mathbf{i}}, t+1\right)=\sum_{i} \mathrm{c}_{\mathbf{i}} n_{i}(X, t)
$$

With the Boltzmann approximation, the Boolean variables $n_{i}$ are replaced by ensemble averaged mean populations $N_{i}$. Since no correlations between particles are assumed, the lattice Boltzmann equation is just a counterpart of Eq.(2.4) with $n_{i}$ replaced by $N_{i}$, i.e.

$$
N_{i}\left(X+c_{i}, t+1\right)=N_{i}(X, t)+\sum_{s s^{\prime}}\left(s_{i}^{\prime}-s_{i}\right) A\left(s \rightarrow s^{\prime}\right) \Pi_{j} N_{j}^{s_{j}}\left(1-N_{j}\right)^{1-s_{j}},
$$


where $A\left(s \rightarrow s^{\prime}\right)$ is the ensemble average of Boolean transition probabilities $\xi_{s s^{\prime}} \cdot \Pi_{j} N_{j}^{s_{j}}(1-$ $\left.N_{j}\right)^{1-s_{j}}$ is intepreted as the probability for configuration $s$. For the two phase fluids, a configuration depends not only on the particle distribution, but also the color distribution. The corresponding probability for a configuration then becomes(Rothman and Zaleski, 1989)

$$
p(s)=\Pi_{i=0}^{i=6} R_{i}^{r_{i}}(X, t) B_{i}^{b_{i}}(X, t)\left[1-N_{i}(X, t)\right]^{1-s_{i}} .
$$

Since the two phase collisions also depends on their nearest neighbours, the microdynamical equations become

$$
R_{i}\left(X+c_{i}, t+1\right)=R_{i}(X, t)+\sum_{s^{\prime} \sigma}\left(r_{i}^{\prime}-r_{i}\right) A\left(s, s^{\prime}, g\right) p(s) w(g),
$$

where $R_{i}$ is the red density, $A\left(s, s^{\prime}, g\right)$ is the probability of an output state $s^{\prime}$ given input state $s$ and input neighbour $g, w(g)$ is the probability for neighbour configuration $g$,

$$
w(g)=\Pi_{j=1}^{j=6} \Pi_{i=0}^{i=6} R_{i}^{r_{i}^{j}}\left(X+\mathbf{c}_{\mathbf{j}}, t\right) B_{i}^{b_{i}^{j}}\left(X+\mathbf{c}_{\mathbf{j}}, t\right)\left[1-N_{i}\left(X+\mathbf{c}_{\mathbf{j}}, t\right)\right]^{1-s_{i}}
$$

There is equation similar to Eq.(2.10) for blue particles. Hence the microdynamic equation for the mean population $N_{i}$ is

$$
N_{i}\left(X+c_{i}, t+1\right)=N_{i}(X, t)+\sum_{s s^{\prime} g}\left(s_{i}^{\prime}-s_{i}\right) A\left(s, s^{\prime}, g\right) p(s) w(g) .
$$

\subsubsection{Derivation of the surface tension formula}

In Eqs.(1.4) and (1.5), we need to know the particle distribution to calculate the surface tension. One direct way is to solve Eqs.(2.10) and (2.12) for the steady state solutions. However the equations are intractable due to the high nonlinearity and many terms involved. An alternative way is to use the perturbation method to linearize the equations. To set up the perturbation procedure, we assume that the original zeroth order state is two separated isotropic systems with red and blue fluids respectively. Obviously the two systems are governed by one phase dynamics. Then the two systems are brought together. If still only one phase collision rules are applied, the two fluids will eventually be totally mixed. Here the two phase collision rules are applied, surface tension is created and the interface is kept. The anisotropic distributions are assumed small and used as perturbation parameter. When the anisotropic particle distribution is created near the interface, let

$$
R_{i}(X, t)=R_{i}^{0}+R_{i}^{1}, \quad B_{i}(X, t)=B_{i}^{0}+B_{i}^{1}, \quad N_{i}(X, t)=d+N_{i}^{1},
$$

where $R_{i}^{0}=\theta_{i}(X, t) d, \quad B_{i}^{0}=\left(1-\theta_{i}(X, t)\right) d, \quad N_{i}^{1}=R_{i}^{1}+B_{i}^{1} . \theta_{i}$ is the color concentration of the assumed zero state, and terms with index 1 represent deviation from zero order state distributions. Also let

$$
A\left(s, s^{\prime}, g\right)=A^{0}\left(s, s^{\prime}\right)+A^{\prime}
$$


where $A^{0}\left(s, s^{\prime}\right)$ is the one phase collision operator and color information is meaningless in configuration $s$ and $s^{\prime}$, and $A^{\prime}=A\left(s, s^{\prime}, g\right)-A^{0}\left(s, s^{\prime}\right)$ is the modification of two phase collisions. Inserting Eqs.(2.13) and (2.14) into Eq.(2.12) and doing Taylor expansion yields

$$
\begin{aligned}
N_{i}\left(X+\mathbf{c}_{\mathbf{i}}, t+1\right)-N_{i}(X, t)= & \sum_{s s^{\prime} g}\left(s_{i}^{\prime}-s_{i}\right) A^{0}\left(s, s^{\prime}\right) p(s) w(g)+\sum_{s s^{\prime} g}\left(s_{i}^{\prime}-s_{i}\right) A^{\prime} p(s) w(g) \\
= & \sum_{s s^{\prime}}\left(s_{i}^{\prime}-s_{i}\right) A^{0}\left(s, s^{\prime}\right) p^{0}(s)+\sum_{j} \Omega_{i j} N_{j}^{1} \\
& +\sum_{s s^{\prime} g}\left(s_{i}^{\prime}-s_{i}\right) A^{\prime} p^{0}(s) w^{0}(g)+\vartheta\left(A^{\prime} N^{1}\right) .
\end{aligned}
$$

Here only up to first order terms are kept. $\Omega_{i j}$ is the one phase linearized collision operator, which is given by

$$
\begin{aligned}
\Omega_{i j} & =\sum_{s, s^{\prime}}\left(s_{i}^{\prime}-s_{i}\right) A^{0}\left(s, s^{\prime}\right) d^{p-1}(1-d)^{6-p} s_{j} \\
& =-\frac{1}{2} \sum_{s, s^{\prime}}\left(s_{i}^{\prime}-s_{i}\right)\left(s_{j}^{\prime}-s_{j}\right) A^{0}\left(s, s^{\prime}\right) d^{p-1}(1-d)^{6-p}
\end{aligned}
$$

The first term of the right hand side of Eq.(2.15) will vanish:

$$
\begin{aligned}
\sum_{s s^{\prime}}\left(s_{i}^{\prime}-s_{i}\right) A^{0}\left(s, s^{\prime}\right) p^{0}(s) & =\sum_{s s^{\prime}}\left(s_{i}^{\prime}-s_{i}\right) A^{0}\left(s, s^{\prime}\right) d^{p}(1-d)^{7-p} \\
& =\sum_{s s^{\prime}} s_{i}^{\prime} A^{0}\left(s, s^{\prime}\right) d^{p}(1-d)^{7-p}-\sum_{s s^{\prime}} s_{i} A^{0}\left(s, s^{\prime}\right) d^{p}(1-d)^{7-p} \\
& =0
\end{aligned}
$$

In the above derivation the semi-detailed balance property of the one phase collision operator is used. Also we have

$$
\sum_{s s^{\prime} g}\left(s_{i}^{\prime}-s_{i}\right) A^{0} p^{0}(s) w^{0}(g)=\sum_{s s^{\prime}}\left(s_{i}^{\prime}-s_{i}\right) A^{0} p^{0}(s)=0,
$$

thus the last term of right hand side of Eq.(2.15) becomes

$$
\sum_{s s^{\prime} g}\left(s_{i}^{\prime}-s_{i}\right) A^{\prime} p^{0}(s) w^{0}(g)=\sum_{s s^{\prime} g}\left(s_{i}^{\prime}-s_{i}\right) A p^{0}(s) w^{0}(g) \equiv q_{i},
$$

where $q_{i}$ is defined for latter convenience. For a steady state with appropriate boundary conditions, we have

$$
\begin{aligned}
\sum_{X} N_{i}\left(X+\mathbf{c}_{\mathbf{i}}, t\right) & -\sum_{X} N_{i}(X, t) \\
& =\sum_{X} N_{i}\left(X+\mathbf{c}_{\mathbf{i}}\right)-\sum_{X} N_{i}(X)=0,
\end{aligned}
$$


where $\sum_{X}$ is a summation over the lattice. Using Eqs.(2.17), (2.18) and (2.19), Eq.(2.15) becomes

$$
\sum_{X} \sum_{j} \Omega_{i j} N_{j}^{1}=-\sum_{X} q_{i}
$$

Eq.(2.20) clearly states that the aniotropic distribution is caused by the two phase collision operator. As pointed out by Gunstensen et al, $\Omega_{i j}$ is a self adjoint operator and $U_{i} \equiv c_{i N}^{2}-c_{i T}^{2}$ is its eigenvector with eigenvalue $\lambda(d)$, Eq.(2.20) can be rewritten as

$$
\sum_{X} \sum_{i} U_{i} N_{i}^{1}=-\frac{1}{\lambda} \sum_{X} \sum_{i} q_{i} U_{i}
$$

By using the above rewritten equation the surface tension can be obtained without solving explicitly for the anisotropic distributions $N_{i}^{1}$. Combining Eqs(1.4),(1.5),(2.21) yields the surface tension formula

$$
\sigma=\frac{\cos \theta_{f}}{n_{y}} \sum_{X} \sum_{i} N_{i} U_{i}=\frac{\cos \theta_{f}}{n_{y}} \sum_{X} \sum_{i} N_{i}^{1} U_{i}=-\frac{\cos \theta_{f}}{n_{y} \lambda(d)} \sum_{X} \sum_{i} q_{i} U_{i},
$$

where $\theta_{f}$ is the angle between color gradient and lattice axis (see Fig.1.8),

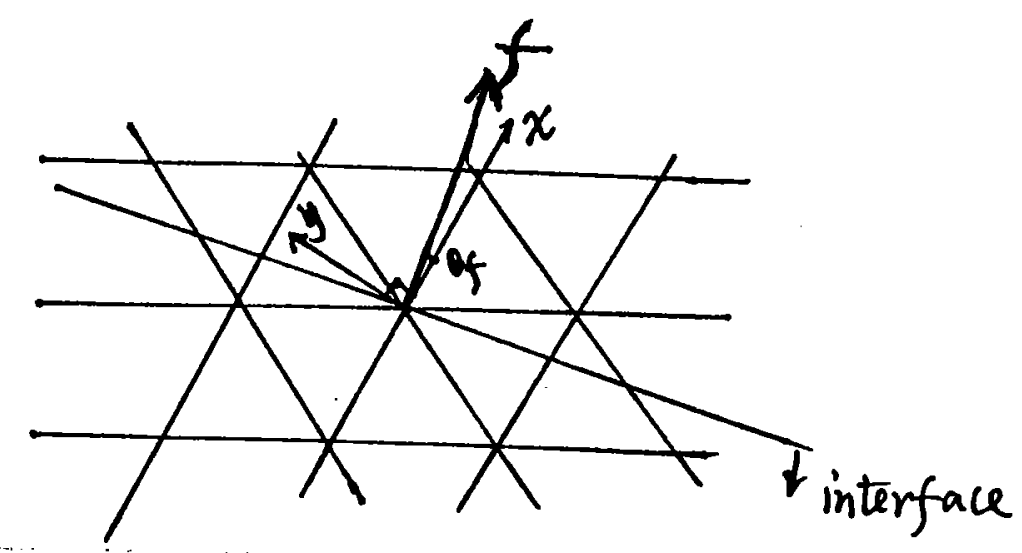

Figure 15.8. A figure to show the cöordinate structure

$n_{y}$ is the number of layers the system has in the y direction. The procedure from Eq. (2.19) to Eq.(2.22) is similar to what used in Gunstensen et al's paper. However the perturbation is replaced by the two phase collisions and the predicted values of surface tension are very different, as discussed at the beginning of the section. Below we will evaluate the formula just derived and compare with previous measurements.

15.3.3 evaluation of the surface tension formula

We consider here a interface as shown below 


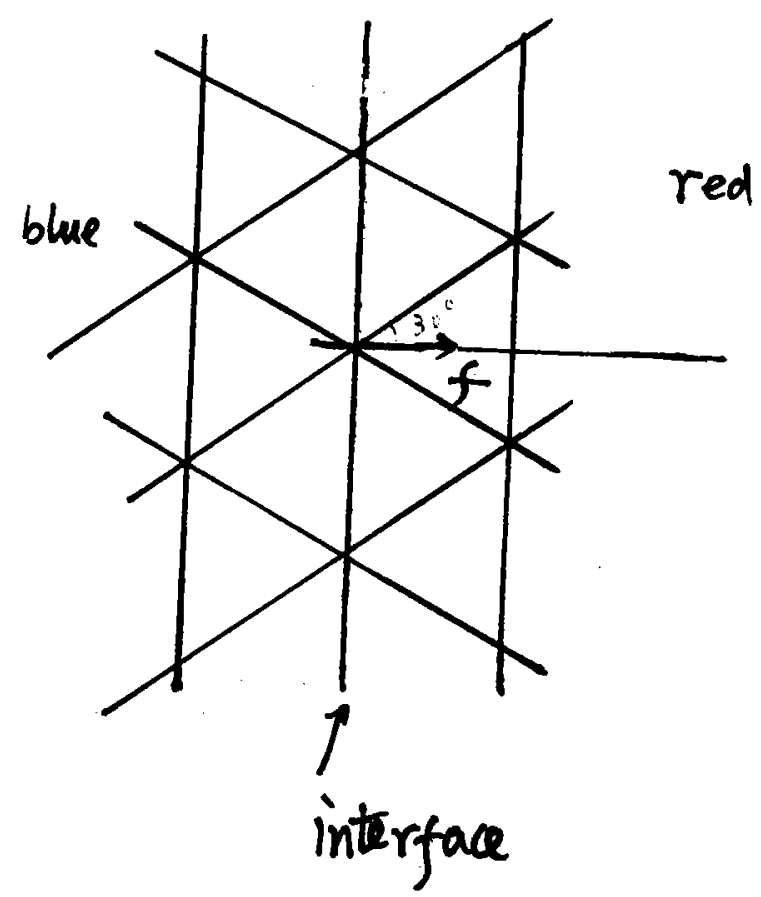

Figure 15.9. The interface used in evaluating the surface tension formula

The interface is assumed very thin and the lattice is designed that the interface is along one lattice axis. Then the summation over the lattice in Eq.(2.22) has only contributions from the sites at the interface. Obviously different sites at the interface has the same contribution, the summation along the y direction is not needed, thus the summation in Eq.(2.22) is reduced to a single site at the interface. At the interface, factor $w(g)$ in $q_{i}$ represents the statistical fluctuation of the color field orientation. To get quick results, we first neglected the color field fluctuation, i.e. $w(g)$ which gives the plot of surface tension vs. density $d$ (see the attached page). In the plot, lower curve is the measured value. Comparing with the measument, the formula gives a reasonable estimate for high densities. When the color field fluctuation is included, better estimate is expected, since fluctuation will bring down the value, and lower densities have larger fluctuations.

\subsection{Discussions and future works}

The approximations made in deriving the formula will cause some errors in the estimate of surface tension. First of all is the Boltzmann approximation which assumes no correlations between particles, while color correlation has been observed in simulations. Another approximation is that we always assumed the existence of an interface, while this is not the case for ILG simulation at low densities. That is the major reason why the formula has large discrepency with measurements at low densities. How accurate this formula will predict surface tension at high density and what is the density range for this formula to be correct needs further testing. Besides the color field fluctuation, different color field orientations need to be considered. Also, if the thin interface used here is relaxed, better predictions will be expected at lower densities since the interface at lower densities is not as sharp as at high densities. Finally, improved measurements are needed for the ILG simulations. 


\section{References}

D.H. Rothman and J.M. Keller (1988), J. stat. phys., 52, 1119.

G. McNamara and G. Zanetti (1988), Phys. Rev. Lett.,61, 2332.

U. Frisch,B. Hasslacher and Y. Pomeau (1986), Phys. Rev. Lett., 56, 1505.

J.O. Hirschfelder, C.F. Curtiss and R. Byron Bird (1954), Molecular theory of Gases and Liquids, PP. 336, (John Wiley and sons, Inc., New York.

U. Frisch, D. d'Humieres, B. Hasslacher, P. Lalleman, Y. Pomeau and J.-P. Rivet (1987), Complex Systems, 1, 648(.

A.K. Gunstensen, D.H. Rothman, S. Zaleski and G. Zanetti (1991), Physical Review A, 43, 4320.

D. H. Rothman and S. Zaleski (1989), J. Phys. France, 50, 2161. 


\title{
16. A continuum model for aeolian sand ripples
}

Rebecca B. Hoyle

Department of Applied Mathematics and Theoretical Physics, Silver Street, Cambridge CB3 9EW, England.

\begin{abstract}
We develop a continuum model for the evolution of aeolian sand ripples, incorporating the effect of shadowing. Linear travelling wave solutions are found for this model, and we show that the ripple amplitude varies directly with the wavelength. It is found that short waves travel faster than long waves, suggesting the selection of long lengthscales. For a fixed volume of sand, the ripple aspect ratio varies directly with the intensity of impacting saltating sand grains. A weakly nonlinear analysis is also presented.
\end{abstract}

\subsection{Introduction}

As the wind blows across a loosely packed sand bed, the sand is organised into propagating waves known as aeolian sand ripples. They have wavelengths of about $5-15 \mathrm{~cm}$, with aspect ratios typically in the range $\frac{1}{15}-\frac{1}{20}$, and the crests lie perpendicular to the wind direction. Aeolian ripples occur both in the desert and at the seashore.

There is a large body of previous work dealing with these bedforms. Bagnold's work (1941) has guided much recent study. There are also field observations, e.g. Sharp (1963), and computer simulations, e.g. Werner (1991). Anderson (1987) has modelled the initial generation of ripples from a flat bed as a continuum process. The objective of the present work is to find a continuum model which adequately describes the evolution of fully developed aeolian sand ripples.

In $\S 2$ we will formulate a model by considering the physical processes occurring on the sand bed. In $\S 3$ we will attempt to find solutions to the equations derived in $\S 2$. In $\S 4$ we will discuss the usefulness of this model, and directions for further work.

\subsection{Processes governing sand ripple development}

The waves are formed by the action of saltating sand grains on surface roughness. In saltation grains are whipped along the surface of the sand bed by the wind, impacting the bed at small angles to the surface, typically $10^{\circ}-15^{\circ}$ (Bagnold 1941), and with high speed. Despite the gusting of the wind, the impact angle remains fairly constant. The saltating grains rebound at slightly higher angles, but still with high speed. It is assumed for the purposes of this model that these grains are sufficiently energetic that they continue in saltation and do not join the bed. The impacts lead to ejection of sand grains from the bed, and these are then 
said to be in 'reptation' (Mitha et al., 1986). The reptating grains have much lower speeds than the saltating grains.

Figure 1 (Werner 1991 figure 1), is a schematic diagram of sand ripples, showing saltating and reptating grains. It also shows the shadow zone, where the bed is shielded from saltating grains. The upwind slope is known as the stoss slope, and tends to be shallower than the downwind or lee slope.

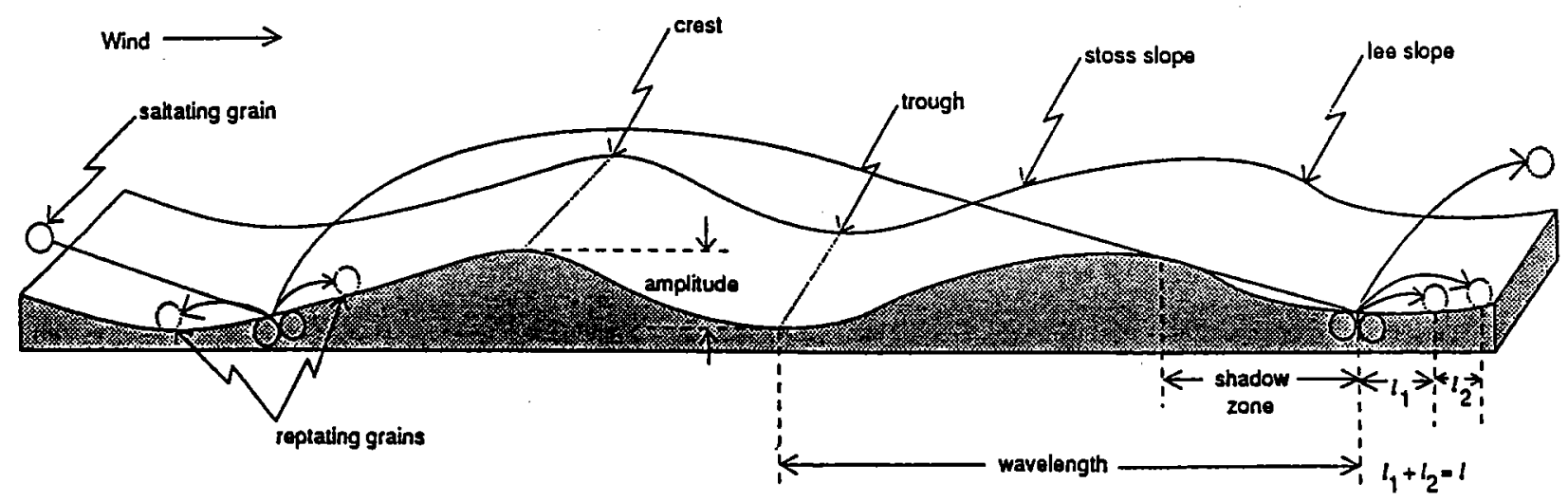

Figure 16.1. Schematic diagram of sand ripples. Werner 1991

The two processes which are considered to be the most important in this model of ripple evolution are the hopping of the reptating grains, and smoothing due to particles rolling down the ripple surface.

\subsubsection{Hopping}

Particles are knocked out of the bed by the bombardment of saltating grains, and hop along the surface, as shown in figure 2. Typically, the distance a reptating particle hops is much less than one ripple wavelength. 


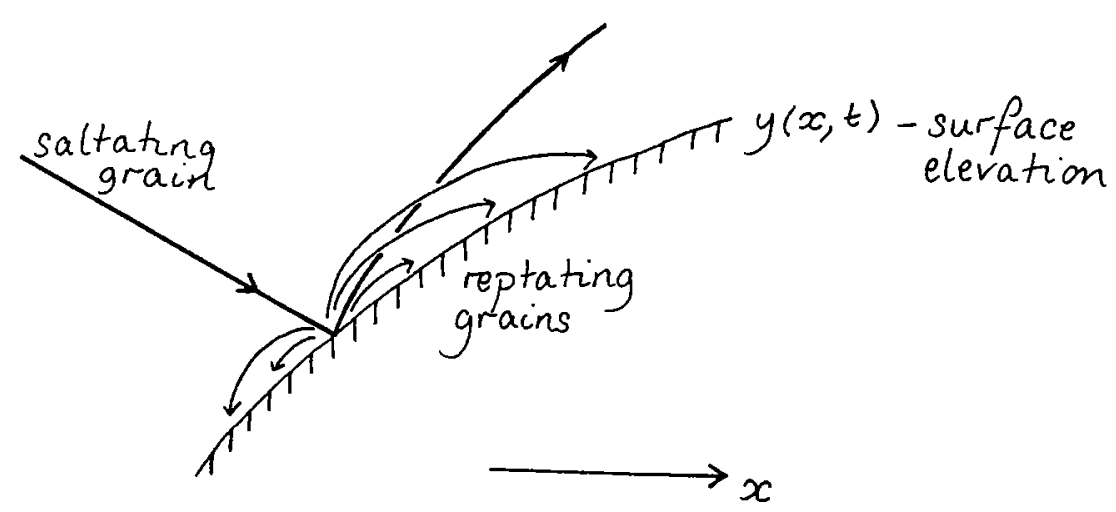

Figure 16.2. The impact of a saltating grain causes several other grains to hop along the surface of the sand.

For simplicity, consider the two-dimensional case, with the flux of saltating particles perpendicular to the ripple crests. Let $x$ be the distance along the bed, $t$ be time, and $y(x, t)$ be the surface elevation of the bed relative to some arbitrary zero level. Then

$$
y_{t}=-a_{p} \frac{\partial Q}{\partial x}
$$

where $a_{p}$ is the area of a sand grain, and $Q(x, t)$ is the horizontal number flux of reptating grains per unit width. In order to calculate $Q(x, t)$, consider $N(x, t)$, the number of particles ejected per unit area of sand bed per unit time. We will assume

$$
N(x, t) \propto \mathbf{I} \cdot \mathbf{n}
$$

where $\mathbf{I}$ is the number flux of saltating grains, and $\mathbf{n}$ is the unit inward normal on the sand bed.

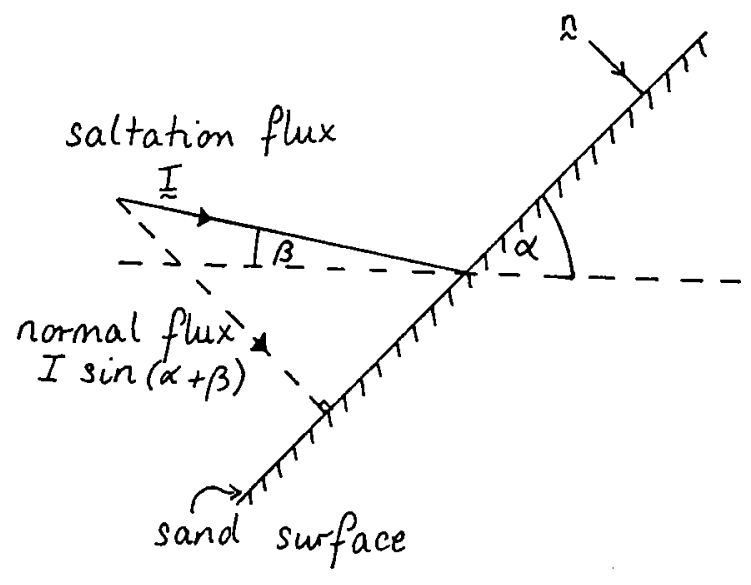

Figure 16.3. The normal component of the saltation flux is related to the impact angle and the local slope of the bed. 
As shown in figure 3 ,

$$
\mathbf{I} \cdot \mathbf{n}=I \sin (\beta+\alpha)
$$

where $\tan \alpha=y_{x}$ and $I=|\mathbf{I}|$. In terms of $y_{x}$,

Hence

$$
\sin (\beta+\alpha)=\frac{y_{x} \cos \beta+\sin \beta}{\left(1+y_{x}^{2}\right)^{\frac{1}{2}}}
$$

$$
N(x, t) \propto \frac{y_{x} \cos \beta+\sin \beta}{\left(1+y_{x}^{2}\right)^{\frac{1}{2}}} .
$$

We will assume that a reptating particle hops a horizontal length $a$ with probability $p(a)$. This probability distribution, $p(a)$, contains all the information about the transfer of momentum from the saltating particles to the reptating particles. It is related to Ungar and Haff's (1987) 'splash function', and some idea of its form can be obtained experimentally e.g., Mitha et al, 1986.

If $a$ were a constant,

$$
Q(x, t)=\int_{x-a}^{x} N(x, t) d x,
$$

since all particles leaving from between $x-a$ and $x$, will hop past $x$. Then (16.1) yields

$$
y_{t} \propto-[N(x, t)]_{x-a}^{x} .
$$

If we now allow a distribution of $a$, we expect

$$
y_{t} \propto-\int_{-\infty}^{\infty} p(a)[N(x, t)]_{x-a}^{x} d a
$$

Assuming that $p(a)$ drops off very rapidly as $a$ increases, and that a typical hop length is much less than a wavelength so that gradients are $O(1)$ on the smooth slopes, we can make a Taylor expansion,

$$
y_{t} \propto-\int_{-\infty}^{\infty} p(a)\left\{N(x, t)-N(x, t)+a N_{x}(x, t)-\frac{a^{2}}{2} N_{x x}(x, t)+\ldots\right\} d a,
$$

and neglect $\int_{-\infty}^{\infty} p(a) a^{2} d a$ compared with $\bar{a}\left(\equiv \int_{-\infty}^{\infty} p(a) a d a\right)$, so that

$$
y_{t} \propto-\bar{a} N_{x} \text {. }
$$

This results in

$$
y_{t}=-\hat{\bar{I}} \bar{a} \frac{\partial}{\partial x}\left\{\frac{y_{x} \cos \beta+\sin \beta}{\left(1+y_{x}^{2}\right)^{\frac{1}{2}}}\right\} \text {. }
$$

\subsubsection{Rolling}

Particles will tend to roll down a slope under the influence of gravity balanced by friction, and this results in smoothing of the ripple profile. As shown in figure 4, we expect a local balance governed by a drag law

$$
r u=g \sin \alpha,
$$

where $g$ is the acceleration due to gravity,

$u$ is the particle speed, and $r \equiv r(\alpha, u$, grain size.... is the drag coefficient. 


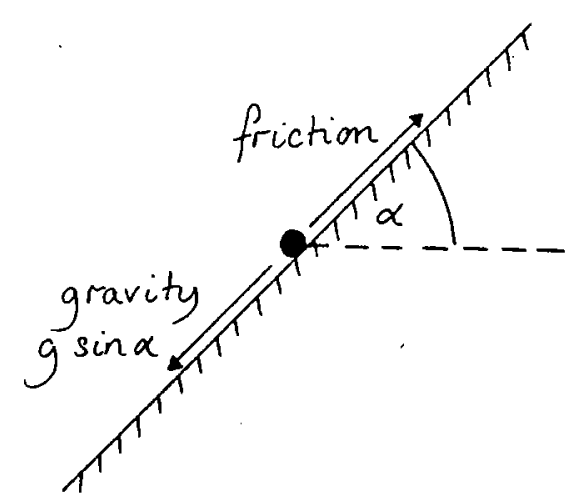

Figure 16.4. Force balance on a rolling particle.

The horizontal component of the particle velocity is

$$
\begin{aligned}
u_{H} & =-u \cos \alpha \\
& =-\frac{g}{r} \sin \alpha \cos \alpha \\
& =-\frac{g}{r} \frac{y_{x}}{\left(1+y_{x}^{2}\right)}
\end{aligned}
$$

For simplicity, we assume that $r$ is constant, and this suggests that

$$
Q(x, t) \propto-\frac{y_{x}}{\left(1+y_{x}^{2}\right)}
$$

which would result in

$$
y_{t}=L \frac{\partial}{\partial x}\left\{\frac{y_{x}}{1+y_{x}^{2}}\right\}
$$

for some constant $L$.

Combining the effects of hopping (16.2) and rolling (16.3) gives

$$
y_{t}=L y_{x x}-\hat{I} \bar{a} y_{x x}\left(\cos \beta-y_{x} \sin \beta\right)
$$

where we have neglected terms $O\left(y_{x}^{2} y_{x x}\right)$. This should be a good approximation, since sand ripples usually have an aspect ratio of around $\frac{1}{20}$ (Cornish, 1914, established a value of $\frac{1}{18}$ ), and we do not expect large gradients.

Initially, as the ripple develops from surface roughness, there will be small gradients everywhere (i.e. $\left|y_{x}\right|<\tan \beta$ ) and (16.4) will hold at all points on the sand bed. However, fully developed ripples have a shadow zone over a large part of the lee slope (figure 5 ), where there is no hopping since no saltating particles can arrive. 


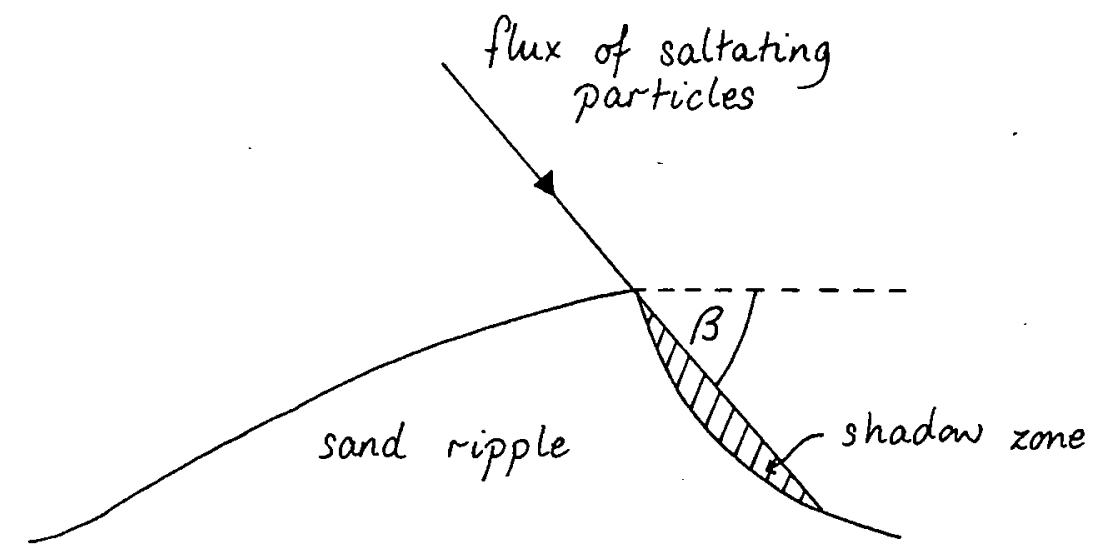

Figure 16.5. When $y_{x}$ exceeds $\tan \beta$ a shadow zone develops.

If we assume that all of the lee slope is in shadow, we can adjust the model to take account of this shielding effect:

$$
\left\{\begin{array}{ll}
y_{t}=L y_{x x}-\hat{I} \bar{a} y_{x x}\left(\cos \beta-y_{x} \sin \beta\right) & \text { stoss slope } \\
y_{t}=L y_{x x} & \text { lee slope }
\end{array} .\right.
$$

\subsection{Solutions of the equations}

\subsubsection{Initial value problem}

During the initial evolution, while gradients are still small, we can linearise in $y_{x}$, and keep $O\left(a^{2}\right)$ in the Taylor expansion so that we have

$$
y_{t}=L y_{x x}-\hat{I} \bar{a} y_{x x} \cos \beta+\frac{\hat{I} \bar{a}^{2}}{2} y_{x x x} \cos \beta,
$$

which is valid everywhere, as long as $\left|y_{x}\right|<\tan \beta$. Anderson (1987) looked at this problem for $L=0$, and the cases

$$
\begin{aligned}
& p(a)=\delta(a-\bar{a}) \\
& p(a)= \begin{cases}A a^{b} e^{-d a} & a>0 \\
0 & a<0\end{cases}
\end{aligned}
$$

keeping all orders in $a$. For our simplified problem, substituting $y=\hat{y} \exp \{i k(x-c t)+\sigma t\}$ gives a growth rate

$$
\sigma=k^{2}(\hat{I} \bar{a} \cos \beta-L),
$$

and a wave speed

$$
c=\frac{\hat{I} \bar{a}^{2}}{2} k^{2} \cos \beta .
$$

However, as soon as $y_{x}=\tan \beta$ at any point on the profile, this analysis breaks down. The hopping term on the lee slope must be neglected, and it is difficult to see how to proceed, so we simplify the problem further. 


\subsubsection{Linear travelling waves}

For the case of fully developed ripples, we can look for travelling wave solutions. Linearising (16.5) results in

$$
\left\{\begin{array}{ll}
y_{t}=y_{x x}(L-I a \cos \beta) & \text { stoss slope } \\
y_{t}=L y_{x x} & \text { lee slope }
\end{array},\right.
$$

where we have written $\hat{I}=I$ and $\bar{a}=a$. Setting $y \equiv y(\phi)$ where $\phi=x-c t$, and letting prime denote differentiation with respect to $\phi$ leads to the equations

$$
\begin{cases}-c y^{\prime}=y^{\prime \prime}(L-I a \cos \beta) & \phi<0 \\ -c y^{\prime}=L y^{\prime \prime} & \phi>0\end{cases}
$$

for a wave with its crest at $\phi=0$. Integrating twice gives

$$
\begin{cases}y=k_{1}+A_{1} \exp \{-c \phi /(L-I a \cos \beta)\} & \phi<0 \\ y=k_{2}+A_{2} \exp \{-c \phi / L\} & \phi>0 .\end{cases}
$$

For physically sensible solutions, the surface elevation and the flux of sand must be matched at the crest. The sand flux is given by

$$
\begin{cases}-(L-I a \cos \beta) y^{\prime} & \phi<0 \\ -L y^{\prime} & \phi>0 .\end{cases}
$$

With these matching conditions, the surface elevation is

$$
\begin{cases}y=y_{1} \exp \{-c \phi /(L-I a \cos \beta)\} & \phi<0 \\ y=y_{1} \exp \{-c \phi / L\} & \phi>0 .\end{cases}
$$

At some value of $\phi$, the lee slope will no longer be in shadow, and the hopping term must be reintroduced. Let the point at which this happens be $\phi=\phi_{1}$, as shown in figure 6 .

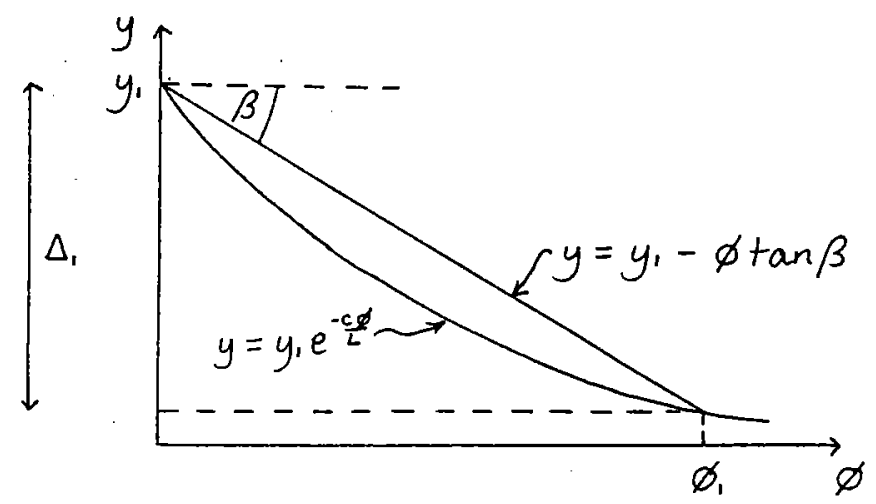

Figure 16.6. The shadow zone ends where saltating particles can once more hit the sand surface. 
So $\phi_{1}$ and $y_{1}$ satisfy the relation

$$
y_{1} \exp \left\{-c \phi_{1} / L\right\}=y_{1}-\phi_{1} \tan \beta
$$

giving

$$
y_{1}\left(1-\exp \left\{-c \phi_{1} / L\right\}\right)=\phi_{1} \tan \beta=\Delta_{1}, \text { the wave amplitude } .
$$

Then $\Delta_{1} \sim \phi_{1}$ for fixed $\beta$. At $\phi=\phi_{1}$ we can match on another wave with its crest at $\phi=\phi_{2}$, and then $\phi_{1}$ is the length of the lee slope. For the second wave

$$
y=h_{1}+y_{2} \exp \left\{-\frac{c\left(\phi-\phi_{2}\right)}{(L-I a \cos \beta)}\right\}, \quad \phi_{1}<\phi<\phi_{2}
$$

Continuity of surface elevation, and sand flux imposes a relation between the amplitudes of successive waves.

$$
y_{2}=y_{1} \exp \left\{-\frac{c \phi_{1}}{L}+\frac{c\left(\phi_{1}-\phi_{2}\right)}{(L-I a \cos \beta)}\right\} \text {. }
$$

In order to have periodic waves with $y_{2}=y_{1}$, the wavelength $\phi_{2}$ must satisfy

$$
\phi_{2}=\frac{I a}{L} \cos \beta \phi_{1}
$$

So for fixed $I, a, L$, and $\beta, \phi_{2} \sim \phi_{1} \sim \Delta_{1}$. Notice that $\phi_{1}$ is the length of the lee slope and hence also the shadow zone, in our model. The dependence of the wavelength $\phi_{2}$ on $\phi_{1}$ is consistent with Sharp's argument that the ripple wavelength is controlled by the length of the shadow region. Also, taller waves are longer in this model, as they are in nature.

In order to find how the wave speed, $c$, varies with the amplitude, $\Delta_{1}$, we need a closure assumption. For a static ripple, there is an angle, known as the angle of repose, which is the maximum that the sand can support. This is simply a feature of the packing of the sand grains, and for dry sand, the angle of repose is about $30^{\circ}$ (von Burkalow, 1945). It seems reasonable to suppose that for travelling ripples, there is an angle, $\theta \leq$ angle of repose, which gives the maximum slope attainable during motion. We will assume that at the crest on the lee side, this angle is always attained. Since the dynamics on the lee slope is governed entirely by the diffusive rolling process, this maximum slope condition is equivalent to fixing the flux of sand at the crest. At $\phi=0_{+}$, we have

$$
y^{\prime}=-\frac{c y_{1}}{L}=-s
$$

with $s$ fixed, and $s=\tan \theta$. Substituting into (16.6) we see that

$$
\frac{\phi_{1}}{y_{1}} \tan \beta=1-\exp \left(-\frac{s \phi_{1}}{y_{1}}\right) \text {. }
$$

If $s>\tan \beta$, this has a unique non-zero solution $\phi_{1} / y_{1}=K(s)$, as shown in figure 7 . 


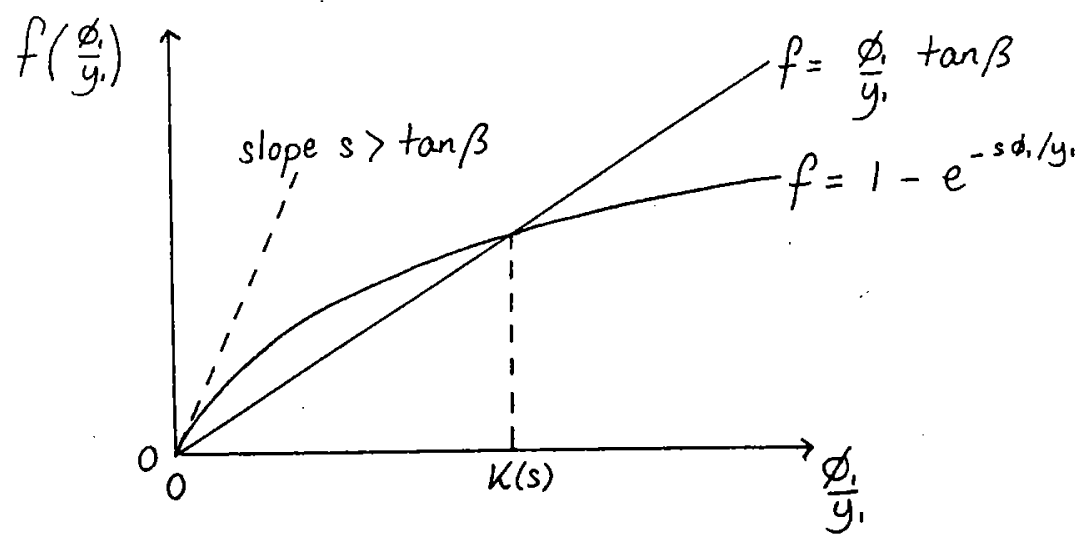

Figure 16.7. The ratio of surface elevation to the length of the lee slope is uniquely determined by $\beta$ and $s$.

In summary

$$
y_{1} \sim \phi_{1} \sim \phi_{2} \sim \Delta_{1} \sim \frac{1}{c}
$$

So shorter waves travel faster. This suggests that short waves will catch up longer waves, and merge into them, so that the system eventually selects the longest wavelength available to it. This agrees with the field observations of Sharp (1963), and the results of Werner's (1991) computer simulation.

In the case where there is a fixed volume of sand in the wave, we can determine the ripple amplitude. This may be relevant physically, since it is likely that only the surface layer of sand is available for transport, the lower layers being densely packed, and relatively immobile. If $A$ is the cross-sectional area of a ripple, then

$$
\begin{aligned}
A & =\int_{0}^{\phi_{2}} y d \phi=\mathrm{constant} \\
& =\frac{I a \cos \beta}{L \tan \beta} \Delta_{1}^{2}\left\{\frac{1}{s K(s)}+1-\frac{1}{K(s) \tan \beta}\right\}
\end{aligned}
$$

Hence for a fixed volume of sand we have the following scalings with impact intensity:

$$
\begin{gathered}
\Delta_{1} \sim \phi_{1} \sim y_{1} \sim I^{-\frac{1}{2}} \\
c \sim \phi_{2} \sim I^{\frac{1}{2}} .
\end{gathered}
$$

The waves have aspect ratio, $R$, given by

$$
R=\frac{\Delta_{1}}{\phi_{2}}=\frac{L \sin \beta}{I a \cos ^{2} \beta} \sim \frac{1}{I}
$$

For increasing impact intensity, the waves become smaller relative to their wavelength. This agrees with Sharp's (1963) observations. The ratio of wavelength to lee slope length provides a measure of the asymmetry of the ripples,

$$
\frac{\phi_{2}}{\phi_{1}} \sim I
$$


The model, therefore, predicts that the profile will become more asymmetrical as the impact intensity becomes stronger. This is in contrast to Sharp's field observations which indicate that the ripples should become more symmetrical as the wind speed increases.

\subsubsection{Weakly nonlinear travelling waves}

Returning to (16.5) and making the substitutions for travelling waves as in the previous section we obtain

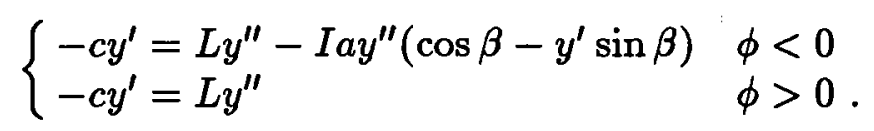

The extra term, in comparison with the linear case, is due to curvature. Integrating for $\phi<0$ gives

$$
k-c y=L y^{\prime}-I a y^{\prime}\left(\cos \beta-\frac{\sin \beta}{2} y^{\prime}\right),
$$

where $k$ is a constant. This function is plotted in figure 8 .

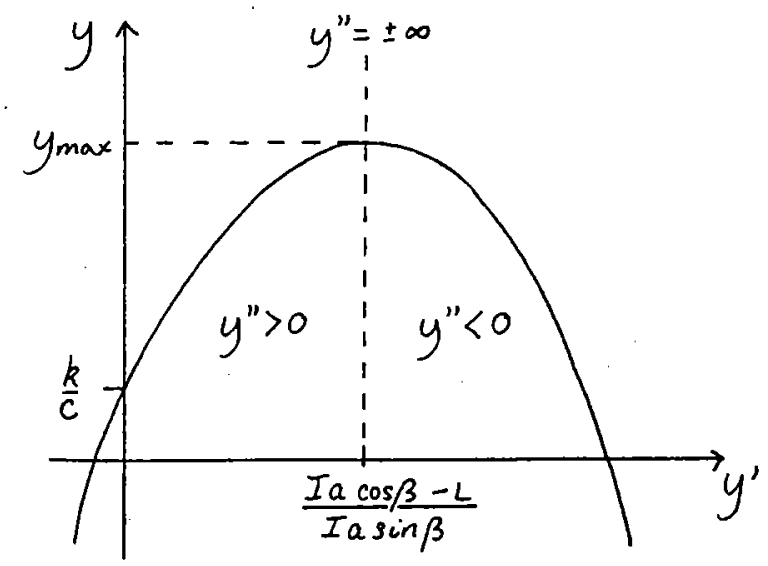

Figure 16.8. Graph of $y$ against $y^{\prime}$ for the weakly nonlinear case. The behaviour of $y^{\prime \prime}$ in the various regions of the plane is shown.

Notice that there is a maximum value of the surface elevation,

$$
\begin{gathered}
y_{\text {max }}=\frac{1}{c}\left\{\frac{(I a \cos \beta-L)^{2}}{2 I a \sin \beta}+k\right\}, \\
\text { at } y^{\prime}=\frac{I a \cos \beta-L}{I a \sin \beta} .
\end{gathered}
$$

In order to be close to the linear case, we must take the branch of solutions where $y^{\prime \prime}>0$. This solution is sketched in figure 9 . 


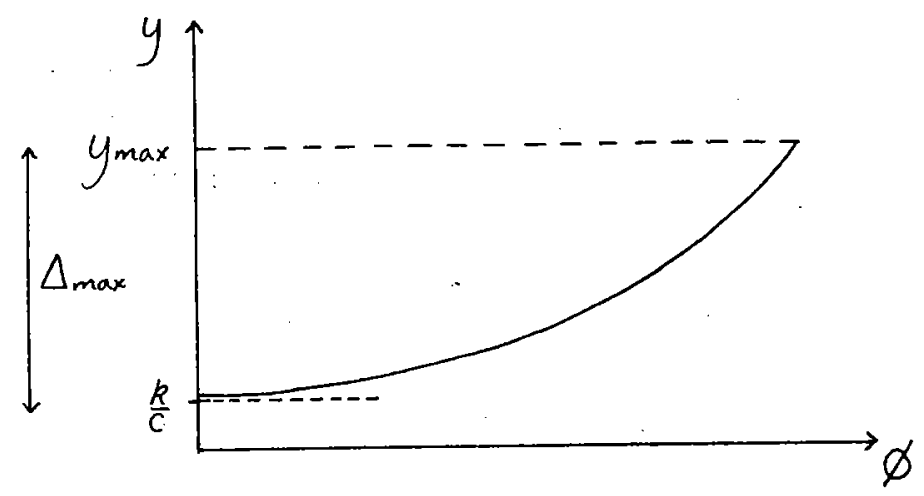

Figure 16.9. Weakly nonlinear solution for the stoss slope of a travelling wave.

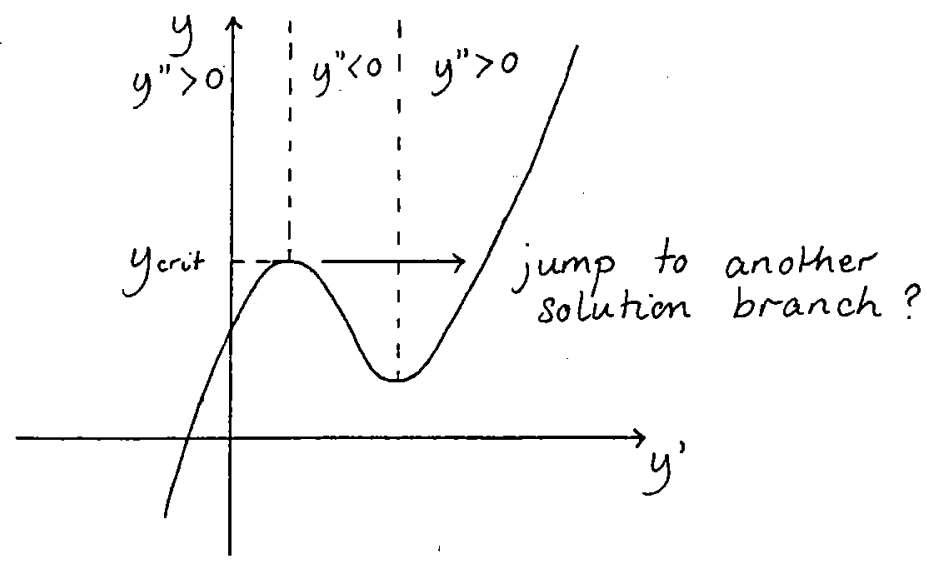

Figure 16.10. Form of $y$ against $y^{\prime}$ with cubic terms retained. There is a possible jump from one solution branch to another at $y=y_{\text {crit }}$.

Consequently, there is a maximum value for the amplitude of the waves, $\Delta_{\max }$, where

$$
\Delta_{\max }=\frac{(\operatorname{Ia} \cos \beta-L)^{2}}{2 \operatorname{Iac} \sin \beta}
$$

However, if we try to match the weakly nonlinear solution for the stoss slope to the solution for the lee slope, we may find that $\Delta<\Delta_{\max }$. When $y>y_{\max }$ we expect $O\left(y^{\prime 2} y^{\prime \prime}\right)$ to become important, and as shown in figure 10, it is possible that the solution jumps to another branch of the curve. This would correspond to a sudden change in shape with increasing ripple amplitude.

It is not clear whether this is a real feature of the physics of sand ripples, or just a mathematical artifact, but it should be noted that there may be other important physical processes occurring which we have not included in the model, and if so this jump may well disappear when we introduce them. In particular, entrainment of grains from the crests by the wind is likely to play an important role in determining the ripple profile near the crest on the stoss slope. 


\subsection{Discussion}

The hopping-diffusion continuum model has many points of agreement with the field observations of Sharp (1963), and the computer simulations of Werner (1991). In particular, we have shown that short waves can be expected to propagate faster than longer waves, which suggests that the system will select the longest wavelength available to it, by a process of ripple merger as short waves catch up with longer waves. The model also predicts that longer waves are taller, which is to be expected, and agrees with observations. Also, the wavelength of the matched linear travelling waves is controlled by the length of the shadow zone, as Sharp has argued that it should be. For a fixed volume of sand, the aspect ratio decreases with increasing impact intensity, which agrees with Sharp's observations. However, this model also suggests that the ripples become more asymetrical as the intensity of bombardment increases, which conflicts with his observations.

At this stage, it appears to be necessary to introduce more physics to the problem. The entrainment of sand grains from the ripple crests by the wind is an obvious candidate for investigation, and inclusion of this effect might lead to a more realistic shape for the ripple profile near the crest on the stoss slope. There is also numerical work in progress, which should illuminate the initial value problem beyond the point when shadowing appears. It would also be interesting to study the process of ripple merger in the framework of this model.

\section{Acknowledgements}

I would like to thank Joe Keller and Andy Woods for their insight into the weird and wonderful behaviour of sand, and for all their help with the work presented here. Paola Cessi has also been invaluable for her help with the numerics, which have not yet come to fruition, owing to the intervention of hurricane Bob. I am grateful to all the inhabitants of Walsh Cottage for making this a very happy summer, and particularly to George for introducing me to softball. Finally, I would like to acknowledge the contribution made towards my travelling expenses by Trinity College, Cambridge. 


\section{References}

Anderson, R.S. (1987) A theoretical model for aeolian impact ripples. Sedimentology, 34, 943-956

Bagnold, R.A. (1941) The physics of blown sand and desert dunes. Methuen and Co., London Cornish, V. (1914) Waves of sand and snow. Fischer-Unwin, London.

Mitha, S., Tran, M.Q., Werner, B.T., and Haff, P.K. (1986) The grain-bed impact process in aeolian saltation. Acta Mechanica, 63, 267-278

Sharp, R.P. (1963) Wind ripples. J. Geol., 71, 617-636

Ungar, J. and Haff, P.K. (1987) Steady state saltation in air. Sedimentology, 34, 289-299

von Burkalow, A. (1945) Angle of repose and angle of sliding friction: an experimental study. Bull. geol. Soc. Am., 56, 669-708

Werner, B.T. (1991) Computer simulation of sand surface self-organization in wind-blown sand transport. (preprint) 


\section{Convective patterns at zero Prandtl number}

Krishna Kumar

Laboratoire de Physique, Ecole Normale Superieure de Lyon

46, Allee d'Italie, 69364 LYON Cedex 07, FRANCE

\subsection{Introduction}

The low Prandtl number (P) convection has been studied by several people in last many years. This limit is interesting to understand convection in liquid metals $\left(P \sim 10^{-2}\right.$ to $\left.10^{-3}\right)$, convective processes in the convective zone of the $\operatorname{sun}\left(P \sim 10^{-8}\right)$, convection in the geophysical situations, e .g., in the earth's core $\left(P\right.$ is approximated to be $\left.\sim 10^{-1}\right)$. In addition it would be interesting to see the behaviour of Boussinesq equations for thermal convection in the asymptotic limit of zero Prandtl number.

Expriments show nearly independece of convective heat flux on $P$ at convection onset for $1<P<10^{3}$ but strong dependence is observed in the low range of Prandtl numbers $\left(10^{-2}<P<1\right)$. In this range of $P$, there are two mechanisms that lead to convective process. The convective patterns arising from the saturation due to the nonlinearity in momentum equation is characterized by a small slope of the heat flux. The patterns generated by the saturation mechanism due to convective nonlinearity $\left(v_{j} \partial_{j} \theta\right)$, known as inertial convection or "fly wheel" effect, are charaterized by a large slope of the heat flux.

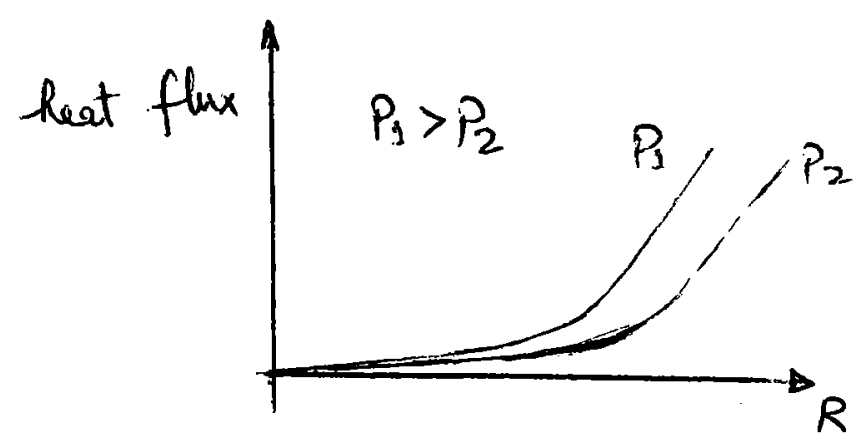

Figure 17.1. Heat flux vs $R$

The Prandtl number dependence of the critical Rayleigh number $\left(R_{*}\right)$, at which the inertial convection sets in, is not known experimentally.

Numerical simulations in two dimension (2D) by Clever and Busse(1981) show that $R_{*}$ increses with decreasing $P$. Proctor(1976) has shown analytically that for $2 \mathrm{D}$ convection in 
circular geometry, $R_{*}$ is finite as $P \rightarrow 0$. This might not be the case for Rayleigh- Benard geometry even in $2 \mathrm{D}$. In other words it is not known whether $R_{*}(P)$ is finite, in general, as $P \rightarrow 0$.

In 3D the older numerical work by Herring(1970), with stress free boudary conditions at the top and bottom, shows a blow up behavior for a large class of initial conditions. But the recent work by Thual(1991) shows that with 3D initial conditions one has intersting behaviour for stress free as well as rigid boundary conditions.

\subsection{Hydrodynamic equations}

We consider an infinite layer of a fluid enclosed by two horizontal surfaces subjected to an adverse temperature gradient. The temperature difference $(\Delta T)$ between the upper and the lower surfaces is assumed to be small and, therefore, Boussinesq approximation would be considered.

\subsubsection{Scaling and equations}

There are two kinds of scaling which are widely used to study thermal convection. In one time is non-dimesionalized by the diffusive time scale $\left(\frac{d^{2}}{\kappa}\right)$, while all the lengths by $d$, the height of the fluid layer. This sets scaling for velocity as $\frac{\kappa}{d}$. It means one can write the velocity fields as $v=\frac{\kappa}{d} F(R, P)$ and the Reynold's number $(\Re)$ as $\Re=\frac{F(R, P)}{P}$. One does not know a priory whether in the limit $P \rightarrow 0, v \rightarrow \frac{\kappa}{d} F_{0}(R)$ and $R e \rightarrow \frac{F_{0}(R)}{P}$. The second scaling uses the viscous time scale $\left(\frac{d^{2}}{\nu}\right)$ to nondimensionalize time and $d$, as before, to scale lengths. This fixes the scaling for the velocity field as $\frac{\nu}{d}$. In this case, the velocity field can be written as $v=\frac{\nu}{d} G(R, P)$ and the Reynold's number as $\Re=G(R, P)$. Again it is a question whether $G(R, P) \rightarrow G_{0}(R)$ as $P \rightarrow 0$.

In the region of inertial convection, the first kind of scaling is the approprate one. In the absence of inertial convection, the second scaling is more relevant. We will consider this scaling, while the temperature field would be scaled by $P \Delta T$, where $\Delta T$ is the temperature difference across the fluid layer. This corresponds to the "limit of high thermal diffusitivity". The hydrodynamic equations for deviations in fields from the conduction profile read

$$
\begin{gathered}
\partial_{t} v_{i}+\left(v_{j} \partial_{j}\right) v_{i}=-\partial_{i} p+\nabla^{2} v_{i}+R \theta \lambda_{i} \\
P \partial_{t} \theta=\lambda_{i} v_{i}+\nabla^{2} \theta \\
\partial_{j} v_{j}=0
\end{gathered}
$$

where $R\left(=\frac{\alpha g d^{3}(\Delta T)}{\kappa \nu}\right)$ is the Rayleigh number, $\alpha$, the thermal expansion coefficient of the fluid and $g$, the acceleration due to gravity, and $\lambda_{i}=(0,0,1)$

Since we intend to study the convection at $P=0$, we shall set $P=0$ in the above hydrodynamic equations in the rest of the paper. The recent numerical simulations of Thual(1991) show that the hydrodynamic equations with $P=0$ does not blow up very large value of $R$ 
and that they describe the $P \rightarrow 0$ limit appropriately. The relevant hydrodynamic equations (Spiegel, 1962) then become

$$
\begin{gathered}
\partial_{t} \nabla^{2} w=\nabla^{4} w+R \nabla_{1}^{2} \theta-\lambda_{i} \epsilon_{i j k} \partial_{j}\left[\left(\omega_{l} \partial_{l}\right) v_{k}-\left(v_{l} \partial_{l}\right) \omega_{k}\right] \\
\partial_{t} \zeta=\nabla^{2} \zeta+\left[\left(\omega_{j} \partial_{l}\right) w-\left(v_{j} \partial_{l}\right) \zeta\right], \\
\nabla^{2} \theta+w=0 \\
\partial_{j} v_{j}=0 .
\end{gathered}
$$

$w$ and $\zeta$ are the z-components of the velocity and the vorticity $\left(\omega_{i}=\epsilon_{i j k} \partial_{j} v_{k}\right)$ fields respectively.

\subsubsection{Boundary conditions}

We consider that the fluid layer is enclosed by stress free and infinitely conducting horizontal surfaces. That means $w=D^{2} w=\theta=D \zeta=0$ at $z=0,1$.

\subsubsection{Linear stability}

Linearizing Eqs.(2), the vorticity field decouples from the rest of the system and the equations read

$$
\begin{gathered}
\partial_{t} \nabla^{2} w=\nabla^{4} w+R \nabla_{1}^{2} \theta, \\
\nabla^{2} \theta+w=0 .
\end{gathered}
$$

Expanding $w$ in normal modes, i.e.,

$$
\begin{gathered}
w=A_{k} e^{\sigma t+i \mathbf{k} . \mathbf{r}} \sin (n \pi z), \\
{\left[\sigma t+\left(n^{2} \pi^{2}+k^{2}\right)\right]\left(n^{2} \pi^{2}+k^{2}\right)^{2}=R k^{2} .}
\end{gathered}
$$

At the marginal stability curve(i.e., for $\sigma=0$ ),

$$
R k^{2}=\left(n^{2} \pi^{2}+k^{2}\right)^{3}
$$

a standard result as expected.

\subsubsection{Principle of exchange of stability}

Taking Chandrasekhar's result, which is valid for stress free as well as rigid horizontal surfaces, we have at $P=0$

$$
\operatorname{Im}(\sigma) \cdot R k^{2} \int_{0}^{1} d z\left(|D W|^{2}+k^{2}|W|^{2}\right)=0,
$$

where $W$ is defined by the relation $w=W(z) e^{\sigma t+i k \cdot r}$ and $D \equiv \partial_{3}$. As $R>0$ to have convection,

$$
\operatorname{Im}(\sigma)=0
$$

for cellular convection (i.e., for $k^{2}>0$ ). But this does not mean that convection is stationary near for $R$ slightly above $R_{c}$. For any $R\left(>R_{c}\right)$ growing rolls are exact solutions of the hydrodynamic equations at $P=0$. These growing rolls become unstable above some crititical value of their amplitude and this leads to oscillatory instability (Busse, 1972). In addition, we shall show that square and hexagonal patterns are unstable for $R>R_{c}$. 


\subsection{Amplitude Equations for square patterns}

In this section we shall derive amplitude equations for squares and study their stability behaviour near the onset.

\subsubsection{Perturbative expansion}

Since we are interested in the behavior near the onset, we set

$$
R=R_{c}\left(1+\epsilon^{2}\right) \text {. }
$$

After defining the small parameter $\epsilon$, we shall expand all the fields in the increasing powers of this parameter, i.e.,

$$
\mathbf{X}=\epsilon\left[\mathbf{X}_{0}+\epsilon \mathbf{X}_{1}+\epsilon^{2} \mathbf{X}_{2}+\ldots\right]
$$

where $\mathbf{X}=\left(\begin{array}{c}w \\ \theta\end{array}\right)$

Introducing a slow time scale $T=\epsilon^{2} t$, and inserting the perturbation expansion in Eqs.(2), we find

at $O(\epsilon)$

$$
\begin{aligned}
\mathcal{L} \mathrm{X}_{\mathbf{0}} & =0, \\
\partial_{j} v_{0 j} & =0,
\end{aligned}
$$

with boundary conditions $w_{0}=D^{2} w_{0}=\theta_{0}=0$ at $z=0,1$.

At next order, i.e., $O\left(\epsilon^{2}\right)$

$$
\begin{gathered}
\mathcal{L} \mathrm{X}_{1}=\mathrm{N}_{0}, \\
\partial_{j} v_{1 j}=0,
\end{gathered}
$$

with boundary conditions $w_{1}=D^{2} w_{1}=\theta_{1}=0$ at $z=0,1$.

And at the $O\left(\epsilon^{3}\right)$

$$
\begin{aligned}
\mathcal{L} \mathrm{X}_{2} & =\mathrm{N}_{1}, \\
\partial_{j} v_{2 j} & =0,
\end{aligned}
$$

with boundary conditions $w_{2}=D^{2} w_{2}=\theta_{2}=0$ at $z=0,1$.

In the above,

$$
\begin{gathered}
\mathcal{L}=\left(\begin{array}{cc}
\nabla^{4} & R_{c} \nabla_{1}^{2} \\
1 & \nabla^{2}
\end{array}\right) \\
\mathbf{N}_{0}=\left(\begin{array}{c}
\epsilon_{3 j k} \partial_{j}\left[\left(\omega_{0 l} \partial_{l}\right) v_{0 k}-\left(v_{0 l} \partial_{l}\right) \omega_{0 k}\right] \\
0
\end{array}\right)
\end{gathered}
$$

and

$$
\mathbf{N}_{1}=\left(\begin{array}{c}
\epsilon_{3 j k} \partial_{j}\left[\left(\omega_{0 l} \partial_{l}\right) v_{1 k}-\left(v_{0 l} \partial_{l}\right) \omega_{0 k}+\left(\omega_{1 l} \partial_{l}\right) v_{0 k}-\left(v_{1 l} \partial_{l}\right) \omega_{k}\right] \\
0
\end{array}\right)
$$


We will treat the generation of the vorticity in $\mathrm{z}$-direction due to the non-linearity later.

\subsubsection{Solutions of the perturbative equations}

For the zeroth order (i.e., the linear problem) we choose the solution as

$$
\theta_{0}=\left[A(T) e^{i(k x+l y)}+c . c .+B(T) e^{i(k x-l y)}+c . c .\right] \sin (\pi z)
$$

and, therefore,

$$
w=\frac{R_{c} a^{2}}{\left(\pi^{2}+a^{2}\right)^{2}}\left[A(T) e^{i(k x+l y)}+\text { c.c. }+B(T) e^{i(k x-l y)}+c . c .\right] \sin (\pi z) .
$$

Here $a^{2}=k^{2}+l^{2}=\frac{\pi^{2}}{2}$, and this choice describe two systems of rolls at any angle between them. When they are at right angle, they represent squares.

The nonlinearity at the $O(\epsilon)$ generates only higher order harmonics and, therefore, there is no problem with the solvability of the differential equations at this order. The solution at this order can be written as

$$
w_{1}=4\left[\left(\pi^{2}+k^{2}\right)\left(A_{1} e^{2 i k x}+\text { c.c. }\right)+\left(\pi^{2}+l^{2}\right)\left(B_{1} e^{2 i l y}+\text { c.c. }\right)\right] \sin (2 \pi z)
$$

and, therefore,

$$
\theta_{1}=\left[A_{1} e^{2 i k x}+c . c .+B_{1} e^{2 i l y}+c . c .\right] \sin (2 \pi z)
$$

where,

$$
A_{1}=-\frac{2 \pi R_{c} k^{2} l^{2}}{a^{2}\left[16\left(\pi^{2}+k^{2}\right)^{3}-R_{c} k^{2}\right]} A B
$$

and

$$
B_{1}=-\frac{2 \pi R_{c} k^{2} l^{2}}{a^{2}\left[16\left(\pi^{2}+l^{2}\right)^{3}-R_{c} l^{2}\right]} A B^{*}
$$

Before applying solvability condition to the system of differential equation of $O\left(\epsilon^{\mathbf{3}}\right)$ let us look at the equation for the z-component of the vorticity field. The continuity equation gives the space derivatives of the horizontal velocity fields $u_{1}$ and $v_{1}$. Thus $u_{1}$ involves a costant of integration $f(x, z, T)$ and similarly $v_{1}$ involves $g(x, z, T)$.

We can break $f$ and $g$ as

$$
f(y, z, T)=f_{1}(y, T) \cos (2 \pi z)+f_{2}(y, T)
$$

and

$$
g(x, z, T)=g_{1}(x, T) \cos (2 \pi z)+g_{2}(x, T)
$$


As we have introduced a slow time scale, i.e., we are looking around the stationary state, the z-component of the vorticity can be generated only at the $O\left(\epsilon^{3}\right)$. The equation for the vertical vorticity field at order $\epsilon^{2}$ is

$$
\nabla^{2} \zeta_{1}=0
$$

with the boundary conditions $D \zeta_{1}=0$ at $z=0,1$. This says that the functions $f_{1}, f_{2}, g_{1}$ and $g_{2}$ would be independent of the spatial coordinates in horizontal direction. And the equation of the $O\left(\epsilon^{3}\right)$, i.e., becomes

$$
\nabla^{2} \zeta_{2}=-\left[\left(\omega_{1 l} \partial_{l}\right) w_{0}+\left(\omega_{0 l} \partial_{l}\right) w_{1}\right]
$$

This does not yield the evolution equations for functions $f$ and $g$ unless and until one includes distorsion in the pattern structure. We will not do that here and, therefore, $f_{1}, f_{2}, g_{1}$ and $g_{2}$ would be constants slowly varying in time only.

Nevertheless, these functions are going to modify the nonlinear terms in the equation for $w_{2}$. Applying the solvability condition on the equations of $O\left(\epsilon^{3}\right)$ leads to the amplitude equations. These are

$$
\begin{aligned}
& \partial_{T} A=\left[\left(\pi^{2}+a^{2}\right)-i / 2 \mathcal{F}_{k l}\right] A-d_{k l}|B|^{2} A \\
& \partial_{T} B=\left[\left(\pi^{2}+a^{2}\right)-i / 2 \mathcal{G}_{k l}\right] B-d_{k l}|A|^{2} B
\end{aligned}
$$

where,

$$
\begin{gathered}
d_{k l}=\frac{8 \pi^{2} k^{2} l^{2} R_{c}}{a^{4}}\left[\frac{l^{2}\left(\pi^{2}+k^{2}\right)}{16\left(\pi^{2}+k^{2}\right)^{3}-R_{c} k^{2}}+\frac{k^{2}\left(\pi^{2}+l^{2}\right)}{16\left(\pi^{2}+l^{2}\right)^{3}-R_{c} l^{2}}\right] \\
\mathcal{G}_{k l}=\left[\left\{\frac{3 \pi^{2}-a^{2}}{\pi^{2}+a^{2}} f_{1}+f_{2}\right\} k+\left\{\frac{3 \pi^{2}-a^{2}}{\pi^{2}+a^{2}} g_{1}+g_{2}\right\} l\right], \\
\mathcal{H}_{k l}=\left[\left\{\frac{3 \pi^{2}-a^{2}}{\pi^{2}+a^{2}} f_{1}+f_{2}\right\} k-\left\{\frac{3 \pi^{2}-a^{2}}{\pi^{2}+a^{2}} g_{1}+g_{2}\right\} l\right] .
\end{gathered}
$$

These equations describe patterns arising from nonlinear superposition of rolls patterns making an angle $\psi$ defined by the expression

$$
\cos \psi=\frac{k^{2}-l^{2}}{k^{2}+l^{2}}
$$

\subsubsection{Squares and their stability}

The "true" squares are obtained by setting $k=l$. Writing $(A, B)=\left(R_{1} e^{i \phi_{1}}, R_{2} e^{i \phi_{2}}\right)$, it is easy to see that the phase decouples. The square fixed points are given by

$$
R_{1 s}^{2}=R_{2 s}^{2}=\frac{\left(\pi^{2}+a^{2}\right)}{d_{k k}}
$$

The squares exist because $d_{k k}$ is positive. If $r_{1}$ and $r_{2}$ represent small perturbations from the stationary solutions, their evolution equation in linearized form read

$$
\begin{aligned}
& \partial_{T} r_{1}=-2\left(\pi^{2}+a^{2}\right) r_{2} \\
& \partial_{T} r_{2}=-2\left(\pi^{2}+a^{2}\right) r_{1} .
\end{aligned}
$$

Clearly the square patterns are unstable and the phase diagram is given below. 


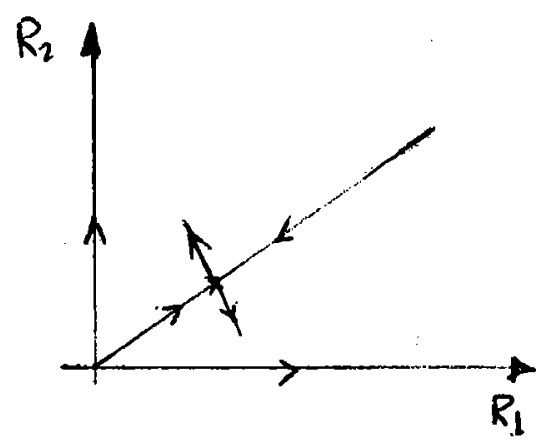

Figure 17.2. Phase diagram

It can be seen immeadiately from Eqs.(4) that other patterns arising from the superposition of rolls at any angle are also unstable.

\subsubsection{Higher order amplitude equations}

One might ask whether higher order terms in amplitude equations can describe other stable solutions.

Using symmetry arguments, the equations to the fifth order are of the form

$$
\begin{aligned}
& \partial_{T} A=A\left[\mu-|B|^{2}+|B|^{4}-\epsilon|A|^{2}|B|^{2}\right], \\
& \partial_{T} B=B\left[\mu-|A|^{2}+|A|^{4}-\epsilon|B|^{2}|A|^{2}\right] .
\end{aligned}
$$

Note that there is no nonlinear term involvig $A$ or $B$ alone because growing rolls are exact solutions of the full nonlinear equations. With the choice of the coefficients we made $(\epsilon<1)$ above square solution exists due to the fifth order terms, but it is unstable too. Indeed, the stationary square solutions are given by

$$
|A|^{2}=|B|^{2}=a_{0}^{2},
$$

where

$$
a_{0}^{2}\left[1-(1-\epsilon) a_{0}^{2}\right]=\mu .
$$

The equations for the pertubations $(a, b)$ are given by

$$
\begin{aligned}
& \dot{a}=\alpha a+\beta b, \\
& \dot{b}=\alpha b+\beta a,
\end{aligned}
$$

with

$$
\alpha=-2 \epsilon a_{0}^{4}
$$

and

$$
\beta=-2 \mu+2 a_{0}^{4} .
$$


Writing

$$
\begin{gathered}
\left(\begin{array}{l}
a \\
b
\end{array}\right) \sim e^{s t} \\
s=\alpha \pm \beta
\end{gathered}
$$

So squares are unstable for small $\epsilon$.

\subsection{Amplitude equations for hexagonal patterns}

Again, because of the translational invariance of the Boussinesq equation in space, the amplitude equations for hexagons to leading order can be written as

$$
\partial_{t} A_{i}=\mu A_{i}-\left(|A|_{j}^{2}+|A|_{k}^{2}\right) A_{i}
$$

where $A_{i}$ (for $i=1,2,3$ ) represent the complex amplitudes of three sets of rolls forming the hexagonal patterns. It is obvious from the equations that the phases can be separated and the equations for real amplitudes can be written, after some scaling, as

$$
\begin{gathered}
\dot{X}=\left[\mu-\left(Y^{2}+Z^{2}\right)\right] X, \\
\dot{Y}=\left[\mu-\left(X^{2}+Z^{2}\right)\right] Y, \\
\dot{Z}=\left[\mu-\left(X^{2}+Y^{2}\right)\right] .
\end{gathered}
$$

The stationary hexagons are given by

$$
X_{s}=Y_{s}=Z_{s}=a
$$

with $a^{2}=\frac{\mu}{2}$. Again doing the linear stability analysis around this state, the growth rate is given by

$$
(s-1)^{2}(s+2)=0 .
$$

So the stationary hexagons are unstable. 


\subsection{Lorenz model at zero Prandtl number}

It is clear from the above discussion that stationary patterns are unstable near the onset for zero Prandtl number convection. The stationary roll solutions are possible only at $R=R_{c}$. Busse showed that the stationary rolls become unstable for a critical amplitude and this leads to oscillatory instability. However, this instability occurs for an order one amplitude of the rolls, and is thus out of reach within our amplitude equation formalism. Therfore, we try to study competing patterns at $P=0$ with a Lorez-like model. Since the oscillatory instability is caused because of the generation of the vertical vorticity, any Lorenz-like model to be successful in capturing essential behavior in this problem must include the relevant vorticity modes. We describe a minimum mode model here, which include such coupling and describe rolls as well as squares. We write the z-components of the velocity and the vorticity fields as

$$
\begin{aligned}
w=\left[a_{1} \cos (k x)+a_{2} \cos (k y)\right] \sin (\pi z) \\
+\left[a_{3} \cos (k x) \cos (k y)+a_{4} \sin (k x) \sin (k y)\right] \sin (\pi z) \\
+a_{5} \cos (k x) \cos (k y) \sin (2 \pi z)
\end{aligned}
$$

and

$$
\begin{aligned}
& \zeta=\left[b_{1} \sin (k x)+b_{2} \sin (k y)\right] \\
& \quad+\left[b_{3} \sin (2 k x)+b_{4} \sin (2 k y)+b_{5} \cos (k x) \sin (k y)+b_{6} \sin (k x) \cos (k y)\right] \cos (\pi z)
\end{aligned}
$$

In the above we have assumed stress-free surfaces in z-direction and periodic and stress free in lateral direction. For thermal boundary condition we assume perfectly conducting horizontal boundaries. With these modes the model reads

$$
\begin{aligned}
& \dot{\mathbf{X}}=\mu \mathrm{X}+\left(\begin{array}{c}
X_{2} \\
X_{1}
\end{array}\right) Z+\frac{b^{\prime}}{4}\left(\begin{array}{c}
-Y_{2} S_{2} \\
Y_{1} S_{1}
\end{array}\right)-\frac{b}{4}\left(\begin{array}{c}
Y_{2} T_{1} \\
Y_{1} T_{2}
\end{array}\right) \\
& \dot{\mathrm{Y}}=-b^{\prime} \mathbf{Y}-\left(\begin{array}{c}
X_{1} U_{1} \\
X_{2} U_{2}
\end{array}\right)-\left(\begin{array}{c}
X_{2} S_{1} \\
X_{1} S_{2}
\end{array}\right)+\left(\begin{array}{c}
X_{2} T_{2} \\
X_{1} T_{1}
\end{array}\right) \\
& \dot{\mathrm{S}}=-d_{1}^{2} \mathbf{S}+b\left(\begin{array}{c}
-X_{2} Y_{1} \\
X_{1} Y_{2}
\end{array}\right)+\left(\begin{array}{c}
U_{1} \\
-U_{2}
\end{array}\right) Z \\
& \dot{\mathbf{T}}=-d_{1}^{2} \mathbf{T}+b\left(\begin{array}{c}
X_{1} Y_{2} \\
X_{2} Y_{1}
\end{array}\right)+\frac{1}{2}\left(\begin{array}{c}
U_{2} \\
-U_{1}
\end{array}\right) Z \\
& \dot{\mathrm{U}}=-d_{2}^{2} \mathrm{U}+b\left(\begin{array}{c}
-X_{1} Y_{1} \\
X_{2} Y_{2}
\end{array}\right)+\frac{3}{2}\left(\begin{array}{c}
-T_{2} \\
T_{1}
\end{array}\right) Z+\frac{3}{2}\left(\begin{array}{c}
S_{1} \\
-S_{2}
\end{array}\right) Z \\
& \dot{Z}=-2 d_{1}^{Z}-\frac{1}{d_{1}^{2}}\left[b X_{1} X_{2}+\frac{3 b}{32}\left(S_{1} U_{1}-S_{2} U_{2}\right)\right]
\end{aligned}
$$

where $b=\frac{8}{3}, b^{\prime}=\frac{1}{3}, d_{1}^{2}=\frac{4}{3}, d_{2}^{2}=2 . X_{1}, X_{2}, Y_{1}, Y_{2}, S_{1}, S_{2}, T_{1}, T_{2}, U_{1}, U_{2}$ and $Z$ are the rescaled versions of $a_{1}, a_{2}, b_{1}, b_{2}, a_{3}, a_{4}, b_{3}, b_{4}, b_{5}, b_{6}$ and $a_{5}$ respectively. 
The exact roll solution at $\mu=0$ is given by $X_{1}^{0}=a$ for rolls parllel to $\mathrm{y}$-axis. The stability of these growing rolls will be decide by the following set of equations:

$$
\begin{gathered}
\dot{X}_{2}=a Z \\
\dot{Y}_{1}=-b^{\prime} Y_{1}-a U_{1} \\
\dot{Y}_{2}=-b^{\prime} Y_{2}-a S_{2}+a T_{1} \\
\dot{S}_{2}=-d_{1}^{2} S_{2}+a b Y_{2} \\
\dot{T}_{1}=-d_{1}^{2} T_{1}+a b Y_{2} \\
\dot{U}_{1}=-d_{2}^{2} U_{1}-a b Y_{1} \\
\dot{Z}=-2 d_{1}^{2} Z-\frac{2 a b}{d_{1}^{2}} X_{2}
\end{gathered}
$$

Assuming the time dependence of all the fields as $e^{s t}$ we find that the growth rate, $\mathrm{s}$, is given by

$$
\left(s+d_{1}^{2}\right)^{2}\left(s+b^{\prime}\right)\left[\{ a ^ { 2 } b + ( s + b ^ { \prime } ) ( s + d _ { 2 } ^ { 2 } ) \} \left\{s\left(s+2 d_{1}^{2}\right)+\frac{2 a^{2} b}{d_{1}^{2}}=0\right.\right.
$$

There is no positive root of the above equation. So this model is not good enough to capture instability generated by vertical vorticity. One needs to incorporate more modes, and particularly relaxing some condition in lateral direction may be enough to capture competition between different patterns.

\subsection{Conclusions}

The main result is that there is no stationary stable patterns above $R_{c}$ for $P=0$. We did derive the coefficient of the amplitude equations up to the leading order in the case of supeposition of rolls. For higher order amplitude equations, we used symmetry properties to derive the amplitude equations in case of squares. We summerize our results on the stability of stationary patterns in the following bifurcation diagram. To make the picture complete, we include Busse's results in the diagram. 


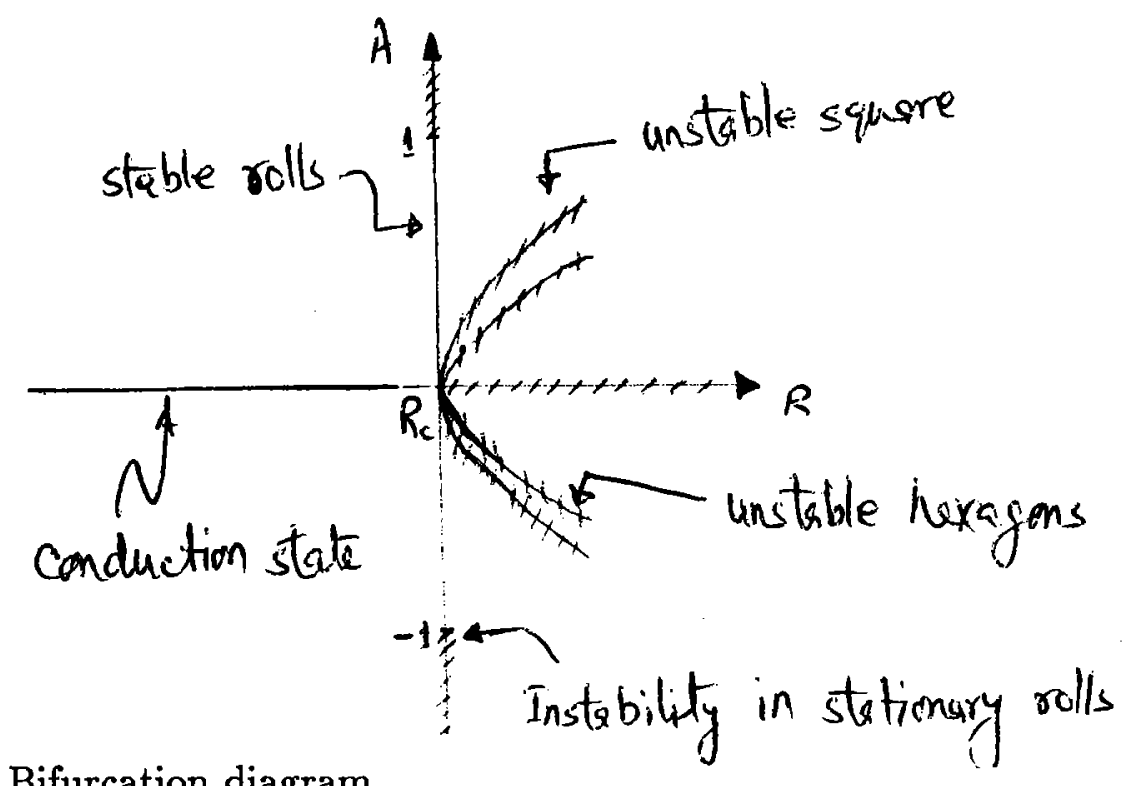

Figure 17.3. Bifurcation diagram

With our study and other results the follwing picture emerges:

There is no stable stationary state near the onset at zero Prandtl number. The growing roll, which is an exact solution of the hydrodynamic equation in this limit goes under oscillatory instability because of the generation of the vertical vorticity at some critical amplitude. This probably leads, as shown by Thual,to competition among the patterns near the onset. Therefore, the hydrodynamic equations [Eqs(2)] at zero Prandtl number represent a very good system to study temporal behaviour, competition of patterns and turbulence close to the convective instabilty.

Acknowledgements I am indebted to Stephan Fauve and George Veronis for discussions I had with them very regularly on this project. It was a great pleasure to work with them. I appreciate friendly atmosphere, where all members were easily available for any discussion. At last I would like to thank my wife, Tara for her constant encouragement during the project.

\section{References}

Busse, F. H. (1972) The oscillatory instability of convection rolls in a layer with stree-free boundaries. J. Fluid Mech. 97

Clever, R. M. and Busse, F.H. (1981) Low-Prandtl-number convection in layer heated from below. J. Fluid Mech. 102, 61-83.

Herring, J. R. (1970) Convection at zero Prandtl number. WHOI-GFD, Vol. 1, 115-118

Proctor, M.R.E. (1977) Inertial convection at low Prandtl number. J. Fluid Mech. 82 97-114.

Spiegel, E.A. (1962) J. Geophysical Res. 67, 3063

Thual, O. (1991) Zero Prandtl number convection. (Preprint.), see also WHOI Technical Report 


\title{
18. Ray Theory of Water Waves on a Current
}

\author{
Paul A. Milewski \\ Department of Mathematics, M.I.T., Cambridge, MA 02139
}

\begin{abstract}
The interaction between a current with vorticity and short surface gravity waves is studied using ray theory. The expansion used is based on both the Froude number of the current and the amplitude of the wave being small and is similar to that of Keller (1979). A sequence of problems results, describing both the evolution of the current and of the waves. We assume that the current is given. The dispersion relation is a doppler shifted version of the dispersion relation in quiescent water. The amplitude equation contains several new terms when compared to that of Longuet-Higgins and Stewart (1961). Three new contributions appear explicitly: the effect of horizontal vorticity, the effect of the undulation of the free surface due to the current and the effect of the first order correction to the current.
\end{abstract}

\subsection{Introduction}

The study of waves propagating on the surface of a fluid in non-uniform motion is not new (see for example Johnson 1947, Ursell 1960). At first, the refractive changes in wavelength and direction of propagation were studied. No dynamical interaction between the wave and the current was considered and therefore the changes in amplitude were thought to be due only to conservation of wave energy. Longuet-Higgins and Stewart (1961) were the first to propose that the current interacted directly with the waves by doing work on them, and calculated its effect. They did so for two special cases (when the change in the primary horizontal current is balanced by vertical upwelling and when it is balanced by a secondary horizontal current) and then inferred a general energy equation. We will show that they ignored certain effects, and we will generalize their equation to include them. We will not make any assumptions about the irrotationality of the velocity fields of the current or of the waves.

There are many physical situations where both vorticity and surface waves are present. Examples occur in the wake of a ship and in the estuary of a river. The effect of vorticity has been studied by several authors both analytically and experimentally (see Thomas 1981 and the references in that paper). We extend the previous work by using a method of asymptotic expansion inspired by Keller (1979). He studied the wave pattern due to a ship. Our results are compared to his and to Longuet-Higgins and Stewart's. 


\subsection{Formulation of the Problem}

We consider a three dimensional fluid domain with a mean free surface close to the $z=0$ plane. The fluid is incompressible, not stratified, inviscid, infinitely deep and surface tension is negligible. The assumption of infinite depth is a relatively weak one, since all that is necessary is that the depth be larger than the typical wavelength. Since we are studying short waves, this is easily satisfied. The atmosphere above the fluid is modeled by the pressure being constant on the surface of the fluid. The force of gravity points in the $-\mathbf{e}_{\mathbf{3}}$ direction. All dependent variables are functions of $(x, y, z, t)=(\mathbf{x}, t)$ except for the surface height $\eta$ which is a function of $(x, y, t)$ only. Vectors are denoted by boldface characters.

The governing equations are:

$$
\begin{gathered}
\left(\partial_{t}+\mathbf{u} \cdot \nabla\right) \mathbf{u}=-\frac{1}{\rho} \nabla \hat{p}-g \mathbf{e}_{\mathbf{3}} \\
\nabla \cdot \mathbf{u}=0 \\
\left(\partial_{t}+u \partial_{x}+v \partial_{y}\right) \eta=w, \quad z=\eta \\
\hat{p}=0, \quad z=\eta .
\end{gathered}
$$

They are supplemented by the condition $\mathbf{u} \rightarrow 0$ as $z \rightarrow-\infty$. We non-dimensionalize the equations using a velocity scale $U$ (a characteristic velocity of the background current), a length scale $L$ (a characteristic length scale over which variations in the velocity occur) and a time scale $L / U$. This choice introduces the Froude number $F=U / \sqrt{g L}$ as a parameter. We define $\epsilon=F^{2}$ and use $\epsilon$ as a small parameter for the basis of a perturbation expansion. The non-dimensionalized equations are:

$$
\begin{gathered}
\left(\partial_{t}+\mathbf{u} \cdot \nabla\right) \mathbf{u}=-\nabla \hat{p}-\frac{1}{\epsilon} \mathbf{e}_{\mathbf{s}} \\
\nabla \cdot \mathbf{u}=0 \\
\epsilon\left(\partial_{t}+u \partial_{x}+v \partial_{y}\right) \eta=w, \quad z=\epsilon \eta \\
\hat{p}=0, \quad z=\epsilon \eta .
\end{gathered}
$$

One can simplify the momentum equation by writing $\hat{p}=-z / \epsilon+p$ where $p$ is the pressure deviation from hydrostatic. The hydrostatic term will reappear in the dynamic boundary condition and forces the rescaling of $\eta$ as $\epsilon \eta$ (see 1d). In the limit $\epsilon \ll 1$ the current causes only small variations of the free surface. We now want to study the propagation of waves on this surface. This is accomplished by the expansion

$$
\begin{aligned}
\mathbf{u} & =\overline{\mathbf{u}}+\delta \mathbf{u}_{\mathbf{w}}+\ldots \\
p & =\bar{p}+\delta p_{\boldsymbol{w}}+\ldots \\
\eta & =\bar{\eta}+\delta \eta_{\boldsymbol{w}}+\ldots
\end{aligned}
$$


Here $\delta$ is the relative amplitude of the wave compared to the current. Since we want to study waves that are short in comparison to variations in the medium (ray theory) we write

$$
\begin{gathered}
\mathbf{u}_{\mathbf{w}}=\exp \left[i \epsilon^{-1} s(\mathbf{x}, t)\right]\left(\mathbf{u}_{0}+\epsilon \mathbf{u}_{1}+\ldots\right) \\
p_{w}=\exp \left[i \epsilon^{-1} s(\mathbf{x}, t)\right]\left(p_{0}+\epsilon p_{1}+\ldots\right) \\
\eta_{w}=\exp \left[i \epsilon^{-1} s(x, y, \epsilon \eta, t)\right]\left(\eta_{0}+\epsilon \eta_{1}+\ldots\right) .
\end{gathered}
$$

We also expand the current as

$$
\begin{aligned}
\overline{\mathbf{u}} & =\overline{\mathbf{u}}_{0}+\epsilon \overline{\mathbf{u}}_{1}+\ldots \\
\bar{p} & =\bar{p}_{0}+\epsilon \bar{p}_{1}+\ldots \\
\bar{\eta} & =\bar{\eta}_{0}+\epsilon \bar{\eta}_{1}+\ldots
\end{aligned}
$$

These expansions are quite general and some explanation of their meaning is in order. The small parameter $\delta$ can be dependent on or independent of $\epsilon$. If $\delta$ is related to $\epsilon$ then the above expansions describe the wave production and motion due to the current, as is the case when waves are created by a ship. This is the case studied by Keller using potential theory. He shows that to obtain wave motion, given the class of boundary conditions in the ship problem one must pick any $\delta$ from the family $\delta=\epsilon^{n+1 / 2}$ where $n=0,1,2 \ldots$ If $\delta$ is independent of $\epsilon$ then the expansions describe the interaction of a current with waves due to another source such as a wave maker. This is the case studied here. The expansion has the following meaning: $\overline{\mathbf{u}}_{0}$ is a flow which is given but might fail to satisfy some boundary condition, $\overline{\mathbf{u}}_{1}$ is the correction to this flow to satisfy the boundary conditions, $\mathbf{u}_{0}$ is the leading order wave and $\mathbf{u}_{1}$ is a correction to $\mathbf{u}_{0}$ due to the interaction of the wave and the current.

\subsection{The Hierarchy of Equations to be Solved}

The substitution of these asymptotic expansions into 1 is quite messy and one must be especially cautious when treating the free surface conditions. The sequence of problems to be solved is as follows.

Order 1:

$$
\begin{gathered}
\left(\partial_{t}+\overline{\mathbf{u}}_{0} \cdot \nabla\right) \overline{\mathbf{u}}_{0}=-\nabla \bar{p}_{0} \\
\nabla \cdot \overline{\mathbf{u}}_{0}=0 \\
\bar{w}_{0}=0, \quad z=0 \\
\bar{p}_{0}=\bar{\eta}_{0}, \quad z=0 .
\end{gathered}
$$

Order $\epsilon$ :

$$
\begin{gathered}
\left(\partial_{t}+\overline{\mathbf{u}}_{0} \cdot \nabla\right) \overline{\mathbf{u}}_{1}+\overline{\mathbf{u}}_{1} \cdot \nabla \overline{\mathbf{u}}_{0}=-\nabla \bar{p}_{1} \\
\nabla \cdot \overline{\mathbf{u}}_{1}=0
\end{gathered}
$$




$$
\begin{gathered}
\left(\partial_{t}+\bar{u}_{0} \partial_{x}+\bar{v}_{0} \partial_{y}\right) \bar{\eta}_{0}=\bar{w}_{1}+\bar{\eta}_{0} \partial_{z} \bar{w}_{0}, \quad z=0 \\
\bar{p}_{1}-\bar{\eta}_{1}+\bar{\eta}_{0} \partial_{z} \bar{p}_{0}=0, \quad z=0 .
\end{gathered}
$$

Order $\delta$ : (here $D=\partial_{t}+\mathbf{u}_{0} \cdot \nabla$.)

$$
\begin{gathered}
i D s \mathbf{u}_{0}+i(\nabla s) p_{0}=0 \\
\mathbf{u}_{0} \cdot \nabla s=0 \\
i \eta_{0} D s-w_{0}=0, \quad z=0 \\
p_{0}-\eta_{0}=0, \quad z=0 .
\end{gathered}
$$

Order $\epsilon \delta$ :

$$
\begin{gathered}
i D s \mathbf{u}_{1}+i(\nabla s) p_{1}=-D \mathbf{u}_{0}-\mathbf{u}_{0} \cdot \nabla \overline{\mathbf{u}}_{0}-\nabla p_{0}-i \mathbf{u}_{0} \overline{\mathbf{u}}_{1} \cdot \nabla s \\
\mathbf{u}_{1} \cdot \nabla s=i \nabla \cdot \mathbf{u}_{0} \\
i \eta_{1} D s-w_{1}=-D \eta_{0}-\mathbf{u}_{0} \cdot \nabla \bar{\eta}_{0}-i \eta_{0}\left(\bar{u}_{1} s_{x}+\bar{v}_{1} s_{y}\right) \\
-i \eta_{0} \bar{\eta}_{0} \partial_{z}\left[\left(\partial_{t}+\bar{u}_{0} \partial_{x}+\bar{v}_{0} \partial_{y}\right) s\right] \\
-i \eta_{0} s_{z} D \bar{\eta}_{0}+\eta_{0} \partial_{z} \bar{w}_{0}+\bar{\eta}_{0} \partial_{z} w_{0}, \quad z=0 \\
p_{1}-\eta_{1}=-\eta_{0} \partial_{z} \bar{p}_{0}-\bar{\eta}_{0} \partial_{z} p_{0}, \quad z=0 .
\end{gathered}
$$

Equations 3 yield the variational or linearized problem for the evolution of disturbances on an inviscid flow. The forcing is in the boundary conditions. We assume that both 2 and 3 have been solved and focus on the lowest order coupling between $\overline{\mathbf{u}}$ and $\mathbf{u}_{\mathbf{w}}$. Therefore we concentrate on the equations for the wave motion 4 and 5 .

\subsection{Wave Motion}

We now solve equations 4 and 5 . Taking the scalar product of equation $4 \mathrm{a}$ and $\nabla s$ and using $4 \mathrm{~b}$ we obtain

$$
(\nabla s)^{2}=0
$$

Using equations $4 \mathrm{c}$ and $4 \mathrm{~d}$ along with the $\mathbf{e}_{3}$ component of $4 \mathrm{a}$ we obtain the dispersion relation

$$
(D s)^{2}=i s_{z}, \quad z=0 .
$$

In terms of the surface wave-vector, 7 becomes the more familiar

$$
(D s)^{2}=\left(s_{x}^{2}+s_{y}^{2}\right)^{1 / 2}, \quad z=0 .
$$

To obtain 8 the condition $\mathbf{u}_{1} \rightarrow 0$ as $z \rightarrow-\infty$ was used to choose the sign of the root. The rays are the characteristic curves of 8 . To solve explicitly for them we write $\mathbf{k}=s_{x} \mathbf{e}_{1}+s_{y} \mathbf{e}_{2}=$ $\nabla_{2} s, k=|\mathbf{k}|$ and $\omega=-s_{t}$. Then the dispersion relation can be written as

$$
\omega=\mathbf{k} \cdot \overline{\mathbf{u}}_{0} \pm \sqrt{k} \equiv W(\mathbf{k}, x, y, t),
$$


where the sign is chosen by a radiation condition specific to the problem. Without loss of generality we choose the + sign from now on.

The rays satisfy the set of ordinary differential equations (Whitham 1974):

$$
\begin{gathered}
\frac{d k_{i}}{d t}=-\frac{\partial W}{\partial x_{i}} \\
\frac{d x_{i}}{d t}=\frac{\partial W}{\partial k_{i}} .
\end{gathered}
$$

Recall that the group velocity $\mathbf{c}_{\mathbf{g}}$ is given by

$$
\mathbf{c}_{\mathbf{g}}=\frac{\partial W}{\partial k_{i}}=\overline{\mathbf{u}}_{0}+\frac{1}{2} k^{-\frac{3}{2}} \mathbf{k}
$$

The equation for the amplitude of the wave involves 4 and 5 . The amplitude equation is the equation for either $\mathbf{u}_{0}, \eta_{0}$ or $p_{0}$, the latter two being equal on the surface by $4 \mathrm{~d}$. We shall choose to solve for $p_{0}$; the other unknowns can be found immediately by substituting back into 4 . Formally the amplitude equation is found by applying the solvability condition that the right hand side of 5 be orthogonal to the null-space of 4 . This is the same thing as eliminating $\mathbf{u}_{1}, p_{1}, \eta_{1}$ from 5 using 6 and 7 .

The momentum-continuity equation is obtained by taking the scalar product of $5 \mathrm{a}$ and $\nabla s$, using 6 and $4 \mathrm{~b}$, and substituting equation $5 \mathrm{~b}$ to yield

$$
-\nabla \cdot \mathbf{u}_{0} D s+\left(D \mathbf{u}_{0}+\mathbf{u}_{0} \cdot \nabla \overline{\mathbf{u}}_{0}\right) \cdot \nabla s=-\nabla p_{0} \cdot \nabla s .
$$

This can be reduced to an equation for $p_{0}$ alone by substituting $4 \mathrm{a}\left(\mathbf{u}_{0}=-p_{0} \nabla s / D s\right)$. After some algebra, using identities such as $\nabla s \cdot D \nabla s=\frac{1}{2} D\left[(\nabla s)^{2}\right]=0$, we obtain

$$
p_{0} D s \triangle s+2 D s \nabla s \cdot \nabla p_{0}+2 p_{0} \nabla s \cdot \nabla \overline{\mathbf{u}}_{0} \cdot \nabla s=0 .
$$

The surface equation is found by multiplying $5 \mathrm{c}$ by $i D s$ and adding the $\mathbf{e}_{\mathbf{3}}$ component of $5 \mathrm{a}$. Then substituting $p_{1}$ from $5 \mathrm{~d}$ and using the dispersion relation 7 we obtain

$$
\begin{array}{rl}
-i s_{z}\left(\eta_{0} \partial_{z} \bar{p}_{0}+\bar{\eta}_{0} \partial_{z} p_{0}\right)=D & s\left[-i D \eta_{0}-i \mathbf{u}_{0} \cdot \nabla \bar{\eta}_{0}+\eta_{0}\left(\bar{u}_{1} s_{x}+\bar{v}_{1} s_{y}\right)\right. \\
& +\eta_{0} \bar{\eta}_{0} \partial_{z}\left(D s-\bar{w}_{0} s_{z}\right)+\eta_{0} s_{z} D \bar{\eta}_{0}+i \eta_{0} \partial_{z} \bar{w}_{0} \\
& \left.+i \bar{\eta}_{0} \partial_{z} w_{0}\right]-D w_{0}-\mathbf{u}_{0} \cdot \nabla \bar{w}_{0}-\partial_{z} p_{0}-i w_{0} \overline{\mathbf{u}}_{1} \cdot \nabla s, \quad z=0 .
\end{array}
$$

Now, to obtain an equation with $p_{0}$ alone we substitute either $w_{0}=-s_{z} p_{0} / D s$ (wherever z-derivatives of $w_{0}$ appear) or $w_{0}=i D s p_{0}$ (valid only at $z=0$ ) and $4 \mathrm{~d}$ to get

$$
\begin{aligned}
2 i D s D p_{0}+\partial_{z} p_{0}=[ & -i D^{2} s+(D s)^{2} \partial_{z} \bar{p}_{0}-i \bar{\eta}_{0} s_{z z}+i \nabla s \cdot \nabla \bar{\eta}_{0} \\
& +D s\left(2 \overline{\mathbf{u}}_{1} \cdot \nabla s-\bar{w}_{1} s_{z}\right)+2 D s \bar{\eta}_{0} D s_{z} \\
& \left.+2 D s \bar{\eta}_{0} \nabla s \cdot\left(\partial_{z} \overline{\mathbf{u}}_{0}\right)-D s \bar{\eta}_{0} s_{z} \partial_{z} \bar{w}_{0}+D s s_{z} D \bar{\eta}_{0}\right] p_{0}, \quad z=0
\end{aligned}
$$


Finally we write an equation in $(x, y, t)$ only by substituting $\partial_{z} p_{0}$ from 11 . We also drop the $\partial_{z} \bar{p}_{0}$ term since by the $\mathbf{e}_{3}$ component of $2 \mathrm{a}$ and the fact that $\bar{w}_{0}=\partial_{t} \bar{w}_{0}=\partial_{x} \bar{w}_{0}=\partial_{y} \bar{w}_{0}=0$ at $z=0$ therefore $\partial_{z} \bar{p}_{0}=0$. Thus we obtain

$$
\begin{aligned}
D p_{0}-\frac{1}{2(D s)^{3}} \nabla_{2} s \cdot \nabla_{2} p_{0}= & \frac{1}{2 D s}\left[\frac{1}{2(D s)^{2}} \Delta s-D^{2} s+\nabla s \cdot \nabla \bar{\eta}_{0}\right. \\
& -i D s\left[\left(2 \overline{\mathbf{u}}_{1} \cdot \nabla s-\bar{w}_{1} s_{z}\right)+s_{z} D \bar{\eta}_{0}\right. \\
& \left.-\bar{\eta}_{0} s_{z} \partial_{z} \bar{w}_{0}+2 \bar{\eta}_{0} \nabla s \cdot\left(\partial_{z} \overline{\mathbf{u}}_{0}\right)+2 \bar{\eta}_{0} D s_{z}\right] \\
& \left.-\bar{\eta}_{0} s_{z z}-\frac{1}{(D s)^{3}} \nabla s \cdot \nabla \overline{\mathbf{u}}_{0} \cdot \nabla s\right] p_{0}, \quad z=0 .
\end{aligned}
$$

This can be rewritten as

$$
\left(\partial_{t}+\mathbf{c}_{\mathbf{g}} \cdot \nabla\right) p_{0}+\gamma p_{0}=0, \quad z=0
$$

where

$$
\begin{aligned}
\gamma=- & \frac{1}{2 D s}\left[\frac{1}{2(D s)^{2}} \Delta s-D^{2} s+\nabla s \cdot \nabla \bar{\eta}_{0}-i D s\left[\left(2 \overline{\mathbf{u}}_{1} \cdot \nabla s\right.\right.\right. \\
& \left.-\bar{w}_{1} s_{z}\right)+s_{z} D \bar{\eta}_{0}-\bar{\eta}_{0} s_{z} \partial_{z} \bar{w}_{0}+2 \bar{\eta}_{0} \nabla s \cdot\left(\partial_{z} \overline{\mathbf{u}}_{0}\right) \\
& \left.\left.+2 \bar{\eta}_{0} D s_{z}\right]-\bar{\eta}_{0} s_{z z}-\frac{1}{(D s)^{3}} \nabla s \cdot \nabla \overline{\mathbf{u}}_{0} \cdot \nabla s\right], \quad z=0 .
\end{aligned}
$$

Equation 12 can now be written in characteristic form as the amplitude along the ray defined by 10

$$
\frac{d p_{0}}{d t}+\gamma p_{0}=0, \quad z=0
$$

The solution is

$$
p_{0}=p_{0}\left(t_{0}\right) \exp \left[-\int_{t_{0}}^{t} \gamma\left(t^{\prime}\right) d t^{\prime}\right], \quad z=0,
$$

where $p_{0}\left(t_{0}\right)$ is the value of $p_{0}$ at a point $\left(x\left(t_{0}\right), y\left(t_{0}\right)\right)$ on the ray.

Equations 9, 10,13, with appropriate initial conditions, complete the solution on the surface. One can then extend the solution into the whole fluid with 11. 


\subsection{Interpretation and Comparison with Previous Results}

Let us now compare these results to the work of other authors. Keller considers the propagation of waves from a ship. The current $\overline{\mathbf{u}}$ is the potential flow around the moving ship; the waves are radiated from non-smooth portions of the vessel such as the bow and the stern. They then interact with this current. Since his expansion is $\Phi=\phi+F^{2} \phi_{1}+$ $F^{3} \exp \left[i F^{-2} s\right]\left(a+F^{2} a_{1}\right)+\ldots$, taking $\mathbf{u}=\nabla \Phi$ yields the scaling $\delta=F=\epsilon^{1 / 2}$. Substituting $\overline{\mathbf{u}}_{0}=\nabla \phi, \overline{\mathbf{u}}_{1}=\nabla \phi_{1}$ and $p_{0}=i a D s$ in 12 one recovers his result. We will also show that the vorticity appears explicitly in our equations. Therefore it is impossible to obtain our equations by substituting the above relations into Keller's equations. Also, if the scaling $\delta=\epsilon^{1 / 2}$ was chosen in the present work from the start, the governing equations for the current would have been different at $\mathrm{O}(\epsilon)$. For example the term $\mathbf{u} \cdot \nabla \mathbf{u}$ in 1a would produce the term $i \exp \left[2 i \epsilon^{-1} s\right]\left(\mathbf{u}_{1} \cdot \nabla s \mathbf{u}_{0}+\mathbf{u}_{0} \cdot \nabla s \mathbf{u}_{1}-i \mathbf{u}_{0} \cdot \nabla \mathbf{u}_{0}\right)$ in 3a.

Another work which allows ready comparison is Longuet-Higgins and Stewart. They have approached the same problem of wave-current interaction with potential theory and a fluid of finite depth. Their generalized interaction equation is written in the form of an energy equation where the wave energy density is propagated along rays. The rate of change of the energy density is due to two terms, one being the focusing or divergence of the rays, and the other the work done by the current on the waves. Their equation for $\hat{E}$ is

$$
\frac{\partial \hat{E}}{\partial t}+\nabla_{2} \cdot\left[\hat{E}\left(\hat{\mathbf{c}}_{\mathbf{g}}+\mathbf{U}\right)\right]+\frac{1}{2} \hat{S}_{i j}\left(\frac{\partial U_{i}}{\partial x_{j}}+\frac{\partial U_{j}}{\partial x_{i}}\right)=0 .
$$

Where $\hat{S}_{i j}$ is a radiation tensor, which for the limit of infinitely deep water is

$$
\hat{\mathbf{S}}=\left(\begin{array}{ccc}
\frac{1}{2} \hat{E} & 0 & 0 \\
0 & 0 & 0 \\
0 & 0 & 0
\end{array}\right)
$$

and $\hat{\mathbf{c}}_{\mathbf{g}}$ is the group velocity of the waves with no current $\left(\hat{\mathbf{c}}_{\mathbf{g}}+\mathbf{U}=\mathbf{c}_{\mathbf{g}}\right)$.

In the present work, introducing the energy density $E=\frac{1}{2} p_{0}^{2}$ in 12 we obtain

$$
\frac{\partial E}{\partial t}+\nabla_{2} \cdot\left(E \mathbf{c}_{\mathbf{g}}\right)+\left(2 \gamma-\nabla_{2} \cdot \mathbf{c}_{\mathrm{g}}\right) E=0
$$

After considerable manipulation we get the equation

$$
\begin{aligned}
\frac{\partial E}{\partial t}+\nabla_{2} \cdot\left(E \mathbf{c}_{\mathrm{g}}\right)=- & \frac{1}{2} S_{i j}\left(\frac{\partial \bar{u}_{0 i}}{\partial x_{j}}+\frac{\partial \bar{u}_{0 j}}{\partial x_{i}}\right)+E\left[\frac{1}{s_{z}} \nabla_{2} s \cdot\left(\partial_{z} \overline{\mathbf{u}}_{0}\right)\right. \\
& +\frac{1}{D s}\left[\nabla s \cdot \nabla \bar{\eta}_{0}-i D s\left(\left(2 \overline{\mathbf{u}}_{1} \cdot \nabla s-\bar{w}_{1} s_{z}\right)+s_{z} D \bar{\eta}_{0}\right.\right. \\
& \left.\left.\left.-\bar{\eta}_{0} s_{z} \partial_{z} \bar{w}_{0}+2 \bar{\eta}_{0} \nabla s \cdot\left(\partial_{z} \overline{\mathbf{u}}_{0}\right)+2 \bar{\eta}_{0} D s_{z}\right)-\bar{\eta}_{0} s_{z z}\right]\right], \quad z=0,
\end{aligned}
$$


where

$$
\mathbf{S}=\frac{E}{2\left(s_{x}^{2}+s_{y}^{2}\right)}\left(\begin{array}{ccc}
s_{x}^{2} & s_{x} s_{y} & 0 \\
s_{x} s_{y} & s_{y}^{2} & 0 \\
0 & 0 & 0
\end{array}\right), \quad z=0
$$

The tensor $\hat{S}_{i j}$ is $S_{i j}$ in a rotated system of coordinates where $\overline{\mathbf{u}}_{0}$ is is the velocity in the direction of the wavevector. Since the wavevectors vary in space this rotation is a function of position. This can be seen in two ways; by setting $s_{y}=0$ in 17 one recovers 15 , or one can write $\hat{\mathbf{S}}=\mathbf{R}^{-1} \mathbf{S R}$ with

$$
\mathbf{R}=\left(\begin{array}{ccc}
1 & 1 & 0 \\
s_{y} / s_{x} & -s_{x} / s_{y} & 0 \\
0 & 0 & 0
\end{array}\right), \quad z=0
$$

The next term in 16 is due explicitly to the horizontal vorticity. Since $\partial_{x} \bar{w}_{0}=\partial_{y} \bar{w}_{0}=0$ at $z=0$ this term is $\mathbf{e}_{\mathbf{3}} \cdot \mathbf{k} \times \overline{\boldsymbol{\zeta}}_{0}$ where $\bar{\zeta}_{0}=\nabla \times \overline{\mathbf{u}}_{0}$ is the vorticity of the principal current. This term is obviously absent from a potential formulation. All of the other terms in 16 are due to either the deformation of the surface due to the current or the correction to the current, $\overline{\mathbf{u}}_{1}$. Both of these are absent from the formulation of Longuet-Higgins and Stewart.

\subsection{Conclusion}

An equation has been obtained for the amplitude of gravity waves, taking account of their interaction with an unsteady, non-uniform, slowly varying current. Neither the current nor the waves was assumed to be irrotational. The equation involves a direct dynamical interaction between the wave and the horizontal vorticity of the current. In addition the effect on the waves of the deformation of the surface due to the current (the effect due to the waves having to "climb" the free surface) and the effect of the correction to the current are included.

There are various points that need to be studied further. We would like to understand how vorticity is exchanged between the current and the wave and how it is transported by the wave. For instance if initially irrotational waves arrive in a region of vorticity, do they transport vorticity out of the region? Another interesting question is to find how much of the governing equation can be put in the form of conservation of wave-action. Specifically, can the vorticity term be put in this form? Lastly, some examples should be worked out and compared to available experimental data.

\section{Acknowledgements}

I would like to thank Joe Keller for his invaluable help. Thank you also to all the other people who made this a memorable summer: the GFD fellows, Bill Young and the steering committee, hurricane Bob, George Veronis for the baseball coaching and Kim for her delicious sandwiches. 


\section{References}

Johnson, J. W. (1947) The refraction of surface waves by currents. Trans. Amer. Geophys. Un., 28, 867-874

Keller, J. B. (1979) The ray theory of ship waves and the class of streamlined ships. J. Fluid Mech., 91, 465-488

Longuet-Higgins, M. S. \& Stewart, R. W. (1961) The changes in amplitude of short gravity waves on steady non-uniform currents. J. Fluid Mech., 10 , 529-549

Thomas, J. P. (1981) Wave-current interactions: an experimental and numerical study. Part 1. Linear Waves. J. Fluid Mech., 110 , 457-474

Ursell, F. (1960) Steady wave patterns on a non-uniform steady fluid flow. J. Fluid Mech., 9, 333-346

Whitham, G. B. (1974) Linear and Nonlinear Waves, Wiley. 


\title{
19. A Simple Model of Pressure Effects on Mush Growth
}

\author{
John F. Olson
}

Department of Earth, Atmospheric, and Planetary Sciences

Massachusetts Institute of Technology, Cambridge, MA 02139

\subsection{Introduction}

When we seek to describe the solidification of a melt, the problem is complicated by a moving boundary between the phases. And when the solidification occurs under conditions such that the solid/liquid interface is morphologically unstable (Sekerka [1973]), the interface not only moves but also deforms into an enormously complex shape. The region wherein crystals and liquid are intermixed has been called a mush, and Worster [1986] derived a set of equations to describe the growth of this mush by considering its locally averaged bulk properties. In this paper, the effect of pressure on the growth of a mush is considered by adding certain terms to a simplified version of Worster's model.

\subsubsection{Morphological instability}

The solid/liquid interface of a pure substance is always morphologically stable, but when a solution freezes, the mismatch between the rates of thermal diffusion (fast) and compositional diffusion (slow) can result in constitutional supercooling, which leads to morphological instability.

When a solution freezes, solute is excluded from the crystal into the solution, so the concentration in the solution near the interface increases (figure 1). Latent heat is also liberated at the interface. If the interface is perturbed, isotherms rapidly smooth out due to diffusion, while contours of concentration remain closely tied to the interface. The consequence is that lines of constant concentration and constant temperature cross, so that the liquid near the interface becomes supercooled.

Mullins and Sekerka [1964] showed that constitutional supercooling will occur whenever

$$
\left.k_{s} \frac{\partial T_{s}}{\partial z}\right|_{\text {Interface }}+\left.k_{l} \frac{\partial T_{l}}{\partial z}\right|_{\text {Interface }}<\left.\left(k_{s}+k_{l}\right) \frac{\partial T_{L i q}}{\partial c} \frac{\partial c}{\partial z}\right|_{\text {Interface }}
$$

where $k_{(s, l)}$ and $T_{(s, l)}$ are thermal diffusion coefficients and temperatures in the solid and liquid phases, $T_{L i q}$ is the liquidus temperature, $\mathrm{c}$ is concentration in the liquid phase and $z$ increases in the direction of motion of the solidifying front. More recently, Woods [1990] 
(a)

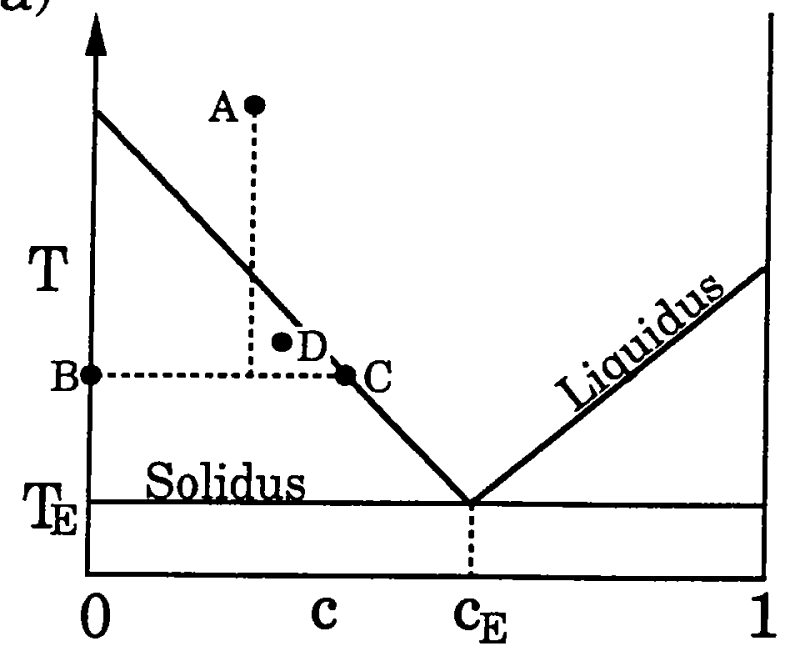

(b)

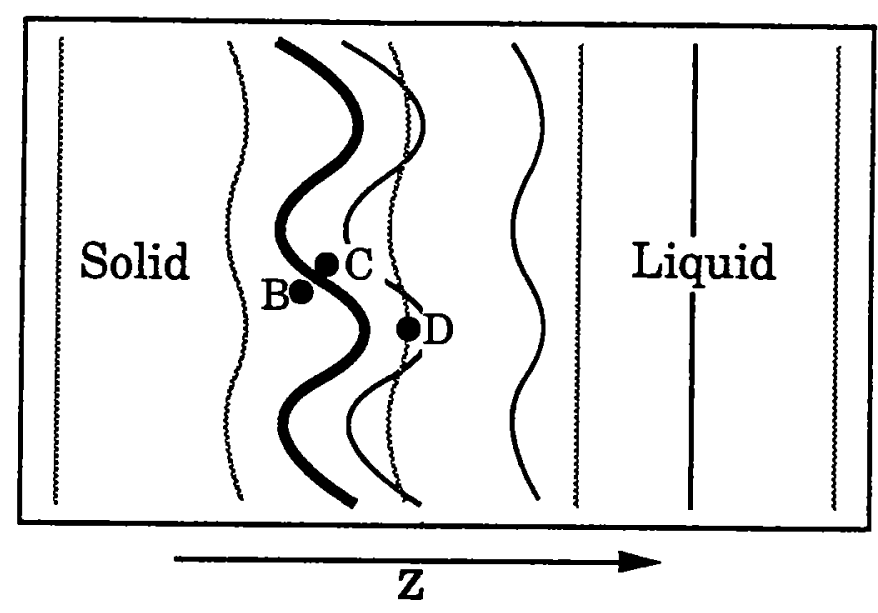

Figure 19.1. The Constitutional Supercooling Mechanism (a) A simplified phase diagram. A liquid solution at temperature and concentration $\mathrm{A}$ is cooled gradually; when the temperature first meets the liquidus curve (here and throughout approximated by a straight line) pure solid solute freezes out, leaving the solution enriched in solvent. The freezing temperature of the solution near the interface decreases because of the extra solvent, so the interface temperature and composition moves down to $\mathrm{C}$ as the solidification proceeds. (b) Heat (grey contours) and solvent (black contours) diffuse away from the solid/liquid interface (heavy curve). Just inside the interface, the composition and temperature match point $B$ on the phase diagram; just outside, they match point C. A little way from the interface, (at point D) the concentration has decreased less than the temperature has increased, so the solution there is supercooled (point $\mathrm{D}$ on the phase diagram), the liquid phase is unstable, and solidification will occur rapidly.

derived a condition for constitutional supercooling that includes the effect of pressure on the liquidus:

$\left.\overline{k_{s}} \frac{\partial T_{s}}{\partial z}\right|_{\text {Interface }}+\left.\overline{k_{l}} \frac{\partial T_{l}}{\partial z}\right|_{\text {Interface }}<F\left(k_{s}, k_{l}\right)\left(\left.\frac{\partial T_{L i q}}{\partial p} \frac{\partial p}{\partial z}\right|_{\text {Interface }}+\left.Q \frac{\partial T_{L i q}}{\partial c} \frac{\partial c_{l}}{\partial z}\right|_{\text {Interface }}\right)$

where the coefficients $\bar{k}_{(}(s, l)$ are related to the thermal diffusion coefficients, and $Q$ is a constant. $\frac{\partial T_{\mathrm{Liq}}}{\partial c}$ and $\frac{\partial C_{l}}{\partial z}$ are always both of the same sign, so the concentration effect always promotes instability. On the other hand $\frac{\partial p}{\partial z}$ can have any sign, and for most substances $\frac{\partial T_{L i q}}{\partial p}$ is positive, so the pressure effect can either promote or suppress the instability. Having established conditions under which morphological instability can occur-and hence, under which mush may form-it is appropriate to consider the equations that describe this mush. 


\subsubsection{Worster's Mush Model}

Consider a solution cooled by contact with a plate at $z=0$. We will suppose that conditions are such that a mush forms, so we take $z=a(t)$ as the position of the solid/mush interface and $z=b(t)$ as the mush/liquid interface. The "solid" phase is the region where all the interstitial liquid has frozen; it can be defined as the region where the temperature is everywhere below the solidus. Similarly, the RliquidS is the region where temperature is everywhere above the liquidus.
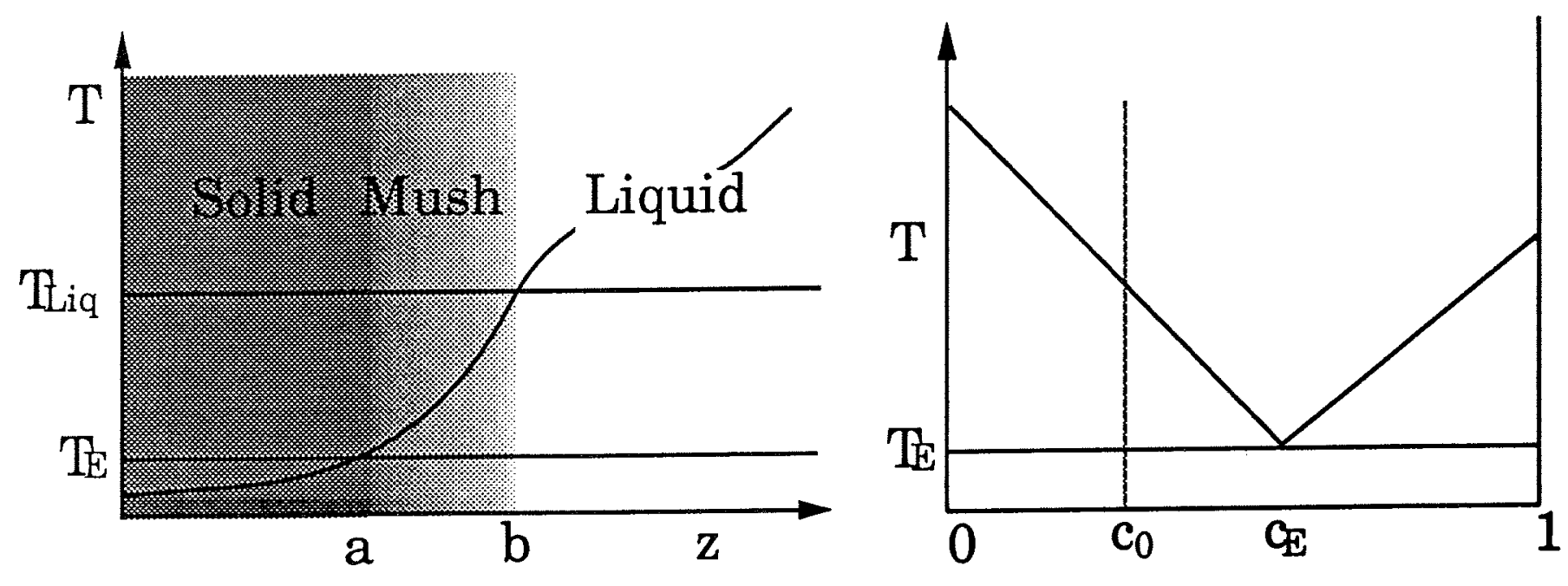

Figure 19.2. Worster's Mush Model When the temperature is below the eutectic, $T_{E}$, we have solid, and when above the liquidus, $T_{L i q}$, we have liquid. In between is a layer of mush. The solid fraction $\phi$ is 1 in the solid, 0 in the liquid, and varies through the mush. The variables $a$ and $b$ are the position of the solid/mush and mush/liquid interfaces, respectively.

The mush is treated as a separate phase with properties that vary with the solid fraction, $\phi$ in the mush. Given a region in the mush much smaller than the whole mush layer but much larger than an individual crystal in the mush, $\phi$ is defined as the ratio of the mass of solid to the total mass in the region. Then, for example, the density of the mush layer is taken to be

$$
\rho_{m}=\phi \rho_{s}+(1-\phi) \rho_{l}
$$

where $\rho_{(s, l)}$ are the densities of the solid and liquid phases. More importantly, the release of latent heat in the mush and the rate of exclusion of solvent from the crystals are determined from the rate of change of the solid fraction in the mush. This leads to these differential equations in the mush layer:

$$
\left(\rho C_{p}\right)_{m} \frac{\partial T}{\partial t}=\frac{\partial}{\partial z}\left(k_{m} \frac{\partial T}{\partial z}\right)+\rho_{s} L \frac{\partial \phi}{\partial t}
$$


for heat, and

$$
(1-\phi) \frac{\partial c}{\partial t}=\frac{\partial}{\partial z}\left(D(1-\phi) \frac{\partial c}{\partial z}\right)+c \frac{\partial \phi}{\partial t}
$$

for composition, where $C_{p}$ denotes heat capacity and $L$ is the latent heat of fusion. Note particularly the $\frac{\partial \phi}{\partial t}$ source terms in these equations. This system of equations is closed by imposing a local phase equilibrium everywhere in the mush: in other words, $\phi$ is evaluated from the phase diagram. The solid and liquid are modeled with simple diffusion:

$$
\rho_{(s, l)} C_{p(s, l)} \frac{\partial T}{\partial t}=k_{(s, l)} \frac{\partial^{2} T}{\partial z^{2}}
$$

for heat and

$$
\frac{\partial c}{\partial t}=D \frac{\partial^{2} c}{\partial z^{2}}
$$

for composition, where compositional diffusion is neglected in the solid. Temperature continuity is imposed at both interfaces, while compositional continuity is imposed only at the mush/liquid interface, not at the mush/solid interface. The solid fraction need not be continuous at either interface, since it is not continuous in the case of simple solid growth. Because of this, a finite amount of heat may be released at either boundary, so the temperature flux need not be continous at either boundary. These interface conditions will be considered in more detail in the next section.

\subsubsection{Simplifications for Small Chemical Diffusivity}

The mechanism of constitutional supercooling relies on the fact that heat diffusion is typically faster than chemical diffusion. We take this argument one step further, and neglect chemical diffusion altogether in this paper. In essence, we say that when solvent is excluded from a solidifying crystal, it remains in the neighbourhood of the interface so that the local average concentration is everywhere the same. This average concentration will be denoted $c_{0}$. Note that the concentration in the liquid fraction of the mush layer will vary from place to place, but the average concentration is everywhere $c_{0}$. With this, we can drop the compositional diffusion equations, and the solid fraction can be evaluated simply from the temperature.

If the liquidus temperature is given by

$$
T_{L i q}(c)=T_{E}+\Gamma\left(c_{E}-c\right)
$$

then the liquidus concentration at a given temperature is

$$
c_{L i q}(T)=c_{E}+\frac{T_{E}-T}{\Gamma} .
$$

By Worster's local equilibrium hypothesis, this will be the concentration in the liquid fraction of the mush. But the average concentration is always $c_{0}$, so

$$
\phi c_{s}+(1-\phi) c_{L i q}=c_{0}
$$


and the concentration of the solid is taken to be 0 , so

$$
\phi=1-\frac{c_{0}}{c_{L i q}}
$$

Defining scaled temperature and concentration:

$$
\tilde{c}=\frac{c}{c_{E}} \quad \tilde{T}=\frac{T-T_{E}}{\Gamma c_{E}}
$$

(see figure 3) simplifies the liquidus relation and solid fraction to

$$
\tilde{T}_{L i q}=1-\tilde{c} \quad \phi=1-\frac{\tilde{c}_{0}}{1-\tilde{T}} .
$$

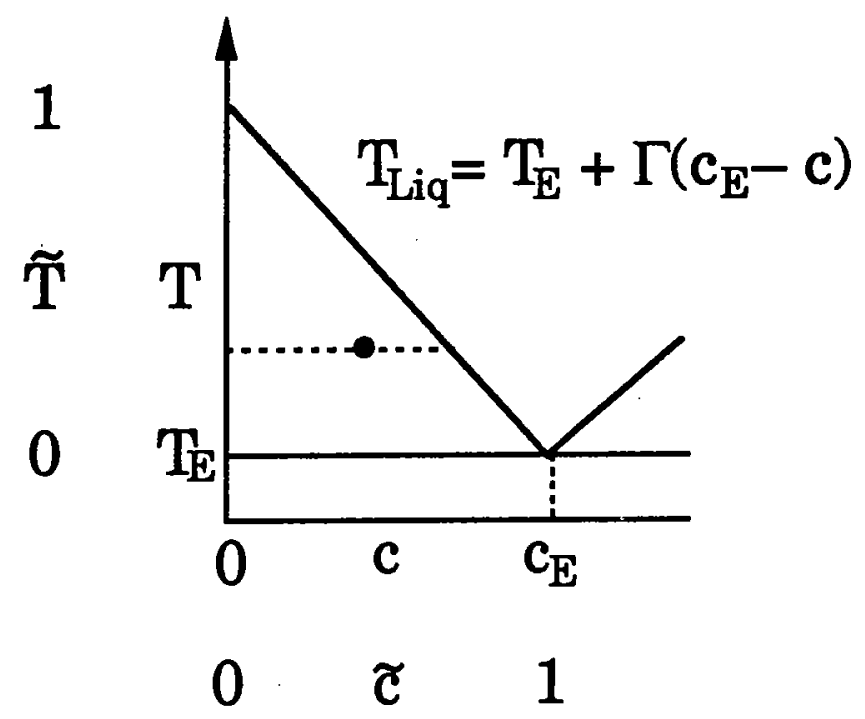

Figure 19.3. Phase Diagram Scalings Concentration is scaled by the eutectic composition, and temperatures are measured relative to the eutectic temperature and scaled by the liquidus slope.

These simplifications reduce the mush equations to

$$
\frac{\partial}{\partial z}\left(k_{m} \frac{\partial \tilde{T}}{\partial z}\right)=\left[\left(\rho C_{p}\right)_{m}+\frac{\rho_{s} L \tilde{c}_{0}}{(1-\tilde{T})^{2}}\right] \frac{\partial \tilde{T}}{\partial t}
$$

Calculations have been done to compare this simplified model to Worster's full equations (Jarvis [1991]); the full set predicts a mush layer two percent thicker than the simplified set, when $\frac{D}{k}$ is $\mathcal{O}\left(10^{-2}\right)$.

In the next section, a simple model of pressure effects on the liquidus will be introduced, and uniformly translating similarity solutions to the resulting equations will be presented. 


\subsection{Pressure Effect}

To consider pressure effects properly, one really ought to include a pressure field in the equations and allow the pressure to evolve dynamically. And one ought to include the thermodynamic effects of expansion work, because the solid will typically contract on freezing. We did not do this. Instead, we took the densities of solid and liquid to be the same, so that no explicit $\mathrm{pV}$ work would occur on freezing; and we modeled both the pressure gradient and the variation of liquidus with pressure as constants. In other words, we took

$$
T_{L i q}=T_{E}+\Gamma\left(c_{E}-c\right)-\Omega z
$$

and

$$
T_{\text {Solidus }}=T_{E}-\Omega z
$$

where $\Omega$ can be thought of as $\left|\left(\frac{\partial T_{i i q}}{\partial p}\right)\left(\frac{\partial p}{\partial z}\right)\right|$. Using the scaling of the previous section, the liquidus and solidus relations reduce to

$$
\tilde{T}_{L i q}=1-\tilde{c}-\tilde{\Omega} z \quad \text { and } \quad \tilde{T}_{\text {Solidus }}=-\tilde{\Omega} z
$$

with $\tilde{\Omega}=\frac{\Omega}{\Gamma c_{E}}$. This addition makes only a small difference in the solid fraction and the mush heat equations:

$$
\phi=1-\frac{\tilde{c}_{0}}{1-\tilde{T}-\tilde{\Omega} z}
$$

and

$$
\frac{\partial}{\partial z}\left(k_{m} \frac{\partial \tilde{T}}{\partial z}\right)=\left[\left(\rho C_{p}\right)_{m}+\frac{\rho_{s} L \tilde{c}_{0}}{(1-\tilde{T}-\tilde{\Omega} z)^{2}}\right] \frac{\partial \tilde{T}}{\partial t}
$$

We make a further simplification by taking the thermal diffusivity and heat capacity of the solid and liquid phases to be the same, along with the density. Then, scaling $t$ by $\frac{k}{\rho C_{p}}$ reduces the equations to

$$
\frac{\partial^{2} \tilde{T}}{\partial z^{2}}=\frac{\partial \tilde{T}}{\partial \tilde{t}}
$$

in the solid and liquid, and

$$
\frac{\partial^{2} \tilde{T}}{\partial z^{2}}=\left[1+\frac{S \tilde{c}_{o}}{(1-\tilde{T}-\tilde{\Omega} z)^{2}}\right] \frac{\partial \tilde{T}}{\partial \tilde{t}}
$$

in the mush, with $\tilde{t}=\frac{t k}{\rho C_{p}}$ and $S=\frac{L}{C_{p} \Gamma c_{E}} . S$ is called the Stefan number, which compares the relative importance of latent heat to specific heat. It can be thought of as an effective specific heat for the mush layer, because if $S$ is large, a great deal of heat has to be withdrawn from the mush to freeze it while only a small temperature change occurs; and if $S$ is small, the mush releases little heat but cools more as it solidifies. It should be noted that this Stefan number is somewhat different from the usual definition; in this case, the temperature scale chosen was inherent in the phase diagram $\left(\Gamma c_{E}\right)$ whereas the temperature scale is usually 
chosen as the difference between some hot boundary condition and the freezing temperature. This latter definition is natural for freezing a pure substance, but the former seems more appropriate in this case.

It is useful now to consider the interface conditions again, and derive explicit forms for them. At the mush/liquid boundary,

$$
\left.\tilde{T}_{m}\right|_{b(\tilde{t})}=\left.\tilde{T}_{l}\right|_{b(\tilde{t})}=1-\tilde{c}_{0}-\tilde{\Omega} b(\tilde{t}) \quad \text { so }\left.\quad \phi\right|_{b(\tilde{t})}=0
$$

while at the solid/mush boundary,

$$
\left.\tilde{T}_{s}\right|_{a(\hat{t})}=\left.\tilde{T}_{m}\right|_{a(\hat{t})}=-\tilde{\Omega} a(\tilde{t}) \quad \text { so }\left.\quad \phi\right|_{a(\hat{t})}=1-\tilde{c}_{0} .
$$

so a finite amount of liquid eutectic must freeze at the solid/mush boundary, releasing a finite amount of heat. The rate of heat production due to this freezing eutectic is $L C_{p}\left(1-\tilde{c}_{o}\right) \dot{a}(\tilde{t})$, so the fluxes at the boundary must mismatch by just this amount:

$$
\left.\frac{\partial \tilde{T}_{m}}{\partial z}\right|_{a(\hat{t})}=\left.\frac{\partial \tilde{T}_{s}}{\partial z}\right|_{a(\hat{t})}+S \tilde{c}_{0} \dot{a}(\tilde{t})
$$

while the fluxes at the mush/liquid interface must simply match.

Worster sought similarity solutions to his equations, using the similarity variable $\eta=\frac{z}{\sqrt{t}}$ because this arises naturally from the dilational symmetry of the diffusion equation. However, adding the pressure effect as we have done breaks this symmetry, so this similarity variable is no longer apropriate. Instead, uniformly translating solutions using the similarity variable $\xi=z-v \tilde{t}$ were sought.

\subsubsection{Translating Similarity Solutions}

These translating solutions model a stream of liquid passing at constant velocity $v$ into a region where pressure and temperature are controlled to make it solidify (figure 4).

Seeking such similarity solutions is slightly more complicated than it appears, because (19.4) was derived under the assumption that the solid fraction only depends on time through $\frac{\partial \tilde{T}}{\partial \tilde{t}}$; now $\frac{\partial \phi}{\partial t}$ also needs a component expressing the variation of pressure effect with time as the liquid is advected. The resulting ordinary differential equations are

$$
\frac{d^{2} \tilde{T}}{d \xi^{2}}=-v \frac{d \tilde{T}}{d \xi}
$$

in solid and liquid and

$$
\frac{d^{2} \tilde{T}}{d \xi^{2}}=-v \frac{d \tilde{T}}{d \xi}\left[1+\frac{S \tilde{c}_{0}}{(1-\tilde{T}-\tilde{\Omega} \xi)^{2}}\right]-\frac{S \tilde{c}_{0} v \tilde{\Omega}}{(1-\tilde{T}-\tilde{\Omega} \xi)^{2}}
$$




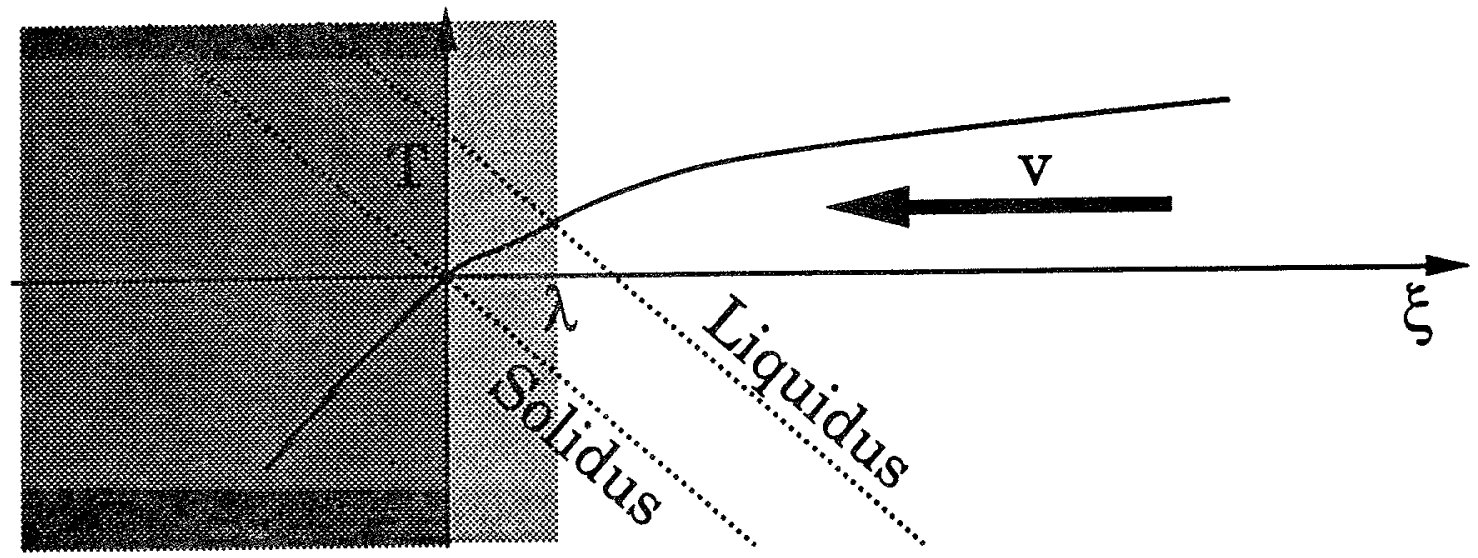

Figure 19.4. Interpretation of the Translating Solutions $T(\xi)$ can be thought of as a stationary temperature (and, implicitly, pressure) distribution into which liquid is advected at a constant velocity $v$. The position $\xi=0$ is chosen (without loss of generality) to be the location of the solid/mush interface, so only one distance, the mush thickness $\lambda$, needs to be determined.

in the mush, with the solid/mush interface condition (19.5) now

$$
\left.\frac{d \tilde{T}_{m}}{d \xi}\right|_{\xi=0}=\left.\frac{d \tilde{T}_{s}}{d \xi}\right|_{\xi=0}-S \tilde{c}_{0} v
$$

Imposing this similarity variable breaks reflection symmetry of the equations, with the consequence that boundary conditions can be imposed only at one end. This is apparent from the solid and liquid equations, which have solutions of the form $T=e^{-v \xi}$ and so can have no boundary condition as $\xi \rightarrow-\infty$. But this need not be a problem if we suppose that some other process limits the temperature at large negative $\xi$.

We can apply the same similarity variable to the equations without pressure effect, (19.2) , and in that case the mush layer ODE is integrable, yielding

$$
\begin{array}{r}
\xi=\frac{1}{v}\left\{\frac{\tilde{T}_{\infty}+S-1}{\sqrt{-\Delta}}\left[\operatorname{arctanh}\left(\frac{2 \tilde{T}-\left(\tilde{T}_{\infty}+S+1\right)}{\sqrt{-\Delta}}\right)-\operatorname{arctanh}\left(\frac{-\left(\tilde{T}_{\infty}+S+1\right)}{\sqrt{-\Delta}}\right)\right]-\right. \\
\left.\frac{1}{2} \ln \left[\frac{\left|\tilde{T}^{2}-\left(\tilde{T}_{\infty}+S+1\right) \tilde{T}+\tilde{T}_{\infty}+S\left(1-\tilde{c}_{0}\right)\right|}{\left|\tilde{T}_{\infty}+S\left(1-\tilde{c}_{0}\right)\right|}\right]\right\}
\end{array}
$$

in the mush zone, where

$$
\Delta=4\left[\tilde{T}_{\infty}+S\left(1-\tilde{c}_{0}\right)\right]-\left(\tilde{T}_{\infty}+1+S\right)^{2}
$$

, which can be shown always to be negative, and $\tilde{T}_{\infty}$ is the limiting temperature as $\xi \rightarrow \infty$. This solution can be plotted (see figure 5 ) and provides a good basis for understanding the solutions to the pressure-modified model. 


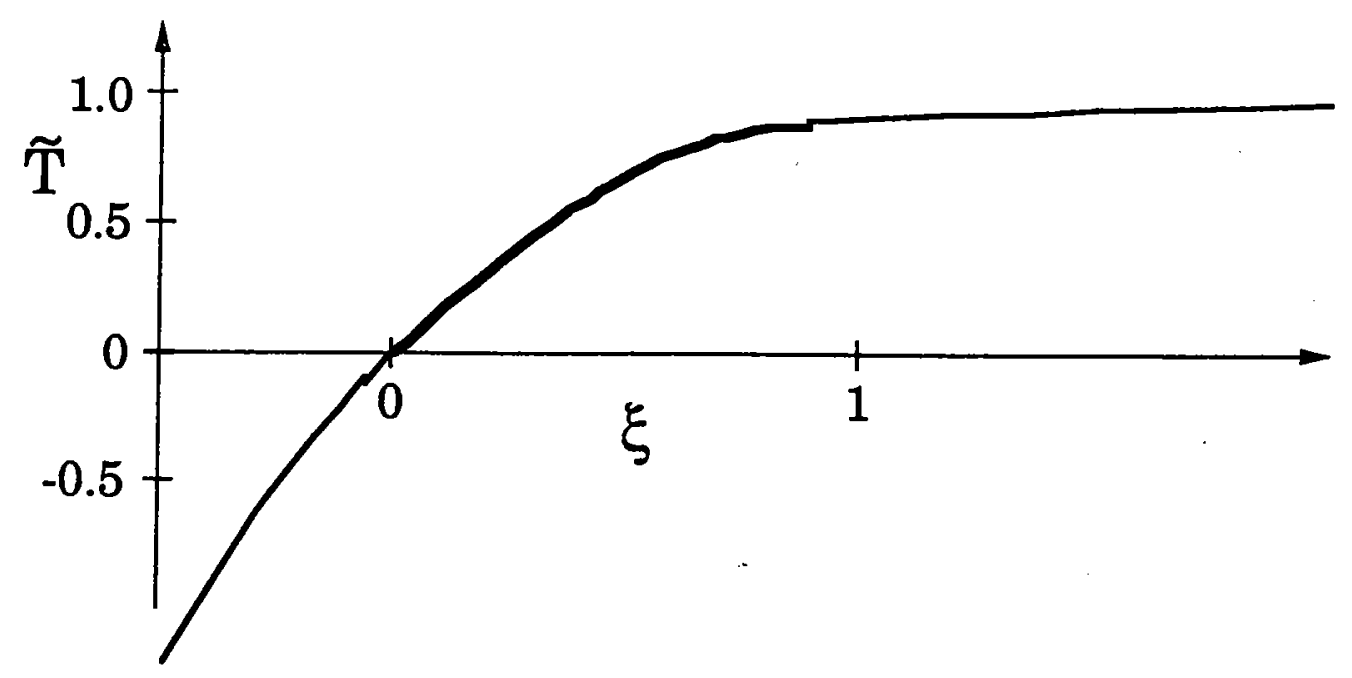

Figure 19.5. Travelling Temperature Profile Without Pressure Effect The heavy line corresponds to the mush zone. Parameters: $\tilde{T}_{\infty}=1, \tilde{c}_{0}=0.1, S=1$, $v=1$. Increasing any of the parameters makes the mush thinner, each one for a different reason.

The equation with pressure effect (19.6) cannot be integrated because of the extra source term, so numerical solutions were sought instead. The observed solutions fall into three broad categories, which can be understood in terms of the balance between diffusive and latent heat fluxes.

In figure 6(a), the temperature profile is essentially the same as in the case without pressure effects, though the mush zone is narrower and the temperature gradient through the mush is steeper. Both effects occur because the pressure induces solidification and consequently the release of latent heat. Figures 6(b) and (c) show solutions that could only occur in the pressure- driven case. Cold liquid is advected into a region of higher pressure, where it solidifies and releases its latent heat to become a hot solid. It is worth noting that Woods' morphological stability criterion (19.1) is likely to be satisfied at such an interface because the temperature gradients are negative. Only if the pressure effect were larger than the concentration effect could the interface be stable. The solution in figure 6(c) emphasizes the importance of pressure-driven solidification. A maximum in temperature can occur in the mush zone if advection causes the release of latent heat faster than the heat can diffuse into the surrounding liquid and solid. It is difficult to imagine a geophysical flow into a region of increasing pressure occuring rapidly enough to create such a temperature distribution.

The local balance between diffusion and production of heat can be understood by comparing the slope and curvature of the temperature profile (figure 7). Where the curvature is negative, the slope and consequently the diffusive flux is decreasing, so more heat leaves one side than enters the other and the temperature should drop. If the slope is positive, then the temperature does drop as fluid is advected, and diffusion dominates the heat at that point. But if the slope is negative, the temperature is increasing and latent heat dominates. 

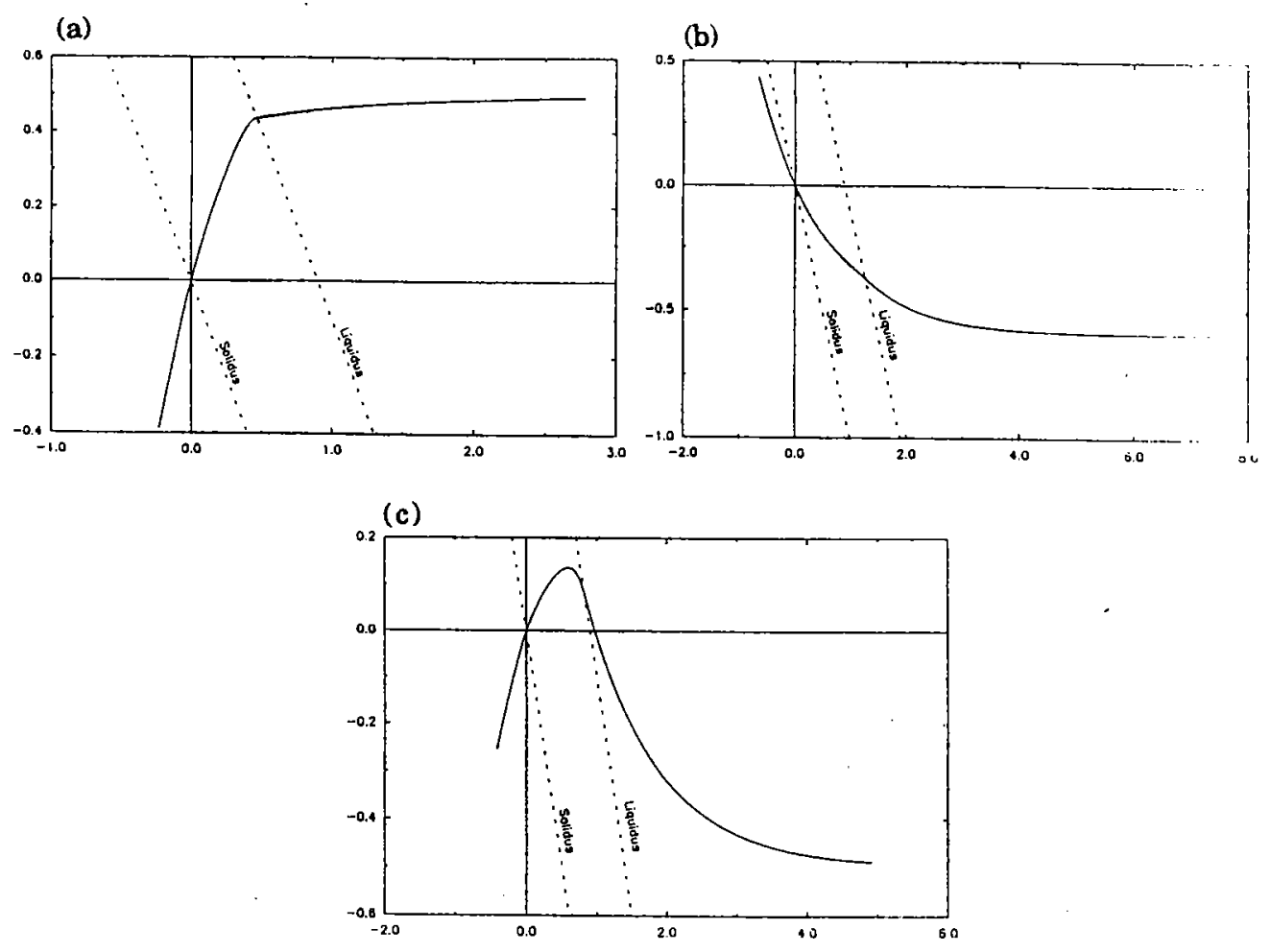

Figure 19.6. Travelling Temperature Profiles With Pressure Effect See text for details. The horizontal axis in each case is $\xi$, and the vertical axis is $\tilde{\mathrm{T}}$. Liquid is advected from the right. Parameters: For all graphs, $\tilde{c}_{0}=0.1$ and $v=\tilde{\Omega}=1$. In (a), $\tilde{T}_{\infty}=0.5$ and $S=1$; in (b), $\tilde{T}_{\infty}=-0.6$ and $S=0.1$; and in (c), $\tilde{T}_{\infty}=-0.5$ and $S=1$.

The behaviour shown in figure 7(d) was not observed in the numerical solutions, because such a curve corresponds to a melting interface. Melting interfaces are always morphologically stable so never form mush layers and are not considered here.

After developing some understanding with the travelling wave solutions, we now seek to apply the pressure modified mush model to a problem of greater geophysical interest, the solidification of the core of a planet.

\subsubsection{Isothermal Sphere Model}

As a grossly simplified first model, consider a sphere of liquid of uniform composition, cooling by a constant heat flux through its surface. We suppose that convection keeps the deviation of temperature from the adiabat small throughout the liquid, and that the liquidus gradient is steeper than the adiabat so that solidification begins at the centre. This is opposite to Kennedy and Higgins' [1973] core paradox, and in line with Stevenson's [1980] results from statistical mechanics considerations.

We take the adiabat to be uniform, though the calculation may work with a less artificial assumption, and we assume that the solidifying portion is uniformly at the same temperature. 

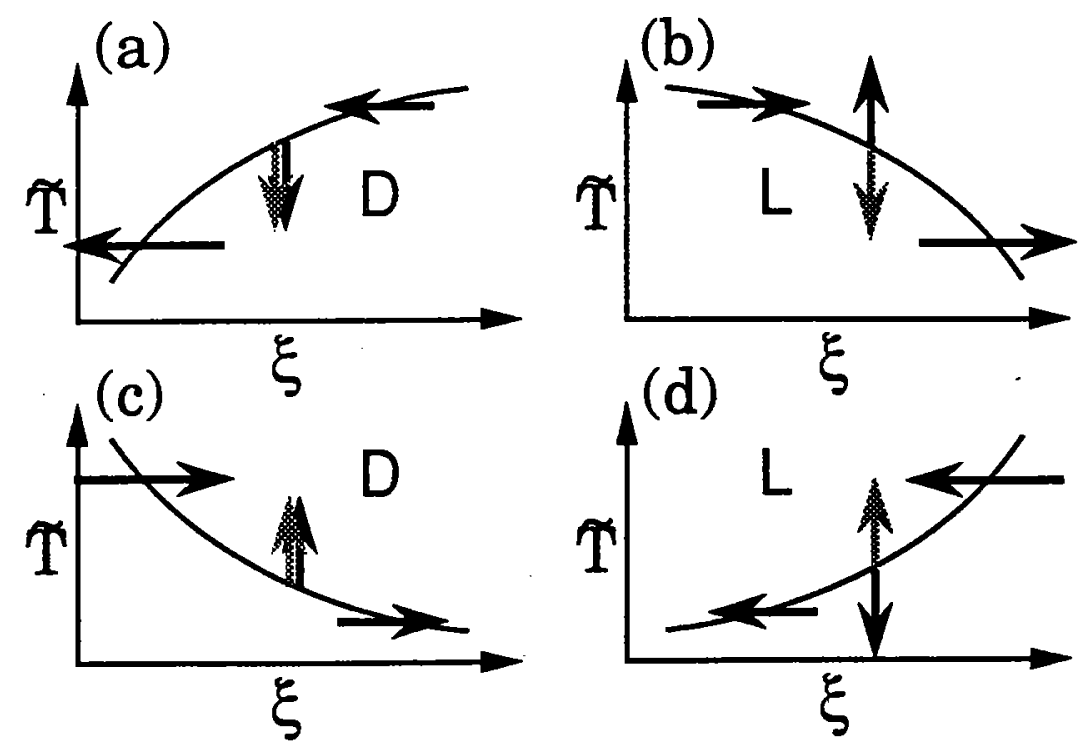

Figure 19.7. Balance Between Heat Diffusion and Latent Heat Curvature and slope can be either positive or negative at a point, leading to four cases. When curvature is positive (cases (a) and (b)) fluxes (horizontal arrows) away from the point exceed fluxes toward it, so the temperature ought to drop (grey arrow). In case (a), the temperature of a parcel of fluid does drop (black arrow) as it is advected to the left, while in case (b) the temperature of the parcel increases. Hence, diffusion dominates profile (a) and latent heat production dominates (b). Similarly, diffusion dominates (c) and latent heat consumption (i.e., melting) dominates case (d).

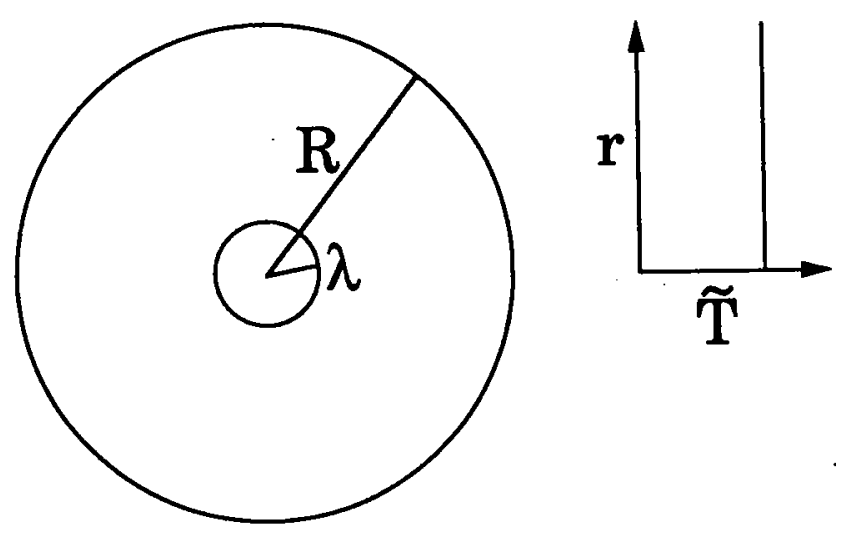

\section{Figure 19.8. Isothermal Sphere Model}

In other words, the entire sphere is everywhere the same temperature. Then it is particularly easy to calculate the specific heat of the sphere, and the latent heat released by solidification:

$$
Q_{\text {specific }}=\frac{4}{3} \pi R^{3} \rho C_{p} \tilde{T} \quad \text { and } \quad Q_{\text {latent }}=4 \pi \rho L \int_{0}^{\lambda} r^{2} \phi(r) d r
$$

where $R$ is the radius of the sphere and $\lambda$ is the radius of the solidifying region (figure 8). 
Within the simple mush model, the solid fraction is given by

$$
\phi(r)=1-\frac{\tilde{c}_{0}}{1-\tilde{T}-\tilde{\Omega} r}
$$

and the radius of the mush region is

$$
\lambda(\tilde{T})=\frac{1-\tilde{T}-\tilde{c}_{0}}{\tilde{\Omega}}
$$

so the total latent heat released depends parametrically on the temperature. In the initial stages of solidification, none of the sphere would have reached the eutectic, so there is no need to include a solid layer. When the scaled temperature $\tilde{\mathrm{T}}$ reaches zero, solid eutectic will start to form at the centre, and at that point the model needs to be adapted. The mush thickness at that point will be

$$
\lambda_{\max }=\frac{1-\tilde{c}_{0}}{\tilde{\Omega}}
$$

which we estimate from values given in Loper [1991] as between 10 and $1000 \mathrm{~km}$ for the Earth's core.

If the radius $R$ of the entire sphere is much larger than $\lambda$, the specific heat will dominate and the temperature will drop linearly with time, since the surface heat flux is taken as constant. The radius of the mushy core varies linearly with temperature, so the mushy core will grow at a constant velocity. But the total amount of solid formed may grow in a more interesting way, since the solid fraction is not uniform throughout the core. The total solid density in the core is

$$
\begin{aligned}
\Phi(\lambda) & =\frac{\int_{0}^{\lambda} r^{2} \phi(r) d r}{\frac{4}{3} \pi \lambda^{3}} \\
& =\frac{3 \tilde{c}_{0}^{2}+(\lambda \tilde{\Omega})^{2}+\frac{9}{2} \tilde{c}_{0} \lambda \tilde{\Omega}}{(\lambda \tilde{\Omega})^{2}}-\frac{3}{(\lambda \tilde{\Omega})^{3}}\left[\left(\tilde{c}_{0}+\lambda \tilde{\Omega}\right)^{2} \tilde{c}_{0} \ln \left(\frac{\tilde{c}_{0}+\lambda \tilde{\Omega}}{\tilde{c}_{0}}\right)\right]
\end{aligned}
$$

which increases more slowly than linearly, as can be seen in figure 9 .

Since $\lambda \propto \tilde{T} \propto t, \Phi(\lambda)$ can be interpreted as the time variation in total solid fraction. If one assumed that the entire volume in which the temperature is colder than the liquidus were pure frozen solute, one would overestimate the amount of solid by a significant amount, particularly at early times. Yet this appears to be what Loper [1991] did in his attempts to calculate the latent heat and compositional buoyancy fluxes in the Earth. It may be that Loper's estimates are too high. 


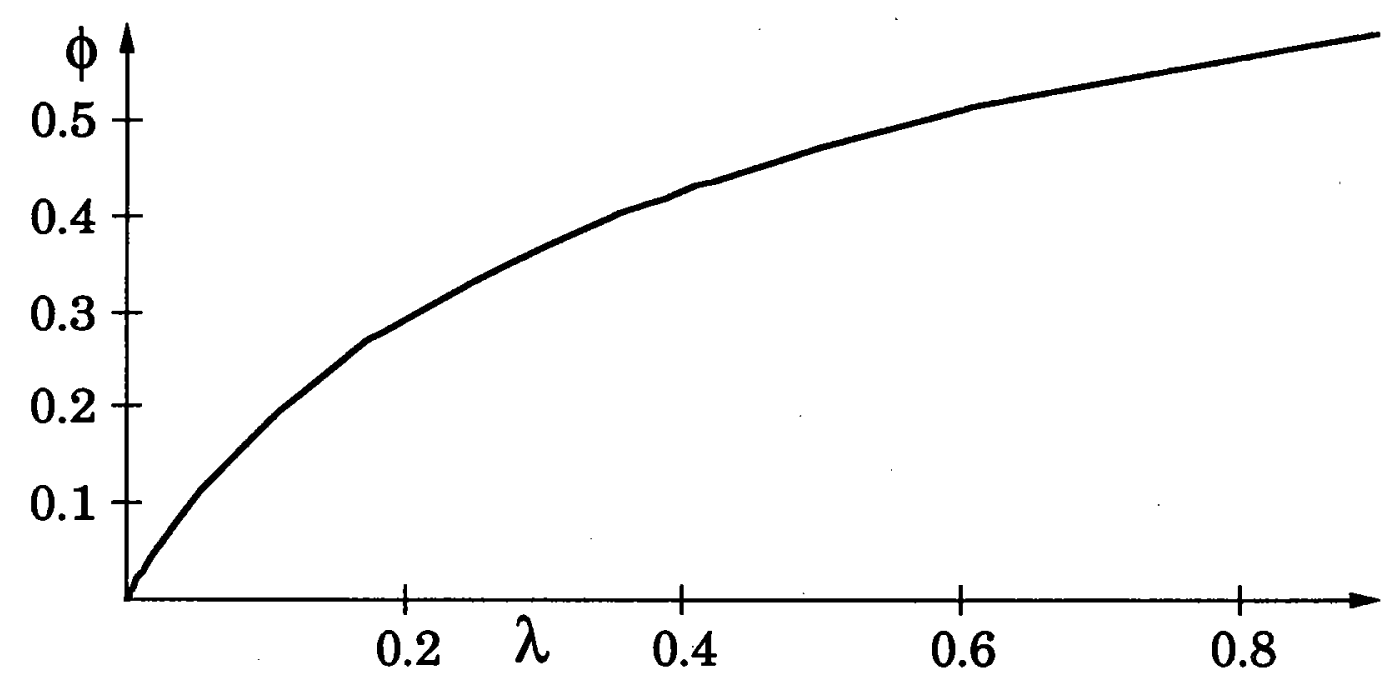

Figure 19.9. Total Solid Density Only a small amount of the mushy volume is actually occupied by solid, even when the mush thickness approaches its maximum.

\subsection{Conclusions}

Most substances will freeze when either cooled or compressed, and solutions will form mushes when frozen by either means under appropriate conditions. We have developed a simple model for the rapid growth of a mush under the dual influences of pressure and temperature, and found uniformly translating numerical solutions to this model. We also considered the consequences of mush growth in the center of a cooled sphere of liquid, and showed that, at least in a simple calculation, the amount of solid formed in the centre is significantly smaller than would be found in a calculation that neglects the mush. Insofar as mush growth has been omitted in previous work on core solifdification, quantities of geophysical significance (such as compositional buoyancy) may have been overestimated, and should be reconsidered in this light.

\section{Acknowledgements}

I am grateful to Andy Woods for many hours of provocative discussion, and the steering committee of the GFD program for making this summer possible. Thanks to George Veronis for softball and a soft heart: it was great not to be chosen last for a change. I am pleased to acknowledge the financial support of the MIT Porous Flow Project. 


\section{- References}

Huppert, H.E. (1990) J. Fluid Mech. 212 210-240

Jarvis, R. (1991) private communication

Kennedy, G.C. and Higgins, G. (1973) J. Geophys. Res. 78 900-904

Kerr, R.C., Woods, A.W., Worster, M.G., and Huppert, H.E. (1990) J. Fluid Mech. 216 $323-342$

Loper, D.E. (1991) J. Geomag. Geoelectr. 43 79-91

Malkus, V.R. (1973) Geophysical Fluid Dynamics 4 267-278

Mullins, W.M. and Sekerka, R.F. (1964) J. Appl. Phys. 35, 444

Sekerka, R.F. (1973) "Morphological Stability" Crystal Growth: an Introduction, P. Hartman, ed., North-Holland, 403-441.

Stevenson, D.J. (1980) Phys. Earth Planet. Inter. 22 42-52

Woods, A.W. (1990) private communication

Woods, A.W. (1991) Phys. Fluids A 3 1393-1404

Worster, M. G. (1986) J. Fluid Mech. 167, 481-501 


\section{Boussinesq convection in the equatorial region of a rotating spherical shell}

Shin-ichi Takehiro

Department of Earth and Planetary Physics, Faculty of Science, University of Tokyo

\subsection{Introduction}

Convection in a rotating spherical shell is an interesting geophysical problem because of its relevance to the motion of atmospheres of planets and stars. We have studied the convection of Boussinesq fluid in a thin rotating spherical shell by using asymptotic expansions. It is well known that a vertical (i.e. parallel to gravity) component of rotation suppresses convection (Chandrasekhar, 1961). From this fact, we suppose that the equatorial region is the most unstable in a rotating shell heated from the inside. Therefore, we will use local orthogonal coordinates whose origin is at the equator to describe convective motion.

A characteristic feature of the equatorial region is that the planetary rotation vector is almost parallel to the horizontal plane. The recent studies of the convection with the horizontal rotation are Busse and Or (1986) and Busse (1986). They studied the rotating cylindrical annulus heated from the inside. The different point between the system they considered and here is the latitudinal dependence of the rotating vector. The fact that rotation varies with latitude suggests the existence of Rossby waves, which have vertical vorticity. In usual studies of convection, modes with vertical vorticity were often forgotton. However, under the slip boundary condition, vorticity modes are excited by the convection motion and change the pattern of the convection. For exampie, it is seen in Sigga and Zippelius (1981) in the case of no rotation. Thus, we will study the convection problem including the effect of vorticity modes.

\subsection{The governing equations}

Let us consider the equatorial region of the shell rotating with the angular velocity $\Omega^{*}$. Figure 1.1 shows the geometry we consider. We take $x$ in the longitudinal direction, $y$ in the latitudinal direction, and $z$ in the vertical direction. The rotation vector is almost in the north, parallel to the horizontal plane, and varies with latitude. Therefore, we describe the rotation vector as

$$
\boldsymbol{\Omega}=\frac{\Omega^{*}}{r} y \mathbf{k}+\Omega^{*} \mathbf{j},
$$

where $\mathbf{i}, \mathbf{j}, \mathbf{k}$ are unit vectors in the east, north and vertical direction and $r$ is the radius of the spherical shell. 

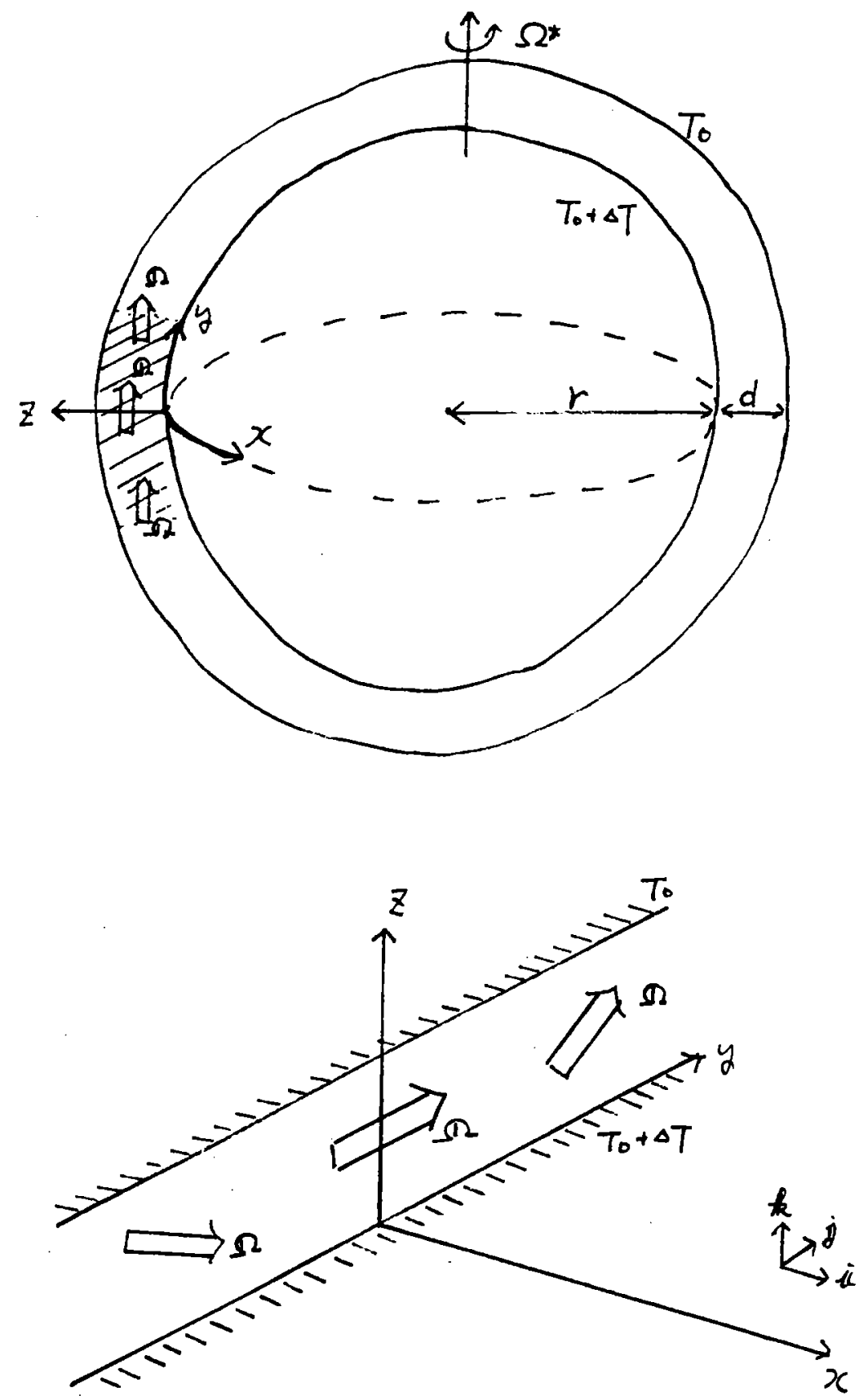

Figure 20.1. Schematic picture of the system considered.

We select the length scale as the thickness of the fluid, $d$, time scale as the thermal diffusive time, $d^{2} / \kappa$, where $\kappa$ is thermal diffusivity, velocity scale, $\kappa / d$, temperature scale as the temperature difference of the boundaries, $\Delta T_{0}$, pressure scale as $\kappa \nu / d^{2}$ where $\nu$ is kinematic viscosity. The governing equations of the Boussinesq fluid in a rotating system are as follows:

$$
\begin{aligned}
& P^{-1}\left[\partial_{t} \mathbf{v}+(\mathbf{v} \cdot \nabla) \mathbf{v}\right]+\beta y \mathbf{k} \times \mathbf{v}+\bar{f}_{0} \mathbf{j} \times \mathbf{v}=-\nabla p+R \theta \mathbf{k}+\nabla^{2} \mathbf{v} \\
& \partial_{t} \theta+(\mathbf{v} \cdot \nabla) \theta=\mathbf{v} \cdot \mathbf{k}+\nabla^{2} \theta \\
& \nabla \cdot \mathbf{v}=0
\end{aligned}
$$


where $\mathbf{v}, p, \theta$ are nondimensional velocity, pressure, and temparature. The definition of the nondimensional parameters are:

$$
\begin{aligned}
& P \equiv \frac{\nu}{\kappa} \quad ; \text { Prandtl number, } \\
& R \equiv \frac{\alpha g \Delta T d^{3}}{\kappa \nu} \quad ; \text { Rayleigh number, } \\
& \bar{f}_{0} \equiv \frac{2 \Omega^{*} d^{2}}{\nu} \quad ; \text { Square Root of Taylor Number. } \\
& \beta \equiv \frac{2 \Omega^{*} d^{3}}{\nu r}=\bar{f}_{0} \frac{d}{r} .
\end{aligned}
$$

$\beta$ corresponds to the $\beta$ parameter used in meteorology and oceanography, describing the latitudinal dependence of the rotation.

20.2.1 The description with the toroidal and poloidal potentials

We describe velocity field with the toroidal and poloidal potentials $\psi$ and $\phi$ such that,

$$
\mathbf{v}=\nabla \times(\psi \mathbf{k})+\nabla \times \nabla \times(\phi \mathbf{k}) \text {. }
$$

Operating with $(\mathbf{k} \cdot \nabla \times)$ and $(\mathbf{k} \cdot \nabla \times \nabla \times)$ on the equation of motion and substituting the expression of the velocity, we get the equations of toroidal and poloidal potentials. Also expressing the thermal equation with the potentials, we have,

$$
\begin{aligned}
& {\left[\left(P^{-1} \partial_{t}-\nabla^{2}\right) \nabla_{H}^{2}+\beta \partial_{x}\right] \psi-\left[\left(\beta y \partial_{z}+\bar{f}_{0} \partial_{y}+\beta\right) \nabla_{H}^{2}+\beta \partial_{z} \partial_{y}\right] \phi} \\
& \quad=P^{-1} \mathbf{k} \cdot[\nabla \times(\mathbf{v} \cdot \nabla) \mathbf{v}], \\
& {\left[\left(P^{-1} \partial_{t}-\nabla^{2}\right) \nabla_{H}^{2} \nabla^{2}+\beta \partial_{z}^{2} \partial_{x}\right] \phi+\left[\left(\beta y \partial_{z}+\bar{f}_{0} \partial_{y}\right) \nabla_{H}^{2}+\beta \partial_{z} \partial_{y}\right] \psi+R \nabla_{H}^{2} \theta} \\
& \quad=-P^{-1} \mathbf{k} \cdot[\nabla \times \nabla \times(\mathbf{v} \cdot \nabla) \mathbf{v}], \\
& \quad\left(\partial_{t}-\nabla^{2}\right) \theta+\nabla_{H}^{2} \phi=-(\mathbf{v} \cdot \nabla) \theta
\end{aligned}
$$

where $\nabla_{H}^{2}$ is a horizontal Laplacian operator, $\nabla_{H}^{2}=\partial_{x}^{2}+\partial_{y}^{2}$.

\subsubsection{Boundary conditions}

We consider free-slip and fixed temperature boundaries. Corresponding boundary conditions are

$$
\partial_{z} u=\partial_{z} v=w=\theta=0 \quad \text { at } \quad z=0,1 .
$$

Or, expressed in terms of potentials, we get

$$
\phi=\partial_{z}^{2} \phi=\partial_{z} \psi=\theta=0, \quad \text { at } \quad z=0,1
$$




\subsection{Asymptotic expansion : derivation of the amplitude equation of convection mode}

We solve the governing equations derived in the previous section by asymptotic expansion method with the assumption of weak effect of rotation. We expand each physical variable with small parameter $\varepsilon$ such that

$$
\begin{aligned}
& \psi=\varepsilon \psi^{(1)}+\varepsilon^{2} \psi^{(2)}+\cdots \\
& \phi=\varepsilon \phi^{(1)}+\varepsilon^{2} \phi^{(2)}+\cdots \\
& \theta=\varepsilon \theta^{(1)}+\varepsilon^{2} \theta^{(2)}+\cdots
\end{aligned}
$$

We also expand Rayleigh number with $\varepsilon$.

$$
R=R^{(0)}+\varepsilon R^{(1)}+\varepsilon^{2} R^{(2)}+\cdots .
$$

\subsubsection{Assumptions and scaling}

We consider the case in which the $\beta$ parameter is small. We assume the order of the parameter $\bar{f}_{0}, \beta, P$ such that:

$$
\begin{aligned}
\bar{f}_{0} & =\mathrm{O}(1), \\
\beta & =\mathrm{O}\left(\varepsilon^{2}\right), \quad \text { or } \beta=\varepsilon^{2} \beta^{(2)}, \\
P & =\mathrm{O}(1) .
\end{aligned}
$$

This is equivalent to assuming that $\beta / \bar{f}_{0}=d / r \sim \mathrm{O}\left(\varepsilon^{2}\right)$.

Next, we introduce slowly varying coordinates $(X, Y, T)$. We consider only slowly varying scale in the $y$ direction. Because of the Taylor Proudman theorem, we can suppose that the structure of the most unstable convection mode does not vary in the north-south direction, which is the direction of the rotating vector. Then, $Y=\varepsilon y$, or $\partial_{y} \longrightarrow \varepsilon \partial_{Y}$. We select the slowly varying scale in the $x$ direction as the size of the shells. Since we assume $\beta \sim O\left(\varepsilon^{2}\right)$, the slowly varying $x$ coordinate $X$ is $X=\varepsilon^{2} x$, or $\partial_{x} \longrightarrow \partial_{x}+\varepsilon^{2} \partial_{X}$. We consider only one slowly varying time scale, $T=\varepsilon^{2} t$, or $\partial_{t} \longrightarrow \varepsilon^{2} \partial_{T}$.

20.3.2 Asymptotic expansion

From $O(\varepsilon)$, we can get the following equations:

$$
\begin{aligned}
& \left(\partial_{x}^{2}+\partial_{z}^{2}\right) \partial_{x}^{2} \psi^{(1)}=0 \\
& \left(\partial_{x}^{2}+\partial_{z}^{2}\right)^{2} \partial_{x}^{2} \phi^{(1)}-R^{(0)} \partial_{x}^{2} \theta^{(1)}=0 \\
& \left(\partial_{x}^{2}+\partial_{z}^{2}\right) \theta^{(1)}-\partial_{x}^{2} \phi^{(1)}=0 .
\end{aligned}
$$

These equations are the same as the case with no rotation. Thus, we have

$$
\begin{aligned}
& \psi^{(1)}=\Psi(X, Y, T) \\
& \phi^{(1)}=\Phi(X, Y, T) \exp (i k x) \sin \pi z+\text { c.c. } \\
& \theta^{(1)}=\frac{k^{2}}{k^{2}+\pi^{2}} \phi^{(1)}=\frac{k^{2}}{k^{2}+\pi^{2}} \Phi(X, Y, T) \exp (i k x) \sin \pi z+\text { c.c. }
\end{aligned}
$$


where the wave number $k$ and Rayleigh number are the critical values,

$$
k=\frac{\pi}{\sqrt{2}}, \quad R^{(0)}=\frac{27}{4} \pi^{4} .
$$

$\Psi(X, Y, T)$ is the amplitude of a vorticity mode and $\Phi(X, Y, T)$ is the amplitude of a convection mode. Note that convection cells are aligned parallel to the $y$ axis.

From $O\left(\varepsilon^{2}\right)$, we get the following results.

$$
\begin{aligned}
\psi^{(2)} & =\frac{\pi}{k^{2}+\pi^{2}} \beta^{(2)} Y \Phi \exp (i k x) \cos \pi z+\bar{f}_{0} \Phi_{Y} \exp (i k x) \sum_{m=0}^{\infty} a_{2 m} \cos 2 m \pi z+\text { c.c. } \\
\phi^{(2)} & =0 \\
\theta^{(2)} & =-\frac{k^{4}}{2 \pi\left(k^{2}+\pi^{2}\right)}|\Phi|^{2} \sin 2 \pi z
\end{aligned}
$$

where the coefficient $a_{2 m}$ is

$$
a_{0}=\frac{4}{\pi^{3}}, \quad a_{2 m}=\frac{8}{\pi^{3}\left(1-4 m^{2}\right)\left(1+8 m^{2}\right)} \quad(m \neq 0) .
$$

From the solvability condition, we have $R^{(1)}=0$. At this order, velocities with a toroidal potential component are excited by the rotation effect. Thermal field appears as in the nonrotating convection problem.

By applying the solvability condition to the $O\left(\varepsilon^{3}\right)$ equations, we get the amplitude equation of the convection mode.

$$
\begin{aligned}
\left(1+P^{-1}\right)\left[\Phi_{T}+i \frac{\pi}{\sqrt{2}} \Psi_{Y} \Phi\right]= & {\left[\frac{2}{9 \pi^{2}} R^{(2)}-\frac{4}{9 \pi^{2}} \beta^{(2) 2} Y^{2}+i \frac{2 \sqrt{2}}{3 \pi} \beta^{(2)}\right] \Phi } \\
& +\frac{4}{9 \pi^{4}} \bar{f}_{0}^{2} \Phi_{Y Y}-\frac{\pi^{4}}{8}|\Phi|^{2} \Phi .
\end{aligned}
$$

In this equation, there are some different terms from the amplitude equation derived by Segal (1967) and Newell and Whitehead (1967). The second term of the left-hand side is the advection of the rolls by vorticity modes. $\beta^{(2) 2} Y^{2}$ and $\bar{f}_{0}^{2} \partial_{Y}^{2}$ on right-hand side are the effect of suppressing the convection by the vertical and horizontal components of the rotation. The $i \beta^{(2)}$-term on the right-hand side describes the propagation of the convection cells by $\beta$ effect. 


\subsection{The Magic Equation and the amplitude equation of vorticity modes}

Next, we derive the amplitude equation of the vorticity modes. Of course, we can get the equation of vorticity modes by further expansion of the governing equations. However, this process is a tedious and complicated calculation. Then, we take another derivation of the equation of vorticity modes with a vertically averaged vertical vorticity equation. This process is much simpler than the former one, and it is easy to get dynamical insights of each term of the equation. The vertically averaged vorticity equation is called 'the magic equation' (Cessi, et al, 1991).

The equation of vertical vorticity $\zeta$ of Boussinesq fluid in an equatorial region is

$$
P^{-1} \partial_{t} \zeta+\beta v-\beta y \partial_{z} w-\bar{f}_{0} \partial_{y} w-\nabla^{2} \zeta=-P^{-1} \mathbf{k} \cdot \nabla \times[(\mathbf{v} \cdot \nabla) \mathbf{v}]
$$

We define $\langle a\rangle$ as the average in the $z$ direction:

$$
<a>\equiv \int_{0}^{1} a d z
$$

Averaging the vorticity equation and applying the free-slip boundary condition, we get

$$
\begin{aligned}
P^{-1} \partial_{t}<\zeta> & +\beta<v>-\bar{f}_{0} \partial_{y}<w>-\nabla_{H}^{2}<\zeta> \\
& =P^{-1}\left[\partial_{x} \partial_{y}\left(<u^{2}>-<v^{2}>\right)+\left(\partial_{y}^{2}-\partial_{x}^{2}\right)<u v>\right] .
\end{aligned}
$$

This is the 'magic equation' of a Boussinesq fluid in an equatorial region. Note that the symmetric and double differentiated form of the nonlinear terms. These terms express the excitation of vertical vorticity by Reynolds stresses.

Substituting the solutions of the $O\left(\varepsilon, \varepsilon^{2}\right)$ equations obtained in the previous section, and averaging with respect to the convection cells, we get from $O\left(\varepsilon^{5}\right)$ that

$$
\begin{aligned}
& P^{-1} \partial_{T} \partial_{Y}^{2} \Psi+\beta^{(2)} \partial_{X} \Psi-\partial_{Y}^{4} \Psi \\
& \quad=-P^{-1} \frac{\pi^{4}}{2} \partial_{X} \partial_{Y}|\Psi|^{2}-P^{-1} \frac{i \pi^{3}}{2 \sqrt{2}} \partial_{Y}^{2}\left(\Phi \Phi_{Y}^{*}-\Phi^{*} \Phi_{Y}\right)+P^{-1} \frac{\pi^{2}}{3} \partial_{Y}^{2}\left(\beta^{(2)} Y|\Phi|^{2}\right) .
\end{aligned}
$$

This is the amplitude equation of the vorticity modes. 


\subsection{Simple solutions of the amplitude equations}

\subsubsection{Rescaling of the amplitude equations}

Now, we have obtained the amplitude equations of vorticty and convection modes. Since the coefficients of these equations are complicated, let us make a rescaling before starting the study of these equations. Selecting the new scales:

$$
\begin{aligned}
& X=\frac{\bar{f}_{0}}{\beta^{(2)}} \tilde{X}, \quad Y=\sqrt{\frac{\bar{f}_{0}}{\pi \beta^{(2)}}} \tilde{Y}, \quad T=\frac{9 \pi^{3}\left(1+P^{-1}\right)}{4 \beta^{(2)} \tilde{f}_{0}} \tilde{T} \\
& \Phi=\sqrt{\frac{32 \beta^{(2)} \bar{f}_{0}}{9 \pi^{7}}} \tilde{\Phi}, \quad \Psi=\frac{9 \pi^{3}\left(1+P^{-1}\right)}{4 \beta^{(2)} \bar{f}_{0}} \sqrt{\frac{\pi \beta^{(2)}}{\bar{f}_{0}}} \tilde{\Psi}, \quad U \equiv \Psi_{Y}=\frac{9 \pi^{3}\left(1+P^{-1}\right)}{4 \beta^{(2)} \bar{f}_{0}} \tilde{U}
\end{aligned}
$$

we have the following normalized amplitude equations:

$$
\begin{aligned}
& \Phi_{T}+i \frac{\pi}{\sqrt{2}} U \Phi=\left[\mu-Y^{2}+i \omega\right] \Phi+\Phi_{Y Y}-|\Phi|^{2} \Phi \\
& \frac{p}{\omega^{2}} U_{Y Y T}+\frac{3 \sqrt{2}}{2 \omega} U_{X}-U_{Y Y Y Y}=-q\left[\frac{2 \omega}{3}|\Phi|_{X Y Y}^{2}+i\left(\Phi \Phi_{Y}^{*}-\Phi^{*} \Phi_{Y}\right)_{Y Y Y}-\frac{2}{\omega}\left(Y|\Phi|^{2}\right)_{Y Y Y}\right] .
\end{aligned}
$$

We omitted () for simplicity. The definition of parameters are as follows:

$$
\mu=\frac{\pi R^{(2)}}{2 \beta^{(2)} \bar{f}_{0}}, \quad \omega=\frac{3 \sqrt{2} \pi^{2}}{2 \bar{f}_{0}}, \quad p=\frac{2}{1+P}, \quad q=\frac{2 \sqrt{2} P^{-1}\left(1+P^{-1}\right)}{\pi}
$$

$\mu$ correseponds to the Rayleigh number, $1 / \omega$ to $\bar{f}_{0}$ which expresses the magnitude of the rotation. $p$ and $q$ are coefficients determined by the Prandtl number.

\subsubsection{Onset of convection}

Firstly, we examine the onset of convection. Linearizing the amplitude equation of convection, we have,

$$
\Phi_{T}=\left[\mu-Y^{2}+i \omega\right] \Phi+\Phi_{Y Y}
$$

We can eliminate $i \omega$ by writing $\Phi=\tilde{\Phi} \exp (i \omega T)$. Further, we assume $\tilde{\Phi} \propto \exp (\eta T)$ and we get,

$$
\tilde{\Phi}_{Y Y}+\left[\mu-\eta-Y^{2}\right] \tilde{\Phi}=0 .
$$

The solutions of this equation are expressed by Hermite polynominals $H_{n}$ such that:

$$
\begin{aligned}
& \tilde{\Phi}=A \exp \left(-\frac{1}{2} Y^{2}\right) H_{n}(Y), \\
& \eta=\mu-(2 n+1), \quad n=0,1,2, \cdots .
\end{aligned}
$$

The most unstable mode is $n=0$ and has a critical paramter $\mu=1$. This corresponds to a critical Rayleigh number

$$
R_{c}=R^{(0)}+\varepsilon^{2} R_{c}^{(2)}=\frac{27 \pi^{4}}{4}+\frac{2 \bar{f}_{0} \beta}{\pi}=\frac{27 \pi^{4}}{4}+\frac{2}{\pi} \bar{f}_{0}^{2} \frac{d}{r} .
$$


Thus, at the onset of convection, convection cells whose amplitude is a Gaussian distribution in the $Y$ direction slowly propagate westward.

From the coefficient of $Y$ in the rescaling procedure, we can find a characteristic length in the $Y$ direction, $L_{Y}$,

$$
L_{Y} \sim \sqrt{\frac{\bar{f}_{0}}{\pi \beta^{(2)}}}=\frac{1}{\varepsilon} \sqrt{\frac{r}{\pi d}},
$$

or, expressed in the dimensional form,

$$
L_{Y}^{*} \sim \sqrt{\frac{r}{\pi d}} d \propto \sqrt{r d} .
$$

Note that this length is independent on the magnitude of rotation, and determined by the ratio between the thickness and the radius of the shell. We can also interpret this length as the distance from equator to the horizon as shown in figure 1.2.
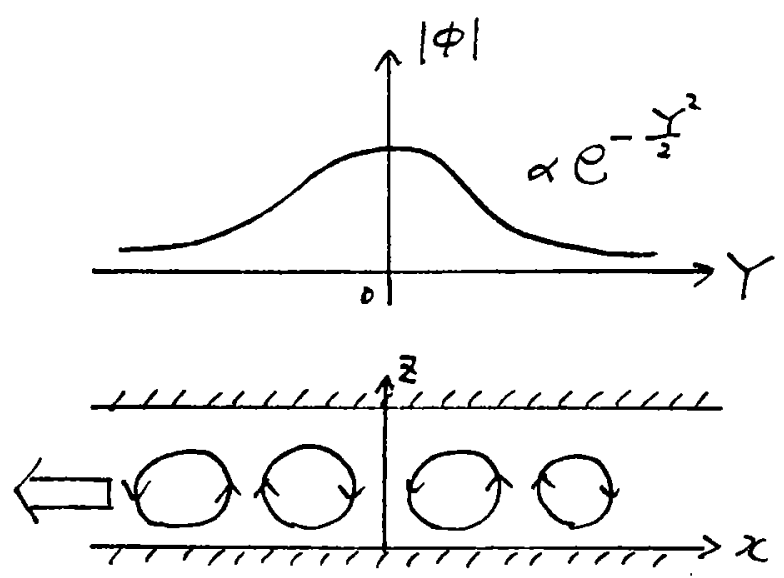

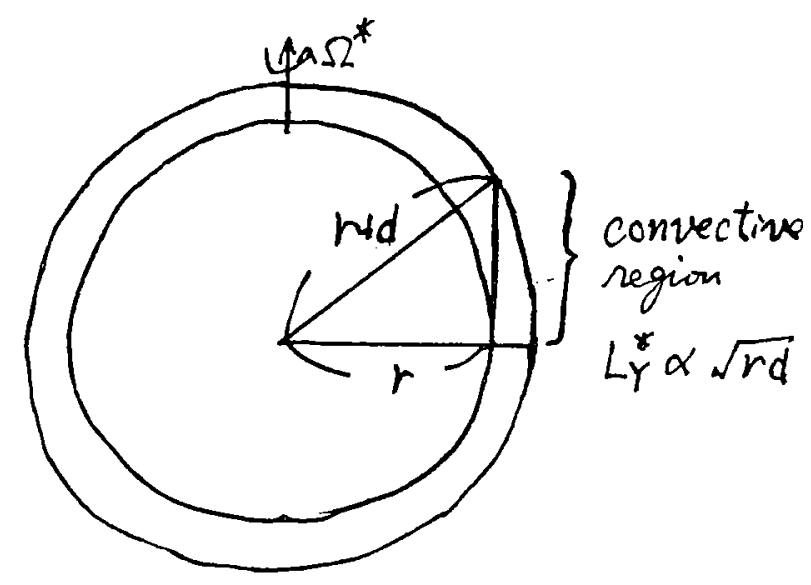

Figure 20.2. The pattern at the onset of convection.

\subsubsection{Homogeneous solutions}

We find that convection cells propagate westward at the onset. But, do nonlinear solutions show similar behaviour? To investigate this property, we will seek the solution of the amplitude equations by asymptotic expansion again, in the case that $\mu$ is slightly larger than the critical value. Let us consider the solution with no $X$ dependence. Expanding $\mu$ in a small parameter $\varepsilon$ again, introducing long time coordinate $\tau=\varepsilon^{2} T$ and expanding $\Phi$ and $\Psi$ by $\varepsilon$ such that:

$$
\begin{aligned}
& \Phi=\left(\varepsilon \Phi^{(1)}+\varepsilon^{3} \Phi^{(3)}+\cdots\right) \exp (i \omega T), \\
& U=\varepsilon^{2} U^{(2)}+\cdots .
\end{aligned}
$$

From the $O(\varepsilon)$ equation of the convection modes, we get

$$
\Phi_{Y Y}^{(1)}+\left(1-Y^{2}\right) \Phi^{(1)}=0 .
$$


Thus, the solution is

$$
\Phi^{(1)}=A(\tau) \exp \left(-\frac{1}{2} Y^{2}\right) .
$$

The $O\left(\varepsilon^{2}\right)$ equation of the vorticity modes is

$$
-U_{Y Y Y Y}^{(2)}=\frac{2 q}{\omega}|A|^{2}\left[Y \exp \left(-Y^{2}\right)\right]_{Y Y Y} .
$$

Thus, we get an eastward zonal flow excited by the convection motion:

$$
U^{(2)}=\frac{q}{\omega}|A|^{2} \exp \left(-Y^{2}\right) .
$$
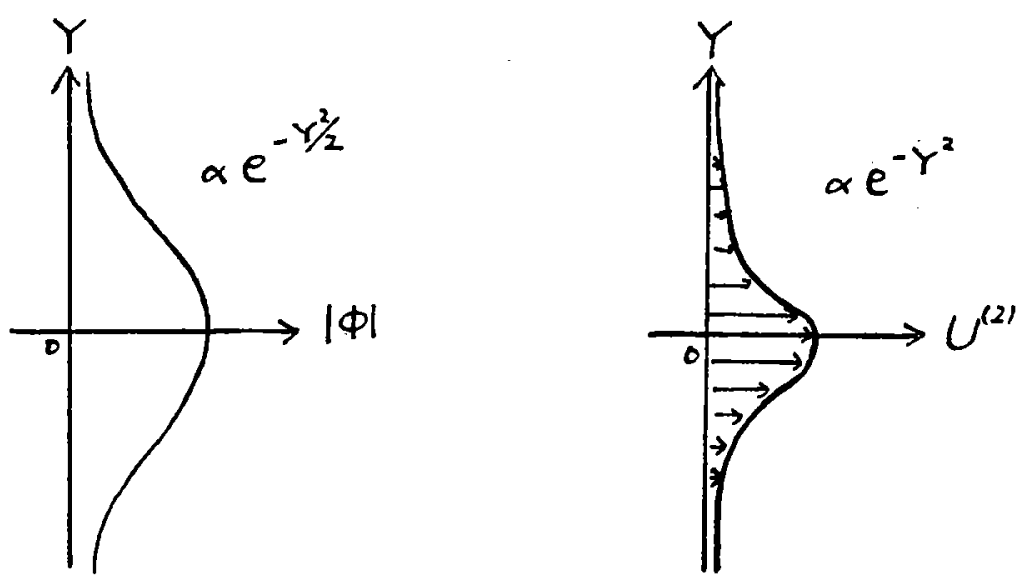

Figure 20.3. Profile of the amplitude of convection and a flow of vorticity modes.

Substituting $U^{(2)}$ into the $O\left(\varepsilon^{3}\right)$ convection equation, we have

$$
\begin{aligned}
A_{\tau} \exp \left(-\frac{Y^{2}}{2}\right) & +i \frac{\pi q}{\sqrt{2} \omega}|A|^{2} A \exp \left(-\frac{3 Y^{2}}{2}\right) \\
& =\mu^{(2)} A \exp \left(-\frac{Y^{2}}{2}\right)-|A|^{2} A \exp \left(-\frac{3 Y^{2}}{2}\right)+\left(1-Y^{2}\right) \Phi^{(3)}+\Phi_{Y Y}^{(3)}
\end{aligned}
$$

Applying the solvability condition, that is, multiplying $\exp \left(-\frac{Y^{2}}{2}\right)$ and integrating from $-\infty$ to $\infty$, we get the equation of the amplitude $A$ :

$$
A_{\tau}+i \frac{\pi q}{2 \omega}|A|^{2} A=\mu^{(2)} A-\frac{1}{\sqrt{2}}|A|^{2} A .
$$

This is the amplitude equation of the solution with no $X$ dependence. This equation has the following solutions:

$$
\begin{gathered}
A=A_{0} \exp \left(-i \frac{\tilde{\omega}}{\varepsilon^{2}} \tau\right)=A_{0} \exp (-i \tilde{\omega} T) \\
A_{0}=2^{1 / 4} \sqrt{\mu^{(2)}} \\
\tilde{\omega}=\varepsilon^{2} \frac{\pi q}{2 \omega}\left|A_{0}\right|^{2}=\frac{\sqrt{2} \pi q}{2 \omega}(\mu-1)
\end{gathered}
$$


or, expressing in terms of the original variables,

$$
\phi^{(1)}=A_{0} \exp \{i[k x-(\tilde{\omega}-\omega) T]\} \sin \pi z+\text { c.c. }
$$

The frequency $\omega$ expresses the westward propagation by the $\beta$ effect. On the other hand, $\tilde{\omega}$ expresses the eastward advection of the cells by the zonal flow induced by convection. When the Rayleigh number increases, the amplitude of the convection grows and zonal flows get strong. Therefore, convection cells move eastward.

We can determine the critical parameter, $\mu_{s}$, where the direction of the propagation changes by the condition $\tilde{\omega}-\omega=0$. Substituting the expressions of $\tilde{\omega}$ and $\omega$, we get

$$
\mu_{s}=1+\frac{\sqrt{2} \omega^{2}}{\pi q}
$$

Corresponding Rayleigh number, $R_{s}$, is

$$
R_{s}=R_{c}+\frac{9}{2} \pi^{3} \frac{\beta}{P^{-1}\left(1+P^{-1}\right) \bar{f}_{0}}=R_{c}+\frac{9}{2} \pi^{3} \frac{1}{P^{-1}\left(1+P^{-1}\right)} \frac{d}{r}
$$

Note that this expression is applicable only for small supercritical case. From the condition $\mu_{s}-1 \ll 1$, we have,

$$
\frac{9 \pi^{4}}{4} \frac{P^{2}}{1+P} \frac{1}{\bar{f}_{0}^{2}} \ll 1
$$

This is the range of parameters where the critical value $R_{s}$ is effective.
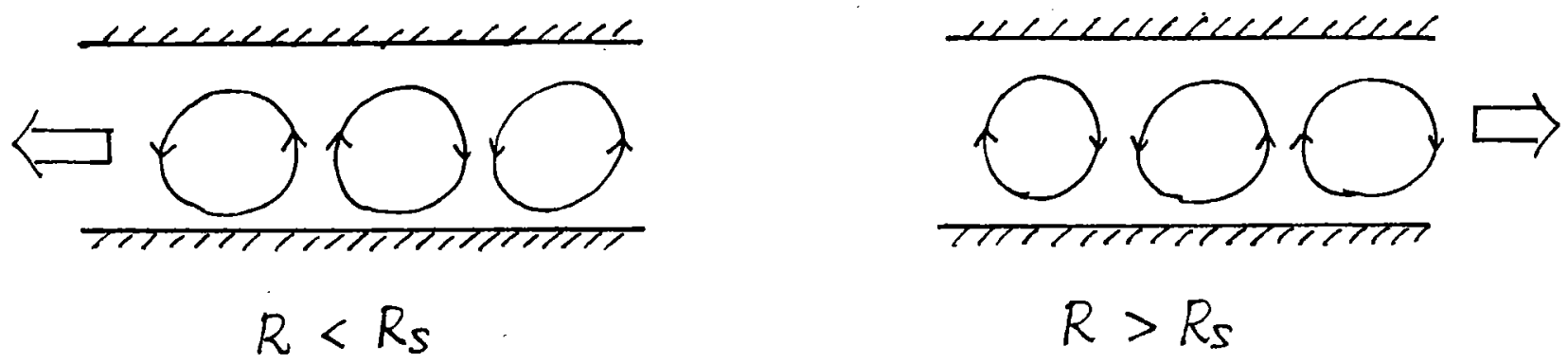

Figure 20.4. The direction of the propagation of convection cells of weak nonlinear solutions. 


\subsubsection{Solutions with a sinusoidal amplitude}

In the same manner, we can get the solutions with a sinusoidal amplitude with respect to $X$. From the $O(\varepsilon)$ equation, we begin with

$$
\Phi^{(1)}=\left[A(\tau) \exp (i K X)+A^{*}(\tau) \exp (-i K X)\right] \exp \left(-\frac{1}{2} Y^{2}\right) .
$$

From $\mathrm{O}\left(\varepsilon^{2}\right)$, we have

$$
\begin{aligned}
U^{(2)}=\frac{2 q}{\omega}|A|^{2} \exp \left(-Y^{2}\right) & -i K \frac{4}{3} q \omega A^{2} \exp (2 i K X) C_{1}\left(\frac{3 \sqrt{2}}{\omega} K, Y\right)+\text { c.c. } \\
& +\frac{q}{\omega} A^{2} \exp (2 i K X) C_{2}\left(\frac{3 \sqrt{2}}{\omega} K, Y\right)+\text { c.c. }
\end{aligned}
$$

where $C_{n}(n=1,2)$ are

$$
C_{n}(K, Y)=\frac{1}{2 \sqrt{\pi}} \int_{-\infty}^{\infty} \frac{l^{2 n}}{l^{4}-i K} \exp \left(-l^{2} / 4+i l Y\right) d l .
$$

Applying the solvability condition to the $O\left(\varepsilon^{3}\right)$ equation, we get

$$
\begin{aligned}
A_{\tau} & =\mu^{(2)} A-\left(\gamma_{r}+i \gamma_{i}\right)|A|^{2} A, \\
\gamma_{r} & =\frac{3 \sqrt{2}}{2}+\frac{4}{3} k q \omega K D_{1 R}\left(\frac{3 \sqrt{2}}{\omega} K\right)-k \frac{q}{\omega} D_{2 I}\left(\frac{3 \sqrt{2}}{\omega} K\right), \\
\gamma_{i} & =k\left[\frac{\sqrt{2} q}{\omega}+\frac{4}{3} q \omega K D_{1 I}\left(\frac{3 \sqrt{2}}{\omega} K\right)+\frac{q}{\omega} D_{2 R}\left(\frac{3 \sqrt{2}}{\omega} K\right)\right],
\end{aligned}
$$

where $D_{n R}$ and $D_{n I}(n=1,2)$ are

$$
\begin{aligned}
& D_{n R}(K)=\frac{1}{\sqrt{\pi}} \int_{-\infty}^{\infty} \operatorname{Re}\left[C_{n}(K, Y)\right] \exp \left(-2 Y^{2}\right) d Y=\frac{1}{2 \sqrt{\pi}} \int_{-\infty}^{\infty} \frac{l^{2 n+4}}{l^{8}+K^{2}} \exp \left(-\frac{l^{2}}{2}\right) d l \\
& D_{n I}(K)=\frac{1}{\sqrt{\pi}} \int_{-\infty}^{\infty} \operatorname{Im}\left[C_{n}(K, Y)\right] \exp \left(-2 Y^{2}\right) d Y=\frac{1}{2 \sqrt{\pi}} \int_{-\infty}^{\infty} \frac{K l^{2 n}}{l^{8}+K^{2}} \exp \left(-\frac{l^{2}}{2}\right) d l
\end{aligned}
$$

When $\gamma_{r}$ is positive, we can get $A=A_{0} \exp (-i \omega \tau)$ type solution. Since $\gamma_{i}$ is always positive, this solution express the eastward propagation of the sinusoidal amplitude, while the convection cells propagte westward. 


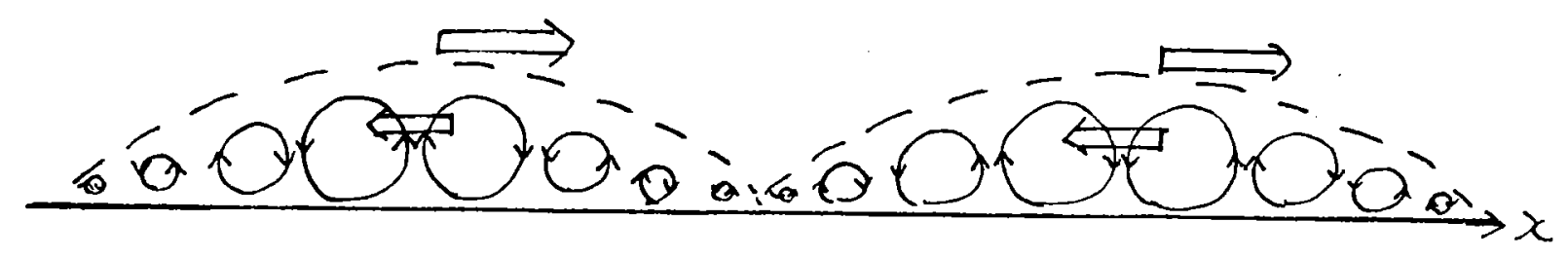

Figure 20.5. The solution with sinusoidal amplitude. The size of the cells express the magnitude of convection motion. The convection cells propagate westward while the sinusoidal amplitude profile propagates eastward.

However, it may be possible that $\gamma_{r}$ becomes negative when $K>0$ and small $\omega$ or $K<0$ and large $\omega$. In this case, the amplitude equation has a subcritical bifurcation point.

\subsubsection{More general cases and further studies}

In the same manner, we can also get the amplitude equation of the modes described by Fourier series such as:

$$
\Phi^{(1)}=\sum_{K=-\infty}^{\infty} A_{K} \exp (i K X) \exp \left(-\frac{Y^{2}}{2}\right)
$$

From the $O\left(\varepsilon^{2}\right)$ equation, we have

$$
\begin{aligned}
U^{(2)}= & -\frac{2}{3} q \omega \sum_{K=-\infty}^{\infty} \sum_{L=-\infty}^{\infty} i L A_{K} A_{K-L}^{*} \exp (i L X) C_{1}\left(\frac{3 \sqrt{2}}{2 \omega} L, Y\right) \\
& +\frac{q}{\omega} \sum_{K=-\infty}^{\infty} \sum_{L=-\infty}^{\infty} A_{K} A_{K-L}^{*} \exp (i L X) C_{2}\left(\frac{3 \sqrt{2}}{2 \omega} L, Y\right) .
\end{aligned}
$$

The amplitude equation derived from the $O\left(\varepsilon^{3}\right)$ equation is

$$
\begin{gathered}
A_{N, \tau}=\mu^{(2)} A_{N}-\sum_{K=-\infty}^{\infty} \sum_{L=-\infty}^{\infty}\left[\gamma_{r}(L)+i \gamma_{i}(L)\right] A_{K} A_{K-L}^{*} A_{N-L} \\
\gamma_{r}(L)=\frac{1}{\sqrt{2}}+\frac{2}{3} k q \omega L D_{1 R}\left(\frac{3 \sqrt{2}}{2 \omega} L\right)-k \frac{q}{\omega} D_{2 I}\left(\frac{3 \sqrt{2}}{2 \omega} L\right) \\
\gamma_{i}(L)=k\left[\frac{2}{3} q \omega L D_{1 I}\left(\frac{3 \sqrt{2}}{2 \omega} L\right)+\frac{q}{\omega} D_{2 R}\left(\frac{3 \sqrt{2}}{2 \omega} L\right)\right]
\end{gathered}
$$

Unfortunately, we have not had time to investigate these solutions in detail. As further investigations, it is necessary to study the stability of the solutions obtained in the previous section by linearizing the above equations. Or, we have to study the amplitude equations by numerical integration. 


\subsection{Acknowledgements}

I would like to thank all GFD staffs and fellows for this enjoyable program. Especially, I would like to thank William Young and Edward Spiegel for many helpful comments and advices during whole summer. I also thank Stephan Fauve for his fundamental lectures and discussions about amplitude equations.

\section{References}

Busse, F. H. (1986) Asymptotic theory of convection in a rotating, cylindrical annulus. $J$. Fluid Mech., 173, 545-556

Busse, F. H. and Or, A. C. (1986) Convection in a rotating cylindrical annulus : thermal Rossby waves. J. Fluid Mech., 166, 173-187

Cessi, P., Spiegel, E. and Young, W. R. (1991) Notes for convection with rotation. with private communication.

Chandrasekher, S. (1961) Hydrodynamic and hydromagnetic stability. Oxford.

Newell, A. and Whitehead, J. A. (1969) Finite bandwidth, finite amplitude convection. $J$. Fluid Mech., 38, 279-303

Segal, L. A. (1969) Distant side-walls cause slow amplitude modulation of cellular convection. J. Fluid Mech., 38, 203-224

Sigga, D. and Zippelius, A. (1981a) Dynamics of defects in Rayleigh-Benard convection. Physical Review A, 24, 1036-49

Sigga, D. and Zippelius, A. (1981b) Pattern selection in Rayleigh-Benard convection near threshold. Physical Review Letters, 47, 835-8 


\title{
21. Forced Vortex Merger
}

Darryn W. Waugh

DAMTP, University of Cambridge, England, CB3 9EW

\begin{abstract}
In this paper we investigate the coalescence of two identical vortices in external flows induced by other vortices. The merger of like-signed vortices in the collision of two equal dipoles and in a uniform straining flow is examined by considering the motion of point vortices and of circular vortices with uniform vorticity. The critical separation below which the vortices merge is shown to be significantly different from that of isolated vortices, and varies with the nature of the forcing. We examine the idea that simple characteristics of the point vortex motion may be used to try and predict the merger of finite-area vortices, and conclude that in general this works only in a limited way.
\end{abstract}

\subsection{Introduction}

The close interaction of two coherent vortices is an important process in two-dimensional turbulence and geophysical flows. Numerical simulations of two-dimensional turbulence have shown that coherent vortices emerge spontaneously from certain types of unstructured initial conditions, and that these vortices then dominate the evolution of the flow (see for example Benzi et. al. 1989, Santangelo et. al. 1989, McWilliams 1990 \& refs.). As the turbulent flow evolves the number of coherent vortices decreases while the size of the vortices increases. The dominant mechanism for this growth in vortex size is the coalescence of like-signed vortices (so called vortex merger).

There have been numerous investigations into the merger of two isolated vortices in twodimensional, inviscid, incompressible fluid, see for example Melander et al. (1988), Waugh (1991) \& refs. These studies have shown that the two identical vortices will merge into a single coherent vortex if they are initially below a critical separation (for two identical circular vortices with uniform vorticity this critical separation is approximately 3.3 times the vortex radii). As the vortices merge filaments are ejected, and some circulation is lost to small scale filamentary vorticity. The resultant coherent vortex has, however, greater circulation than either of the initial vortices (Waugh 1991), and the merger of vortices is a process that transfers energy from smaller to larger scales. For initial separations much larger than this critical separation the motion of finite-area vortices is the same as point vortices, and the vortices rotate about their centre vorticity at a fixed separation. Between these two regimes there is a fairly narrow intermediate regime where the vortices join together to form a compound vortex which subsequently breaks into two new vortices, each containing 
fluid from both the original vortices. The coalescence of two unequal vortices is more varied than that of equal vortices (Melander et al. 1987, Dritschel \& Waugh 1991, Yasuda \& Flierl 1991). Two vortices can still merge to form a single larger compound vortex, although there are regimes where the interaction forms two vortices of different sizes than the original vortices. If one vortex is much larger than the other it is possible for the smaller vortex to be 'strained-out' by the larger vortex with no growth in vortex size.

Two coalescing vortices in two-dimensional turbulence flow and geophysical flows are, however, rarely isolated - they are influenced by external forcing or by other vortices in the flow. This 'forcing' alters the evolution of the coalescing vortices and hence the above merger process. In this paper we consider the merger of two identical vortices which are not isolated, and investigate the variation of merger process with the forcing flow.

One mechanism in turbulent flows for bringing two like-signed vortices together is the collision of dipole pairs. Dipoles (pairs of opposite-signed vortices) form readily in turbulent flows (Couder \& Basdavent 1986) and translate rapidly through the flow. They are usually shortlived - their destruction occurs when the ambient strain pulls the vortices apart, or when they collide with other vortices (either isolated or in other dipoles). These collisions therefore bring like-signed vortices close together. Another mechanism for bringing vortices together is the advection by distant vortices. The multitude of surrounding vortices in a turbulent flow causes a straining flow which can push two (initially well separated) vortices together.

We investigate the merger of two identical vortices in both the above types of 'forcing'. Merger via dipole collisions is examined by considering the collision of two equal dipole pairs. A preliminary investigation into the collision of (unequal as well as equal) finite-area dipoles by McWilliams \& Weiss (1991) has shown that such collisions can indeed cause vortex merger. The forcing due to distant vortices is investigated by considering the merger of two equal vortices in a spatially-uniform straining flow. This straining flow is an approximation of the flow due to the multitude of surrounding vortices in a turbulent flow. It is also a leading order effect of the external forcings in many atmospheric/oceanic flows. Both the flows to be considered are highly simplified compared with the flow encountered in a turbulent flow - the vortices are in general of unequal size, the collisions may involve only a dipole with a single vortex, and the straining flow due to distant vortices is time dependent. These simplifications have been made so that the problems are symmetric, and can then be compared with the much studied isolated, symmetric vortex merger (Melander et al. (1988), Waugh (1991) \& refs).

To examine both problems we use the motion of point vortices as a first approximation of the motion of finite-area vortices. The equations of motion for a collection of interacting point vortices forms a Hamiltonian dynamical system (Aref 1983), and in the above cases the motion can be determined analytically. Although two point vortices do not merge (except in rare cases such as collapse of three vortices, Aref 1983 \& refs) their motion is the same as well separated finite-area vortices, and the closeness of approach of point vortices gives a useful guide for predicting vortex merger. If the separation of the the point vortices is less 
than the critical merger separation (i.e. 3.3 radii for identical circular vortices), then the corresponding finite-area vortices will be strongly deformed and will start to merge. If the point vortices are held close for long enough time then the finite-area vortices will merge, otherwise they will move apart as in point vortex dynamics. The closeness and the length of time required for merger varies with the forcing flow, and is a central focus of this paper.

The evolution of the finite-area vortices is examined using the numerical method of 'contour surgery' (Dritschel 1989 \& refs), a refinement of contour dynamics developed by Zabusky et al. (1979). For simplicity we consider circular vortices with uniform vorticity (although this seems a severe assumption, recent calculations by Dritschel \& Legras (1991) suggest that the straining due to distant vortices will form vortices with sharp edges and so vortices in a turbulent flow can be well approximated by vortices with uniform vorticity). The evolution of the vortices is determined for different forcing 'amplitudes', and is classified into different regimes. The critical forcing amplitude for merger is then linked with the minimum separation of the singular point vortices.

In $\S 2$ the collision of two equal dipoles is investigated, while the evolution of two identical vortices in a spatially-uniform straining flow is considered in $§ 3$. The results for the forcing flows are then compared in $\S 4$, and related to recent 'point vortex' models of two-dimensional turbulence.

\subsection{Dipole collisions}

In this section we consider the collision of two equal dipoles. Each dipole consists of two vortices with equal area but opposite signed vorticity (and circulation). Initially the two dipoles are heading towards each other along horizontal trajectories which are offset a distance $I$ (impact parameter), see figure 21.1. The initial horizontal separation of the dipoles $L$ is assumed large compared with the impact parameter $I$ and the intercentroid separation of the vortices in each dipole $b$. Without loss of generality we assume that the closest likesigned vortices have positive circulation (which corresponds to $I>0$, see figure 21.1). We first consider the motion of point vortex dipoles, and then we consider the motion of circular vortices with radius $R$ and uniform vorticity $\omega$ (and the same circulation $\Gamma=\pi \omega R^{2}$ as the point vortices). In the following we rescale all lengths by $b$, and $\tilde{l}$ denotes the ratio of a length $l$ to $b$ (e.g. $\tilde{I} \equiv I / b)$. It should be noted that although dipoles consisting of circular vortices are not steadily translating states (Wu et al. $1984 \&$ refs), the circular vortices deform to states similar to these steadily translating V-states.

\subsubsection{Point vortices}

The system of $N$ interacting point vortices in a two-dimensional inviscid, incompressible fluid forms a Hamiltonian dynamical system. The equations of motion of $N$ point vortices with circulation $\Gamma_{\alpha}(\alpha=1,2, \ldots, N)$, and position $\left(x_{\alpha}, y_{\alpha}\right)$ can be written as

$$
\Gamma_{\alpha} \dot{x_{\alpha}}=\frac{\partial H}{\partial y_{\alpha}} \quad, \quad \Gamma_{\alpha} \dot{y_{\alpha}}=-\frac{\partial H}{\partial x_{\alpha}}
$$




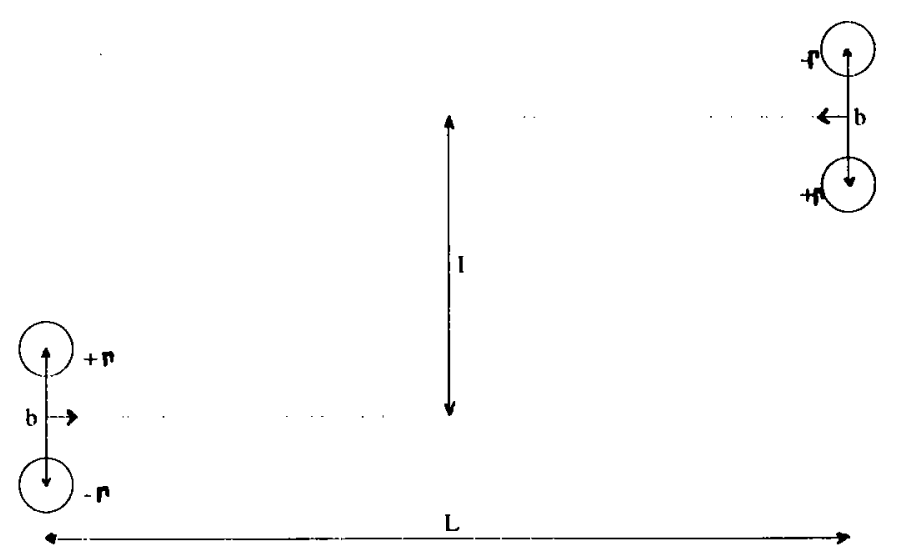

Figure 21.1. Initial condition for the collision of two equal dipoles

where

$$
H=-\frac{1}{2 \pi} \sum_{\alpha<\beta} \Gamma_{\alpha} \Gamma_{\beta} \log \left(l_{\alpha \beta}\right),
$$

is the Hamiltonian of the system $\left(l_{\alpha \beta}\right.$ is the distance between vortices $\alpha$ and $\beta$ ) and is a constant of the motion.

For the collision of two equal dipoles (see figure 21.1) the above system is integrable, and has been examined in detail by Eckhardt \& Aref (1988) (they also consider the general case of two unequal dipoles). The interaction of the dipoles results in a 'scattering' event the outgoing dipoles travel along different trajectories than those of the incoming dipoles. Depending on the initial configuration the scattering is either direct (outgoing dipoles have the same partners as the incoming dipoles) or exchange (dipoles swap partners during the collision). Figure 21.2 shows typical trajectories for direct and exchange scattering.

(a)

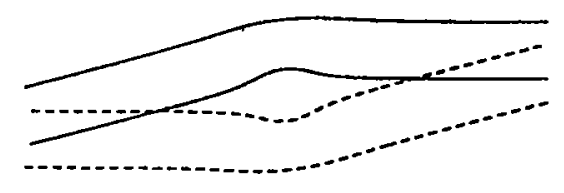

(b)

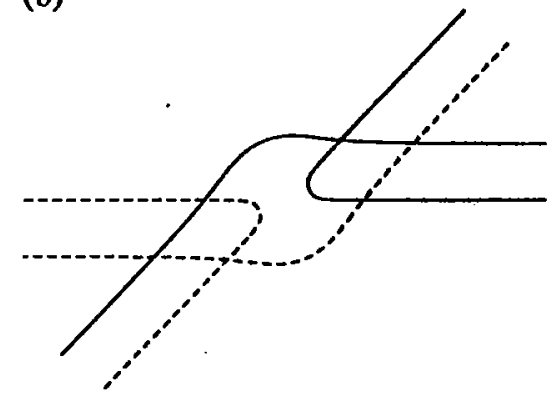

Figure 21.2. Point vortex trajectories for (a) direct $(I=1.55 b)$ and (b) exchange $(I=0.978 b)$ scattering. In both cases $L=10 b$. Solid curves correspond to the initially left travelling vortices.

The type of scattering is determined by the value of the constant of the motion (see Eckhardt \& Aref 1988)

$$
\chi=\frac{4 \exp \left(4 \pi H / \Gamma^{2}\right)}{J^{2}}
$$


where $J=\sum_{\alpha} \Gamma_{\alpha}\left(x_{\alpha}^{2}+y_{\alpha}^{2}\right)$ is the angular impulse. For two equal dipoles with large initial separation $(L>>I, b)(21.3)$ reduces to

$$
\chi=\tilde{I}^{2}
$$

and there is direct scattering if $\tilde{I}>1$ and exchange scattering when $\tilde{I}<1$, see Eckhardt $\&$ Aref (1988) for details. At the boundary between the two scattering events $(\tilde{I}=1)$ the vortices become 'trapped' in a steadily rotating state in which the vortices form a rhombus.

In both types of scattering the separation between the positive vortices decreases until a minimum value $d_{\min }$ is reached, and then the separation increases as the dipoles move apart. Figure 21.3 shows the variation of the minimum separation $d_{\min }$ with the impact parameter $I$ when the initial separation is $\tilde{L}=10$ (this curve was determined by numerically integrating the equations of motion (21.1)). The transition between exchange and direct scattering occurs at $\tilde{I} \approx 0.99$ (as $\tilde{L} \rightarrow \infty$ the transition occurs at $\tilde{I} \rightarrow 1$ ). As $I$ (greater than $b$ ) increases the minimum separation increases and in the limit of large $I$ the effect of the dipoles on each other is weak and $d_{\min } \rightarrow I$. In the next section, the variation of $d_{\min }$ with $I$ is used to predict the merger of finite-area vortices.

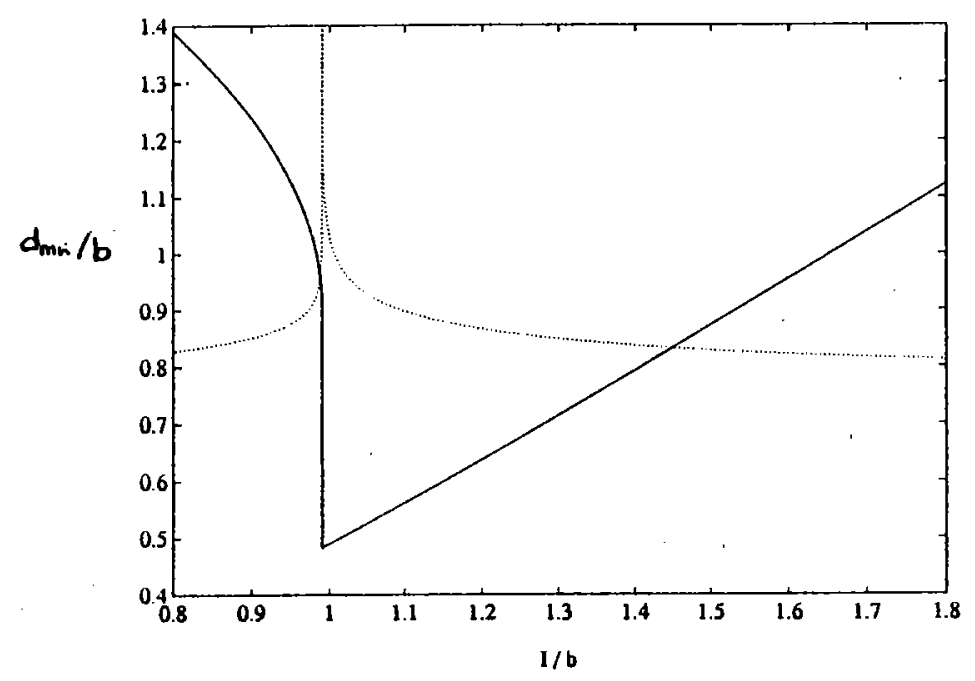

Figure 21.3. Variation of dimensionless minimum separation between positive point vortices $d_{\min } / b$ with dimensionless impact parameter $\hat{I}$. Also plotted is the variation of the close approach time $\tau$ (dashed curve is $\tau / 20$ ), see text for details.

Also shown in figure 21.3 is the variation of the 'close approach' time $\tau$, which is defined as the time that the vortices are closer than 3.3 (the critical merger separation for circular vortices with unit radius). This is different from the usual measure of the scattering time (which is the time the vortices are closer than their initial separation, see Eckhardt \& Aref 1988), and was chosen because it gives an estimate of the time that finite-area vortices have been with the critical merger separation (see next section for details). The qualitative variation of this time is, however, the same as the usual scattering time, i.e. the time increases as the 
minimum separation decreases and there is a singularity at the boundary between direct and exchange scattering at which point the vortices are trapped.

\subsubsection{Circular vortices}

For two-dimensional flow in an inviscid, incompressible fluid the vorticity $\omega=\nabla^{2} \psi$ ( $\psi$ is the streamfunction) is materially conserved, and completely determines the velocity at any point in the flow. Furthermore, for piecewise constant vorticity distributions the velocity at a point $\mathbf{x}$ is given by the contour integrals

$$
\frac{\mathrm{d} \mathbf{x}}{\mathrm{d} t}=\mathbf{u}(\mathbf{x})=-\sum_{k} \tilde{\omega}_{k} \oint_{C_{k}} G\left(\left|\mathbf{x}-\mathbf{x}_{k}\right|\right) \mathrm{d} \mathbf{x}_{k}
$$

where

$$
G(r)=\frac{1}{2 \pi} \log r
$$

is the Green function for unbounded flow, $C_{k}$ is the boundary of a region of uniform vorticity, $\tilde{\omega}_{k}$ is the jump in vorticity across $C_{k}$, and $\mathbf{x}_{k}$ is a point on $C_{k}$. The numerical method of contour dynamics developed by Zabusky et al. (1979) determines the evolution of the contours $C_{k}$ (and therefore the flow) from these contour integrals.

In this section we consider the motion of dipoles which consist of vortices with uniform vorticity and have initial position as shown in figure 21.1. The evolution of the vortices is determined numerically using 'contour surgery' (Dritschel $1989 \&$ refs), a refined version of contour dynamics. In all calculations, each vortex has unit radius and uniform vorticity $2 \pi$.

McWilliams \& Weiss (1991) have performed (pseudo-spectral) calculations of the collision of finite-area dipoles, and have shown that in both scattering regimes it is possible for the likesigned vortices to merge. In this section we investigate in detail the collision of finite-area dipoles, and compare the evolution to that of point vortex dipoles.

For large intercentroid separations the motion of finite-area vortices is the same as point vortex dynamics, and the early time evolution of the uniform vorticity dipoles is as described above. This is true even for dipoles consisting of close vortices - the speed of steadily translating dipoles with intercentroid separation equal to three vortex radii is within $2 \%$ of that for the corresponding point vortex dipoles (Wu et al. 1984). As the finite-area vortices get very close their motion is no longer the same as that of point vortices, and the vortices may merge.

Point vortex dynamics can still, however, be used to predict whether merger is possible. From calculations of the coalescence of isolated, identical circular vortices we know that the vortices will make contact with each other only for separations less than 3.45 radii, while merger occurs only when the separation is less than 3.3 radii (Waugh 1991). Hence if the minimum separation of the point vortices $d_{\min }$ (in the remainder of this paper $d_{\min }$ will represent the minimum separation of the point vortices even if we are discussing the evolution of finite-area vortices) is always greater then 3.45 then the motion of circular vortices (with 
unit radius) will be almost the same as the point vortices. Moreover, vortex merger is likely to occur only if $d_{\min }$ is less than 3.3 at some stage of the evolution. Hence we have an outer bound on the impact parameter $I$ for which merger can occur. For example, if the initial intercentroid separation $b$ is 3 times the vortex radii $R$ then merger can only occur if $\tilde{d}_{\min }<3.3 \tilde{R}=1.1$ which corresponds to $0.96<\tilde{I}<1.77$, see figure 21.3. From figure 21.3 we also see that if $\tilde{R}<0.1465$ the minimum separation is always outside the merger separation $\left(\tilde{d}_{m i n}>0.4835\right)$ and merger will not occur (i.e. if the separation of the vortices in the dipoles is large the flow due to the negative vortices is not strong enough to push the positive vortices together, and a dipole collision will not cause vortex merger).

We now examine in detail the evolution for dipoles with $\tilde{R}=1 / 3$, for impact parameter within the above range. We first consider the case corresponding to direct point vortex scattering. Although the point vortex separation is less than 3.3 radii for $\tilde{I}<1.77$, merger does not occur unless $\tilde{I}<1.55$ (which corresponds to $d_{\min }<2.7 R$ ). For $\tilde{I} \geq 1.55$ the finitearea vortices follow point vortex dynamics (see figure 21.2(a)) until the positive vortices are very close. Figure 21.4 shows the later time evolution of the finite-area vortices, and the corresponding point vortices. As the finite-area vortices approach each other they deform and join together to form a compound vortex. This elongated vortex is pulled apart by the straining flow due to the negative vortices, and breaks into two new vortices containing fluid from both original positive vortices. These new vortices are joined by a thin ribbon of vorticity which is stretched out as the newly formed dipoles move apart (note that the new dipoles are moving on different trajectories from the point vortex dipoles). The initial stage of the coalescence shown in figure 21.4 is very similar to that of the merger of isolated vortices. But whereas in isolated merger the compound vortex ejects filaments to form a stable elliptical vortex, the compound vortex in the above example is torn in two by the straining flow of the opposite-signed vortices.

For slightly smaller impact parameter the initial stage of the collision is as above. The positive vortices join to form a compound vortex which is again pulled apart by the straining flow due to the negative vortices. Now, however, the ribbon joining the new positive vortices is thicker and rolls up in to a central vortex (see figure 21.5). The resultant configuration is now a five-vortex state. The long-time evolution of this state is discussed later.

As the vortices are pushed closer together (i.e. smaller $d_{\min }$ ) they form a less elongated compound vortex which does not get pulled apart. The evolution of this compound vortex is then very similar to that in the isolated merger, i.e. the vortex ejects filaments and becomes roughly elliptical (figure 21.6). The resultant state then consists of a large central positive vortex with two smaller negative vortices (plus surrounding filaments of positive vorticity). This configuration is very similar to the steadily rotating tripolar states determined by Polvani \& Carton (1991). Polvani \& Carton investigated the stability of these tripolar vortices to finite amplitude disturbances, and showed that it is possible for the tripolar vortex to break up into two outgoing dipoles. This break-up is very similar to the reverse of the collision shown in figure 21.6 (although there are more filaments generated in the dipole 


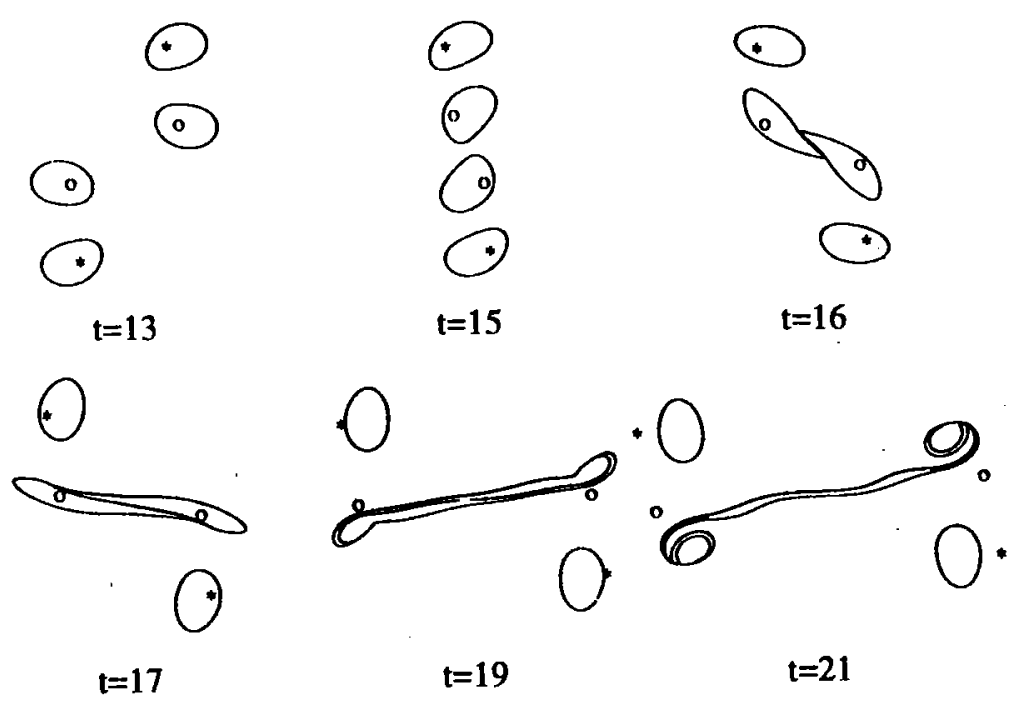

Figure 21.4. Contour surgery calculation of the collision of two equal dipoles. The vortices have vorticity $2 \pi$, are initially circular with unit radius and the intercentroid separation within each dipole is $b=3 R$. The initial state is as in figure 21.1 with horizontal separation $L=10 b$ and impact parameter $I=1.55 b$ (which corresponds to $d_{\min }=2.74 R$ ). The symbols correspond to the positions of point vortices with the same circulation as the finite-area vortices, see figure 21.2 for full trajectories.

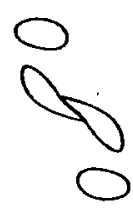

$t=16$

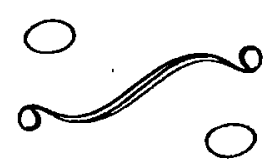

$\mathrm{t}=\mathbf{2 0}$

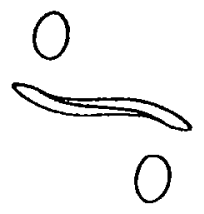

$t=17$

$t=18$
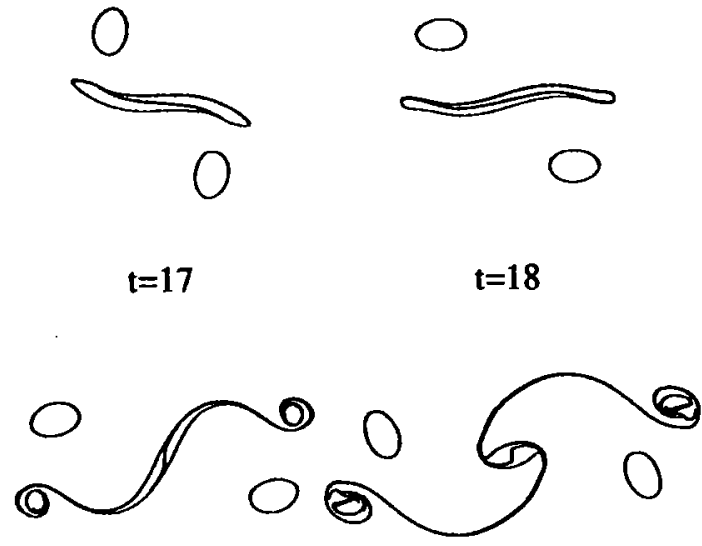

$\mathrm{t}=\mathbf{2 2}$

$t=25$

Figure 21.5. As in figure 21.4, except $I=1.53 b\left(d_{\min }=2.70 R\right)$. The point vortex positions have been omitted.

collision). Note that the formation of a tripole structure from the collision of (unequal) dipoles has already been observed by Larichev \& Reznik (1983). 


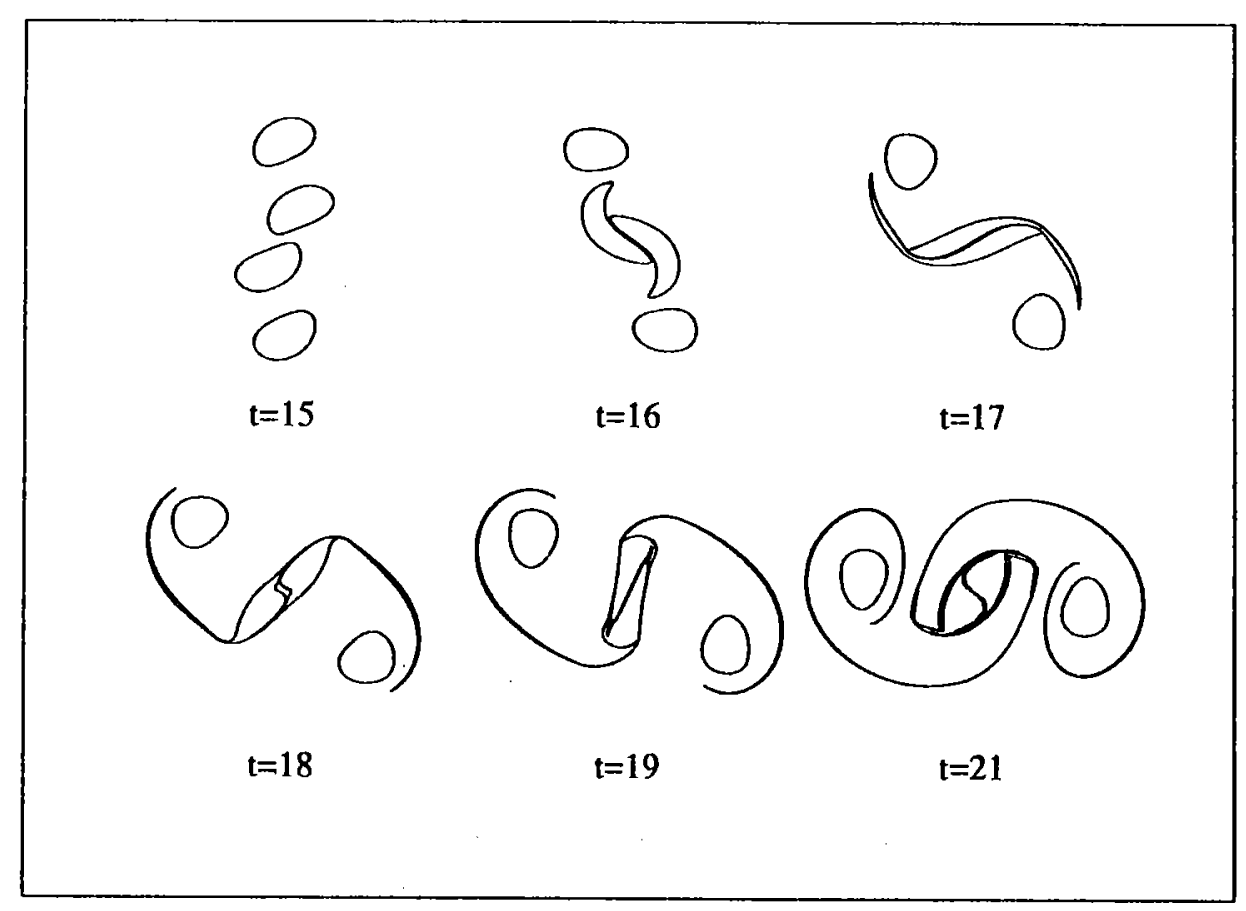

Figure 21.6. As in figure 21.4, except $I=1.33 b\left(d_{\min }=2.22 R\right)$

The above results show that there are at least three possible outcomes from the collision between two equal (finite-area) dipoles: two outgoing dipoles, a 5-vortex state, or a tripolar vortex. The transition between the states is gradual (e.g. as the positive vortices are pushed closer together the size of the satellite vortices decreases and the state changes from the 5vortex to the tripolar state). Preliminary investigations suggest, however, that the long time evolution of the 5-vortex state leads to either a tripolar vortex or two dipoles. As the whole structure rotates about the origin the satellite positive vortices rotate around the negative vortices. This brings the satellite vortices between the negative vortices and the central positive vortex, and the three positive vortices may join together. It is unclear whether these three vortices will merge to form one central vortex or whether the interaction will form multiple positive vortices. It should be noted that the above process takes around five times as long as the merger of isolated vortices (fifteen eddy turn-around times compared two or three eddy turn-around times).

The above calculations correspond to collisions in the point vortex direct scattering regime. We now investigate the evolution in the exchange scattering regime. As above we find that the point vortex separation must be less than $3.3 R$ for vortex merger to occur. For $\tilde{I}<0.977$ (which corresponds to $d_{\min }>3.09 R$ ), the vortices form a compound vortex which then breaks in two. This collision generates only a few filaments and the evolution of the two positive vortices is very similar to the pulsation with exchange regime that occurs in isolated merger (Waugh 1991). If the vortices are pushed closer together $(\tilde{I}>0.978$, for which $d_{\min }<3.08 R$ ) the compound vortex does not break in two and the final state is a tripolar vortex (see figure 21.7). This critical value of $d_{\min }$ for merger is different from that 
for direct scattering. The difference is due to the different vortex trajectories (compare figure 21.2(a) and (b)), and hence the different straining flow imposed on the merging vortices.

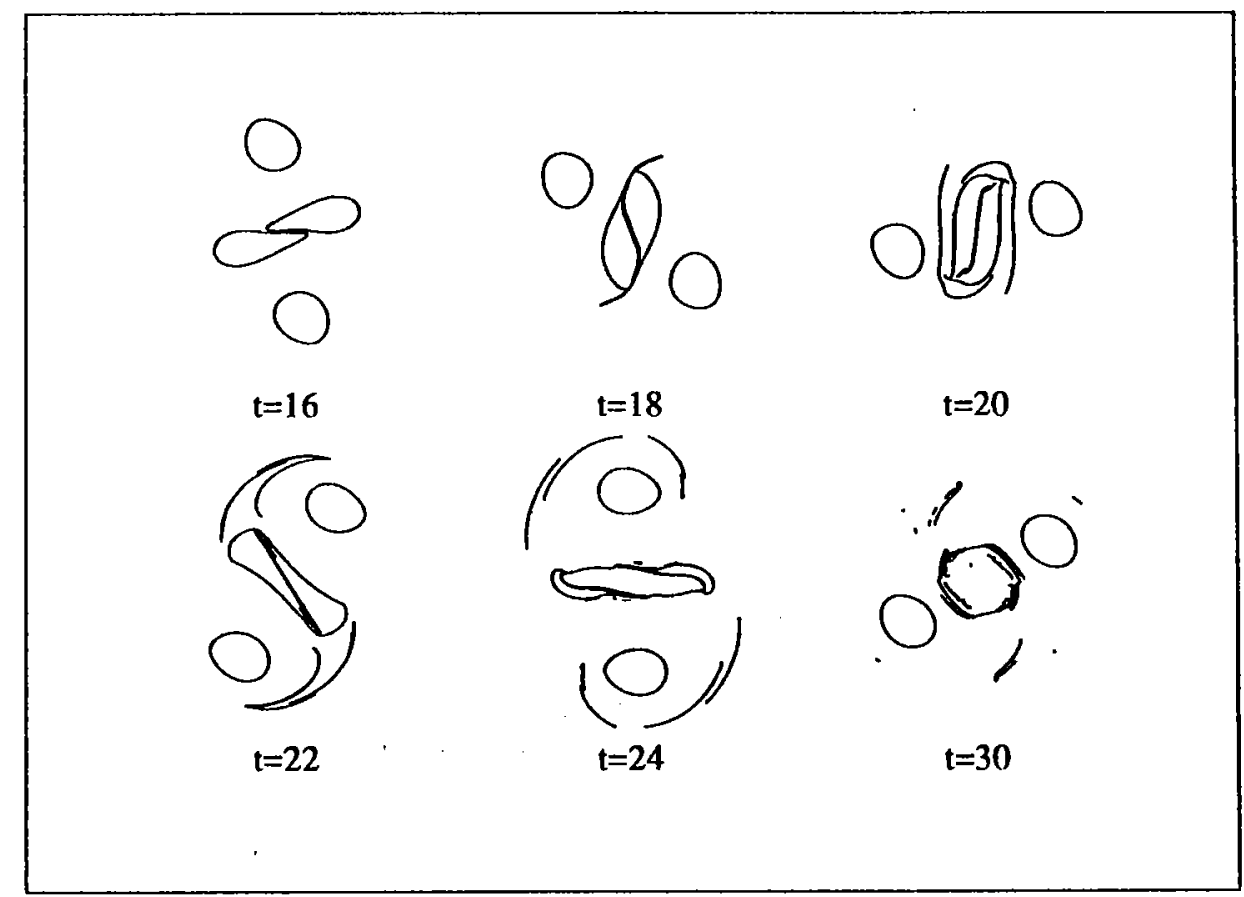

Figure 21.7. As in figure 21.4, except $I=0.978 b\left(d_{\min }=3.08 R\right)$

The above calculations for dipoles with $\tilde{R}=1 / 3$ have shown that if the two positive vortices are to merge into a central vortex, then the point vortex dynamics has to push the vortices significantly closer than the isolated critical merger separation. Furthermore, the critical separation depends on the type of scattering. We now investigate the variation in collision (and vortex merger) with the separation of the vortices in each dipole. For simplicity we consider only collisions in the direct scattering regime.

The evolution for all values of $\tilde{R}$ considered is qualitatively the same as above. The flow can be classified into the same regimes, although the critical impact parameters (and the corresponding $\tilde{d}_{\min }$ ) vary with $\tilde{R}$. As $\tilde{R}$ increases the critical $\tilde{d}_{\min }$ for merger decreases (because the negative vortices are closer to the merging vortices and the forcing flow pulling the vortices apart is stronger).

An estimate of the forcing on the merging vortices can be determined by calculating the instantaneous strain due to the negative vortices when the positive point vortices are at their minimum separation. We define strain $S$ as

$$
S^{2}=S_{1}^{2}+S_{2}^{2},
$$

where

$$
S_{1}=\frac{1}{2}\left\{\frac{\partial u}{\partial x}-\frac{\partial v}{\partial y}\right\} \quad, \quad S_{2}=\frac{1}{2}\left\{\frac{\partial v}{\partial x}+\frac{\partial u}{\partial y}\right\}
$$


The strain at the midpoint between two circular vortices with unit radius, constant vorticity $\omega$, and intercentroid separation $l$ is then $S=4 \omega / l^{2}$. From conservation of angular impulse $J$ we have that during the dipole collision the separation of the negative point vortices $d_{-}$ is related to that of the positive vortices, $d_{+}$, by

$$
d_{-}^{2}=4 I b+d_{+}^{2} \text {. }
$$

Hence the strain at the origin due to the negative vortices when the positive point vortices are at their minimum separation is

$$
\hat{S} \equiv S / \omega=\left(I b+\frac{1}{4} d_{\min }^{2}\right)^{-1} .
$$

In figure 21.8 the outcome of the finite-area vortex collisions have been plotted on a graph of $d_{\text {min }} / R$ versus the dimensionless strain $\hat{S}$ given by (21.5). A cross represents merger (we say the vortices have merged if they form a coherent central vortex), whereas a circle corresponds to no merger. This plot shows that for 'direct scattering' collisions as the strain increases the vortices have to be pushed closer together for merger to occur. The solid curve has been drawn by hand and represents the critical $d_{\min }$ for merger in a direct scattering collision.

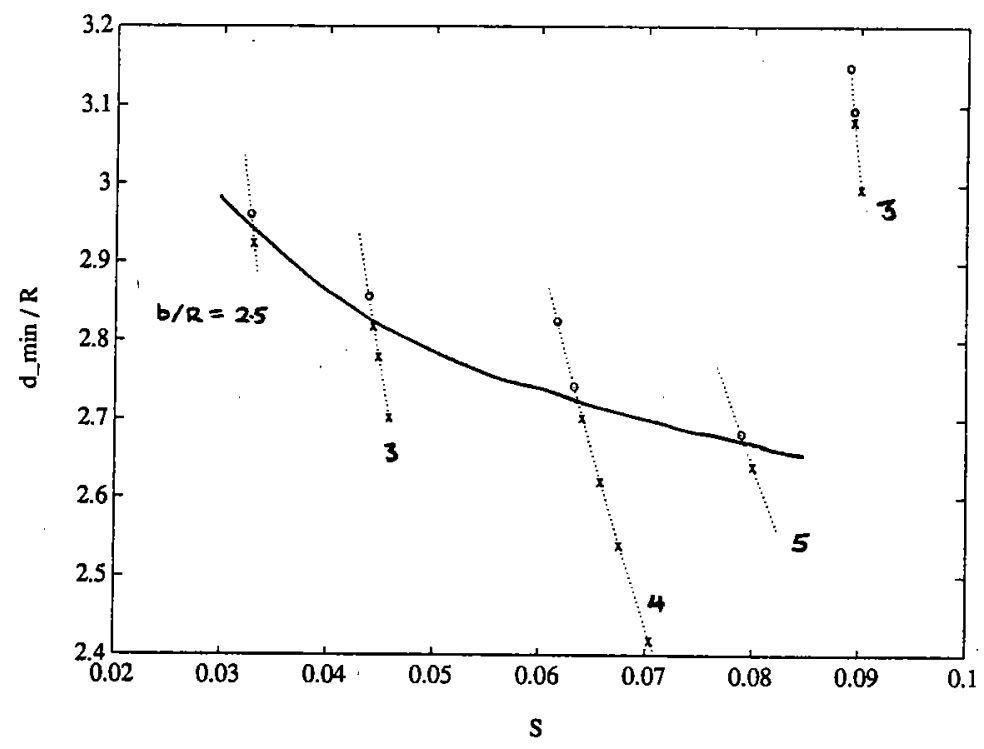

Figure 21.8. Results of Contour Surgery calculations for the collision of two equal dipoles. The outcome of each calculation is plotted on a graph of the minimum separation $d_{m i n} / R$ versus dimensionless strain $\hat{S}$ for the corresponding point vortex dipole collision. A cross represents merger, whereas a circle corresponds to no merger. Results with $\hat{S} \approx 0.09$ correspond to 'exchange scattering', while all others correspond to 'direct scattering'. 
The direct scattering calculations cover only part of the range of $d / R$ for which merger is possible. If $\tilde{R}$ is greater than 0.4 the corresponding steadily translating dipoles are significantly noncircular and calculations have not been performed, while for $\tilde{R}$ smaller than $1 / 6$, approximately, there is no merger.

From figure 21.8 we see that the calculations for 'exchange scattering' do not agree with the results for 'direct scattering'. The strain on the merging vortices is larger than the direct scattering collisions, yet the vortices merge at a larger $d_{\min }$. This discrepancy between the two types of collisions shows that the instantaneous strain is not a good measure for predicting merger in a forcing flow. As can be seen from figure (21.2), the two types of dipole collisions bring the vortices together along different trajectories, and the merging vortices are held 'close' for different lengths of time and experience varying strain.

A better measure of the forcing flow is, perhaps, the time that the vortices are held 'close'. From the collision of point vortex dipoles we can calculate the time that the vortices are kept within the critical separation for isolated merger (e.g. close approach time $\tau$, figure 21.3), and this can be used as a measure of the forcing flow. Small $\tau$ corresponds to a strong forcing flow (i.e. vortices pushed close and pulled apart very rapidly), whereas large $\tau$ corresponds to weak forcing (e.g. $\tau$ equals infinity for two isolated vortices).

In figure 21.9, we have replotted the results of the finite-area vortex collisions on a graph of $r$ versus $d_{\min }$. The solid line $\tau_{c}(d)$ has been drawn by hand, and gives an estimate of the critical close approach time for merger. If the point vortex dynamics pushs the two like-signed vortices to within $d_{\min }$ of each other and $\tau$ is greater than $\tau_{c}\left(d_{\min }\right)$ then the finite-area dipole collision leads to vortex merger.

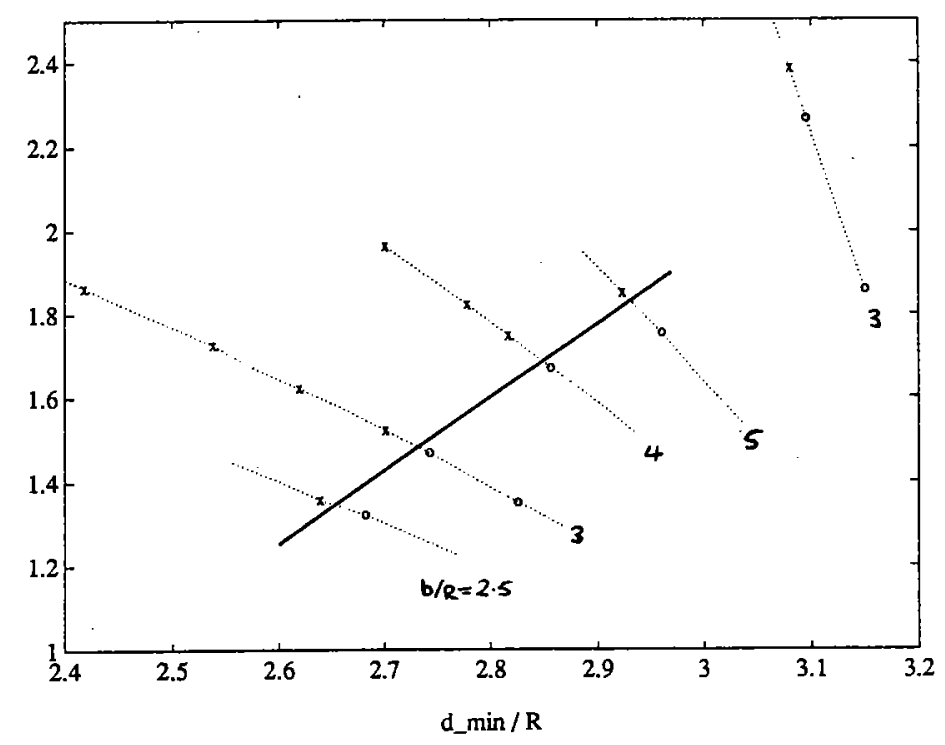

Figure 21.9. Results of Contour Surgery calculations for the collision of two equal dipoles. The outcome of each calculation is plotted on a graph of close approach time $\tau$ versus minimum separation $d_{\min } / R$ for the corresponding point vortex dipole collision. A cross represents merger, whereas a circle corresponds to no merger. 
As expected, if the vortices are not pushed very close then the point vortex dynamics must hold the vortices there for a longer time if merger is to occur (i.e. $\tau_{c}$ increases with $d_{m i n}$ ). Note that the time they must be held together is about one eddy turnaround time $(4 \pi / \omega=2$ in above calculations), while the merger of two isolated vortices takes between two and three eddy turnaround times (the time for isolated merger also increases with separation).

The results for the calculations of the 'exchange scattering' now agree better with the 'direct scattering' results, although there is still a significant difference between the two sets of results. The reason for the difference could be that $\tau_{c}$ increases rapidly as $d=3.3 R$ is approached (for $d>3.3 R \tau_{c}=\infty$ ). Or it could be because the results from different types of forcing do not have the same $\tau-d_{m i n}$ dependence. In the next section we investigate this further by examining the merger of vortices in a different forcing flow.

\subsection{Uniform shear flow}

In the previous section we examined the merger of vortices in a forcing flow due to close vortices. We now examine vortex merger in a forcing flow due to distant vortices. We consider an external flow of the form

$$
\begin{aligned}
& u_{s}=\gamma x-\Omega y, \\
& v_{s}=\Omega x-\gamma y
\end{aligned}
$$

where $\gamma$ is the strain rate and $\Omega$ is the rotation rate of the strain axes. This flow is the leading order distortional effect of a population of distant vortices. In general the strain rate and the rotation rate of the strain axes are time-dependent, but for simplicity we consider $\gamma$ and $\Omega$ constant, equal and opposite so that the flow is simple shear flow.

As shown by Dritschel (1989, figure 27), the flow (21.6) can bring two distant vortices close enough together so that they merge into a single coherent vortex. In this section we examine in detail the evolution of two identical vortices, for various shear rates. As in the previous section, we investigate the evolution by considering the point vortex dynamics and then the evolution of circular vortices with uniform vorticity. We consider only the case where the strain rate $\gamma$ is the same sign as the merging vortices.

\subsubsection{Point vortices}

The motion of point vortices in the straining flow (21.6) is still a Hamiltonian dynamical system. The equations of motion can still be written in the form (21.1), except now the Hamiltonian is

$$
H=-\frac{1}{2 \pi} \sum_{\alpha<\beta} \Gamma_{\alpha} \Gamma_{\beta} \log \left(r_{\alpha \beta}\right)+\gamma \sum_{\alpha} x_{\alpha} y_{\alpha}-\frac{1}{2} \Omega \sum_{\alpha}\left(x_{\alpha}^{2}+y_{\alpha}^{2}\right) .
$$

In this paper we consider the motion of two identical point vortices $\left(\Gamma_{1}=\Gamma_{2}=\Gamma\right)$, and so the equations of motion can be solved to determine the trajectories of the vortices. Defining new variables

$$
\xi=x_{1}-x_{2} \quad, \quad \eta=y_{1}-y_{2},
$$


we can rewrite (21.1) as

$$
\dot{\xi}=\frac{\partial H}{\partial \eta} \quad, \quad \dot{\eta}=-\frac{\partial H}{\partial \xi}
$$

where

$$
H=-\frac{\Gamma}{\pi} \hat{H} \equiv-\frac{\Gamma}{\pi}\left\{\log r-\hat{\gamma} \xi \eta+\frac{1}{2} \hat{\Omega} r^{2}\right\}
$$

$\hat{\gamma}=\pi \gamma / \Gamma$, and $\hat{\Omega}=\pi \Omega / \Gamma$. The level curves of $\hat{H}$ correspond to the trajectories of the two point vortices (we consider symmetric initial conditions, $x_{1}=-x_{2}$, and so $x_{1}=\frac{1}{2}(\xi, \eta)$ ).

We now consider the special case of adverse shear, $\hat{\Omega}=-\hat{\gamma}$. Figure 21.10 is the 'phase plane' diagram of (21.7) in this case. There are stationary points at the origin and at

$$
\xi=\eta= \pm 0.5 \hat{\gamma}^{-1 / 2}
$$

Inside the separatrix the vortex-vortex interaction dominates the straining flow and the vortices rotate about the origin in a closed orbit. Whereas outside the separatrix the straining flow brings the vortices close together (arrows correspond to the direction of motion of the vortices), the vortex-vortex interaction then becomes important before the strain again dominates and sweeps the vortices away. Note that although this forcing is different from that on the positive vortices in the dipole collisions, the trajectories of the vortices are similar to those in direct scattering.

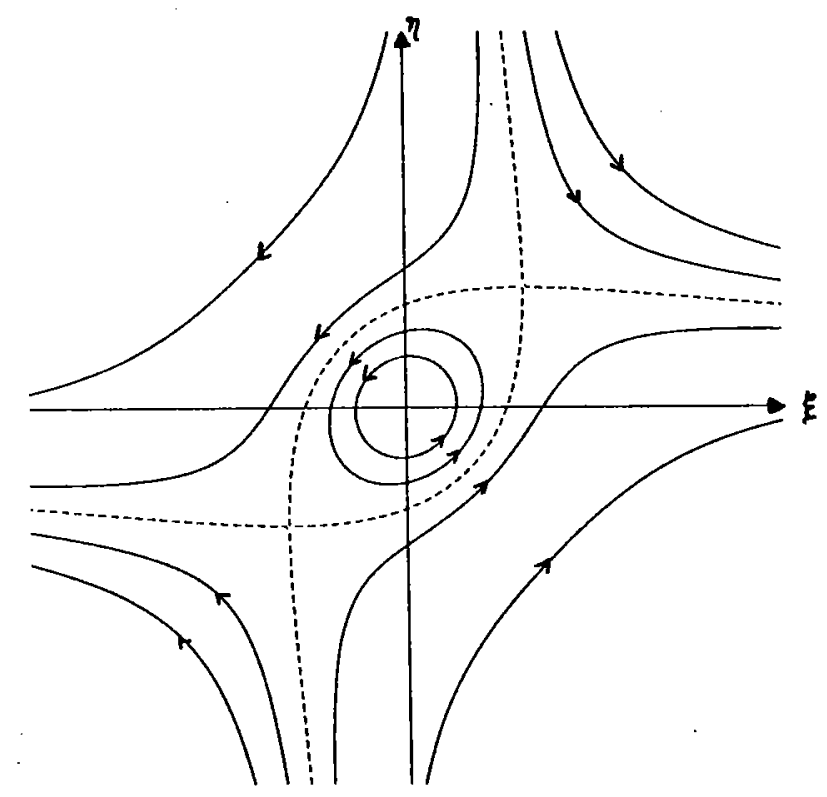

Figure 21.10. 'Phase plane' diagram in the case of adverse shear, $\hat{\gamma}=-\hat{\Omega}$. Arrows correspond to the direction of motion of the vortices. 
For given initial position (and hence $\hat{H}$ ), we can determine the minimum separation of the vortices. It can be easily shown that the extremum of the vortex separation $d$ for given $\hat{H}$ occur along the lines $\xi= \pm \eta$. For a given Hamiltonian $\hat{H}_{0}$ (with minimum separation at $\xi=-\eta$ ) the minimum separation $d_{\min }$ is given by

$$
d_{\min }^{2}=\exp \left\{-2 \hat{H}_{0}\right\} .
$$

For two vortices on the separatrix the minimum separation is then

$$
d_{\min }^{2}=\exp \{-1-\log 2 \hat{\gamma}\}
$$

For a given strain rate $\hat{\gamma}$, equation (21.9) gives the smallest separation within which two initially distant vortices can be pushed together.

From (21.9) we have that the minimum separation $d_{\min }$ is greater than 3.3 for $\hat{\gamma}<0.017$, and that as the strain rate is increased the minimum separation decreases. Therefore, as in the dipole collisions, weak forcing will not bring two circular vortices within merger distance, and as the forcing is increased the two vortices can be pushed closer together.

\subsubsection{Circular vortices}

The above point vortex dynamics has shown that it is possible for adverse shear to push two initially distant vortices within the critical merger separation. We now investigate the evolution of circular vortices with uniform vorticity using contour surgery simulations. The initial position of the vortices, $\pm \mathbf{x}_{0}$, is chosen so that point vortex dynamics will bring the vortices within $d_{\min }$ of each other (see equation (21.8)). In all calculations we have set $y_{0}=5$ and varied $x_{0}$ so that the vortices lie on the trajectory with minimum separation $d_{\min }$. The vortices have unit radius (which means that $\hat{\gamma}=\gamma / \omega R^{2}$ is the ratio of the strain rate to the vorticity of the vortices) and the numerical parameters used are the same as in $\S 2$.

From the point vortex dynamics we know that weak shear $(\hat{\gamma}<0.017)$ will not bring two distant vortices close enough together to merge. For $\hat{\gamma}=0.02$, the point vortex dynamics can bring the vortices within 3.03 radii (see equation (21.9)). As with merger induced by dipole collisions, we find that the point vortex dynamics has to bring the vortices closer than the critical separation for isolated merger before the vortices merge. For $d_{\min } \geq 3.15 R$ the vortices do not touch each other and motion is the same as point vortex dynamics (although the vortex shape is significantly deformed when the vortices are close). For smaller minimum separation, $d_{\min } \leq 3.125 R$, the vortices join together to form an elongated compound vortex which breaks into two new vortices. This process is very similar to the pulsation with exchange regime for isolated vortices (Waugh 1991). The subsequent evolution is, however, quite different from the coalescence of isolated vortices. Whereas the new vortices formed in the isolated case never merge to form a single vortex (although they might reconnect and break apart several times), the new vortices in weak strain remerge to form a single elliptical vortex (plus filaments), see figure 21.11. 


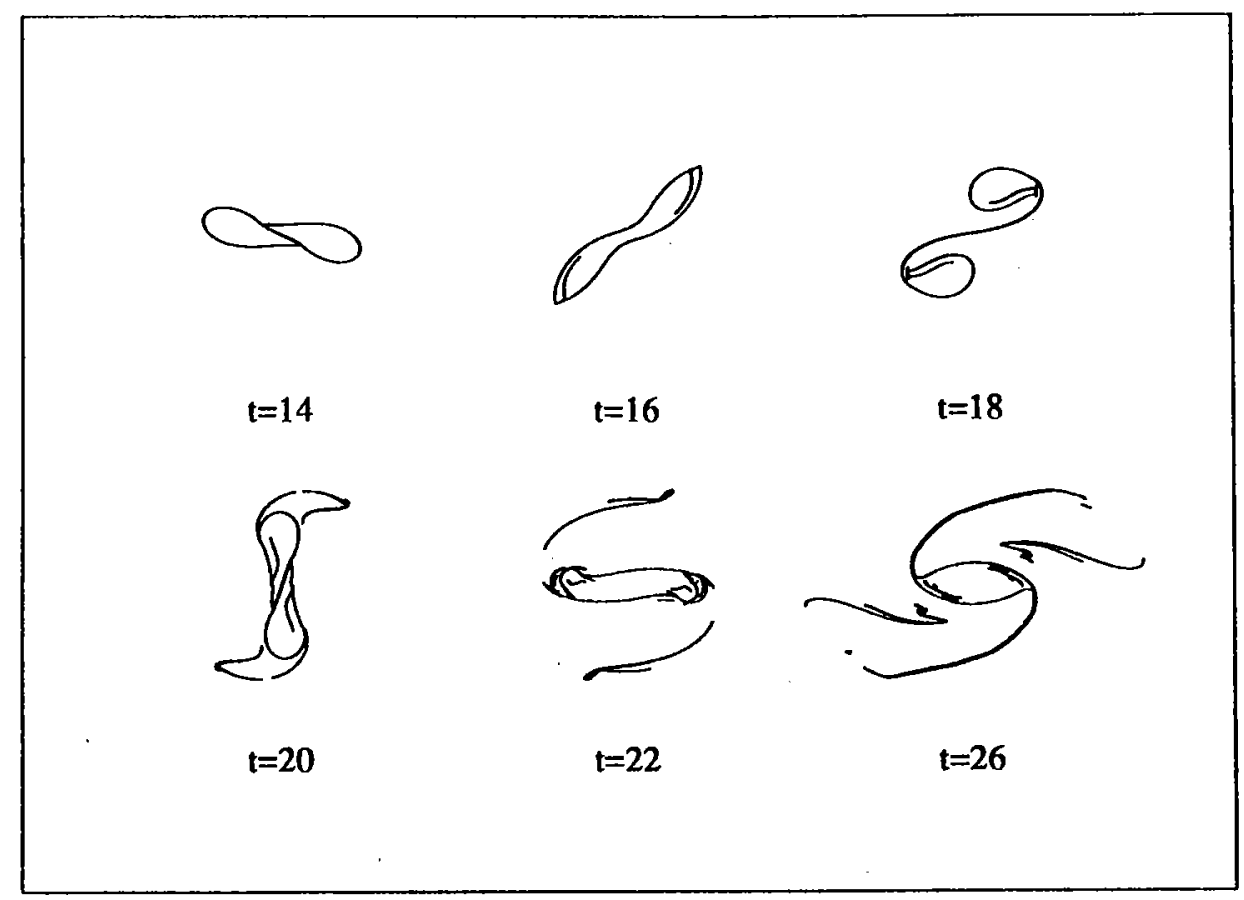

Figure 21.11. Contour surgery calculation of two identical vortices in adverse shear $\hat{\gamma}=0.02$. The vortices are initially circular with unit radii and are positioned at $\left(x_{0}, y_{0}\right)= \pm(0.458,5.0)$. The crosses represent the motion of the corresponding point vortices, whose minimum separation is $d_{\min }=3.05$.

The two new vortices formed in the above evolution remerge because their centroids are inside the separatrix of the phase diagram 21.10, and point vortex dynamics brings the vortices back inside the merger separation. For example, when $\hat{\gamma}=0.02$ and $d_{\min }=3.05 R$, the vortices formed after the first interaction are at, approximately, 45 degrees to the $x$-axis and their intercentroid separation is $4.02 R$, and so the vortices are inside the separatrix ( $d=5.05 R$ at 45 degrees) and will be pushed closer together. The evolution for $d_{\min } \approx 3.03$ is the same as shown in figure 21.11 , and so although adverse shear $\hat{\gamma}=0.02$ can induce the merger of distant vortices, the vortices do not merger during the initial interaction.

For slightly stronger shear $\hat{\gamma}=0.025$, the point vortex dynamics has to push the vortices closer together for merger to occur. For $d_{\min } \geq 3.125 R$ the finite-area vortices do not make contact and the motion is the same as that of point vortices. While for $3.125 R>d_{\min } \geq$ $3.075 R$ the vortices form a compound vortex which breaks into two new vortices. But unlike the weaker shear calculations, these two new vortices are outside the separatrix and the straining flow pushes them apart before they can remerge, see figure 21.12. Note that as $\hat{\gamma}$ increases the separatrix gets smaller (c.f. equation 21.9), and the point vortices have to be closer together for bounded motion. If initial position of the vortices is such that the forcing pushes the vortices closer together $\left(d_{\min } \leq 3.025 R\right)$, the vortices merge together in the same way as isolated vortices, see figure 21.13. 


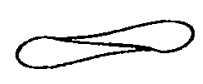

$t=10$

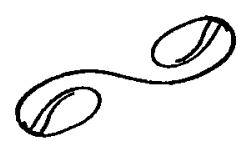

$t=14$

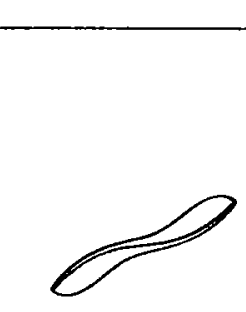

$t=11$

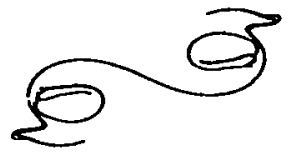

$t=16$

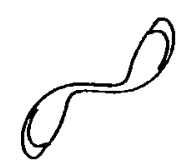

$t=12$
Yr

$t=18$

Figure 21.12. As 21.11, except $\hat{\gamma}=0.025,\left(x_{0}, y_{0}\right)=\mp(0.143,5.0)$ and $d_{\min }=$ $3.075 R$.

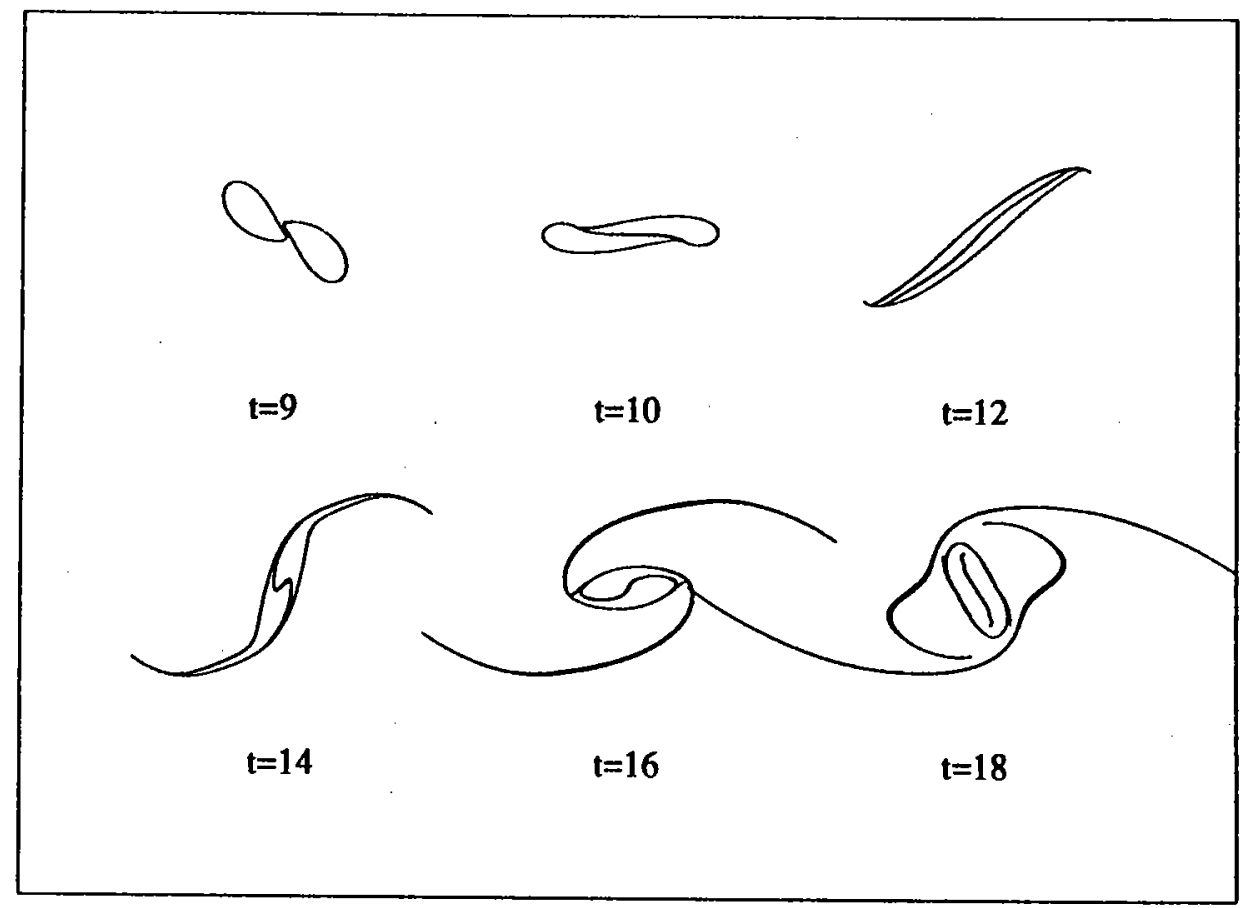

Figure 21.13. As 21.11, except $\hat{\gamma}=0.025,\left(x_{0}, y_{0}\right)=\mp(0.109,5.0)$ and $d_{\min }=$ $3.025 R$.

As the straining is increased further the evolution of the vortices is qualitatively the same although the vortices have to be pushed even closer together for merger to occur (e.g. for $\hat{\gamma}=0.035, d_{\min } \leq 2.85 R$ for merger). As the strain becomes too large there are, however, 
significant strain-induced deformations to the finite-area vortices, and the evolution of the vortices changes.

It has been shown by Kida (1981) that there exist exact, time-dependent solutions of elliptical vortices with uniform vorticity in the straining flow (21.6), see also Dritschel (1990) and Meacham et al. (1990). From Kida's exact solutions it can be shown that a circular vortex will be infinitely stretched out (and hence destroyed) if $\hat{\gamma} \geq 0.074 \ldots$. Therefore as $\hat{\gamma}$ approaches this critical value the shape of the merging vortices becomes significantly noncircular, and the merger process changes. For $\hat{\gamma}=0.05$, vortices can still merge to form a coherent elliptical vortex if $d_{\min }$ is small enough. For larger $d_{\min }$ the vortices form an elongated compound vortex with filamentary arms, as in isolated vortex merger. This compound vortex is now, however, torn apart by the shear, see figure 21.14.

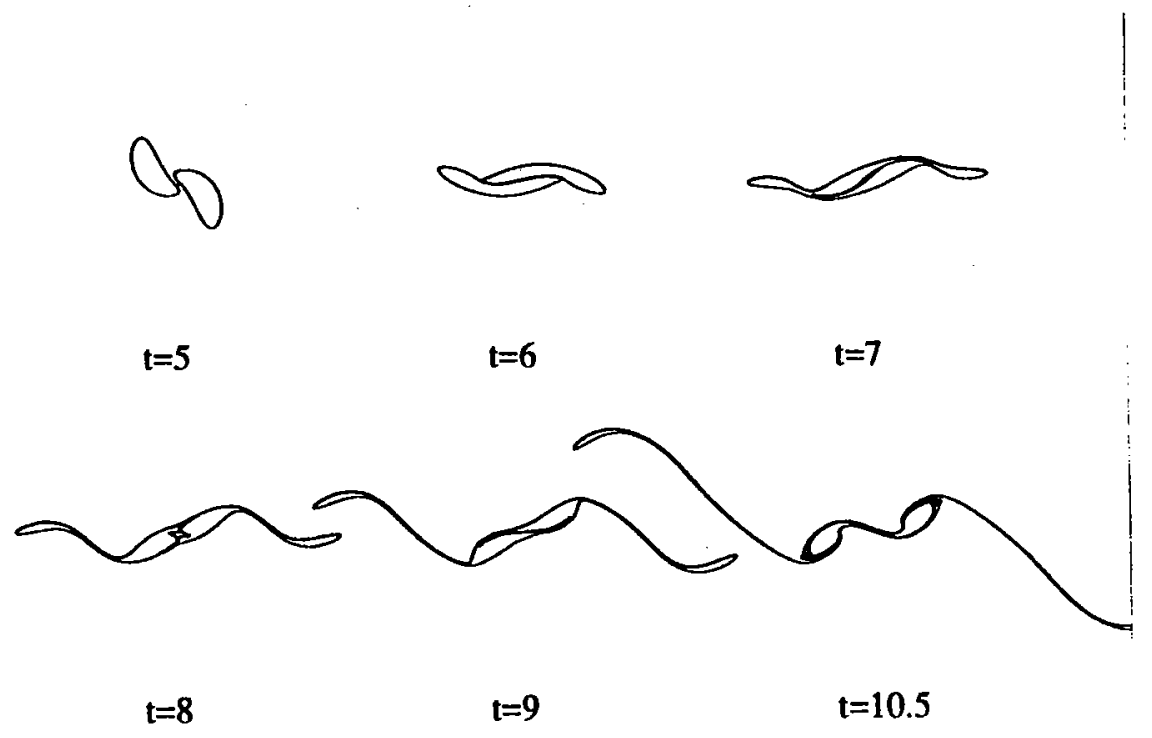

Figure 21.14. As 21.11, except $\hat{\gamma}=0.05$ and $d_{\min }=2.8$.

The outcome of all the contour surgery calculations is shown in figure 21.15 which plots $d_{\min } / R$ versus $\hat{\gamma}$. For the flow (21.6) we have $\hat{S}=\hat{\gamma}$, and so as in dipole collisions the critical merger separation decreases with the instantaneous strain at $d_{\min }$ (see figure 21.8). But as shown in the dipole collisions the instantaneous strain is not a good measure for predicting the merger of two vortices. Figure 21.16 replots the outcome of the calculations for the alternative measure of the forcing flow, the close approach time $\tau$. Comparing figure 21.16 with figure 21.9 we see that the critical $\tau-d_{\min }$ curve for vortices in adverse shear is very different from that in dipole collisions. The time that the vortices have to close for merger is larger for smaller $d_{\min }$. It therefore appears that $\tau\left(d_{\min }\right)$ is not sufficient to predict vortex merger. This is because, as shown above, strain induced deformations to the vortices can play an important role in the vortex merger and $\tau$ can not measure this effect. 


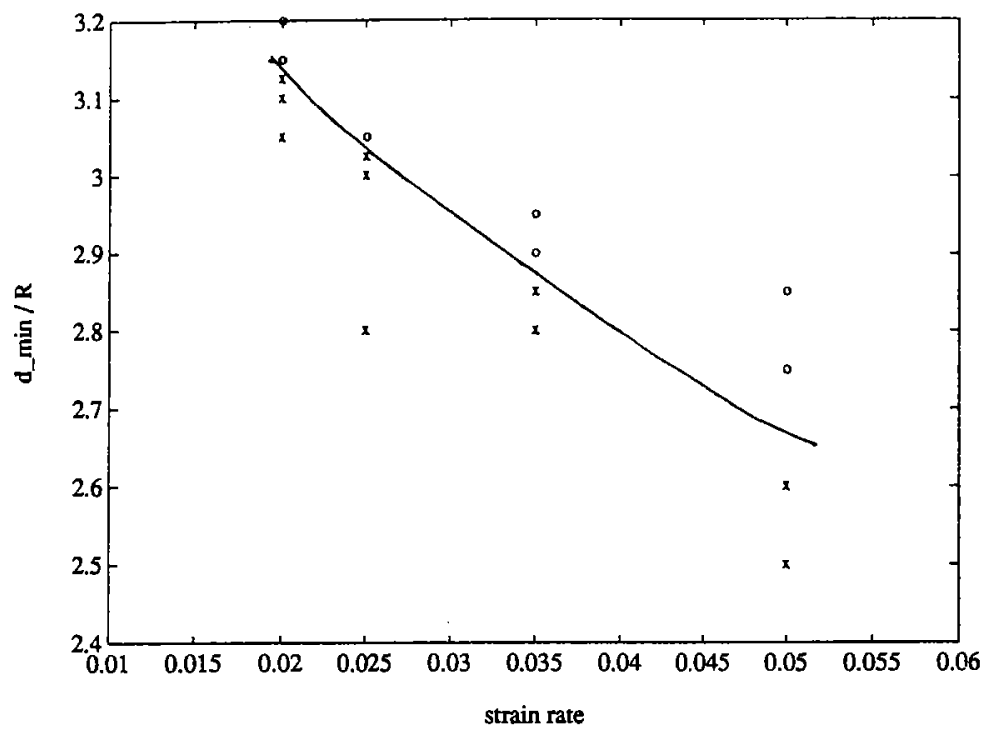

Figure 21.15. Results of Contour Surgery calculations for merger in adverse shear. The outcome of each calculation is plotted on a graph of $d_{\min } / R$ versus $\hat{\gamma}$ for the corresponding point vortex dynamics. A cross represents merger, whereas a circle corresponds to no merger.

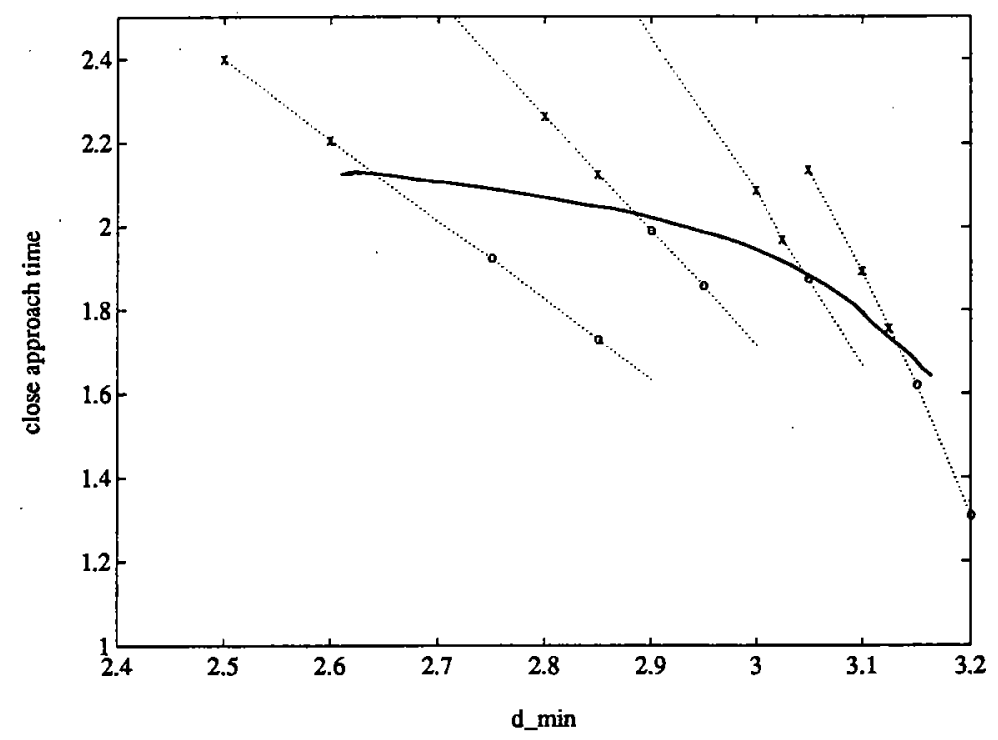

Figure 21.16. Results of Contour Surgery calculations for merger in adverse shear. The outcome of each calculation is plotted on a graph of close approach time $\tau$ versus minimum separation $d_{\min } / R$ for the corresponding point vortex dynamics. A cross represents merger, whereas a circle corresponds to no merger. 


\subsection{Discussion}

In this paper the merger of identical vortices in different forcing flows has been investigated. Although the merger process is qualitatively the same, the critical separation below which two vortices will merge is shown to vary drastically with the nature of the forcing flow. The conditions within which the vortices merge is of importance to our understanding of many geophysical flows, and also in the development of simple models of two-dimensional turbulence.

Recently, non-conservative point vortex models of two-dimensional turbulence have been developed (e.g. Benzi et al. 1991, Carnevale et al. 1991). In these models the coherent vortices are represented by circular vortices with (the same) uniform vorticity. For separations outside 3.3 times the average radii of the vortices the motion of the vortices is the same as that of point vortices, but when the vortices move within this separation a non-conservative transformation is performed. This transformation replaces the two vortices with a single vortex with less circulation than the total circulation of the two initial vortices (this transformation is a parametrization of the merger process). This simple procedure does not take into account the variation of the merger process with relative vortex sizes and intercentroid separation (Dritschel \& Waugh 1991). Furthermore, as has been shown in this paper, identical vortices do not necessarily merge when they are within 3.3 radii. To improve these point vortex models a simple criterion involving the external forcing is needed to determine whether two vortices will merge.

In this paper we have tried to use the point vortex motion to predict the merger of circular vortices. Unfortunately, neither the instantaneous strain at minimum separation, nor the time within which the vortices are close, provide such criterion. The 'close approach' time does, however, give an estimate of the minimum time the vortices need to be close if they are going to merge (i.e. between a half and one eddy turn-around time). Furthermore, if we consider only the weak strain forcing (i.e. less than half the value of the strain which would destroy a circular vortex) and the dipole collisions, we then have general trend where the minimum time the vortices have to be close decreases with the minimum separation. Whether the straining due to distant vortices in a turbulent flow is weak (in which case considering only weak strain may be valid) is presently under consideration. We are also investigating further the merger in uniform strain flows, and also other measures for predicting merger.

\section{Acknowledgements}

I thank Steve Meacham, Jeff Weiss, and Bill Young for many helpful discussions during the summer. I also thank Bill, the steering committee, and the other participants for making this a very enjoyable summer. I gratefully acknowledge contributions to my travel expenses from the Association of Commonwealth Universities and DAMTP, University of Cambridge. Finally, special thanks to hurricane Bob for testing my sketching ability. 


\section{References}

Aref H. 1979 Motion of three vortices. Phys. Fluids. 22, 393-400.

Benzi R., Patarnello S. \& Santangelo P. (1988) Self similar, coherent structures in 2D, decaying turbulence. J. Phys. A: Math. Gen. 21, 1221-1237.

Benzi R., Briscolini M., Colella M. \& Santangelo P. 1991 A simple point vortex model for two-dimensional decaying turbulence. (preprint)

Carnevale G.F., McWilliams J.C., Pomeau Y., Weiss J.B. \& Young W.R. 1991 Evolution of vortex statistics in two-dimensional turbulence, Phys. Rev. Lett. 66, 2735-2737.

Couder Y. \& Basdavent C. 1986 Experiments and numerical studies of vortex couples in two-dimensional flows. J. Fluid Mech. 173, 225-251.

Dritschel D.G. 1989 Contour dynamics and contour surgery: Numerical algorithms for extended, high-resolution modelling of vortex dynamics in two-dimensional, inviscid, incompressible flows. Comp. Phys. Rep.. 10, 77-146.

Dritschel D.G. 1990 The stability of elliptical vortices in an external straining flow. J. Fluid Mech. 210, 223-262

Dritschel D.G. \& Legras B. 1991. Vortex Stripping (in preparation).

Dritschel D.G. \& Waugh D.W. 1991 A simple model for vortex growth in two-dimensional turbulence (in preparation).

Eckhardt B. \& Aref H. 1988 Integrable and chaotic motion fo four vortices, II. Collision dynamics of vortex pairs Phil. Trans. Roy. Soc. Lond. A. 326 655-696.

Kida S. 1981 Motion of an elliptic vortex in a uniform shear flow. J. Phys. Soc. Japan 50, 3517-3520.

Larichev V.D. \& Reznik G.M. 1983 On collisions between two dimensional solitary Rossby waves. Oceanology 23, 545.

McWilliams, J.C. 1990 The vortices of two-dimensional turbulence. J. Fluid Mech. 219, 361-385.

McWilliams, J.C. \& Weiss J.B 1991 The search for simplified models of two-dimensional, structured turbulence. Proceedings of IMA workshop on nonlinear phenomena in atmospheric and oceanic sciences.

Meacham S.P., Flierl G.R. \& Send U. 1990. Vortices in shear. Dyn. Atmos. Oceans 14, 333-386.

Melander M.V., McWilliams J.C. \& Zabusky N.J. 1987a Axisymmetric vortex merger in two-dimensions: which vortex is 'victorious'? Phys. Fluids 30 2610-2612.

Melander M.V., Zabusky N.J. \& McWilliams J.C. 1988 Symmetric vortex merger in two dimensions: causes and conditions. J. Fluid Mech. 195 303-340.

Polvani L.M. \& Carton X. 1990 The tripole: a new coherent structure of incompressible two-dimensional flows. Geophys. Astrophys. Fluid Dyn. 51, 87-102.

Santangelo, R., Benzi, R. \& Legras, B. 1989 The generation of vortices in high-resolution, two-dimensional decaying turbulence and the influence of initial conditions on the breaking of self-similarity. Phys. Fluids A 1, 1027-1034.

Waugh D.W. 1991. The efficiency of symmetric vortex merger. J. Fluid Mech. (submitted), and this volume. 
Wu H.M., Overman E.A. \& Zabusky N.J. 1984 Steady-state solutions of the Euler equation in two dimensions. Rotating and translating V-states with limiting cases. I. Numerical algorithm and results. J. Comp. Phys. 53, 42-71.

Yasuda T. \& Flierl G.R. 1991 Two dimensional asymmetric vortex merger : Part 1. Contour dynamics experiments. (preprint)

Zabusky N.J., Hughes M.H: \& Roberts K.V. 1979 Contour dynamics of the Euler equations in two-dimensions. J. Comp. Phys. 30, 96-106. 


\title{
22. Primitive equation models of Gulf Stream mean- ders
}

\author{
Richard Williams \\ Scripps Institution of Oceanography, La Jolla, Ca 92093-0230
}

\subsection{Introduction}

The meandering of strong oceanic jets, such as the Gulf Stream, is a long-standing oceanographic problem. Much theoretical work on this problem has been done within the framework of quasigeostrophic theory, which has certain features that are not realistic for the processes under study. The fundamental difficulty is that quasigeostrophic theory linearizes the governing equations about an ambient stratification by assuming that the Rossby number of the jet is small and that changes in depth of any particular layer of fluid are small.

There is considerable observational evidence that these conditions do not hold in a strong current such as the Gulf Stream (c.f. Hall, 1986; Bower, 1989), and the purpose of this work is to develop a theoretical model of the meandering of a strong current that includes effects neglected in quasigeostrophic theory. We have chosen to study the development of meanders rather than the instability processes that lead to the formation of the meanders. Thus we will use a basic flow that is stable and will study the properties of both small and large amplitude waves on that flow.

The basic flow we have chosen to study is a jet at a potential vorticity front in a layer of fluid overriding an infinitely deep stagnant layer of slightly greater density. This configuration has the advantage that it is reasonably simple to approach analytically, and at the same time is a reasonable approximation to the actual conditions in the Gulf Stream. The quasigeostophic version of this system has been studied using contour dynamics by Pratt and Stern (1986). Here we explore the behavior of waves on a potential vorticity front when the restrictions of the quasigeostrophic approximation are loosened.

In $\S 2$ we state the governing equations, perform a suitable nondimensionalization, give the form of the basic jet and discuss the scale of observed meanders. In $\S 3$ we explore the dynamics of linear waves on the jet, deriving expressions for the dispersion of linear waves. In $\S 4$ the analysis is extended to weakly nonlinear waves and in $\S 5$ a method of finding large amplitude waves using contour dynamics is outlined. 


\subsection{Governing Equations}

The problem to be considered is sketched in figure 1. There is a geostrophic jet at the interface between two regions of different constant potential vorticity but equal density $\rho$ which overrides stagnant fluid of infinite depth and density $\rho+\Delta \rho$. The rotation rate is taken to be constant. The governing equations for this $1 \frac{1}{2}$ layer flow are

$$
\begin{aligned}
u_{t}+u u_{x}+v u_{y}-f v & =-g^{\prime} h_{x} \\
v_{t}+u v_{x}+v v_{y}+f u & =-g^{\prime} h_{y} \\
h_{t}+(u h)_{x}+(v h)_{y} & =0
\end{aligned}
$$

which can be combined to find that potential vorticity

$$
q=\frac{v_{x}-u_{y}+f}{h}
$$

is conserved. Here $u$ and $v$ are the $x$ and $y$ velocities, $h$ is the upper layer depth, $f$ is the Coriolis parameter and g' is the reduced gravity. The upper layer depth goes to $H_{1}$ as $y \rightarrow \infty$ and $H_{2}$ as $y \rightarrow \infty$; subscripts 1 and 2 will be used to refer to the two regions of different potential vorticity throughout but, as in (1-2) above, will be omitted when not needed.

We scale h with $H=\left(H_{1}+H_{2}\right) / 2$, u with $u_{0}, \mathrm{x}$ and $\mathrm{y}$ with the deformation radius $R_{d}=$ $\sqrt{g^{\prime} H} / f$ and define $Q_{i}=H / H_{i}$ and the Rossby number $\epsilon=u_{0} / f R_{d}$. It is convenient to define $h_{i}=Q_{i}^{-1}+\epsilon \psi_{i}$ in the nondimensional system. Notice also that $Q_{1}^{-1}+Q_{2}^{-1}=2$. The nondimensional momentum, continuity and potential vorticity equations are then

$$
\begin{aligned}
\epsilon\left(u_{t}+u u_{x}+v u_{y}\right)-v & =-\psi_{x} \\
\epsilon\left(v_{t}+u v_{x}+v v_{y}\right)+u & =-\psi_{y} \\
\epsilon\left(\psi_{t}+(u \psi)_{x}+(v \psi)_{y}\right)+Q^{-1}\left(u_{x}+v_{y}\right) & =0 \\
v_{x}-u_{y} & =Q \psi
\end{aligned}
$$

and the boundary conditions at infinity are that $u, v, \psi \rightarrow 0$. The boundary conditions at the interface between the two regions are crucial in determining the behavior of the interface. We will specify a basic state in which the velocity is continuous across the interface, so writing the position of the interface as $y=\eta(x)$, we will require that pressure and both velocity components remain continuous across the interface.

In quasigeostrophic approximation (c.f. Pedlosky, 1979), the velocity and layer depth are expanded in powers of Rossby number and the first order equations give a solvability condition on the lowest order fields, which are simple in geostrophic balance. If the expansion of $(1-2)$, written as $u=u^{(0)}+\epsilon u^{(1)}+\ldots$ etc., is carried to higher order, the order $\mathrm{n}+1$ equations give a solvability condition on the order $n$ variables:

$$
\frac{\partial}{\partial t} q^{(n)}+\sum_{i=0}^{n} \nabla \cdot\left(\mathbf{u}^{(i)} q^{(j)}\right)=0 ; \quad j=n-i
$$




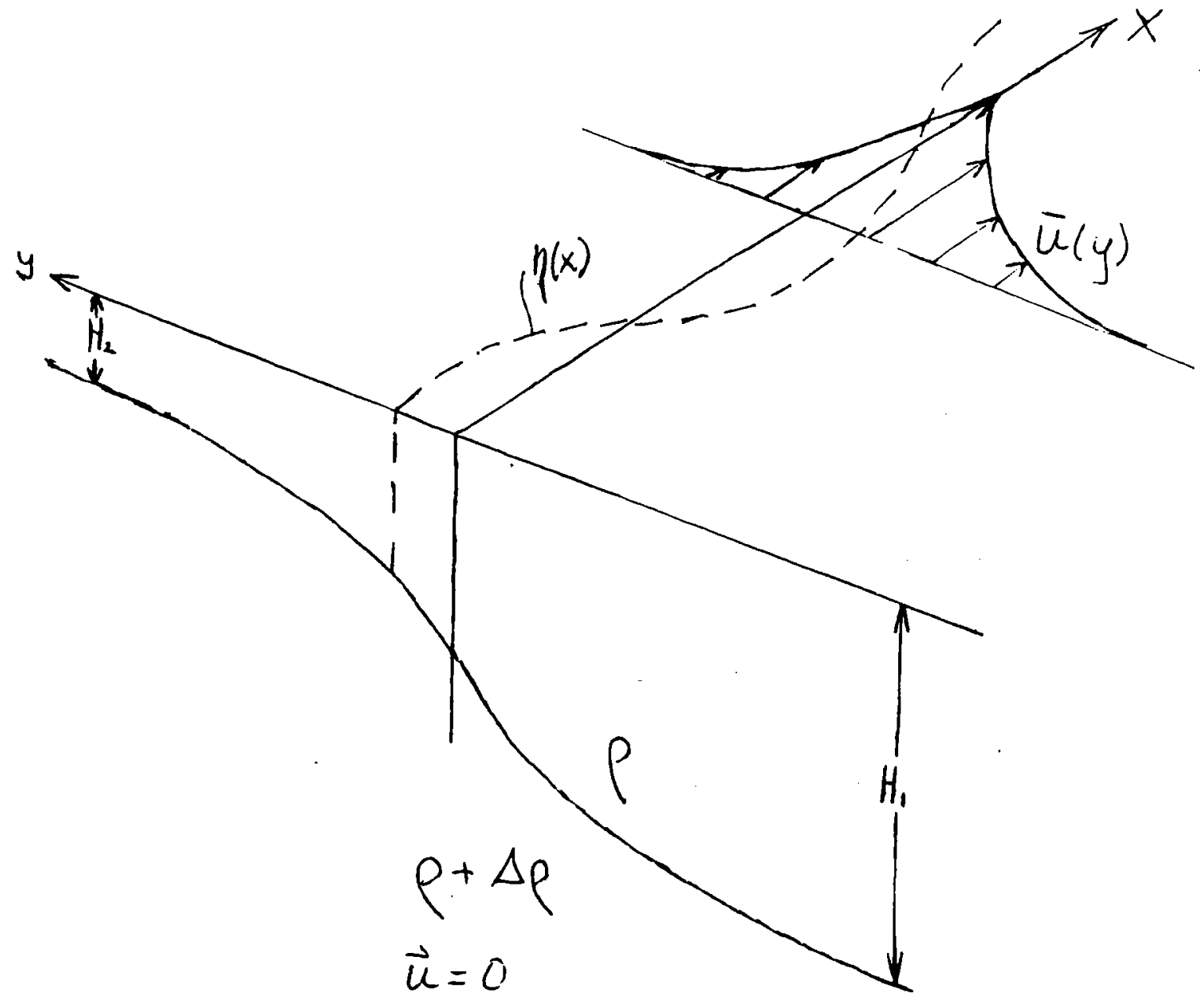

Figure 22.1. A meandering potential vorticity front.

where $q^{(i)}=v_{x}^{(i)}-u_{y}^{(i)}-Q \psi^{(i)}$.

This gives, to $O(\epsilon)$,

$$
\frac{D}{D t}\left(\nabla^{2} \psi^{(0)}-Q \psi^{(0)}\right)=0
$$

where $\frac{D}{D t}=\frac{\partial}{\partial t}+\frac{\partial\left(\psi^{(0)}, \bullet\right)}{\partial(x, y)}$ and the velocity field is given by the $O(1)$ equations: $u^{(0)}=-\psi_{y}^{(0)}$, $v^{(0)}=\psi_{x}^{(0)}$. 
The solvability condition at $O\left(\epsilon^{2}\right)$ is,

$$
\frac{D}{D t}\left[\nabla^{2} \psi^{(1)}-Q \psi^{(1)}+2\left(\psi_{x y}^{(0)}{ }^{2}-\psi_{x x}^{(0)} \psi_{y y}^{(0)}\right)\right]=0
$$

while the $O(\epsilon)$ velocities can be found from

$$
u^{(1)}=-\psi_{y}^{(1)}-\frac{D v^{(0)}}{D t}, \quad v^{(1)}=\psi_{x}^{(1)}+\frac{D u^{(0)}}{D t}
$$

In what follows, there is assumed to be a steady basic flow in which the interface between the two regions is at $\mathrm{y}=\mathbf{0}$ and there is a purely zonal velocity field that is continuous at $\mathrm{y}$ $=0$ and is given by

$$
\begin{gathered}
\bar{u}_{i}=-\bar{\psi}_{i y}=e^{ \pm \alpha_{i} y}, \quad \bar{v}_{i}=0 \\
\bar{\psi}_{1}=-\frac{1}{\alpha_{1}} e^{\alpha_{1} y}, \quad \bar{\psi}_{2}=\frac{1}{\alpha_{2}} e^{-\alpha_{2} y}
\end{gathered}
$$

where $\alpha_{i}=\sqrt{Q_{i}}, \mathrm{i}=1,2$ are the nondimensional deformation radii in each region. Since the deformation radius is smaller in the region $y>0$ than in the region $y<0$, the velocity profile is asymmetric and falls off more rapidly as $y \rightarrow \infty$ than as $y \rightarrow-\infty$. At $y=0$, pressure continuity requires that there be no step in layer depth, or $\epsilon\left(\psi_{1}-\psi_{2}\right)=Q_{2}^{-1}-Q_{1}^{-1}$. This relates the Rossby number to the deformation radii on either side of the interface:

$$
\epsilon=\frac{1}{\alpha_{1}}-\frac{1}{\alpha_{2}}
$$

and, since $\alpha_{1}^{-2}+\alpha_{2}^{-2}=2$, we will use $\epsilon$ is the only free parameter.

Measurements in the Gulf Stream give $\epsilon=.3-.5$ and a deformation radius of $45 \mathrm{~km}$ (Bower, 1989). Meander scales, observed through float tracking (Bower, 1989) or from satellite images of sea surface temperature (Legekis, 1978) give a range of meander wavelengths and amplitudes, typical scales are $\lambda \approx 300-600 \mathrm{~km}$, amplitude $\approx 75-150 \mathrm{~km}$. Defining the wavenumber $k=2 \pi R_{d} / \lambda, k \approx .5-1$. Thus we must work towards a theory that models finite amplitude, finite wavelength meanders. 


\subsection{Linear Waves}

We look at the behavior of linear waves by substituting

$$
\begin{aligned}
u(x, y, t) & =\bar{u}(y)+u(y) e^{i k(x-c t)} \\
v(x, y, t) & =v(y) e^{i k(x-c t)} \\
\psi(x, y, t) & =\bar{\psi}(y)+\psi(y) e^{i k(x-c t)}
\end{aligned}
$$

into (3a,b,d) and linearizing. By also taking $\hat{v}=v / i k$, we obtain

$$
\begin{aligned}
\epsilon(\bar{u}-c)+\epsilon \hat{v} \bar{u}_{y}-\hat{v} & =-\psi \\
-k^{2} \epsilon(\bar{u}-c) \hat{v}+u & =-\psi_{y} \\
-k^{2} \hat{v}-u_{y} & =Q \psi .
\end{aligned}
$$

The boundary conditions require that the pressure and normal velocity are continuous at $y=0$, that is $[\psi]=0$ and $[\hat{v}]=0$ at $\mathbf{y}=0$, where the square bracket denotes the jump in value from region 1 to region 2 .

Expanding in Rossby number

$$
\begin{aligned}
& u=u^{(0)}+\epsilon u^{(1)}+\ldots \\
& \hat{v}=\hat{v}^{(0)}+\epsilon \hat{v}^{(1)}+\ldots \\
& \psi=\psi^{(0)}+\epsilon \psi^{(1)}+\ldots \\
& c=c^{(0)}+\epsilon c^{(1)}+\ldots
\end{aligned}
$$

the lowest order terms are combined to give

$$
\psi_{y y}^{(0)}-\left(k^{2}+\alpha^{2}\right) \psi^{(0)}=0
$$

and writing $\beta^{2}=k^{2}+\alpha^{2}$,

$$
\psi_{i}^{(0)}=A e^{ \pm \beta y} .
$$

At this order, $\hat{v}=\psi^{(0)}$ so the boundary condition on the normal velocity is trivially satisfied. At the next order, (8a) gives

$$
\hat{v}^{(1)}=u^{(0)}\left(\bar{u}-c^{(0)}\right)+\hat{v}^{(0)} \bar{u}_{y}+\psi^{(1)}
$$

so the condition $\left[\hat{v}^{(1)}\right]=0$ gives

$$
\left[u^{(0)}\right]\left(1-c^{(0)}\right)+\hat{v}^{(0)}\left[\bar{u}_{y}\right]=0
$$

and using the results from the lowest order we find the dispersion relation

$$
c^{(0)}=1-\frac{\alpha_{1}+\alpha_{2}}{\beta_{1}+\beta_{2}} .
$$


This lowest order solution has quasigeostrophic dynamics in both regions but the deformation radii in the two regions are not equal. This results in the dispersion relation (9) rather than the quasigeostrophic dispersion relation

$$
c=1-\alpha / \beta
$$

found by Pratt and Stern (1986).

Continuing the analysis at this order, the terms of $O(\epsilon)$ in $(8 \mathrm{a}-\mathrm{c})$ are combined to give

$$
\psi_{y y}^{(1)}-\beta^{2} \psi^{(1)}=2 k^{2} \bar{u}_{y} \psi^{(0)}
$$

The solution that satisfies the boundary condition $[\psi]=0$ at $\mathrm{y}=0$ is

$$
\psi^{(1)}=\frac{2 A k^{2}}{\alpha+2 \beta}\left(e^{(\alpha+\beta) y}-e^{\beta y}\right)
$$

To find the $O(\epsilon)$ correction to the dispersion relation, we must consider the $\mathrm{x}$-momentum equation at the next order,

$$
\hat{v}^{(2)}=\psi^{(2)}+u^{(1)}\left(\bar{u}-c^{(0)}\right)-u^{(0)} c^{(1)}+\hat{v}^{(1)} \bar{u}_{y} .
$$

The boundary condition $\left[\hat{v}^{(2)}\right]=0$ at $y=0$ becomes

$$
\left[u^{(1)}\right]\left(1-c^{(0)}\right)+\hat{v}^{(1)}\left[\bar{u}_{y}\right]-[u(0)] c^{(1)}=0
$$

and the resulting correction to the dispersion relation is

$$
c^{(1)}=\frac{\left(\alpha_{1} \beta_{2}-\alpha_{2} \beta_{1}\right)\left(\alpha_{1}+\alpha_{2}\right)}{\left(\beta_{1}+\beta_{2}\right)^{2}}\left[\frac{4 k^{2}}{\left(\alpha_{1}+2 \beta_{1}\right)\left(\alpha_{2}+2 \beta_{2}\right)}-1\right]
$$

The dispersion relation for linear waves has also been found exactly by solving (8a-c) numerically using a shooting technique. The exact dispersion relation is plotted for various Rossby numbers in figure 2 , with $\epsilon=0$ corresponding to quasigeostrophic theory (10). The exact relation and the relations given by (9), (10) and (11) are plotted for $\epsilon=.4$ in figure 3. The trend in all forms of the dispersion relation is the same, with wave speed always in the direction of the mean current and increasing as wavenumber increases. The first order correction to quasigeostrophic theory, in which the dynamics are quasigeostrophic but with different deformation radii in each region, over-corrects the dispersion relation and the exact solution falls between the quasigeostrophic result and the corrected result. Including the $O(\epsilon)$ effect in the dispersion relation in turn over-corrects the lowest order result and the dispersion curve is moved close to the quasigeostrophic result. 


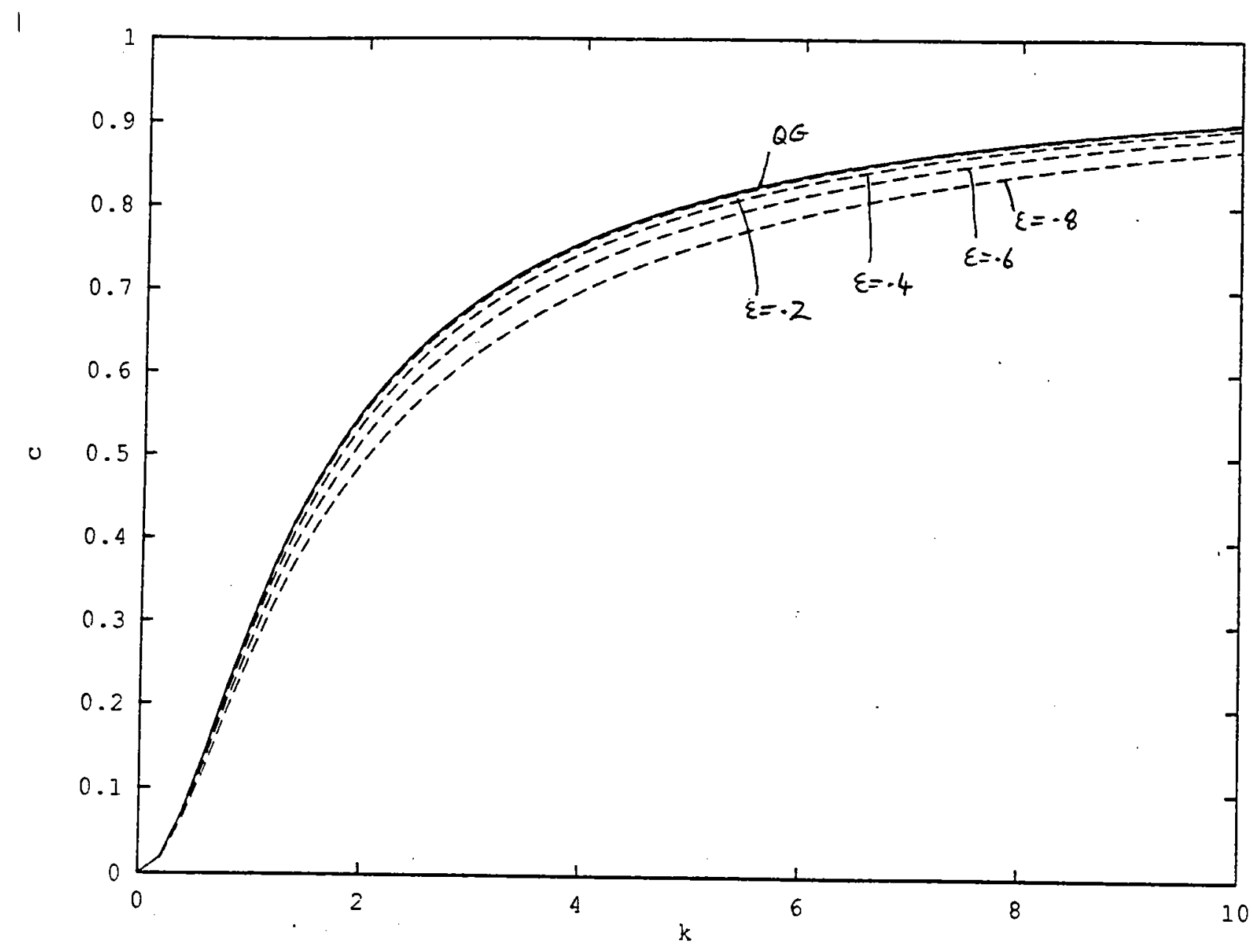

Figure 22.2. Dispersion relation $c(k)$ for $\epsilon=0, .2, .4, .6, .8$.

\subsection{Weakly Nonlinear Waves}

We are interested in the behavior of finite amplitude meanders and here we approach this problem by studying the shape of the interface between the two regions for small but finite amplitude waves in the simple case of quasigeostrophic dynamics with two deformation radii.

We suppose that $\psi$ consists of a background flow plus a wave field that satisfies (4), the first order equation:

$$
\psi=(\bar{A}+A) e^{\alpha y}+\left(B+B^{\prime}\right) e^{\beta y} \cos k(x-c t)+C e^{\gamma y} \cos 2 k(x-c t) .
$$

Here $\gamma^{2}=4 k^{2}+\alpha^{2}$. The equations for regions 1 and 2 are found by taking $\alpha=\alpha_{1},-\alpha_{2}$ respectively and equivalently for $\beta$ and $\gamma$. The amplitude scale is $\delta$, and $B \sim O(\delta), A \sim$ $O\left(\delta^{2}\right), C \sim O\left(\delta^{2}\right)$ and $B^{\prime} \sim O\left(\delta^{3}\right)$ while the background flow (7) gives $\bar{A}=-1 / \alpha$.

If the position of the interface $\eta$ is of the form

$$
\eta=\eta^{(1)} \cos k(x-c t)+\eta^{(2)} \cos 2 k(x-c t)
$$




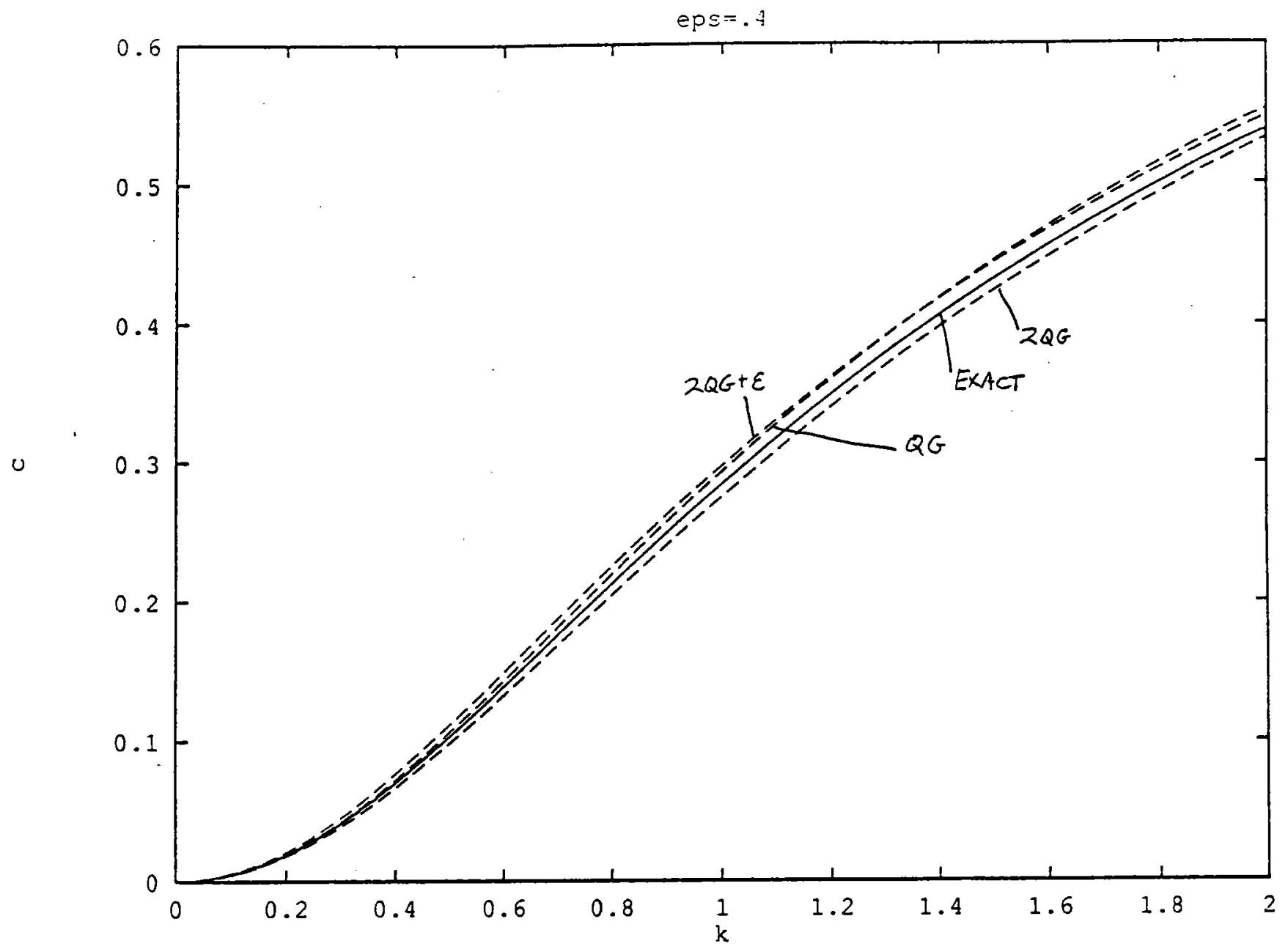

Figure 22.3. Dispersion relations at $\epsilon=.4$ for exact theory, quasigeostrophy (QG), quasigeostrophy with 2 deformation radii (QG2), and with $O(\epsilon)$ correction $(Q G 2+\epsilon)$.

where $\eta^{(1)} \sim O(\delta)$ and $\eta^{(2)} \sim O\left(\delta^{2}\right)$, the problem is one of applying the various boundary conditions on $\psi$ at $y=\eta$. These are that $[\psi]=0,\left[\psi_{y}\right]=0$ and, since the velocity field is geostrophic, that $\psi+c \eta$ is constant.

Since $\eta$ is assumed to be small, the y-dependent terms in (12) are expanded as Taylor series about $\mathrm{y}=0$. The resulting expression for $\psi(\eta)$, grouped by wavenumber and by amplitude within each wavenumber, is

$$
\begin{gathered}
\psi(\eta)=\left(A+\frac{1}{4} \bar{A} \alpha^{2} \eta^{(1)^{2}}+\frac{1}{2} B \beta \eta^{(1)}\right)+ \\
\cos k(x-c t)\left(\bar{A} \alpha \eta^{(1)}+B+\frac{1}{8} \bar{A} \alpha^{3} \eta^{(1)^{3}}+\frac{1}{2} \bar{A} \alpha^{2} \eta^{(1)} \eta^{(2)}+A \alpha \eta^{(1)}\right. \\
\left.\quad+\frac{3}{8} B \beta \eta^{(1)^{2}}+\frac{1}{2} B \beta \eta^{(2)}+B^{\prime}+\frac{1}{2} C \gamma \eta^{(1)}\right)+ \\
\cos 2 k(x-c t)\left(\bar{A} \alpha \eta^{(2)}+\frac{1}{4} \bar{A} \alpha^{2} \eta^{(1)^{2}}+\frac{1}{2} B \beta \eta^{(1)}+C\right)
\end{gathered}
$$


and similarly for $\psi_{y}(\eta)$

$$
\begin{gathered}
\psi_{y}(\eta)=\left(A \alpha+\frac{1}{4} \bar{A} \alpha^{3} \eta^{(1)^{2}}+\frac{1}{2} B \beta^{2} \eta^{(1)}\right)+ \\
\cos k(x-c t)\left(\bar{A} \alpha^{2} \eta^{(1)}+B \beta+\frac{1}{8} \bar{A} \alpha^{4} \eta^{(1)^{3}}+\frac{1}{2} \bar{A} \alpha^{3} \eta^{(1)} \eta^{(2)}+A \alpha^{2} \eta^{(1)}\right. \\
\left.\quad+\frac{3}{8} B \beta^{2} \eta^{(1)^{2}}+\frac{1}{2} B \beta^{2} \eta^{(2)}+B^{\prime} \beta+\frac{1}{2} C \gamma^{2} \eta^{(1)}\right)+ \\
\cos 2 k(x-c t)\left(\bar{A} \alpha^{2} \eta^{(2)}+\frac{1}{4} \bar{A} \alpha^{3} \eta^{(1)^{2}}+\frac{1}{2} B \beta^{2} \eta^{(1)}+C \gamma\right)
\end{gathered}
$$

Applying the boundary conditions $[\psi]$ and $\left[\psi_{y}\right]$ at $y=\eta$ to (14) and (15) allows the various amplitudes in (12) to be found in terms of the amplitudes of the interfacial displacements.

To $O(\delta)$ at wavenumber k:

$$
B_{1}=B_{2}=B=\eta^{(1)} \frac{\alpha_{1}+\alpha_{2}}{\beta_{1}+\beta_{2}}
$$

To $O\left(\delta^{2}\right)$ at wavenumber 0 :

$$
A_{1}=\eta^{(1)^{2}}\left(-\frac{1}{4} \alpha_{2}+\Delta\right), \quad A_{2}=\eta^{(1)^{2}}\left(\frac{1}{4} \alpha_{1}+\Delta\right)
$$

where $\Delta=\frac{1}{4}\left(\alpha_{1}-\alpha_{2}\right)+\frac{1}{2}\left(\beta_{1}-\beta_{2}\right)$.

To $O\left(\delta^{2}\right)$ at wavenumber $2 \mathrm{k}$ :

$$
\begin{gathered}
C_{1}=\left(\frac{\alpha_{1}+\alpha_{2}}{\gamma_{1}+\gamma_{2}}\right)\left[\eta^{(2)}+\eta^{(1)^{2}}\left(-\frac{1}{4} \gamma_{2}+\Delta\right)\right], \\
C_{2}=\left(\frac{\alpha_{1}+\alpha_{2}}{\gamma_{1}+\gamma_{2}}\right)\left[\eta^{(2)}+\eta^{(1)^{2}}\left(\frac{1}{4} \gamma_{1}+\Delta\right)\right] .
\end{gathered}
$$

To $O\left(\delta^{3}\right)$ at wavenumber $\mathrm{k}$ :

$$
B_{1}^{\prime}=\frac{f+\beta_{2} g}{\beta_{1}+\beta_{2}}, \quad B_{2}^{\prime}=\frac{f+\beta_{1} g}{\beta_{1}+\beta_{2}}
$$

where

$$
\begin{gathered}
f=\left(\alpha_{1}+\alpha_{2}\right)\left[\eta^{(1)^{3}}\left(\Delta+\frac{1}{8}\left(\beta_{1}-\beta_{2}\right)\right)+\frac{1}{2} \eta^{(1)} \eta^{(2)}\right] \\
g=\frac{1}{8}\left(\alpha_{1}^{3}+\alpha_{2}^{3}\right) \eta^{(1)^{3}}+\eta^{(1)}\left(A_{2} \alpha_{2}^{2}-A_{1} \alpha_{1}^{2}\right)+\frac{1}{2} \eta^{(1)}\left(C_{2} \gamma_{2}^{2}-C_{1} \gamma_{1}^{2}\right)- \\
\frac{1}{2} \eta^{(1)} \eta^{(2)}\left(\alpha_{2}^{2}-\alpha_{1}^{2}\right)+\frac{1}{2} B \eta^{(2)}\left(\beta_{2}^{2}-\beta_{1}^{2}\right)-\frac{3}{8} B \eta^{(1)^{2}}\left(\beta_{1}^{3}+\beta_{2}^{3}\right)
\end{gathered}
$$


The condition that the interface is a streamline is now applied in a similar manner. The phase speed is expanded in amplitude, $c=c^{(0)}+c^{(2)}$ where $c^{(2)} \sim O\left(\delta^{2}\right)$. Again grouping by wavenumber and amplitude, and setting the contribution to $\psi+c \eta$ at non-zero wavenumber equal to zero, we obtain a sequence of relations.

To $O(\delta)$ at wavenumber $\mathrm{k}$ we find

$$
c^{(0)}=1-\frac{\alpha_{1}+\alpha_{2}}{\beta_{1}+\beta_{2}}
$$

as was found in the previous section (9).

To $O\left(\delta^{2}\right)$ at wavenumber $2 \mathrm{k}$ we find

$$
\eta^{(2)}=\eta^{(1)^{2}}\left[\frac{\frac{1}{2} \beta\left(c^{(0)}-1\right)+\frac{1}{4} \alpha_{1}-\left(\frac{\alpha_{1}+\alpha_{2}}{\gamma_{1}+\gamma_{2}}\right)\left(-\frac{1}{4} \gamma_{2}+\Delta\right)}{c^{(0)}-1+\frac{\alpha_{1}+\alpha_{2}}{\gamma_{1}+\gamma_{2}}}\right]
$$

To $O\left(\delta^{3}\right)$ at wavenumber $\mathrm{k}$ we find

$$
c^{(2)}=\frac{1}{8} \alpha_{1}^{2} \eta^{(1)^{2}}+\frac{1}{2} \alpha_{1} \eta^{(2)}-A_{1} \alpha_{1}-B\left(\frac{3}{8} \beta_{1}^{2} \eta^{(1)^{2}}+\frac{1}{2} \beta_{1} \eta^{(2)}\right)-\frac{B^{\prime}}{\eta^{(1)}}-\frac{1}{2} C_{1} \gamma_{1} .
$$

These results can be applied to the case of a quasigeostrophic jet by setting $\alpha_{1}=\alpha_{2}=\alpha$, $\beta_{1}=\beta_{2}=\beta$ and $\gamma_{1}=\gamma_{2}=\gamma$. We find

$$
\begin{gathered}
B=\eta^{(1)} \frac{\alpha}{\beta} \\
A_{1}=C_{1}=-\eta^{(1)^{2}} \frac{1}{4} \alpha \\
A_{2}=C_{2}=\eta^{(1)^{2}} \frac{1}{4} \alpha \\
\eta^{(2)}=0 \\
B_{1}^{\prime}=B_{2}^{\prime}=\eta^{(1)^{3}} \frac{1}{8} \alpha, \\
c=c^{(0)}+c^{(2)}=1-\frac{\alpha}{\beta}+\eta^{(1)^{2}}\left(\frac{3}{8} \alpha^{2}-\frac{1}{2} \alpha \beta+\frac{1}{8} \alpha \gamma\right) .
\end{gathered}
$$

The strong symmetry of the quasigeostrophic jet about $y=0$ causes there to be no wavenumber $2 \mathrm{k}$ component in the interface position.

The problem considered above is of a jet that is quasigeostrophic on either side of the interface but with different deformation radii in each region. While we have not considered the nonquasigeostrophic effects discussed for linear waves, we still see that the interface location is asymmetric. Writing (16) as $\eta^{(2)}=\eta^{(1)^{2}} F(k, \epsilon)$, gives the $F(k, .4)$ shown in figure 4; we see 
that the asymmetry is small at the wavenumbers of interest. Observed meanders have large amplitudes and the asymmetry in the weakly nonlinear theory suggests that asymmetry could occur in the large amplitude meanders of ocean currents. The amplitude dependent correction to the phase speed (17) can be written $c^{(2)}=\eta^{(1)^{2}} G(k, \epsilon)$, and $G(k, \epsilon)$ is plotted in figure 5 for $\epsilon=0$ and $\epsilon=.4$. The phase speed decreases with wave amplitude and the effect is larger at larger Rossby numbers, since the effects of finite Rossby number and finite amplitude both decrease the phase speed.

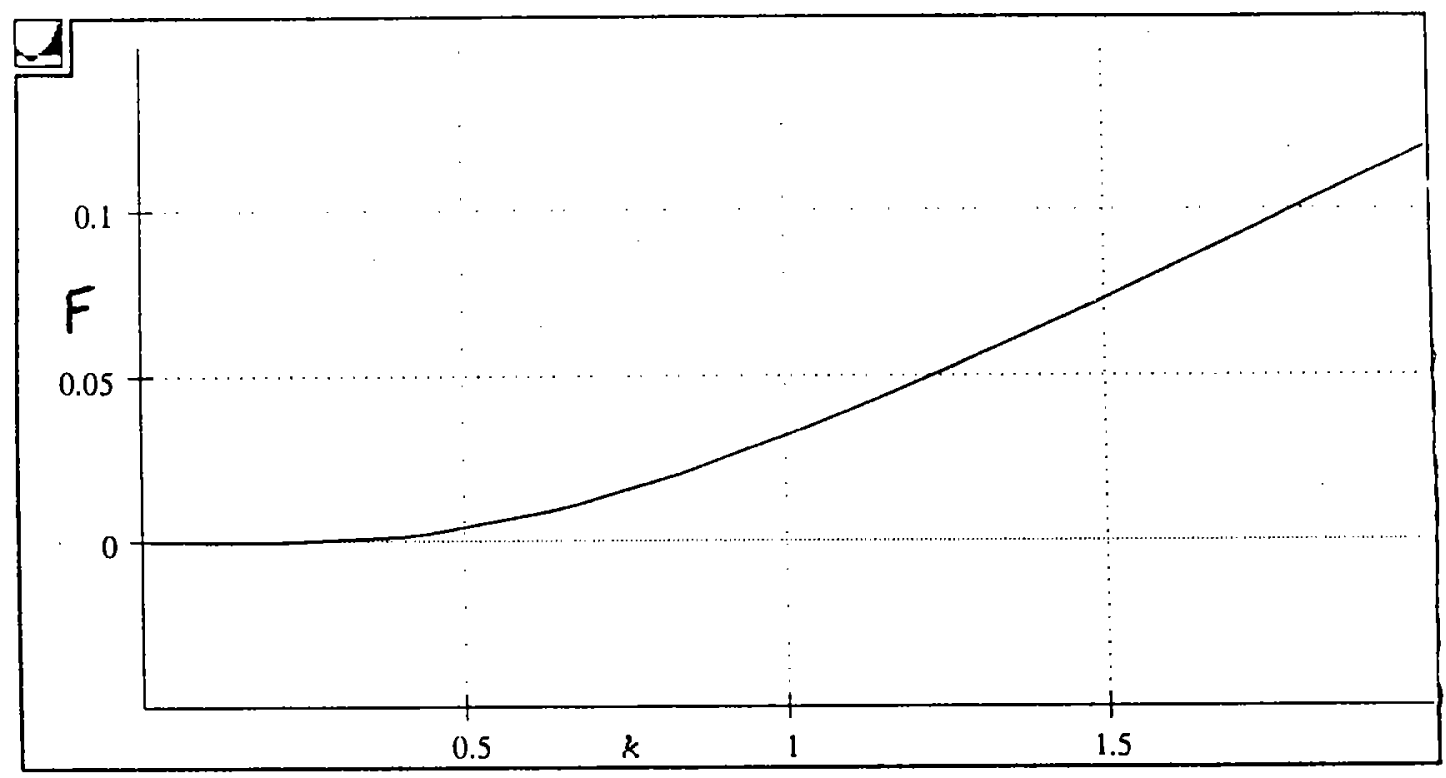

$\varepsilon=0.4$

Figure 22.4. $F(k, .4)$ - amplitude of wavenumber $2 \mathrm{k}$ contribution to the interface displacement.

This approach can be extended to include the effects of finite Rossby number considered in the previous section for linear waves. We again consider a lowest order solution of the form

$$
\psi^{(0)}=\bar{\psi}+\psi=A e^{\alpha y}+B e^{\beta y} \cos k(x-c t)+C e^{\gamma y} \cos 2 k(x-c t)
$$

which satisfies the lowest order equation (4). The coefficients are $A=\bar{A}+A^{(2)}+\ldots, B=$ $B^{(1)}+B^{(2)}+\ldots, C=C^{(2)}+\ldots$ where $A^{(2)} \approx O\left(\delta^{2}\right)$ etc. The equation at the next order (5) becomes, retaining terms to $O\left(\delta^{2}\right)$,

$$
\nabla^{2} \psi^{(1)}-\alpha^{2} \psi^{(1)}=-2 k^{2} B^{2} \beta^{2} e^{2 \beta y}+2 k^{2} B \alpha e^{(\alpha+\beta) y} \cos k x+8 k^{2} C \alpha e^{(\alpha+\gamma) y} \cos 2 k x .
$$

Writing

$$
\psi^{(1)}=\psi^{(1,0)}(y)+\psi^{(1,1)} \cos (k x)+\psi^{(1,2)} \cos (2 k x)
$$



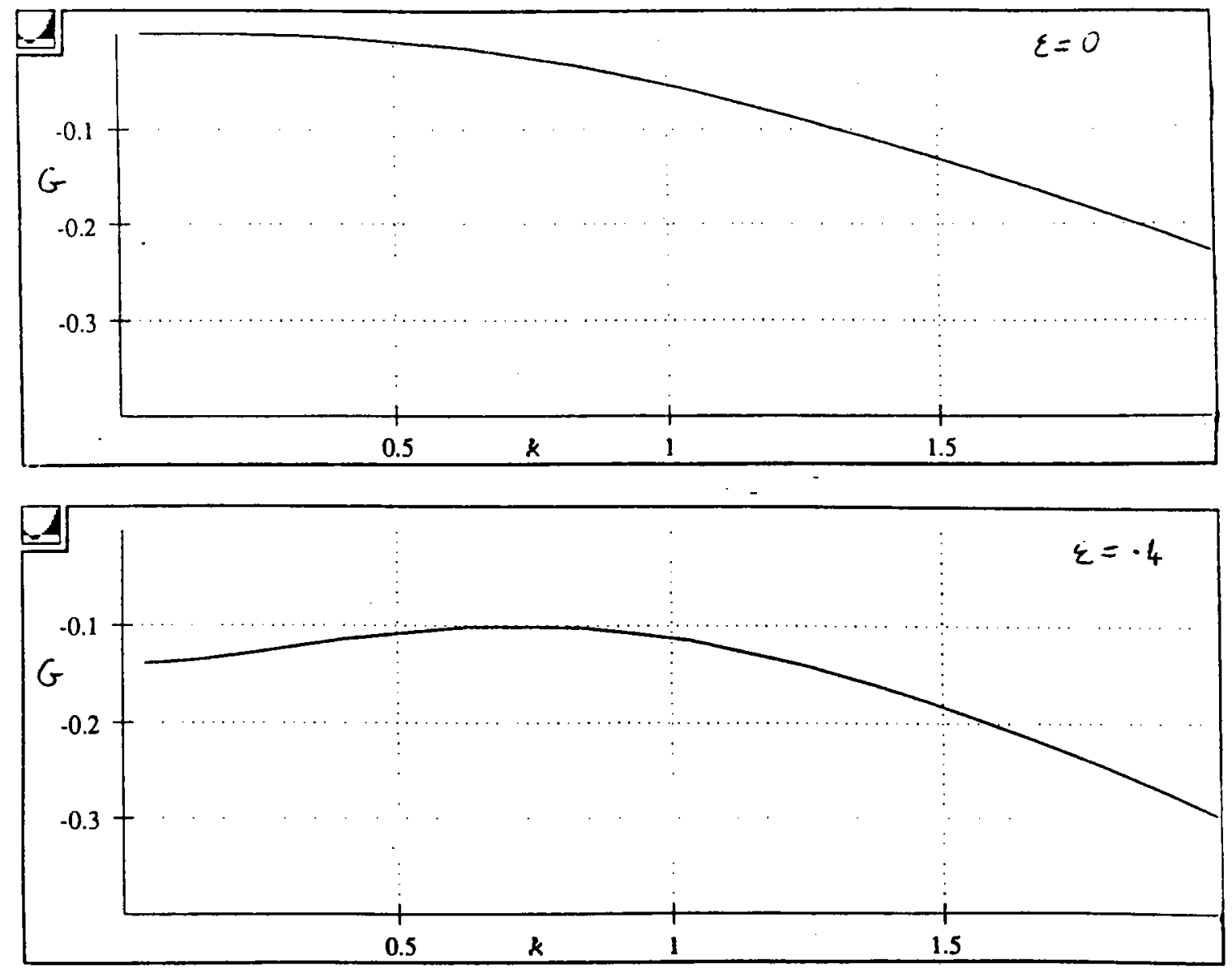

Figure 22.5. $G(k, \epsilon)$ - correction to dispersion relation due to finite amplitude effects.

grouping (18) by wavenumber and solving we find

$$
\begin{aligned}
& \psi^{(1,0)}=-\frac{2 k^{2} B^{2} \beta^{2}}{4 \beta^{2}-\alpha^{2}}\left(e^{2 \beta y}-e^{\alpha y}\right), \\
& \psi^{(1,1)}=\frac{2 k^{2} B}{2 \beta+\alpha}\left(e^{(\alpha+\beta) y}-e^{\beta y}\right), \\
& \psi^{(1,2)}=\frac{8 k^{2} C}{2 \gamma+\alpha}\left(e^{(\alpha+\gamma) y}-e^{\gamma y}\right) .
\end{aligned}
$$

We then combine the $O(1)$ and $O(\epsilon)$ expressions and write

$$
\begin{aligned}
\psi=\psi^{(0)}+\psi^{(1)} & =A e^{\alpha y}+D B^{2}\left(e^{2 \beta y}-e^{\alpha y}\right) \\
& +B\left[e^{\beta y}+E\left(e^{(\alpha+\beta) y}-e^{\beta y}\right)\right] \cos k(x-c t) \\
& +C\left[e^{\gamma y}+F\left(e^{(\alpha+\gamma) y}-e^{\gamma y}\right)\right] \cos 2 k(x-c t)
\end{aligned}
$$

where

$$
D=-\epsilon \frac{2 k^{2} \beta^{2}}{4 \beta^{2}-\alpha^{2}}, \quad E=\epsilon \frac{2 k^{2}}{2 \beta+\alpha}, \quad F=\epsilon \frac{8 k^{2} C}{2 \gamma+\alpha}
$$


As before, we take the position of the interface to be of the form (13), expand the $y$ dependent term in Taylor series about $y=0$, evaluate $\psi$ and $\psi_{y}$ at $y=\eta$ and group terms by wavenumber.

In order to proceed with applying the boundary conditions at $y=\eta$ we must choose a relationship between $\delta$ and $\epsilon$. If we choose $\delta^{2} \approx O(\epsilon)$, the results found above are unchanged until $O\left(\delta^{3}\right)$, where we find finite Rossby number effects as well as finite amplitude effects enter the $O\left(\delta^{2}\right)$ term of the dispersion relation.

\subsection{Finite Amplitude Waves}

We outline here work in progress on a procedure for finding steadily propagating finite amplitude solutions. The problem of large amplitude waves can be approached with techniques of contour dynamics for the case of quasigeostophic dynamics with two deformation radii. To this order, in each region $D_{i}$

$$
\nabla^{2} \psi_{i}+\alpha_{i}^{2} \psi_{i}=0
$$

We define Green's functions for both domains

$$
\begin{gathered}
\nabla^{2} G_{i}\left(x, x^{\prime}\right)+\alpha_{i}^{2} G_{i}\left(x, x^{\prime}\right)=\delta\left(x-x^{\prime}\right) \quad x, x^{\prime} \text { in } D_{i} \\
G_{i}\left(x, x^{\prime}\right)=-\frac{1}{2 \pi} K_{0}\left(\alpha\left|x-x^{\prime}\right|\right)
\end{gathered}
$$

where $K_{0}$ is the zero order modified Bessel function of the second kind.

Defining the front as the curve $\mathrm{C}$ and applying Green's theorem we find

$$
\frac{1}{2} \psi=\int_{C}\left(\psi\left(x^{\prime}\right) \frac{\partial G}{\partial n}-G \frac{\partial \psi}{\partial n}\left(x^{\prime}\right)\right) d s^{\prime}
$$

where the normal derivatives point out from the domain. This integral can be discretized and if $N$ points are used, each domain yields $N$ linear equations with $2 N$ unknowns $\psi_{i}\left(x_{n}\right)$ and $\frac{\partial \psi_{i}}{\partial n}\left(x_{n}\right)$. The boundary conditions on pressure and velocity allow these to be combined into $2 \mathrm{~N}$ linear equations for the $2 \mathrm{~N}$ unknowns. This gives $\psi_{i}\left(x_{n}\right)$ and $\frac{\partial \psi_{i}}{\partial n}\left(x_{n}\right)$ for a particular choice of $\mathrm{C}$. The final condition that must be met is that $\psi+c \eta$ is constant on $\mathrm{C}$. The deviation from meeting this condition is used to adjust the position of $\mathrm{C}$ and the procedure is iterated to convergence.

\subsection{Acknowledgements}

I would like to thank Glenn Flierl for suggesting this problem and guiding my work and Steve Meacham for educating me about contour dynamics. 


\section{References}

Bower, A. S. (1989). Potential vorticity balances and horizontal divergences along particle trajectories in Gulf Stream meanders east of Cape Hatteras. J. Phys. Oceanog. 19, 16691681.

Hall, M. M. (1986). Horizontal and vertical structure of the Gulf Stream velocity field at 68 W. J. Phys. Oceanog. 16, 1814-1828.

Legekis, R. (1978). A survey of worldwide sea surface temperature fronts detected by environmental satellites. J. Geophys. Res. 83, 4501-4522.

Pedlosky, J. (1979). Geophysical Fluid Dynamics. Springer-Verlag, 624pp.

Pratt, L. J. and Stern, M. E. (1986). Dynamics of potential vorticity fronts and eddy detachment. J. Phys. Oceanog. 16, 1101-1120. 


\title{
23. Shear Dispersion and Thermal Reconnection
}

\author{
Thomas P. Witelski \\ Caltech, Department of Applied Mathematics
}

\subsection{Abstract}

The phenomenon of shear dispersion in advection-diffusion-reaction systems is examined for a system confined to a horizontal channel. Starting from a periodic initial condition consisting of vertical stripes of positive and negative temperature fluctuation, the effect of the shear with fixed flux boundary conditions is to distort and and break the rolls to form rows of thermal pockets at the walls. These pockets move with the shear velocity at the walls and lead to a periodic thermal reconnection process. The effect of an instability term due to nonlinear heating can be to reduce the shear enhanced diffusion decay rate of the oscillatory pattern; by properly balancing shear and heating instability, patterns can be sustained for arbitrarily long periods of time, though they ultimately decay. This study has applications to stirred chemical reactions, thermal instabilities in astrophysics and trait propagation in biological systems.

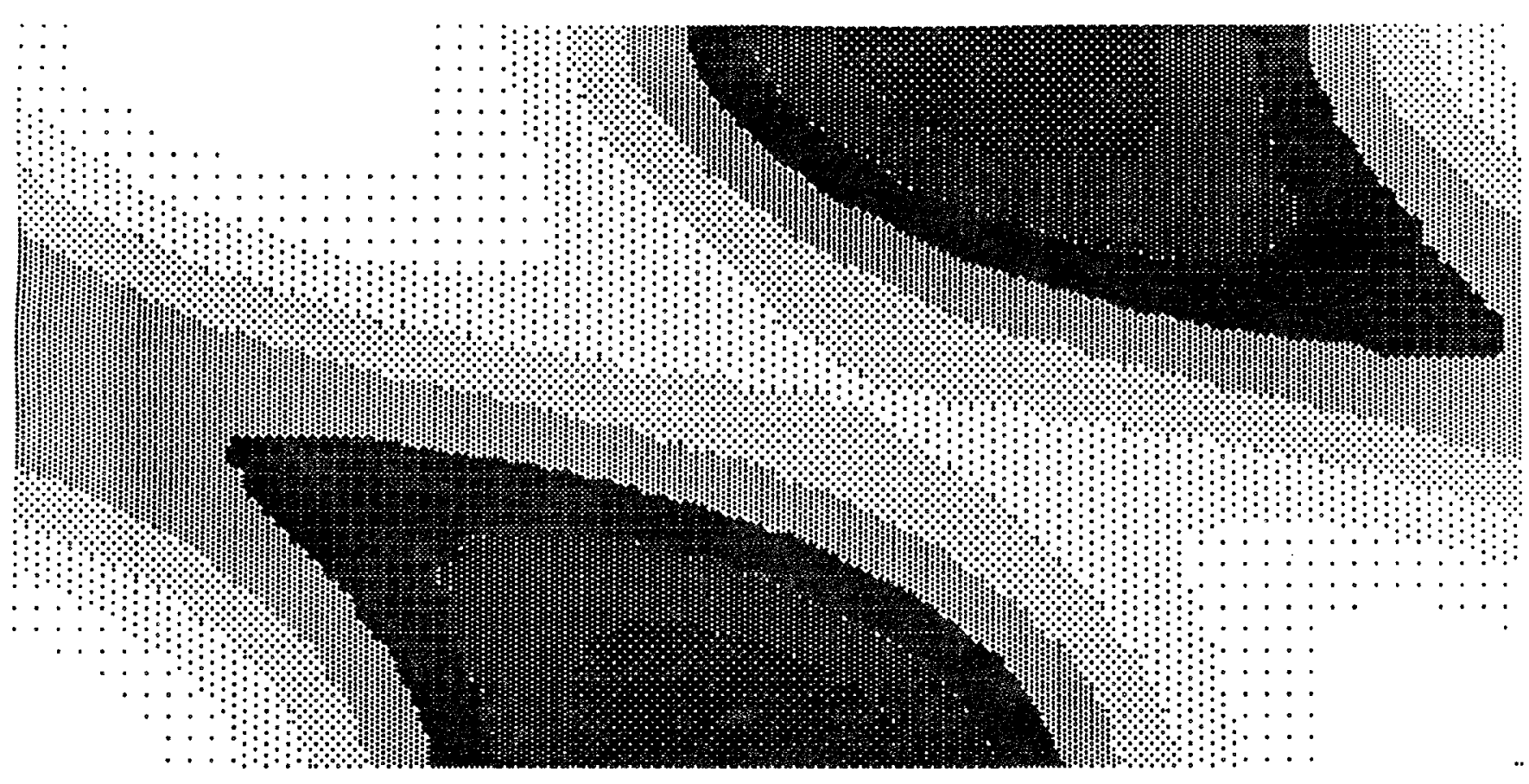

Figure 23.1. Temperature field during the thermal reconnection process. 


\subsection{Introduction}

Many physical systems of interest in astrophysics, chemical engineering and biology are described by advection-diffusion-reaction equations. Examples include stirred chemical reactions, thermal instability in the interstellar medium and trait propagation in biological systems (Cohen and Alexander 1986; Elphick et al. 1990b; Kolmogorov et al. 1937). The possibility of balances between shear enhanced diffusion and nonlinear production leading to the formation of lasting patterns will be examined here. Large bodies of research exist on the individual effects of front propagation (diffusion-reaction) and shear dispersion (Young and Jones,1991). Some additional aspects of each phenomena and the results of their interactions will be studied here.

The study of front propagation dates back to the 1930's and has applications to penetrationdiffusion, flame propagation, melting, and crystal growth processes. As described in the model of reproduction-diffusion of genes in a biological system (Kolmogorov et al., 1937), the nonlinear source term generally drives the system away from an unstable equilibrium with high potential energy to a stable saturated state. More recently, analysis of propagation and stability of collections of localized structures was studied by Elphick, et al. (1990a,b; 1991a,b).

The effect of shear on diffusion was first examined by G.I. Taylor in 1953. Taylor examined the diffusion of a dye in a pipe under the influence of Poiseuille flow. The variation in velocity across the pipe cross-section leads to an increased effective diffusivity which is commonly referred to as the effect of shear dispersion. Taylor's analysis was extended by Aris using equations for the moments of the concentration distribution. More recently, Young, Rhines and Garrett studied oscillatory shear dispersion in the context of oceanography. In 1984, Spiegel and Zaleski examined multi-component reacting systems under shear; their analysis will be used and extended here.

\subsection{Formulation of the Problem}

We formulate the problem by successively adding terms to a simple model. Landau's equation (1) is a simple model of uniform heating of a medium with a saturation temperature.

$$
\frac{d T}{d t}=\alpha T-T^{3}
$$

For $\alpha>0$, Landau's equation has a supercritical bifurcation with stable states $T= \pm \sqrt{\alpha}$. To add considerations for spatial structure of the temperature field we rewrite (1) with a diffusive term (2). Steady state solutions to this equation include periodic states and solutions joining the fixed points (3).

$$
\begin{gathered}
\frac{\partial T}{\partial t}=\kappa \frac{\partial^{2} T}{\partial x^{2}}+\alpha T-T^{3} \\
T(x, t)=H(x)=\sqrt{\alpha} \tanh \sqrt{\frac{\alpha}{2 \kappa}} x
\end{gathered}
$$


Solutions of the form (3) are variously called kinks, fronts, heteroclinic orbits, or topological solitons; these localized structures will be examined later in a discussion of the front dynamics for equation (2). Finally, to determine the effect of shearing on pattern formation in two dimensions we will add an advection term to complete the model. The goal of this study is to understand the effect of shear : can it form and sustain patterns alone or in combination with nonlinear terms?

\subsection{The Model}

The model equation that will be examined is (the PDE),

$$
\frac{\partial T}{\partial t}+\beta y \frac{\partial T}{\partial x}=D\left(\frac{\partial^{2} T}{\partial x^{2}}+\frac{\partial^{2} T}{\partial y^{2}}\right)+\alpha T-\gamma T^{3}
$$

The function $T(x, y, t)$ can represent either a temperature field for analysis of heat transfer in a sheared medium with a nonlinear source, or in the context of chemical engineering, $T$ can be a concentration field in a diffusion-reaction problem. The domain of the experiment is a channel with periodic boundary conditions in the direction of flow (Figure 2). The PDE is examined with fixed flux boundary conditions, corresponding to the physical constraints of nonpermeable (or insulating) walls.

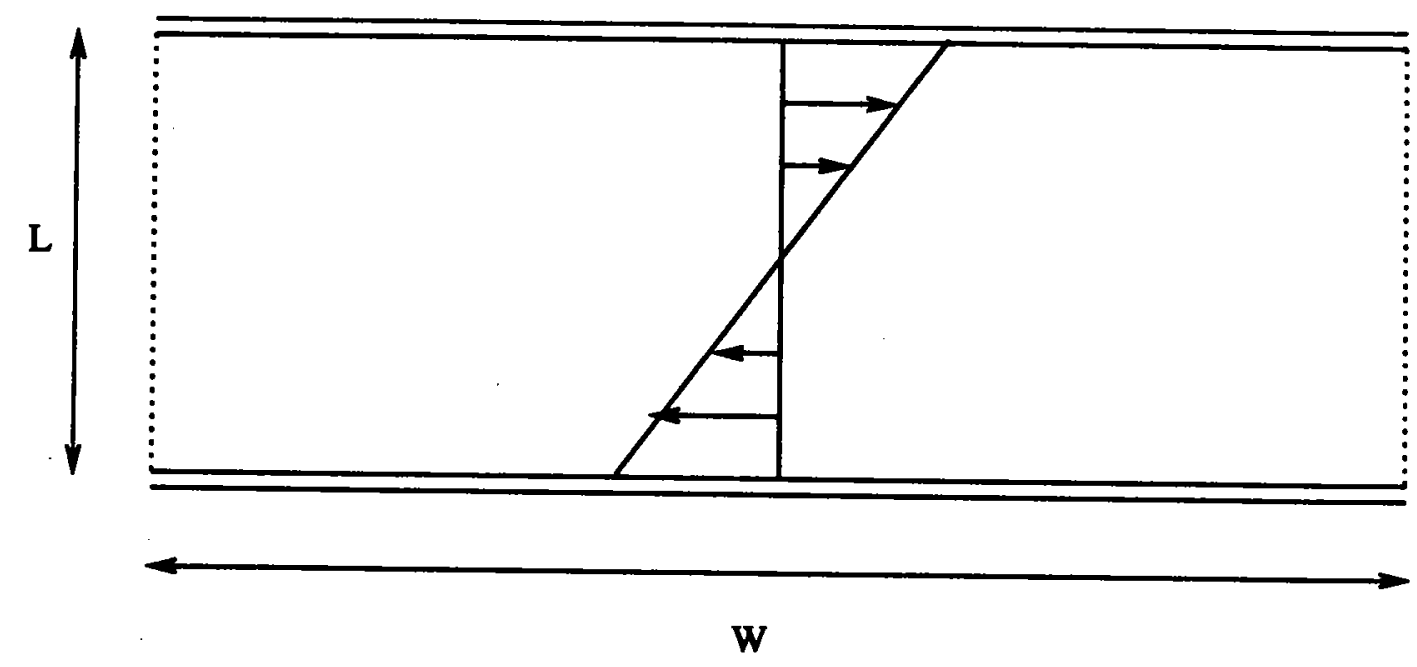

Figure 23.2. Domain for the model. 


\subsection{Analysis}

Nondimensionalizing (4) using characteristic length scales $W$ and $L / 2$ for $x$ and $y$, and characteristic temperature $1 / \sqrt{\gamma}$ yields

$$
\begin{gathered}
x=L_{x} x^{\prime} \quad y=L_{y} y^{\prime} \quad T=\frac{1}{\sqrt{\gamma}} T^{\prime} \quad t=t^{*} t^{\prime} \\
\alpha^{*}=\frac{\alpha L_{y}^{2}}{D} \quad \beta^{*}=\frac{\beta L_{y}^{2}}{D} \quad t^{*}=\frac{L_{y}^{2}}{D} \quad r=\frac{L_{y}}{L_{x}} \\
\frac{\partial T^{\prime}}{\partial t^{\prime}}+\beta^{*} r y^{\prime} \frac{\partial T^{\prime}}{\partial x^{\prime}}=r^{2} \frac{\partial^{2} T^{\prime}}{\partial x^{\prime 2}}+\frac{\partial^{2} T^{\prime}}{\partial y^{\prime 2}}+\alpha^{*} T^{\prime}-T^{3} \\
\left.x^{\prime} \in[-\pi, \pi] \quad y^{\prime} \in[-\pi / 2, \pi / 2] \quad \frac{\partial T^{\prime}}{\partial y^{\prime}}\right|_{y^{\prime}= \pm \pi / 2}=0
\end{gathered}
$$

Here we are using a diffusion time scale and $r$ is an aspect ratio. Our system now depends on three parameter; $\beta^{*}$, the magnitude of the shear; $\alpha^{*}$, the strength of the instability due to the heating term, and $r$, the aspect ratio of the channel. A Peclet number, the ratio of the advection to the diffusion terms, can also be defined for the system as $P e=\beta L / D$. Numerical simulations of this system were done using a pseudo-spectral method with a Fourier series in the $x$-direction and a cosine expansion in $y$, thus automatically satisfying the boundary conditions. The behavior of the system for the parameters $r=0.5, \alpha^{*}=1.5$, $\beta^{*}=3.5$ is typical for a broad range of parameter values. (From now on, we will use the $\mathrm{PDE}$ in the form given by (5), dropping all primes and stars.)

\subsubsection{Homogeneous Solutions}

In the homogeneous case, the PDE reduces to Landau's equation (6), with general solution (7).

$$
\begin{gathered}
\frac{d T}{d t}=\alpha T-T^{3} \\
T(t)=\frac{\sqrt{\alpha C} e^{\alpha t}}{\sqrt{1+C e^{2 \alpha t}}}
\end{gathered}
$$

The fixed points of (6) are $T=0$ and $T= \pm \sqrt{\alpha}$ with the zero solution being unstable and the $\pm \sqrt{\alpha}$ states being linearly stable.

\subsubsection{Linear Theory}

The initial evolution of infinitesimal perturbations about the unstable $T=0$ state will be governed by the growth rates of the normal modes of the linearized PDE (8).

$$
\frac{\partial T}{\partial t}+\beta r y \frac{\partial T}{\partial x}=r^{2} \frac{\partial^{2} T}{\partial x^{2}}+\frac{\partial^{2} T}{\partial y^{2}}+\alpha T
$$


The modes can be found in the form (9), with the fixed flux boundary conditions corresponding to $g^{\prime}( \pm \pi / 2)=0$ :

$$
T(x, y, t)=e^{i k x} e^{(s+\alpha) t} g(y)
$$

This ansatz reduces (8) to an ordinary differential equation, an eigenvalue problem for $s$ and $g(y)$,

$$
\frac{d^{2} g}{d y^{2}}-\left(s+(r k)^{2}+i \beta(r k) y\right) g(y)=0
$$

For $k=0$ the analytic solution is:

$$
\begin{aligned}
& s=-n^{2} \quad n=0,1,2, \ldots \\
& g(y)= \begin{cases}\cos n y, & \mathrm{n} \text { even } \\
\sin n y, & \mathrm{n} \text { odd }\end{cases}
\end{aligned}
$$

Solving numerically, we find that the dispersion relation is given by the graph (Figure 3). For increasing wavenumber $k$, we see that pairs of modes with real growth rates come together and form conjugate pairs of oscillatory modes. It was found that this bifurcation wavenumber, $k_{b}$, was inversely proportional to $\beta$, the magnitude of the shear.

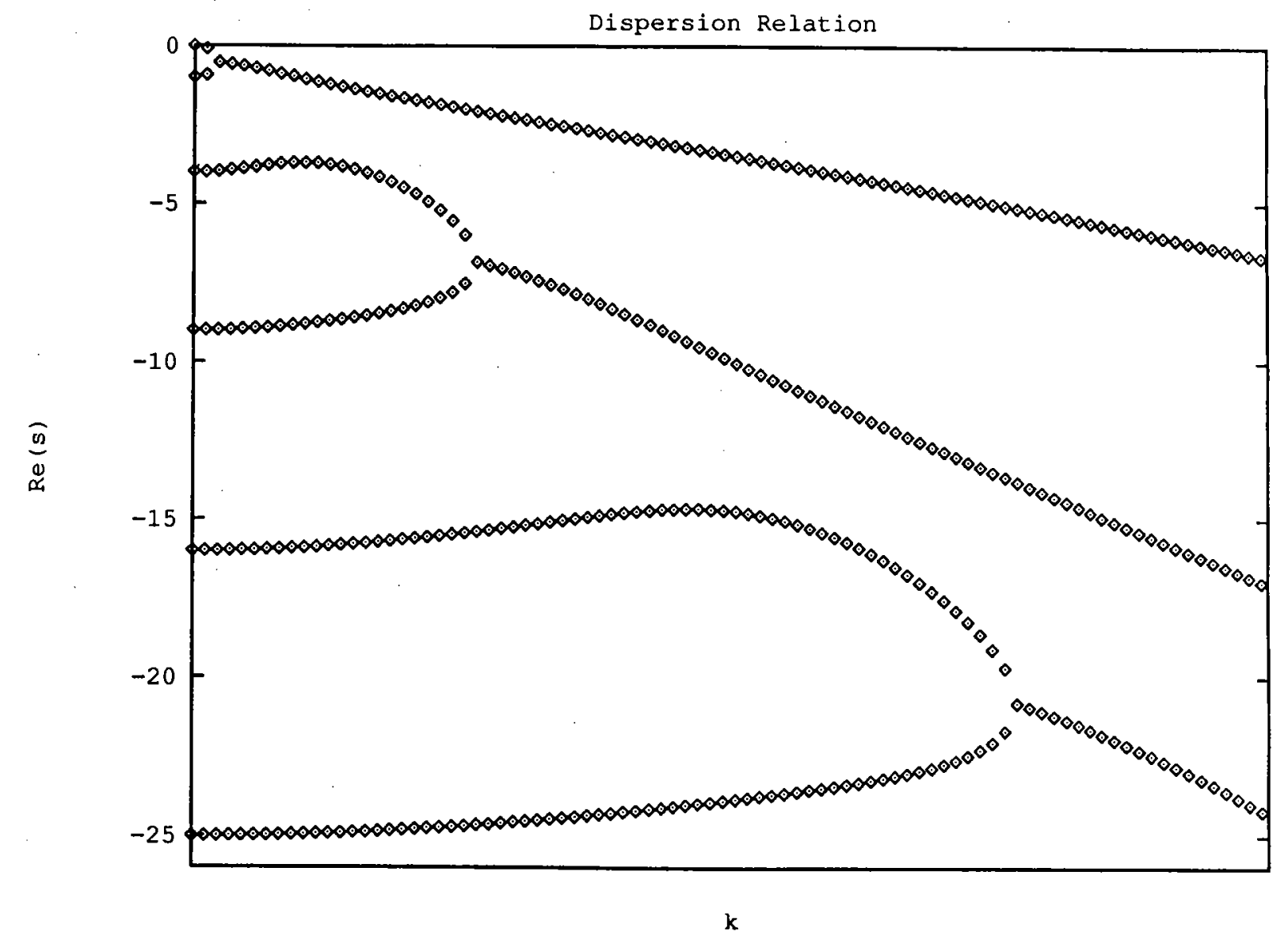

Figure 23.3. The Dispersion relation. 
The periodic boundary conditions in the $x$-direction quantize $k$, so modes must have $k=$ $0,1,2, \ldots$. The parameter $\alpha$ controls the instability of the system and can be adjusted to shift the dispersion graph down or up making modes more or less stable. To simplify comparison of the numerics with the analytics, the system was examined for values of the parameters that produced only four unstable modes: two real growing modes at $k=0$ and two conjugate oscillating modes at $k=1$. Contour lines for the oscillating eigenfunctions are given in (Figure 4). Taking the sum and the difference of these eigenfunctions yields states that closely match the numerical simulations.
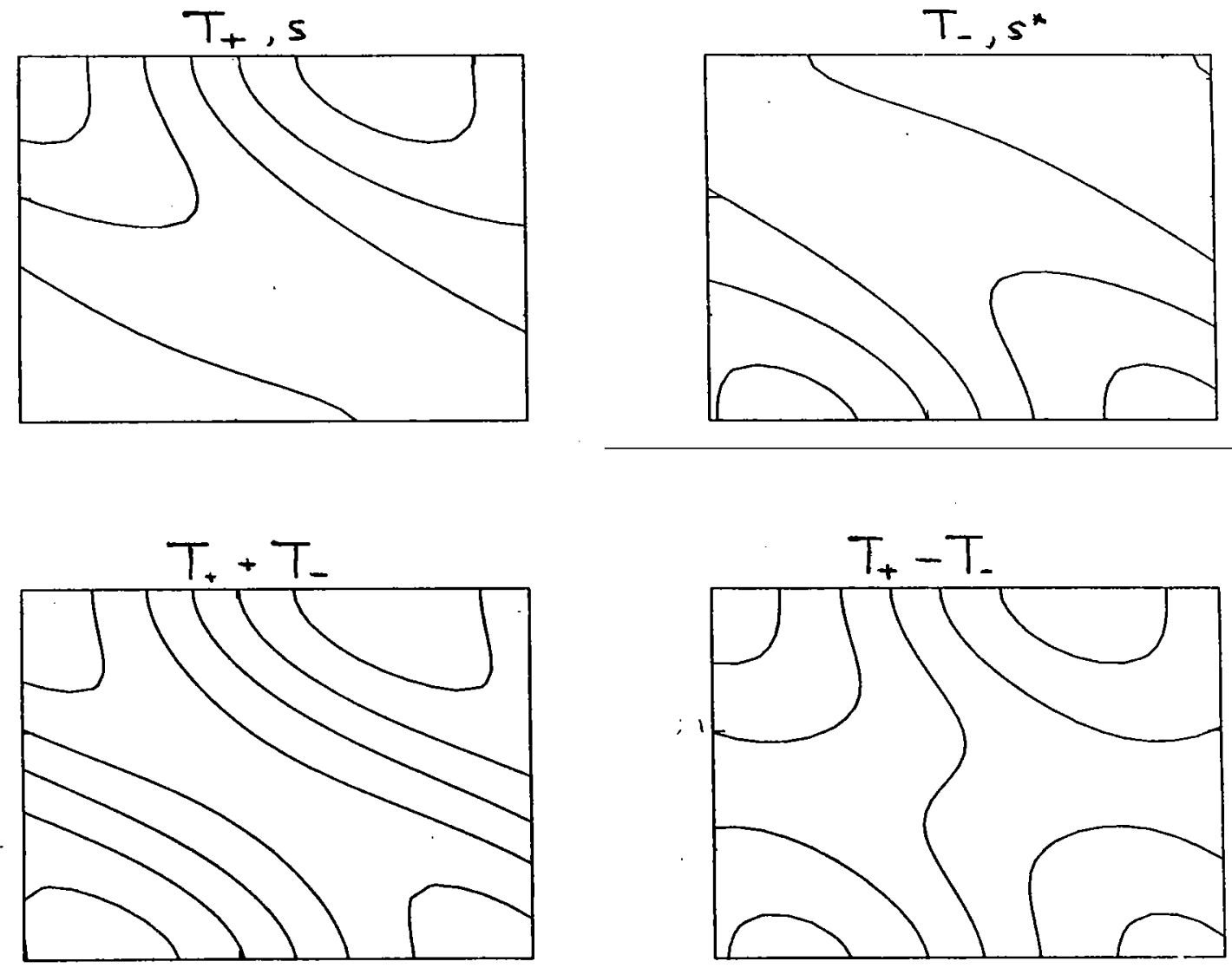

Figure 23.4. Linear eigenmodes.

\subsubsection{Truncated Expansion}

Equation (10) can be solved in terms of the Airy functions $A i(z)$ and $B i(z)$, but these solutions are difficult to deal with in this setting. Alternatively, the stability analysis of the PDE can be examined by expanding the temperature field and shear in truncated Fourier series and writing coupled amplitude equations. Shifting the domain of the system from $y \in[-\pi / 2, \pi / 2]$ to $y \in[0, \pi]$ allows us to use $-\cos y$ as the leading term in a cosine expansion of the shear velocity field, yielding the modified model

$$
\frac{\partial T}{\partial t}-\beta r \cos y \frac{\partial T}{\partial x}=r^{2} \frac{\partial^{2} T}{\partial x^{2}}+\frac{\partial^{2} T}{\partial y^{2}}+\alpha T-T^{3}
$$


In the simplest expansion, we can express the temperature field as the sum of two modes at wavenumber $k$.

$$
T(x, y, t)=A(t) \cos k x+B(t) \sin k x \cos y
$$

The form (12) leads to the set of coupled amplitude equations

$$
\begin{gathered}
\frac{d A}{d t}-\frac{\beta}{2} r k B=-r^{2} k^{2} A+\alpha A-\frac{3}{4} A^{3}-\frac{3}{8} A B^{2} \\
\frac{d B}{d t}+\beta r k A=-r^{2} k^{2} B-B+\alpha B-\frac{3}{4} A^{2} B-\frac{9}{16} B^{3} .
\end{gathered}
$$

$A=0, B=0$ is a fixed point of $(13 a, b)$. Searching for perturbations $a, b$ about this fixed point that grow exponentially as $\exp (s+\alpha) t$ yields an equation for the growth rates of the modes that qualitatively matches the behavior of the full dispersion, pairs of real modes bifurcating to produce pairs of oscillatory modes above some $k_{b}$ that is inversely proportional to $\beta$ (Figure 5):

$$
s=-\frac{1}{2}-r^{2} k^{2} \pm \sqrt{1-2 \beta^{2} r^{2} k^{2}} .
$$

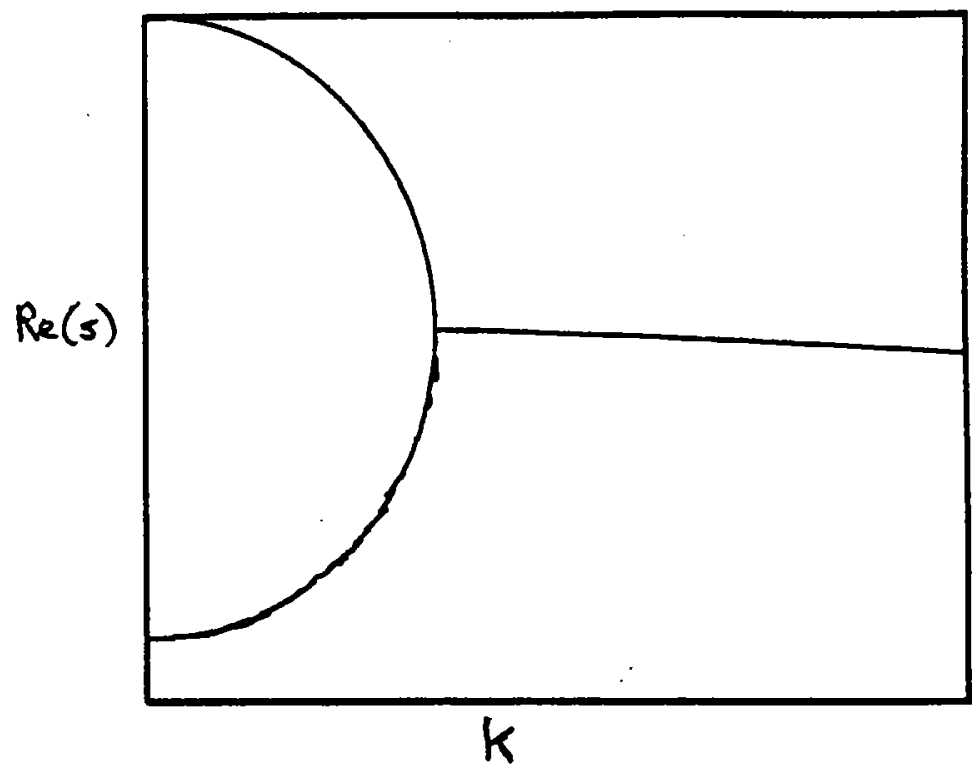

Figure 23.5. Truncated expansion dispersion relation.

\subsubsection{Semi-empirical Dispersion Relation}

The qualitative agreement of the simple truncated expansion result with the full dispersion relation suggests fitting the form (14) to model the leading order behavior of the full system by including two empirical parameters $a$ and $c$ as in

$$
s=\alpha-\frac{1}{2}-a r^{2} k^{2} \pm \sqrt{\frac{1}{4}-c \beta^{2} r^{2} k^{2}} .
$$

It was found that for $c \simeq 0.74$ and $a=f(\beta)$ (positive, and of order one), (15) fits the exact dispersion relation for a wide range of shears (Figure 6) quite well. 

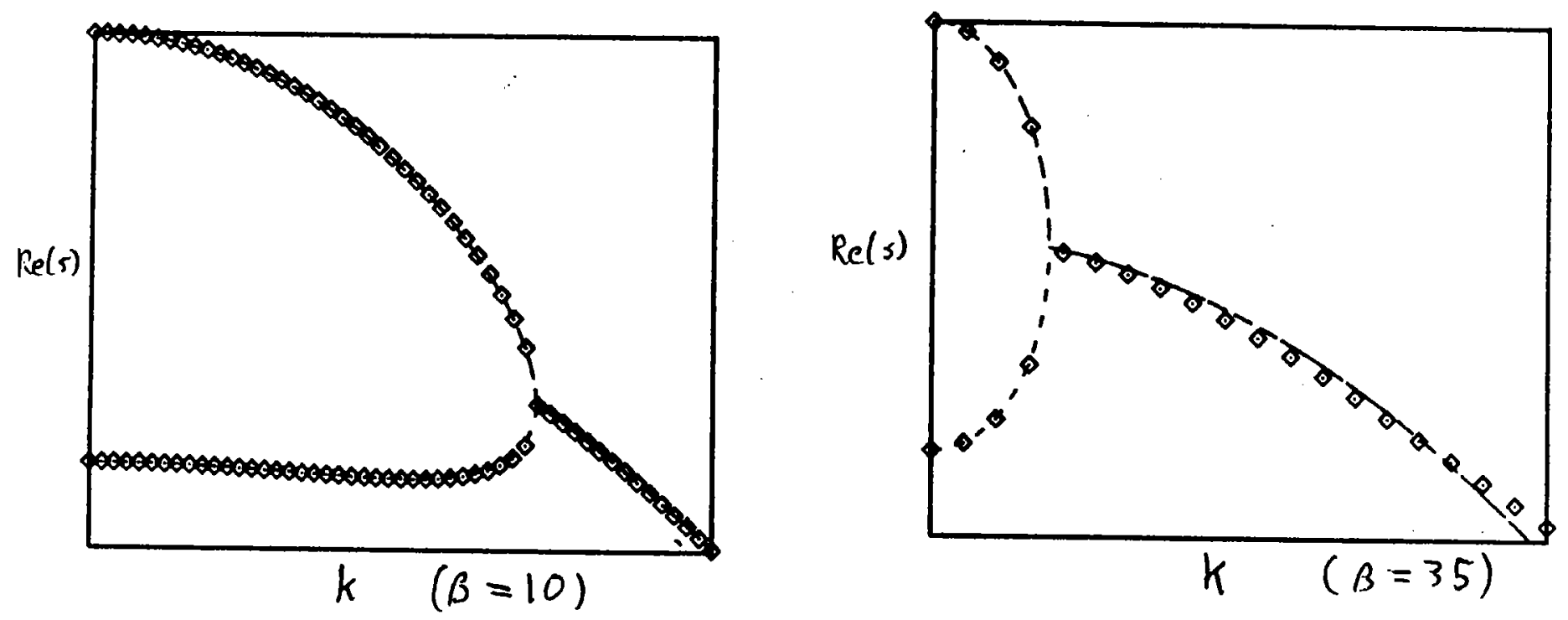

Figure 23.6. Comparison of the semiempirical and exact dispersion relations.

Multiplying (15) by an amplitude function $A(x, t)$ and taking the inverse Fourier transform yields an evolution equation for the $x$ structure of small perturbations (16).

$$
\begin{gathered}
\left(\left(\frac{\partial}{\partial t}-\alpha+\frac{1}{2}-a r^{2} \frac{\partial^{2}}{\partial x^{2}}\right)^{2}-\frac{1}{4}-c \beta^{2} r^{2} \frac{\partial^{2}}{\partial x^{2}}\right) A(x, t)=0 \\
T(x, y, t)=\left(A(x, t) g(y) e^{i k x}+c . c .\right)
\end{gathered}
$$

Using the symmetries of the PDE, we can identify the allowable forms for the nonlinear terms that can be added to the amplitude equation (16). Some of the symmetries present in the model include:

1) $T \rightarrow-T$, an inversion symmetry.

2) $x \rightarrow x+x_{0}$, translation invariance in the $\mathrm{x}$-direction.

3) $t \rightarrow t+t_{0}$, time shift invariance.

4) $x \rightarrow-x$ and $y \rightarrow-y$, a point reflection symmetry which corresponds to the reflection $x \rightarrow-x$ and reversing the shear flow $\beta \rightarrow-\beta$.

5) $x \rightarrow x+\beta r a t$ and $y \rightarrow y-a$, (for any arbitrary constant $a$ ), a translation invariance in the $x y$ plane that represents a translation in the $y$ direction and a change to a corresponding moving reference in the $x$ direction.

Symmetries 1,2 and 3 require that the amplitude equation be invariant under rotations in the complex plane, $A \rightarrow A e^{i \phi}$. Symmetries 2 and 3 mean that $x$ and $t$ cannot appear explicitly in any terms. Symmetry 4 forces differential terms to be of even order. Hence, we conclude 
that the nonlinear terms can be of form (18), where $f$ is an algebraic function, and since $A$ is a slowly varying function $|A|^{2} A$ will be leading nonlinear term.

$$
\text { Nonlinear terms }=f\left(\partial_{x^{2}},|A|^{2}\right) A=|A|^{2} A+\ldots
$$

\subsubsection{Thermal Instability}

The initial condition used for the numerical simulations was a periodic sequence of stripes of positive and negative temperature fluctuations, aligned along the $y$ axis. Various profiles for the $x$ structure of the stripes used included sinusoidal (as in the comparison with Rhines and Young), modified-gaussian, and square pulse, but the time evolution for all of these states was fundamentally the same. For initial conditions with zero spatial average, it was observed that the system tended toward the unstable uniform zero state. If the spatial average is nonzero then the system will converge to the like sign $\sqrt{\alpha}$ stable saturated state.

The properties of the initial condition that decide whether the system will go to a stable or unstable homogeneous state can be examined using a truncated expansion and model (11). In this case we will use the expansion

$$
T(x, y, t)=A(t)+B(t) \cos y
$$

which yields the amplitude equations

$$
\begin{gathered}
\frac{d A}{d t}-\frac{\beta}{2} r B=\alpha A-A^{3}-\frac{3}{2} A B^{2} \\
\frac{d B}{d t}=-B+\alpha B-3 A^{2} B .
\end{gathered}
$$

$A(t)$, the amplitude of the homogeneous mode, is coupled linearly only to $B(t)$. If $A(0)=0$ (i.e. the average of the initial temperature distribution is zero), then the system can reach one of the homogeneous stable solutions only if $B(t)$ is nonzero. $B(t)$ in general is linearly coupled to higher frequency $\cos (n y)$ terms. All of these modes will be zero if the the initial condition is independent of $y$ and has zero average. More general conditions on the class of initial conditions that lead to stable or unstable states can be found by examining the spatial average of $T$, which leads to the system of equations:

$$
\begin{gathered}
\langle T\rangle_{t}=\alpha\langle T\rangle-\left\langle T^{3}\right\rangle \\
\frac{1}{3}\left\langle T^{3}\right\rangle_{t}=-2 r^{2}\left\langle T T_{x}^{2}\right\rangle-2\left\langle T T_{y}^{2}\right\rangle+\alpha\left\langle T^{3}\right\rangle-\left\langle T^{5}\right\rangle
\end{gathered}
$$

\subsubsection{Perturbation Expansion}

The nonlinear growth of perturbations can be examined by expanding $T$ in a perturbation series using the method of multiple scales. With the scalings

$$
X=\epsilon x \quad \tau=\epsilon^{2} t \quad \alpha=\epsilon^{2} a
$$


$T$ can be expanded as a perturbation series

$$
T=\epsilon \hat{T}=\epsilon T^{(0)}+\epsilon^{2} T^{(1)}+\ldots
$$

and yield an amplitude equation for the perturbations as a solvability condition. With this set of scales, the PDE becomes equation

$$
\frac{\partial^{2} \hat{T}}{\partial y^{2}}=\epsilon^{2}\left(\frac{\partial \hat{T}}{\partial \tau}-r^{2} \frac{\partial^{2} \hat{T}}{\partial X^{2}}-a \hat{T}+\hat{T}^{3}\right)+\epsilon \beta r y \frac{\partial \hat{T}}{\partial X} .
$$

At leading order, using the ansatz $T^{(0)}=f^{(0)}(y) G(X, \tau)$ with the boundary conditions $f^{\prime}( \pm \pi / 2)=0$ yields $T^{(0)}=G(X, \tau)$.

$$
\mathcal{L} T^{(0)}=\frac{\partial^{2} T^{(0)}}{\partial y^{2}}=0
$$

At first order, the perturbation is given by (25) with the solvability condition for the selfadjoint operator $\mathcal{L}$ being automatically satisfied.

$$
\begin{gathered}
\mathcal{L} T^{(1)}=\beta r y \frac{\partial T^{(0)}}{\partial X}=\beta r y \frac{\partial G}{\partial X} \\
\int_{-\pi / 2}^{\pi / 2} r \beta y \frac{\partial G}{\partial X} d y=0
\end{gathered}
$$

Solving (25) with $T^{(1)}=f^{(1)}(y) G_{X}(X, \tau)$ yields

$$
T^{(1)}=r \beta \pi^{3}\left(\frac{1}{48}\left(\frac{2 y}{\pi}\right)^{3}-\frac{1}{16}\left(\frac{2 y}{\pi}\right)\right) \frac{\partial G}{\partial X}+H(X, \tau)
$$

At third order, all of the terms from the PDE are present, equation (28), and the nontrivial solvability condition yields an amplitude equation that determines the form of $G(X, \tau)$, equation (30).

$$
\begin{gathered}
\mathcal{L} T^{(2)}=\frac{\partial T^{(0)}}{\partial \tau}-r^{2} \frac{\partial^{2} T^{(0)}}{\partial X^{2}}-a T^{(0)}+T^{(0)^{3}}+\beta r y \frac{\partial T^{(1)}}{\partial X} \\
\int_{-\pi / 2}^{\pi / 2} \frac{\partial G}{\partial \tau}-r^{2} \frac{\partial^{2} G}{\partial X^{2}}-a G+G^{3}+r \beta y f^{(1)}(y) \frac{\partial^{2} G}{\partial X^{2}} d y=0 \\
\frac{\partial G}{\partial \tau}=r^{2}\left(1+\frac{\beta^{2} \pi^{4}}{120}\right) \frac{\partial G}{\partial X^{2}}+a G-G^{3}
\end{gathered}
$$

Since $T=G(X, \tau)$ at leading order, we can interpret the coefficient of the diffusive term in (30) as the effective diffusivity. The increase in the diffusivity by $\beta^{2} \pi^{4} / 120$ is the Taylor shear dispersion (Spiegel and Zaleski, Taylor). We also observe that the coefficients of the heating terms have not changed, with the physical interpretation that the shear has no effect on effective rates of heating or chemical reaction.

The case of a sinusoidal shear as used in the truncated expansion can be handled by a similar perturbation process and yields a diffusivity enhancement of $\beta^{2} / 2$. We define a sinusoidal shear to have an equivalent constant shear it has the same shear dispersion.

$$
\beta_{c}=\frac{\pi^{2}}{\sqrt{60}} \beta_{\sin }
$$




\subsection{Examination of Shear Dispersion}

More comments on the behavior of the model can be made by comparison of the system with related solvable systems. Rhines and Young (1983) give an analytic expression for a concentration field under the influence of shear dispersion in the unbounded $x y$ plane.

$$
\begin{gathered}
\frac{\partial T}{\partial t}-\beta r \cos y \frac{\partial T}{\partial x}=r^{2} \frac{\partial^{2} T}{\partial x^{2}}+\frac{\partial^{2} T}{\partial y^{2}} \\
T(x, y, t)=\exp \left(-r^{2} k^{2} t-\frac{1}{3} \beta^{2} r^{2} k^{2} t^{3}\right) \cos k(x-\beta r y t)
\end{gathered}
$$

$T$ given by (32) at $t=0$ is simply $\cos (k x)$, a periodic sequence of hot and cold stripes parallel to the $y$ axis. We will look at the spatially averaged gradient squared for (32) and compare its time evolution to that of the PDE. For (32), $\left\langle|\nabla T|^{2}\right\rangle$ is

$$
\left\langle|\nabla T|^{2}\right\rangle=\frac{1}{2} k^{2}\left(1+\beta^{2} r^{2} t^{2}\right) \exp \left(-2 r^{2} k^{2} t-\frac{2}{3} \beta^{2} r^{2} k^{2} t^{3}\right) .
$$

The time evolution of this quantity is affected by two processes: stretching of the field due to the shear, which increases the average gradient squared, and diffusive shmearing which causes the gradient to decay. For the model, the evolution of the $x$ and $y$ contributions to the average gradient are governed by

$$
\begin{gathered}
\frac{1}{2}\left\langle T_{x}^{2}\right\rangle_{t}=-r^{2}\left\langle T_{x x}^{2}\right\rangle-\left\langle T_{x y}^{2}\right\rangle-\left\langle T_{x}^{2}\left(3 T^{2}-\alpha\right)\right\rangle \\
\frac{1}{2}\left\langle T_{y}^{2}\right\rangle_{t}=-r^{2}\left\langle T_{x y}^{2}\right\rangle-\left\langle T_{y y}^{2}\right\rangle-\beta r\left\langle T_{x} T_{y}\right\rangle-\left\langle T_{y}^{2}\left(3 T^{2}-\alpha\right)\right\rangle .
\end{gathered}
$$

Without the heating terms, equations $(34,35)$ describe the process of shear dispersion and we see that $\left\langle T_{x}^{2}\right\rangle$ is monotonically decreasing. $\left\langle T_{y}^{2}\right\rangle$ is not necessarily decreasing for shear dispersion in a channel, and neither term is forced to be decreasing once the instability due to $\alpha$ is added.

A plot of the time evolution of the average gradient squared for Rhines and Young and for the PDE (Figure 7) shows that the boundary effects in a channel can significantly affect the behavior of the solution. In the unbounded case, there is initially an increase in the gradient due to shear, but after some characteristic time diffusion will monotonically smooth out the field. For shear dispersion in a channel (given by the numerical simulation without the heating term), the decay due to diffusion is not as rapid and the decay is also not monotonic! The long term time evolution of the gradient shows that there is an oscillatory phenomenon occurring. The response of the PDE with the heating terms shows an even slower decay with more prominent oscillations, suggesting that a proper balance can be found between shear-dispersion promoted oscillatory decay and heating instability induced growth of the temperature gradients to sustain patterns for an arbitrarily long time. 

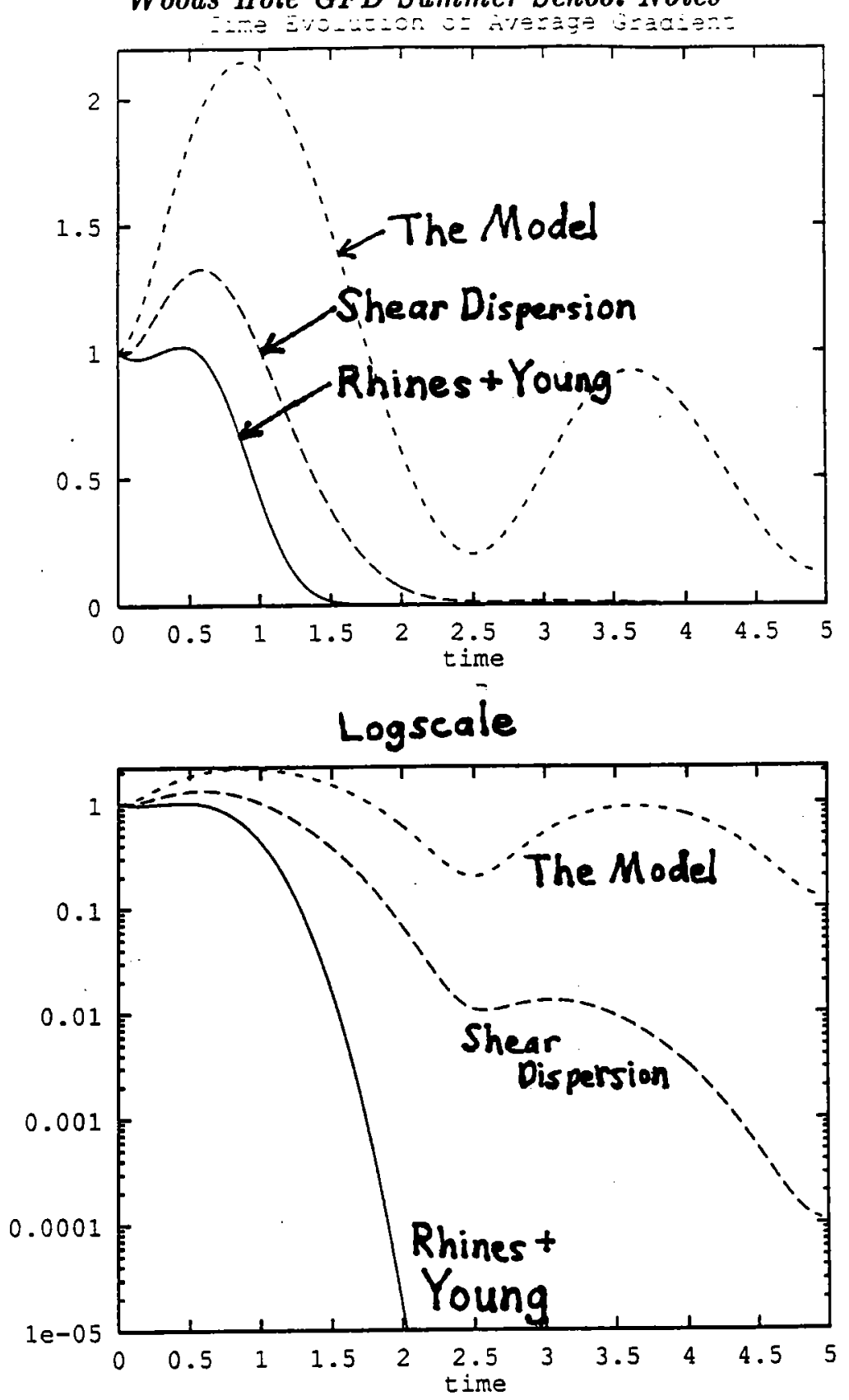

Figure 23.7. Comparison of the model to results for shear dispersion.

\subsection{Thermal Reconnection}

The physical processes underlying the oscillatory behavior can be understood by further comparisons with the case of shear dispersion in the unbounded case. For (32), the isotherms are given by

$$
y=\frac{1}{\beta r t}(x+c) .
$$

We see that as time increases the isotherms tilt with slope proportional to $1 / t$ and also stretch, becoming narrower in the $x$ direction, with stripe width also being proportional to $1 / t$. In a channel, the fixed flux boundary conditions at the walls force the temperature rolls 
to be perpendicular to the walls, so if the rolls behave similarly to the unbounded case near the centerline of the channel, then they must distort to obey the boundary conditions.

What is observed from the numerical simulations is that the rolls initial tilt under the under the influence of the shear while remaining perpendicular to the walls. Past some critical level of tilting, the rolls will pinch off in the middle forming rows of thermal fluctuation pockets at the top and bottom walls that move with the shear velocity. As the rows of alternating hot and cold pockets move by each other at velocities $\pm \beta r \pi / 2$, there will be periodic instances of separation (when opposite fluctuation pockets pass by) and thermal reconnection (when like sign pockets pass by and rejoin across the centerline) (Figure 8). For low shears, the instances of reconnection form structures very similar to the initial set of rolls.

This process is very accurately described by the linear combination of oscillatory eigenfunctions found in the linear stability analysis (Figure 4); the sum of the two modes corresponds to a moment shortly after reconnection (as marked by the roll-like mostly parallel tilted isotherms). The difference of the two modes is representative of the system during a separation (midway between instances of reconnection).

As described in the section on Thermal Instability, the temperature field can decay to the unstable zero amplitude state while retaining the oscillatory thermal reconnection behavior. If the initial condition leads to a uniform temperature saturation state of $T= \pm \sqrt{\alpha}$ then the thermal reconnection processes is still observed, but it will end in a finite time. The oscillatory reconnection-separation goes on while the homogeneous mode grows larger favoring either the hot or the cold thermal pockets. Eventually either the hot side or the cold side dominates and the weaker side becomes surrounded and engulfed by the stronger. Unable to reconnect due to sparsity of like sign pockets, the weaker side will disperse in a manner similar to that described by Elphick and Spiegel for the annihilation of "clouds". This motivates the analysis of the thermal reconnection/instability process in terms of front dynamics.

\subsection{Front Dynamics}

The stability of spatial patterns formed by a differential equation can be examined by decomposing the patterns into combinations of localized structures that are exact solutions of the equation. This approach is similar to the linear combination of atomic orbitals (LCAO) method used to describe large atomic systems in quantum chemistry and solid state physics. The solution will be a linear combination of local solutions plus a time dependent term describing the perturbations to the sum of local structures due to their mutual interactions. In the quantum mechanics context, the assumption that the perturbation to the linear combination will be small is called the tight binding approximation. Following the methods of Elphick, et al., using solvability conditions for the time evolution of the perturbation, the stability of the linear combination of local structures can be determined, and equations can be written for the effective forces of interaction between them. 


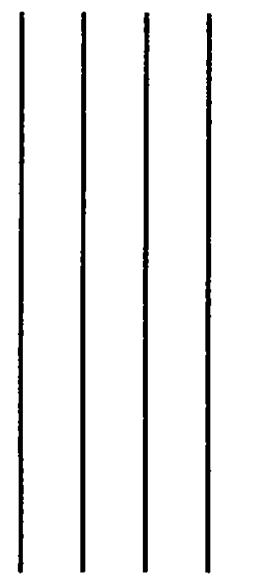

Tilting and

Stretching

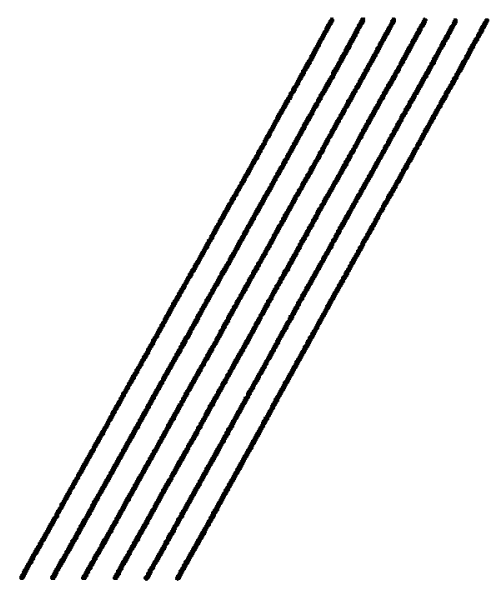

Shear Dispersion in the Unbounded Case

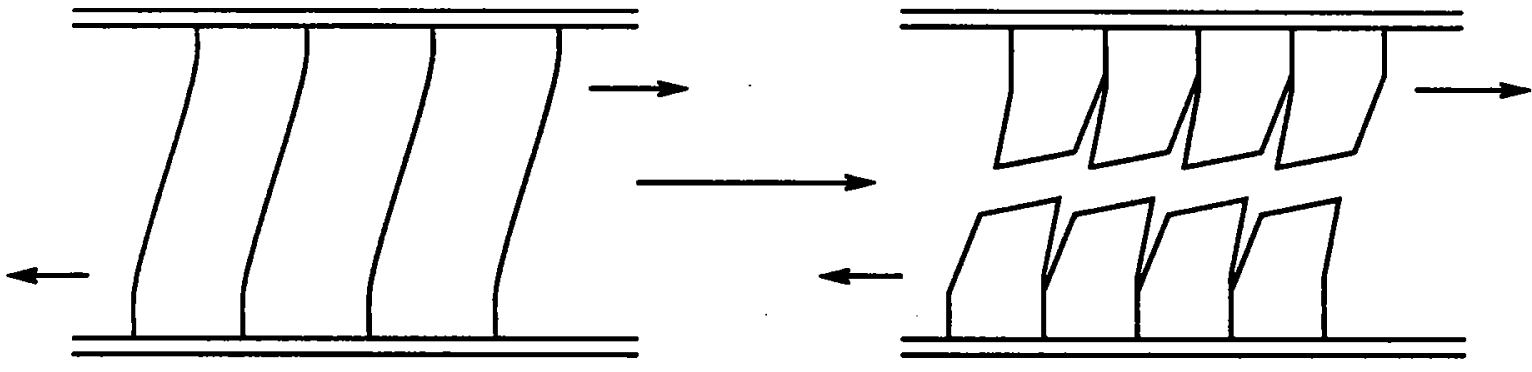

Shear Dispersion in a Channel: Rolls tilt, pinch off in the middle and form rows of thermal pockets that move with the shear along the walls

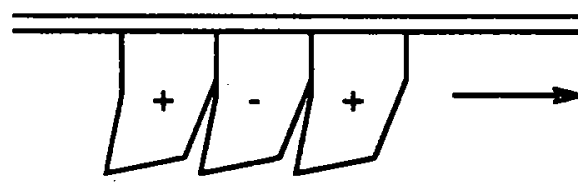

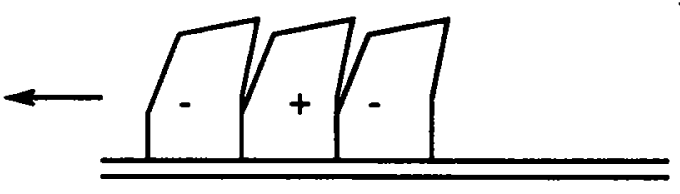

Separation

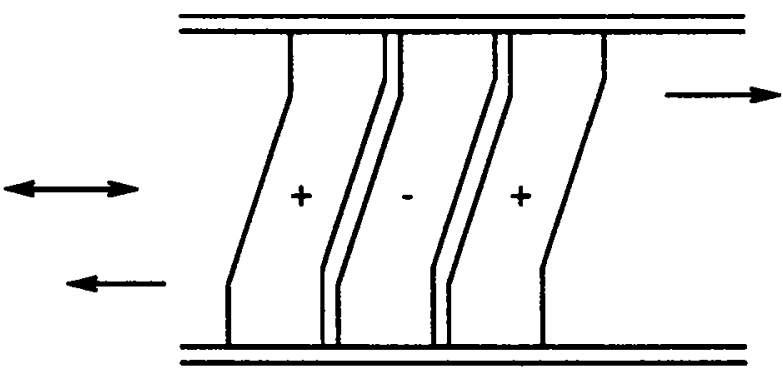

Thermal Reconnection

Figure 23.8. Shear dispersion: the physical processes in the unbounded case and for dispersion in a channel. 
23.8.1 The Amplitude Equation

$$
\frac{\partial G}{\partial \tau}=\kappa \frac{\partial G}{\partial X^{2}}+\alpha G-G^{3}
$$

Equation (36) is the general form of the one dimensional amplitude that is closely tied to the basic structure of the solutions to the PDE. We will examine the front dynamics for this equation.

Equation (36) shows up in the analysis of the PDE at several points. We have seen that a perturbation expansion yields this form with the effect of shear dispersion enhanced diffusivity (30). The PDE also reduces directly to this form in $y$ for solution that are independent of $x$. An examination of the PDE at the upper or lower walls gives with the boundary layer assumption that gradients in $y$ are negligible yields

$$
\frac{\partial T}{\partial t} \pm \beta r \frac{\pi}{2} \frac{\partial T}{\partial x}=r^{2} \frac{\partial^{2} T}{\partial x^{2}}+\alpha T-T^{3}
$$

Travelling wave solutions of $(37), T(x, t)=H(x-c t)$ subject to the periodic boundary conditions in $x$ of the $\mathrm{PDE}$, require that the velocity $c= \pm \beta r \frac{\pi}{2}$. This restriction yields the equation for evolution of the wave profile, which is (36) with $\kappa=r^{2}$.

\subsubsection{The Front Solution}

Equation (36) has the localized front solution

$$
H(x)=\sqrt{\alpha} \tanh \sqrt{\frac{\alpha}{2 \kappa}} x .
$$

The height of the front is twice the saturation temperature and directly with the instability parameter $\alpha$. The width of the front is inversely related to the diffusivity $\kappa$. The translational invariance of (36) means that a front $H\left(x-x_{0}\right)$ can occur at any position. Since (36) is invariant under $H \rightarrow-H,-H(x)$ is also a solution. We will call $H(x)$ a kink and $-H(x)$ (or $H(-x)$ ) an antikink solution.

\subsubsection{The Lyaponuv Functional}

The behavior of solutions to (36) can be explained in terms of the existence of a Lyaponuv functional for the equation. Multiplying (36) across by $G_{\tau}$ and integrating over the domain, shows that a functional decreasing in time exists for the system.

$$
\begin{gathered}
\int\left(\frac{\partial G}{\partial \tau}\right)^{2} d X=-\frac{\partial}{\partial \tau} \int \frac{\kappa}{2}\left(\frac{\partial G}{\partial X}\right)^{2}-\frac{\alpha}{2} G^{2}+\frac{1}{4} G^{4} d X \\
-\int\left(\frac{\partial G}{\partial \tau}\right)^{2} d X=\frac{\partial}{\partial \tau} F[G] \leq 0 \\
F[G]=\int \frac{\kappa}{2}\left(\frac{\partial G}{\partial X}\right)^{2}-\frac{\alpha}{2} G^{2}+\frac{1}{4} G^{4} d X
\end{gathered}
$$


That $F[G]$ is bounded from below can be shown by examining homogeneous solutions $\left(G_{X}=\right.$ 0 ) and observing that the functional derivative of $F$ with respect to $G$ is minimized by setting $G= \pm \sqrt{\alpha}$. The Lyaponuv functional forces the system to converge to either the absolute minima $G= \pm \sqrt{\alpha}$ or to a function $G(x)$ that is a local relative minimum of $F$.

\subsubsection{The Stability of Pulse Solutions}

The periodic boundary conditions imposed by the PDE in the $x$ direction preclude the front solution. The simplest periodic structure that can be formed is a pulse joining $\sqrt{\alpha}$ to $-\sqrt{\alpha}$ and back to $\sqrt{\alpha}$ (as $\left.x_{0} \rightarrow \infty, x_{0}>0\right)$,

$$
G(x)=H\left(x-x_{0}\right)-H\left(x+x_{0}\right)+\sqrt{\alpha} .
$$

For simplicity, we examine pulses that are symmetric about $x=0$. The pulses given by (42) are not exact time independent solutions of (36), but for sufficiently large $x_{0}$ they closely approximate the fixed points. The stability of these states to perturbations $g(x) \exp s t$ is determined by solving the eigenvalue problem

$$
s g=\kappa \frac{d^{2} g}{d x^{2}}+\alpha g-3 G(x)^{2} g .
$$

Solving numerically in a periodic domain, we find that such pulses are unstable and have at least one unstable eigenmode (Figure 9). The common growing eigenmode will be an even function corresponding to an increase or decrease of the pulse width by a symmetric of both fronts in or out. All pulse solutions will also have a zero eigenvalue that corresponds to a translational eigenmode; the eigenfunction will be an odd function, causing both fronts in the pulse to move either to the left or to the right.

\subsubsection{Front Dynamics Analysis}

More insight into the dynamics of the system can be gained by studying the time evolution of a pulse in terms of the movements of its fronts and the growth of the interaction term. The time dependent pulse solution to (36) can be written as

$$
G(x, t)=H_{+}\left(x-x_{0}(\epsilon t)\right)-H_{-}\left(x+x_{0}(\epsilon t)\right)+\sqrt{\alpha}+\epsilon R(x, \epsilon t)
$$

where we allow the fronts to move according to $x_{0}(e t)$ and we assume that the interaction term $\epsilon R$ is small. Substituting (43) into (36) yields

$$
\epsilon \mathcal{L} R=-\epsilon\left(H_{+}^{\prime}+H_{-}^{\prime}\right) x_{0}^{\prime}+3\left(H_{+}-H_{-}\right)\left(H_{+}+\sqrt{\alpha}\right)\left(H_{-}+\sqrt{\alpha}\right)+O\left(\epsilon^{2}\right)
$$

where

$$
\mathcal{L}=\kappa \partial_{x^{2}}-3\left(H_{+}-H_{-}+\sqrt{\alpha}\right)^{2}+\alpha .
$$

We note that $\mathcal{L}$ is a self-adjoint operator and that the derivative of $H_{+}-H_{-}+\sqrt{\alpha}$ is the eigenfunction corresponding to the marginally stable translational mode, hence $H_{+}^{\prime}+H_{-}^{\prime}$ is 

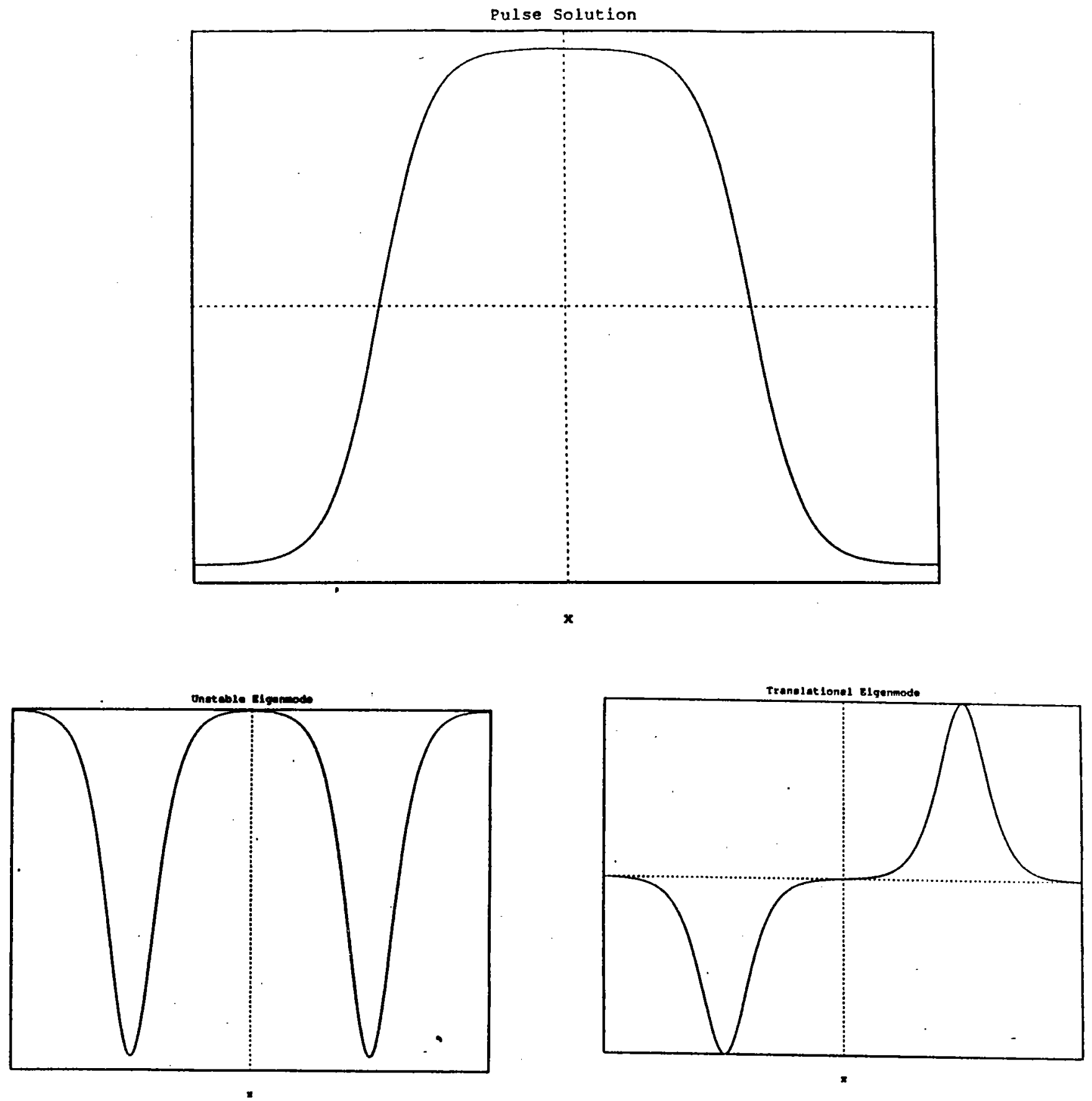

Figure 23.9. A pulse solution of the amplitude equation and its translational and unstable linear eigenmodes.

an element of the nullspace of $\mathcal{L}$. For sufficiently localized fronts, the last term of (44) will be of order $\epsilon$ everywhere. Similarly, it can be shown that

$$
\mathcal{L} \sim \kappa \partial-3 H_{+}^{2}-3 H_{-}^{2}-3 \alpha+\alpha .
$$

Defining $\mathcal{L}_{+}, \mathcal{L}_{-}$by

$$
\mathcal{L}_{*}=\kappa \partial_{x^{2}}-3 H_{*}^{2}+\alpha \quad *=+,-
$$


we see that $\mathcal{L}_{*} H_{*}^{\prime}=0$ and that $\mathcal{L} H_{*}^{\prime}=O(\epsilon)$. Hence, the solvability condition at leading order is

$$
\int H_{+}^{\prime} \mathcal{L} R d x=0
$$

or

$$
\frac{d x_{0}}{d t}=\frac{3 \int H_{+}^{\prime}\left(H_{+}-H_{-}\right)\left(H_{+}+\sqrt{\alpha}\right)\left(H_{-}+\sqrt{\alpha}\right) d x}{\epsilon \int H_{+}^{\prime 2} d x}
$$

Using the approximation methods of Elphick et al. (1990b), (45) can be simplified to

$$
\frac{d x_{0}}{d t} \propto-e^{-\sqrt{\frac{2 \alpha}{\kappa}} x_{0}} .
$$

Hence we see that the pulse is doomed to collapse in on itself. Therefore we can conclude that one dimensional patterns can not be sustained by this amplitude equation.

\subsection{Conclusions}

We have observed the behavior of thermal instabilities and shear dispersion for advectiondiffusion-reaction systems confined to a channel. After going through an oscillatory "thermal reconnection" phase, the system will settle down to either the stable uniform saturated solution or the unstable zero solution depending on the nature of the initial conditions. The examination of the front dynamics for the underlying one dimensional amplitude equation leads us to believe that if any lasting patterns are possible in this system then they must be fully two dimensional.

Further study of this system will include attempts to verify the predictions of the front dynamic analysis for the rate of pulse collapse and the development of a front dynamics analysis with a moving reference frame for use in analysis of the thermal reconnection process. Extensions to the PDE and the numerical simulations will include examination of nonlinearly coupled multi-component reacting systems, examination of the behavior of time dependent shear and analysis of pattern formation in systems where the flow field is affected by the pattern development.

\subsection{Acknowledgments}

I thank Dr. E.A. Spiegel and Dr. G.R. Ierley for their patience and guidance. I also thank Dr. W. Young, Dr. S. Fauve, Dr. S. Meacham and Dr. J. B. Keller for their helpful suggestions. 


\section{References}

R. Aris (1956). On the dispersion of a solute in a fluid through a tube. Proc. Roy. Soc. London,A235, 67-77.

D.S. Cohen and R. Alexander (1986). Chemical reactor theory and problems in diffusion. Physica D,20, 122-141.

C. Elphick, G.R. Ierley, Oded Regev, and E.A. Spiegel (1991a). Interacting localized structures with Galilean invariance. Physical Review $A$, vol 44, no 2, preprint.

C. Elphick, E. Meron, J. Rinzel, and E.A. Spiegel (1990a). Impulse patterning and relaxational propagation in excitable media. J. Theor. Biol.,146,249-268.

C. Elphick, E. Meron, and E.A. Spiegel (1990b). Patterns of propagating pulses. SIAM J. Appl. Math.,vol 50 2, 490-503.

C. Elphick, O. Regev, and E.A. Spiegel (1991b). Complexity from thermal instability. Mon. Not. R. Astro. Soc.,259,617-628.

A. Kolmogorov, I. Petrovsky and N. Piscounoff (1937). Study of the diffusion equation with growth of the quantity of matter and its application to a biology problem. Dynamics of Curved Fronts, P. Pelce editor, Academic Press, NY, 1991.

P.B. Rhines and W.R. Young (1983). How rapidly is a passive scalar mixed within closed streamlines? J. Fluid Mech., 133, 133-145.

E.A. Spiegel and S.Zaleski (1984). Reaction-Diffusion instability in a sheared medium. Physics Letters, 106A,7, 335-338.

G.I. Taylor (1953). Dispersion of soluble matter in solvent flowing slowly through a tube. Proc. Roy. Soc. London,A219, 186-203.

W.R. Young, P.B. Rhines and C.J.R. Garrett (1982). Shear-Flow Dispersion, Internal Waves and Horizontal Mixing in the Ocean. J. Phys. Oceanography, 12, 515-517.

W.R. Young and S. Jones (1991). Shear Dispersion. Physics of Fluids A,3(5), 1087-1091. 


\section{Abstracts of Seminars}




\title{
24. Almost Symmetric Solitary Eddies in Two Layer Models
}

\author{
George G. Sutyrin and William K. Dewar
}

P. P. Shirshov Institute of Oceanology, USSR Academy of Sciences, Krasikova No. 23, 117218, Moscow, USSR (GGS), and Dept. of Oceanography, Florida State University, Tallahassee, FL, 32306 (WKD).

\subsection{Abstract}

An asymptotic theory of two-dimensional solitary waves is presented. Previous studies in one and one-half layer models have discovered special classes of radially symmetric structure which are associated with permanent form eddies. We generalize these studies by including an active lower layer and by considering the effects of azimuthal structure. Accordingly, we stress two principle results; namely, (1) permanent form two layer eddies with essentially arbitrary radial structure exist, provided the eddy includes a weak imbedded dipolar asymmetry and an appropriate counter-rotating deep flow, and (2) fluid trapped under an eddy in Taylor columns can significantly affect eddy properties if the trapped fluid possesses non-trivial potential vorticity.

The structural permanency in our solutions arises from a balance between nonlinear steepening, driven by the continuity equation, and planetary dispersion. The structural asymmetries affect eddy propagation, either by dipole interaction within the layer (as occurs in modons) or by pressure forces acting between layers. The primary role of the deep counter-rotating flow is to balance the net upper layer transport. The interesting layer-layer interaction, however, involves higher order dynamics and is sensitive to the continuity of the potential vorticity field. In general, these eddies trap fluid both in the upper thermocline and in the lower layer.

The dominance of oceanic anticyclones over cyclones is relatively well-known. A principle conclusion of this study is that the class of long-lived anticyclones is considerably broader than previously realized. This may help explain the observed bias toward anticyclonic eddies. A second conclusion is that estimates of material transport by eddies may need to account for the movement of fluid outside the main bowl of the eddies. 


\title{
25. Applications of contour dynamics to eddies
}

\author{
Glenn R. Flierl
}

$M I T$

\subsection{Introduction}

The contour dynamics procedure represents the conserved dynamical field, the potential vorticity, as built up from patches of constant value. Thus for an eddy, we have

$$
q=\left(\nabla^{2}+\frac{\partial}{\partial z} \frac{f^{2}}{N^{2}} \frac{\partial}{\partial z}\right) \psi=\sum \Delta_{i}(z) \mathcal{H}\left(r_{i}+\eta_{i}(\theta, z, t)-r\right)
$$

If the dynamics has an invertability principle, as QG dynamics does, the flow can be expressed as a functional of the locations of the boundaries of the patches - the $\eta_{i}$ functionals. But the boundaries are themselves material surfaces so that the evolution equation

$$
\frac{\partial}{\partial t} \eta_{i}=-\frac{1}{r_{i}+\eta_{i}} \frac{d}{d x} \psi\left(r_{i}+\eta_{i}(\theta, z, t), \theta, z, t\right)
$$

can be thought of as an integro-differential equation for the changes in a set of functions $\eta_{i}$. The numerical modelling work solves these equations using various time-stepping and integration formulae. But the basic ansatz of making the PV field piecewise constant can also be of great use in analytical theory as well.

\subsection{Linearized dynamics:}

Here, the contour dynamics viewpoint demonstrates a very direct procedure for turning a stability problem into a standard matrix eigenvalue problem. Linearizing the potential vorticity expression (1) for small displacements of the interfaces gives

$$
q^{\prime}=\sum \Delta_{i} \eta_{i} \delta\left(r-r_{i}\right)
$$

or (inverting and presuming the disturbances have the form $\exp (\imath[\theta-\Omega t]))$ gives

$$
\psi^{\prime}\left(r_{i}\right)=\sum G_{m}\left(r_{i} \mid r_{j}\right) \Delta_{j} \eta_{j}
$$

with $G_{m}$ the Greens function. Linearizing (2) with the exponential form gives

$$
\Omega \eta_{i}=\sum\left[\frac{\bar{V}\left(r_{i}\right)}{r_{i}} \delta_{i j}+G_{m}\left(r_{i} \mid r_{j}\right) \Delta_{j}\right] \eta_{j}
$$


with the basic state vortex velocity given by

$$
\bar{V}\left(r_{i}\right)=-\sum G_{1}\left(r_{i} \mid r_{j}\right) \Delta_{j}
$$

The simplest procedure, then, is to select the set of $r_{i}$ values and the desired velocities $\bar{V}\left(r_{i}\right)$ at these radii. From the radii, we can evaluate the matrix elements on the right hand side of (5) and invert to find the vorticity jumps $\Delta_{i}$. We can then calculate the matrix in (4) and use a standard eigenvalue/ eigenvector package to examine the stability properties. Although the discussion above has neglected vertical structure, it can be incorporated easily (Flierl, 1988). Some examples were given.

\subsection{Nonlinear dynamics:}

For nonlinear calculations, in particulr weakly nonlinear theory, Phil Morrison, George Bell, and I have been studying the Hamiltonian formulation of contour dynamics. Using invertability, we write

$$
\psi(\xi, \theta, z)=\frac{1}{2} \sum \int d z \Delta_{i}\left(z^{\prime}\right) \int d \theta^{\prime} \int_{0}^{\xi} d \xi^{\prime} G\left(\xi, \xi^{\prime}, z, z^{\prime}, \theta-\theta^{\prime}\right)
$$

(Here $\xi=r^{2}$.) One could then find the nonlinear evolution equations for $\xi_{i}$ by Taylorexpanding (6) around $\xi_{i}=r_{i}^{2}$ to some order in amplitude. One then evaluates the resulting streamfunction on the curve $\xi_{j}$, again expanding to a consistent order. Fourier-expanding the boundary displacements leads to integrals which can be done multiplying products of the Fourier amplitudes.

However, we have gone a step further and defined a Hamiltonian

$$
H=-\frac{1}{8} \sum \sum \iint \Delta_{i}\left(z^{\prime}\right) \int_{0}^{\xi_{i}} \iint \Delta_{j}\left(z^{\prime \prime}\right) \int_{0}^{\xi_{j}} G\left(\xi^{\prime}, z^{\prime}, \xi^{\prime \prime}, z^{\prime \prime}, \theta^{\prime}-\theta^{\prime \prime}\right)
$$

For small but finite amplitude motions, the Hamiltonian can be approximated by amplitude and Fourier expansion. The equations of motion,

$$
\frac{d}{d t} \xi_{i}=\left\{\xi_{i}, H\right\}=\frac{4}{\Delta_{i}} \frac{d}{d \theta} \frac{\delta H}{\delta \xi_{i}}
$$

can also be simplified by the Fourier expansion to give

$$
\frac{d}{d t} \xi_{i, m}=\frac{\imath 2 m}{\pi \Delta_{i}} \frac{d H}{d \xi_{i,-m}}
$$


We are using this approach to demonstrate that barotropic instability of a circular vortex is subcritical and to examine the nonlinear interaction between ellipticity and a baroclinic "tilting" mode.

\section{References}

Flierl, G.R. (1988) On the instability of geostrophic vortices. J. Fluid Mech., 197, 349-388. 


\section{Surface Tension}

Joseph B. Keller

Dept. of Math. and Mech Eng., Stanford University

Some problems involving surface tension are treated. First the equilibrium free surface of a wedge-shaped region is considered in the absence of gravity. It is shown that the height of the free surface at the corner tends to infinity as the wedge angle decreases to critical value dependent upon the contact angle. This result is extended to the case of different contact angles on the two walls of the wedge. Then the motion of this liquid and its interface is examined when the liguid is released from a non-equilibrium state. Next the macroscopic equations governing surface tension phenomena are derived from a molecular theory. The usual terms involving the mean curvature of the liquid surface are obtained, together with correction terms depending upon second derivatives of the curvature. The corresponding boundary behavior, where the liquid surface meets a solid boundary, is considered also. 


\section{Molecular Dynamics of Interfaces and Spreading Drops}

Joel Koplik

Levich Institute, City College of New York

We have used molecular dynamics simulations of 4000-20,000 Lennard-Jones molecules to study several situations of interface rupture and coalescence, and of drop spreading on solid surfaces. The interface rupture phenomenon studied, the Raleigh instability of a liquid thread and the detachment of a pendant drop from a solid support by gravity, behave in qualitative accord with continuum calculations despite the nicroscopic size and time scales of the systems. Bulk quantities such as rupture time and detached mass are given to order of magnitude by estimates using macroscopic parameters, but local information such as velocity and stress fields are too noisy to resolve. The spreading of liquid drops on solid surfaces (with molecular structure) exhibits the full range of spreading mechanisms as the solid-liquid interaction strength varies. We see partial wetting, complete wetting and terraced spreading as the interaction strength increases. The dynamics are not in agreement with estimates based on lubrication hydrodynamics or other models of layering. (Work done in collaboration with Ju-Xing Yang and Jayanth Banavar.) 


\section{Influence of Periodic and Stochastic Modulation on Transcritical and Flip Bifurcations in Iterated Maps}

Stefan J. Linz

Department of Engineering Sciences and Applied Mathematics, Northwestern University, Evanston IL 60208

Since R. May's seminal article (Nature 261, 459 (1976)) the study of iterated one dimensional maps $x_{n+1}=f\left(x_{n} ; r\right)$ attracts growing interest. From the biological point of view such maps are the most simple nontrivial models describing the evolution of populations $x_{n}$ with non-overlapping generations where all external influences on the growth of $x_{n}$ are absorbed in the control parameter $r, n$ represents a discretized time. Usually $r$ is assumed to be constant in time. For the evolution law there are several phenomenological models discussed in the literature, the most prominent ones are

(a) Logistic Map, i.e. $f(x, r)=r x(1-x)$, and

(b) Ricker Map, i.e. $f(x, r)=x \exp (r(1-x))$.

Typical for both systems is the existence of stable fixed points for low $r$, the bifurcation of period-doubling solutions when $r$ is increased, and chaotic behavior for large $r$.

This abstract briefly reviews the effects of time dependent variations of the control parameter $r$ according to $r \rightarrow r_{n}=r\left(1+\Delta \zeta_{n}\right)$ and the resulting changes of the structure of the first bifurcation in these systems. Here $\Delta$ measures the strength of the perturbations and $\zeta_{n}$ is a normalized periodic or random variable. Rather detailed investigations can be found for the logistic map in the paper by Linz and Luecke (1986) and for the Ricker map in two recent publications by Kornadt, Linz and Luecke (1991). Let us first of all note that the structure of the first bifurcation differs in system (a) and (b) already without perturbations. The logistic map first undergoes a transcritical bifurcation, i.e. exchange of stability of two existing fixed points, at $r=1$, whereas the first bifurcation in the Ricker map at $r=2$ is a flip bifurcation, i.e. a fixed point becomes unstable towards a period two limit cycle.

Some of the main effects of parametric perturbations are:

(1) The basic fixed point, i.e $\bar{x}=0$ for the logistic map and $\bar{x}=1$ for the Ricker map, remain fixed points of the modulated system.

(2) For small perturbation strength $|\Delta|$ the stability of the fixed points as function of $r$ is enhanced universally. The stabilization is proportional to $\Delta^{2}$ for small $|\Delta|$. For large $|\Delta|$ there is a destabilization. For given noise distributions the stability thresholds can 
be determined analytically for all $\Delta$. Details depend on the model and the probability distribution of the $\zeta_{n}$.

(3) In the case of the logistic map, small parametric noise leads above the stability threshold to a probability distribution of $x_{n}$ roughly centered about $1-1 / r$, the second fixed point of the unperturbed system. In comparison to this fixed point the mean value of the distribution is lowered due to noise. Analytical techniques were used to calculate the first two moments and the shape of the distribution for small $|\Delta|$. Finally it was shown that additive coupling of noise totally destroys the bifurcation structure at the $r=1$.

(4) In the case of the Ricker map we also studied the dependence of the bifurcation structure for periodic modulation. Depending upon $\Delta$ and whether the period is even or odd, harmonic or subharmonic bifurcations are generated. Stochastic modulation with small amplitudes lead above the stability threshold of $\bar{x}=1$ to a probability distribution of $x_{n}$ which consists of two distinct single hump contributions. They are centered roughly about the period two fixed points of the unperturbed Ricker map. Introducing the mean width of the pitchforklike bifurcation structure as order parameter, we could show that parametric noise reduces the growth of this order parameter in comparison to the unmodulated case. The response to aperiodic modulation, e.g. $\zeta=\cos n$, is quite different from the results for stochastic modulation.

\section{References}

S.J. Linz and M. Luecke, Phys.Rev.A., 33, 2694 (1986).

O. Kornadt, S.J. Linz, and M. Luecke, Phys.Rev.A, 44, 940 (1991), and to be published. 


\section{Three-dimensional, quasigeostrophic vortices}

Steve Meacham

$M I T$

Using the quasigeostrophic approximation, we examine the behavior of a three-dimensional vortex in a continuously stratified fluid of infinite extent. We consider the case in which the buoyancy frequency, $N$, is spatially uniform and model the vortex as a singly-connected blob, $D$, of uniform perturbation potential vorticity, $q$. In the absence of an ambient flow, the flow field is given by a streamfunction, $\psi$ satisfying

$$
\begin{aligned}
\left(\partial_{x}^{2}+\partial_{y}^{2}+\frac{f^{2}}{N^{2}} \partial_{z}^{2}\right) \psi & =q, & & \mathbf{x} \in D \\
& =0, & & \mathbf{x} \notin D
\end{aligned}
$$

We can make the left-hand operator isotropic by stretching the $z$ coordinate by a factor $f / N$. As a result, for oceanic values of $N$, a sphere in the stretched coordinates is really a very shallow lense in physical coordinates.

Non-spherical, steadily rotating equilibria

Beginning with the spherical case, we can obtain numerically a series of families of steadily rotating equilibria, each family having a particular azimuthal symmetry (Meacham, 1990). Some vertically symmetric examples of these are illustrated in Figure 15.1. The sizes of these vortices are normalized by requiring that the area of the horizontal section through the middle of the vortex, $z=0$, be $\pi$, the same as the area of the mid-section of a unit sphere. For each vertically symmetric series, the shape of the mid-section is non-circular and so we can find a maximum radius, $R_{m}>1$. We define a bifurcation parameter

$$
\mu=R_{m}-1
$$

As $\mu$ increases, the $n$-fold azimuthally symmetric states with $n>2$ eventually develop cusp in the plane of the mid-section, rather like the $\mathrm{V}$-states found by Zabusky and co-workers in 2-D Euler flow. As $\mu \rightarrow 0$, each series tends to the spherical limit. For small $\mu$, the equilibria can be approximated by a sphere plus a linear superposition of a small number of the normal modes of the sphere. 

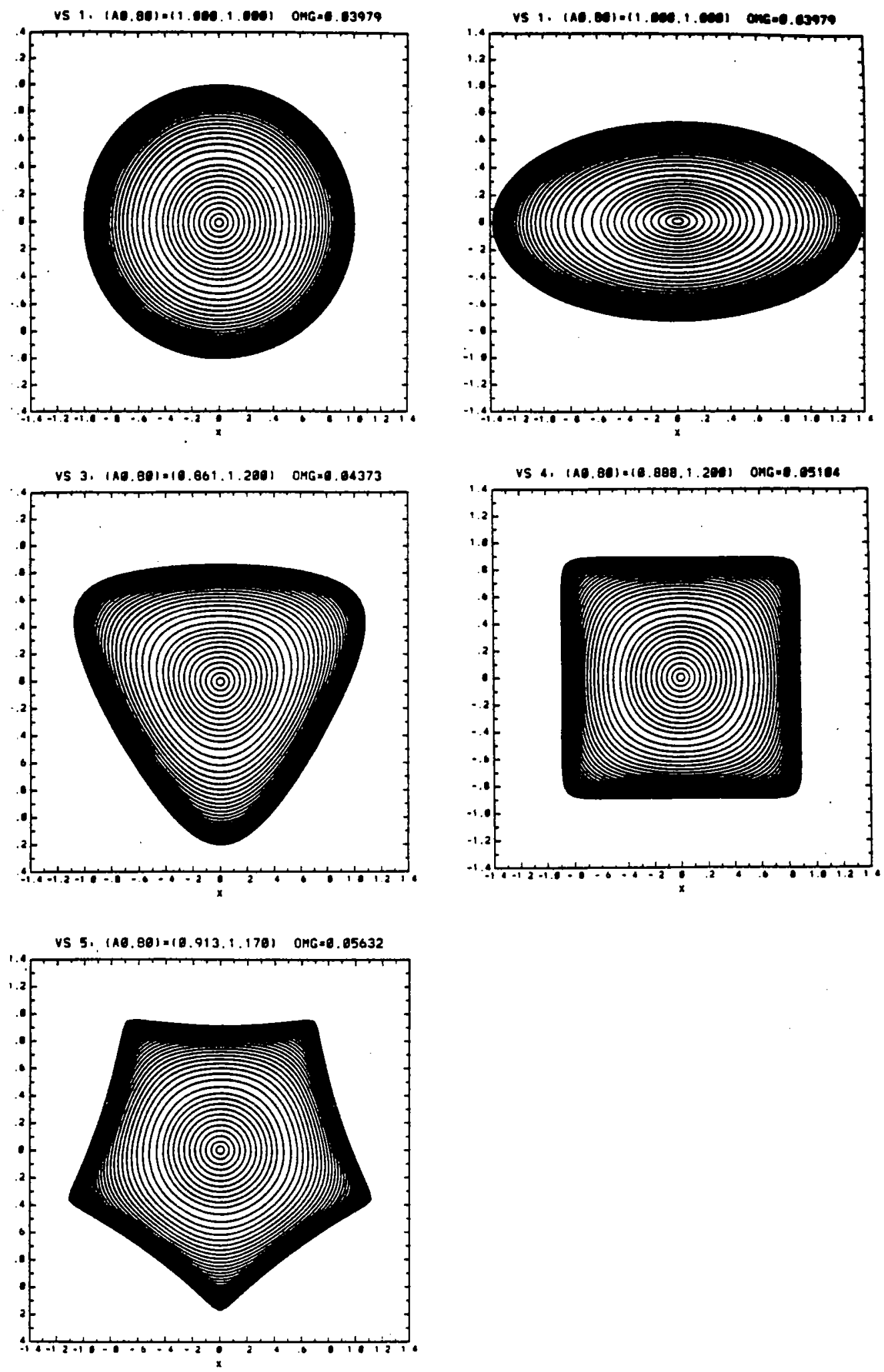

Figure 29.1. Examples of rotating equilibria with azimuthal symmetries of $\infty, 2$, 3,4 and 5. Contours are isolines of surface elevation as seen from above.

For the series illustrated in Figure 15.1, in the limit of small $\mu$, the vortices can be represented as the superposition of a unit sphere, a single normal mode at small amplitude and higher order corrections. 


\section{Weakly nonlinear perturbations to a sphere}

The normal modes of the sphere are all neutral and the sphere itself is linearly stable (a result which can be obtained by applying a theorem due to Dritschel, 1988.) We analyzed the stability of small $\mu$ equilibria by considering weakly nonlinear perturbations to the sphere in which two or three normal modes (one of which, when combined with the sphere, represented the leading order structure of a particular, steadily rotating equilibrium) were allowed to interact resonantly. From this analysis we discovered that the combination of the sphere plus a small amplitude, vertically symmetric mode with an even number of azimuthal lobes was unstable to a mode with half that number of azimuthal lobes (and a different vertical structure.) The implication of this is that the members of those families of vertically symmetric, steadily rotating equilibria that have an even number of azimuthal lobes are unstable even in the limit of small $\mu$, i.e. the almost-spherical limit, and that the growth rate of these instabilities should scale with $\mu$. The weakly nonlinear analysis also showed that the four-lobed, nearly spherical equilibrium was unstable to a resonant triad interaction.

In both types of instability, the nonlinear system formed by the amplitude equations for the modes in the pair or triad is bounded and the long term evolution is a periodic vacillation. The interpretation as instability comes from considering the other member(s) of the pair (triad) to be initially present as small amplitude perturbations to the mode that represents the non-axisymmetric part of the equilibrium. Consequently, we expect that the corresponding instabilities of the steadily rotating vortices (in the small $\mu$, near-spherical limit) to equilibrate at a moderate amplitude and result in a vortex with time-dependent shape, rather than grow to such large amplitudes that they break up the vortex. This can be clarified by a simple example. The vertically symmetric, two-fold symmetric steadily rotating solutions have an elliptical cross-section (in fact, they are ellipsoids, with a vertical axis of a length intermediate between those of the two horizontal axes ) and, since they have an even number of lobes, are unstable to an azimuthal sub-harmonic. The latter is a vertically anti-symmetric mode and corresponds to tilting the vertical axis of the ellipsoid away from the vertical. As this unstable mode grows, the tilt from vertical increases. At the same time, the nonlinear interaction of this perturbation with itself feeds back on the amplitude of the ellipsoidal deformation and weakens it so that horizontal sections become more circular. The amplitude of the ellipsoidal deformation and the tilting are coupled in a vacillation cycle so the increase in vertical tilt eventually stops, reverses and the vortex becomes more upright and more elliptical once more. This sequence just corresponds to an elliptical vortex that was initially slightly tilted from the vertical, wobbling periodically about an axis tilted from the vertical. 
FREQ VS. ROIFF

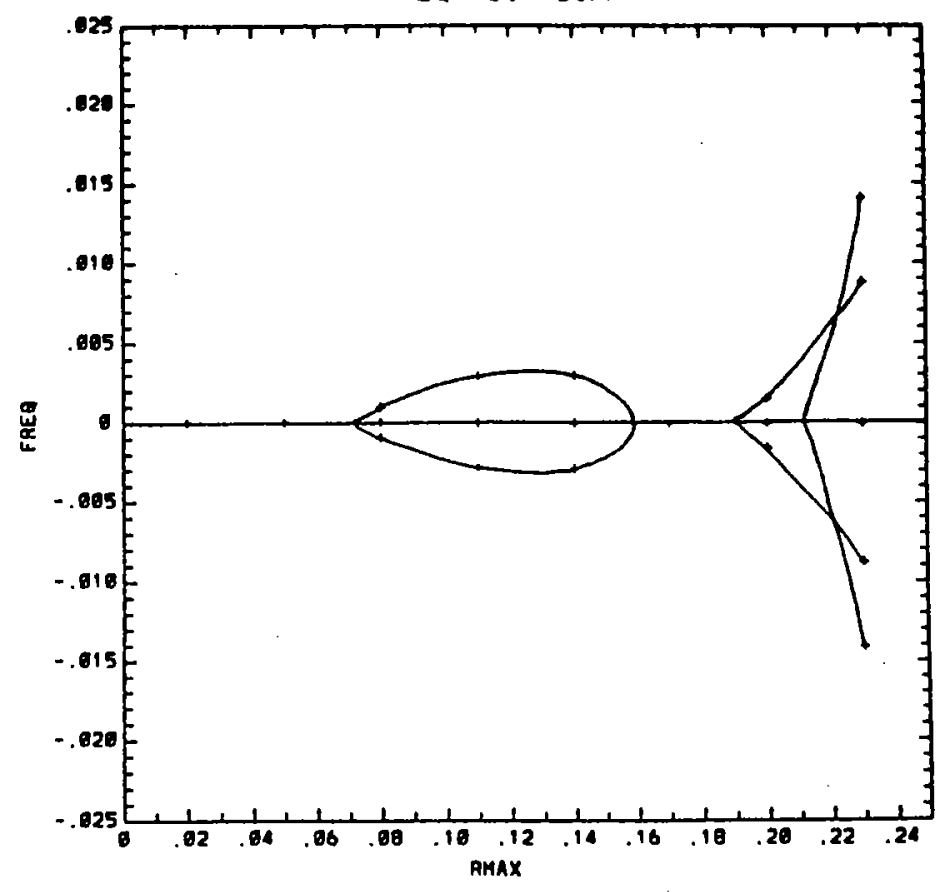

(a)

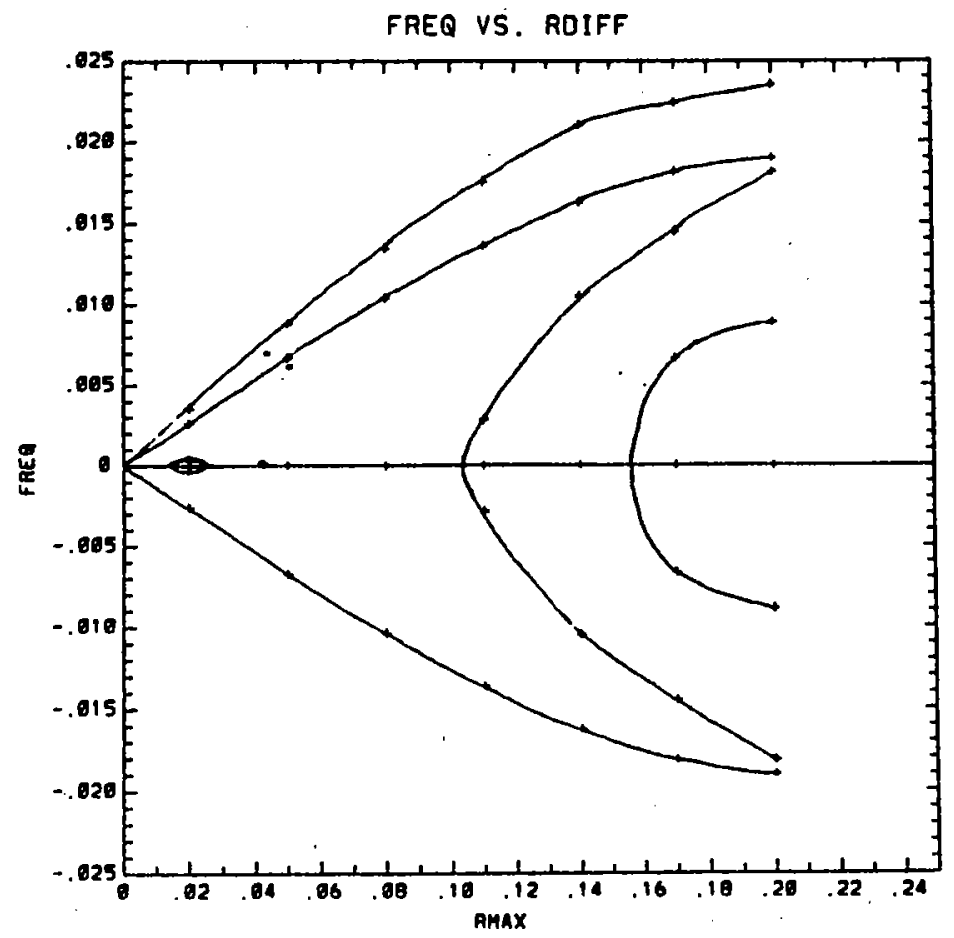

(b)

Figure 29.2. Growth/decay rates of complex eigenmodes on rotating equilibria.

(a) - threefold symmetric, (b) - fourfold symmetric.

Eigenmodes of non-spherical equilibria

These results from weakly nonlinear theory should be valid for small $\mu$. The eigenfrequencies 
of the normal modes of individual members of the series on steadily rotating equilibria can be computed numerically for general $\mu$. Eigenfrequencies for members of the 2-, 3-, and 4-fold azimuthally symmetric series are shown in Figure 15.2. At small $\mu$, members of the 2- and 4 -fold symmetric series are unstable to the respective azimuthal subharmonic as predicted by the theory described above. In addition, members of the four-fold symmetric series have two other unstable modes (with equal growth rate) at small $\mu$ which correspond to two sidebands in a resonant triad (the third element of the triad being the 4-fold symmetric distortion that gives the vortex equilibrium shape.) In contrast, members of the 3-fold symmetric series are stable to low order modes for $0<\mu<\sim 0.06$

(a)
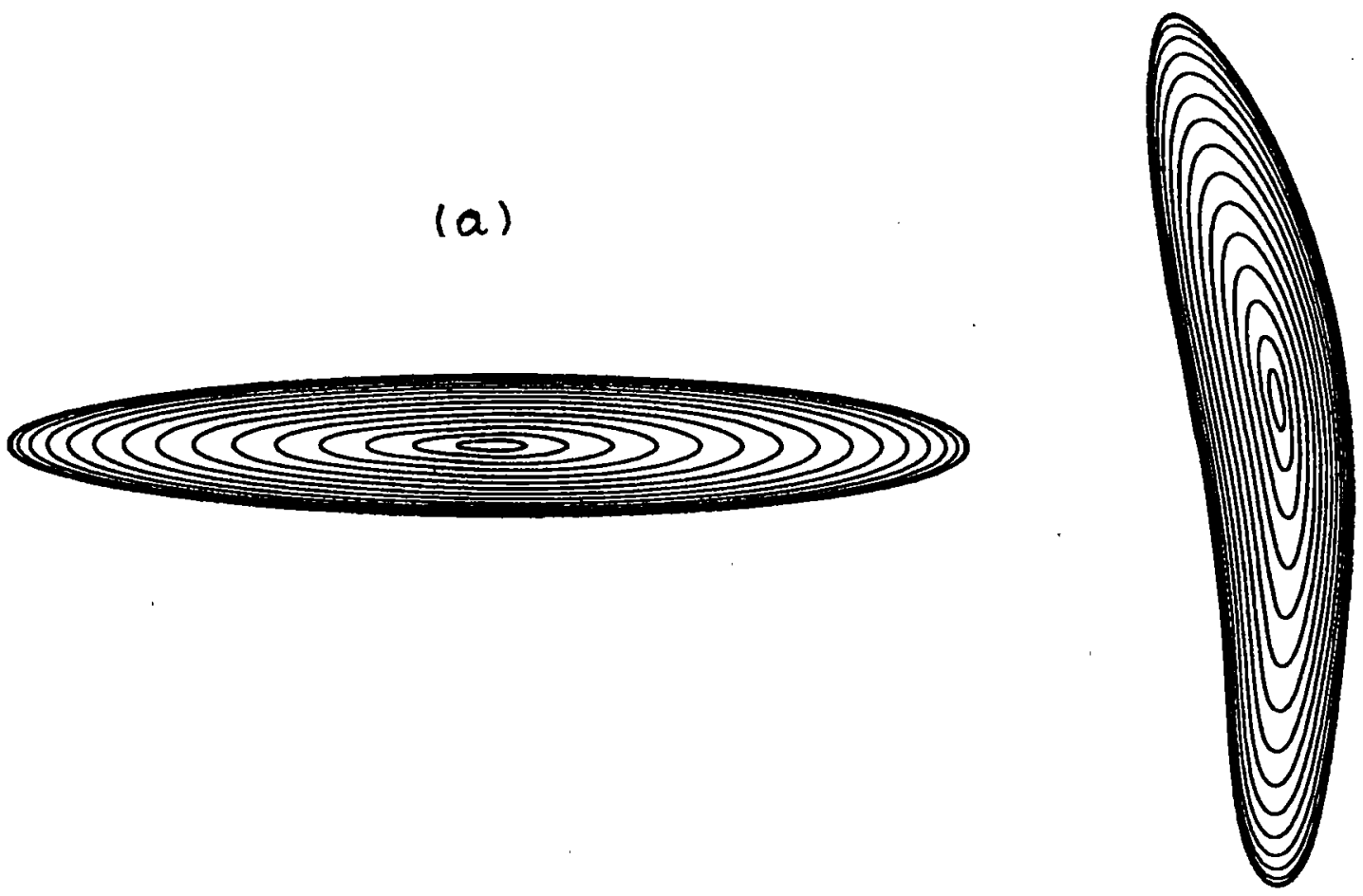

(b)

\section{(c)}
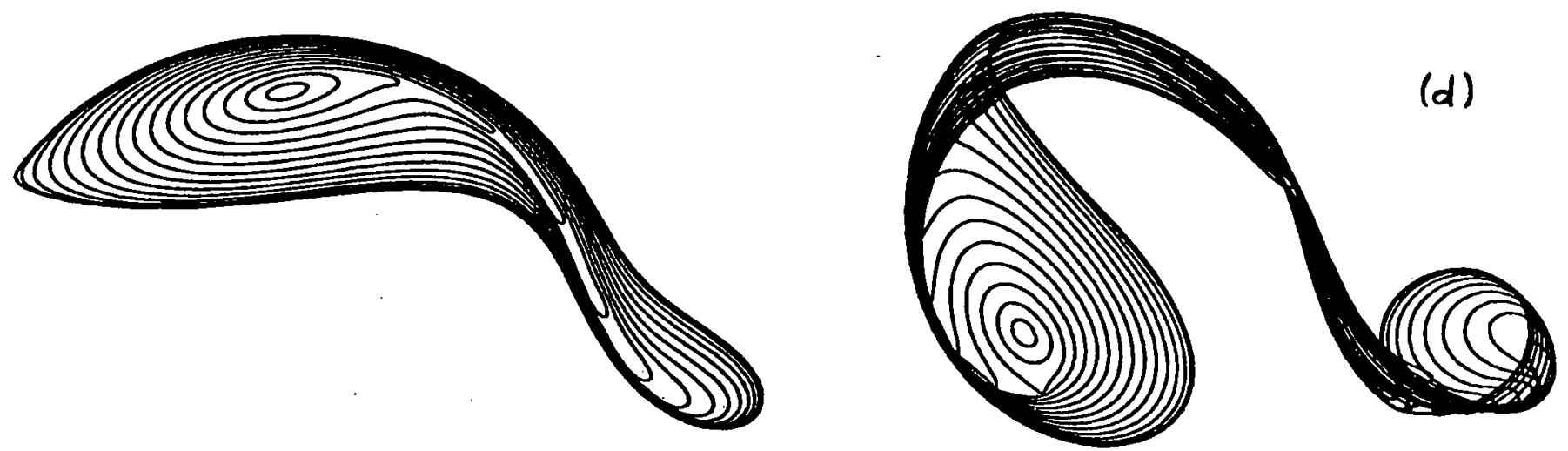

Figure 29.3. Numerical simulation of the growth of an unstable perturbation to an ellipsoid. The ellipsoid is viewed from above. 


\section{Ellipsoidal vortices}

One can show that ellipsoidal blobs of uniform vorticity constitute exact, steadily rotating equilibria (Zhmur, 1989; Meacham, 1990) Moreover, in analogy with Kida's result for the Kirchhoff ellipse (Kida, 1981), they remain exact solutions in the presence of horizontal shear and strain flows with uniform vorticity (Zhmur and Pankratov, 1989), vertically sheared flows (Zhmur and Shchepetkin, 1991) and in a background flow with a general, quadratic streamfunction (Meacham et al., 1991) In all three types of background flow, the geometric parameters (horizontal and vertical aspect ratios) of the ellipse, as well as the rotation rates, are time-dependent. In the latter type of flow the range of possible behavior is quite complicated as both horizontal and vertical shears are present. Nevertheless, provided that these shears are not too strong, a range of initially ellipsoidal vortices will remain ellipsoidal.

The stability of ellipsoidal vortices in the absence of background flows can be examined analytically (Meacham, 1991) using a technique similar to that of Love (1893). When one axis if the vortex is vertical, we find that nearly spherical vortices are unstable if the vertical axis has a length intermediate between those of the other two. This instability just corresponds to a wobbling motion around the vertical direction of the sort alluded to above. Vortices with more elliptical horizontal cross sections have additional unstable modes, some of which do not appear to equilibrate at finite amplitude but instead grow until vorticity is pinched off from the main vortex. Figure 15.3 shows a numerical example of this. The stability of some of the time-dependent, ellipsoidal solutions that occur in the presence of background shear is discussed in Meacham et al. (1991) In particular, in horizontal strain flows, solutions in which one axis of the vortex is vertical - analogues of the nutating and tumbling solutions in the 2D Kida problem - are frequently unstable to perturbations that tilt that axis away from the vertical.

\section{References}

Dritschel, D.G. (1988) J. Fluid Mech. 191, 575-581.

Love, A.E.H. (1893) Proc. Lond. Math. Soc. 25, 18-42.

Meacham, S.P. (1990) In Topological fluid mechanics: Proceedings of the IUTAM Symposium. Eds.H.K. Moffatt and A. Tsinober. Cambridge University Press, Cambridge, pp805.

Meacham, S.P. (1991) Dyn. Atmos. and Oc., 16

Meacham, S.P., Pankratov, K.K., Shchepetkin, A.F. and Zhmur, V.V. (1991) In preparation Zhmur, V.V. (1989) Okeanologia.

Zhmur, V.V. and Pankratov, K.K. (1989) Okeanologia 29 205-211.

Zhmur, V.V. and Shchepetkin, A.F. (1991) Okeanologia 31. 


\title{
30. The merger of three-dimensional, quasi- geostrophic vortices
}

\author{
K. K. Pankratov \\ Center for Meteorology and Physical Oceanography, MIT
}

In this work we study strong interaction of baroclinic quasigeostrophic (QG) vortices in a continuously stratified ocean (on f-plane with constant buoyancy frequency $\mathrm{N}$ ). Such process of interaction of two like-signed vortices was recently a subject of many investigations in two-dimensional or two-layer QG models as well as numerous laboratory experiments. It was observed that two like-signed vortices merge into a bigger one when put sufficiently close together and this tendency to merge is very robust in the sense that it does not depend significantly onparticular internal structure of the vortices, viscosity, etc.

An important parameter in the merger process is a critical intercentroid separation in the initial configuration of vortices - a threshold below which merger does occur.

Merger process can not happen in a system of two point vortices because they lack internal degrees of freedom and can only rotate around each other at a constant distance. Instead, a suitable analytical model to study the merger (only its initial stage) used in 2D was that of two Kirchoff elliptical vortices (Melander et al., 1986). Kirchoff vortex remains exactly elliptical in time-dependent flow with velocities linearly depending on coordinates (Kida, 1981). So that linear approximation of velocity, induced by one vortex in the vicinity of another can be used to study their interaction. The elliptical (or moment) model captures main features of initial tendency to merge: initially circular vortices become deformed and oriented with their bigger semiaxes toward each other so that their vorticity cores become effectively closer. Then they exchange their vorticity and merge which is beyond the ability of the moment model to describe. But still the critical intercentroid sepa! ration in the moment model (3.2r, In three-dimensional QG case (f-plane, $N=$ const) there is also an exact solution for ellipsoidal region of constant potential vorticity similar to elliptical vortex in 2D (Zhmur and Pankratov, 1989, Meacham, 1991.) In a barotropic background flow with linear of coordinates velocities it remains ellipsoidal and behaves similar to the Kirchoff vortex, only self-rotation rate is different and depend on a vertical axis of the ellipsoid (as well as on the horizontal axes ratio). For such vortices an analogous moment model can be constructed. For initially circular (spheroidal) vortices the critical merger distance can be calculated using an energy and angular momentum conservation (and a symmetry of the system). That is at each moment of time following relations must be satisfied: 
For energy:

$$
\frac{1}{R}+\frac{r^{2}}{10 R^{3}}\left[\nu-3 \tilde{\nu} \cos (2 \phi-2 \theta)-2 \mu^{2}\right]+E=\frac{1}{R_{0}^{3}}+\frac{r^{2}}{10 R_{0}^{3}}\left(2-2 \mu^{2}\right)+E_{0}
$$

where

$$
E=\frac{3}{5 r} \int_{0}^{\infty} \frac{a b c}{\sqrt{\left(a^{2}+\tau\right)\left(b^{2}+\tau\right)\left(c^{2}+\tau\right)}} d \tau
$$

is the energy of an isolated vortex (without presence of another).

(Here also a,b,c - semiaxes of the ellipsoid, $r=\sqrt{a b}, \mu=c / r, \nu=a / b+b / a, \tilde{\nu}=a / b-b / a$, $\mathrm{R}$ is the distance between the centers of the vortices.)

For angular momentum:

$$
R^{2}+\frac{4}{5} r^{2} \nu=R_{0}^{2}+\frac{8}{5} r^{2}
$$

From these two equations the critical merger distance is obtained, its dependence on parameter $\mu$ (vertical extent of the vortex) is shown in figure 1 .

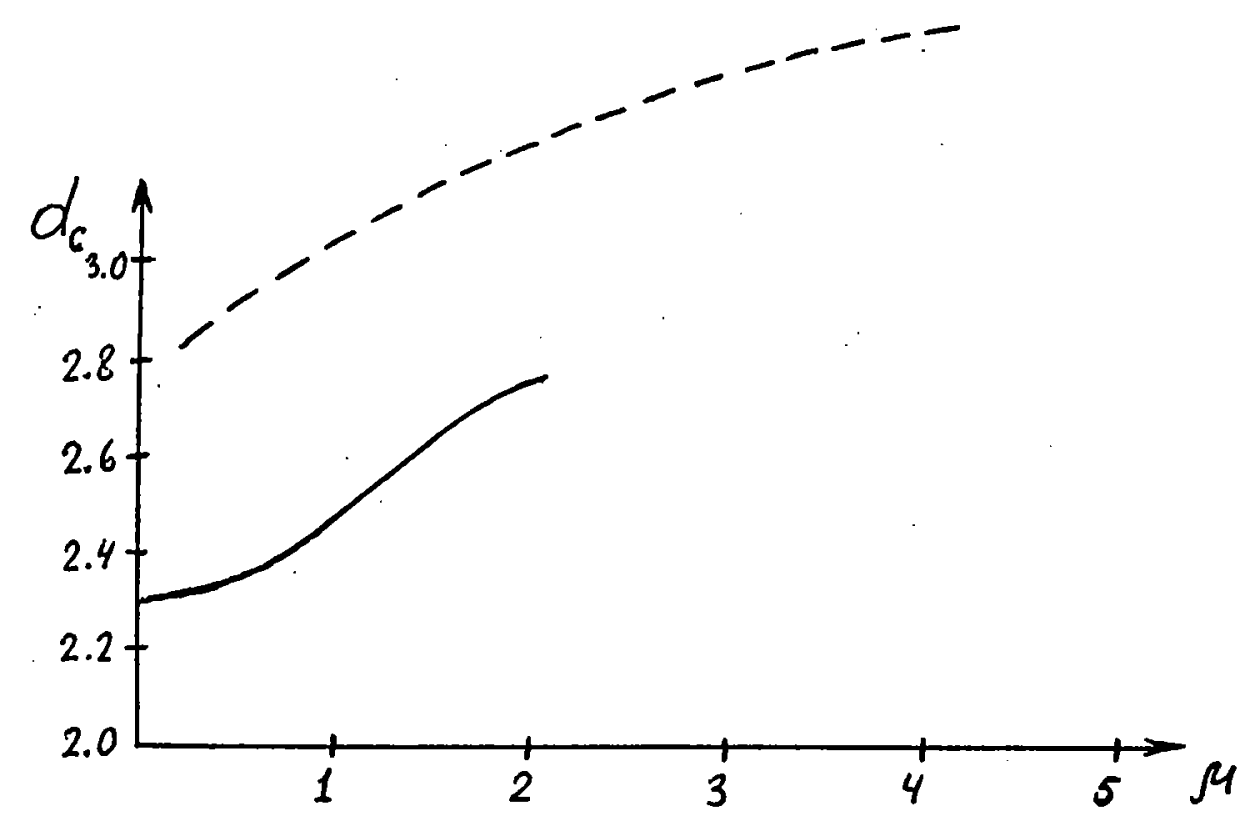

Figure 30.1. Critical merger distance for the ellipsoidal model (solid line) and for CD simulations

To verify the results of the moment model numerically a multi-level contour dynamics simulations were carried out (numerical code was developed by S. Meacham). Results of these simulations confirmed a tendency of the critical merger distance to increase with growth of parameter $\mu$ (barotropization of the vortices). But in numerical simulations we found an important process which is not present in 2D and not captured by the ellipsoidal model. This is the process of angular momentum transfer vertically. Because different levels rotate with unequal angular velocities after certain time centroids at different levels become relatively 
displaced so that the mid-levels of two vortices become effectively clother together while the upper (and lower) levels - further apart. Thus the angular momentum is transferred from the mid- to outer levels. This process enhances merger which occurs first at mid-levels. Because of this process the critical merger distance in the numerical simulations is significantly larger th

One example of numerical simulations is shown in figure 2 (only the positions of mid- and uppermost level are shown). Our results therefore suggest the importance of these baroclinic processes and 3D structure for the interaction of the vortices in the oceanic and atmospheric applications.
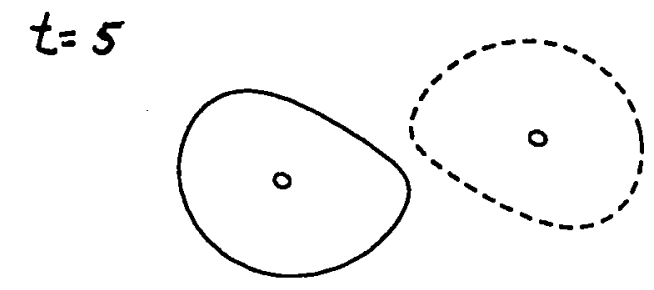

15

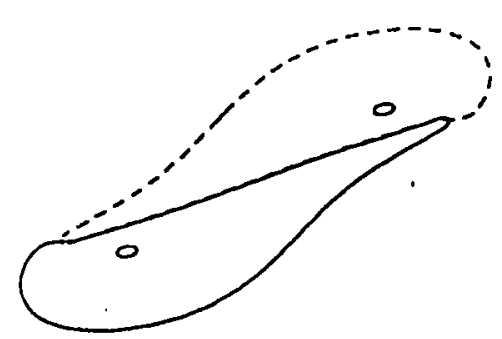

30

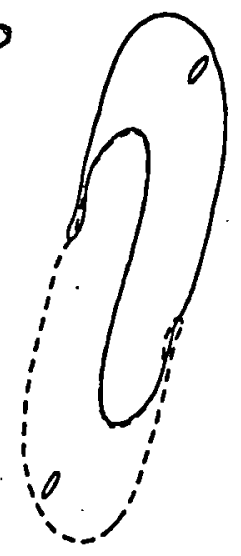

50

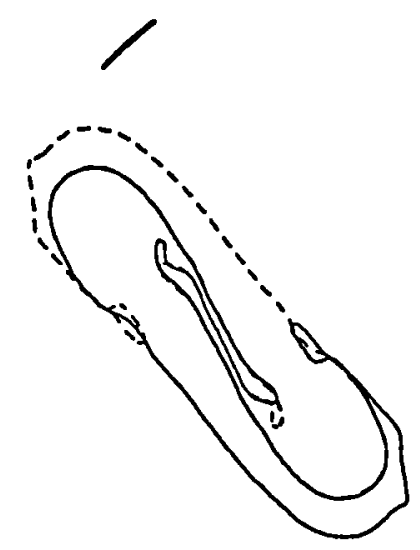

Figure 30.2. Example of the evolution of two merging vortices. Initial separation $R_{0}=2.6, m=1$.

\section{References}

Kida S., 1981. Motion of an elliptic vortex in iniform shear flow. J. Phys. Soc.Japan, 50, (10) 3517-20.

Meacham S. P., 1991. Ellipsoidal vortices in a stratified ocean. Dyn. Atmos. Oceans, 16.

Melander M. V., Zabusky N. J., Styczec A. S., 1986. A moment model for vortex interaction of the two-dimensional Euler equations. Part 1. Computational validation of a Hamiltonian elliptical representation. J. Fluid Mech., 167, 95-115.

Zhmur V. V., Pankratov K. K., 1989. Dynamics of hemiellipsoidal surface vortex in a nonuniform flow. Oceanologia, 29, (2) 205-211 (in Russ.). 


\title{
31. Development of Axisymmetric Singular solutions to the incompressibles 3-d Euler equations
}

\author{
Alain Pumir and Eric D. Siggia \\ Laboratory of Atomic and Solid State Physics, Cornell University, Ithaca, \\ N.Y. 14853-2501
}

\subsection{Abstract}

In an effort to understand how do the rate of strain tensor and the vorticity couple together to produce large, possibly divergent gradients of the velocity field in the 3d Euler equations, axisymmetric flows have been simulated numerically. A finite difference, adaptive mesh code has been used in this work.

Away from the axis of symmetry, a strong similarity exists between the 3d Euler equations and the $2 \mathrm{~d}$ equations of convection in the Boussinesq approximation. In the Euler case, the azimuthal velocity plays the role of temperature : $\theta \rightarrow\left(r u_{\phi}\right)^{2}$, and the 'gravity' depends upon $r$ as $1 / r^{3}$. This analogy with Boussinesq convection becomes quantitatively better as one works further away from the symmetry axis.

In the presence of a swirl, the evolution leads to the formation of sharp gradients of the azimuthal velocity field, at the leading edges of 'hot bubbles' (i.e., regions with localized azimuthal velocities) and to spirals rolling up on their trailing edges. The tips of the bubbles eventually undergo 'tip-splitting' instabilities, thus leading to smaller and smaller radii of curvature. The latter instability is essential for generating diverging gradients, as we have observed in the Boussinesq case. There, the gradients of the temperature field grow approximatly like $1 /\left(t^{*}-t\right)^{2}$. We have observed an amplification of the gradients of the order $10^{4}$, compatible with this asymptotic law. In the case of axisymmetric flows, it corresponds to a growth of the vorticity $\|\omega\|_{\infty} \sim 1 /\left(t^{*}-t\right)$. The velocity field remains finite close to the singularity.

Analytic estimates for the rate of growth of the gradients can be derived, and provide a semi-quantitative explanation of our numerical results. 


\section{Zero Prandtl Number Convection}

Olivier Thual $N C A R$

The zero Prandtl number limit $(P=0)$ of the Oberbeck-Boussinesq equations is compared to small Prandtl number Rayleigh-Bénard convection through numerical simulations. Both no-slip and free-slip boundary conditions, imposed at the top and bottom of a small aspect ratio, horizontally periodic box are considered. A rich variety of regimes are observed as the Rayleigh number is increased: supercritical oscillatory instabilities for various values of the aspect ratios, competition between 2D rolls, squares and hexagonal patterns, competition between traveling and standing waves, transition to chaos, and scaling laws for the first Rayleigh number decade. This multiplicity of regimes can be attributed to the close interaction between the stationary and oscillatory instabilities at zero Prandtl number.

(a)
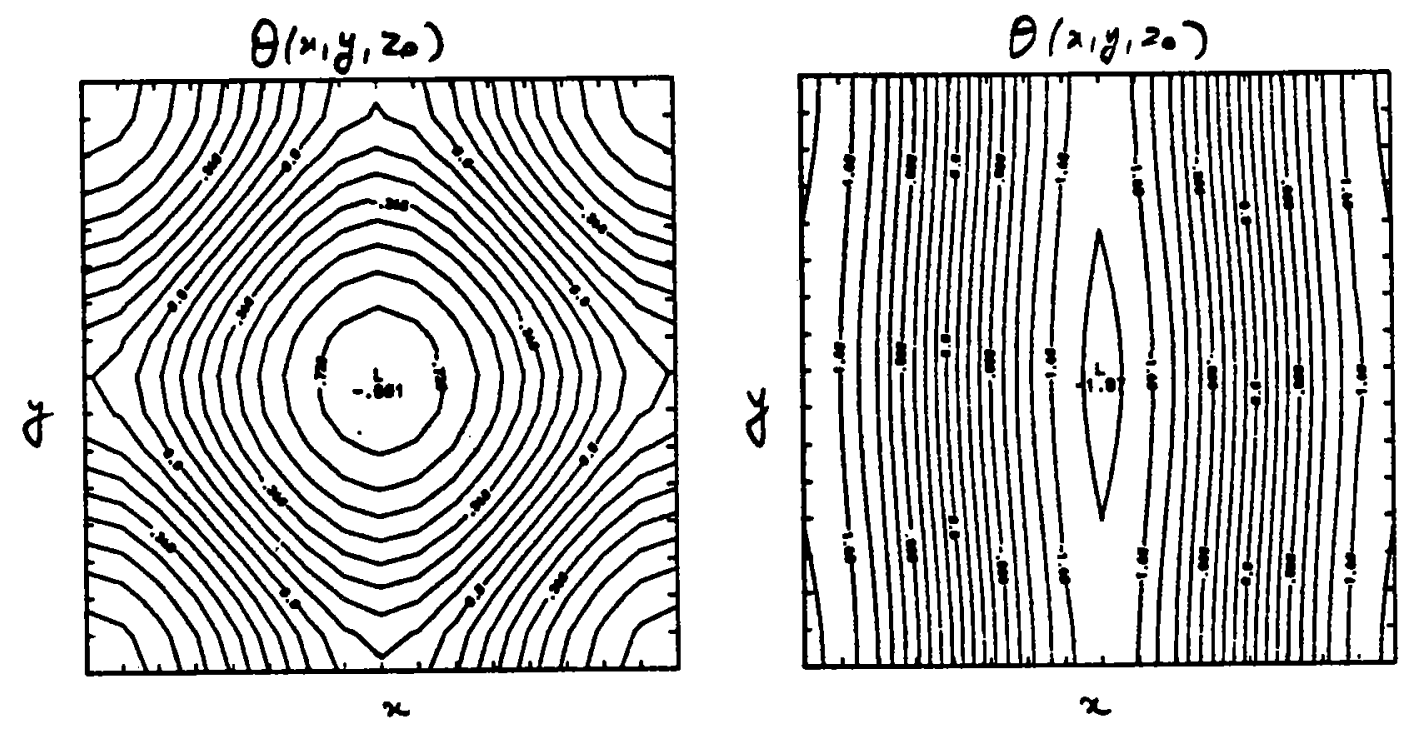

Figure 32.1. Relaxation oscillations regime (SQOR) with basic square pattern for free-slip boundary conditions, $P=0, R=700$ and $k_{x}=k_{y}=\pi / \sqrt{2}$. (a) Horizontal contours of $\theta$ at $z_{0}=1 / 2$ during the quiescent periods. (b) The same contour during a burst. 

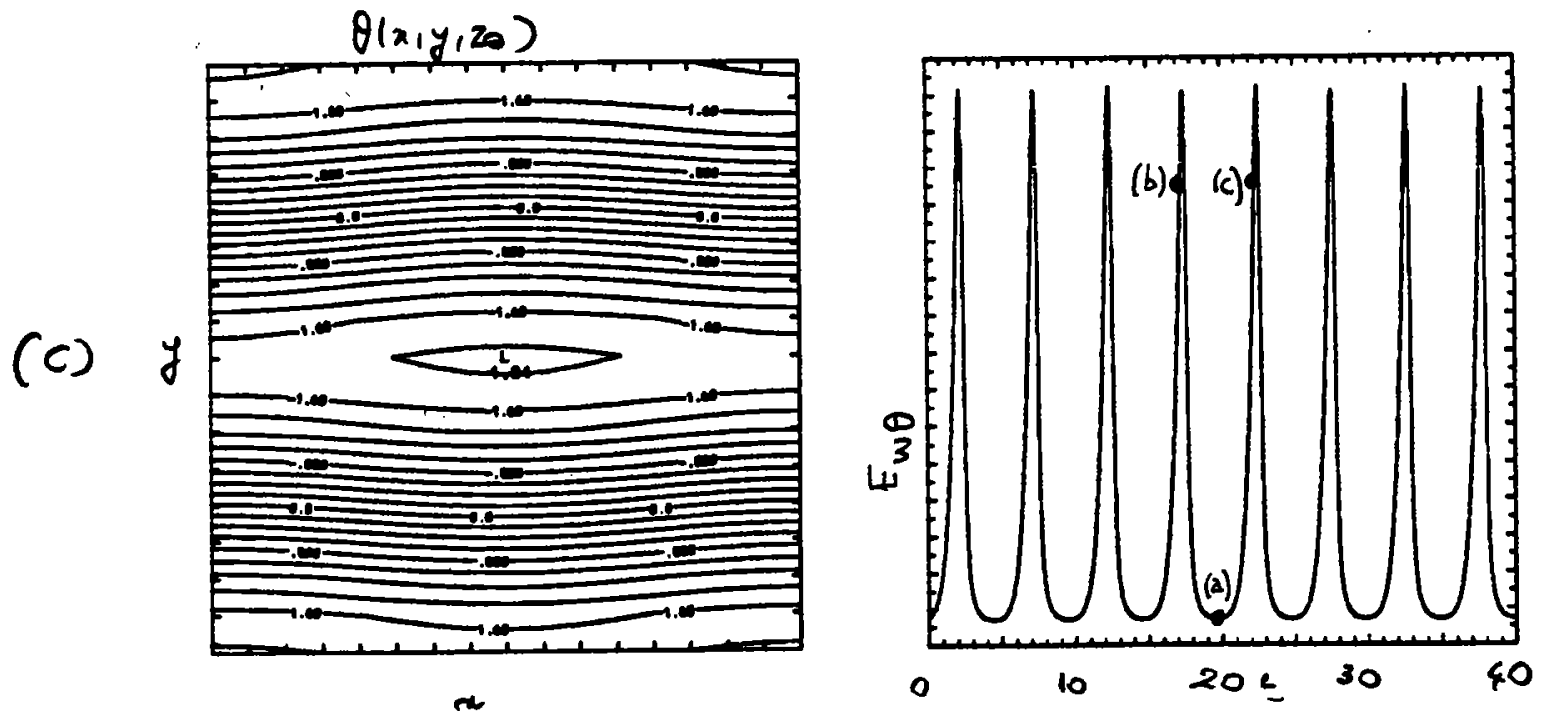

(d)

Figure 32.2. As in preceding figure, (c) The same contour during the next burst. (d) Time evolution of $E_{w \theta}$.

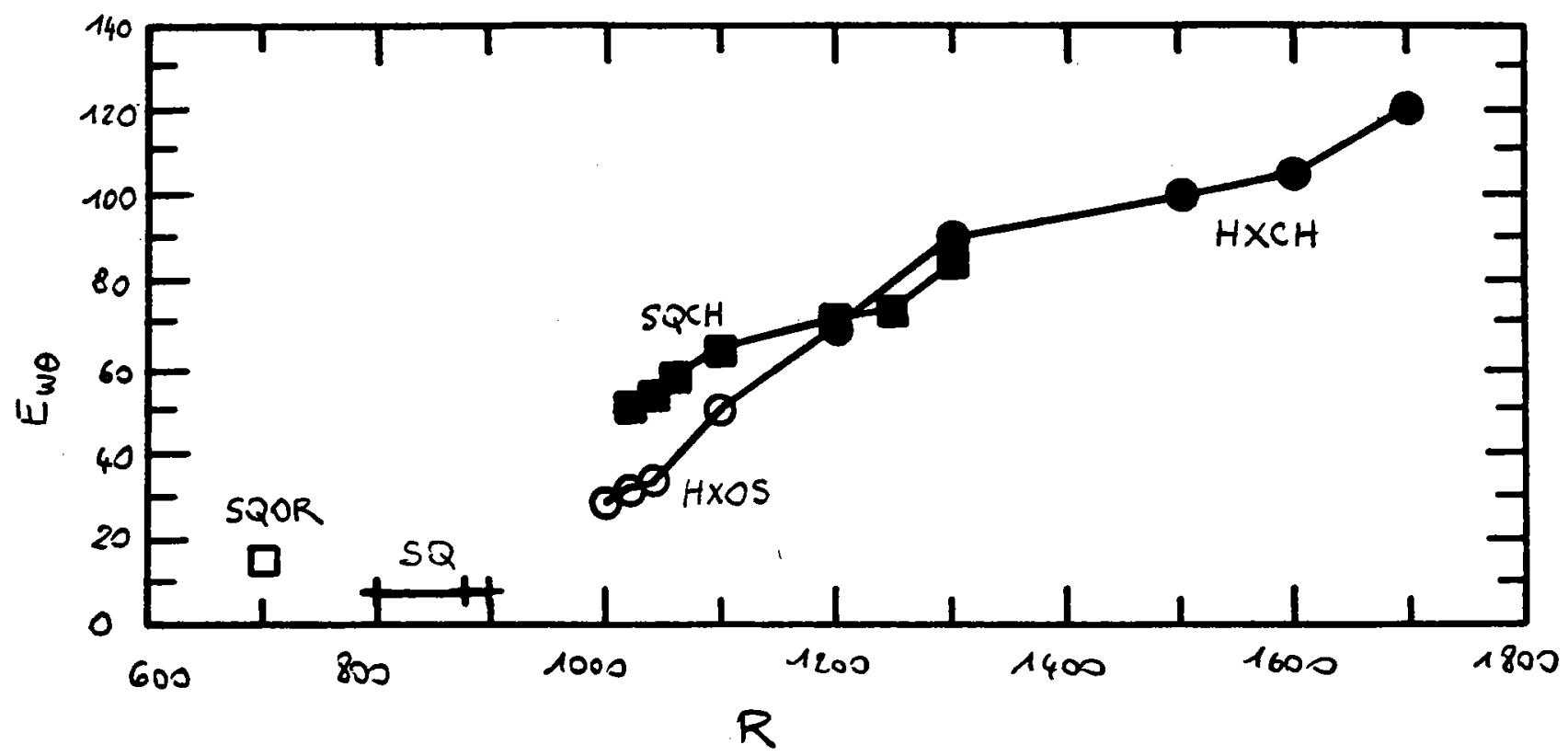

Figure 32.3. Partial bifurcation diagrams for free-slip boundary conditions, $P=$ 0 and $k_{x}=k_{y}=k_{c}$. Regimes: stationary squares (SQ), relaxation oscillating squares (SQOR), chaotic squares (SQCH), oscillating hexagons (HXOS), and chaotic hexagons (HXCH). 


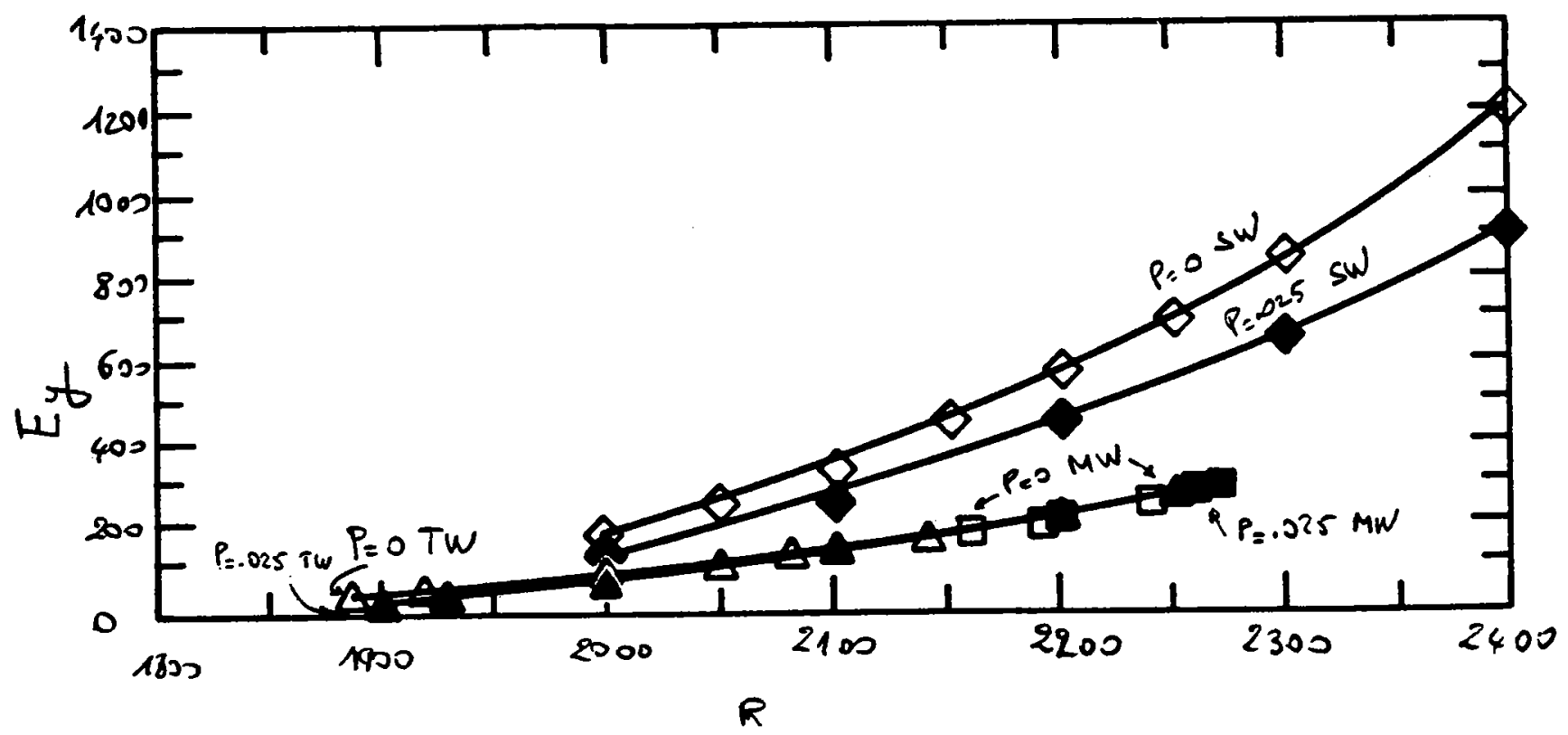

Figure 32.4. Bifurcation diagram of the traveling waves (TW) and standing waves (SW) competition, for no-slip boundary conditions, $P=0$ (white symbols) and $P=$ 0.025 (black symbols), with aspect ratios $k_{x}=3.177$ and $k_{y}=2.2$. Longitudinal energy $E_{y}=\left\langle v^{2}\right\rangle^{x y z}$ versus Rayleigh number $R$.

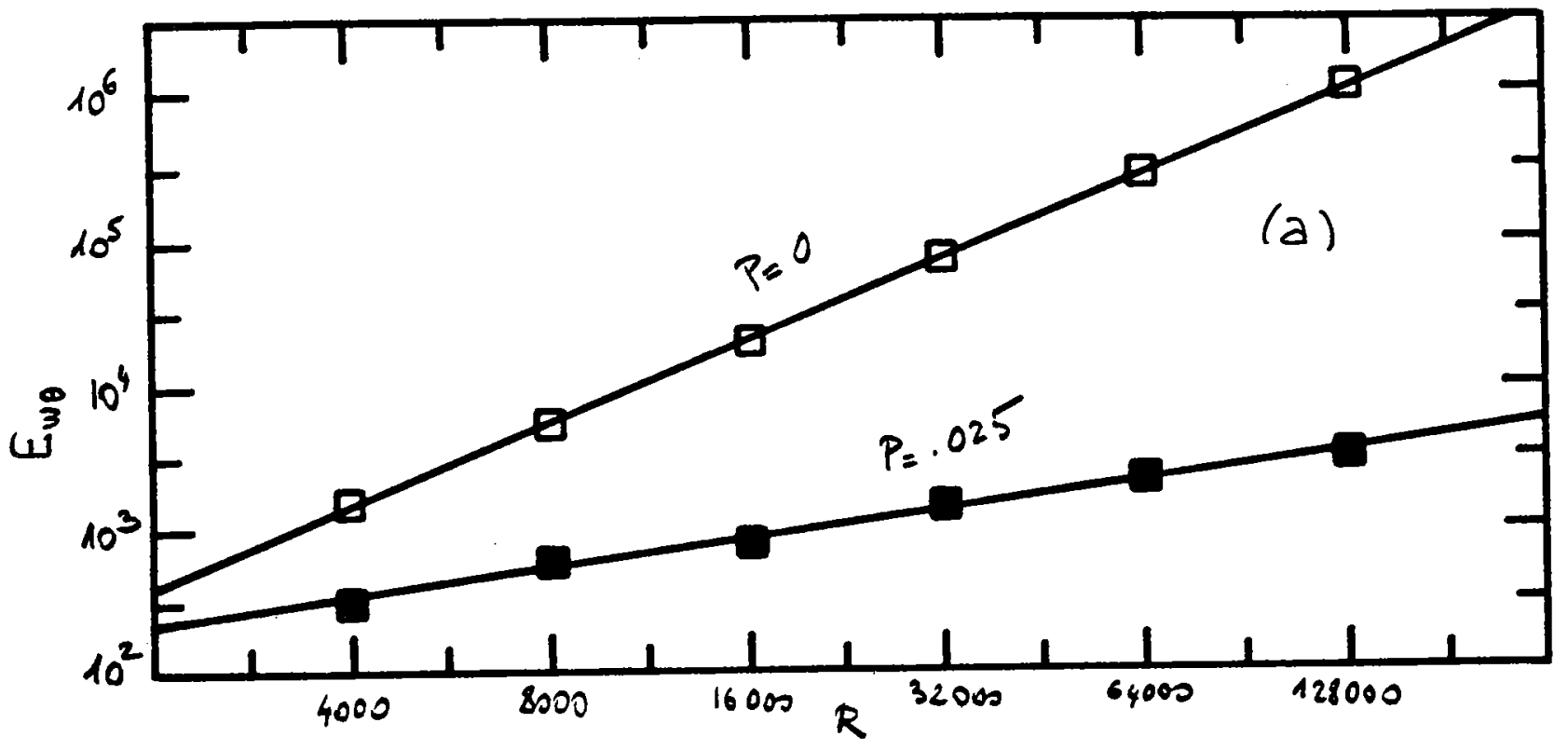

Figure 32.5. Scaling law of $E_{w \theta}=\langle w \theta\rangle^{x y z}$, for no-slip boundary conditions, $P=0$ (white symbols) and $P=0.025$ (black symbols), with $k_{x}=3.177$ and $k_{y}=2.2$. 


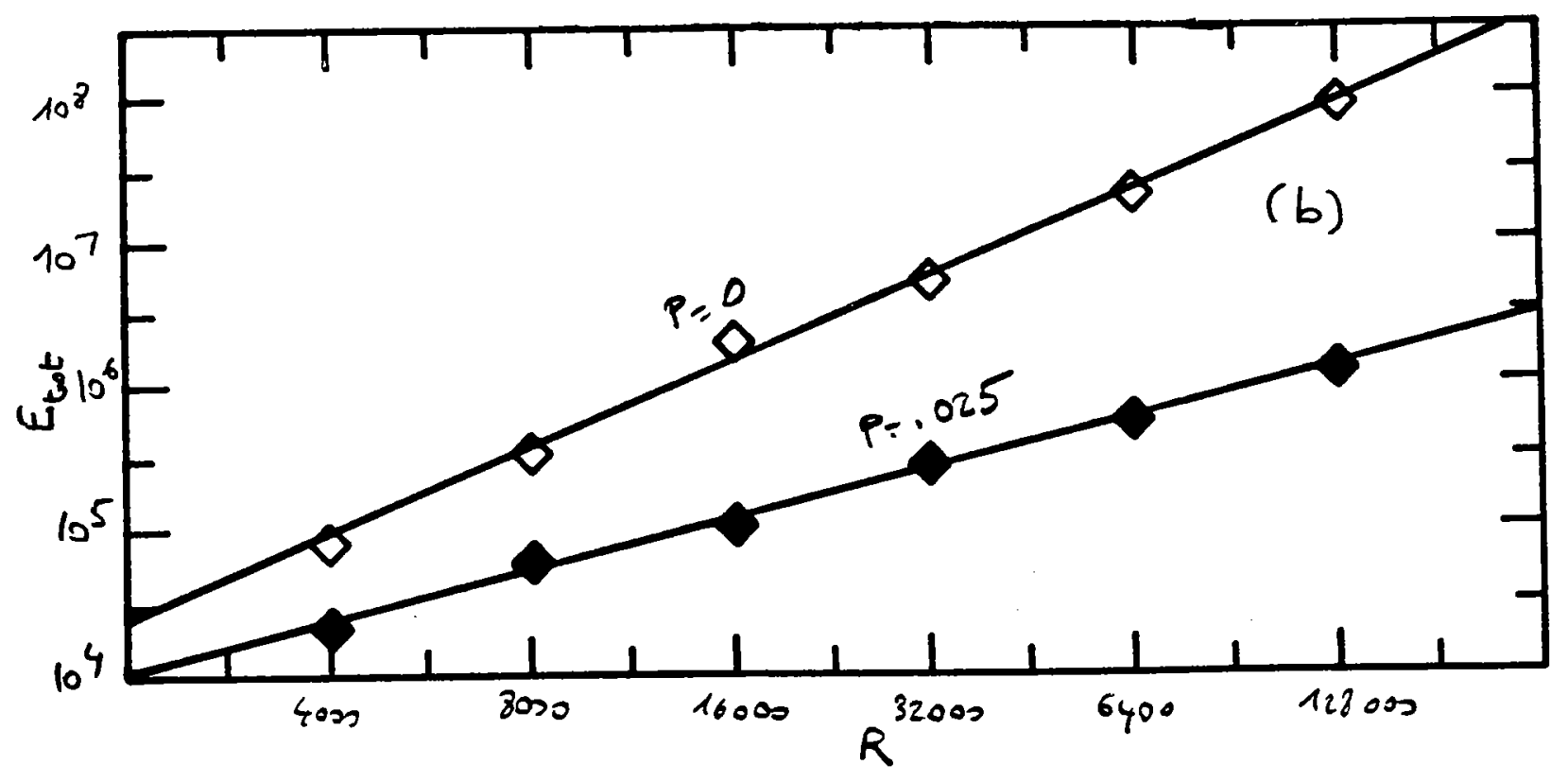

Figure 32.6. Parameters as in preceding figure, scaling law of $E_{\text {tot }}$ 


\title{
33. Instability, finite amplitude and unsteady salt fin- gers
}

\author{
G. Veronis \\ Geology and Geophysics Department, Yale University
}

An array of long, vertically uniform salt fingers in an environment with salt input from above, fresh input from below, a vertically constant, stabilizing temperature gradient and negligible salt diffusion is unstable to perturbations with vertical structure. The maximum growth rate and the form of the instability are derived for fingers with widths that yield maximum buoyancy flux in the unperturbed state. The dependance of the instability on the magnitude of the salt difference imposed over a given finite depth is obtained for the heat-salt system. A direct (non-oscillatory) mode with a vertical scale of the order of the buoyancy layer thickness is the most unstable when the amplitude of the vertical velocity of the fingers is large. The instability is due to the shear flow between rising and sinking fluid in adjacent fingers and is relatively unaffected by the perturbation buoyancy. When the driving is weaker, the dominant instability involves the same processes as the basic fingers do, i.e., perturbation buoyancy, viscosity and diffusion, and the mode becomes oscillatory in time. All of the most unstable modes have a vertical scale of the order of the buoyancy layer thickness. Both the direct and the oscillatory modes have net horizontal flows that vary with the vertical coordinate and time and in finite amplitude could cause the fingers to incline toward the horizontal. The oscillatory mode involves pairs of fingers so the emerging behavior could include a kind of period doubling.

Laboratory experiments of salt-sugar fingers evolving from an initially two-layer configuration in a Hele Shaw cell indicate that the fingers are initially unsteady and somewhat irregular but eventually settle to long relatively stable features that lengthen and widen as the mean stabilizing gradient diminishes. The increase of width appears to be due to the penetration into the finger zone of wider fingers which are generated in the outer regions of the finger zone.

Quasi-steady, regular fingers, obtained in a numerical simulation of the heat-salt case, alter the mean salinity gradient which is positive ( ) only in the middle quarter of the salt finger zone. Recirculation occurs in the upper and lower $3 / 8$ of the finger zone where the salinity contours have umbrella-like shapes which reflect the accumulation of salinity anomalies at the "ends" of the fingers. Noise superimposed on the system leads to an instability which appears to be due to intrusions of alternate fingers into the reservoirs. This instablity is completely different from the one obtained by perturbing the long fingers as described in the first paragraph. 


\title{
34. The Efficiency of Vortex Merger
}

\author{
Darryn W. Waugh \& David G. Dritschel \\ DAMTP, University of Cambridge, England, CB3 9EW
}

In simulations of two-dimensional turbulence, coherent vortex structures are observed to emerge spontaneously and dominant the evolution of the flow (see for example Benzi et. al. 1989, McWilliams 1990 \& refs.). Understanding the interactions of these coherent vortices is therefore important in understanding turbulence itself. In this presentation we look at, perhaps, the most fundamental of these vortex interactions, the coalescence of two structures with like-signed vorticity (so called vortex merger).

The results of a detailed, quantitative investigation into the coalescence of two circular vortices with uniform vorticity in two-dimensional inviscid, incompressible vortex dynamics are discussed. High resolution simulations using the numerical method of 'contour surgery' (Dritschel 1989 \& refs) show that the evolution of the vortices may be classified into different regimes. The efficiency of the merger of two vortices is determined by calculating the loss of globally-conserved quantities to filaments and small scale structures.

In the case of identical vortices (symmetric merger, see Melander at al. (1988) \& refs) the evolution is classified into three regimes depending on the initial intercentroid separation. For initial separation less than 3.3 radii the two vortices merge together to form a single elliptical-like coherent vortex. During this merger process filaments are ejected from the vortices and circulation is lost to small scale structures. For initial separations near the upper limit of this regime the evolution is in two stages. In the first stage the vortices merge to form a near elliptical core, with very few filaments. This elongated core is unstable, and in the second stage filaments are shed as the eccentricity of the vortex decreases until a stable vortex is formed. For initial separation between 3.3 and 3.45 radii a second regime occurs in which the vortices merge, exchange fluid and then separate to form two new vortices. These new vortices may reconnect several times but after sufficient exchanges the separation of the vortices is such that they will not come in contact with each other. In the third, and final, regime (separation greater than 3.45 radii) the two vortices rotate about their centre of vorticity without coming in contact.

Using the surgical section of the contour surgery algorithm fine scale structures are removed from the resultant vorticity distribution, and the aspect ratio and globally conserved quantities of the central vortex are determined. The resultant central vortex is non-circular (with aspect ratio greater than 1.5) and has at least $56 \%$ more circulation than either of the initial 
vortices, showing that the merger process forms larger scales. On the other hand, the angular momentum of the resultant coherent vortex is only between 30 and $60 \%$ of the original vortices. Hence, although very little circulation is transferred to filamentary vorticity there is a large transfer of angular momentum. The loss of globally conserved quantities during the second regime is very small (two orders of magnitude less than the merger regime).

Analytical calculations of the energetics of two identical elliptical vortices show that an inviscid transition from two identical elliptical vortices to a single elliptical vortex is possible, and the separation of the two vortices is very close to the critical merger separation obtained from numerical calculations.

The evolution of two circular vortices with unequal radii (but the same uniform vorticity) is more varied than that of identical vortices. The evolution is classified into five different regimes depending on both the initial intercentroid separation and the vortex size ratio. In general, the coalescence of the two vortices produces two vortices, one at least as large as the the largest original vortex and one smaller than (or equal to) the smallest original vortex.

For large initial separation (with distance between inner edges of the vortices greater than, approximately, 1.4 times the radii of the larger vortex) the resultant coherent vortices are the same size as the original vortices. For closer initial separation, vorticity is removed from the smaller original vortex. If the ratio of initial vortex sizes is large, the larger vortex tears away part of (partial straining-out regime), or all of (complete straining-out), the smaller vortex without gaining vorticity. Hence the close interaction of a large vortex with a smaller vortex does not cause vortex growth. For two more evenly matched vortices there is, however, vortex growth. The vorticity removed from the smaller vortex is incorporated into the larger vortex and the largest resultant vortex is larger than either original vortices (partial merger). As the initial separation of the vortices is decreased the smaller vortex losses more vorticity, and if the vortices are sufficiently close the vortices merge into a single coherent vortex (complete merger).

These efficiency results enable a simple parametrization of the coalesce of two like-signed vortices. This can then be incorporated into a non-conservative point vortex model of twodimensional turbulence similar to that developed by Carnevale et al. (1991).

A more detailed discussion of the above results can be found in Waugh (1991) and Dritschel \& Waugh (1991). 


\section{References}

Benzi R., Patarnello S. \& Santangelo P. (1988) Self-similar, coherent structures in 2D, decaying turbulence. J. Phys. A: Math. Gen. 21, 1221-1237.

Carnevale G.F., McWilliams J.C., Pomeau Y., Weiss J.B. \& Young W.R. (1991) Evolution of vortex statistics in two-dimensional turbulence, Phys. Rev. Lett. 66, 2735-2737.

Dritschel D.G. (1989) Contour dynamics and contour surgery: Numerical algorithms for extended, high-resolution modelling of vortex dynamics in two-dimensional, inviscid, incompressible flows. Comp. Phys. Rep.. 10, 77-146.

Dritschel D.G. \& Waugh D.W. (1991) A simple model for vortex growth in two-dimensional turbulence (in preparation).

McWilliams, J.C. (1990) The vortices of two-dimensional turbulence. J. Fluid Mech. 219, 361-385.

Melander M.V., Zabusky N.J. \& McWilliams J.C. (1988) Symmetric vortex merger in two dimensions: causes and conditions. J. Fluid Mech. 195 303-340.

Waugh D.W. (1991). The efficiency of symmetric vortex merger. J. Fluid Mech. (submitted). 


\section{2D Decaying Structured Turbulence}

Jeffrey B. Weiss

NCAR, P.O. Box 3000, Boulder, CO 80307

Numerical simulations of two-dimensional decaying turbulence starting from random initial conditions exhibit self-organization of the fluid into coherent vortices. The subsequent evolution of the fluid is dominated by two processes: 1 ) the nearly conservative mutual advection of the vortices, and 2) the merger of same-sign vortices during close approach, accompanied by the dissipation of enstrophy. It is found that the energy of the fluid and peak vorticity of the vortices are conserved as the fluid evolves, while other properties evolve algebraically with various exponents. In this lecture we describe a scaling theory and a point vortex model, both of which give results in good agreement with numerical simulations of decaying turbulence (Carnevale, et.al., 1991).

A scaling theory can be constructed which predicts the evolution of the various vortex and fluid properties. The theory assumes that energy and peak vorticity are conserved, and that the vortex number decays as $t^{-\xi}$. Turbulence simulations show $\xi \approx 0.75$. The scaling theory then predicts the exponents for other properties in terms of $\xi$. These exponents are in agreement with those found in turbulence simulations.

A model of two-dimensional decaying structured turbulence is constructed in terms of point vortices, with each coherent vortex represented by a single point vortex. The model evolves according to the Hamiltonian dynamics of point vortices until two same-sign vortices come close together. The two close same-sign vortices are then merged, forming a new vortex, according to merger rules which conserve self-energy and peak vorticity. The critical merger distance used is that found in the second order moment model, in which vortices are represented as ellipses. Through this punctuated Hamiltonian dynamics the system evolves, decreasing the number of vortices, and increasing their size. The exponents obtained match both the scaling theory and turbulence simulations. Through a renormalization procedure based on scaling theory, a configuration with few vortices can be used to create a new configuration with many vortices, which is then allowed to decay. By successive renormalizations, the asymptotic evolution of the collection of vortices can be studied. It is found that the distribution of vortex sizes reaches an asymptotic distribution which exhibits self-similar evolution. 


\section{References}

G.F. Carnevale, J.C. McWilliams, Y. Pomeau, J.B. Weiss, and W.R. Young, Phys. Rev. Lett., 66, 2735, (1991). 


\title{
36. The Hydraulics of Stratified and Planetary Flows
}

\author{
Andrew W. Woods \\ Institute of Theoretical Geophysics, DAMTP, Cambridge, England, CB3 9EW
}

In this presentation, the hydraulics of stratified channel flow over topography and through a constriction were discussed. The presentation commenced with a discussion of single layer hydraulics, identifying some of the basic concepts of sub and super critical flow, hydraulic control flow and hydraulic jumps from super to sub critical flow downstream of a control. The analysis was then generalised to consider multi-layer hydraulics, following Wood (1968) and Armi (1986), and it was shown that similar control phenomena arise in a two-layer system. We focused upon the case where the controls are relative to the propagation of interfacial waves, so that the depth of the whole system remained constant.

We then considered continuously stratified fluid flowing along a channel. We discussed the flows which preserve their shape as they pass through a contraction, as originally discussed by Wood (1968) and Benjamin (1981). We showed that these shape preserving flows can become critical with respect to the slowest internal mode of the system at the minimum width of the contraction, but pass through a sequence of virtual controls upstream of this point; at each of these points the flow becomes critical to faster propagating internal modes. Therefore, on leaving such a contraction, the flow will tend to bifurcate from this shape-preserving solution and remains super-critical to a number of the slower internal modes.

We then developed a simple analogy between the flow of a stratified fluid along a channel and the motion of a zonal current upon an equatorial $\beta$-plane, following Ball(1954). We showed that the variation of the earth's rotation with latitude exerts a force upon a zonal planetary current analogous to that of gravity upon a stratified fluid, in the particular case in which the density is a linear function of the streamfunction (Woods, 1991).

By applying this analogy, we then developed a theory for the hydraulic control of planetary scale zonal flows propagating to the east over topography. Using the earlier results obtained for the flow of stratified fluid through a contraction, we showed that on passing over topography, an inertial zonal current on an equatorial $\beta$-plane may pass through a control at which the flow changes from a sub-critical to a super-critical solution branch, critical to the slowest internal mode of the system. The internal modes correspond to planetary waves rather than gravity waves. As the flow passes through the point of minimum depth, the solution may bifurcate from the self-similar solution, and remain supercritical to some of the lowest internal modes of the system. Donwstream of this point, the flow may pass through a transition back to the sub-critical solution branch; this is effected through the generation 
of radiating planetary waves or planetary eddies. We discussed the relevance of this simple, phenomenological theory for a number of atmospheric and oceanic phenomena. We also showed that the self-similar flow solutions on a beta-plane may be simply derived by using the conservation of potential vorticity. Further details are given by Woods (1991).

\section{References}

Armi, L., 1986, The hydraulics of two flowing layers of different density, J. Fluid Mech., 163, 27-58.

Ball, R.K., 1954, Long waves, lee waves and gravity waves, Q.J.R.M.S., 85, 24-30.

Benjamin, T.B., 1981, Steady flows drawn from a stably stratified reservoir, J. Fluid Mech., 106, 245-260.

Wood, I., 1968, Selective withdrawal from a stably stratified reservoir, J. Fluid Mech., 32, 209-223.

Woods, A.W., 1991, The topographic control of planetary scale flows by analogy with a stratified fluid, sub-judice. 


\title{
37. Bioconvection and Photoelutriation
}

\author{
E.A. Spiegel \\ Dept. Astronomy, Columbia University
}

Certain microorganisms exhibit negative geotaxis, that is, they swim upwards. Since they are somewhat denser than the ambient soup, they thus cause a density inversion. The ensuing instability leads to the formation of interesting patterns. For slightly unstable conditions, amplitude equations can be develoned and these indicate that the corresponding bifurcation is subcritical. This is connected to the nonBoussinesq character of this problem. One of the simplrest patterns that arises is the polka dot pattern in which round spots array themselves in a regular manner. The nonlinear development of the spot strucuture can be studied as a dynamical extension of the Stommel problem in which particles in a convecting fluid can be levitated by the flow. The added bioconvective ingredient is the swimming. This work is described in a paper with S. Childress, submitted to J.F.M. (1978).

This work has also inspired an astrophysical analogue in which the heavier particles that move upward are ions that are forced upward through the ambient fluid by radiation pressure resulting from a strong resonance line. This time, the accumulation of impurity near the top does not produce a density inversion but it does reshape the radiative levitation and leads to an instability like that of bioconvection. This work has been featured in a thesis by $\mathrm{J}$. Lin and a paper with him is in preparation. 


\section{DOCUMENT LIBRARY}

March 11, 1991

\section{Distribution List for Technical Report Exchange}

Attn: Stella Sanchez-Wade

Documents Section

Scripps Institution of Oceanography

Library, Mail Code C-075C

La Jolla, CA 92093

Hancock Library of Biology \&

Oceanography

Alan Hancock Laboratory

University of Southern California

University Park

Los Angeles, CA 90089-0371

Gifts \& Exchanges

Library

Bedford Institute of Oceanography

P.O. Box 1006

Dartmouth, NS, B2Y 4A2, CANADA

Office of the International

Ice Patrol

c/o Coast Guard R \& D Center

Avery Point

Groton, CT 06340

NOAA/EDIS Miami Library Center

4301 Rickenbacker Causeway

Miami, FL 33149

Library

Skidaway Institute of Oceanography

P.O. Box 13687

Savannah, GA 31416

Institute of Geophysics

University of Hawaii

Library Room 252

2525 Correa Road

Honolulu, HI 96822

Marine Resources Information Center

Building E38-320

MIT

Cambridge, MA 02139

Library

Lamont-Doherty Geological

Observatory

Columbia University

Palisades, NY 10964

Library

Serials Department

Oregon State University

Corvallis, OR 97331
Pell Marine Science Library

University of Rhode Island

Narragansett Bay Campus

Narragansett, RI 02882

Working Collection

Texas A\&M University

Dept. of Oceanography

College Station, TX 77843

Library

Virginia Institute of Marine Science

Gloucester Point, VA 23062

Fisheries-Oceanography Library

151 Oceanography Teaching Bldg.

University of Washington

Seattle, WA 98195

Library

R.S.M.A.S.

University of Miami

4600 Rickenbacker Causeway

Miami, FL 33149

Maury Oceanographic Library

Naval Oceanographic Office

Stennis Space Center

NSTL, MS 39522-5001

Marine Sciences Collection

Mayaguez Campus Library

University of Puerto Rico

Mayaguez, Puerto Rico 00708

Library

Institute of Oceanographic Sciences

Deacon Laboratory

Wormley, Godalming

Surrey GU8 5UB

UNITED KINGDOM

The Librarian

CSIRO Marine Laboratories

G.P.O. Box 1538

Hobart, Tasmania

AUSTRALIA 7001

Library

Proudman Oceanographic Laboratory

Bidston Observatory

Birkenhead

Merseyside L43 7 RA

UNITED KINGDOM 


\begin{tabular}{|c|c|c|}
\hline $\begin{array}{l}\text { REPORT DOCUMENTATION } \\
\text { PAGE }\end{array}$ & $\begin{array}{l}\text { 1. REPOAT NO. } \\
\qquad \text { WHOI-92-16 }\end{array}$ & 3. Recipient's Accession No. \\
\hline \multirow{2}{*}{\multicolumn{2}{|c|}{$\begin{array}{l}\text { 4. Title and Subttle } \\
1991 \text { Summer Study Program in Geophysical Fluid Dynamics - Patterns in } \\
\text { Fluid Flow }\end{array}$}} & $\begin{array}{l}\text { 5. Report Date } \\
\text { September } 1991\end{array}$ \\
\hline & & 6. \\
\hline \multicolumn{2}{|c|}{ 7. Author(s) Steve Meacham, Editor } & $\begin{array}{l}\text { 8. Performing Organization Rept. No. } \\
\text { WHOI-92-16 }\end{array}$ \\
\hline \multirow{2}{*}{\multicolumn{2}{|c|}{$\begin{array}{l}\text { 9. Performing Organization Name and Address } \\
\text { Woods Hole Oceanographic Institution } \\
\text { Woods Hole, Massachusetts } 02543\end{array}$}} & 10. Project/Task/Work Unit No. \\
\hline & & $\begin{array}{l}\text { 11. Contract(C) or Grant(G) No. } \\
\text { (C) OCE } 8901012 \\
\text { (G) }\end{array}$ \\
\hline \multicolumn{2}{|c|}{ 12. Sponsoring Organization Name and Address } & $\begin{array}{l}\text { 13. Type of Report \& Period Covered } \\
\text { Technical Report }\end{array}$ \\
\hline & & 14. \\
\hline
\end{tabular}

15. Supplementary Notes

This report should be cited as: Woods Hole Oceanog. Inst. Tech. Rept., WHOI-92-16.

16. Abstract (Limit: 200 words)
The GFD program in 1991 focused on pattern forming processes in physics and geophysics. The principal lecturer, Stephan Fauve, discussed a variety of systems, including our old favorite, Rayleigh-Bénard convection, but passing on to exotic examples such as vertically vibrated granular layers. Fauve's lectures emphasize a unified theoretical viewpoint based on symmetry arguments. Patterns produced by instabilities can be described by amplitude equations, whose form can be deduced by symmetry arguments, rather than the asymptotic expansions that have been the staple of past Summer GFD Programs. The amplitude equations are far simpler than the complete equations of motion, and symmetry arguments are easier than asymptotic expansions. Symmetry arguments also explain why diverse systems are often described by the same amplitude equation. Even for granular layers, where there is not a universally accepted continuum description, the appropriate amplitude equation can often be found using symmetry arguments and then compared with experiment.

Our second speaker, Daniel Rothman, surveyed the state of the art in lattice gas computations. His lectures illustrate the great utility of these methods in simulating the flow of complex multiphase fluids, particularly at low Reynolds numbers. The lattice gas simulations reveal a complicated phenomenology much of which awaits analytic exploration.

The fellowship lectures cover broad ground and reflect the interests of the staff members associated with the program. They range from the formation of sand dunes, through the theory of lattice gases, and on to two dimensional-turbulence and convection on planetary scales. Readers desiring to quote from these reports should seek the permission of the authors (a partial list of electronic mail addresses is included on page v). As in previous years, these reports are extensively reworked for publication or appear as chapters in doctoral theses. The task of assembling the volume in 1991 was at first facilitated by our newly acquired computers, only to be complicated by hurricane Bob which severed electric power to Walsh Cottage in the final hectic days of the Summer.

17. Document Analysis a. Descriptors

pattern formation

convection

dynamical system

b. Identifiers/Open-Ended Terms

c. COSATI Field/Group

18. Availability Statement

Approved for public release; distribution unlimited.

\begin{tabular}{|c|}
$\begin{array}{c}\text { 19. Security Class (This Report) } \\
\text { UNCLASSIFIED }\end{array}$ \\
\hline 20. Security Class (This Page)
\end{tabular}

21. No. of Pages

22. Price 


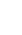

